\title{
Les configurations familiales aux dernières étapes de la vie
}

Myriam Girardin

\section{ㄴ.. PETER LANG}




\section{Myriam Girardin}

\section{Les configurations familiales aux dernières étapes de la vie}

Cet ouvrage se base sur l'approche configurationnelle pour étudier les familles dans la vieillesse. Cette approche conçoit les familles comme des configurations, à savoir des réseaux formés de membres significatifs de la famille qui, par les ressources qu'ils s'échangent, sont dépendants les uns des autres. Les analyses révèlent une diversité de configurations familiales. Celles-ci se distinguent dans leur composition et dans le type de capital social qu'elles génèrent ainsi que dans leur gestion de l'ambivalence familiale. En somme, la diversité et la complexité caractérisent les liens familiaux dans la vieillesse. Ce constat nuance l'image homogène et positive de la famille longtemps défendue dans les modèles dominants de la gérontologie sociale.

Myriam Girardin est titulaire d'un doctorat en sociologie et travaille comme sociologue de la famille au Centre Interfacultaire de Gérontologie et d'Études des Vulnérabilités de l'Université de Genève. Ses domaines de recherche sont les réseaux familiaux dans la vieillesse, leur dynamique, les conflits et l'ambivalence qui les caractérisent. 
Les configurations familiales aux dernières étapes de la vie 


\section{Population, Family, and Society Population, Famille et Société}

\section{Volume 29}

Edited by / Edité par

Michel Oris

Editorial Board / Comité de lecture

Guy Brunet (Démographie historique, Université Lyon II)

Martin Dribe (Economic History, Lund University)

Georg Fertig (Geschichte, Universität Münster)

Vincent Gourdon (Histoire, Université de Paris-Sorbonne)

Matteo Manfredini (Biology and Demography, Università degli Studi di Parma)

Jon Mathieu (Geschichte, Universität Luzern)

Muriel Neven (Histoire, Université de Liège)

Emiko Ochiai (Sociology, Kyoto University)

Diego Ramiro Farinas (Demography, Spanish National Research Council)

Gilbert Ritschard (Econométrie, Université de Genève)

Clémentine Rossier (Démographie, Université de Genève)

Paul Servais (Histoire, Université catholique de Louvain-la-Neuve)

Frans van Poppel (Demography, The Hague)

Philippe Wanner (Démographie, Université de Genève)

Eric D. Widmer (Sociologie, Université de Genève)

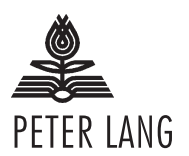

Bern • Berlin • Bruxelles • New York • Oxford - Wien 
Myriam Girardin

\section{Les configurations familiales aux dernières étapes de la vie}

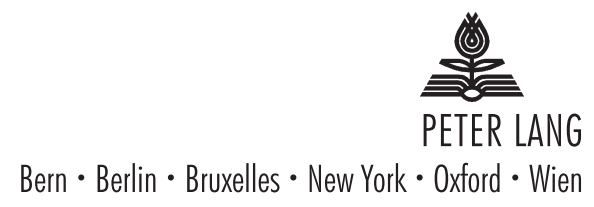




\section{Information bibliographique publiée par « Die Deutsche Nationalbibliothek »}

« Die Deutsche Nationalbibliothek » répertorie cette publication

dans la « Deutsche Nationalbibliografie »; les données bibliographiques

détaillées sont disponibles sur Internet sous

$\langle$ http ://dnb.d-nb.de .

L'étape de la prépresse de cette publication a été soutenue par le Fonds national suisse de la recherche scientifique.

Cette publication a également reçu le soutien du Fonds Rappard de la Faculté des Sciences de la Société de

I'Université de Genève.

Illustration de la couverture : Photo de ma grand-mère, Ida Hêche.

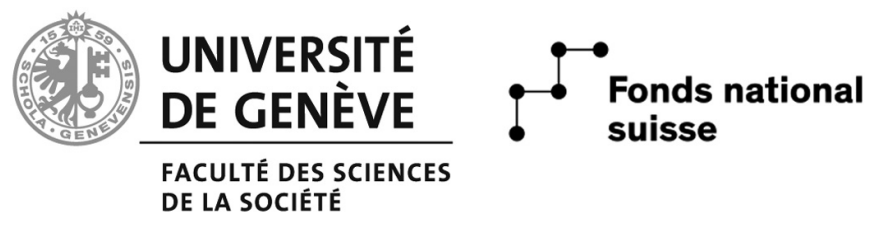

ISSN 1660-6043

ISBN 978-3-0343-3200-2 (Print)

E-ISBN 978-3-0343-3197-5 (E-Book)

E-ISBN 978-3-0343-3198-2 (EPUB)

$10.3726 / \mathrm{b} 12273$

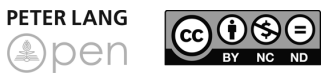

Open Access : Cette oeuvre est mise à disposition selon les termes de la Licence Creative Commons Attribution - Pas d'Utilisation

Commerciale - Pas de Modification 4.0. Pour consulter une copie de cette licence, visitez le site internet

https ://creativecommons.org/licenses/by-nc-nd/4.0/

Cette publication a fait l'objet d'une évaluation par les pairs.

(C) Myriam Girardin, 2022

Peterlang SA Editions Scientifiques internationales, Berne bern@peterlang.com, www.peterlang.com 
A ma grand-maman, Ida, née le 29 octobre 1915 à Cornol, Jura 



\section{Avant-propos}

La notion du «convoi », bien connue en gérontologie sociale, se réfère à l'ensemble des membres d'un réseau personnel qui accompagnent l'individu au cours de son parcours de vie et qui, par le support qu'ils lui apportent, l'aident à traverser au mieux les différentes étapes de sa vie. La trajectoire du doctorant ressemble à bien des égards au parcours de vie avec ses nombreuses étapes et ses événements-clé. Ayant terminé mon parcours de doctorante, je souhaite remercier chaleureusement tous les membres de mon « convoi » qui m'ont soutenue tout au long de cette grande aventure et qui m'ont permis d'arriver au bout de ma recherche et d'aboutir à ce présent ouvrage!

Je remercie plus particulièrement Eric Widmer, mon directeur de thèse, qui m'a offert la possibilité de participer à la recherche " Vivre/ Leben / Vivere » (VLV) et de réaliser mon doctorat. Je le remercie pour son soutien constant, son amitié et sa passion de la recherche qui m'ont donné l'envie et la motivation d'aller plus loin et de finaliser ce projet. A toutes les étapes de mon parcours, j'ai apprécié sa disponibilité, son écoute, son grand intérêt pour mon sujet de thèse ainsi que la justesse et la finesse de ses analyses. Je remercie également Michel Oris de m'avoir intégrée à l'équipe du CIGEV [Centre Interfacultaire de Gérontologie et d'Études des Vulnérabilités de l'Université de Genève] en charge de l'étude «VLV ». Grâce à son énergie et son investissement dans cette vaste enquête interdisciplinaire, nous avons, toutes et tous, eu accès à une base de données solide et originale qui nous a permis de produire des résultats intéressants, base sur laquelle reposent de nombreux travaux de recherche réalisés au sein du CIGEV. Je le remercie aussi pour son soutien, son accueil toujours chaleureux et l'infrastructure dont j'ai bénéficié au CIGEV.

Par ailleurs, l'étude «VLV» a reçu le soutien du pôle de recherche national LIVES - «Overcoming Vulnerability : Life Course Perspectives »et du programme Sinergia ( ${ }^{\circ}$ CRSII1_129922/1), tous deux financés par le Fonds national suisse de la recherche scientifique. Cette étude a également bénéficié de l'appui financier de Pro Senectute Suisse. Je les remercie pour leur soutien sans lequel cette vaste enquête interdisciplinaire n'aurait pas pu avoir lieu. 
Je remercie également Anna-Maija Castrén et Jean-François Bickel d'avoir accepté de faire partie de mon jury de thèse et qui ont, par leurs suggestions et commentaires, contribué à améliorer et compléter mon manuscrit de thèse. Experts dans les configurations familiales (Anna-Maija Castrén), en gérontologie sociale et en politiques sociales (Jean-François Bickel), j'ai été particulièrement ravie et honorée de leur présence au sein de mon jury de thèse.

Les résultats présentés dans cet ouvrage ont donné lieu à plusieurs articles scientifiques et ceux-ci sont à la base de mon travail de thèse. Je remercie Eric Widmer qui a initié et co-écrit l'ensemble de ces articles mais aussi Ingrid Connidis, Anna-Maija Castrén, Rita Gouveia et Barbara Masotti qui ont participé activement à l'article portant sur l'ambivalence familiale dans la vieillesse ainsi que Catherine Ludwig pour sa collaboration à l'article sur les conflits familiaux et la santé des personnes âgées. Par ailleurs, je souhaite aussi remercier Emmanuel Rousseaux pour sa disponibilité, sa fiabilité et son soutien pour tout ce qui concerne R et LateX. Son aide m'a été très précieuse au cours de ces années de thèse et je lui en suis très reconnaissante. Je tiens aussi à remercier tous mes collègues du CIGEV qui ont collaboré de loin ou de près à l'étude «VLV ». Merci à Aline, Alessandra, Aude M., Aude T., Barbara, Catherine, Claire, Delphine, Eduardo, Greg, Julia H., Julia S., Laure, Laurie, Marie, Marjorie, Marthe, Nathalie, Oana, Nora, Rainer, Sylvie et Stefano pour leur travail appliqué, leur persévérance et leur soutien. Un grand merci aussi à mes collègues d'Uni-Mail, et plus particulièrement à Jana, Manuela et Rita ainsi qu'à Marie-Eve, Olga, Sabrina, mes collègues de l'Observatoire des familles de l'Université de Genève, qui m'ont énormément soutenue lors de cette dernière année de thèse. Finalement, je souhaiterais remercier Stéphane, mon compagnon, ma famille et mes amis qui n'ont jamais cessé de m'encourager! Et finalement, j'adresse mes derniers remerciements à Ida, ma grand-mère, née en 1915, qui m'a accompagnée tout au long de ce travail de recherche et à qui je dédie cet ouvrage. 


\section{Table des matières}

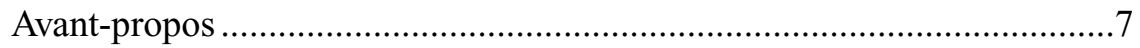

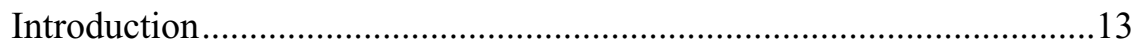

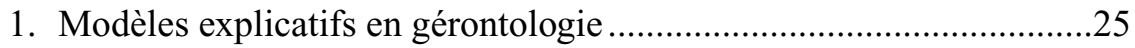

1.1 Les réseaux personnels et le modèle du convoi des relations

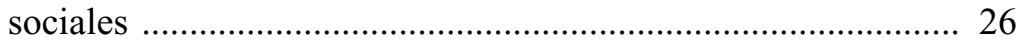

1.1.1 Le modèle du convoi des relations sociales .....................27

1.1.2 Les limites du modèle du convoi des relations sociales.....31

1.2 Les relations intergénérationnelles et le modèle de la solidarité intergénérationnelle ........................................................... 33

1.2.1 Le modèle de la solidarité intergénérationnelle .................36

1.2.2 Les limites du modèle de la solidarité

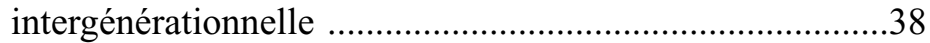

1.3 Les normes de réciprocité et le « Support bank » ................... 41

1.3.1 la réciprocité équilibrée versus généralisée .......................43

1.3.2 La banque de support (« support bank») .........................45

1.3.3 Les limites de l'approche dyadique de la réciprocité et

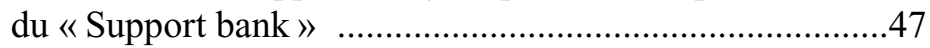

1.4 La sélection comme mode d'adaptation dans la vieillesse ....... 48

1.4.1 La sélection au cours du parcours de vie ..........................49

1.4.2 La théorie de la sélectivité socio-émotionnelle .................50

1.4.3 Les limites de la théorie de la sélectivité

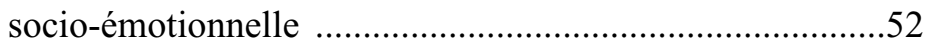

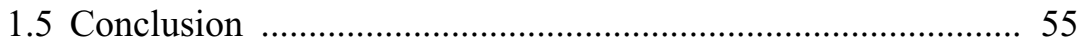

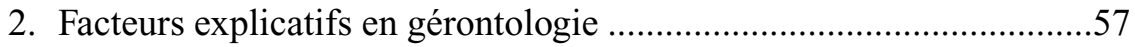

2.1 Le réservoir de parenté ......................................................... 57

2.1.1 L'évolution et la pluralité des réservoirs de parenté ..........59 
2.1.2 Les limites des indicateurs socio-démographiques ...........63

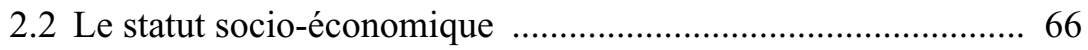

2.2.1 Des réseaux personnels différenciés ................................67

2.2.2 Des relations intergénérationnelles distinctes ...................68

2.2.3 Des questions restées en suspens dans la littérature ...........72

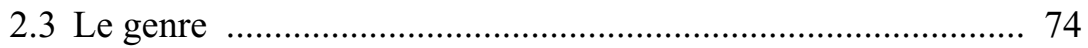

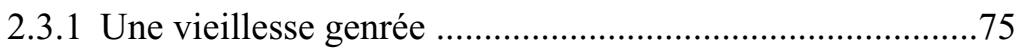

2.3.2 Les inégalités socio-économiques entre hommes et

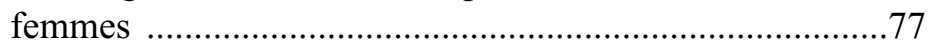

2.3.3 Les relations sociales et familiales selon le genre ..............81

2.3.4 De nouvelles dimensions à explorer dans la recherche .....86

2.4 L'âge et la santé ................................................................ 88

2.4.1 Les « jeunes-vieux » (65-74 ans) ...................................91

2.4.2 Les « vieux-vieux » (75-84 ans) ......................................93

2.4.3 Les « très-vieux » (85 ans et plus) ......................................96

2.4.4 Des aspects non abordés dans la littérature .......................98

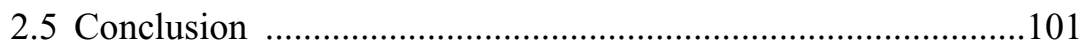

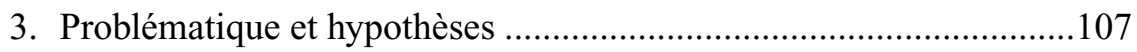

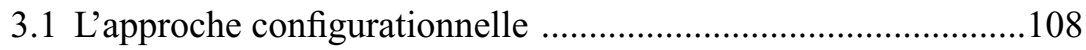

3.1.1 Les configurations d'interdépendances ..........................109

3.1.2 Les configurations familiales ......................................111

3.1.3 Les apports de l'approche configurationnelle .................113

3.2 Les membres significatifs de la famille dans la vieillesse ........123

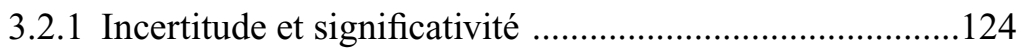

3.2.2 Une re-définition des liens familiaux et non familiaux ...129

3.2.3 L'influence du parcours de vie .........................................135

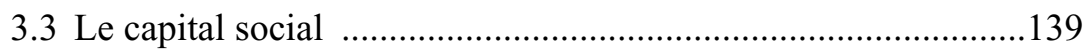

3.3.1 Le capital social et ses différents types ..........................140

3.3.2 La composition des configurations familiales ..................148

3.3.3 Le profil socio-démographique d'ego .............................156

3.4 Les conflits et l'ambivalence structurelle ...................................164

3.4.1 L’ambivalence intergénérationnelle ................................165 
3.4.2 L'ambivalence dans les configurations familiales ...........170

3.4.3 La typologie des modes de conflit et de support ..............174

3.4.4 Les conditions d'émergence des modes de conflit et de

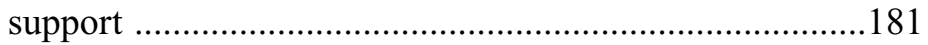

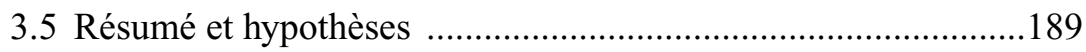

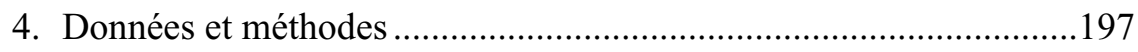

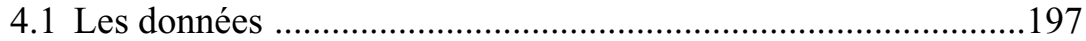

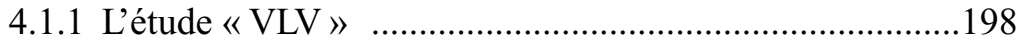

4.1.2 L'échantillon genevois et son contexte socio-historique .201

4.1.3 Genève et sa politique de la vieillesse .............................206

4.1.4 Description de l'échantillon genevois ............................211

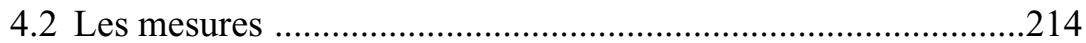

4.2.1 Les membres significatifs de la famille ..........................215

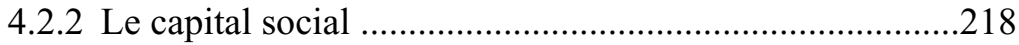

4.2.3 Les modes de conflit et de support .................................223

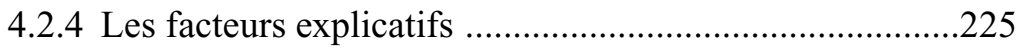

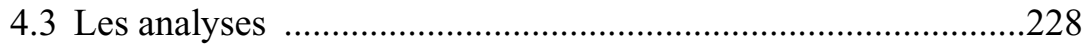

4.3.1 L'analyse de classification hiérarchique ........................228

4.3.2 L'analyse de variance (ANOVA) et la régression linéaire 232

4.3.3 L'analyse des correspondances multiples (ACM) ...........235

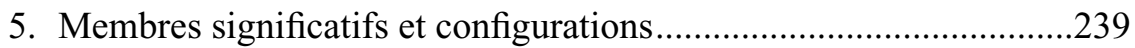

5.1 Les membres significatifs de la famille ..................................239

5.1.1 L'état du réservoir de parenté .......................................240

5.1.2 Le nombre et l'identité des membres significatifs de la

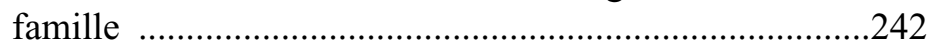

5.1.3 Les membres de la famille non cités ...............................246

5.1.4 Conclusion sur les membres significatifs de la famille ...247

5.2 La composition des configurations familiales ............................250

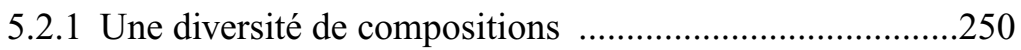

5.2.2 Les facteurs explicatifs de la composition ......................257

5.2.3 Le profil des membres des configurations .......................264 
Table des matières

5.2.4 Conclusion sur la composition des configurations familiales .270

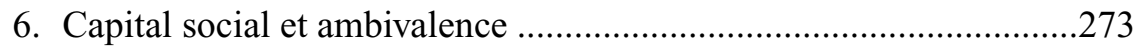

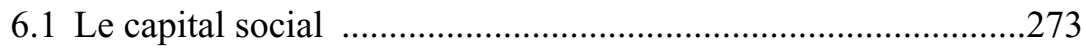

6.1.1 Le capital social dans les configurations familiales ........275

6.1.2 Le capital social selon le profil des répondants ...............283

6.1.3 Le capital social selon le réservoir de parenté .................289

6.1.4 Les facteurs déterminants du capital social .....................294

6.1.5 Illustrations de types de capital social .............................305

6.1.6 Conclusion sur le capital social .......................................310

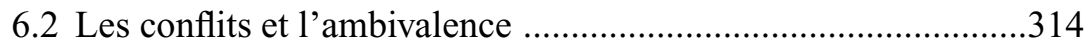

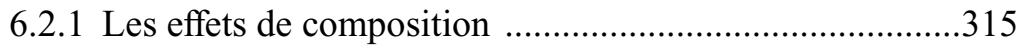

6.2.2 La prise en compte des ressources dans l'analyse ............318

6.2.3 Illustrations de modes de conflit et de support ................328

6.2.4 Conclusion sur les conflits et l'ambivalence ...................337

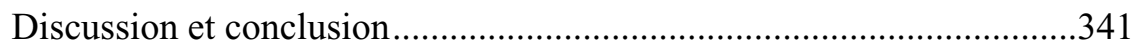

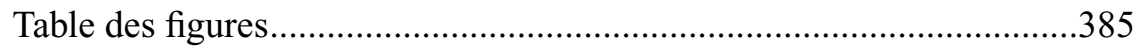

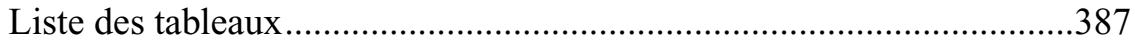

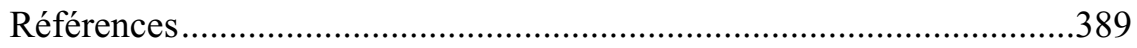




\section{Introduction}

Étudier les relations familiales des personnes âgées peut paraître a priori ennuyeux et moins «passionnant» que d'observer des problèmes de société plus médiatisés tels que la migration, la délinquance ou l'insertion des jeunes sur le marché de l'emploi. Nous montrons dans cet ouvrage que l'étude des individus âgés, et qui plus est dans le contexte familial, est un sujet fascinant et cela à plusieurs égards. Contrairement aux idées reçues, la vieillesse est loin d'être une phase de vie «tranquille» où le temps s'arrête et rien ne se passe. La vieillesse est au contraire une période pendant laquelle l'individu est bousculé, confronté à de nombreux changements auxquels il doit s'adapter. Face à ces changements, il doit se re-définir et ré-évaluer son rôle et sa place dans son contexte familial et social qui évolue rapidement. D'une part, sa mise en retraite l'astreint à repenser son rôle dans la société, son activité et son identité sociale. D'autre part, le départ de ses enfants et l'arrivée des petits-enfants impliquent des changements de rôles au sein de la famille, invitant la personne âgée à revoir sa position au sein de ses relations familiales.

Outre ce repositionnement dans le contexte familial et social, les dernières étapes de la vie se ponctuent d'événements négatifs tels que le veuvage, le décès des membres de la famille ou celui de ses amis qui amenuisent de manière drastique son potentiel relationnel. Par ailleurs, la vieillesse se caractérise aussi par le processus de fragilisation qui, inéluctable, aboutit au déclin de la santé et affecte de plein fouet l'aptitude à la réciprocité : l'individu fragilisé ne peut plus rendre ce qu'il reçoit, ce qui perturbe l'équilibre des échanges au sein de son réseau familial et amical. Hormis la capacité à la réciprocité, le processus de fragilisation menace aussi le maintien de l'autonomie - fonctionnelle mais aussi relationnelle - qui est l'un des enjeux les plus centraux aux dernières étapes de la vie. Sa perte bouscule les liens familiaux et place l'individu dans une situation de vulnérabilité. Celui-ci, fragilisé dans sa santé, devient dépendant de l'aide des autres, et notamment de celle de la famille. La peur de perdre son autonomie et de dépendre de l'aide familiale est source de stress chez les personnes âgées et cela d'autant plus que le contexte familial évolue 


\section{Introduction}

rapidement lors de la vieillesse en raison du décès des proches et des changements de rôles au sein de la famille. De plus, le soutien familial est devenu incertain compte tenu de la complexification des trajectoires de vie que connaissent les membres de la famille en termes de mobilité, de divorce ou encore d'instabilité professionnelle. Ce stress est d'autant plus marqué chez les personnes âgées qui ont de fortes attentes vis-à-vis des membres «proches» de leur famille, comme le partenaire ou les enfants, et sur lesquels elles comptent pour obtenir une aide "inconditionnelle» qu'elles estiment justifiée en raison des nombreuses ressources qu'elles leur ont octroyées par le passé. Ces attentes créent une forte pression sur les membres de la famille qui doivent s'organiser pour répondre au mieux aux demandes d'aide du parent âgé.

A cela s'ajoute l'imminence de la mort qui donne une valeur, un sens profond aux choses qui nous entourent, aux activités que l'on exerce et aux relations que l'on entretient, sachant que toutes se termineront dans un avenir proche. Le temps étant fortement limité, il ne peut être gaspillé. Par conséquent, les relations que l'on maintient aux dernières étapes de la vie sont celles qui font sens; elles sont empruntes d'une forte significativité et sont émotionnellement investies. Face à l'urgence de la fin proche, certaines personnes âgées parviennent à développer des stratégies d'adaptation, souvent non conscientes, qui leur permettent de maintenir un certain bien-être malgré les aléas de la vieillesse en s'entourant des membres de leur famille qu'elles jugent les plus importants, les plus significatifs. La vieillesse n'est donc pas une étape de vie de tout repos ni pour l'individu qui doit s'adapter ni pour les membres de sa famille qui l'entourent. Changements de rôles familiaux, décès des membres de la famille et des amis, déclin de la santé, aptitude à la réciprocité de plus en plus limitée, plus grande dépendance à l'aide familiale, attentes de soutien des membres proches de la famille, imminence de la mort et mécanismes individuels d'adaptation font de la vieillesse une période durant laquelle les liens familiaux sont bousculés tout en étant porteurs de fortes attentes et de significativité. La vieillesse constitue, en somme, un «laboratoire privilégié » des dynamiques familiales.

La «famille » est généralement peu étudiée en tant que telle en gérontologie sociale. Elle fait certes l'objet de nombreux travaux en gérontologie sociale mais elle est souvent abordée sous l'angle du support social, scrutée dès lors dans son aptitude à aider l'individu à s'adapter aux pertes 
auxquelles il doit faire face durant la vieillesse. Cette centration sur le soutien familial est devenue particulièrement marquante depuis les années 90 lorsque de nombreux chercheurs se sont interrogés sur la pérennité de la famille et sur sa capacité à offrir du soutien. Perçus comme les conséquences d'un individualisme exacerbé, les changements sociaux qui ont caractérisé les années 60 tels que l'augmentation du taux de divorce, la mobilité et l'émergence de nouvelles formes familiales (familles monoparentales, recomposées, etc.) ont souvent été interprétés tant dans la recherche que dans le public comme des signes avant-coureurs de la fin de la solidarité familiale (Popenoe, 1993). Face à cette incertitude, le soutien familial devint le cœur de nombreuses recherches en gérontologie sociale (Marshall, Matthews, \& Rosenthal, 1993; Silverstein \& Bengtson, 1997) et c'est dans ce contexte que de nombreux modèles théoriques se sont développés avec pour objectif de mieux comprendre les relations de soutien dans la vieillesse et leur impact sur le bien-être des individus âgés.

Le modèle du «convoi des relations sociales » (Antonucci, 1990, 2001; Kahn \& Antonucci, 1980), par exemple, considère la famille comme l'une des composantes structurelles d'un réseau personnel plus large. La famille constitue dans ce modèle le cœur du réseau d'aide, prometteur d'un support indéfectible et inconditionnel. Elle est perçue comme une «ressource » indispensable, visant à protéger les individus âgés de l'isolement social et à leur assurer tout le support dont ils ont besoin pour affronter les aléas de la vieillesse (Antonucci, 1990, 2001; Kahn \& Antonucci, 1980). D'autres modèles théoriques, comme le modèle de la «solidarité intergénérationnelle » (Bengtson \& Roberts, 1991 ; Roberts \& Bengtson, 1990) ou celui du «support bank» (Antonucci, 1990, 2001; Antonucci, Akiyama, \& Sherman, 2007; Antonucci \& Jackson, 1989), se sont focalisés sur la relation intergénérationnelle entre le parent âgé et l'enfant adulte qu'ils décrivent comme la dyade la plus centrale en matière de soutien. Ces modèles scrutent les fondements de la solidarité intergénérationnelle dans sa complexité (modèle de la "solidarité intergénérationnelle») et dans le temps (modèle du « support bank ») en cherchant à dégager les éléments qui la favorisent. Finalement, le modèle de la "sélectivité socio-émotionnelle» (Carstensen, 1991, 1992) se centre sur l'individu et sur sa capacité à gérer ses relations de soutien en fonction de ses besoins. Les relations familiales sont à nouveau considérées comme des « ressources » que l'individu âgé gère en fonction de ses besoins et de ses capacités afin de s'adapter au 


\section{Introduction}

mieux au processus de fragilisation et préserver ainsi son bien-être jusqu'à la fin de sa vie.

Bien que ces divers modèles théoriques nous éclairent sur le soutien familial, ils ont d'une certaine manière biaisé la recherche sur les relations familiales dans la vieillesse. En effet, la famille est principalement perçue comme une entité extérieure à l'individu, comme une " ressource » sur laquelle l'individu âgé s'appuie pour faire face aux événements négatifs qui ponctuent son parcours de vie. En conséquence, la famille, en tant qu' « objet d'étude », n'a jamais été réellement explorée ni dans sa dynamique ni dans sa complexité, comme l'ont souligné certains auteurs faisant le bilan critique de la gérontologie sociale (Allen, Blieszner, \& Roberto, 2011; Connidis, 2015; Marshall et al., 1993; Silverstein \& Giarrusso, 2010). Nous proposons dans cet ouvrage de changer d'angle d'analyse et de recentrer l'analyse sur les liens qui relient entre eux tous les membres de la famille. Pour ce faire, nous appliquons l'approche configurationnelle qui permet de replacer les dynamiques familiales au centre de la recherche (Castrén \& Ketokivi, 2015; Widmer, Castrén, Jallinoja, \& Ketokivi, 2008; Widmer, 2016). Cette approche n'a jamais été utilisée pour étudier les relations familiales dans la vieillesse et, pourtant, elle comporte de nombreux avantages.

D'abord, cette approche considère les définitions personnelles de la famille, ne se contentant pas d'une définition «classique » de la famille. Ce sont en effet les individus eux-mêmes qui désignent au sein de leur réservoir de parenté, et parfois au-delà, les membres de leur famille qu'ils considèrent comme significatifs. Dans de nombreux travaux en gérontologie sociale, les définitions personnelles des individus âgés de « leur» famille significative - c'est-à-dire de leur famille « qui compte vraiment »- ne sont jamais considérées. La famille est au contraire définie « a priori» reposant sur le modèle « idéal » de la famille «nucléaire », c'est-à-dire une famille composée d'un couple marié avec des enfants et dont les comportements d'aide sont prescrits par des statuts et des rôles clairement définis, sexués, hiérarchisés et régis par de fortes obligations familiales. Et, selon cette définition "classique » de la famille, les obligations auxquelles sont tenus les membres de la famille nucléaire garantissent la cohésion et la stabilité familiale, quelles que soient les difficultés rencontrées (Murdock, 1949; Parsons \& Bales, 1956). Se basant sur cette définition «classique» de la famille, les indicateurs démographiques utilisés pour approcher la famille font essentiellement référence à une palette limitée de statuts et de rôles 
qui sont «a priori» définis comme importants (Widmer, 2006; Widmer, Aeby, \& Sapin, 2013; Widmer et al., 2008). Cette définition, tout comme les indicateurs démographiques qui y font référence, est rarement remise en question. Or, comme le montrent des études sur des populations plus jeunes, la définition personnelle de la famille, de celle « qui compte vraiment », est plurielle et ne correspond que pour une minorité de personnes à la définition «classique » de la famille proposée par le chercheur (Widmer, 2016; Widmer \& Jallinoja, 2008). Celle-ci n'est dès lors plus suffisamment opératoire pour analyser les relations familiales contemporaines. Plus encore, elle néglige la valeur émotionnelle et symbolique - la significativité que revêt la famille pour les individus interviewés. Pourtant, cette « significativité » est importante dans la vieillesse, sa recherche rendue pressante par l'imminence de la mort. Dans cet ouvrage, nous proposons de remédier à ce manque en s'intéressant aux définitions personnelles de la famille des personnes âgées. Nous identifierons les membres de la famille que ces dernières considèrent, elles-mêmes, comme «significatifs » et sur la base desquels nous dégagerons plusieurs types de configurations familiales.

Ensuite, l'approche configurationnelle offre un angle d'analyse qui va au-delà de la structure, des dyades ou de l'individu. Elle considère en effet la famille comme une configuration de liens d'interdépendance, à savoir un réseau complexe d'individus liés les uns aux autres par des échanges de diverses ressources telles que le soutien. Dans cette approche, ce sont les liens d'interdépendance qui unissent les différents membres de la configuration familiale qui «font» famille. Une telle perspective dépasse le traitement habituel de la famille en vigueur dans les modèles dominants de la gérontologie sociale. En effet, la famille renvoie dans le modèle du « convoi des relations sociales » à la composante d'un réseau personnel plus large (Antonucci, 1990, 2001; Kahn \& Antonucci, 1980); elle est appréhendée par sa structure, c'est-à-dire par le nombre et le profil des membres de la famille mais les liens entre les différents membres ne sont jamais pris en considération. Dans les modèles de la «solidarité intergénérationnelle » (Bengtson \& Roberts, 1991; Roberts \& Bengtson, 1990) ou celui du « support bank » (Antonucci, 1990, 2001; Antonucci et al., 2007; Antonucci \& Jackson, 1989), la famille est réduite à la dyade intergénérationnelle et le contexte familial plus large dans lequel elle s'insère est rarement pris en compte. Finalement, dans le modèle de la « sélectivité socio-émotionnelle» (Carstensen, 1991, 1992), l'individu, acteur, utilise la famille comme une 


\section{Introduction}

«ressource » et semble indépendant des contraintes relationnelles qui soustendent les liens familiaux et desquelles il dépend pourtant. Ces divers modèles théoriques peinent donc à nous offrir une vision plus large, au niveau familial, qui tient compte à la fois des individus, des dyades et de l'ensemble des relations familiales qui relient entre eux les individus et les dyades. En utilisant l'approche configurationnelle, nous pouvons aller plus loin que ces divers modèles théoriques. La prise en compte des liens d'interdépendance ouvre en effet de nouvelles perspectives de recherche telles que l'analyse de réseaux qui permet, en s'appuyant sur des indicateurs spécifiques, de mesurer le capital social au sein des configurations familiales et d'en identifier les différentes formes (Bourdieu, 1980, 1986; Portes, 1998; Widmer, 2016). Nous reviendrons plus longuement sur le concept de capital social, ses mécanismes de production et ses différents types dans le chapitre consacré à l'approche configurationnelle. Le capital social au sein de la famille est rarement évoqué en gérontologie sociale parce que les liens d'interdépendance entre les différents membres familiaux ne sont jamais considérés. Nous proposons donc d'y pallier et de mesurer le capital social au sein des différentes configurations familiales que nous identifierons. Nous nous interrogerons, ensuite, sur les diverses formes de capital social produites au sein des familles dans la vieillesse et leurs facteurs.

De plus, l'approche configurationnelle tient compte non seulement des liens de soutien entre les différents membres qui composent les configurations familiales mais aussi des tensions et des conflits (Widmer, 2016). En effet, les liens d'interdépendance ne créent pas que du capital social, ils produisent aussi du conflit et de l'ambivalence. Grâce aux outils de l'analyse des réseaux, la conflictualité de chaque configuration familiale peut être évaluée au même titre que son potentiel de soutien, c'est-à-dire son capital social. Motivés principalement par la problématique du soutien, les modèles dominants de la gérontologie sociale ont pendant longtemps défini la famille comme une source de soutien inconditionnel. Dès lors, les tensions et les conflits étaient davantage perçus comme des dysfonctionnements ou comme le fait d'individus «nocifs » (Antonucci et al., 2007). Les travaux sur l'ambivalence intergénérationnelle ont bousculé la conception "idéalisée » de la famille en soulignant que les tensions et les conflits sont des dimensions intrinsèques des relations familiales au même titre que les relations de soutien (Connidis, 2012, 2015; Connidis \& McMullin, 2002; Lüscher, 2002; Lüscher \& Pillemer, 1998). A ce jour, l'ambivalence demeure toujours en gérontologie sociale une propriété 
structurelle principalement dyadique et majoritairement étudiée dans les relations intergénérationnelles. Nous proposons dans cet ouvrage de sortir l'ambivalence de son carcan dyadique habituel et, en utilisant l'approche configurationnelle, de la mesurer au niveau plus large des configurations familiales. Pour ce faire, nous allons construire, en nous inspirant de la typologie des stratégies de gestion de l'ambivalence intergénérationnelle de Kurt Lüscher (Lüscher, 2000, 2002; Lüscher \& Hoff, 2013), quatre « modes de conflit et de support» qui allient le soutien et le conflit de manière distincte. Nous observerons ensuite comment ces différents « modes de conflit et de support» varient en fonction non seulement de la composition des configurations familiales des individus âgés mais aussi en fonction du profil socio-démographique de ces derniers et des différentes ressources dont ils disposent.

Bien que certains chercheurs en gérontologie sociale reconnaissent depuis cette dernière décennie la pluralité des formes familiales et leur complexité - comme en témoigne le nombre de travaux sur des structures familiales particulières telles que les couples de même sexe ou encore les familles recomposées et les nombreuses études sur l'ambivalence dans les relations intergénérationnelles - la gérontologie sociale anglo-saxonne, empêtrée dans la problématique du soutien, peine à changer d'angle d'analyse et à placer les dynamiques familiales au cœur de ses interrogations. Selon certains auteurs, la plupart des chercheurs en gérontologie sociale se doivent de dépasser l'influence prédominante des principaux modèles théoriques et d'innover tant dans les approches que dans les méthodes pour aborder la diversité et la complexité des réalités familiales d'aujourd'hui (Carr \& Moorman, 2011; Silverstein \& Giarrusso, 2010). L'approche configurationnelle et les outils d'analyse de réseaux qui lui sont associés répondent pleinement à cette quête en remettant au centre de l'analyse les liens d'interdépendance, positifs (soutien) et négatifs (conflit), entre les différents membres significatifs de la famille et sur la base desquels se forment les configurations familiales. Le soutien est certes aussi considéré mais il ne constitue pas, en soi, le centre de l'analyse; il ne sert que de prétexte pour observer les liens d'interdépendance qui sous-tendent les configurations familiales. Seule une telle approche permet d'appréhender la complexité des relations familiales qui caractérise les dernières étapes de la vie.

Par ailleurs, cet ouvrage allie l'approche configurationnelle initiée, entre autres, par Norbert Elias (Elias, 1991) et la théorie de l'échange social qui modélise les échanges dyadiques en s'inspirant de la logique 


\section{Introduction}

économique (Blau, 1964; Cropanzano \& Mitchell, 2005; Kelley \& Thibaut, 1978). Suivant la perspective configurationnelle, l'analyse porte en effet sur l'ensemble des liens d'interdépendance, positifs et négatifs, qui sous-tendent les configurations familiales dans la vieillesse. Ces liens d'interdépendance s'associent aux échanges de soutien mais aussi aux contraintes puisque l'individu qui s'y insère n'est pas totalement «libre » d'agir, son comportement dépendant étroitement des autres membres familiaux avec qui il est en lien et des normes qui régissent leurs échanges. Cependant, l'individu dispose d'une certaine marge de manœuvre au sein de sa configuration familiale. Acteur, il peut influencer par ses actions l'organisation des liens d'interdépendance au sein de sa configuration familiale. Pour évoquer ces contraintes et la marge de manœuvre dont dispose l'individu au sein de sa famille, nous nous référons à la terminologie de la théorie de l'échange social (Blau, 1964; Cropanzano \& Mitchell, 2005 ; Kelley \& Thibaut, 1978). Il sera donc question dans cet ouvrage de « besoins », d' « échanges de ressources », des «normes de réciprocité », d' « investissement » ou de « désinvestissement », d' ' engagement» ou de « désengagement», de « stratégies », de « dettes» et de « négociations ». Cette terminologie est largement utilisée par les tenants des modèles dominants de la gérontologie sociale, ceux-ci ayant développé leur théorie en se basant sur les échanges sociaux de type dyadique. Par exemple, Toni Antonucci se réfère au «support bank » et aux « dettes » des enfants adultes à l'égard de leurs parents (Antonucci, 1990, 2001; Antonucci et al., 2007; Antonucci \& Jackson, 1989), Laura Carstensen évoque dans sa théorie de la «sélectivité socio-émotionnelle» des "stratégies de sélection » et « de désinvestissement» (Carstensen, 1991, 1992) et, finalement, Kurt Lüscher conceptualise différentes «stratégies de gestion » de l'ambivalence intergénérationnelle (Lüscher, 2000, 2002; Lüscher \& Hoff, 2013). Même si ces termes suggèrent l'intentionnalité des individus, les actions ou les stratégies individuelles au sein des configurations familiales ne sont pas toujours conscientes ou intentionnelles, fruit d'un choix rationnel. Ces actions peuvent répondre à des attentes ou, plus globalement, aux normes d'échanges (réciprocité généralisée) qui sont intériorisées par les individus qui participent aux échanges familiaux. D'autres peuvent être « réactives » ou «adaptatives » à des changements qui touchent l'individu lui-même - en cas de problèmes de santé, par exemple - ou qui déstabilisent sa configuration familiale - notamment, lors du décès d'un des membres de sa famille. 
Ces changements déséquilibrent les échanges familiaux et poussent les individus à agir en vue de rééquilibrer les liens d'interdépendance au sein de leur configuration familiale.

Les données quantitatives sur lesquelles portent les analyses de cette recherche permettent de rendre compte de ces deux aspects. D'une part, elles décrivent de manière factuelle l'organisation des liens d'interdépendance au sein des configurations familiales et mettent en exergue les contraintes relationnelles dans lesquelles baignent les individus sans que ceux-ci en aient pleinement conscience (Castrén \& Ketokivi, 2015). D'autre part, elles signalent la position et l'activité des individus au sein de leur configuration familiale, révélant ainsi leur degré d'engagement dans les échanges familiaux. Autrement dit, ces données rendent aussi compte de l'ampleur de la marge de manœuvre des individus au sein de leur famille. De telles données sont cependant limitées pour saisir les motivations profondes qui justifient les choix, les décisions et les actions des individus au sein de leur configuration familiale. Seuls des entretiens approfondis, par le biais d'enquêtes qualitatives, permettraient de mettre à jour les motivations, même inconscientes, qui expliquent les actions et les choix relationnels des individus au sein de leur famille (Castrén \& Ketokivi, 2015). Dans la « discussion et conclusion » de cet ouvrage, nous reviendrons sur cette limite.

Cet ouvrage est divisé en sept chapitres. Les trois premiers sont essentiellement théoriques. Dans le premier, nous reprenons, en les explicitant davantage, les principaux modèles théoriques de la gérontologie sociale, à savoir le modèle du « convoi des relations sociales », le modèle de la « solidarité intergénérationnelle », le modèle du «support bank » et la théorie de la «sélectivité socio-émotionnelle». Ces divers modèles ont pendant longtemps orienté la recherche en gérontologie sociale anglo-saxonne et leur influence est aujourd'hui encore prédominante dans de nombreuses études gérontologiques portant sur les relations sociales et familiales dans la vieillesse. Nous soulignons leurs apports dans le domaine des relations sociales et familiales mais aussi leurs limites et les manques que cet ouvrage vise à combler. Dans le deuxième chapitre, nous exposons les principaux facteurs explicatifs des relations sociales et familiales souvent avancés en gérontologie sociale tels que l'état du réservoir de parenté, le statut socioéconomique, le genre, l'âge et la santé. Nous décrivons les résultats obtenus dans la littérature en lien avec ces divers facteurs et nous soulignons à nouveau les questions auxquelles ne répondent pas les modèles dominants de 


\section{Introduction}

la gérontologie sociale. Nous clôturons ce deuxième chapitre en posant les principales questions de recherche qui ont orienté notre investigation. Dans le troisième chapitre, "Problématique et hypothèses ", nous présentons l'approche configurationnelle en mettant en exergue ses avantages aussi bien théoriques que méthodologiques pour étudier les relations familiales dans la vieillesse. Ensuite, nous reprenons divers concepts de l'approche configurationnelle qui sont centraux pour appréhender les dynamiques familiales à savoir les «membres significatifs de la famille», le «capital social» et l'«ambivalence ». Pour chacun de ces concepts, nous précisons ce que la gérontologie sociale nous apprend mais aussi ce qu'elle ne traite pas et comment l'approche configurationnelle permet de combler ces manques. Finalement, nous présentons les différentes hypothèses opérationnelles que nous testons ensuite dans les analyses.

Le quatrième chapitre est consacré aux données et aux méthodes. Dans un premier temps, l'étude «Vivre/Leben/Vivere» (VLV) y est décrite avec ses objectifs et ses procédures d'enquête. Dans un deuxième temps, nous évoquons les raisons qui nous ont poussés à privilégier l'échantillon genevois sur les cinq cantons sondés. Après avoir décrit les caractéristiques de ce dernier, nous introduisons la « Family Network Method» (Widmer, 2016; Widmer et al., 2013) utilisée pour récolter les données sur les réseaux familiaux ainsi que les mesures et les analyses statistiques sur lesquelles nous nous sommes appuyés dans cet ouvrage. L'ensemble des résultats obtenus sont présentés dans le cinquième et sixième chapitre. Nous exposons d'abord la liste les membres significatifs de la famille qui sont les plus cités dans la vieillesse ainsi que la typologie des six configurations familiales obtenues après avoir effectué une analyse de classification hiérarchique sur l'ensemble des termes de parenté cités. Ensuite, dans le sixième chapitre, nous examinons les liens d'interdépendance, positifs et négatifs, qui soustendent ces six configurations familiales. En premier lieu, nous abordons les différentes formes du capital social (« chaîne » et «pont») produites au sein de ces configurations familiales et nous mettons en exergue leurs différents facteurs. Puis, nous présentons les résultats ayant trait à l'ambivalence et aux quatre «modes de conflit et de support». L'analyse de correspondances multiples et une étude de cas nous enseignent que ces quatre modes s'associent à différents types de configurations familiales et à la présence ou à l'absence de différentes ressources. Dans le septième chapitre, «Discussion et conclusion», nous reprenons les principaux résultats 
de ce travail et soulignons en quoi ils contribuent à la recherche gérontologique dans le domaine des relations familiales. Ce faisant, nous mettons en exergue les points forts de cette recherche, les quelques implications pratiques auxquelles elle aboutit ainsi que ses limites. 



\section{Modèles explicatifs des relations familiales en gérontologie sociale}

Ce premier chapitre vise à présenter un état des lieux de la littérature gérontologique concernant les relations familiales dans la vieillesse. Nous ne cherchons pas à rendre compte de toutes les études qui ont été menées sur ce thème mais l'objectif de ce chapitre est de présenter les principaux modèles théoriques qui ont dominé la recherche gérontologique dans le domaine de relations familiales et sociales. Nous faisons plus particulièrement référence au modèle du « convoi des relations sociales » (Antonucci, 1990, 2001 ; Kahn \& Antonucci, 1980), au paradigme de la « solidarité intergénérationnelle » (Bengtson \& Roberts, 1991; Roberts \& Bengtson, 1990), au modèle du «support bank» (Antonucci, 1990; Antonucci \& Jackson, 1989) et à la théorie de la sélectivité socio-émotionnelle (Carstensen, 1991). Ces différents modèles théoriques sont importants puisqu'ils ont influencé la recherche sur les relations sociales et familiales dans la vieillesse ces dernières décennies et orientent toujours un grand pan de la recherche gérontologique anglo-saxonne. Ils sont en effet souvent cités dans les études scientifiques comme modèles explicatifs et leur référence est incontournable. Leur prégnance est telle dans le domaine gérontologique qu'ils sont relativement peu ébranlés par les perspectives plus critiques de certains auteurs - tels que Allen et al. (2011); Connidis (2015); Marshall et al. (1993) - qui remettent en question les fondements théoriques sur lesquels ils reposent. Il est clair que ces divers modèles ont apporté à la gérontologie sociale de nouvelles perspectives d'analyse et ont contribué à une meilleure connaissance des relations sociales et familiales dans la vieillesse. Cependant, ils comportent de nombreuses limites. Nous allons dans ce chapitre les présenter en soulignant non seulement leurs apports mais aussi leurs faiblesses. La critique de la gérontologie sociale que nous faisons tout au long de ce travail concerne principalement ces divers modèles qui ont encore aujourd'hui une forte prégnance dans de nombreux travaux portant sur la vieillesse. 


\subsection{Les réseaux personnels et le modèle du convoi des relations sociales}

Un premier grand pan de la recherche gérontologique s'est particulièrement focalisé sur les réseaux personnels des personnes âgées. Dans la littérature, le réseau personnel renvoie à l' « environnement interpersonnel significatif » de l'individu (Litwin, 2001); il a trait à l'ensemble des personnes qui - quel que soit le cercle de sociabilité auquel elles appartiennent (famille, parenté, amis, voisins et milieux associatifs) - sont perçues par l'individu comme affectivement "proches » et «importantes » en fonction du support qu'elles lui apportent (Fiori, Antonucci, \& Akiyama, 2008; Fiori, Antonucci, \& Cortina, 2006; Kahn \& Antonucci, 1980). Dans la littérature gérontologique, la diversité des membres qui composent le réseau personnel est mise en avant et les membres de la famille ne constituent qu'une part de ses différentes composantes. Dans cette section, nous présentons plus avant comment les réseaux personnels sont abordés en gérontologie sociale et nous nous focalisons plus spécifiquement sur le modèle théorique du convoi des relations sociales de Toni Antonucci qui a particulièrement marqué la recherche sur les réseaux personnels dans la vieillesse puisque la majorité des études gérontologiques sur les relations sociales s'y réfèrent systématiquement.

L'intérêt marqué des gérontologues pour l'étude des réseaux personnels aux dernières étapes de la vie fait écho aux nombreuses études en épidémiologie et en psychologie qui ont montré l'effet protecteur de la diversité des relations sociales sur la santé tant physique que mentale, et cela dans des populations de tous âges (Berkman, Glass, Brissette, \& Seeman, 2000; House, 2001; Moren-Cross \& Lin, 2006; Song, Son, \& Lin, 2011). S'inspirant de cette tradition de recherche, les gérontologues ont fait de l'insertion de l'individu âgé dans plusieurs cercles de sociabilité l'un des facteurs-clé du « successful aging» (Antonucci et al., 2007). D'une part, la diversité des membres du réseau personnel témoigne de l'engagement multiple de l'individu au sein de la société et de sa bonne intégration. Ce dernier y endosse de nombreux rôles sociaux (époux, parent, ex-collègue, ami, voisin, membre d'une association, etc.), ce qui contribue au maintien de sa santé aussi bien physique que mentale (estime de soi) (Antonucci et al., 2007; Berkman et al., 2000; Moren-Cross \& Lin, 2006). D'autre part, cette diversité assure à l'individu âgé un accès privilégié à 
une variété de ressources et de support social (soutien émotionnel, aide pratique, financière, information, etc). Ces ressources permettent à l'individu de mieux gérer le stress inhérent aux transitions de vie difficiles qu'il traverse, telles que le veuvage, le déclin de la santé ou encore une baisse de revenu, et de préserver ainsi sa santé physique et mentale (Berkman et al., 2000; Cohen, 2004; Lefrançois, Leclerc, Hamel, \& Gaulin, 2000; Song et al., 2011). Les données empiriques attestent que les réseaux personnels de grande taille et composés d'une variété de membres s'associent à un bon niveau de bien-être alors que les réseaux composés de peu de membres ou homogènes (centrés sur la famille) se conjuguent à un bas niveau de bien-être (Antonucci, 2001; Jang, Haley, Small, \& Mortimer, 2002; Wenger, 1997). Ces résultats confirment ceux d'études épidémiologiques ou psychologiques précédentes qui montrent l'importance d'être socialement intégré et d'avoir du support social pour se maintenir en bonne santé. Au vu de ces observations, de nombreux gérontologues se sont interrogés sur l'évolution des réseaux personnels et ont cherché à mieux comprendre comment certains individus âgés développent de larges réseaux personnels " fonctionnels » alors que d'autres présentent des réseaux de petite taille ou homogènes, relativement peu fonctionnels.

\subsubsection{Le modèle du convoi des relations sociales}

Pour répondre à ces questions, Toni Antonucci et son équipe (Antonucci \& Akiyama, 1995; Antonucci et al., 2007; Kahn \& Antonucci, 1980) proposent un modèle théorique explicatif - le modèle du convoi des relations sociales ( «Convoy Model of Social Relations ») - qui tient compte à la fois du profil socio-démographique des individus âgés mais aussi des étapes précédentes de leur parcours de vie. Ce modèle postule que tout individu est, tout au long de sa vie, entouré de personnes qui lui fournissent les ressources (support) dont il a besoin pour se développer socio-affectivement et pour faire face aux difficultés qui ponctuent son parcours de vie (Antonucci, 1990; Antonucci et al., 2007; Kahn \& Antonucci, 1980). Ces personnes lui offrent un «cadre protecteur» qui lui permet de «poursuivre» son parcours de vie sans encombre et de préserver sa santé et son bien-être jusqu'aux dernières étapes de la vie (Antonucci, 2001; Antonucci et al., 2007; Fiori et al., 2006). Selon ce modèle, le nombre et l'identité des personnes qui accompagnent l'individu varient 


\section{Modèles explicatifs en gérontologie}

selon le profil socio-démographique de ce dernier, les transitions de sa vie passées et les rôles qu'il a été amenés à jouer tout au long de sa vie (Antonucci, 2001; Kahn \& Antonucci, 1980; Van Tilburg \& Thomése, 2010). Les transitions de vie (devenir époux ou parent, etc) que l'individu et les membres de son réseau ont traversées influencent la structure de son réseau personnel; certaines transitions entraînent la perte de relations (veuvage, divorce, déménagements, etc.) alors que d'autres (devenir grandparent, par exemple) s'accompagnent de l'arrivée de nouveaux membres et, par là même, de nouvelles opportunités de développer des relations personnalisées. Par conséquent, à l'orée de la vieillesse, certains réseaux sont petits; d'autres au contraire sont beaucoup plus larges. Certains se centrent surtout sur la famille, d'autres sur les amis alors que d'autres encore comprennent une diversité de membres tels que des membres de la famille, des amis, des voisins ou encore d'anciens collègues (Antonucci et al., 2007; Fiori et al., 2008, 2006).

En plus des changements dans la structure, ces diverses transitions conduisent à des changements de rôles (sociaux et familiaux) et à l'émergence de nouveaux besoins, entraînant une ré-évaluation des liens existants et de leurs apports (ressources) de la part non seulement de l'individu mais aussi des membres de son réseau. Ce processus de ré-évaluation peut tout aussi bien aboutir à la rupture (désengagement) qu'à l'activation d'une relation jusque-là laissée en berne (anciens liens), à la valorisation d'une relation périphérique, au renforcement d'un lien existant ou encore à l'investissement dans de nouveaux liens (Antonucci, 2001; Kahn \& Antonucci, 1980; Van Tilburg, 1998). Un voisin, par exemple, peut lors d'un événement difficile devenir un ami par le soutien émotionnel dont il fait preuve et qui « outrepasse » son rôle de voisin qui, généralement, se limite à rendre de petits services. Certaines transitions de vie rendent ce mécanisme de ré-évaluation particulièrement manifeste, notamment lors du déclin de la santé qui caractérise les dernières étapes du parcours de vie. Généralement, les individus dont la santé se détériore réduisent leur réseau personnel (désengagement) alors que les membres proches de la famille, au contraire, se mobilisent (renforcement). Toute relation est donc amenée à être re-définie et négociée au fil du temps, en fonction des transitions du parcours de vie et des nouveaux besoins qu'elles génèrent (Connidis, 2010; Finch, 2007). Ces différentes dynamiques relationnelles (activation, désengagement, renforcement, etc) rendent compte du travail de ré-évaluation 
mis en œuvre par l'individu âgé et les membres de son réseau personnel tout au long du parcours de vie (Van Tilburg, 1998). En principe, de telles modifications devraient procurer à l'individu les relations les mieux adaptées à ses besoins qui évoluent au fil du temps, selon son développement physique et psychologique (Antonucci, 2001; Van Tilburg, 1998).

Le modèle du convoi tient donc compte du caractère dynamique des relations d'aide qui sont amenées à être investies et négociées différemment selon les besoins des individus au cours du temps (Connidis, 2010). Certaines données confirment ces processus en montrant que les individus qui disposent d'un même potentiel - à savoir un réseau d'aide à structure équivalente en termes de taille et de composition - n'obtiennent pas forcément le même montant ni les mêmes types de soutien (Fiori et al., 2008; Litwin \& Landau, 2000). Ces résultats révèlent que, même avec un réseau personnel à structure similaire, les individus ne développent pas forcément les mêmes relations d'aide et de soutien avec les mêmes personnes (Fiori et al., 2008; Litwin \& Landau, 2000). Autrement dit, ils investissent leurs relations et les négocient différemment selon leurs besoins qui évoluent au gré du temps et des circonstances (Campbell, Connidis, \& Davies, 1999; Connidis, 2010). Selon le modèle du convoi, les réseaux personnels peuvent donc s'avérer fort différents selon les individus, non seulement en fonction de leur profil socio-démographique, de leur trajectoire de vie et de celles de leurs proches (mariage, divorce, veuvage, déménagement, enfants, nombre et proximité géographique des enfants, etc) mais aussi en fonction de la façon dont ils ont - selon leur profil socio-démographique et familial-investi et négocié certaines de leurs relations. Le modèle du convoi souligne donc la pluralité des contextes relationnels et leur dynamique à l'entrée de la vieillesse.

La diversité des réseaux personnels est, dans le modèle du convoi, mesurée en fonction de leur taille (nombre de membres) et de leur composition (profil socio-démographique des membres, leur rôle social par rapport à l'individu âgé, leur proximité géographique, la fréquence et la durée de leur relation et les types de support que les membres du réseau offrent à la personne étudiée). Ces deux indicateurs sont considérés comme des éléments essentiels pour estimer non seulement le degré d'intégration de l'individu âgé dans la société mais aussi le potentiel d'aide (montant et qualité des ressources) qu'il a à disposition (Ajrouch, Blandon, \& Antonucci, 2005; Antonucci, 2001). Les membres du réseau personnel sont généralement 
catégorisés en fonction du rôle social (partenaire, ami, voisin, etc.) qu'ils jouent par rapport à l'individu focal. Et en fonction de ce rôle, il leur est associé «a priori » un degré de proximité affective avec l'individu étudié et une qualité de support.

Dans le cercle périphérique du réseau se situent, généralement, ceux qui sont considérés comme «proches» («the close»), il s'agit de la parenté, des amis, de voisins ou d'anciens collègues. Ceux-ci rendent quelques services ou s'avèrent être de bons compagnons lors d'activités diverses. Ceci dit, ces relations, particulièrement sensibles aux transitions de vie de l'individu (déménagements, etc.), sont instables au cours du temps. Ensuite, viennent les plus proches («the closer») tels que certains membres de la fratrie et les amis intimes. Bien que soumises à une certaine ré-évaluation au cours du temps (une ré-activation du lien fraternel, par exemple), ces relations sont relativement stables, source de support émotionnel, parfois instrumental, et de compagnonnage (Antonucci et al., 2007; Campbell et al., 1999; Eriksen \& Gerstel, 2002).

Finalement, le cercle des très proches («the closest») — usuellement, le partenaire et les enfants - constitue le cœur des réseaux personnels (Antonucci, 2001; Fiori et al., 2008, 2006; Kahn \& Antonucci, 1980). Perçus comme l'une des sources majeures de support social (émotionnel, instrumental, financier, soins, conseils, etc.) dans la vieillesse (Krause, 2006; Ryan \& Willits, 2007; Walker, Pratt, \& Eddy, 1995), les membres primaires de la famille sont définis «a priori » comme des « autruis significatifs » auxquels l'individu est fortement attaché (Thoits, 2011). Ils sont décrits comme fiables et stables au cours du temps, toujours disposés à offrir le support nécessaire, quels que soient les événements stressants que l'individu ait été amené à rencontrer comme le décès de proches ou le déclin de la santé à la fois physique et mentale (Antonucci, 2001 ; Shor, Roelfs, \& Yogev, 2013; Thoits, 2011). Par le support « inconditionnel» qu'ils offrent «a priori », les membres primaires de la famille, qui se situent au cœur du réseau personnel, contribuent activement à la satisfaction des besoins tant psychologiques que physiques du parent âgé et participent ainsi au maintien de son identité et de sa santé jusqu'aux dernières étapes de la vie (Merz \& Huxhold, 2010; Thoits, 2011). Par l'importance qu'ils revêtent «a priori » pour les individus, les membres primaires de la famille semblent donc, selon le modèle du convoi, aller de soi et sont peu soumis à un travail de ré-évaluation par l'individu et les membres de son réseau. 


\subsubsection{Les limites du modèle du convoi des relations sociales}

La manière dont les membres primaires de la famille sont « a priori » perçus et catégorisés dans le modèle du convoi constitue une première limite. Le partenaire et les enfants sont définis comme les membres «très proches » du réseau, ils en composent le cœur et sont «a priori » qualifiés de stables, fiables et immuables. Ils sont désignés comme ceux qui accompagnent l'individu âgé jusqu'aux dernières étapes de sa vie, quels que soient les événements auxquels il est confronté. Dans cette perspective, leur soutien, perçu comme «allant de soi », n'est pour ainsi dire jamais questionné. De même, le travail de ré-évaluation et de négociation qui touche les relations périphériques ne semble pas concerner les liens familiaux primaires. Définie «a priori » comme relativement homogène et stable, la composante familiale du réseau personnel est finalement peu explorée dans de nombreuses études consacrées aux relations sociales. Dans cet ouvrage, nous irons au-delà de cette conception "idéalisée » en montrant que le réseau familial est loin d'être homogène, étant soumis, lui aussi, au travail de ré-évaluation des relations personnelles. Ce travail accompagne toutes transitions de vie et est susceptible de donner lieu à une diversité de configurations familiales dans la vieillesse.

Ensuite, le modèle du convoi n'explore pas toutes les facettes des réseaux personnels; il se contente de s'appuyer sur la composition et la taille des réseaux personnels pour inférer le degré d'intégration des individus et, plus encore, le potentiel et la qualité de leur support, et d'en déduire ensuite leurs effets sur la santé. Cependant, la façon dont les liens de soutien s'organisent au sein du réseau personnel entre les différents membres qui le constituent ne sont jamais questionnés. En effet, le réseau personnel est considéré comme un ensemble de dyades - indépendantes les unes des autres - impliquant ego (l'individu focal) et chacun des membres de son réseau personnel pris séparément (Antonucci, 2001; Fiori et al., 2008, 2006). Chaque dyade est étudiée en termes de fréquence des contacts, d'échanges de soutien et de durée de la relation (Antonucci et al., 2007; Broese Van Groenou \& Van Tilburg, 2007; Moren-Cross \& Lin, 2006) mais les liens entre les dyades ne sont jamais considérés.

Pourtant, comme l'ont révélé les précurseurs de l'analyse de réseau tels qu'Élisabeth Bott (Bott, 1955), le degré de connexion entre les membres du réseau - c'est-à-dire à quel point les membres du réseau sont liés les uns aux autres - a un effet important sur le fonctionnement des dyades qui le 


\section{Modèles explicatifs en gérontologie}

constituent et le comportement des individus, et indirectement sur la santé de ces derniers (Berkman et al., 2000; Bott, 1955; Moren-Cross \& Lin, 2006). De plus, il est probable que le travail de ré-évaluation des relations qui constituent le réseau personnel ne dépend pas uniquement de la volonté des individus mais il est aussi tributaire des liens d'interdépendance qui sous-tendent l'ensemble du réseau personnel et de la position des individus en son sein. Il est vrai que, depuis peu, le degré de connexion des liens et la position des individus dans leur réseau sont davantage considérés en gérontologie sociale dans le domaine des réseaux personnels (Broese Van Groenou \& Van Tilburg, 2007; Cornwell, 2009b; Moren-Cross \& Lin, 2006). Certains reconnaissent en effet que c'est la manière dont sont reliés les membres du réseau personnel qui influence le comportement des individus et leur santé (Berkman et al., 2000; Cornwell, Schumm, Laumann, \& Graber, 2009; Moren-Cross \& Lin, 2006). Mais ces indicateurs sont surtout cantonnés dans les études portant sur l'intégration sociale au sein des réseaux personnels (Ashida \& Heaney, 2008; Cornwell, Laumann, \& Schumm, 2008; Cornwell, 2009b). Ils n'ont jamais été exploités pour explorer les liens de soutien au sein des configurations familiales dans la vieillesse. De même, le terme de « capital social» est rarement nommé en gérontologie sociale même si les études sur les réseaux personnels l'explorent en dégageant les différentes propriétés structurelles de ces derniers (Moren-Cross \& Lin, 2006).

Dans cet ouvrage, nous proposons d'aller plus loin, de dépasser l'approche classique du modèle du convoi et de nous intéresser à la façon dont s'organisent les liens de soutien au cœur même du réseau personnel, à savoir les liens qui relient les membres significatifs de la famille. La prise en compte de ces liens est nécessaire et primordiale pour comprendre comment les échanges de soutien sont organisés au sein des configurations familiales. Nous postulons en effet que l'organisation des liens de soutien au sein du réseau familial donne une meilleure indication du potentiel de support que seules la taille et la composition du réseau. Pour ce faire, nous utiliserons les outils de l'analyse de réseaux (Freeman, 2004; Kadushin, 2012; Moren-Cross \& Lin, 2006) qui permettent de dégager certaines propriétés structurelles - telles que la centralité d'ego dans son réseau ou encore la densité du soutien (degré de connexion entre les différents membres du réseau personnel) - indiquant, pour chaque réseau 
étudié, la façon dont le soutien s'organise au sein de l'ensemble des relations qui le constituent et d'en mesurer ainsi le capital social (cf. chapitre «Problématique et hypothèses »).

Prendre en compte les interdépendances relationnelles entre les différents membres du réseau personnel permet aussi de considérer les tensions et les conflits qui y surgissent. La ré-évaluation de certains liens, qui se manifeste par exemple par le désengagement de certaines relations ou l'activation d'autres, n'est pas sans conséquence sur les autres relations qui forment le réseau. Il est probable qu'un tel processus s'accompagne d'un réajustement des liens de support dans l'ensemble du réseau, processus qui n'est pas sans créer des tensions entre les différents membres. Ces aspects-là ne sont jamais pris en compte dans les études sur les réseaux personnels en gérontologie sociale, tant le soutien qu'ils offrent et leurs effets positifs sur la santé des individus âgés sont considérés comme primordiaux. Certes, les chercheurs reconnaissent que certains membres du réseau, même parmi les plus proches, sont parfois nocifs pour les parents âgés, générant du stress chez ces derniers avec des conséquences négatives sur leur santé (Antonucci et al., 2007). Imputables à des individus toxiques, les tensions ne sont jamais perçues dans le modèle du convoi comme les conséquences de réajustements ou de ré-équilibrages relationnels au sein du réseau. Dans ce travail, nous proposons de prendre en compte l'ensemble des relations d'interdépendance, tant positives (soutien) que négatives (tensions), au sein des configurations familiales et de considérer les tensions comme des indicateurs de ré-équilibrage des échanges au sein de la famille.

\subsection{Les relations intergénérationnelles et le modèle de la solidarité intergénérationnelle}

Dans cette section, les relations intergénérationnelles - entre parents âgés et enfants adultes - et le modèle de la solidarité intergénérationnelle proposé par Vern Bengtson et son équipe (Roberts \& Bengtson, 1990; Bengtson \& Roberts, 1991) sont au cœur de notre questionnement. Comme celui du convoi pour les réseaux personnels, le modèle de la solidarité intergénérationnelle a dominé pendant des décennies - et domine encore - la 


\section{Modèles explicatifs en gérontologie}

recherche sur les relations intergénérationnelles dans la vieillesse (Lawton, Silverstein, \& Bengtson, 1994b; Silverstein, Gans, \& Yang, 2006; Schmeeckle, Giarrusso, Feng, \& Bengtson, 2006). Ce modèle est souvent cité dans les travaux en gérontologie sociale et a inspiré de nombreuses études sur les relations intergénérationnelles, non seulement entre les parents âgés et leurs enfants adultes mais aussi entre les grands-parents et leurs petits-enfants (Giarrusso, Feng, Silverstein, \& Bengtson, 2001; Silverstein \& Marenco, 2001) ou encore entre les individus âgés et les enfants adultes de leur conjoint (Schmeeckle et al., 2006). Ce modèle a aussi servi de référence dans des programmes de recherche axés sur les relations intergénérationnelles (Suitor, Sechrist, Gilligan, \& Pillemer, 2011).

Avant de présenter ce modèle, il est nécessaire de souligner que la dyade " parents-enfants » est perçue dans les courants dominants de la recherche gérontologique comme l'une des dyades familiales les plus importantes, voire la plus centrale, dont dépendent étroitement la santé et le bien-être des individus âgés (Lowenstein \& Katz, 2010; Pyke \& Bengtson, 1996; Suitor et al., 2011). C'est pourquoi elle est depuis longtemps au cœur de la recherche en gérontologie sociale. Comme l'ont souligné de nombreux chercheurs, les enfants adultes sont considérés - avec les conjoints - comme les principaux prestataires d'aide et de soins («caregiving ») dans la vieillesse, et cela même dans les pays où l'aide formelle est passablement développée (Lowenstein, Katz, \& Daatland, 2004; Pyke \& Bengtson, 1996; Suitor et al., 2011). L'une des caractéristiques des relations entre parents âgés et enfants adultes a trait aux obligations familiales qui les sous-tendent et qui sont perçues dans la littérature gérontologique comme particulièrement prégnantes (Ganong \& Coleman, 2006; Litwak, 1985; Rossi \& Rossi, 1990). Sorte de « contrats tacites », ces obligations orientent en effet les comportements d'aide entre parents et enfants tout au long du parcours de vie (Ganong \& Coleman, 2006; Rossi \& Rossi, 1990; Silverstein \& Bengtson, 1997). Intériorisées, elles se manifestent sous la forme de «sentiments » d'obligation et de responsabilité parentale ou filiale (Ganong \& Coleman, 2006; Lawton et al., 1994b; Silverstein et al., 2006). Celles-ci poussent autant les parents que les enfants à accomplir leurs devoirs de solidarité mais elles suscitent aussi, en contrepartie, de fortes attentes d'aide et de soutien de part et d'autre de la relation (Ganong \& Coleman, 2006; Lawton et al., 1994b; Silverstein et al., 2006).

Dans les années 90, certains auteurs ont remis en question la prégnance de ces normes, associant les changements sociaux qui ont marqué 
la fin du 20e siècle - dont, entre autres, la montée du divorce, l'émergence de nouvelles formes familiales, la diminution de la co-résidence intergénérationnelle, la mobilité géographique, la plus grande participation des femmes sur le marché du travail, le développement des mouvements féministes et l'essor de l'État social (accusé de se substituer à la famille) - au « déclin » de « la famille ». Perçus comme le résultat d'un individualisme exacerbé, ces changements sociaux annonçaient ainsi la fin de la solidarité familiale et l'avènement de l'isolement social des personnes âgées (Popenoe, 1993). Ce discours négatif reposait sur l'idée qu'en remettant en question la primauté du sang et de l'alliance et en privilégiant l'individu et son autonomie, les valeurs et les obligations familiales tendaient à s'affaiblir, prétéritant de fait la solidarité intergénérationnelle. Cette supposition fut largement relayée par les médias, relatant, par exemple, l'abandon des individus âgés par leurs enfants n'ayant plus le temps ni l'envie de passer du temps avec leurs parents (Szydlik, 2008). Les données empiriques ont contesté cette prédiction des plus pessimistes en mettant au contraire en évidence l'amélioration, au cours de la deuxième moité du $20 \mathrm{e}$ siècle, des contacts intergénérationnels et de la solidarité familiale, offrant, même aux plus âgés, aide et soutien en cas de besoin (Bengtson, 2001 ; Lowenstein \& Katz, 2010; Suitor et al., 2011).

Bien que les familles contribuent toujours aux fonctions d'éducation et de soutien envers leurs membres, elles sont aujourd'hui très différentes dans leur structure, leur organisation, leurs pratiques et leurs valeurs (Bengtson, 2001; Lowenstein \& Katz, 2010). L'émergence de nouvelles formes familiales a contribué à rendre plus flous non seulement les frontières - se limitant dans la famille nucléaire aux relations bio-légales mais aussi les statuts et les rôles au sein de la famille. Ce faisant, les obligations familiales qui jusqu'ici orientaient les comportements d'aide et garantissaient la solidarité au sein de la famille nucléaire sont devenues plus incertaines (Allan, 2008; Treas \& Marcum, 2011; Van Tilburg \& Thomése, 2010). La pluralité des définitions du rôle de «beau-parent » (partenaire du parent biologique), allant de parent à membre de la famille (sans être parent), à ami et même à étranger, témoigne de ce flou normatif (Schmeeckle et al., 2006). Un tel flou engrange dans ces familles une grande incertitude quant aux comportements d'aide à adopter vis-à-vis des autres membres de la famille, ceux-ci n'étant plus régis par des obligations familiales précises (Ganong \& Coleman, 2006; Schmeeckle et al., 2006; Van Tilburg \& Thomése, 2010). Certaines données 


\section{Modèles explicatifs en gérontologie}

montrent en effet que l'absence de définitions claires des rôles et des statuts dans les familles recomposées rend complexes les liens de soutien non seulement entre beaux-parents et beaux-enfants mais aussi entre parents et enfants biologiques (Ganong \& Coleman, 2006; Van der Pas \& Van Tilburg, 2010). En conséquence, le flou normatif qui caractérise ces nouvelles formes familiales créée de la complexité non seulement en leur sein mais aussi dans l'ensemble des relations familiales qui y sont connectées. Plus complexes et incertaines, les relations familiales - y compris les liens de soutien entre parents et enfants de même sang - sont amenées à être davantage négociées au cours du temps et des circonstances, créant davantage de tensions, de conflit et d'ambivalence (Allan, 2008; Connidis, 2015; Silverstein et al., 2006). Les relations intergénérationnelles au sein des familles contemporaines se sont donc fortement diversifiées et complexifiées (Allan, 2008; Schmeeckle et al., 2006; Van der Pas \& Van Tilburg, 2010) et les obligations familiales qui les sous-tendent sont, même si elles sont encore fortes aujourd'hui, loin de garantir, à elles seules, la solidarité intergénérationnelle (Ganong \& Coleman, 2006; Silverstein et al., 2006; Silverstein \& Bengtson, 1997).

\subsubsection{Le modèle de la solidarité intergénérationnelle}

Face à cette complexification grandissante, certains gérontologues se sont interrogés sur la «capacité » des familles contemporaines à apporter à leurs membres âgés aide et soutien jusqu'à la fin de leur vie (Pyke \& Bengtson, 1996; Silverstein \& Giarrusso, 2010; Suitor et al., 2011). C'est dans ce contexte d'incertitudes que Vern Bengtson et de son équipe (Roberts \& Bengtson, 1990; Bengtson \& Roberts, 1991) proposent, dans les années 90, le modèle théorique de la solidarité intergénérationnelle qui vise à mieux saisir les échanges d'aide entre parents âgés et enfants adultes aux dernières étapes de la vie. Dans leur modèle, Vern Bengtson et ses collègues définissent la solidarité intergénérationnelle comme un « construit» multidimensionnel composé de six dimensions indépendantes. Celles-ci reflètent aussi bien les aspects affectifs, normatifs, comportementaux, cognitifs que structuraux des relations intergénérationnelles (de sang) (Bengtson \& Roberts, 1991; Parrott \& Bengtson, 1999; Silverstein 
\& Bengtson, 1997). Dans ce modèle, les obligations familiales, qui constituent l'une de ses dimensions (normatif), ne sont plus au centre de la solidarité intergénérationnelle.

Parmi ces différentes dimensions, il y a la dimension fonctionnelle qui renvoie aux échanges de soutien (pratique et émotionnel) entre parents âgés et enfants adultes et celle, dite associative, qui se rapporte aux contacts et aux activités partagées (réunions familiales, loisirs, etc). Les sentiments d'obligation et les attentes qui caractérisent les relations intergénérationnelles constituent la dimension normative. Quant à la dimension consensuelle, elle se réfère au degré d'adhésion aux mêmes valeurs, croyances et styles de vie entre les parents âgés et leurs enfants. La dimension affective a trait à l'attachement affectif entre les parents âgés et leurs enfants adultes. Elle renvoie plus particulièrement à l'ensemble des sentiments positifs (proximité affective, intimité, confiance, respect, compréhension, etc.) ressentis à l'égard de l'autre. Finalement, la dimension structurale se rapporte à tous les facteurs structurels, qui facilitent - ou qui limitent - les opportunités d'interactions tels que la proximité (distance) géographique, le statut marital des protagonistes, les ressources à disposition, la santé ou le besoin d'aide qui peut activer la relation intergénérationnelle. Plus ces opportunités sont grandes, plus elles stimulent la solidarité intergénérationnelle et à l'inverse, plus elles sont limitées, plus elles l'entravent (Bengtson \& Roberts, 1991; Parrott \& Bengtson, 1999; Silverstein \& Bengtson, 1997).

Ce modèle comporte plusieurs atouts. Son caractère multidimensionnel permet de rendre davantage compte de la complexité des relations intergénérationnelles. En effet, la solidarité comporte aussi bien un aspect «manifeste » (contacts et échanges) qu'un aspect « latent» (affection, normes et valeurs) qu'il faut nécessairement considérer. Dans sa forme «latente ou potentielle», la solidarité représente une ressource importante qui peut - ou ne pas - être mobilisée en cas de besoin (Riley, 1983; Riley \& Riley, 1996; Silverstein et al., 2006). Son activation dépendra, entre autres, des "opportunités structurelles » qui initient, facilitent ou limitent les échanges d'aide (Silverstein et al., 2006; Silverstein \& Bengtson, 1997). Ce modèle permet aussi de mettre en évidence la pluralité des relations intergénérationnelles dans la vieillesse. Sur la base de ces diverses dimensions, Vern Bengtson et son équipe construisent une typologie des relations intergénérationnelles (Bengtson, 2001; Silverstein, Lawton, \& Bengtson, 1994; Silverstein \& Bengtson, 1997). Certaines d'entre elles se 


\section{Modèles explicatifs en gérontologie}

caractérisent par de l'affection et des échanges d'aide fréquents, d'autres affichent un échange d'aide soutenu mais peu d'affection, d'autres sont peu actives mais reposent sur une forte potentialité (affection et consensus) et, finalement, certaines se distinguent par leur absence totale d'affection et de solidarité (Bengtson, 2001; Lawton, Silverstein, \& Bengtson, 1994a; Silverstein et al., 1994). Ces divers types sont distribués de manière égale dans la population âgée américaine, ce qui confirme la grande diversité des relations intergénérationnelles dans la vieillesse, certaines étant marquées par une forte solidarité alors que d'autres en sont totalement exemptes (Bengtson, 2001; Silverstein \& Bengtson, 1997).

\subsubsection{Les limites du modèle de la solidarité intergénérationnelle}

Malgré sa prégnance, ce modèle a fait l'objet de nombreuses controverses dans la littérature gérontologique (Connidis, 2012; Connidis \& McMullin, 2002; Lowenstein, 2007; Lüscher, 2000; Silverstein \& Giarrusso, 2010). On lui reproche de reposer sur une conception idéalisée de la famille, formant une unité harmonieuse sans heurts et contradictions (Connidis \& McMullin, 2002; Lüscher \& Hoff, 2013; Lüscher \& Pillemer, 1998). Le terme même de « solidarité » conduit à ne regarder les relations intergénérationnelles que dans leur facette positive (Connidis \& McMullin, 2002; Lüscher, 2000; Marshall \& Mueller, 2003). Plus concrètement, seuls les affects positifs sont considérés pour approcher la dimension affective (affection, chaleur, la confiance, etc.) alors que les aspects négatifs (conflits, tensions, contrôle, jalousie, haine, ressentiment, violence, etc) sont tout simplement négligés dans sa formulation (Connidis \& McMullin, 2002; Lüscher, 2000; Marshall et al., 1993). De fait, les scores bas sur l'ensemble des affects positifs indiquent seulement une absence de solidarité mais ne disent rien quant aux aspects négatifs de la vie familiale (Connidis \& McMullin, 2002; Lüscher, 2000; Lüscher \& Pillemer, 1998). Ces derniers, comme les conflits ou la violence, font l'objet d'autres études et, comme pour la solidarité intergénérationnelle, ils ne sont scrutés que sous leur angle négatif, faisant fi des aspects positifs qui caractérisent les relations familiales, même conflictuelles (Connidis \& McMullin, 2002; Lüscher \& Hoff, 2013; Marshall et al., 1993). 
Critiquant cette polarisation, de nombreux chercheurs relèvent que les aspects tant positifs que négatifs définissent les relations intergénérationnelles (Lüscher \& Hoff, 2013; Lüscher \& Pillemer, 1998; Szydlik, 2008). Se basant sur diverses études empiriques, Lüscher et Pillemer (1998) rapportent, par exemple, que les relations intergénérationnelles qui atteignent les scores les plus élevés sur la plupart des dimensions de la solidarité (affection, proximité géographique, contacts et échanges de soutien fréquents) sont aussi celles qui affichent un plus haut degré de conflictualité. D'ailleurs, plus les relations sont considérées comme proches affectivement, plus elles sont décrites par les répondants comme problématiques et conflictuelles (Connidis, 2015; Fingerman, Hay, \& Birditt, 2004; Widmer, 2016). Les tensions et les conflits sont, pour ainsi dire, si intégrés dans les relations intergénérationnelles qu'ils n'ont aucun impact sur le montant total de l'aide échangée (instrumentale, financière et émotionnelle) et sa réciprocité (Parrott \& Bengtson, 1999). Parce que de telles contradictions sont inhérentes aux relations intergénérationnelles, elles doivent être observées sous leurs différentes facettes afin de mieux rendre compte de toute leur complexité (Lüscher, 2000; Lüscher \& Pillemer, 1998). Pour ce faire, ces différents auteurs proposent l' « ambivalence» comme modèle alternatif à celui de la solidarité intergénérationnelle (Lüscher, 2000). Le modèle de l'« ambivalence intergénérationnelle » permet de concevoir les sentiments mixtes, les attitudes et les comportements contradictoires comme parties intégrantes des relations intergénérationnelles. Les tensions et le conflit ne sont plus considérés comme des signes de dysfonctionnement mais plutôt comme faisant partie des dynamiques familiales (Connidis \& McMullin, 2002; Lüscher, 2002; Lüscher \& Hoff, 2013) et doivent, de fait, être analysés ensemble puisqu'ils se rapportent à la même réalité familiale (Lüscher, 2002).

Bien que le conflit ait été ensuite intégré dans le modèle de la solidarité intergénérationnelle en tant que septième dimension, donnant ainsi lieu au modèle de solidarité-conflit (Bengtson, Giarrusso, Mabry, \& Silverstein, 2002; Clarke, Preston, Raksin, \& Bengtson, 1999; Schmeeckle et al., 2006), il n'en demeure pas moins que la solidarité et le conflit sont considérés de manière séparée dans ce nouveau modèle. Ce dernier postule en effet que la solidarité et le conflit sont indépendants l'un de l'autre, répondant chacun à des facteurs différents. Par ailleurs, les mesures du conflit restent focalisées sur des attitudes spécifiques telles que des divergences par rapport à l'éducation des petit-enfants ou des choix politiques mais ne 


\section{Modèles explicatifs en gérontologie}

reflètent en aucun cas des processus relationnels (Connidis \& McMullin, 2002; Lüscher, 2002). En somme, le conflit est perçu comme un indicateur de dysfonctionnement, décrivant des relations intergénérationnelles difficiles. Autrement dit, il est rarement lié à la dynamique relationnelle qui sous-tend toutes relations interpersonnelles (Connidis \& McMullin, 2002; Widmer, 2016). De fait, le modèle de la solidarité intergénérationnelle - même dans sa version actualisée (modèle de solidarité-conflit) reste un modèle trop restrictif qui ne parvient pas à mettre en lumière toute la diversité et la complexité des relations intergénérationnelles.

Ce qui n'est pas le cas du modèle de l'ambivalence intergénérationnelle qui admet, dans sa formulation, la simultanéité de la solidarité et du conflit au sein des relations intergénérationnelles, faisant de l'ambivalence un concept premier, surplombant celui de la solidarité et du conflit (Lüscher, 2002). Ce modèle permet d'aller plus loin que le modèle de la solidarité intergénérationnelle dans la compréhension des relations entre enfants adultes et parents âgés dans la vieillesse. Toutefois, il reste, comme le modèle de la solidarité intergénérationnelle, principalement limité à l'étude des dyades intergénérationnelles. Autrement dit, le réseau complexe de liens d'interdépendance affective et pratique dans lequel toute dyade familiale s'insère n'est, pour ainsi dire, jamais pris en compte. Pourtant, les relations qui constituent les configurations familiales et auxquelles les différentes dyades sont rattachées peuvent impacter la façon dont les dyades s'organisent, s'échangent du soutien et gèrent leurs tensions. En effet, en raison des étroites interdépendances affectives et pratiques qui sous-tendent les configurations familiales, il est probable que la manière dont s'organise le soutien familial et la façon dont se gèrent les conflits dans l'ensemble du réseau familial influencent le soutien et le conflit au sein de ces dyades. Or, l'influence du réseau familial n'est jamais étudiée et rarement évoquée en gérontologie sociale comme si les dyades intergénérationnelles constituaient des entités à part, pouvant sans autre être extirpées de leur contexte familial et social. Pourtant, un tel contexte doit être pris en compte pour saisir toute la complexité des relations intergénérationnelles (Bott, 1955; Marshall et al., 1993; Widmer, 2016).

Alors que la solidarité et l'ambivalence dans les relations intergénérationnelles ont été largement étudiées en gérontologie sociale, nous proposons dans cet ouvrage de prendre de la hauteur et de sortir du cadre dyadique pour nous intéresser à l'ambivalence au niveau du contexte familial en tenant compte de l'ensemble des liens d'interdépendance qui 
sous-tendent les configurations familiales. Ce faisant, nous nous intéresserons aussi bien à l'organisation des liens de soutien qu'aux tensions au sein des configurations familiales. Soutien et tensions sont ici considérés comme faisant partie intégrante des dynamiques familiales. Dans cette perspective, le conflit n'est pas un indicateur de difficultés spécifiques mais un signe d'ajustement ou de ré-équilibrage relationnel au sein du contexte familial. L'articulation des deux - soutien et conflit - nous permet d'observer comment les membres familiaux gèrent les déséquilibres relationnels au sein de leur configuration familiale, ce qui n'a jamais été étudié en gérontologie sociale, du moins de manière quantitative.

\subsection{Les normes de réciprocité et le «Support bank»}

Les obligations familiales qui sous-tendent les relations intergénérationnelles ont été évoquées au début de la section précédente. Dans cette prochaine section, nous allons aborder d'autres types de normes qui n'en sont pas moins importantes pour le maintien des relations familiales à savoir les normes de réciprocité dans les échanges interpersonnels. Dans la théorie de l'échange social, qui s'appuie sur des principes économiques, toute mise en relation, quelle que soit sa nature, est «a priori » stratégique : les individus s'engagent dans une relation en vue d'obtenir les ressources nécessaires à la satisfaction de leurs besoins, faisant de la relation à l'autre une exigence (Blau, 1964; Kelley \& Thibaut, 1978; Offer, 2012). Ainsi, le lien entre deux protagonistes se tisse, se développe et se renforce au gré de leurs échanges, initiés et motivés par leur désir commun de satisfaire leurs besoins personnels (Cropanzano \& Mitchell, 2005). Ces échanges qui caractérisent les relations dyadiques, y compris familiales, n'ont rien d'aléatoires, ils sont régis par des normes de réciprocité (Blau, 1964; Gouldner, 1960; Kelley \& Thibaut, 1978; Molm, Collett, \& Schaefer, 2007). Celles-ci structurent les relations interpersonnelles en exigeant que l'échange qui se produit soit équilibré, équitable ou perçu comme tel, pour que la relation dyadique se développe et se maintienne, et cela même dans les relations les plus proches (Blau, 1964; Cropanzano \& Mitchell, 2005; Gouldner, 1960). Autrement dit, tout ce que donne un individu à son partenaire relationnel, en termes de services ou de biens, doit lui être rendu 
sous une forme jugée équivalente, quel que soit son statut, son rôle familial ou social. Si ces normes sont respectées, elles génèrent au sein du lien dyadique une reconnaissance mutuelle (de soi et de l'autre) qui, indépendamment de la "volonté propre» des deux membres de la dyade, influence le cours de leur relation en l'intensifiant et en la consolidant (Kadushin, 2012).

L'équilibre, réel ou perçu, dans les échanges réciproques répond au besoin de cohérence des individus, gage de leur bien-être psychologique. En effet la non-réciprocité dans les échanges dyadiques génère, de part et d'autre de la relation, un malaise, de la culpabilité, de la frustration, une perte de sentiment de contrôle et la dépression (Antonucci et al., 2007; Cornwell, 2009a; Offer, 2012). Cette norme assure aussi la stabilité dans les relations, l'équité dans les échanges et, en prévenant l'exploitation de certains, elle contribue à renforcer la cohésion et l'intégration sociale (Gouldner, 1960; Johnson, 1988; Molm et al., 2007). En termes de support, aider ceux qui nous ont aidés ou qui nous aident développe la solidarité entre les individus (Molm et al., 2007; Offer, 2012). La norme de réciprocité insère les individus dans un cycle d'échanges qui les maintient dépendants les uns des autres. En ce sens, cette norme assure une meilleure stabilité des liens que ceux qui sont uniquement régis par des règles et des obligations liées à des rôles et à des statuts familiaux ou sociaux (Antonucci \& Jackson, 1989; Offer, 2012).

Cependant, bien qu'implicite, la norme de réciprocité est exigeante, porteuse de fortes obligations et d'attentes à l'égard des autres. D'une part, pour initier et maintenir le lien, il faut être « solvable », c'est-à-dire être en mesure de pouvoir rendre ce que l'on a reçu pour soutenir l'échange (Offer, 2012). D'autre part, il est fortement attendu de régler ses dettes au risque de déséquilibrer l'échange et de se placer dans une situation de dépendance vis-à-vis de son interlocuteur (Antonucci \& Jackson, 1989; Molm et al., 2007; Silverstein, Conroy, Wang, Giarrusso, \& Bengtson, 2002). En cas de grande vulnérabilité, l'incapacité récurrente de rendre son dû peut conduire les individus dans une situation délicate et générer un énorme stress même dans le contexte familial (Offer, 2012). Ces derniers doivent faire face aux reproches, aux critiques et aux mépris des autres. La rupture, même d'avec les plus proches, devient dès lors fort possible (Offer, 2012). Certains sont exclus de la relation, d'autres s'y retirent volontairement afin d'éviter les jugements négatifs et de préserver une image positive d'eux-mêmes (Offer, 2012). S'appuyant sur la tradition anthropologique 
(Sahlins, 1965), la littérature gérontologique distingue généralement plusieurs formes de réciprocité en fonction de la nature de la relation et de l'échange : la réciprocité équilibrée («balanced reciprocity») et la réciprocité généralisée (Antonucci et al., 2007; Antonucci \& Jackson, 1989; Johnson, 1988; Offer, 2012).

\subsection{1 la réciprocité équilibrée versus généralisée}

La réciprocité équilibrée repose sur un échange équitable de ressources se produisant dans un laps de temps relativement court (Antonucci \& Jackson, 1989; Molm et al., 2007). Les échanges sont négociés, c'est-à-dire que les ressources échangées sont définies et soumises à l'accord des deux protagonistes (Molm et al., 2007). Ces ressources sont, en général, perçues comme équivalentes (Antonucci \& Jackson, 1989; Molm et al., 2007), satisfaisant, dans l'immédiat ou à court terme, les deux interlocuteurs. Ici, les échanges n'induisent pas d'interdépendance entre les deux protagonistes puisque la réciprocité et l'équivalence des ressources sont respectées. La réciprocité équilibrée est de mise soit dans les relations qui sont peu investies affectivement comme celles qui se situent en dehors du contexte familial (associations, voisinage, etc.) soit dans des relations affectives, familiales ou amicales, basées sur l'affinité et motivées par des intérêts et un style de vie commun. Il peut s'agir de relations entre frères et sœurs adultes, entre personnes ayant des liens de parenté éloignée (cousins, etc) ou d'alliance (belle-famille, etc) ou encore entre amis proches (Johnson, 1988; Offer, 2012). Ce sont des relations personnalisées, investies affectivement, initiées et maintenues grâce aux bénéfices (activités communes, compagnonnage, soutien, etc.) que la relation procure à chacun des deux partenaires.

Le strict respect de la norme de réciprocité permet aux deux interlocuteurs d'occuper une place équivalente dans l'échange. Aucun d'eux ne peut prétendre à avoir le pouvoir sur l'autre puisque ni l'un ni l'autre n'est le principal prestataire. Aucun d'eux ne se sent redevable, leurs dettes étant réglées rapidement (Molm et al., 2007). Par conséquent, ces relations sont perçues comme égalitaires, vécues comme choisies et non obligatoires (Johnson, 1988). Le sentiment de redevabilité étant faible, les deux protagonistes se sentent libres de quitter la relation si elle ne leur convient plus, si leurs besoins ne sont plus satisfaits ou s'ils perçoivent leurs échanges 
comme déséquilibrés (Johnson, 1988). Par conséquent, la recherche d'alternatives plus avantageuses peut entraîner la rupture de la relation sans générer pour autant de graves conséquences sur le bien-être des individus (Fingerman et al., 2004). Les relations axées sur la réciprocité équilibrée sont donc, par définition, plus fragiles (Johnson, 1988; Offer, 2012).

Contrairement à sa forme équilibrée, la réciprocité généralisée est beaucoup plus flexible. En effet, les ressources ne sont pas strictement équivalentes; elles peuvent être multiples et de différentes natures. De même, les ressources données n'exigent pas d'être rendues dans l'immédiat; elles peuvent être rendues bien plus tard dans le temps (Antonucci \& Jackson, 1989; Molm et al., 2007; Sahlins, 1965), ce qui contribue à rendre interdépendants les protagonistes de l'échange (Antonucci et al., 2007; Molm et al., 2007; Offer, 2012). Par ailleurs, la réciprocité généralisée ne requiert pas nécessairement que ce soit l'individu bénéficiaire qui rende ce qu'il a reçu. Il peut s'agir d'une tierce personne, en lien aussi bien avec le bénéficiaire qu'avec le prestataire, qui soutient indirectement l'échange; la réciprocité peut donc être indirecte (Molm et al., 2007). Les relations dyadiques de type familial, se caractérisant par une longue histoire d'échanges de ressources multiples et variées, aussi bien affectives que pratiques, sont généralement régulées par cette forme de réciprocité (Antonucci \& Jackson, 1989; Silverstein et al., 2006).

Les relations conjugales et intergénérationnelles en constituent un bon exemple. Dans ce type de relations, le contrôle strict de la réciprocité et de l'équilibre des échanges est impossible puisque le laps de temps entre le bien donné et son rendu peut être long ou octroyé de manière indirecte (Johnson, 1988; Molm et al., 2007). Les enfants adultes peuvent, par exemple, rendre divers services à leurs parents âgés et, en échange, ces derniers soutiennent financièrement leurs petits-enfants. Diffuse et sans limite dans le temps, la réciprocité généralisée rend incertain ce qui est dû par les uns et par les autres (dettes), induisant inévitablement chez les protagonistes un fort sentiment de redevabilité à l'égard de l'autre (Johnson, 1988; Molm et al., 2007). Redevables, ils se sentent « obligés » de maintenir le lien et les échanges, ce qui crée entre eux un solide lien d'interdépendance tant affective que pratique (Johnson, 1988; Offer, 2012). Dans les relations " parents-enfants », un tel lien d'interdépendance contribue à renforcer l'adhésion des protagonistes aux normes de solidarité intergénérationnelle, celles-ci ne pouvant pas être conçues en dehors de relations affectivement investies, personnalisées (Lawton et al., 1994b). 
Dans un tel contexte, enfants et parents (ou partenaires) sont certains d'obtenir l'aide dont ils ont besoin ou auront besoin le moment venu. Cette certitude contribue encore davantage à développer entre eux des relations de confiance et d'affection (Antonucci \& Jackson, 1989; Johnson, 1988; Molm et al., 2007). Soutenue par ce sentiment de redevabilité, la norme de réciprocité généralisée est efficace dans l'activation de comportements d'aide collective au sein de la famille (Johnson, 1988) et nécessaire au maintien de la cohésion familiale (Silverstein \& Bengtson, 1997). Cette norme pousse les membres familiaux de différentes générations à répondre aux demandes de soutien de ceux qui en ont le plus besoin (enfants en bas âge, parent âgé, etc.) (Bowling, 1994; Silverstein \& Bengtson, 1997; Silverstein et al., 2006). Selon le rôle familial des individus au sein de la famille (mère, fille, etc), cette norme de réciprocité peut être particulièrement prégnante et valorisée, se manifestant généralement sous la forme d'obligations familiales à l'égard des autres membres de la famille (Silverstein et al., 2006). Pour certains auteurs, la solidarité familiale ( "gratuite») ne serait que la manifestation, observée à un moment donné, de la réciprocité généralisée, directe ou indirecte, diffuse et différée dans le temps (Antonucci et al., 2007; Johnson, 1988; Offer, 2012). Mesurés à un moment précis - par exemple, en situation de crise (veuvage, accidents, hospitalisation, etc) - le service rendu, le prêt d'argent ou l'aide octroyée peuvent être perçus comme " gratuits » et interprétés comme des actes de générosité ou des manifestations d'altruisme (Lüscher \& Pillemer, 1998). Or, il n'en est rien si l'on considère le flux des échanges tout au long du parcours de vie; une telle aide pourrait être le rendu d'une ressource donnée, due depuis longtemps.

\subsubsection{La banque de support (« support bank»)}

Dans de nombreux travaux en gérontologie sociale, la norme de réciprocité généralisée a été conceptualisée sous la notion de «banque de support» (support bank). Proposée par Toni Antonucci (Antonucci, 1990; Antonucci et al., 2007; Antonucci \& Jackson, 1989), cette notion renvoie aux échanges de support dans les relations dyadiques (intergénérationnelles ou conjugales) qui ont lieu tout au long du parcours de vie. Selon cette perspective, la norme de réciprocité généralisée serait utilisée de manière «stratégique » par les individus au milieu de leur parcours de vie afin de 


\section{Modèles explicatifs en gérontologie}

s'assurer le soutien des membres proches de leur famille (surtout des enfants et du partenaire) dans les dernières étapes de leur parcours de vie (Offer, 2012; Silverstein et al., 2002). Ce concept suggère donc qu'au milieu du parcours de vie, les parents, jeunes et en pleine santé, investissent de nombreuses ressources (argent, temps, affection, etc.) dans l'éducation et les soins de leurs enfants (Antonucci, 1990; Antonucci \& Jackson, 1989; Silverstein et al., 2002). Tant qu'ils le peuvent, ils continuent jusqu'à leur vieillesse à offrir de multiples ressources et à rendre de nombreux services tant à leurs enfants adultes qu'à leurs petits-enfants. A priori, ils donnent sans compter et sans attendre un quelconque retour de leur part, du moins dans l'immédiat (Antonucci, 1990; Antonucci et al., 2007; Antonucci \& Jackson, 1989).

Or, ces ressources octroyées ne sont pas pour autant gratuites; elles représentent une sorte d'investissement « inconscient», une sorte de « compte épargne » de support que les parents pourront mobiliser aux dernières étapes de leur vie lorsqu'ils en auront besoin (Antonucci et al., 2007; Antonucci \& Jackson, 1989; Silverstein et al., 2002). A ce moment-là, soucieux de s'acquitter de leurs dettes, les enfants adultes redonneront à leurs parents âgés toute l'aide et le soutien qu'ils auront reçus tout au long de leur parcours de vie (Antonucci et al., 2007; Antonucci \& Jackson, 1989; Silverstein et al., 2006). S'ils ne le font pas, ils prennent le risque d'être au prise d'un fort sentiment de culpabilité et de honte (Lüscher \& Pillemer, 1998; Rappoport \& Lowenstein, 2007). Quant aux parents âgés devenus bénéficiaires, ils ne se sentent pas à ce moment-là redevables s'ils reçoivent plus qu'ils n'en donnent - sauf si l'aide requise est importante (voir le chapitre sur l'ambivalence) - puisqu'ils interprètent le soutien reçu comme le juste retour de leur investissement passé (Antonucci et al., 2007).

Parce que la norme de réciprocité généralisée est si prégnante, les parents âgés, qui ont investi beaucoup de ressources dans leurs enfants et qui, ce faisant, ont construit un « compte épargne » de support, perçoivent le soutien de leurs enfants comme «allant de soi », fiable et inconditionnel, quelles que soient les difficultés qu'ils rencontrent (Antonucci \& Jackson, 1989; Krause, 1997a ; Pinquart \& Sörensen, 2000). Par les interdépendances affectives et pratiques qu'elle crée, la réciprocité généralisée contribue à pérenniser les liens et les échanges intergénérationnels, rendant la rupture « a priori » difficilement envisageable. Même si les relations intergénérationnelles ou conjugales peuvent être tendues, conflictuelles 
ou ambivalentes (Lüscher \& Pillemer, 1998), tant les enfants que les parents ont la certitude qu'ils obtiendront toujours l'aide escomptée par les membres proches de leur famille (Antonucci \& Jackson, 1989; Krause, 1997a, 1997b). Certains auteurs évoquent le terme de support «anticipé » ou « potentiel » (Ashida \& Heaney, 2008; Krause, 2006, 1997a) et c'est cette certitude, rassurante et source d'espoir, qui explique le lien positif entre support et santé aussi bien physique que mentale chez les personnes âgées (Jang et al., 2002; Krause, 1997a, 1997b; Shaw \& Janevic, 2004).

\subsubsection{Les limites de l'approche dyadique de la réciprocité et du «Support bank»}

La prise en compte des normes d'échanges, telles que la réciprocité «généralisée », est fondamentale pour comprendre les liens d'interdépendance pratique et affective qui se tissent entre les différents membres des réseaux familiaux et qui produisent en leur sein un potentiel de support ou, comme le décrit Toni Antonucci, une sorte de compte épargne de soutien. Dans cette perspective, la solidarité intergénérationnelle s'inscrit dans le « structurel » ou le « relationnel» plutôt que dans le «statutaire». Cette perspective va donc plus loin que la conception uniquement «statutaire» des normes de solidarité intergénérationnelle qui lie les sentiments d'obligations familiales aux rôles et aux statuts au sein de la famille (" enfants » versus « parents ») (Silverstein et al., 2006). Si l'on considère au contraire la norme de réciprocité généralisée, les obligations familiales naissent de ce fort sentiment latent de redevabilité ; elles ne dépendent plus exclusivement des statuts familiaux mais elles résultent du jeu complexe des échanges intergénérationnels.

Bien que de nombreuses études en gérontologie sociale considèrent les normes de réciprocité dans les échanges familiaux et reconnaissent leur impact sur la construction des liens d'interdépendance, elles ne dépassent pas dans leur analyse le cadre dyadique. La notion de "support bank» fait, en effet, principalement référence au support latent ou potentiel au sein des relations conjugales et surtout intergénérationnelles. Comme pour le modèle de la solidarité intergénérationnelle, l'ensemble des liens d'interdépendance dans lequel s'insèrent ces dyades n'est jamais considéré. Dans cet ouvrage, nous proposons d'aller plus loin en suggérant que les normes de réciprocité régissent le flux des échanges dans l'ensemble du 


\section{Modèles explicatifs en gérontologie}

réseau familial et, ce faisant, elles génèrent en son sein une sorte de sentiment latent de redevabilité, prometteur d'un soutien collectif en cas de besoin. Nous passerons de la notion de « support bank », utilisée dans une majorité de travaux en gérontologie pour définir le potentiel de support dyadique, à celle de « capital social » qui tient compte de l'ensemble des liens d'interdépendance au sein du réseau familial.

Le concept de «capital social», qui fera l'objet d'un prochain chapitre (cf. chapitre 6), renvoie à l'ensemble des ressources individuelles (y compris le soutien) produites au sein de réseaux personnels et auxquelles peuvent accéder les membres qui en ont besoin (Bourdieu, 1980, 1986; Moren-Cross \& Lin, 2006). Le capital social dépend étroitement des normes de réciprocité qui structurent les échanges au sein du réseau personnel (Bourdieu, 1980, 1986; Moren-Cross \& Lin, 2006). Selon le type de réciprocité qui les régit (« équilibrée» vs " généralisée »), les échanges au sein des réseaux personnels s'organisent de manières différentes, donnant lieu à plusieurs types de capital social. Bien qu'il soit généralement attendu que les échanges familiaux soient régis par la réciprocité «généralisée », nous verrons dans ce travail que plusieurs types de capital social peuvent être générés au sein des configurations familiales, sous-entendant dès lors l'existence de normes d'échanges différentes, et cela même dans les familles. En analysant les propriétés structurelles des réseaux familiaux, nous pourrons déterminer la façon dont s'organisent les échanges en leur sein et identifier, pour chacun d'entre eux, le type de capital social produit. La notion de capital social permet de prendre de la hauteur et de quitter le niveau dyadique pour observer les échanges de soutien à un niveau plus large, celui du réseau familial. Dans la prochaine section, nous aborderons le dernier modèle théorique qui a dominé la gérontologie sociale ces dernières décennies, à savoir la «sélectivité socio-émotionnelle» de Laura Carstensen.

\subsection{La sélection comme mode d'adaptation dans la vieillesse}

De nombreux chercheurs en psychologie ont souligné l'existence de mécanismes d'adaptation socio-cognitifs qui aident les individus à faire face au processus de fragilisation auquel ils sont confrontés aux dernières étapes 
de leur parcours de vie (Baltes \& Baltes, 1990; Carstensen, 1991, 1992). Ces différents mécanismes ou stratégies d'adaptation permettent aux individus âgés de compenser l'effet délétère du déclin de la santé sur leur bien-être (Baltes \& Baltes, 1990; Freund, Li, \& Baltes, 1999). Le modèle théorique «SOC », avancé par Baltes (Baltes \& Baltes, 1990; Freund et al., 1999), met en exergue plusieurs de ces stratégies : la « sélection», l'« optimisation» et la «compensation ». Ces différents mécanismes permettent aux individus de s'adapter aux étapes successives du parcours de vie (Baltes \& Smith, 2003; Freund et al., 1999) et le maintien du bien-être subjectif lors de transitions difficiles témoigne de leur efficacité (Baltes \& Smith, 2003; Freund et al., 1999). Parmi ces mécanismes, la sélection est non seulement prégnante dans la vieillesse mais elle s'avère être aussi une stratégie efficace, notamment dans le domaine relationnel (Baltes \& Smith, 2003; Freund et al., 1999), au point que Laura Carstensen et son équipe ont modélisé ce mécanisme en proposant la théorie de la « sélectivité socio-émotionnelle » (Carstensen, 1991, 1992). Contrairement aux autres modèles exposés précédemment, cette théorie se centre sur l'individu âgé et sa capacité à «gérer » ses ressources relationnelles pour s'adapter au mieux au déclin de la santé. En somme, la sélection relève davantage de stratégies individuelles d'adaptation que de dynamiques relationnelles. Nous la présentons ici brièvement en montrant ses apports mais aussi ses limites.

\subsubsection{La sélection au cours du parcours de vie}

Comme les autres mécanismes d'adaptation, le processus de sélection débute bien avant la vieillesse et s'intensifie progressivement au fil des ans. Ce sont d'abord les contacts avec les connaissances ou les « copains » qui tendent à s'affaiblir lors des premières années de la vie adulte (vers 30 ans) alors que les contacts avec la famille et les amis proches (affectivement) tendent peu à peu à s'intensifier. Cet approfondissement progressif des liens avec les proches s'accompagne au gré du temps d'une plus grande proximité émotionnelle et d'une plus grande satisfaction relationnelle. Ce processus permettrait à l'individu de se construire un réseau d'aide et de reconnaissance, composé de membres particulièrement fiables, aptes à l'accompagner tout au long de son parcours de vie (Carstensen, 1991, 1992). Aux dernières étapes de la vie, ce mécanisme s'intensifie avec le processus de fragilisation, donnant lieu à une réduction significative de la 


\section{Modèles explicatifs en gérontologie}

vie relationnelle des personnes âgées. Ne disposant plus des ressources nécessaires (santé, énergie, capacités psychologiques, etc) pour entretenir des liens réciproques avec tous les membres de leur réseau personnel et, plus encore, avec l'imminence de leur propre mort (temps restreint), les individus vieillissants «sélectionnent» les relations qui sont, à leurs yeux, les plus importantes, les plus significatives, celles qu'ils associent à la satisfaction «immédiate » de leurs besoins tant identitaires qu'affectifs (Carstensen, 1992; Carstensen, Pasupathi, Mayr, \& Nesselroade, 2000; Shaw, Krause, Liang, \& Bennett, 2007). Étant le fruit d'une stratégie, l'affaiblissement de la vie relationnelle ne s'accompagne pas d'un déclin du bien-être; elle s'associe au contraire à une plus grande satisfaction relationnelle (Carstensen, 1991, 1992).

\subsubsection{La théorie de la sélectivité socio-émotionnelle}

La « sélection » des autruis significatifs ne se fait pas à la légère; les individus âgés doivent satisfaire leurs besoins non seulement identitaires mais aussi émotionnels. Ils choisissent, par exemple, des membres de leur réseau personnel qui sont valorisants à leur égard, qui leur renvoient une image positive d'eux-mêmes et se délestent des personnes qui - par leurs propos, leurs attitudes ou leur situation - nuisent à l'image qu'ils se font d'euxmêmes (self-concept) (Carstensen, 1991, 1992; Carstensen et al., 2000). Non seulement ils accordent plus d'importance à la qualité de leurs relations mais leur bien-être en dépend aussi davantage (Pinquart \& Sörensen, 2000). Plus que le maintien de l'identité, la régulation des émotions devient centrale dans la vieillesse - et plus encore à l'approche de la mort - si bien que les moments de vie riches en émotions positives sont particulièrement valorisés (Carstensen, 1992; Carstensen et al., 2000; Shaw et al., 2007). En conséquence, les très âgés préfèrent passer le peu de temps qui leur reste avec des personnes qu'ils connaissent bien et qu'ils apprécient, des personnes avec lesquelles ils ont partagé des événements forts aussi bien heureux que malheureux, plutôt que d'épuiser leur temps et leur énergie à entretenir des relations avec des personnes qu'ils connaissent peu et dont l'issue est incertaine (Carstensen, 1992; Carstensen et al., 2000; Shaw et al., 2007). Ce faisant, ils déclarent se sentir moins proches affectivement des membres de leur réseau personnel (Cornwell et al., 2008, 2009) et 
jugent leurs relations non familiales de moins bonne qualité (Merz \& Huxhold, 2010). Ils se concentrent dès lors davantage sur des relations qu'ils perçoivent de haute qualité, potentiellement aidantes, et auxquelles ils sont affectivement attachés - généralement les membres primaires de la famille (partenaire et enfants, parfois, fratrie), définis dans la littérature comme affectivement proches, ou des amis intimes - ceux en qui ils ont une pleine confiance et avec qui ils souhaitent vivre pleinement les derniers moments de leur vie (Carstensen, 1992; Carstensen et al., 2000; Merz \& Huxhold, 2010). Les individus âgés ayant un statut socio-économique élevé - majoritairement des hommes - ont une plus forte propension, avec l'âge avançant, de sélectionner les relations qui leur sont le plus gratifiantes, généralement le partenaire et les enfants (Shaw et al., 2007).

En l'absence de tels membres, les individus âgés peuvent aussi s'investir affectivement dans une nouvelle relation, sans lien de sang ni d'alliance (un voisin ou une aide professionnelle, par exemple) invitant au cœur même de leur réseau personnel un nouveau membre. Une telle relation, même très récente, peut donc être surinvestie affectivement par les individus âgés qui, à l'approche de la mort, survalorisent les dernières expériences de vie (Carstensen et al., 2000). Les interactions avec les membres qu'ils ont sélectionnés sont perçues comme émotionnellement gratifiantes, leur procurant des émotions positives (affection, plaisir, joie, confiance et sécurité) qui, aux yeux de ceux dont le temps est compté, prennent davantage de valeur et de signification (Carstensen, 1992; Carstensen et al., 2000; Shaw et al., 2007). Le besoin de maintenir des relations affectives qu'ils jugent positives est tel qu'ils n'en perçoivent plus - ou moins - les aspects négatifs (tensions, conflits, etc.). Alors qu'à d'autres étapes du parcours de vie, les relations avec les membres proches de la famille sont décrites comme ambivalentes, elles sont, aux ultimes étapes de la vie, estimées comme essentiellement positives (Fingerman et al., 2004).

Afin de pouvoir investir pleinement («optimisation») le peu de ressources dont ils disposent encore (énergie, temps, etc.) dans les relations qu'ils perçoivent non seulement comme les plus gratifiantes mais aussi comme les plus fiables (Carstensen, 1991; Freund et al., 1999), les individus âgés se désengagent des relations qu'ils ne considèrent pas ou plus significatives. Ils « compensent», en somme, la quantité par la qualité relationnelle en optant pour des relations riches émotionnellement et particulièrement gratifiantes, c'est-à-dire celles qui leur renvoient une image 


\section{Modèles explicatifs en gérontologie}

positive d'eux-mêmes. Résultat de ces divers modes d'adaptation (sélection, désengagement et investissement), la diminution des liens et de la vie relationnelle reflète la capacité des individus âgés à agir sur leur environnement social afin d'en garder le contrôle le plus longtemps possible. Selon la théorie de la sélectivité socio-émotionnelle, la perte de ce contrôle peut générer une baisse importante du bien-être chez les très âgés (Carstensen, 1991).

\subsubsection{Les limites de la théorie de la sélectivité socio-émotionnelle}

La théorie de la sélectivité socio-émotionnelle est intéressante car elle met en évidence la capacité d'agir des individus sur leur environnement et d'influer, même à des âges très avancés, leur contexte relationnel. Dans sa formulation, le processus de sélectivité socio-émotionnelle s'arrête, aux dernières étapes de la vie, à un nombre restreint de membres de la famille, généralement les membres dit " primaires » de la famille, à savoir le partenaire et les enfants les plus proches (sauf s'ils sont inexistants). Ces derniers sont désignés dans la littérature comme significatifs pour les individus âgés en raison de la valeur émotionnelle qu'il leur est attribuée de manière « automatique ». Or, ce présent ouvrage remettra en question cette idée préconçue en montrant que, pour certains individus âgés (y compris les plus âgés), les membres sélectionnés comme émotionnellement gratifiants ou significatifs ne renvoient pas forcément à des membres de la famille « nucléaire ». Ils peuvent faire référence à une diversité de membres familiaux ou non familiaux. Certains, certes, se centreront sur leur partenaire et leurs enfants les plus proches, mais d'autres choisiront des membres de la famille qui sortent du cadre bio-légal attendu. A notre connaissance, la théorie de la sélectivité socio-émotionnelle n'a jamais été utilisée pour observer le processus de sélection au sein même du réseau familial. Il est pourtant probable que, même au sein de la famille, les personnes âgées recourent activement à ce mécanisme, les amenant à se construire des configurations familiales distinctes, diversifiées et fortement significatives, qui répondent au mieux à leurs besoins émotionnels.

Bien que dominante dans le champ de la gérontologie sociale, la théorie de la sélectivité socio-émotionnelle a essuyé quelques critiques dans la littérature. Pour certains auteurs, par exemple, l'individu très âgé ne « choisit » pas les liens qu'il investit affectivement mais il se contente plutôt des 
relations dont il dispose encore à l'approche de la mort. Ce seraient plutôt la rareté des réserves démographiques, c'est-à-dire avoir un nombre restreint de membres de la famille encore en vie, et surtout l'absence d'alternatives (absence de parenté ou d'amis) qui pousseraient les individus âgés à investir davantage les relations familiales restantes, à minimiser leurs éventuels aspects négatifs et à les considérer comme des liens significatifs (Fingerman \& Birditt, 2003). Bien-sûr, certaines personnes âgées ont peu de famille ou de parenté (réserves démographiques) pour faire un «vrai » choix et ne préserver, en somme, que les relations qu'elles considèrent comme gratifiantes. Cependant, disposer d'un réservoir démographique restreint, c'est-à-dire avoir un nombre limité de membres de la famille ou de la parenté encore en vie, ne signifie pas pour autant que l'on développe des relations affectives fortes avec eux et que l'on évalue ces liens familiaux comme valorisants et gratifiants. Des données empiriques confirment en effet que l'absence d'alternatives dans la vieillesse - c'est-à-dire avoir un réseau personnel composé uniquement de quelques membres de la famille - ne s'associe pas à un plus grand investissement affectif, ni à un meilleur bien-être subjectif (Cornwell et al., 2008; Fiori et al., 2008, 2006; Litwin, 2001 ; Wenger, 1997). L'existence de membres familiaux et la proximité affective ne sont donc pas forcément corrélées, notamment si les relations familiales ne sont pas activées et affectivement investies (Antonucci et al., 2007; Cornwell et al., 2008; Pahl \& Spencer, 2004). L'absence d'alternatives relationnelles dans le grand âge ne suffit donc pas à rendre compte du mécanisme de la sélectivité socio-émotionnelle. Ce processus de sélection se met en place quel que soit l'état des réserves démographiques.

Plus que l'état des réserves démographiques, c'est la non prise en compte des liens d'interdépendance affective et pratique dans lequel l'individu âgé s'insère qui pose problème. La théorie de la sélectivité socioémotionnelle s'axe sur l'individu et sa capacité à « choisir et à gérer» ses ressources relationnelles; mais elle néglige que ce dernier baigne dans un réseau complexe de liens d'interdépendance relationnelle qui se modèle et se renforce tout au long de son parcours de vie. La non prise en compte de ces liens constitue une sérieuse limite à ce modèle. En effet, l'individu âgé n'est pas seul; il dépend étroitement des membres qui constituent son réseau personnel et qui l'influencent dans ses choix et ses stratégies. Sa marge de manœuvre est donc relativement limitée si l'on tient compte de l'ensemble des relations dont il dépend. Par conséquent, les stratégies qu'il déploie - la sélection et le désengagement - ne peuvent se mettre en 


\section{Modèles explicatifs en gérontologie}

place que si certaines propriétés structurelles de son réseau familial le lui permettent.

Si les liens d'interdépendance au sein de son réseau familial sont denses, c'est-à-dire si tous les membres de son réseau se connaissent ou s'échangent des ressources, l'autonomie de l'individu est fortement réduite. Ce dernier ne peut que difficilement mettre en place des stratégies de sélection ou de désengagement s'il s'insère dans un réseau dense d'interdépendances. C'est le cas notamment lorsque les membres du réseau familial se mobilisent autour du parent âgé malade et se coordonnent pour lui apporter l'aide dont il a besoin, la marge de manœuvre de l'individu âgé est ici particulièrement limitée. Dépendant directement de l'aide de certains membres de son réseau, il ne peut pas se désengager d'une relation qu'il juge négative et non gratifiante au risque de déstabiliser le réseau d'aide dont il dépend étroitement. Par ailleurs, selon l'état de sa santé, il n'a plus l'énergie suffisante pour chercher ailleurs l'aide dont il aurait besoin (auprès d'amis, par exemple). Autrement dit, s'investir dans une relation plutôt qu'une autre (sélection) et se désengager de certaines relations (désengagement) ne sont pas sans conséquence dans un réseau au sein duquel tous les membres sont en lien. Ces mécanismes peuvent générer des déséquilibres, des jalousies, des tensions et du conflit dans l'ensemble du réseau, et cela d'autant plus s'il est composé de membres qui se connaissent bien et depuis longtemps comme dans le contexte familial.

Par contre, si les membres du réseau familial sont peu reliés entre eux, l'individu âgé bénéficie d'une plus grande autonomie. Il peut dès lors agir et moduler sa configuration familiale afin qu'elle réponde au mieux à ses besoins émotionnels sans qu'il y ait des risques d'y déséquilibrer les échanges. Il peut sélectionner les relations qui lui semblent le plus bénéfiques et s'y investir pleinement, tout en se désengageant de celles qu'il considère comme moins importantes. La marge de manœuvre des individus dépend donc étroitement de l'organisation des liens d'interdépendance qui sous-tendent leur configuration familiale. La prise en compte des propriétés structurelles des réseaux familiaux est nécessaire pour mieux comprendre les variations interindividuelles dans la mise en œuvre de ces diverses stratégies d'adaptation et leur efficacité dans la vieillesse. Grâce aux outils de l'analyse de réseau, nous pouvons mesurer les propriétés structurelles des réseaux familiaux dans lesquels les individus âgés s'insèrent. Ce faisant, nous pouvons évaluer pour chacun d'entre eux l'étendue de leur marge 
de manœuvre, c'est-à-dire leur degré d'autonomie au sein de leur configuration familiale respective. En mesurant l'autonomie relationnelle des personnes âgées dans leur réseau familial, nous pouvons identifier celles qui, relativement autonomes, peuvent mettre en œuvre la sélectivité socioémotionnelle et cela jusqu'à des âges avancés, et celles qui, insérées et contraintes dans un réseau familial relativement dense, peinent à mettre en place une telle stratégie d'adaptation.

\subsection{Conclusion}

Dans ce chapitre, nous avons abordé les principaux modèles explicatifs qui ont guidé, ces dernières décennies, la recherche en gérontologie sociale dans le domaine des relations sociales et familiales. Bien qu'ils comportent de nombreuses limites et de sérieux manques, tous ces modèles ont néanmoins contribué à une meilleure connaissance des relations sociales et familiales dans la vieillesse en y apportant, chacun, un éclairage spécifique. Certains, comme le modèle du convoi de Toni Antonucci et son équipe, expliquent l'évolution de la structure (taille et composition) des réseaux personnels au cours du parcours de vie, en mettant en exergue leur diversité en fonction des profils des individus âgés et de la pluralité de leurs trajectoires familiales. D'autres modèles se sont focalisés sur les relations dyadiques, notamment intergénérationnelles, comme celui de la solidarité intergénérationnelle de Vern Bengtson ou encore le «support bank » de Toni Antonucci. L'un comme l'autre ont montré toute la complexité de la solidarité intergénérationnelle, celle-ci ne pouvant pas s'expliquer uniquement par la prégnance des obligations familiales qui régissent les comportements d'aide. Ces modèles suggèrent en effet que d'autres dimensions, affective, consensuelle ou structurelle (cf. Vern Bengtson), mais plus encore relationnelle (réciprocité) (cf. Toni Antonucci), contribuent à maintenir les relations intergénérationnelles. Finalement, la théorie de la sélectivité socio-émotionnelle de Laura Carstensen explique l'évolution des relations personnelles comme la mise en œuvre de stratégies d'adaptation que l'individu met en place pour faire face au déclin de sa santé. Dans ce modèle, l'individu occupe une place centrale, étant le «gestionnaire » de ses relations. 


\section{Modèles explicatifs en gérontologie}

Tout au long de ce chapitre, des limites importantes ont été relevées. Celles-ci sont récurrentes dans l'ensemble de ces modèles théoriques. Premièrement, la conception de la «famille» repose généralement sur le modèle de la famille «nucléaire » qui privilégie les liens bio-légaux. Dans cette perspective, les liens conjugaux et intergénérationnels (parents âgés et enfants adultes) sont considérés comme centraux au point que, parfois, la famille semble réduite à ces dyades. Pourtant, comme le montrera cet ouvrage, les individus ont une définition propre, personnelle, de "leur» famille et y incluent une variété d'individus qui vont bien au-delà des liens de sang ou d'alliance. Une deuxième limite a trait à la non considération de la famille comme un réseau complexe de liens d'interdépendance relationnelle. Dans ces divers modèles explicatifs, la famille renvoie soit à une « dyade » soit à une « composante» d'un réseau personnel plus large. Par conséquent, la famille n'est jamais vraiment considérée dans la complexité des liens qui la constituent. Plus encore, trop centrés sur le soutien et son impact positif sur le bien-être, la plupart de ces modèles négligent les tensions et les conflits au sein des relations familiales. Pourtant, le conflit est un indicateur essentiel des échanges au sein de la famille. Cet ouvrage vise donc à dépasser ces diverses limites, d'une part, en définissant la famille comme une réalité subjective plutôt qu'objective et, d'autre part, en la considérant comme un réseau complexe d'interdépendances relationnelles générant aussi bien du soutien que du conflit. Avant d'introduire l'approche configurationnelle utilisée dans cet ouvrage, nous présentons dans le prochain chapitre cinq facteurs explicatifs qui sont souvent mis en avant dans la littérature gérontologique pour expliquer la dynamique des relations sociales et familiales dans la vieillesse. 


\section{Facteurs explicatifs des relations familiales en gérontologie sociale}

Dans ce deuxième chapitre, nous présenterons cinq facteurs explicatifs dont l'influence déterminante sur les relations sociales et familiales dans la vieillesse a souvent été mise en avant dans littérature gérontologique. Il s'agit du réservoir de parenté, du statut socio-économique, du genre, de l'âge et de la santé. Le premier renvoie à l'ensemble des réserves démographiques dont disposent les individus âgés au cours de la vieillesse. Quant au statut socio-économique, au genre et à l'âge, ils désignent plutôt des positions spécifiques dans la société, auxquelles s'associent non seulement des rôles et des statuts particuliers mais aussi des ressources distinctes (moyens financiers, capacités relationnelles et physiques, etc.). La santé fait partie de ces ressources. Bien que la santé soit liée aussi bien au statut socioéconomique qu'au genre et à l'âge, nous en ferons mention lorsque nous traiterons de l'âge puisque l'avancée en âge et le déclin de la santé sont fortement associés. Ces divers facteurs sont importants puisqu'ils contribuent à modeler l'ensemble des relations familiales, amicales et sociales au cours de la vie. On peut donc s'attendre, en tenant compte de l'ensemble de ces facteurs, à une variété de situations familiales aux dernières étapes de la vie. Dans ce chapitre, nous recenserons les principaux résultats en gérontologie sociale qui portent sur l'influence de ces différents facteurs sur le soutien et les relations familiales dans la vieillesse. Ce faisant, nous mettrons en exergue les aspects qui sont rarement évoqués dans la littérature gérontologique.

\subsection{Le réservoir de parenté}

Le premier facteur explicatif a trait au réservoir de parenté dans la vieillesse (Coenen-Huther et al., 1994; De Carlo, Aeby, \& Widmer, 2014). Ce facteur est important puisqu'il renvoie à l'ensemble des réserves démographiques 


\section{Facteurs explicatifs en gérontologie}

dont disposent les individus âgés pour faire face aux aléas de leur vieillesse. Le terme de "réservoir de parenté » se réfère à la composition et au nombre de membres de la parenté qui constituent les réserves démographiques. Avoir un partenaire, des enfants, des petits-enfants, des sœurs, des frères, des belles-sœurs, des beaux-frères, des cousines ou des cousins, etc. représentent d'importantes réserves démographiques (Widmer, 2016). L'existence de ces membres dans le réservoir de parenté offre un ensemble d'opportunités relationnelles; les individus âgés peuvent y recourir en cas de besoin alors que leur absence limite de telles opportunités (CoenenHuther et al., 1994; De Carlo et al., 2014; Fingerman \& Birditt, 2003). En offrant des ressources relationnelles, le réservoir de parenté influence non seulement la vie familiale des individus âgés mais aussi la définition qu'ils se font de leur famille proche. Si les personnes âgées ont un grand réservoir de parenté, elles mentionnent plus de membres familiaux comme affectivement proches que celles qui ne disposent que d'un petit réservoir de parenté (Fingerman \& Birditt, 2003). Cependant, certaines études montrent aussi que les individus âgés ne désignent qu'un petit nombre des membres de leur réservoir de parenté comme émotionnellement proches et significatifs, même si leur réservoir est grand (Coenen-Huther et al., 1994; De Carlo et al., 2014; Fingerman \& Birditt, 2003).

Bien que le réservoir de parenté et la définition personnelle de la famille soient étroitement liés, ils ne s'équivalent pas, l'un renvoyant à une réalité " objective et démographique » alors que l'autre comporte une grande part de subjectivité (Coenen-Huther et al., 1994; De Carlo et al., 2014; Fingerman \& Birditt, 2003). Pourtant, dans la recherche gérontologique, la définition subjective, personnelle, de la famille est souvent laissée de côté, seul le réservoir de parenté est pris en compte pour appréhender la famille. Dans de nombreuses études, tous les membres familiaux existants - ceux qui constituent le réservoir de parenté - sont souvent considérés comme importants par les chercheurs alors qu'ils ne le sont pas en réalité pour les individus. Dans cette section, nous remettrons en question cet amalgame qui est souvent fait en gérontologie sociale entre le «réservoir de parenté » et la «famille », celle qui compte vraiment aux yeux des personnes âgées. Mais, avant cela, nous allons décrire, en nous basant sur la littérature gérontologique, la diversité des réservoirs de parenté dans la vieillesse. Nous évoquerons plusieurs changements sociétaux qui ont contribué à cette diversification : les changements démographiques, 
l'émergence de nouvelles formes familiales et la contraction du réservoir de parenté aux dernières étapes de la vie.

\subsubsection{L'évolution et la pluralité des réservoirs de parenté}

L'allongement de l'espérance de vie et la baisse de natalité sont parmi les changements socio-démographiques qui ont le plus modelé la structure familiale actuelle. Grâce aux divers progrès techniques et scientifiques, associés à l'industrialisation et à la croissance économique de l'après-guerre, l'espérance de vie a augmenté de manière marquante alors que, dans le même laps de temps, la natalité a baissé. Résultant de ces deux changements importants (longévité croissante et faible natalité), l'augmentation du nombre de personnes âgées et la diminution du nombre d'enfants ont eu - et ont toujours - des effets directs sur la structure familiale (Bengtson, 2001 ; Lowenstein \& Katz, 2010). D’une part, la longévité croissante a permis à des membres de plusieurs générations de se côtoyer au sein d'une même famille (Bengtson, 2001; Lowenstein \& Katz, 2010; Silverstein \& Giarrusso, 2010). Et, d'autre part, la baisse de natalité s'est associée à la réduction du nombre de membres de mêmes générations au sein de la famille, processus que les chercheurs en gérontologie sociale ont nommé la « contraction intragénérationnelle » (Bengtson, 2001; Bengtson, Rosenthal, \& Burton, 1990; Lowenstein \& Katz, 2010). Globalement, la structure familiale s'est donc verticalisée et a pris la forme d'un échalas (cf. «beanpole family »); elle s'est donc à la fois allongée (plusieurs membres répartis sur plusieurs générations) et amincie (membres peu nombreux par générations) (Bengtson, 2001; Bengtson et al., 1990; Lowenstein \& Katz, 2010).

Le contrôle des naissances depuis les années soixante a par ailleurs eu d'autres effets sur la structure familiale que seule la baisse de natalité. Les transitions à la parentalité se sont notamment « individualisées », donnant lieu à une pluralité des structures familiales, parfois fort contrastées (Bengtson et al., 1990; Bengtson, Rosenthal, \& Burton, 1995; Lowenstein \& Katz, 2010). La fertilité précoce dans certaines familles s'est traduite par un nombre d'enfants potentiellement plus grand et par de petites différences d'âge entre générations, permettant à de nombreuses générations de se côtoyer. Dans d'autres familles au contraire, la fertilité tardive liée, en partie, aux carrières féminines plus fréquentes dans les classes 
moyennes et supérieures - a eu, pour conséquence, la réduction du nombre d'enfants mais aussi la présence de membres ayant des différences d'âge importantes, laissant moins de place à l'intergénérationnalité au sein de la famille (Bengtson et al., 1995; Lalive d'Epinay, Bickel, Maystre, \& Vollenwyder, 2000; Lowenstein \& Katz, 2010). Finalement, la baisse de la fertilité ainsi que la parentalité retardée ont contribué à l'augmentation des individus sans enfant parmi les différentes cohortes d'âgés (Dykstra \& Hagestad, 2007; Schnettler \& Wöhler, 2014).

Outre ces divers changements démographiques, le processus de déstandardisation du parcours de vie a entraîné, depuis les années soixante, une diversification des trajectoires familiales, celles-ci ne suivant plus un même modèle prescrit, reposant sur des étapes chronologiques précises (Allan, 2008; Cavalli, 2007; Kohli \& Meyer, 1986; Widmer \& Ritschard, 2009). Se manifestant par un moindre engagement des individus dans les institutions (mariage, famille, église, travail, communauté, etc.), qui traditionnellement leur garantissaient la stabilité et la continuité, le processus de déstandardisation a contribué à libérer les individus du carcan institutionnel (Allan, 2008). Ne suivant plus à la lettre les étapes de la vie familiale prescrites et son organisation modèle, bon nombre d'individus ont modelé et individualisé leur propre trajectoire familiale. Résultat de ce processus, le taux de divorces a connu une forte augmentation depuis les années soixante et de nouvelles formes familiales ont émergé, telles que les couples sans enfant, les familles recomposées, les familles monoparentales, les unions hors mariage, les familles vivant dans des ménages différents ou encore les couples homosexuels avec ou sans enfant (Manning \& Brown, 2011; Smart, 2007; Treas \& Marcum, 2011). Reflétant des styles de vie différents, personnalisés (Allan, 2001; Widmer, 2016), ces diverses formes familiales se sont normalisées et « concurrencent » aujourd'hui la famille nucléaire qui renvoie à la famille «modèle», axée sur des liens de sang ou d'alliance (Murdock, 1949; Parsons \& Bales, 1956). Désormais, la famille «nucléaire » ne représente plus qu'une forme familiale parmi d'autres (Bengtson, 2001; Finch, 2007; Smart, 2007). Se construisant, pour certaines, au delà des frontières de la relation maritale et, pour d'autres, en y insérant en leur sein des liens autres que biologiques, ces nouvelles formes familiales ont contribué et contribuent toujours à diversifier les structures familiales des individus, y compris celles des plus âgés (Manning \& Brown, 2011; Smart, 2007; Treas \& Marcum, 2011). 
Alors que certains «jeunes-vieux» (âgés aujourd'hui entre 65 et 74 ans) ont eux-mêmes connu au cours de leur vie ces diverses transitions maritales telles que le divorce, le remariage ou encore la cohabitation (Elder \& Johnson, 2003; Van der Pas \& Van Tilburg, 2010; Van Tilburg \& Thomése, 2010), les données montrent que les individus plus âgés ont été relativement épargnés par ces nouvelles formes familiales, ayant adopté, pour une grande majorité d'entre eux, le modèle familial de la famille nucléaire. Rares sont ceux qui ont divorcé comme le révèlent certaines études. A Genève, par exemple, la proportion de personnes âgées (65-94 ans) divorcées ou séparées est passée de $8 \%$ en 1979 à $16 \%$ en 2011 , ce qui représente en 30 ans une très légère augmentation (Baeriswyl, 2016). La faible proportion de personnes divorcées parmi les cohortes plus âgées s'explique non seulement par leur remariage mais aussi par la forte stigmatisation du divorce lors de leur jeunesse (Carr \& Moorman, 2011). De même, la cohabitation hors mariage et vivre dans des lieux séparés (« living apart together ») sont des formes de relations maritales particulièrement rares chez les 65 ans et plus (Carr \& Moorman, 2011).

Ceci dit, même si les individus âgés n'ont pas eux-mêmes «expérimenté » ces diverses formes familiales, ils ont été témoins de ces transformations au sein même de leur famille, assistant à l'émergence de ces nouvelles formes familiales dans la vie de leurs proches (enfants, petitenfants mais aussi frères et sœurs). Le divorce et la recomposition familiale des membres de l'entourage familial modifient indirectement la structure familiale des individus âgés, ceux-ci étant dépendants - selon le concept de « vies liées » («linked lives ») (Elder, 1991 ; Elder \& Johnson, 2003; Heinz, 2001) - de ceux avec qui ils sont étroitement liés. En conséquence, toutes et tous sont confronté(e)s à l'insertion au sein de leur réservoir de parenté de nouveaux membres sans lien de sang, ni d'alliance. De fait, la structure familiale des âgés est amenée à se modifier; elle peut s'enrichir ou s'appauvrir en fonction des trajectoires familiales des membres de leur famille. En raison de la pluralisation des formes familiales, qui touche directement et indirectement les jeunes et les anciennes générations, on peut faire l'hypothèse que les personnes âgées connaissent actuellement des structures familiales diverses.

Finalement, la «contraction » du réservoir de parenté, au cours des dernières étapes de la vie, altère inévitablement sa structure. Le nombre restreint des membres d'une même génération au sein de la famille, résultant de la « verticalisation» de la structure familiale, s'accompagne dans la 


\section{Facteurs explicatifs en gérontologie}

vieillesse d'un fort risque de perdre une partie ou la totalité de ces derniers (comme le conjoint, les sœurs, les frères ou les cousin(e)s) et même parfois, au très grand âge, de voir disparaître ses propres descendants (Bickel \& Girardin, 2008; Dykstra \& Hagestad, 2007). Ces changements sont par ailleurs très rapides : Johnson et Troll (1996) relèvent dans leur étude que la part des individus n'ayant plus de fratrie double entre 70 et 90 ans. Les données Swilsoo, étude longitudinale portant sur des octogénaires en Suisse, montrent que, sur un laps de temps de 5 ans, ils sont presque la moitié (45\%) à avoir été confrontés au décès d'un de leurs proches (conjoint, fratrie, mais aussi enfants) (Bickel \& Girardin, 2008). Globalement, cependant, la taille du réservoir de parenté ne varie pas significativement; l'arrivée des nouveaux membres par le biais des naissances compense la perte des anciens (Antonucci, 2001; Van Tilburg, 1998). L'étude Swilsoo révèle en effet que, sur la même période de 5 ans, les individus âgés déclarent autant un décès (45\%) qu'une naissance de petits-enfants et d'arrière-petits-enfants (46\%). Bien que la taille du réservoir évolue peu, sa composition change rapidement exigeant de la part de l'individu et de son entourage une grande capacité d'adaptation (Johnson \& Troll, 1996; Van Tilburg, 1998).

Alors que la composition du réservoir de parenté se modifie pour les individus âgés qui ont des enfants, la contraction du réservoir de parenté a des effets néfastes pour les individus qui n'ont pas de descendance. En effet, le décès des membres familiaux de même génération réduit considérablement et rapidement la structure familiale des personnes très âgées sans enfants, celles-ci ne pouvant compter, dès lors, que sur un réseau d'aide potentielle réduit et instable (Dykstra \& Hagestad, 2007; Johnson, 1988). Sachant que les enfants adultes sont considérés dans la littérature gérontologique comme les principaux prestataires de soins et de support des parents âgés (Armi, Guilley, \& Lalive d'Epinay, 2008; Lowenstein et al., 2004 ; Silverstein et al., 2006), les individus âgés sans enfants sont de fait désignés comme une population à « haut» risque d'isolement (Fiori et al., 2008, 2006). Ils ont en effet une forte probabilité d'être les seuls survivants de leur réservoir de parenté après avoir perdu leur conjoint, leur fratrie et les membres de leur parenté éloignée (Johnson \& Troll, 1996; Lalive d'Epinay et al., 2000). Ils entrent donc dans la grande vieillesse dépourvus de toutes réserves démographiques (Bickel \& Girardin, 2008; Dykstra \& Hagestad, 2007).

Ces divers changements socio-démographiques (longévité grandissante, baisse de natalité, contrôle des naissances, augmentation du taux 
de divorce, émergence de nouvelles formes familiales, etc.) qui ont marqué ces dernières décennies ont profondément diversifié et pluralisé les réservoirs de parenté des personnes âgées (Bengtson et al., 1990; Manning \& Brown, 2011; Treas \& Marcum, 2011). Certains individus ont un partenaire alors que d'autres n'en ont pas ou plus. Certains ont un ou plusieurs enfants alors que d'autres n'en ont jamais eus ou n'en ont plus. Certains sont intégrés dans une famille à structure «monogénérationnelle » disposant d'un ou de plusieurs membres de même génération (un conjoint, des membres de la fratrie ou de la parenté éloignée). D'autres, au contraire, présentent une famille "multigénérationnelle» comprenant trois à quatre générations, pouvant prendre diverses formes selon les types de membres qui la composent (enfants, beaux-enfants, petits-enfants, arrière-petits-enfants, fratrie, et même parfois, selon l'âge, d'ascendants). D'autres encore sont des "survivants », seuls représentants de leur famille après avoir perdu leur conjoint, leur fratrie et même parfois leurs enfants (Bengtson et al., 1990; Manning \& Brown, 2011; Treas \& Marcum, 2011). Selon la taille et la composition de leur réservoir de parenté, les individus âgés ont des réserves démographiques fort variables. Autrement dit, certains ont l'opportunité de développer une diversité de relations familiales grâce à un réservoir de parenté bien fourni alors que d'autres sont « a priori » limités par la pauvreté de leurs réserves démographiques (Coenen-Huther et al., 1994; De Carlo et al., 2014; Widmer, 2016).

\subsubsection{Les limites des indicateurs socio-démographiques}

Pour approcher la diversité des réservoirs de parenté, de nombreuses études en gérontologie sociale - comme dans d'autres domaines de recherche (Widmer et al., 2013) - disposent d'indicateurs démographiques qui font référence à un nombre restreint de liens de parenté jugés comme importants (par exemple, le partenaire, les enfants, les petits-enfants, les frères et sœurs, etc.). Ces indicateurs permettent de recenser l'ensemble des réserves démographiques (Manning \& Brown, 2011). Ce faisant, les auteurs de ces études se contentent de lister les membres du réservoir de parenté mais ils ne se demandent pas si les membres recensés sont considérés par les individus étudiés comme des membres importants de leur famille, s'ils font partie de leur "famille significative». En effet, comme le montrent des données sur des populations plus jeunes, la famille significative et 


\section{Facteurs explicatifs en gérontologie}

le réservoir de parenté, bien qu'ils soient étroitement liés, ne renvoient pas aux mêmes réalités (Coenen-Huther et al., 1994; Fingerman \& Birditt, 2003; Widmer, 2016). Fingerman et Birditt (2003) mentionnent par exemple que les participants de leur étude citent deux-tiers à trois-quart (en moyenne, $73 \%$ ) des membres de leur réservoir de parenté comme des membres proches (émotionnellement) de leur famille. Or, dans de nombreuses études en gérontologie sociale, cette distinction n'est pas vraiment considérée : la définition de la « famille» renvoie en tout point à la structure familiale (réservoir) et est appréhendée exclusivement par des indicateurs démographiques. En d'autres termes, la famille « significative», celle qui compte vraiment pour les individus, est rarement considérée.

Bien que ces indicateurs donnent un aperçu des structures familiales et permettent de distinguer les individus âgés qui disposent d'un grand réservoir de parenté de ceux qui n'en ont pas, leur seule prise en compte pour approcher la famille pose plusieurs problèmes. D'abord, ils sont limités pour rendre compte de toute la diversité des structures familiales. Étant formatés pour n'approcher que les membres familiaux qui répondent à la définition «classique » de la famille, c'est-à-dire ceux qui ont des liens de sang ou d'alliance, ces indicateurs peinent à identifier les membres familiaux qui, issus de nouvelles formes familiales, s'inscrivent au-delà du cadre bio-légal (Widmer, 2006, 2016). Ensuite, ces indicateurs ne suffisent pas à définir ce qu' " est» la famille, celle-ci étant beaucoup plus complexe qu'une simple liste de membres familiaux, perçus « a priori » par le chercheur comme importants en raison des rôles familiaux auxquels ils font référence. Il est vrai que certains individus disposent de larges réservoirs de parenté, multigénérationnels, mais rien ne garantit qu'ils développent des relations affectives avec l'ensemble des membres de leur réservoir, et cela même s'ils sont de même sang. Ceci est d'autant plus vrai dans la vieillesse puisque la composition du réservoir de parenté change rapidement en raison des décès des membres du même âge (conjoint, fratrie, etc.), ce qui rend difficile le maintien de liens familiaux significatifs. Ceux-ci sont difficilement remplaçables par l'arrivée de nouveaux venus, qu'ils soient de même sang ou non.

Même si les individus âgés ont un partenaire ou des enfants dans leur réservoir démographique, leur seule présence ne suffit pas à garantir qu'ils soient considérés comme significatifs. Des chercheurs ont montré en effet que des individus âgés qui ont un riche réservoir de parenté, comprenant un partenaire et des enfants, ne sont pas à l'abri du sentiment de 
solitude puisque ce dernier ne dépend pas de l'absence de relations personnelles (quantité) mais plutôt du manque de satisfaction et de soutien (qualité) qu'ils en retirent (Carr \& Moorman, 2011; De Jong Gierveld, Broese van Groenou, Hoogendoorn, \& Smit, 2009). Or, dans bon nombre d'études gérontologiques, les aînés qui s'insèrent dans des familles à structures multigénérationnelles sont perçus comme avantagés, ayant accès à des membres familiaux (par exemple, les enfants et les petits-enfants) considérés «a priori » comme essentiels car étant de même sang (Widmer, 2006; Widmer et al., 2013). Jugés comme avantagés, ces individus sont systématiquement opposés à ceux qui sont sans partenaire et sans enfants. $\mathrm{Ne}$ disposant pas des réserves démographiques les plus importantes, ces derniers sont perçus comme vulnérables (Bickel \& Girardin, 2008; Dykstra \& Hagestad, 2007; Johnson \& Troll, 1996). La possibilité qu'ils se construisent « leur» famille significative en dehors de la descendance n'est rarement, voire jamais, évoquée. Ceci dit, l'insertion dans le réservoir de parenté d'individus issus de nouvelles formes familiales, c'est-à-dire sans lien de sang ni d'alliance, ne garantit pas non plus qu'ils soient automatiquement perçus comme des membres significatifs de la famille, même s'ils constituent des aides potentielles à même de renflouer le réservoir de parenté (Riley, 1983 ; Riley \& Riley, 1996; Van Tilburg \& Thomése, 2010).

La définition «statutaire et démographique » de la famille ne suffit pas à appréhender la « famille» dans la recherche en gérontologie sociale. Nous postulons donc dans ce travail que ce qui «fait» la famille n'est pas tant la présence et le nombre des membres familiaux «présumés » importants, comme le partenaire, les enfants ou les petits-enfants, mais plutôt le sens qu'ils revêtent pour les individus concernés. Dans cet ouvrage, nous nous intéressons aux membres de la famille qui comptent pour les individus âgés, à savoir ceux qu'ils perçoivent comme significatifs, ceux auxquels ils sont émotionnellement attachés et envers lesquels ils se sentent affectivement « engagés ».

Dans la littérature scientifique, les « autruis » significatifs au sein de la famille sont perçus comme « essentiels » dans le quotidien, à même de guider les individus dans leurs choix de vie, aussi bien positivement que négativement (Carstensen, 1991; Thoits, 2011; Widmer, 1999a). Leur importance est telle que, lorsqu'ils disparaissent (décès), il est difficile pour la personne âgée de compter sur le support des autres membres de son réservoir de parenté, à savoir ceux qui ne font pas partie des autruis significatifs, 
parce que les liens ont été moins investis et même parfois rompus (Van Tilburg \& Thomése, 2010). L'absence des autruis significatifs peut être source d'angoisse et de dépression (Carstensen, 1991). Ici, la fréquence des interactions ou la distance géographique ne sont pas très importantes (Widmer, 2016, 1999a). Ce qui compte, c'est la perception que l'«autre » est important pour soi et que l'on soit important pour lui. Des études portant sur diverses populations révèlent que les membres significatifs de la famille sont fort variés et s'inscrivent bien au delà des liens de sang et d'alliance (De Carlo et al., 2014; Widmer, 1999a; Widmer \& La Farga, 2000; Widmer, 2006). Pour l'heure, nous savons peu de choses sur les membres significatifs de la famille dans la population âgée. Comme le souligne Victor Marshall, la significativité des liens familiaux a souvent été négligée en gérontologie sociale, trop centrée sur l'étude du soutien et du « caregiving » (Marshall et al., 1993).

L'un des objectifs de cet ouvrage est donc de s'écarter de la conceptualisation «statutaire et démographique» de la famille, fort présente dans de nombreux travaux en gérontologie sociale, et d'identifier « qui », parmi les membres du réservoir de parenté ou en dehors, fait partie de la famille «significative » dans la vieillesse; de mettre ainsi en exergue les définitions personnelles de la famille chez les aînés. Certes, les données quantitatives sur lesquelles repose cette recherche ne nous éclairent pas quant aux raisons profondes qui expliquent l'insertion de certains membres, plutôt que d'autres, dans la famille significative. Seuls des entretiens approfondis pourraient nous éclairer à ce sujet (Castrén \& Ketokivi, 2015). Les données quantitatives, cependant, permettent de recenser les principaux membres de la famille que les individus âgés perçoivent comme "significatifs ». C'est une première étape de recherche qui est nécessaire et qu'il faudra, à l'avenir, compléter par une étude qualitative afin de mieux comprendre le « sens » que donnent les individus âgés à leur famille qui « compte».

\subsection{Le statut socio-économique}

Le statut socio-économique est souvent avancé dans la littérature gérontologique comme l'un des facteurs-clé des relations sociales et familiales dans la vieillesse (Antonucci, 2001; Berkman et al., 2000; Litwin, 2001 ; 
Pinquart \& Sörensen, 2000). Il est généralement mesuré soit par le statut socio-professionnel (avant la retraite), soit le niveau d'éducation ou le revenu. Il signale si l'individu dispose (ou non) d'un certain nombre de ressources non seulement économiques mais aussi relationnelles et psychologiques. S'il est élevé, l'individu étudié appartient à une catégorie socio-professionnelle prestigieuse et a les moyens financiers pour s'assurer des conditions de vie adéquates et résoudre certains problèmes (payer certains services comme ceux d'une femme de ménage, accéder à des traitements alternatifs, etc.). De plus, il dispose d'une bonne formation et a acquis des ressources non seulement relationnelles (communication, négociation, etc.) mais aussi psychologiques (cognitives, intellectuelles, etc) qui lui ont permis de développer des stratégies de coping efficaces et de préserver une bonne santé tant physique que mentale (Broese Van Groenou \& Van Tilburg, 2003; Guilley \& Lalive d'Epinay, 2008; Krause, 1997b; Pinquart \& Sörensen, 2000). Le statut socio-économique s'associe donc à une multitude de ressources qui, toutes, influencent d'une manière ou d'une autre le développement et le maintien de relations sociales variées (Ajrouch et al., 2005; Carr \& Moorman, 2011; Pinquart \& Sörensen, 2000; Broese Van Groenou \& Van Tilburg, 2003). Dans cette revue de la littérature sur le statut socio-économique et son impact sur les relations personnelles, nous ferons référence aux deux champs dominants de la gérontologie sociale présentés précédemment, à savoir les réseaux personnels et les relations intergénérationnelles.

\subsubsection{Des réseaux personnels différenciés}

Dans la vieillesse, l'impact du statut socio-économique se manifeste dans la structure des réseaux personnels (Ajrouch et al., 2005 ; Broese Van Groenou \& Van Tilburg, 2003; Cornwell et al., 2008, 2009; Stevens \& Van Tilburg, 2011). De nombreuses études montrent que les individus âgés ayant un statut socio-économique élevé ont des réseaux personnels de grande taille, composés d'une diversité de membres (des amis, pour la plupart) rencontrés au cours des études, de la carrière professionnelle ou par le biais d'activités extra-professionnelles (associations, engagement politique, etc) (Ajrouch et al., 2005; Antonucci, 2001; Stevens \& Van Tilburg, 2011). Quant aux individus ayant un bas statut socio-économique, ils affichent 
des réseaux personnels de petite taille, homogènes, essentiellement composés de membres de la famille (Ajrouch et al., 2005; Cornwell et al., 2008, 2009; Fiori et al., 2006; Litwin, 2001).

Dans la littérature scientifique, plusieurs explications ont été avancées. D'une part, les individus ayant un statut socio-économique élevé sont plus mobiles au cours de leur vie, devant se déplacer davantage pour trouver des emplois correspondants; ils entretiennent, dès lors, moins de contacts avec leur famille (Broese Van Groenou \& Van Tilburg, 2003; Fiori et al., 2006; Stevens \& Van Tilburg, 2011). De plus, les professions qu'ils exercent leur offrent davantage d'opportunités de rencontrer des personnes variées (collègues, clients, etc.) que ceux ayant un bas statut socio-économique. Les conditions de travail associées aux professions de bas statut socio-économique sont en effet moins propices à la diversification des liens (Ajrouch et al., 2005). A cela s'ajoutent les aptitudes cognitives (apprendre, comprendre, chercher des informations, etc.) et relationnelles (communication, négociation, etc.) - acquises au gré d'un long cursus scolaire et consolidées au cours de leur parcours de vie - qui permettent aux individus des milieux privilégiés d'initier et de maintenir des relations interpersonnelles dans divers cercles de sociabilité (Ajrouch et al., 2005; Broese Van Groenou \& Van Tilburg, 2003; Krause, 2006; Stevens \& Van Tilburg, 2011). A la retraite, ils sont par conséquent mieux disposés à s'engager dans des activités sociales diverses (Pinquart \& Sörensen, 2000). A l'opposé, le manque de ressources (revenu, éducation, prestige, etc) qui caractérise les individus socialement désavantagés a contribué à restreindre leurs opportunités relationnelles tout au long de leur parcours de vie. Dès lors, ils peinent dans la vieillesse à développer et à maintenir des relations en dehors du contexte familial, ce qui pourrait expliquer l'absence d'amis au sein de leur réseau personnel (Ajrouch et al., 2005; Krause, 2006; Pinquart \& Sörensen, 2000).

\subsubsection{Des relations intergénérationnelles distinctes}

Alors que le statut socio-économique influence la composition des réseaux personnels, il a également un impact sur les échanges entre parents âgés et enfants adultes. Les données révèlent que la solidarité intergénérationnelle est plus faible dans les familles nanties que dans celles qui ont moins de ressources socio-économiques. En effet, lorsque les parents et leurs enfants 
ont un statut socio-économique élevé et de bonnes ressources (par exemple, avoir un(e) partenaire et être en bonne santé), les contacts et les échanges sont moins intenses que dans les familles issues des milieux populaires. Vivant plus généralement à distance de leurs parents âgés, les enfants adultes socialement favorisés affichent un faible niveau d'échanges, même si leur degré d'affection n'en est pas moins élevé (Lawton et al., 1994b; Parrott \& Bengtson, 1999; Silverstein et al., 1994; Silverstein \& Bengtson, 1997). Ces résultats mettent en exergue que les individus privilégiés (parents et enfants) font peu appel à l'aide et au soutien familial (par exemple, instrumental), bénéficiant de suffisamment de ressources (pratiques, financières, relationnelles, etc) au sein de leur réseau personnel pour satisfaire l'ensemble de leurs besoins (Ajrouch et al., 2005; Broese Van Groenou \& Van Tilburg, 2003). Les parents âgés recourent à l'aide professionnelle si nécessaire alors que leurs enfants se procurent tous les services dont ils ont besoin (garderies, aide ménagère, etc) à l'extérieur de la famille. Cette plus grande autonomie économique et pratique des enfants adultes et de leurs parents leur permet ainsi, aussi bien d'un côté que de l'autre, de s'affranchir de la dépendance familiale (Krause, 1997b; Pinquart \& Sörensen, 2000; Silverstein et al., 1994). La structure de leur réseau personnel, plus diversifiée et moins axée sur les membres de leur famille atteste, de fait, de leur plus grande autonomie vis-à-vis des membres de la famille (Ajrouch et al., 2005; Cornwell et al., 2008).

Dans les milieux populaires, la situation est tout autre. Comme le dévoile la structure de leurs réseaux personnels, les membres de la famille y occupent une place dominante. En effet, les personnes âgées ayant un faible statut socio-économique développent des liens de solidarité plus intenses au sein de leur famille (avec les enfants, notamment) que celles qui sont plus aisées financièrement (Antonucci, 2001; Bowling, 1994; Cornwell, 2009b; Krause, 1997b). Par ailleurs, les relations d'aide y sont davantage encouragées (Ganong \& Coleman, 2006). Cette plus grande solidarité familiale peut s'expliquer aussi bien par le «besoin » que par le «manque d'alternatives » (Antonucci, 2001; Broese Van Groenou \& Van Tilburg, 2003 ; Krause, 1997b). D’une part, les individus âgés socialement désavantagés ont une plus forte probabilité d'avoir des problèmes financiers et d'être en mauvaise santé, nécessitant davantage de soutien de la part de leurs proches («besoin ») (Krause, 2006; Pinquart \& Sörensen, 2000). D'autre part, ayant peu de ressources à disposition (financières, relationnelles, etc.), ils n'ont pas d'autres choix que de solliciter le soutien des 


\section{Facteurs explicatifs en gérontologie}

membres de leur famille, notamment de leurs enfants («manque d'alternatives ») (Antonucci, 2001; Krause, 1997b). Ces deux aspects expliqueraient la composition exclusivement familiale de leur réseau personnel et la densité de leurs relations.

Du côté des enfants adultes, le constat est le même. Ceux qui ont un bas revenu ont une probabilité plus élevée de développer des relations intergénérationnelles solidaires que ceux qui ont un revenu élevé (Silverstein \& Bengtson, 1997; Suitor et al., 2011). L'argent offert ou prêté par les parents contribue à activer les contacts et l'aide au sein des relations intergénérationnelles (Parrott \& Bengtson, 1999; Szydlik, 2008); il stimule les enfants adultes à rendre ce qu'ils ont reçu sous la forme d'aide et de soutien. D'autres ressources telles que le temps, l'écoute, et le soutien émotionnel peuvent être proposées si les enfants adultes en expriment le besoin (Silverstein \& Bengtson, 1997; Suitor et al., 2011). De telles ressources s'avèrent essentielles pour les enfants adultes qui traversent certaines difficultés comme le chômage, des difficultés financières, un divorce, ou encore des problèmes de santé, ou expérimentent certaines transitions de vie importantes comme celle de la parentalité (Suitor et al., 2011). C'est notamment le cas des filles adultes qui développent des relations proches avec leur mère, celles-ci répondant présentes aux demandes d'aide de leur fille quant à l'éducation et à la garde de leurs petits-enfants (Silverstein \& Bengtson, 1997; Silverstein et al., 1994). En l'absence d'alternatives peu coûteuses (structures d'accueil extra-familial), les filles ne peuvent compter que sur leurs parents et deviennent fortement dépendantes de l'aide familiale. De même, les enfants adultes non mariés (divorcés, veufs ou célibataires) reçoivent plus d'aide de leurs parents âgés que les enfants mariés, notamment lorsqu'ils cumulent divers problèmes tels que le chômage ou des fragilités au niveau de la santé (Suitor et al., 2011).

Parfois, le manque de ressources incite les parents âgés et leurs enfants adultes à partager un même logement; ce qui semble être de plus en plus le cas aux États-Unis avec la crise économique et le taux de chômage actuel (Suitor et al., 2011). Ces résultats révèlent la forte interdépendance entre des enfants adultes dans le besoin (chômage, divorce, mère célibataires, etc) et leurs parents âgés qui, en échange de leurs faibles ressources (pension, logement, services, etc.), reçoivent de l'aide de leurs enfants. Bien que signe d'une forte solidarité, la co-résidence intergénérationnelle n'en est pas moins source de stress et de conflits (Suitor et al., 2011; 
Willson, Shuey, Elder, \& Wickrama, 2006). Elle peut même s'associer à un faible degré d'attachement affectif, la proximité géographique n'étant pas garante d'un fort lien affectif (Silverstein et al., 1994; Suitor et al., 2011).

D'autres recherches montrent aussi que les difficultés financières peuvent être à l'origine de grandes tensions et de conflits (Antonucci, 2001; Suitor et al., 2011), entraînant parfois la rupture des liens familiaux dans les cas de grande pauvreté (Antonucci, 2001; Krause, 1997a, 2006; Offer, 2012). Les individus sans ressources sont fortement dépendants de l'aide des membres de leur famille, n'ayant aucune autre alternative. Cette forte dépendance génère du stress non seulement chez l'individu âgé dans le besoin mais aussi chez les membres de sa famille. N'étant pas solvable et ne pouvant garantir la réciprocité, la personne âgée n'est pas sûre d'obtenir de sa famille le soutien dont il a besoin, créant chez lui de l'incertitude et du stress (Offer, 2012). Parallèlement, le besoin financier chronique du parent âgé peut représenter une menace à l'encontre de l'équilibre économique précaire des autres membres du réseau familial (Krause, 1997a), sachant que ces derniers proviennent généralement du même milieu socioéconomique que lui (Bourdieu, 1980, 1986; Portes, 1998). Ceux-ci peuvent à la longue se sentir dépouillés, et cela d'autant plus si le «retour» de l'aide qu'ils octroient est incertain, l'absence de ressources (santé, argent, etc.) du parent âgé rendant la réciprocité plus qu'improbable (Coyne, Wortman, \& Lehman, 1988). Leur sécurité économique menacée, stress, tensions et conflits s'immiscent au sein des relations familiales (Krause, 2006, 1997a). Le support ne pouvant être assuré à long terme; ce dernier s'épuise et disparaît (Krause, 2006; Offer, 2012; Pinquart \& Sörensen, 2000).

Certaines données confirment en effet l'absence de solidarité dans des situations de forte précarité en montrant que les enfants adultes, ayant un très faible statut socio-économique, présentent davantage que les autres des relations intergénérationnelles peu solidaires, se caractérisant par un éloignement géographique, peu de contacts et un certain détachement affectif (Silverstein et al., 1994). Les échanges semblent ici totalement interrompus. L'absence de ressources - socio-économiques, relationnelles mais aussi affectives - peut compromettre gravement les liens intergénérationnels dans les familles particulièrement défavorisées. La pauvreté est 
donc bel et bien davantage synonyme d'absence de support que de grande solidarité (Offer, 2012; Parrott \& Bengtson, 1999).

\subsubsection{Des questions restées en suspens dans la littérature}

Ces diverses études montrent l'impact du milieu socio-économique sur la composition des réseaux personnels et la solidarité familiale, intergénérationnelle. Leurs résultats sont éloquents mais restent relativement limités sur plusieurs aspects concernant la famille. Premièrement, ces études relèvent que les aînés socialement privilégiés ont des réseaux personnels plus larges et plus variés que ceux qui sont issus des milieux populaires, mais elles n'indiquent rien quant à la composition de leur famille significative. On peut effectivement se demander si les ressources dont ils disposent influencent leur définition personnelle de la famille, s'ils considèrent comme membres significatifs de leur famille une variété de personnes n'ayant ni lien de sang ou d'alliance ou si, au contraire, ils s'appuient sur le modèle de la famille nucléaire pour définir leur famille significative. De même, certains résultats montrent qu'en raison de problèmes financiers chroniques, les aînés perdent le lien avec les membres "proches » de leur famille (les enfants, notamment). Cependant, il se peut que ces individus se soient construits une famille de substitution qui s'écarte, dans sa conception, de la définition "classique » de la famille. En d'autres termes, même si certains n'ont plus d'échanges avec leur famille de sang, cela ne signifie pas pour autant qu'ils n'ont pas de famille significative. Or, la question de la famille significative n'est jamais considérée dans un grand nombre de recherches en gérontologie sociale. Pourtant, c'est bien cette famille-là qui «compte » pour les individus. Dans ce travail, différentes conceptions de la famille significative chez les âgés seront identifiées ainsi que les différents facteurs qui les expliquent, dont le statut socio-économique.

Deuxièmement, la littérature met en évidence des dynamiques intergénérationnelles différentes selon le statut socio-économique des parents âgés et de leurs enfants. Dans les milieux privilégiés, l'indépendance économique rend les échanges intergénérationnels peu intenses alors que, dans les milieux populaires, la solidarité intergénérationnelle y est forte, étant nécessaire. Malheureusement, ces études ne dépassent 
pas le cadre dyadique et nous ne savons pas si le flux des échanges au sein de l'ensemble de la configuration familiale diffère aussi selon le milieu social. On peut postuler que les relations d'aide au sein de la famille sont plus denses dans les milieux populaires que dans les milieux privilégiés. Par ailleurs, les recherches précédentes montrent qu'un statut socio-économique élevé est prometteur d'une plus grande autonomie relationnelle dans les relations intergénérationnelles. Dès lors, on peut se demander s'il en est de même dans un réseau complexe d'interdépendances familiales. Grâce à l'approche configurationnelle et ses outils d'analyse, nous pourrons répondre à ces questions. Premièrement, nous observerons si la dynamique des liens au sein des configurations familiales dépend du statut socio-économique des aînés. Puis, nous mesurerons la centralité et l'engagement des individus âgés dans leur réseau familial. Ces deux indicateurs reflètent l'autonomie relationnelle des individus dans leurs échanges familiaux. Nous pourrons ainsi observer si la centralité et l'engagement des personnes âgées dans leur famille varient en fonction de leur statut socio-économique. Ces divers éléments nous permettront ensuite de déterminer si les aînés ont accès à différents types de capital social au sein de leur configuration familiale selon leur milieu social.

Troisièmement, la littérature a mis en évidence une plus grande solidarité intergénérationnelle au sein des familles populaires, mais aussi l'émergence de fortes tensions lorsque les ressources socio-économiques viennent à manquer. Dans la recherche en gérontologie sociale, soutien et conflit sont rarement étudiés ensemble dans un contexte relationnel plus large que celui des dyades; et leur articulation au sein des configurations familiales n'est jamais scrutée selon le statut socio-économique des individus âgés. Pour y remédier, nous observerons dans ce travail comment le conflit et le soutien se combinent en fonction de la position sociale des aînés. Il est fort probable que les personnes âgées qui ont des ressources socio-économiques et une certaine autonomie économique gèrent de manière différente les tensions et l'ambivalence au sein de leur configuration familiale que ceux qui, ayant peu de ressources socio-économiques, dépendent davantage de l'aide familiale. On peut donc s'attendre à l'émergence de manières différentes de gérer les conflits et l'ambivalence au sein des configurations familiales en fonction du statut socio-économique des individus âgés et des membres de leur famille. 


\subsection{Le genre}

Tout comme le statut socio-économique, le genre est considéré dans la littérature scientifique comme un important facteur influençant les relations sociales et familiales dans la vieillesse. Quelle que soit la discipline de recherche dans laquelle ils s'inscrivent - médecine, biologie, psychologie ou sociologie - de nombreux chercheurs s'accordent sur un point : hommes et femmes ne vieillissent pas de la même manière (Barer, 1994; Moen, 1996). Ceux-ci disposent en effet de ressources distinctes, à la fois biologiques, psychologiques, socio-économiques et relationnelles, pour faire face au déclin de la santé qui caractérise les dernières étapes de la vie. Alors que certaines différences sont biologiques, d'autres sont construites socialement, la société attribuant dès la naissance des rôles, des tâches, des responsabilités, des devoirs, un statut et un pouvoir distincts aux individus selon leur sexe. Ce faisant, elle leur octroie ainsi une identité distincte et les oriente dans des parcours de vie socialement différenciés, genrés (Krüger \& Levy, 2001; Levy, Widmer, \& Kellerhals, 2002; Moen, 2001). Le genre implique non seulement une division sexuée des rôles et des tâches en fonction du sexe des individus mais il renvoie aussi à un système hiérarchisé de statuts au sein de la société. Et, généralement, les statuts conférés aux femmes sont socialement perçus comme inférieurs à ceux des hommes (Calasanti \& Kiecolt, 2012). Intériorisé depuis l'enfance par le biais de la socialisation, ce système d'inégalités est peu questionné par les individus et est, pour beaucoup, invisible et associé a priori à la "nature» plus qu'à la culture (socialisation) (Calasanti \& Kiecolt, 2012).

Influençant les hommes et les femmes tout au long de leur parcours de vie, le genre donne lieu à des différences importantes quant aux rôles qu'ils doivent jouer au sein de la société mais aussi quant aux ressources dont ils disposent pour faire face aux aléas de leur vieillesse (Moen, 2001). Par ailleurs, ces différences de genre s'inscrivent dans des contextes sociaux divers; elles varient ainsi en intensité selon les milieux socio-économiques, les communautés ethniques, les cohortes de naissance (socialisation différente, par exemple), les étapes et les transitions du parcours de vie. L'interaction entre ces différents contextes - $l^{\prime}$ ' intersectionnalité »-produit une diversité de situations au sein même des catégories "femmes" et « hommes », notamment en termes de perceptions et de ressources à 
disposition, faisant de la vieillesse une expérience fort variée selon le profil socio-démographique et le parcours de vie des individus (Calasanti \& Kiecolt, 2012; Moen, 2001).

Dans cette section sont présentés les principaux résultats concernant les différences de genre dans la recherche en gérontologie sociale. Aussi bien biologiques, socio-économiques que relationnelles, ces différences de genre sont considérées dans la littérature comme des facteurs importants des relations sociales et familiales dans la vieillesse. Aux dernières étapes de la vie, ces différences de genre peuvent, selon les situations, tout aussi bien avantager que désavantager les hommes et les femmes. A certains égards, cette section peut paraître « caricaturale», faisant fi de la variabilité intra-genre et négligeant les similitudes inter-genre (intersectionnalité). En effet, hommes et femmes peuvent présenter de nombreuses similitudes en raison de leur inscription dans des contextes socio-historiques similaires (milieu socio-économique, communauté ethnique, cohorte de naissance, etc.). Mais, au risque de manquer de nuances et de minimiser l'« intersectionnalité », cette section vise à mettre en exergue les différences de genre car elles sont au cœur des inégalités entre hommes et femmes dans la vieillesse, et celles-ci sont susceptibles d'influer l'organisation des liens de soutien et les tensions au sein des configurations familiales. Cette section termine sur certains aspects qui ne sont jamais considérés en gérontologie sociale et qui, pourtant, pourraient expliquer certaines variations dans les relations familiales entre hommes et femmes dans la vieillesse.

\subsubsection{Une vieillesse genrée}

L'une des différences les plus marquantes entre hommes et femmes est celle de l'espérance de vie plus longue des femmes et de leur plus mauvaise santé, notamment fonctionnelle. Plusieurs études ont en effet mis en évidence le «gender paradox », à savoir le lien entre la plus grande longévité des femmes et leur plus mauvaise santé. Bien qu'elles aient une espérance de vie plus grande, les femmes affichent un niveau plus élevé de morbidité, un risque plus grand de souffrir de maladies chroniques, d'incapacités fonctionnelles et de dépendance que les hommes du même âge (Arber \& Cooper, 1999; Barer, 1994; Guilley, Guillet, \& Girardin, 2008; Johnson \& Troll, 1996). Plus encore, les femmes âgées ont, en moyenne, un niveau de bien-être plus bas, avec un risque plus grand de dépression que 


\section{Facteurs explicatifs en gérontologie}

les hommes âgés (Fiori et al., 2006; Jang et al., 2002; Litwin, 2001; Pinquart \& Sörensen, 2000). Le «gender paradox » s'explique par la mortalité précoce des hommes souffrant davantage de maladies cardiovasculaires que les femmes. Ainsi, les hommes qui, au grand âge, ont survécu à de telles maladies constituent une sorte d'élite, se caractérisant - par rapport aux femmes du même âge - par une meilleure santé physique et mentale, avec un moindre risque de souffrir de dépression, d'atteintes fonctionnelles et de devenir dépendants. Autrement dit, plus les hommes et les femmes avancent en âge, plus ils se distinguent du point de vue de leur santé physique et mentale, les femmes connaissant davantage d'atteintes fonctionnelles et de symptômes dépressifs que les hommes (Arber \& Cooper, 1999; Johnson \& Troll, 1996). Les personnes âgées vivant en Suisse connaissent aussi ce "gender paradox » comme le relèvent les données longitudinales Swilsoo récoltées à Genève et en Valais entre 1994 et 2004 (Guilley, Guillet, \& Girardin, 2008; Guilley \& Lalive d'Epinay, 2008).

Outre leur santé plus mauvaise, les femmes âgées qui vivent plus longtemps ont de fait une plus grande probabilité que les hommes de perdre leur partenaire (Arber \& Cooper, 1999; Barer, 1994; Waite \& Das, 2010). Généralement plus jeunes que leur époux, elles sont confrontées au veuvage plus tôt que les hommes. Ces derniers, à l'inverse, disposent de leur épouse, plus jeune, jusqu'à des âges avancés (Arber \& Cooper, 1999; Barer, 1994; Bickel \& Girardin, 2008; Johnson \& Troll, 1996). Par ailleurs, la plupart des hommes tendent, lorsqu'ils perdent leur partenaire, à davantage se remarier (Barer, 1994; Carr \& Moorman, 2011; Dykstra \& Hagestad, 2007; Pudrovska, Schieman, \& Carr, 2006). Précisons qu'être «(re)marié » est généralement plus probable chez les hommes que chez les femmes non seulement en raison de l'espérance de vie plus longue des femmes mais aussi en raison de la meilleure santé des hommes âgés en général et surtout de leur statut socio-économique plus élevé (cf. la prochaine section portant sur les inégalités socio-économiques) (Dykstra \& Hagestad, 2007; Pudrovska et al., 2006; Robles \& Kiecolt-Glaser, 2003). Les individus âgés ayant un bon statut socio-économique parviennent non seulement à davantage pérenniser leur relation conjugale mais se remarient aussi davantage que ceux dont le statut socio-économique est bas (Dykstra \& Hagestad, 2007; Pudrovska et al., 2006; Robles \& Kiecolt-Glaser, 2003).

Les femmes, ayant en moyenne un statut socio-économique plus bas, se remarient moins et se retrouvent souvent seules à un âge où se dégrade leur santé fonctionnelle (Johnson \& Troll, 1996). En conséquence, elles 
sont davantage institutionnalisées que les hommes, ceux-ci pouvant toujours compter sur l'aide de leur épouse (Barer, 1994; Bickel \& Girardin, 2008; Johnson \& Troll, 1996). Cependant, même avec un partenaire, les femmes sont plus nombreuses à être institutionnalisées que les hommes (Arber \& Cooper, 1999), ce qui révèle la plus grande difficulté des époux, plus âgés, à s'occuper de leur épouse souffrant d'incapacités, rôle qui, revenant habituellement aux femmes, constitue pour les hommes des cohortes les plus anciennes une grande source de stress (Barer, 1994). A noter par ailleurs que les hommes âgés seuls, non mariés, sont - excepté aux âges très avancés - davantage institutionnalisés que les femmes seules alors qu'à âge égal leur niveau d'incapacité est plus faible (Arber \& Cooper, 1999). Les femmes seules parviennent donc plus facilement à retarder leur institutionnalisation que les hommes seuls puisqu'elles sont moins nombreuses que ces derniers à entrer en institution malgré leur santé fonctionnelle plus dégradée (Arber \& Cooper, 1999).

Les hommes vivant en couple sont donc de loin les plus avantagés : ils bénéficient jusqu'à des âges très avancés et à des degrés divers d'incapacités, même les plus sévères, de l'aide et du soutien de leur épouse (Arber \& Cooper, 1999; Bowling, 1994). Outre les soins qu'elles donnent à leur mari âgé, les épouses, même très âgées, prennent en charge la plupart des tâches domestiques (courses, vaisselle, ménage, etc) (Armi et al., 2008; Masotti, 2016). A l'opposé, ce sont les hommes seuls qui sont les moins bien lotis : ils sont davantage institutionnalisés que les femmes ayant un profil similaire, même si leur niveau d'incapacités est plus faible. Quant aux femmes seules, elles parviennent à mobiliser leur entourage et à compenser l'absence du conjoint, ce qui leur permet - si leur santé n'est pas trop atteinte - de retarder leur entrée en institution (Barer, 1994). Comment expliquer que les femmes bénéficient davantage d'aide de leur entourage que les hommes? La prise en compte de leurs rôles et trajectoires de vie différents peut nous donner quelques pistes.

\subsubsection{Les inégalités socio-économiques entre hommes et femmes}

Au delà des aspects biologiques et de leurs répercussions dans la vieillesse, le genre renvoie à des parcours de vie différenciés selon le sexe des individus. Selon Moen (2001), deux transitions marquantes du parcours de 


\section{Facteurs explicatifs en gérontologie}

vie déterminent le parcours de vie des femmes et des hommes : le mariage et la parentalité. Ces deux transitions tendent à «fixer» les hommes dans leur trajectoire professionnelle alors qu'elles bouleversent celle des femmes, les renvoyant à la sphère familiale. Cette situation renvoie à la perspective du "statut-maître », selon laquelle le marché du travail, les normes sociales ainsi que certaines institutions définissent, aujourd'hui encore, la famille comme le domaine privilégié des femmes et la carrière professionnelle, comme celui des hommes (Krüger \& Levy, 2001; Levy et al., 2002; Widmer \& Ritschard, 2009). Sous l'effet de ces normes, les femmes s'investissent, en priorité, dans la vie familiale, étant responsables - en tant que «mère et épouse »- des tâches domestiques (ménage, éducation, soins) même si elles sont actives professionnellement (Dykstra \& Hagestad, 2007; Moen, 2001; Rossi \& Rossi, 1990). Ce sont elles qui généralement prennent soin des membres familiaux des autres générations (enfants, mais aussi parents âgés) ou du moins elles s'assurent que l'aide et les soins leur soient octroyés (Connidis \& McMullin, 2002; Pyke \& Bengtson, 1996; Silverstein et al., 2006). En plus de l'organisation et de la réalisation des tâches domestiques, elles se doivent, ayant le rôle de « kinkeeper », de maintenir les relations au sein de la famille afin d'en garantir la cohésion familiale (Finch \& Mason, 1993). Quant aux hommes, dont le rôle est de pourvoir aux besoins économiques de la famille, ils privilégient d'abord leur carrière professionnelle. Celle-ci leur octroie - aussi bien dans la société que dans la famille - de l'autonomie et du pouvoir.

Les effets du «statut-maître » sur le parcours de vie des hommes et des femmes sont clairs : ils s'accompagnent inévitablement d'une insertion différenciée des femmes et des hommes dans les sphères professionnelle et familiale; les hommes s'investissant essentiellement dans l'espace public et professionnel et les femmes, d'abord «mères et épouses », dans la sphère privée et familiale (Krüger \& Levy, 2001; Levy, Gauthier, \& Widmer, 2006; Levy et al., 2002; Levy, 2013). En Suisse, cette spécialisation sexuée des sphères de vie donne lieu à des trajectoires de vie professionnelles genrées (Krüger \& Levy, 2001; Levy, 2013; Levy et al., 2002; Widmer \& Ritschard, 2009). Des données suisses montrent que les femmes disposent, selon leur degré d'insertion professionnelle, de plusieurs modèles de trajectoires professionnelles alors que les hommes poursuivent leur parcours de vie de façon relativement linéaire (Levy et al., 2006; Levy, 2013; Widmer \& Ritschard, 2009). Certaines travaillent à plein temps, d'autres à mi-temps, d'autres suspendent temporairement leur 
emploi, d'autres encore cessent définitivement de travailler pour s'occuper de leur famille (Levy et al., 2006). Cette dernière trajectoire féminine, modèle standardisé « traditionnel », est fréquente parmi les femmes âgées de 60 ans et plus (Levy et al., 2006) et est particulièrement prédominante chez les femmes de 75 ans et plus (Dykstra \& Hagestad, 2007). En effet, avec l'ouverture du marché du travail aux femmes dans les années 60 en Suisse comme ailleurs (Dykstra \& Hagestad, 2007; Levy et al., 2006), la plupart des femmes âgées des cohortes les plus récentes (de moins de 74 ans) ont pour la plupart connu une expérience de travail (Arber \& Cooper, 1999). Mais, contrairement au parcours de vie masculin, peu perturbé par les contraintes familiales, les trajectoires professionnelles féminines sont majoritairement modelées par leur vie familiale, tributaires des événements qui rythment la famille (naissances, soins aux enfants, soins aux plus vieux, etc) et de la trajectoire professionnelle de leur époux (changement de postes, déménagements, etc) (Moen, 2001). Quelle que soit la situation, c'est en effet aux femmes de jongler entre emploi et famille, en s' " arrangeant» de telle manière à ce que les tâches domestiques et de soins soient remplies puisqu'elles en sont responsables (Connidis \& McMullin, 2002). Les trajectoires professionnelles féminines sont, de ce fait, entrecoupées, marquées par l'instabilité et la discontinuité, ce qui les distingue radicalement du parcours de vie masculin, continu et stable (Moen, 1996, 2001; Levy, 2013; Widmer \& Ritschard, 2009).

Par ailleurs, l'insertion des femmes sur le marché du travail est peu comparable à celle des hommes, tant le marché de l'emploi est sexué, hiérarchisé à la faveur des hommes, et cela en Suisse comme dans d'autres pays occidentaux (Ajrouch et al., 2005; Arber \& Cooper, 1999; Moen, 1996; Levy, 2013). Occupant des professions généralement peu valorisées, dites «féminines 》 (soins, éducation, accueil, et domestique), exigeant des formations de plus courte durée et moins qualifiantes que les professions dites "masculines" (techniques ou scientifiques), les femmes qui travaillent ont en moyenne un niveau d'éducation plus bas que celui des hommes, sont moins payées et ont peu de perspectives de carrière professionnelle, et cela d'autant plus dans les cohortes plus anciennes (Broese Van Groenou \& Van Tilburg, 2003; Kuehni, Rosende, \& Schoeni, 2013; Levy, 2013). En revanche, ces professions leur procurent une grande flexibilité; elles peuvent suspendre leur emploi lors des naissances et réduire leur temps de travail (temps partiel) afin de concilier vie professionnelle et familiale. Si bien que peu de femmes, et encore moins parmi les plus 


\section{Facteurs explicatifs en gérontologie}

âgées, ne peuvent prétendre à des postes à responsabilité exigeant un investissement total (Moen, 1996; Levy et al., 2006; Levy, 2013; Widmer $\&$ Ritschard, 2009). Seuls les hommes sont en mesure de briguer de tels postes, leur octroyant des avantages à la fois sociaux et financiers. L'ouverture du marché de l'emploi aux femmes n'a donc pas offert, excepté pour une minorité, l'autonomie économique totale des femmes. En Suisse notamment, la structure de l'emploi, hiérarchisée et inégalitaire, n'a fait que confirmer la dépendance économique des femmes à leur conjoint et la contingence du travail féminin (Levy et al., 2006; Levy, 2013; Widmer \& Ritschard, 2009).

En conséquence, à l'entrée de la vieillesse, les femmes ont - y compris celles qui ont travaillé - un statut socio-économique plus bas que celui des hommes, les rendant d'un point de vue économique plus vulnérables. Les femmes âgées sont en effet sur l'ensemble des dimensions socioéconomiques largement défavorisées par rapport aux hommes, et cela aussi en Suisse : elles ont généralement un niveau d'éducation plus bas, un statut professionnel inférieur - les professions féminines étant relativement peu valorisées - et ont davantage connu l'instabilité socio-professionnelle (Kuehni et al., 2013 ; Levy, 2013; Moen, 2001). A ce propos, la description de l'échantillon genevois et de son contexte socio-historique est assez parlant (cf. Chapitre «Données et méthodes »). Ayant moins cotisé que leur époux en raison de leur parcours professionnel discontinu (Kuehni et al., 2013; Moen, 1996), nombre de femmes âgées - notamment en Suisse touchent à la retraite un revenu (pension) nettement plus bas que celui des hommes au point que certaines présentent un fort risque de paupérisation à la séparation d'avec leur conjoint (divorce, veuvage) et sont généralement en moins bonne santé (Arber \& Cooper, 1999; Carr \& Moorman, 2011; Kuehni et al., 2013; Moen, 1996, 2001). Cette corrélation est telle que, selon certains auteurs, elle explique en grande partie les inégalités de santé aux dernières étapes de la vie, notamment le risque plus grand des femmes âgées de souffrir d'incapacités fonctionnelles; les individus ayant, quel que soit leur sexe, travaillé dans des professions sous-qualifiées ont en effet un plus grand risque de souffrir d'incapacités fonctionnelles (Arber \& Cooper, 1999; Guilley, Guillet, \& Girardin, 2008; Moen, 2001). Conformément au processus cumulatif des (dés)avantages socio-économiques au cours de la vie (Dannefer, 2003), les femmes tendent donc à cumuler les désavantages (santé, revenu, partenaire, etc.) aux dernières étapes de la vie (Moen, 2001). 


\subsubsection{Les relations sociales et familiales selon le genre}

La différence de statut socio-économique entre hommes et femmes tend à avoir un impact sur la structure de leur réseau personnel (Ajrouch et al., 2005 ; Bowling, 1994 ; Litwin, 2001 ; Stevens \& Van Tilburg, 2011). Alors que les hommes ont généralement entretenu, grâce à leur insertion continue sur leur marché du travail et la sphère publique, une diversité de relations sociales (travail, club, associations, etc.), les femmes, centrées sur la sphère familiale, présentent au contraire un nombre limité de membres extrafamiliaux dans leur réseau personnel (Ajrouch et al., 2005). Les femmes au foyer, par exemple, ont significativement moins d'amis au sein de leur réseau personnel que celles et ceux qui travaillent (Ajrouch et al., 2005). Ceci dit, même si elles exercent une activité professionnelle, elles ont cependant peu de temps à consacrer, en raison de leurs obligations familiales, au maintien de leurs relations extra-familiales (amis, collègues) (Ajrouch et al., 2005). Ainsi, à l'entrée de la vieillesse, la plupart des femmes bénéficient d'un réseau personnel moins étendu et moins diversifié que celui des hommes qui ont pu profiter de leur insertion professionnelle et de ses avantages (financiers, opportunités relationnelles, etc.) pour diversifier leurs relations sociales (Ajrouch et al., 2005 ; Bowling, 1994 ; Litwin, 2001 ; Stevens \& Van Tilburg, 2011). Généralement, l'insertion des hommes sur le marché du travail les favorise à plusieurs niveaux : ils bénéficient des ressources non seulement socio-économiques - qu'ils ont cumulées au gré de leur parcours professionnel (niveau de formation, statut professionnel et revenus supérieurs) - mais aussi sociales (structure du réseau personnel plus large et diversifiée) (Ajrouch et al., 2005 ; Cornwell et al., 2009; Litwin, 2001; Stevens \& Van Tilburg, 2011). A la retraite cependant, et l'âge avançant, les hommes perdent peu à peu les avantages qu'ils avaient acquis au cours de leur carrière professionnelle et tendent à se centrer davantage sur le noyau familial (Shaw et al., 2007).

Ceci dit, la centration de la plupart des femmes sur la vie familiale au cours de leur parcours de vie ne comporte pas que des désavantages. En tant que mères et épouses, elles occupent une place centrale dans la famille. Elles sont chargées des tâches d'éducation, de soutien et de soins auprès des membres de la famille mais elles sont aussi responsables des liens («kinkeeper ») (Finch \& Mason, 1993; Silverstein et al., 2006). Davantage socialisées que les hommes aux valeurs familialistes et aux obligations d'aide envers les membres de la famille, elles s'engagent plus que les 
hommes dans le soutien au sein de la famille (Antonucci et al., 2007; Finch \& Mason, 1993; Silverstein et al., 2006; Silverstein \& Bengtson, 1997), dans le maintien des relations familiales inter- et intra-générationnelles et dans la promotion des échanges de support entre les différents membres de la famille afin d'assurer la cohésion familiale (Pinquart \& Sörensen, 2000; Rossi \& Rossi, 1990; Silverstein et al., 1994, 2006). De fait, lorsqu'un problème familial surgit, il leur appartient de rechercher des solutions (Ajrouch et al., 2005). Par ailleurs, les femmes sont aussi responsables du maintien des relations avec la parenté élargie et les amis de la famille (Barer, 1994; Finch \& Mason, 1993); elles relient notamment leur époux non seulement aux autres membres de la famille mais aussi aux amis proches du couple (Ajrouch et al., 2005; Antonucci, 2001; Bowling, 1994). Grâce à leur rôle dans la famille, elles ont plus d'opportunités de développer des relations affectives avec les membres de la famille dont elles ont la charge, ce qui a des répercussions évidentes sur la qualité des relations qu'elles entretiennent plus tard avec leurs proches (Antonucci, 2001; Fingerman et al., 2004). Quant aux hommes, dont le rôle est de pourvoir aux besoins économiques de la famille, ils portent relativement peu d'intérêt aux relations familiales, étant davantage centrés sur leur carrière professionnelle, ce qui a, pour certains auteurs, contribué à péjorer la qualité des relations que certains hommes âgés entretiennent avec les membres de leur famille - les enfants, notamment - tant du point de vue des contacts, de l'aide que du soutien affectif (Silverstein et al., 1994). Bien que le statut socio-économique plus avantagé des hommes s'associe à un potentiel de ressources plus important, il se corrèle pour certains à une moindre qualité relationnelle au sein de la famille (Silverstein et al., 1994).

Dans la vieillesse, la meilleure qualité des relations familiales chez les femmes se manifeste de plusieurs manières. Elles ont des contacts plus fréquents avec leurs enfants que les hommes, leur donnent et reçoivent davantage de soutien de leur part (Kawachi \& Berkman, 2001; Lawton et al., 1994b; Parrott \& Bengtson, 1999; Suitor et al., 2011). En cas de besoin, elles mobilisent davantage de support que les hommes, mais elles en procurent aussi davantage (Antonucci et al., 2007; Kawachi \& Berkman, 2001). Elles sont aussi plus nombreuses que les hommes à mentionner un enfant comme le principal «donneur de soins» (Barer, 1994). En moyenne, les liens que les mères âgées entretiennent avec leurs enfants adultes sont davantage investis émotionnellement que ceux entretenus par les pères, les 
enfants adultes (notamment, les filles) se sentant plus proches affectivement de leur mère que de leur père (Antonucci, Akiyama, \& Lansford, 1998; Pyke \& Bengtson, 1996; Silverstein \& Bengtson, 1997; Silverstein et al., 2006). Les relations intergénérationnelles impliquant la mère se caractérisent, en moyenne, par une plus forte proximité affective, des contacts fréquents et du soutien alors que celles qui intègrent le père sont davantage marquées par le détachement affectif, la faiblesse des contacts et le moindre soutien (Bengtson, 2001; Lawton et al., 1994b; Pyke \& Bengtson, 1996; Silverstein et al., 1994; Silverstein \& Bengtson, 1997). Il en est de même pour les contacts avec la fratrie. Généralement, les femmes entretiennent avec les membres de leur fratrie, notamment les sœurs, des liens affectifs plus étroits et échangent avec eux davantage de soutien émotionnel et d'aide pratique que les hommes (Campbell et al., 1999; Eriksen \& Gerstel, 2002; Voorpostel \& Van Der Lippe, 2007).

Cet investissement affectif plus intense des femmes dans le relationnel est aussi perceptible dans le domaine de l'amitié. Elles conçoivent plus leurs amis proches comme des confidents que les hommes (Campbell et al., 1999; Voorpostel \& Van Der Lippe, 2007), ces derniers associant davantage l'amitié aux activités extérieures (clubs, associations, voisinage, etc.) qu'à l'intimité dyadique, typique des amitiés féminines. Globalement, ils ont moins d'amis « dits » intimes (Carstensen, 1991; Stevens \& Van Tilburg, 2011). Par ailleurs, avec l'avancée en âge, ils perdent peu à peu leurs " copains » au fur et à mesure qu'ils se désengagent de leurs activités extérieures pour se replier sur leur foyer (Shaw et al., 2007; Stevens \& Van Tilburg, 2011) alors que la plupart des femmes maintiennent leurs amitiés reposant, pour certaines (amies d'enfance, par exemple), sur une longue histoire d'échanges de soutien varié, notamment affectif, et de confidences (Antonucci et al., 2007; Cornwell et al., 2009; Stevens \& Van Tilburg, 2011). Plus encore, les femmes sont plus enclines à s'insérer dans le réseau personnel de leur époux (belle-famille et amis) alors que ces derniers sont plus réticents à s'investir dans le réseau de leur épouse. Par conséquent, un grand nombre de femmes développent un réseau personnel qui leur est propre, leur permettant de conserver dans la vieillesse une certaine indépendance relationnelle vis-à-vis de leur époux (Cornwell, 2011; Kearns \& Leonard, 2004).

Pour toutes ces raisons, les femmes mentionnent, en moyenne, un nombre plus grand de relations proches (affectivement parlant) que les hommes, considérant davantage des relations non familiales comme des 
liens de haute qualité (Merz \& Huxhold, 2010), ce qui élargit le cœur de leur réseau personnel - celui-ci étant composé généralement des membres familiaux très proches (conjoint, enfants, fratrie, petits-enfants) - puisqu'elles y insèrent un nombre plus grand et plus varié de membres significatifs, pouvant désigner aussi bien leur époux, leurs enfants, les membres de leur parenté éloignée ou de leur belle-famille ou encore des amis (Antonucci et al., 2007; Cornwell et al., 2009; Fingerman et al., 2004; Merz \& Huxhold, 2010). Par ailleurs, elles sont davantage que les hommes considérées comme des membres significatifs de la famille (Antonucci et al., 1998; Barer, 1994; Campbell et al., 1999; Voorpostel, 2013). Quant aux hommes âgés, ils se soumettent davantage que les femmes du même âge au processus de sélectivité socio-émotionnelle (Cornwell, 2011; Shaw et al., 2007). N'ayant plus besoin de liens « instrumentaux » pour progresser dans leur trajectoire professionnelle, ils se centrent, par conséquent, sur les membres primaires de leur famille et surtout sur leur épouse qu'ils considèrent souvent comme la principale - voire la seule - confidente et source de support émotionnel et pratique (Cornwell, 2011, 2009b; Pudrovska et al., 2006; Shaw et al., 2007) au point même que, pour certains hommes âgés, avoir une épouse a un impact plus positif sur leur bien-être que d'avoir des enfants (Dykstra \& Hagestad, 2007). En effet, l'épouse répond à leurs besoins affectifs, instrumentaux et relationnels puisqu'en tant que gardienne des liens, elle leur sert d'intermédiaire aussi bien à l'intérieur qu'à l'extérieur de la famille (amis) (Antonucci, 2001; Barer, 1994; Johnson \& Troll, 1996).

Symbolisant, pour beaucoup, la perte du lien affectif le plus gratifiant au sein de leur réseau personnel (Bowling, 1994; Carr \& Moorman, 2011; Cornwell et al., 2008), le décès de l'épouse s'avère être un événement fort critique, s'associant chez un grand nombre d'hommes âgés au déclin de leur santé, au stress, au sentiment de solitude, à la dépression et à un risque plus grand de mortalité (Bowling, 1994; Carr \& Moorman, 2011; Pudrovska et al., 2006). Cette association s'explique non seulement par la perte d'un être cher mais aussi par le changement radical que ce décès implique dans les pratiques quotidiennes : un certain nombre de veufs, n'étant plus « sous le contrôle » de leur épouse, adoptent davantage de conduites à risque pour leur santé (alcool, cigarettes, etc.) (Carr \& Moorman, 2011; Dykstra \& Hagestad, 2007; Pudrovska et al., 2006). Par ailleurs, à la perte de l'épouse, certains deviennent plus dépendants de leurs enfants pour les tâches ménagères et ces derniers reprennent parfois le rôle de surveillance 
de l'épouse décédée. Une telle situation peut créer des tensions pouvant nuire à la longue aux relations qu'ils entretiennent entre eux (Dykstra \& Hagestad, 2007; Silverstein \& Bengtson, 1997).

De plus, n'ayant plus d'intermédiaire relationnel, les hommes veufs ont une probabilité plus grande d'être socialement isolés, ayant généralement plus de difficultés que les femmes veuves à entretenir des contacts avec leur entourage familial et amical, à demander du soutien auprès de leurs proches et à l'accepter, mais aussi à nouer de nouveaux liens (Barer, 1994; Carr \& Moorman, 2011; Johnson \& Troll, 1996; Silverstein \& Bengtson, 1997). En effet, confrontés au veuvage à des âges relativement avancés et non préparés à cette éventualité, la plupart des hommes dans cette situation ne disposent plus de suffisamment de ressources physiques (santé, énergie) pour réactiver d'anciennes amitiés ou pour connaître de nouvelles personnes par le biais d'activités sociales (Barer, 1994; Johnson \& Troll, 1996). Le veuvage représente donc pour un certain nombre d'hommes âgés une perte de connexion aux autres, affectant leur intégration sociale (Carr \& Moorman, 2011). Ce risque est, d'ailleurs, tout aussi fort pour les hommes qui n'ont jamais été mariés ou qui sont divorcés (Antonucci et al., 2007), mais il est particulièrement important lorsqu'ils sont sans enfant, faisant des hommes veufs, célibataires ou divorcés sans enfant une population particulièrement vulnérable (Dykstra \& Hagestad, 2007). Dans de telles circonstances, le remariage - plus fréquent chez les hommes que chez les femmes (Carr \& Moorman, 2011) - constitue, pour la plupart des hommes veufs ou divorcés, une bonne stratégie pour remédier à l'isolement. Ce faisant, ils bénéficient ainsi d'une épouse « aidante» et socialement « connectée » (Antonucci et al., 2007; Barer, 1994; Carr \& Moorman, 2011).

Quant aux femmes, veuves relativement tôt dans la vieillesse, la plupart d'entre elles parviennent à bien s'adapter à leur nouvelle vie en solo (Barer, 1994; Cornwell, 2011; Johnson \& Troll, 1996; Stevens \& Van Tilburg, 2011). D'une part, elles maintiennent des relations affectives avec leurs enfants et petits-enfants, nourries par des contacts fréquents et du soutien (Silverstein et al., 1994). D'autre part, libérées de leur charge stressante de « caregiver» auprès de leur mari malade, plus âgé, elles ont davantage de temps pour réactiver et intensifier certaines de leurs relations familiales (fratrie, parenté éloignée, etc.) et amicales de leur réseau personnel (Carstensen, 1991; Cornwell, 2011 ; Stevens \& Van Tilburg, 2011). Certaines, actives et en bonne santé, développent de nouveaux intérêts ou 
de nouvelles activités qui, propices à des rencontres, leur permettent de nouer de nouvelles amitiés avec, entre autres, des personnes de même profil (veuves) (Barer, 1994; Cornwell, 2011; Johnson \& Troll, 1996; Stevens \& Van Tilburg, 2011). Par la réactivation ou le développement de relations d'amitié - personnalisées, axées sur l'affectivité, la confidence et la confiance - certaines veuves comblent leurs besoins émotionnels en recevant de la part de leurs ami(e)s tout le soutien dont elles ont besoin (Barer, 1994). Par conséquent, et contrairement aux hommes veufs, les femmes veuves disposent généralement de réseaux personnels plus larges, axés sur des liens tant intergénérationnels (enfants) qu'horizontaux (fratrie et amis) qui leur apportent le soutien émotionnel nécessaire pour faire face aux difficultés de leur quotidien (Antonucci et al., 2007; Campbell et al., 1999; Cornwell, 2011; Ha, 2008).

\subsubsection{De nouvelles dimensions à explorer dans la recherche}

Ces différents résultats montrent qu'en raison de leur parcours de vie différent les hommes et les femmes ne disposent pas des mêmes ressources pour affronter les aléas de leur vieillesse. Globalement, les ressources avantageuses des hommes comme avoir un revenu décent, une bonne santé, un réseau personnel diversifié, et une épouse active dans les soins et le relationnel, leur permettent de sauvegarder, tout au long de leur vie, une certaine autonomie tant dans la société qu'au sein de la famille. Cependant, le manque d'investissement des hommes dans la sphère familiale au cours de leur vie a des effets négatifs dans la vieillesse puisqu'il compromet le développement de liens d'affection avec les membres de leur famille. A l'inverse, les femmes âgées qui disposent, en moyenne, de peu de ressources, ayant un risque plus grand d'être veuves, en mauvaise santé fonctionnelle, et d'avoir un réseau personnel peu diversifié, peuvent néanmoins compter sur le soutien de leur entourage familial pour obtenir ce dont elles ont besoin. Le rôle de mère, d'épouse et de sœur qu'elles ont joué tout au long de leur parcours de vie leur assure le soutien dont elles ont besoin aux dernières étapes de leur vie («support bank»).

Ces diverses données nous amènent au constat suivant : les femmes et les hommes entretiennent une vie familiale distincte. Certes, mais, comment définissent-ils leur famille significative, celle qui - parce qu'elle 
compte à leurs yeux - influence leur façon d'agir et leur bien-être? La plupart des études gérontologiques portant sur les relations familiales ne nous disent rien à ce sujet. On peut imaginer que leurs expériences distinctes de la vie familiale influencent leur conception de la famille significative. Il est possible que les hommes âgés, étant plus détachés affectivement des membres de leur famille, s'investissent davantage dans leur réseau personnel plus diversifié et considèrent certains de ses membres comme faisant partie de leur famille significative, même s'ils n'ont ni lien de sang ni d'alliance. On peut aussi supposer qu'en raison du rôle significatif de leur épouse dans la vieillesse, les hommes âgés construisent leur famille significative autour de cette dernière, privilégiant de fait une définition de la famille sous sa forme nucléaire. Quant aux femmes, parce qu'elles ont un risque plus grand d'être veuves dans la vieillesse et de s'investir dans un nombre plus grand de relations familiales et amicales, il est probable qu'elles aient une conception particulière de leur famille significative en y intégrant une variété de liens familiaux et non familiaux. Nous explorerons dans cet ouvrage si, dans la vieillesse, les hommes et les femmes ont - parce qu'ils ont suivi un parcours de vie différent et accumulé des ressources distinctes - une définition différente de leur famille significative.

Notons encore que le plus grand investissement affectif des femmes âgées dans leurs relations familiales a été largement souligné en gérontologie sociale. Cependant, ces observations ne dépassent pas le niveau dyadique et nous ne savons pas si cet investissement plus marqué des femmes influence l'organisation des liens de soutien au sein de leur configuration familiale. On peut en effet imaginer que les femmes soient plus engagées et plus actives que les hommes au sein de leur configuration familiale, influençant même la façon dont les échanges de soutien s'organisent au sein de l'ensemble de leur configuration. Plus encore, en raison de leur rôle au sein de la famille, il est fort possible qu'elles y occupent une position centrale. Bien qu'elles soient globalement perçues comme actives au sein le leur famille, la littérature relève néanmoins que les femmes ont moins de ressources que les hommes. Elles ont donc un risque plus grand de dépendre du soutien familial que les hommes, ce qui peut influencer la façon dont les échanges de soutien s'organisent au sein de leur configuration familiale et, ce faisant, modifier la position « centrale» qu'elles y occupent. Par ailleurs, leur plus grande dépendance à l'aide familiale peut 
être source de stress, de tensions et, ce faisant, susciter davantage d'ambivalence que dans les configurations familiales des hommes, ceux-ci ayant assez de ressources pour garantir leur propre autonomie.

Pour l'heure cependant, aucune étude en gérontologie sociale ne s'est aventurée à observer l'ambivalence au niveau «meso » du réseau familial. Nous ne savons donc rien quant à l'effet du genre sur les liens d'interdépendance et l'ambivalence au sein des configurations familiales. Pourtant, en raison de leur rôle familial différent et de leurs ressources distinctes - statut socio-économique, santé, réserves démographiques (avoir un époux(se)) - il est probable que les femmes et les hommes présentent des configurations familiales aux propriétés structurelles distinctes et qu'ils y occupent des positions différentes. On peut, par conséquent, s'attendre à ce qu'ils aient accès au sein de leur configuration familiale à des types différents de capital social et qu'ils négocient de manière différente l'ambivalence inhérente aux relations familiales. Les outils de l'analyse de réseaux que propose l'approche configurationnelle permettront de tester ces différentes hypothèses. Nous pourrons en effet comparer les propriétés structurelles des configurations familiales des hommes et des femmes, ce qui nous renseignera quant aux types de capital social qu'elles produisent. De même, nous observerons si les hommes et les femmes présentent au sein de leur configuration familiale des modes distincts de gestion de l'ambivalence. En somme, nous explorerons les différences de genre dans la façon dont les hommes et les femmes définissent leur configuration familiale dans la vieillesse, comment ils s'y intègrent et négocient leur liens familiaux, ce qui n'a jamais été fait en gérontologie sociale. Dans la dernière section de ce chapitre, nous aborderons l'âge et la santé qui sont perçus dans la littérature scientifique comme des déterminants importants des relations sociales et familiales dans la vieillesse.

\subsection{L'âge et la santé}

L'âge est un facteur complexe et multidimensionnel puisque son impact sur les relations sociales et familiales dans la vieillesse peut être attribué à différents « effets » d'ordre aussi bien biologique que sociohistorique (Attias-Donfut, 1991; Sapin, Spini, \& Widmer, 2007). L'âge 
renvoie, en premier lieu, au processus de fragilisation qui s'accentue au fur et à mesure que les individus vieillissent. Inéluctable avec l'avancée en âge, ce processus consiste en une dégradation multidimensionnelle de la santé (par exemple, l'émergence de multi-morbidités, la baisse des capacités sensorielles, la baisse d'énergie, l'apparition des troubles physiques, des limites fonctionnelles et des atteintes cognitives) (Baltes \& Smith, 2003; Lalive d'Epinay \& Spini, 2008). Ce processus affecte progressivement les réserves physiologiques et sensorimotrices des individus, les vulnérabilisant fortement face à divers accidents de santé comme les chutes, les maladies ou les hospitalisations (Guilley, Armi, Ghisletta, \& Lalive d'Epinay, 2008). Les « effets d'âge » renvoient par conséquent aux conséquences de ce processus à savoir la baisse progressive d'énergie, le déclin de la santé, et, aux ultimes étapes de ce processus, à l'imminence de la mort.

Ensuite, le facteur «âge » a trait aussi au contexte socio-historique durant lequel les individus nés à une même époque, c'est-à-dire appartenant à une même cohorte de naissance, ont grandi et suivi les différentes étapes de leur parcours de vie (Attias-Donfut, 1991; Baeriswyl, 2016; Sapin et al., 2007). Cet ancrage socio-historique des individus d'une même cohorte permet de mieux comprendre leur histoire et leur spécificité propre. Ils ont en effet suivi un même type de socialisation et ont été confrontés aux mêmes événements historiques alors qu'ils traversaient les mêmes étapes de leur parcours de vie, ce qui a généré chez eux un système commun de valeurs et de pratiques (Baeriswyl, 2016; Lalive d'Epinay et al., 2000). La comparaison de plusieurs cohortes de naissance montre en effet que les individus qui les représentent affichent des attitudes différentes en raison de l'inscription spécifique de leur cohorte dans des contextes socio-historiques distincts. Des différences en termes de socialisation, en termes de systèmes éducatifs et de santé, en termes de contextes socio-politiques et économiques peuvent expliquer des valeurs et des comportements individuels différents, bien que mesurés au même âge. Il s'agit ici non plus d'effets d' «âge » mais plutôt d'effets de « cohorte » (Attias-Donfut, 1991; Baeriswyl, 2016; Sapin et al., 2007).

Les effets d'âge et de cohorte $^{1}$ sont par ailleurs fortement imbriqués et peuvent se cumuler : les individus des cohortes de naissance les

1 Outre les effets d'âge et de cohorte, notons encore les effets de période et les effets d'étape de vie (Attias-Donfut, 1991; Sapin et al., 2007). Les effets de période se 


\section{Facteurs explicatifs en gérontologie}

plus récentes connaissent un processus de fragilisation moins rapide que ceux des cohortes plus anciennes parce qu'ils ont acquis de meilleures ressources comme, par exemple, un niveau d'éducation plus élevé, une meilleure santé, un meilleur statut socio-économique, et une espérance de vie plus longue, ayant bénéficié d'un contexte socio-historique et économique plus favorable (Attias-Donfut, 1991). Bien qu'étroitement imbriqués aux effets de cohortes $^{2}$, ce sont principalement les effets d'âge qui seront au centre de cette section parce que la santé et son déclin, conséquence du processus de fragilisation, ainsi que l'imminence de la mort sont souvent mis en exergue dans les travaux en gérontologie sociale portant sur les relations familiales. En effet, la santé déclinante des âgés représente un challenge pour l'équilibre des échanges au sein de la famille quelle que soit leur cohorte d'appartenance. Les problèmes de santé affectent non seulement la capacité des âgés à maintenir la réciprocité mais ils s'accompagnent aussi d'un besoin accru de soutien auquel les membres de la famille doivent faire face. Quant à l'imminence de la mort, elle s'associe, selon le modèle de la sélectivité socio-émotionnelle (Carstensen, 1991, 1992), à une réduction significative des relations interpersonnelles.

Conformément à la littérature gérontologique (Suzman \& Riley, 1985), trois classes d'âge seront distinguées dans ce chapitre : les «jeunesvieux » (« young-old»), entre 65 et 74 ans, les « vieux-vieux » (les « oldold»), entre 75 et 84 ans, et finalement les « très-vieux » (les « oldest-old»), âgés de 85 ans et plus. Dans la littérature, les individus âgés appartenant à ces différentes classes d'âge se différencient en fonction de leur état de santé (Suzman \& Riley, 1985) : les « jeunes-vieux » sont en moyenne en meilleure santé et plus actifs que les « vieux-vieux » alors que ces derniers, bien que fragilisés sur de nombreuses dimensions de santé, connaissent en moyenne moins de limitations fonctionnelles que les «très-vieux » (Guilley, Armi, \& Ghisletta, 2008; Lalive d'Epinay et al., 2000). Ces variations s'expliquent aussi bien par des effets d' «âge »- les individus se

manifestent lorsque les individus de différentes cohortes de naissance ont des comportements similaires au cours d'une même période en réponse à un événement social particulièrement marquant (par exemple, une crise économique ou une guerre). Quant aux effets d'étape de vie, ils renvoient aux effets liés à une position spécifique dans le parcours de vie (Attias-Donfut, 1991; Sapin et al., 2007).

2 Dans le chapitre «Données et méthodes », nous décrirons plus avant l'évolution du contexte socio-historique suisse et genevois, et son impact sur la population genevoise sur laquelle se basent les analyses présentées dans cet ouvrage. 
situent à des stades différents dans le processus de fragilisation - mais aussi par des effets de cohorte puisque chaque classe d'âge se différencie par des ressources distinctes, acquises au gré de contextes socio-historiques spécifiques. Ceci dit, même au sein d'une même classe d'âge, il y a une forte variabilité inter-individuelle car le processus de fragilisation n'atteint pas tous les individus de la même manière ni à la même vitesse (Guilley, Armi, \& Ghisletta, 2008; Lalive d'Epinay et al., 2000). Ainsi, sur la base d'études suisses et internationales, nous mettrons en évidence dans le présent chapitre comment l'état de santé qui caractérise, en moyenne, chacune de ces différentes classes d'âge influence les relations sociales et familiales des individus qui s'y insèrent.

\subsubsection{Les «jeunes-vieux»(65-74 ans)}

Dans la littérature scientifique, les «jeunes-vieux» sont perçus comme les mieux lotis du point de vue de la santé, comparés aux « vieux-vieux » et aux «très-vieux ». Libérés, pour la plupart, de leurs obligations professionnelles et familiales (enfants autonomes), les « jeunes-vieux » disposent de suffisamment de temps, de capacités physiques (santé, énergie), cognitives et mentales pour approfondir les relations déjà existantes ou pour étendre leur réseau personnel en y intégrant de nouveaux liens. Ayant du temps libre et jouissant d'une bonne santé, ils ont en effet les ressources nécessaires pour maintenir les liens et profiter au maximum des relations au sein de leur réseau personnel (Cornwell, 2009a). En ce sens, ils « optimisent » les relations déjà présentes au sein de leur réseau personnel. Ils réactivent, par exemple, des relations, restées en latence pendant une longue période, avec certains membres de la famille ou des amis. Tel est notamment le cas des relations avec certains membres de leur fratrie qui sont ré-investies après la retraite alors qu'elles étaient mises en berne au milieu du parcours de vie, l'éducation des enfants et l'activité professionnelle ne leur permettant pas de maintenir des contacts réguliers (Carstensen, 1991). Hormis la ré-activation de liens d'ores et déjà existants, certains redéfinissent et valorisent des relations jugées auparavant périphériques en s'y investissant davantage (par exemple, des connaissances deviennent des amis ou un ami devient un partenaire) (Van Tilburg, 1998). 
Les «jeunes-vieux» ont aussi le temps et l'énergie pour s'investir davantage dans les relations avec les membres proches de leur réseau personnel en renforçant les liens qu'ils entretiennent avec eux. Certains, par exemple, intensifient les contacts avec leurs enfants non seulement directement mais aussi indirectement en jouant un rôle actif auprès de leurs petits-enfants. Dans certains cas, le rôle de grand-parent reste optionnel alors que, dans d'autres cas, il devient indispensable lorsque les deux parents travaillent ou lorsqu'ils ne peuvent pas subvenir aux besoins de leurs enfants (Bengtson, 2001; Suitor et al., 2011; Van Tilburg \& Thomése, 2010). Ce renforcement des liens avec les membres très proches de leur réseau personnel est perçu dans la littérature comme une sorte d'investissement à long terme, visant à s'assurer dans le le soutien familial dont ils auront besoin pour faire face au déclin de leur santé (Antonucci, 1990; Antonucci et al., 2007; Antonucci \& Jackson, 1989; Silverstein et al., 2002). En d'autres termes, ils tendent, en rendant des services à leurs proches, à « compenser» le déclin physique à venir (Freund et al., 1999) et à garantir le soutien futur des proches qui doivent, selon les normes de la réciprocité généralisée, rendre l'aide qu'ils ont reçue lorsque la situation l'exigera (Antonucci, 1990; Antonucci et al., 2007; Antonucci \& Jackson, 1989; Silverstein et al., 2002). Il s'agit ici, en somme, de construire ou plutôt de renforcer ce que Antonucci nomme le « social support bank » (Antonucci, 1990; Antonucci et al., 2007; Antonucci \& Jackson, 1989).

Outre l'approfondissement des liens avec les membres proches du réseau personnel ou la ré-activation d'anciennes relations, les «jeunesvieux » peuvent aussi enrichir la structure de leur réseau en y insérant de nouveaux membres issus, entre autres, de nouvelles rencontres (par exemple, un nouveau partenaire et ses enfants) mais aussi des nouveaux liens tissés par leurs proches (par exemple, les conjoints des enfants ou les partenaires des frères et des sœurs). Santé et participation sociale allant de pair, ils peuvent aussi, étant en bonne santé, se lancer dans de nouvelles activités sociales ou maintenir les anciennes (associations, clubs, etc). Par ce biais, ils rencontrent de nouvelles personnes dont certaines entreront dans le cercle privilégié des amis, s'intégrant de fait dans leur réseau personnel (Cornwell et al., 2008; Carstensen, 1991). En résumé, les «jeunes-vieux », qui ont en moyenne de l'énergie et une bonne santé physique et mentale, se caractérisent par leur investissement ou leur engagement actif dans la sphère sociale et familiale. Par conséquent, ils affichent, généralement, des réseaux personnels plus larges et plus diversifiés que les individus plus âgés 
ou ayant des problèmes de santé (Ajrouch et al., 2005; Antonucci, 2001; Cornwell et al., 2009; Litwin \& Landau, 2000).

\subsubsection{Les «vieux-vieux» $(75-84$ ans $)$}

Pour ce qui est des « vieux-vieux », leur situation semble, dans la littérature, moins claire que précédemment. Bien qu'ils soient nombreux à être en bonne santé et actifs au sein de leur réseau personnel, un nombre grandissant d'entre eux est cependant confronté au processus de fragilisation qui les affecte peu à peu sur de nombreuses dimensions de la santé. Malgré la forte variabilité inter-individuelle qui prévaut dans cette catégorie d'âge, l'impact du processus de fragilisation sur la vie relationnelle des « vieux-vieux » n'en est pas moins visible puisqu'ils sont, en moyenne, moins actifs dans leur réseau personnel que les «jeunes-vieux » (Ajrouch et al., 2005 ; Carstensen, 1991). Certains problèmes de santé, tels que la baisse de l'ouie, de la vue, l'apparition de troubles physiques ou de douleurs, entravent peu à peu la capacité des « vieux-vieux » à maintenir la réciprocité (" équilibrée ») des échanges avec les membres de leur réseau personnel (Klein Ikkink \& Van Tilburg, 1999). Les services, les visites, et même parfois, les téléphones reçus ne peuvent plus être rendus, ce qui déséquilibre les échanges, générant un sentiment de malaise chez les "vieux-vieux » qui, se sentant redevables, ne parviennent plus à rendre ce qui leur a été donné (Johnson, 1988; Johnson \& Troll, 1994; Offer, 2012). Ce faisant, ils tendent peu à peu à se désengager des relations qu'ils perçoivent dès lors comme plus laborieuses qu'enrichissantes (Johnson, 1988; Johnson \& Troll, 1994; Offer, 2012).

Dans un premier temps, ce désengagement touche non seulement les relations périphériques, c'est-à-dire celles avec des amis non intimes, des « copains » ou des connaissances qui, sans un long passé commun et un attachement profond, exigent une réciprocité « équilibrée » (Antonucci, 2001; Cornwell et al., 2008; Pinquart \& Sörensen, 2000; Van Tilburg, 1998), mais aussi les relations qui sont coûteuses en termes d'efforts physiques ou d'énergie comme, par exemple, des amis proches qui sont éloignés géographiquement ou qui sont institutionnalisés (Johnson \& Troll, 1994). Parallèlement, les membres du réseau personnel qui appartiennent à la même génération que l'individu âgé comme les membres de sa fratrie, les membres de sa parenté éloignée ou des amis sont aussi soumis 
au processus de fragilisation. Par conséquent, ils deviennent moins accessibles, souffrant eux aussi de problèmes de santé ou sont institutionnalisés, et parfois même disparaissent (décès) (Bickel \& Girardin, 2008; Dykstra \& Hagestad, 2007; Johnson \& Troll, 1996; Johnson, 1988). Dans de telles conditions, les échanges deviennent de plus en plus difficiles de part et d'autre de la relation (Johnson, 1988; Dykstra \& Hagestad, 2007). C'est le cas notamment des relations avec des amis de longue date, ceux avec qui les individus âgés ont tissé des liens affectifs solides axés sur la confidence et les échanges tant pratiques qu'affectifs. De tels liens s'affaiblissent aussi avec le temps en raison de la difficulté grandissante des personnes âgées et de leurs amis à se rendre mutuellement visite ou à s'échanger des services (Armi et al., 2008; Bickel \& Girardin, 2008; Johnson \& Troll, 1994; Lalive d'Epinay et al., 2000), et cela même si ces liens de profonde amitié n'exigent pas de réciprocité immédiate (Johnson \& Troll, 1994).

Ce désengagement progressif des relations périphériques ou l'affaiblissement des relations avec les amis proches ne signifient pas pour autant la fin de la vie relationnelle. De nouvelles amitiés peuvent se créer (Johnson \& Troll, 1994). En effet, certains individus âgés, sans doute les moins atteints par le processus de fragilisation, parviennent à maintenir leur capacité à nouer des contacts et à se lier d'amitié. Ceci dit, contrairement aux étapes précédentes du parcours de vie, les critères de choix des amis se sont élargis, contraints par les exigences de l'âge et de la santé déclinante. De fait, ce sont surtout les membres du réseau personnel qui sont proches géographiquement, accessibles et disponibles qui font dès lors partie des amis, même si certains étaient auparavant perçus comme des relations périphériques. Le lien d'amitié est donc re-défini ou re-négocié en fonction d'impératifs pratiques (proximité et disponibilité) et s'avère moins exigeant qu'auparavant, ne répondant plus aux critères absolus d'un vécu commun ou d'un profil similaire (même âge, même sexe, même statut socio-économique et mêmes valeurs) (Johnson \& Troll, 1994). C'est le cas de connaissances rencontrées dans des associations de personnes âgées, à l'église, ou des voisins avec qui l'on discute régulièrement et avec lesquels on échange de petits services (Bickel \& Girardin, 2008). Par ailleurs, certaines relations peuvent être initiées dans un contexte plus formel telles que celles entretenues avec une aide-ménagère, une dame de compagnie ou une infirmière à domicile. Ces nouvelles relations peuvent s'approfondir au fil du temps, à travers les discussions et les échanges réguliers, et évoluer en amitié (Johnson \& Troll, 1994). Même si ces nouvelles amitiés 
ne sont pas «a priori» définies comme proches par les personnes âgées, certaines d'entre elles peuvent néanmoins le devenir et intégrer leur réseau personnel (Bickel \& Girardin, 2008; Bowling, 1994; Van Tilburg, 1998).

Alors que les relations amicales des « vieux-vieux » sont soumises à un travail de re-définition ou de re-négociation débouchant globalement à une baisse de la vie relationnelle, la vie familiale est relativement épargnée (Antonucci, 2001; Carr \& Moorman, 2011; Cornwell et al., 2008; Pinquart \& Sörensen, 2000). En effet, la plupart des « vieux-vieux » maintiennent, même s'ils sont fragilisés, des relations actives avec les membres de leur famille en termes de contacts (visites ou téléphones) mais aussi en termes de services échangés (Armi et al., 2008; Bickel \& Girardin, 2008; Lowenstein et al., 2004). En effet, bien qu'ils soient nombreux à recevoir de l'aide de leur famille, et plus particulièrement de leurs enfants, la plupart maintiennent activement la réciprocité des échanges tant pratiques qu'émotionnels (Armi et al., 2008; Bickel \& Girardin, 2008; Lowenstein et al., 2004; Parrott \& Bengtson, 1999). Ce faisant, ils continuent ainsi à alimenter leur « support bank» en vue de s'assurer un soutien familial futur lorsque celui-ci s'avérera nécessaire (Antonucci, 1990; Antonucci \& Jackson, 1989; Silverstein et al., 2002; Van Tilburg, 1998). Avec le temps cependant, les visites et les services que les «vieux-vieux » rendent baissent peu à peu (Armi et al., 2008; Bickel \& Girardin, 2008) mais, contrairement aux amis, la famille (les enfants, plus particulièrement) se mobilise dès les premiers signes de fragilité du parent âgé, en octroyant plus de soutien et en intensifiant les contacts, et cela même si ce dernier est toujours en mesure de jouer un rôle actif dans les échanges (Armi et al., 2008; Bickel \& Girardin, 2008; Bowling, 1994; Parrott \& Bengtson, 1999). Par cette mobilisation précoce, la famille anticipe, en somme, l'incapacité grandissante du parent âgé à entretenir le lien. Même si les « vieux-vieux » perdent peu à peu leur capacité à la réciprocité, les relations familiales se maintiennent et restent stables au cours du temps (Armi et al., 2008; Parrott \& Bengtson, 1999). Contrairement aux « jeunesvieux » qui s'investissent dans leur réseau personnel, y compris familial, les « vieux-vieux » se désengagent ou re-négocient leurs relations amicales, tout en maintenant néanmoins leurs relations familiales. Ces mécanismes permettent aux individus âgés de s'adapter au processus de fragilisation qui atteint peu à peu leur capacité relationnelle (réciprocité). En conséquence, les réseaux personnels des « vieux-vieux » se contractent, c'est-à-dire que 
les membres périphériques du réseau disparaissent alors que les liens familiaux s'intensifient (Antonucci, 2001; Carr \& Moorman, 2011; Cornwell et al., 2008; Van Tilburg, 1998).

\subsubsection{Les «très-vieux» (85 ans et plus)}

Au grand âge, le processus de fragilisation s'accentue encore davantage, affectant de plein fouet la santé physique et mentale des « très vieux ». Certes, certains se démarquent des autres en affichant non seulement une bonne santé mais aussi une bonne capacité relationnelle, leur permettant de maintenir jusqu'à un âge avancé une vie relationnelle satisfaisante (Johnson \& Troll, 1994). Cependant, ils sont plus nombreux que ceux appartenant aux classes d'âge précédentes à connaître un déclin sévère de leur santé pouvant déboucher sur des atteintes fonctionnelles. Leurs relations personnelles sont donc encore davantage chamboulées nécessitant encore de nouveaux ré-aménagements. La littérature montre en effet que les atteintes fonctionnelles s'associent à une baisse drastique des relations sociales et amicales. En effet, les personnes âgées qui sont limitées dans leur santé fonctionnelle rendent moins de services, se déplacent moins pour rencontrer leurs amis et ont moins d'échanges téléphoniques (Armi et al., 2008; Bickel \& Girardin, 2008). Il en est de même avec les relations de voisinage qui sont significativement moins fréquentes en cas d'incapacités fonctionnelles (Bickel \& Girardin, 2008).

De telles atteintes affectent aussi les relations familiales. En effet, les individus âgés fortement limités dans leur santé ne parviennent plus à jouer un rôle actif au sein de leur famille au travers d'échanges réciproques. Non seulement ils rendent moins de services mais ils sont aussi moins enclins à rendre visite et à téléphoner à leur famille (Armi et al., 2008; Bickel \& Girardin, 2008). La fragilisation croissante érode peu à peu, même chez les plus sociables, leur aptitude non seulement à créer du lien, mais aussi à le maintenir de manière active (Bickel \& Girardin, 2008; Johnson \& Troll, 1994). Les femmes, par exemple, ayant généralement des échanges familiaux plus intenses, atteignent un niveau de sociabilité comparable à celui des hommes en cas de dépendance fonctionnelle (Bickel \& Girardin, 2008). Dans de telles situations, les membres de la famille - les enfants, notamment - intensifient leur mobilisation en rendant davantage de visites et de services pratiques au parent âgé dont la santé s'est fortement détériorée 
(Armi et al., 2008; Bickel \& Girardin, 2008; Cornwell, 2009a, 2009b; Johnson, 1988). Grâce à cette mobilisation familiale, les relations avec la famille demeurent globalement stables et tendent même à s'intensifier (Armi et al., 2008; Bickel \& Girardin, 2008).

Outre cette plus forte mobilisation familiale, la littérature relève qu'avec l'avancée en âge, les «très-vieux », de plus en plus limités par leur santé ou conscients d'une fin de vie imminente, tendent à se focaliser sur les relations avec les membres proches (émotionnellement parlant) de leur réseau personnel, ceux qui «a priori » leur procurent le plus d'émotions positives et gratifiantes. Ce faisant, ils se retirent des relations - même familiales - qui, selon eux, ne sont pas ou plus significatives (Carstensen, 1991, 1992; Shaw et al., 2007; Van Tilburg, 1998). Avec le temps, les liens perçus comme importants se réduisent peu à peu, se limitant presque exclusivement aux membres "primaires » de la famille (conjoint, enfants, et parfois la fratrie) (Ajrouch et al., 2005; Antonucci \& Akiyama, 1995; Antonucci, 2001; Van Tilburg, 1998). Sachant que leur temps est compté, ils préfèrent le passer en compagnie de celles et ceux avec lesquels ils se sentent affectivement proches (Carstensen, 1991, 1992; Carstensen et al., 2000). Van Tilburg (1998) confirme dans son étude que les «très-vieux » se focalisent davantage sur les liens de sang (plus particulièrement, les enfants) que les « jeunes-vieux» qui, eux, valorisent aussi bien les liens de sang que les liens d'alliance comme les conjoints de leurs enfants ou ceux de leurs frères ou sœurs.

Cette centration progressive sur les membres primaires de la famille est, dans un premier temps, favorisée par la mobilisation accrue de ces derniers autour du parent fragilisé (Armi et al., 2008; Cornwell, 2009a, 2009 b) mais elle est surtout interprétée dans la littérature comme le résultat de la sélectivité socio-émotionnelle (cf. chapitre précédent), mécanisme d'adaptation mis en place par les «très-vieux » qui vise à ne préserver que les relations qui sont émotionnellement proches, gratifiantes et soutenantes (Carstensen, 1991, 1992; Carstensen et al., 2000; Shaw et al., 2007). Cette centration familiale permet à l'individu vieillissant, même très fragile, d'investir le peu de ressources (énergie, temps, etc.) qui lui restent dans un nombre limité de liens (Carstensen, 1991, 1992; Freund et al., 1999). En échange du soutien reçu, par exemple, il peut rendre quelques services comme prêter de l'argent mais aussi apporter son soutien affectif. Il peut ainsi préserver l'équilibre des échanges jusqu'à un âge avancé et même fortement fragilisé (Armi et al., 2008; Van Tilburg, 1998). De plus, 


\section{Facteurs explicatifs en gérontologie}

le maintien d'un rôle actif au sein de la relation, si tenu soit-il, peut contribuer à revaloriser une image de soi mise à mal par le déclin physique, car le maintien de ce rôle constitue un objectif de vie atteint (Freund et al., 1999). Mobilisation familiale, sélectivité et centration sur les membres les plus proches de la famille caractérisent le réseau personnel des «très-vieux » face au déclin de leur santé.

\subsubsection{Des aspects non abordés dans la littérature}

Cette revue de la littérature portant sur les trois classes d'âges usuellement citées en gérontologie - «jeunes-vieux », « vieux-vieux » et « très-vieux » - montre comment l'âge et la santé influencent de manière déterminante les relations sociales et familiales. La santé est une ressource primordiale, essentielle, pour initier et maintenir le lien social, amical et familial. Une bonne santé permet non seulement de maintenir la réciprocité au sein du réseau personnel mais aussi d'alimenter le « support bank » familial, prometteur d'aide future en cas de besoin. Par conséquent, toute atteinte à la santé s'accompagne d'effets importants sur le réseau personnel des individus âgés. Lorsque le déclin de la santé est sévère, la capacité de l'individu âgé à entretenir la réciprocité des liens au sein de son réseau personnel est sérieusement compromise, entraînant un réaménagement important de sa vie relationnelle, notamment amicale. Contraint de s'adapter pour faire face aux exigences de sa fragilisation, l'individu âgé se désengage de certaines relations périphériques ou trop coûteuses en termes d'énergie, privilégiant celles qui sont le plus accessibles, lui garantissant le maintien d'une certaine réciprocité. Plus tard, conscient de l'imminence de sa mort, il ne préserve que celles qui lui sont le plus gratifiantes d'un point de vue émotionnel, notamment celles, selon la littérature, qu'il entretient avec les membres très proches de sa famille.

Bon nombre d'études en gérontologie sociale montrent que cette redéfinition ou ce réaménagement des liens touche de manière différente les diverses composantes du réseau personnel : celle des amis est davantage chamboulée que celle de la famille, cette dernière demeurant relativement stable et immuable, quels que soient l'âge et l'état de santé des individus âgés étudiés. Dans ces diverses études, les relations amicales et familiales sont clairement distinguées et opposées. Les premières sont, en raison de leur nature, amenées à s'évanouir alors que les secondes, maintenues par 
des normes de réciprocité " généralisée », demeurent solides, stables, accessibles, intenses, celles-ci faisant «forcément» partie des relations les plus gratifiantes du point de vue émotionnel. Le support familial y est en quelque sorte garanti comme en témoigne la mobilisation familiale (surtout, celle des enfants) qui se manifeste dès les premiers signes de fragilisation du parent âgé. Quant aux amis, ils subissent eux aussi les affres de la vieillesse et tendent à disparaître; ils ne peuvent plus garantir une aide ou un support à long terme. En opposant les amis, «soumis à un réaménagement profond », à la famille, jugée solide et stable, la définition de la famille n'est pas remise en question dans la plupart de ces études. Centrées sur la dichotomie « famille » et " amis », ces études, qui s'inspirent des modèles dominants de la gérontologie sociale, se contentent de définir la famille comme un ensemble homogène, immuable, axé sur les liens bio-légaux, et cela pour l'ensemble des individus âgés. Nous montrerons dans cet ouvrage que ces travaux font fausse route, car, à l'instar des amis, la famille n'est pas épargnée par les réaménagements et les re-définitions qui bouleversent le réseau personnel des individus âgés. Ces réaménagements se reflètent aussi dans la construction des configurations familiales des individus âgés qui doivent, en prenant de l'âge, peu à peu s'adapter au déclin de leur santé.

Comme souligné plus haut, le déclin de la santé pousse les individus âgés à se centrer sur des liens qui sont accessibles, disponibles et qui requièrent peu d'énergie pour être maintenus. Or, les membres de la famille proches (affectivement), comme les enfants, ne sont pas toujours disponibles ou accessibles, ceux-ci étant pris par leurs obligations familiales et professionnelles. Cette indisponibilité pourrait encourager certains individus âgés à développer des relations affectives avec d'autres membres de leur réseau personnel. Ce faisant, ils pourraient les intégrer au sein de leur famille significative. Par ailleurs, le désengagement et la sélection socio-émotionnelle invitent les individus vieillissant à se détacher des membres les moins satisfaisants et à se concentrer sur les relations affectives les plus gratifiantes. Ces deux mécanismes sont sans doute aussi présents dans la sphère familiale. La famille de sang n'étant pas toujours celle du cœur, il est possible qu'avec l'âge et l'imminence de la mort, certains âgés se défont de relations familiales qu'ils jugent conflictuelles ou insatisfaisantes pour se centrer sur celles qu'ils jugent émotionnellement gratifiantes, quel que soit le lien de parenté. Bien que ce travail de réaménagement et de re-définition concerne une grande majorité des individus âgés qui se fragilisent, il n'aboutit pas forcément aux mêmes résultats. Il 
est probable en effet qu'en fonction de l'âge et la santé des individus, nous ayons une variété de configurations familiales, traduisant des définitions personnelles distinctes de la famille. Ces variations dans la composition des configurations familiales peuvent être interprétées comme le signe de réaménagements relationnels au sein même du réseau familial ayant pour objectif une meilleure adaptation au processus de fragilisation qui se veut de plus en plus pressant aux dernières étapes de lavie.

De même, nous ne savons pas grand chose quant à l'organisation des liens de soutien au sein des configurations familiales en fonction de l'âge et de l'état de santé des individus âgés. Les résultats mentionnés plus haut montrent que la dynamique des échanges de soutien entre les individus âgés et les différents membres de leur réseau personnel se modifie en fonction de l'âge et de la santé. Dans la littérature, la bonne santé des «jeunes-vieux » leur permet d'être actifs au sein de leur réseau personnel, de s'y investir et d'y maintenir une certaine autonomie. Lorsqu'ils vieillissent et que leur santé décline - faisant dès lors partie des «vieux-vieux 》 - ils se désengagent des relations périphériques (connaissances, amis, etc.). Ce faisant, ils limitent le nombre des relations dans lesquelles ils s'investissent (sélection). Parallèlement, ils bénéficient d'une mobilisation familiale accrue, ce qui met à mal l'autonomie qu'ils avaient jusque-là. La recherche en gérontologie sociale a révélé ces différents mécanismes - c'est-à-dire l'investissement dans les relations, la sélection des liens et le désengagement de certaines relations et la mobilisation familiale - dans les réseaux personnels des personnes âgées et elle a montré qu'ils sont activés différemment selon l'âge et la santé des individus. Toutefois, elle ne nous renseigne pas si ces différents mécanismes opèrent aussi au sein des configurations familiales et, le cas échéant, s'ils ont un impact sur l'ensemble des liens d'interdépendance qui relient entre eux les différents membres significatifs de la famille.

Si ces mécanismes sont bel et bien mis en œuvre au sein des configurations familiales, il est probable qu'ils s'associent à des organisations différentes des liens de soutien et qu'ils influencent le type de capital social qui y est produit. L'investissement actif dont font preuve les « jeunesvieux » leur permet de se lier à une variété de membres familiaux qui n'entretiennent pas forcément des liens de soutien entre eux. Ce faisant, les «jeunes-vieux » peuvent préserver une certaine autonomie relationnelle au sein de leur configuration familiale. Chez les « très-vieux », la mobilisation accrue des membres primaires de la famille est susceptible de resserrer davantage les liens familiaux autour de la personne âgée dont la santé 
décline, avec le risque que le parent âgé perde peu à peu son autonomie relationnelle. Autrement dit, on peut faire l'hypothèse, en se basant sur les propriétés structurelles de chaque configuration familiale, que le capital social produit en leur sein varie dans sa forme et son volume en fonction de l'âge et de l'état de santé des individus âgés. Grâce aux outils de l'analyse de réseaux, il est possible de mesurer les propriétés structurelles de chaque configuration familiale et d'identifier pour chacune d'elles le type de capital social qui y est produit. Nous pourrons dès lors observer si ces diverses propriétés structurelles - et le type de capital social - varient en fonction de l'âge et de la santé des individus interrogés. Si tel est le cas, ces variations pourront être interprétées comme la manifestation de ces différents mécanismes d'adaptation au sein même des réseaux familiaux.

Par ailleurs, la mise en place de ces divers mécanismes d'adaptation peut générer des déséquilibres dans les échanges de soutien, créer des tensions et de l'ambivalence au sein des configurations familiales. Le déclin sévère de la santé ou l'âge très avancé peuvent - parce qu'ils nécessitent un grand travail de réaménagement des liens de soutien au sein de la configuration familiale (mobilisation et désengagement) - s'accompagner de plus de tensions et d'ambivalence au sein des configurations familiales des «très-vieux » que dans celles des plus jeunes ou de ceux qui sont en meilleure santé. En plus de créer du déséquilibre dans les liens de soutien et des tensions, l'âge très avancé et le déclin de la santé peuvent prétériter la capacité des plus vieux à gérer les conflits et l'ambivalence inhérente au relations familiales. Dans cet ouvrage, nous observerons l'ambivalence au niveau « meso », c'est-à-dire au niveau de l'ensemble des relations au sein de la configuration familiale, ce qui est rarement étudié dans la littérature gérontologique. Et nous analyserons comment les modes de conflit et de support, qui reflètent des manières différentes de gérer l'ambivalence au sein des configurations familiales, varient selon l'âge et l'état de santé des individus âgés.

\subsection{Conclusion}

Dans le premier chapitre sont recensés les modèles théoriques ou perspectives d'analyse qui ont marqué la gérontologie sociale ces dernières 


\section{Facteurs explicatifs en gérontologie}

années et qui ont orienté de manière déterminante les travaux gérontologiques sur les relations sociales et familiales. Leurs apports tout comme leurs limites ont été soulignés. En décrivant les conditions de leur émergence, nous avons mis en exergue que ces modèles ont été influencés par des traditions ou des discours scientifiques ambiants et, pour ces raisons, ils ont privilégié certaines dimensions plutôt que d'autres. Par exemple, le soutien familial et son impact positif sur la santé et le bien-être ont été en effet largement mis en avant alors que les tensions familiales ont été relativement peu traitées. En conséquence, la famille "nucléaire », reposant sur des liens familiaux bio-légaux et prometteuse d'un soutien indéfectible, n'a jamais été remise en question. Les familles significatives, c'est-à-dire celles qui importent et qui font sens aux yeux des individus âgés, ne sont jamais explorées. De même, seules les dyades intergénérationnelles, perçues comme centrales dans les échanges de soutien, sont étudiées et souvent au détriment du réseau complexe de liens d'interdépendance dans lequel elles s'insèrent et dont elles dépendent pourtant.

Le deuxième chapitre traite des facteurs-clé de la dynamique des relations sociales et familiales dans la vieillesse, tels qu'ils sont abordés dans la littérature gérontologique. Leur analyse reste cependant limitée aux réseaux plus larges des relations personnelles ou aux relations dyadiques. Autrement dit, l'influence de ces différents facteurs sur la façon dont les aînés définissent la famille significative, sur l'organisation des liens d'interdépendance au sein de leur configuration familiale et sur la position qu'ils y occupent n'est jamais questionnée. De même, on ne sait pas si le type de capital social, produit au sein des configurations familiales, diffère en fonction des réserves démographiques que les aînés ont à disposition, de leur état de santé et de leur profil socio-démographique. Nous ne savons pas non plus si la manière de gérer l'ambivalence familiale varie en fonction de ces différents facteurs. Dans cet ouvrage, nous irons plus loin que les travaux actuels en proposant de nouvelles questions de recherche qui permettront d'approfondir les connaissances sur les relations familiales dans la vieillesse.

L'une des premières questions qui guidera ce travail est l'identification des membres significatifs de la famille dans la vieillesse. Les modèles dominants de la gérontologie et les nombreux travaux qui s'y réfèrent se sont reposés sur des indicateurs démographiques axés sur des rôles familiaux pré-définis - comme l'époux(se), les enfants ou les petits-enfants pour définir la famille chez les personnes âgées. Ces indicateurs permettent 
certes de mesurer l'état des réserves démographiques dont disposent les individus âgés dans la vieillesse mais, en aucun cas, ils définissent la famille qui « compte » pour les aînés, celle sur laquelle ils s'appuient en cas de difficultés. Le réservoir de parenté peut offrir un large potentiel de ressources relationnelles. Cependant, seules certaines d'entre elles sont investies affectivement par les aînés alors que d'autres, au contraire, n'ont jamais été investies ou ont été désinvesties au cours du temps. Pour l'heure, nous ne savons pas quels membres du réservoir de parenté sont perçus comme significatifs par les individus âgés. Cette question n'est jamais évoquée dans la recherche gérontologique portant sur la famille, du moins dans des enquêtes quantitatives à large échelle, telles que VLV.

La question sur l'identité des membres significatifs de la famille amène celle de la diversité des définitions personnelles de la famille dans la population âgée. En fonction de leur parcours de vie, de leur profil sociodémographique et des ressources dont ils disposent, il est probable que les individus âgés affichent des perceptions différentes de leur famille significative. Selon les liens d'interdépendance affective et pratique qu'ils ont développés au cours de leur parcours de vie et des transitions de vie qu'ils ont été amenés à traverser, on peut supposer que certains aînés ont une conception de la famille plutôt « classique » axée sur le modèle nucléaire de la famille alors que d'autres au contraire y insèrent des membres significatifs qui vont bien au-delà du cadre bio-légal. Les modèles dominants de la gérontologie sociale se sont reposés sur le modèle de la famille nucléaire, privilégiant les liens biologiques ou d'alliance, pour définir «a priori » la famille dans la vieillesse. Ils ont de fait négligé la diversité des définitions personnelles de la famille données par les personnes âgées elles-mêmes. Dans ce travail, nous dégagerons les diverses définitions personnelles de la famille des aînés en nous basant sur les membres de la famille qu'ils ont eux-mêmes cités comme significatifs. Sur cette base, nous construirons une typologie de configurations familiales, chacune d'elle reflétant une conception particulière de la famille dans la vieillesse.

Une troisième question a trait aux liens d'interdépendance qui relient entre eux les différents membres significatifs de la famille au sein des configurations familiales. Cette question a été négligée dans la recherche en gérontologie sociale. Bien que les réseaux personnels soient l'objet de nombreuses études, leur composante familiale n'est jamais en soi étudiée ni dans sa composition ni dans ses liens. En somme, nous ne savons rien quant aux relations et aux échanges qui connectent les différents membres 
de la famille et par lesquels s'organise le soutien. Certes, la solidarité et les normes de réciprocité ont été étudiées en gérontologie sociale mais seulement dans le cadre dyadique, et plus précisément au sein des relations intergénérationnelles. Or, pour mieux comprendre comment se construit la solidarité familiale dans la vieillesse, il est nécessaire de tenir compte de l'ensemble du réseau complexe de liens d'interdépendance dans lequel s'insèrent les dyades familiales. Cette question est importante car la façon dont s'organise le soutien au sein des configurations familiales donne accès à différents types de capital social dont les effets sur le bien-être des individus ont été soulignés dans la littérature (voir le prochain chapitre). Grâce aux outils de l'analyse de réseau, nous pourrons identifier les propriétés structurelles de chaque configuration familiale et mesurer le type de capital qu'elle génère.

Quant à la quatrième question de recherche, elle concerne l'ambivalence au sein des configurations familiales. L'ambivalence qui associe les aspects positifs (amour, soutien ou affection) et négatifs (jalousies, frustrations, tensions ou conflits) des relations interpersonnelles fait partie intégrante des relations familiales en raison de la forte interdépendance relationnelle qui les caractérise. Dans bon nombre de travaux en gérontologie sociale, l'ambivalence a été surtout traitée dans les relations dyadiques, intergénérationnelles. L'observation de l'ambivalence au niveau de l'ensemble du réseau familial n'a jamais été considérée en gérontologie sociale, du moins sur des données quantitatives. Pourtant, l'équilibre des échanges de soutien est difficile à respecter au sein des configurations familiales, créant de la frustration et des tensions lorsque le déséquilibre s'installe. Ces tensions sont particulièrement manifestes lorsque les ressources sont rares, l'obligation d'aider un membre dans le besoin ou de rendre ce que l'on a reçu crée du stress et génère de fortes tensions dans l'ensemble du réseau familial. Latente ou manifeste, l'ambivalence est indissociable des relations familiales et nécessite d'être négociée au sein des configurations familiales. Nous observerons dans cet ouvrage comment l'ambivalence se négocie au sein des configurations familiales. Pour ce faire, nous dégagerons plusieurs modes de conflit et de support qui seront interprétés comme les résultats de gestion différente de l'ambivalence au sein même des configurations familiales.

Finalement, nous nous interrogerons sur l'impact des principaux facteurs mentionnés dans ce chapitre (réserves démographiques, statut socioéconomique, genre, âge et santé) sur la composition des configurations 
familiales et sur les liens d'interdépendance qui les sous-tendent. Ces divers facteurs s'associent à différentes ressources (démographiques, économiques, physiques, cognitives ou relationnelles) qui ont un impact sur la « capacité » des individus âgés à entrer en lien et à maintenir des relations interpersonnelles, notamment familiales. Le montant et la qualité de ces ressources peuvent se révéler fort divers en fonction du profil sociodémographique des aînés et de leur parcours de vie. La recherche en gérontologie sociale a montré que les personnes privilégiées en termes de réserves démographiques, de statut socio-économique, de genre, d'âge et de santé ont davantage d'opportunités relationnelles que celles qui sont moins bien loties. Cependant, nous ne savons rien quant à l'impact de ces différents facteurs sur les définitions personnelles de la famille significative dans la population âgée. On peut par exemple se demander si la diversité qui caractérise les réseaux personnels des individus avantagés socio-économiquement se reflète aussi dans la conception qu'ils ont de leur famille significative. De même, on peut aussi s'interroger sur l'influence de ces facteurs sur l'organisation des liens de soutien qui caractérise les configurations familiales. On peut en effet postuler que, selon les ressources que les individus âgés ont à disposition, ils développent des liens d'interdépendance différents au sein de leur configuration familiale et, plus encore, qu'ils y occupent une position distincte, leur donnant accès à différents types de capital social. Il est aussi probable que la manière de gérer l'ambivalence au sein des configurations familiales diffère aussi en fonction de l'ensemble de ces facteurs, donnant lieu à l'émergence de différents modes de conflit et de support selon le profil socio-démographique et le parcours de vie des aînés.

Ces questions sont rarement évoquées dans la recherche gérontologique. Pourtant, étudier les configurations familiales et leurs particularités permettrait d'en savoir plus sur la vie familiale aux dernières étapes de la vie. Pour répondre à l'ensemble de ces questions, nous utiliserons la perspective configurationnelle qui aborde les relations familiales sous un nouvel angle, celui des liens d'interdépendance au sein des configurations familiales. Les outils de l'analyse de réseaux que cette approche propose permettent d'analyser les propriétés structurelles de chaque configuration familiale. Dans le prochain chapitre «Problématique et hypothèses », nous décrirons plus avant la perspective configurationnelle et, à cette occasion, nous introduirons les différents points explorés dans cet ouvrage à savoir l'identification des membres significatifs de la famille, les différentes 
Facteurs explicatifs en gérontologie

définitions personnelles de la famille, les types de capital social et les diverses formes de gestion de l'ambivalence familiale dans la vieillesse. Chemin faisant, nous présenterons les différentes hypothèses qui ont guidé cette recherche. 


\section{Problématique et hypothèses}

Dans les chapitres précédents, nous avons discuté des principaux modèles théoriques qui ont dominé la gérontologie sociale ces dernières décennies et les facteurs qui sont avancés dans la littérature gérontologique pour expliquer les variations dans les relations sociales et familiales des personnes âgées. Bien que la recherche en gérontologie sociale se soit davantage souciée de la diversité et de la complexité des relations familiales ces dernières années, elle peine cependant à innover tant dans ses concepts que dans ses outils, la pénalisant dans son exploration des liens familiaux (Allen et al., 2011; Silverstein \& Giarrusso, 2010). Afin d'aller plus loin, nous avons proposé à la fin du chapitre précédent de nouvelles questions de recherche. Celles-ci visent non seulement à investiguer les nombreux aspects que les modèles dominants de la gérontologie sociale négligent lorsqu'il est question de la famille mais aussi à innover dans le champs gérontologique en proposant un nouvel angle d'analyse qui permet de mieux saisir la complexité des relations familiales. Nous proposons en effet de recourir à l' " approche configurationnelle » qui a été développée en Europe par des sociologues de la famille au sein du réseau de recherche «Sociology of Families and Intimate Lives » de l'Association Européenne de Sociologie (Castrén \& Ketokivi, 2015; Widmer et al., 2008; Widmer, 2016). Comme en témoigne l'ouvrage collectif «Beyond the nuclear family: Families in a configurational perspective » (Widmer \& Jallinoja, 2008), cette approche a été utilisée par des chercheurs en provenance de toute l'Europe sur des données aussi bien quantitatives que qualitatives, portant sur la population "générale » (Jonas \& Le pape, 2008; Wall, Aboim, \& Marinho, 2008) ou sur des populations particulières (Castrén, 2008; Castrén \& Ketokivi, 2015; Sapin, Widmer, \& Radulescu, 2008; Widmer \& Sapin, 2008).

Cette perspective a été peu utilisée dans la recherche gérontologique, du moins d'un point de vue quantitatif. Seules quelques recherches européennes portant sur des données qualitatives se sont intéressées aux configurations familiales dans le cadre du «caregiving » ou de l'ambivalence aux dernières étapes de la vie (Hillcoat-Nallétamby \& Phillips, 2011; Le Bihan \& Martin, 2008). Dans ce chapitre, nous présenterons les atouts de cette approche, ce qu'elle amène de nouveau par rapport aux modèles 


\section{Problématique et hypothèses}

théoriques dominants introduits dans le premier chapitre. Nous en montrerons la pertinence pour étudier les familles dans la vieillesse. Trois thèmes centraux de l'approche configurationnelle seront particulièrement développés dans ce chapitre : les «membres significatifs de la famille», le « capital social», les « conflits et l'ambivalence ». Sur la base de la littérature existante, nous mettrons en évidence que ces thèmes - qui ne sont jamais ou rarement évoqués en gérontologie sociale ou traités au niveau du réseau familial (ambivalence) - offrent un regard nouveau, original, sur les dynamiques familiales dans la vieillesse. C'est sur ces thèmes que porteront les différentes hypothèses de recherche qui guideront ce travail.

\subsection{L'approche configurationnelle}

Contrairement au modèle du convoi de Toni Antonucci qui conçoit la famille comme l'une des nombreuses composantes d'un réseau personnel, l'approche configurationnelle définit la famille comme une "configuration ", à savoir un réseau de relations familiales significatives (Castrén \& Ketokivi, 2015; Widmer, 2016; Widmer et al., 2008). Cette approche offre une nouvelle définition de la famille et l'explore sous un angle original. Dans cette perspective, ce ne sont pas tant les statuts et les rôles au sein de la famille qui sont perçus comme déterminants mais les relations d'interdépendance qui relient entre eux les membres significatifs qui forment une même configuration familiale. Les recherches précédentes, qui ont utilisé cette approche pour étudier les familles dans divers contextes, ont montré sa pertinence pour mettre à jour la diversité et la complexité des relations familiales (Castrén, 2017; Castrén \& Ketokivi, 2015; Hauri, 2011; De Carlo et al., 2014; Widmer, 2016, 2006; Widmer \& Jallinoja, 2008). Leurs résultats révèlent en effet que les individus développent une variété de configurations familiales en y intégrant une diversité de membres qui vont bien au-delà des critères de sang et d'alliance usuellement utilisés dans la recherche pour définir «la famille». Avant de mettre en évidence les différents atouts de cette perspective pour étudier les relations familiales dans la vieillesse, nous définirons dans la prochaine section le concept général de «configuration», proposé en psychologie par l'un des précurseurs de l'analyse de réseaux, Jacob Levy Moreno (Moreno, 1934), et en sociologie 
par Norbert Elias (Elias, 1991), et celui plus spécifique de « configuration familiale », développée par des sociologues de la famille de l'Association Européenne de Sociologie (Castrén \& Ketokivi, 2015; Widmer, 2016; Widmer et al., 2008).

\subsubsection{Les configurations d'interdépendances}

La perspective configurationnelle s'appuie sur le concept de « configuration » qui renvoie à tout réseau d'individus, unis entre eux par des relations d'interdépendance (Duvoux, 2011; Elias, 1991; Moreno, 1934). Le psychologue et psychiatre Jacob Lévy Moreno (Moreno, 1934) et le sociologue Norbert Elias (Elias, 1991) ont utilisé le terme de « configuration » comme un outil conceptuel et méthodologique pour étudier les relations humaines. Ce faisant, ils ont tous deux œuvré au développement de l'approche configurationnelle (Castrén \& Ketokivi, 2015; Widmer, 2016; Widmer et al., 2008). Jacob Levy Moreno (Moreno, 1934) utilise, au début du 20e siècle, le concept de «configuration sociale » pour étudier la dynamique des groupes. Pour Moreno, ce sont les motivations affectives comme l'amitié, souvent orientées par des objectifs identitaires, qui sont à la base des liens d'interdépendance entre les individus. Ces liens se développent pour former peu à peu des réseaux d'individus interdépendants, c'est-à-dire des configurations sociales (Freeman, 2004; Parlebas, 1992; Scott, 2000). Dans l'approche de Moreno, l'individu est acteur mais il dépend aussi étroitement des autres avec qui il est en lien au sein de son réseau. Ces derniers l'influencent dans ses attitudes, ses choix et son comportement en lui offrant des possibilités de s'épanouir au gré de ses choix relationnels mais aussi en le cadrant, contribuant ainsi au développement de son identité personnelle, familiale, communautaire et sociale (Freeman, 2004; Parlebas, 1992; Scott, 2000). Pour s'aider dans ses investigations, Moreno développe la sociométrie, méthode à la fois quantitative et clinique (outil thérapeutique), qui lui permet d'observer les liens d'interdépendance d'un individu avec les membres de sa configuration sociale à l'aide d'un sociographe (représentation graphique). Il construit également des indices sociométriques tels que la distance, la fréquence, la réciprocité, la densité et la position des individus au sein de leur réseau afin de mesurer les propriétés structurelles des relations interpersonnelles, positives et négatives, des membres d'une même configuration sociale comme, par exemple, au 


\section{Problématique et hypothèses}

sein d'une famille, d'une classe d'école, d'un quartier ou d'un régiment à l'armée (Freeman, 2004; Kadushin, 2012; Parlebas, 1992). En raison de ses travaux novateurs centrés sur la sociométrie, Moreno est considéré dans la littérature comme l'un des précurseurs de l'analyse de réseaux (Freeman, 2004; Parlebas, 1992; Scott, 2000).

Le concept de «configuration » est aussi fort présent dans la sociologie de Norbert Elias comme le montre son ouvrage "Qu'est-ce que la sociologie? »(Elias, 1991). Il est l'un des concepts-clé de sa théorie sociologique. Pour cet auteur, l'individu dépend, dès sa naissance, des relations qui le relient à de nombreux autres individus, notamment aux membres de sa famille. Ces liens d'interdépendance évoluent en fonction des multiples échanges de ressources - telles que l'affection, le soutien, ou encore la reconnaissance - qui se développent entre les individus sans que ces derniers en aient pleinement conscience. Outre la construction de leur identité personnelle, ces ressources leur sont indispensables pour faire face à l'environnement qui les entoure et aux aléas de leur parcours de vie (Elias, 1991; Quintaneiro, 2005). Au fur et à mesure que les liens se créent, les configurations s'allongent peu à peu et se complexifient pour passer d'une configuration de petite taille à des configurations plus larges et plus complexes. Les configurations n'ont pas de limites ni de frontières, elles peuvent prendre diverses formes et atteindre divers degrés de complexité (Elias, 1991; Quintaneiro, 2005). Autrement dit, les interdépendances qui relient les individus sont dynamiques, fluctuant au gré des circonstances individuelles et sociales, et font que la configuration a sa propre réalité avec des caractéristiques qui lui sont propres et qui vont bien au-delà des individus qui la composent ou de la forme figée d'organisation, sous-entendue par la notion de structure (Ducret, 2011; Elias, 1991; Quintaneiro, 2005). Comme pour Moreno, le chercheur peut, par l'analyse des liens d'interdépendance, saisir les particularités de chaque configuration, quelles que soient sa taille et sa forme (Elias, 1991).

Contrairement à la sociologie classique qui considère l'individu et son contexte social comme deux objets distincts, homogènes et statiques, Elias ne conçoit pas l'individu et son contexte comme deux entités séparées (Bax, 1978; Duvoux, 2011; Elias, 1991). Selon cet auteur, le contexte social se développe par les individus qui le forment au gré de leurs diverses interactions et échanges (Bax, 1978; Ducret, 2011; Elias, 1991; Quintaneiro, 2005). Quant à l'individu, il ne peut être extrait de son réseau d'interdépendances dans lequel il s'insère, dépendant étroitement des 
membres de son réseau pour construire son identité personnelle (Elias, 1991; Quintaneiro, 2005). L'individu se définit en effet à partir de ses relations avec les autres : il agit, réagit en fonction du comportement et des actions des autres avec lesquels il a développé des liens d'interdépendance (Elias, 1991; Ducret, 2011 ; Quintaneiro, 2005). Ce faisant, l'individu n'est pas totalement libre ni déterminé par sa seule conscience (l'homo « clausus ») (Elias, 1991). Relié aux autres individus, il est soumis au regard et au contrôle des autres dont il dépend et qui l'invitent à se conformer aux normes collectives qui sous-tendent la configuration d'interdépendances à laquelle il appartient (Ducret, 2011). Tout écart aux normes peut être facilement repéré et sanctionné. Les interdépendances sont donc à la fois source d'opportunités et de contraintes pour les individus qui s'y insèrent. Dans une telle perspective, il n'est pas possible d'appréhender la configuration uniquement par ses composantes (individus) ou sa forme globale. L'approche configurationnelle se centre en effet essentiellement sur les processus relationnels qui sous-tendent les configurations (Duvoux, 2011; Elias, 1991 ; Paulle, Van Heerikhuizen, \& Emirbayer, 2012). En s'axant sur les liens d'interdépendance, cette perspective permet de traiter ensemble et au même niveau l'individu, la dyade et le contexte. De ce point de vue, elle dépasse les principaux modèles théoriques en gérontologie sociale présentés auparavant puisque ceux-ci se centrent uniquement soit sur l'individu (le modèle de la sélectivité socio-émotionnelle), soit sur la dyade (les modèles de la solidarité intergénérationnelle et du « support bank»), soit sur la structure (le modèle du convoi).

\subsubsection{Les configurations familiales}

Dans les années 2000, Eric Widmer, Riitta Jallinoja, Anna-Maija Castrén et Kaisa Ketokivi du réseau de recherche «Sociology of Families and Intimate Lives » de l'Association Européenne de Sociologie (Castrén \& Ketokivi, 2015; Widmer et al., 2008) reprennent le concept de « configuration» pour approcher la famille. Ils constatent dans une série de travaux portant sur différentes classes d'âge que les critères usuellement utilisés pour mesurer la famille, tels que la communauté de ménage et les liens de sang, ne suffisent plus à appréhender la famille, celle-ci s'étendant bien au-delà du ménage et des liens de sang (Castrén \& Ketokivi, 2015; Widmer, 1999a, 2006; Widmer et al., 2008). Dans leurs travaux, ils mettent en évidence que 


\section{Problématique et hypothèses}

la famille ne peut plus être définie comme un petit groupe de personnes, formaté institutionnellement par des rôles et des statuts, et axé sur des liens de sang ou d'alliance, mais plutôt comme un réseau de personnes qui, sans définition institutionnelle pré-établie, sont dépendantes les unes des autres par les multiples relations et échanges qui les relient (Castrén \& Ketokivi, 2015; De Carlo et al., 2014; Widmer, 1999a; Widmer et al., 2008). Pour preuve, la complexification des liens d'interdépendance due à la remise en couple et à la recomposition rend difficile, voire impossible, l'étude des familles recomposées avec des indicateurs classiques en raison de la complexité qui les caractérise (Castrén, 2008; Castrén \& Ketokivi, 2015; Castrén, 2017; De Carlo et al., 2014). Seule l'approche configurationnelle permet de rendre compte de la diversité et de la complexité des nouvelles formes familiales d'aujourd'hui.

Dans cette approche, la famille est définie comme un réseau complexe de liens d'interdépendance qui relient entre eux les différents membres significatifs de la famille. Ce sont les liens d'interdépendance qui font la «famille » et non des critères institutionnels pré-définis (Castrén \& Ketokivi, 2015; Widmer et al., 2008; Widmer, 2016). Les configurations familiales se distinguent des autres types de configurations sociales sur plusieurs aspects. D'une part, les ressources échangées sont multiples comme l'affection, le soutien émotionnel, la reconnaissance sociale, le temps, la communication, l'aide pratique ou encore le soutien financier. Elles répondent ainsi aux multiples besoins individuels tant pratiques, affectifs, émotionnels, financiers qu'identitaires. D'autre part, les échanges de ressources familiales s'inscrivent généralement dans la durée et l'histoire familiale, faisant des liens d'interdépendance qui forment les configurations familiales des connexions particulièrement solides et fortes. La norme de réciprocité généralisée qui sous-tend les échanges familiaux favorise le développement de la confiance entre les membres de la configuration familiale et tend à renforcer le sentiment d'appartenance à une identité familiale commune (Widmer, 2006, 1999a, 2016). Par l'affectivité qui caractérise les liens familiaux, ces ressources sont porteuses d'une grande valeur symbolique (Cropanzano \& Mitchell, 2005; Foa \& Foa, 1980). L'affectivité contribue en effet à donner un sens particulier à l'ensemble des échanges familiaux, même financiers ou matériels. Un service rendu, un prêt d'argent, le temps passé ensemble peuvent être perçus et interprétés comme des preuves d'amour et des signes de reconnaissance. Ces 
échanges nourrissent le sentiment individuel d'être important et de compter pour les autres, renforçant encore davantage les liens d'interdépendance au sein de la famille. A l'inverse, leur non octroi peut être perçu comme un signe de désamour, de trahison, de non reconnaissance, et cela d'autant plus si l'individu demandeur est fortement dépendant des ressources familiales, ne disposant pas d'autres alternatives (Widmer, 2016). En raison de la significativité qu'elles revêtent pour les individus, ces ressources sont particulièrement convoitées et difficilement accessibles en dehors du contexte familial, faisant de la famille une configuration d'interdépendances de premier plan qui ne peut être que difficilement brisée et remplacée (Widmer, 2016).

En considérant les liens d'interdépendance, l'approche configurationnelle permet de se centrer sur le réseau familial en tant qu'objet d'étude et d'aller au-delà des modèles dominants de la gérontologie sociale (cf. premier chapitre). Alors que le modèle du convoi conçoit la famille comme une composante relativement homogène et statique, cette perspective montre au contraire que la famille est plurielle et dynamique. Plus encore, les échanges qui s'y produisent sont multiples, impliquant des dimensions aussi bien affectives, pratiques que cognitives qui rendent les relations familiales particulièrement complexes. Globalement, la recherche en gérontologie sociale a jusqu'à présent omis de considérer la famille comme un réseau de liens d'interdépendance et, ce faisant, elle a négligé toute la diversité et la complexité qui caractérisent aujourd'hui les relations familiales.

\subsubsection{Les apports de l'approche configurationnelle}

L'approche configurationnelle offre de nombreux avantages pour étudier les familles dans la vieillesse. Dans cette section, nous les évoquons l'un après l'autre et soulignons en quoi ces avantages sont innovants et surpassent les modèles théoriques utilisés dans la recherche gérontologique. Premièrement, en se centrant sur ces liens d'interdépendance, cette approche permet d'identifier les membres de la famille qui sont significatifs aux yeux des individus. Se baser uniquement sur les rôles et les statuts dans la famille pour définir «a priori» qui est important dans la famille ne permet pas d'identifier les membres significatifs de la famille (Castrén \& Ketokivi, 2015; De Carlo et al., 2014; Widmer et al., 2008; Widmer, 


\section{Problématique et hypothèses}

2016). Une telle démarche est réductrice car elle suppose que toutes les familles sont organisées de la même manière et que toutes « devraient » être composées des membres de la famille nucléaire (mère, père et enfants biologiques), ceux-ci étant considérés «a priori» comme « significatifs» en raison de leur rôle et de leur statut au sein de la famille. La présence de tels membres peut être corrélée à de forts liens d'interdépendance ou renvoie à un caractère hautement symbolique mais ces termes, seuls, ne suffisent pas à identifier les liens significatifs dans les familles (De Carlo et al., 2014; Widmer et al., 2008; Widmer, 2016). Or, aujourd'hui, cette approche « classique » des familles est rarement remise en question en gérontologie sociale (Allen et al., 2011; Marshall et al., 1993; Silverstein \& Giarrusso, 2010). Les indicateurs démographiques utilisés dans la recherche listent les membres du réservoir de parenté, c'est-à-dire les réserves démographiques, et en fonction des rôles et des statuts auxquels ces membres renvoient, ils sont automatiquement catégorisés comme membres « importants» ou «peu importants » de la famille alors qu'en réalité, ces indicateurs ne donnent qu'une indication sur la composition du réservoir de parenté mais en aucun cas sur la famille qui « compte», celle qui fait sens aux yeux des individus interrogés (Castrén \& Ketokivi, 2015; De Carlo et al., 2014; Widmer et al., 2008; Widmer, 2016).

Comme le montrent divers travaux, les membres significatifs de la famille sont variés (Widmer \& Jallinoja, 2008; Widmer, 2016). Certains correspondent aux membres de la famille nucléaire alors que d'autres s'en éloignent nettement. En effet, les membres de la parenté élargie, de la bellefamille (famille du conjoint), le ou les partenaire(s) des parents et leurs enfants, les conjoints des frères et sœurs, et même les amis peuvent être cités comme membres significatifs de la famille, tout comme le partenaire, le père, la mère, les enfants ou les membres de la fratrie (Castrén, 2017; Widmer, 2016). Ces résultats confirment que le recours à une définition pré-établie de la famille ne suffit pas à appréhender la famille significative; seule l'appartenance à une configuration de liens d'interdépendance mais aussi le caractère symbolique de certains membres, c'est-à-dire ce qu'ils représentent pour les individus, permettent d'identifier les membres significatifs de la famille (Castrén \& Ketokivi, 2015; Widmer et al., 2008; Widmer, 2016). Autrement dit, les personnes que l'individu désigne luimême comme faisant partie de sa configuration familiale peuvent être considérées comme des membres significatifs de sa famille, à savoir des 
personnes qui comptent réellement pour lui (Castrén \& Ketokivi, 2015; Widmer, 1999a; Widmer \& La Farga, 2000; Widmer, 2006).

Deuxièmement, les membres significatifs de la famille se combinent différemment selon les individus. Par leurs combinaisons diverses, ils forment une variété de configurations familiales, traduisant des conceptions personnelles plurielles de la famille (Castrén \& Ketokivi, 2015; Castrén, 2017; Hauri, 2011; Sapin et al., 2008; Wall et al., 2008; Widmer, 2006; Widmer \& Sapin, 2008). Les «logiques» personnelles qui les sous-tendent sont diverses : certains individus privilégient le sang, mentionnant comme membres significatifs essentiellement des liens biologiques, d'autres préfèrent l'alliance, citant le partenaire et sa famille, d'autres encore sélectionnent des liens horizontaux, principalement les frères, les sœurs et leur famille, ou les amis (Castrén, 2017; De Carlo et al., 2014; Widmer, 2016). Généralement, la citation des premiers, les plus significatifs, appelant la mention de leurs alliés. Par exemple, si la sœur est mentionnée en premier lieu, il est probable que le partenaire de la sœur et ses enfants soient également cités. Même au sein d'un couple, les partenaires peuvent avoir des logiques différentes, donnant lieu à des définitions distinctes de leur famille significative (Widmer, 2016). Cette diversité dans les définitions personnelles de la famille s'explique en grande partie par le parcours de vie (Castrén \& Ketokivi, 2015; Castrén, 2017; Wall et al., 2008; Widmer, 2016). Le réservoir de parenté dans lequel les individus puisent les relations familiales qu'ils investissent affectivement se transforme au fil du temps, celui-ci étant tributaire des transitions de vie que les individus et leur proches ont été amenés à traverser au cours de leur vie tels que le mariage, le remariage, le divorce, la parentalité ou les décès des membres de leur famille. Selon leur trajectoire familiale, certains ont un riche réservoir de parenté leur offrant de nombreuses possibilités relationnelles alors que d'autres ont un réservoir de parenté plus limité ayant connu davantage de pertes en termes de décès, de divorces ou d'infertilités. Certains ont donc un choix relativement limité de relations familiales à investir alors que d'autres disposent d'une variété de choix relationnels (Widmer, 1999a; Widmer \& La Farga, 2000; Widmer, 2006).

La diversité des réservoirs de parenté explique en partie la pluralité des configurations familiales, mais elle n'en constitue pas la seule explication. Comme le suggère le modèle du convoi, les transitions de vie s'accompagnent de changements de rôles au sein du réseau personnel nécessitant une ré-évaluation des investissements relationnels. Certaines 


\section{Problématique et hypothèses}

relations peuvent être désengagées alors que d'autres sont ré-investies, répondant mieux aux besoins d'adaptation du moment des individus concernés (Antonucci, 2001; Fiori et al., 2008, 2006; Kahn \& Antonucci, 1980; Van Tilburg, 1998). Dans cet ouvrage, nous faisons l'hypothèse qu'il en est de même dans la famille. Avec des réserves démographiques identiques, c'est-à-dire avec un même réservoir de parenté, les individus ne s'investissent pas dans les mêmes relations familiales, ni de la même manière. Ils disposent donc d'une certaine marge de manœuvre. Ce travail de réévaluation au niveau du réseau familial a été négligé par le modèle du convoi, la famille étant perçue comme une composante du réseau personnel comme relativement figée et homogène. L'approche configurationnelle va donc plus loin en reconnaissant ce travail individuel de ré-évaluation au sein même de la famille. Ce mécanisme de ré-évaluation consiste doncdans les limites, certes, du réservoir de parenté et parfois au delà (amis) - à modeler au fil du temps sa propre famille significative (Castrén \& Ketokivi, 2015 ; De Carlo et al., 2014; Widmer, 2016). La pluralité des configurations familiales témoigne de ce mécanisme et nous montrerons dans cette recherche qu'il est toujours présent même aux dernières étapes de la vie.

Troisièmement, considérer la famille comme une configuration permet de recontextualiser les dyades dans un réseau complexe de liens d'interdépendance, puisque ces dernières, tout comme les individus, font partie intégrante des configurations familiales (Bott, 1955; Castrén \& Maillochon, 2009; Castrén \& Ketokivi, 2015; Giudici \& Widmer, 2017; Milardo, 2007; Widmer, Le Goff, Levy, Hammer, \& Kellerhals, 2006). Or, dans la recherche en gérontologie sociale, les dyades conjugales ou intergénérationnelles sont souvent étudiées en dehors de leur contexte relationnel. Leur insertion dans un complexe de liens d'interdépendance est rarement considérée. Une telle approche ne donne qu'une vision tronquée des liens dyadiques dont le fonctionnement et la dynamique dépendent étroitement du contexte relationnel dans lequel ils s'intègrent (Bott, 1955; Marshall et al., 1993; Milardo, 2007; Widmer \& La Farga, 2000; Widmer et al., 2006). Par exemple, les conflits entre différents membres de la configuration familiale peuvent affecter les relations dyadiques en y générant des tensions. A l'inverse, le soutien apporté par les membres du réseau familial peut atténuer les conflits au sein des dyades, sauf si celui-ci s'associe à des tentatives de contrôle (interférence), elles-mêmes sources de tensions (Widmer, Kellerhals, \& Levy, 2004; Widmer et al., 2006; Widmer, Giudici, Le Goff, $\&$ Pollien, 2009). De même, supervisées par le réseau familial, les dyades 
se doivent de se conformer aux normes collectives, ce qui influence inévitablement leurs décisions et leur fonctionnement (Bott, 1955; Castrén \& Maillochon, 2009; Castrén \& Ketokivi, 2015; Giudici \& Widmer, 2017; Milardo, 2007; Widmer et al., 2006). Celles-ci sont donc influencées par les liens familiaux dont elles dépendent, mais elles les influencent aussi en retour. Ceci est particulièrement le cas des dyades qui sont centrales dans les configurations familiales comme les dyades conjugales ou entre «parent-enfant ». Celles-ci jouent un rôle déterminant dans la formation et le maintien des liens d'interdépendance au sein des configurations familiales (Castrén, 2017; Widmer et al., 2004, 2009; Widmer, 2016). Un changement ou une atteinte dans l'une de ces dyades comme une rupture, la remise en couple ou le décès d'un des protagonistes peut déséquilibrer les échanges de ressources au sein de la configuration originale, entraînant une réorganisation des liens d'interdépendance au sein de l'ensemble de la configuration familiale (Castrén \& Ketokivi, 2015; Castrén, 2017; De Carlo et al., 2014; Widmer, 1999a).

En concevant les dyades comme interdépendantes les unes des autres et comme les parties intégrantes d'un réseau familial plus large, l'approche configurationnelle permet de sortir du cadre dyadique dans lequel se situe un bon nombre de travaux en gérontologie sociale et de considérer la solidarité et la réciprocité dans un contexte relationnel plus large que celui des dyades (Castrén \& Ketokivi, 2015; Castrén, 2017; Widmer et al., 2008; Widmer, 2016). Un tel changement dans le niveau d'observation ouvre de nouvelles perspectives à la fois théoriques mais aussi méthodologiques qui n'ont jamais été considérées dans la recherche sur la famille en gérontologie sociale. Dans la perspective configurationnelle, les normes de réciprocité régulent aussi bien les échanges dyadiques que le flux des échanges dans l'ensemble de la configuration familiale. Ces normes sont centrales puisqu'elles modèlent les liens d'interdépendance entre les différents membres significatifs de la configuration familiale (Widmer, 2016). Ces normes de réciprocité, notamment celles de réciprocité généralisée, sont particulièrement prégnantes dans les familles; elles assurent aux membres significatifs du réseau familial un accès privilégié à toutes les ressources dont ils ont besoin - c'est-à-dire au « capital social» - leur garantissant ainsi la sécurité, une identité collective, le sentiment d'appartenance, le maintien de leur santé et de leur bien-être (Bourdieu, 1980, 1986; Portes, 1998; Widmer, 2016). Le concept de « capital social» va audelà des notions de «solidarité intergénérationnelle » et de « support bank » 


\section{Problématique et hypothèses}

utilisés en gérontologie sociale pour décrire les échanges de soutien entre enfants adultes et parents âgés. Le terme de « capital social» comprend la « solidarité » comme le produit d'un processus relationnel à l'échelle de la configuration familiale et élargit la notion de «support bank » à l'ensemble $\mathrm{du}$ réseau familial. En ce sens, la notion de capital social est première, intégrant aussi bien celle de « solidarité » que celle de « support bank».

Du point de vue méthodologique, l'approche configurationnelle offre une palette d'outils statistiques provenant de l'analyse de réseaux qui permettent de mesurer les propriétés structurelles de chaque configuration familiale. Grâce à ces outils, il est possible de décrire avec précision la façon dont s'organisent les liens de soutien et de mesurer le type et le volume de capital social produit dans chaque configuration (Widmer, 2006; Widmer \& La Farga, 2000; Widmer et al., 2013). Ces mesures vont au-delà des indicateurs généralement utilisés en gérontologie sociale qui renseignent quant au montant ou à la fréquence des échanges (effectifs) de soutien entre l'individu âgé et les membres de son réseau (Ashida \& Heaney, 2008; Krause, 1997a; Van Tilburg, 1998). Ceci dit, ils ne permettent pas d'appréhender le capital social qui relève davantage du potentiel de soutien généré au sein des réseaux personnels ou familiaux que de l'aide effective (Bourdieu, 1980, 1986; Portes, 1998; Widmer, 2016). Grâce aux outils de l'analyse de réseaux, il est possible de détecter les variations du capital social tant dans sa forme que dans son volume en fonction de la composition des configurations familiales. Cette approche permet de mettre en exergue que les familles varient non seulement dans leur forme mais aussi dans le potentiel de support qu'elles produisent (Widmer, 2016). Nous montrerons dans cet ouvrage que c'est aussi le cas dans la vieillesse. Nous remettrons ainsi en question l'idée, relativement dominante en gérontologie sociale, que « la famille» est un groupe homogène et cohésif, producteur d'un soutien inébranlable en toutes circonstances (Bowling, 1994; Pinquart \& Sörensen, 2000; Shor et al., 2013; Silverstein \& Bengtson, 1994). Nous reviendrons plus tard sur la notion de capital social et nous en soulignerons la pertinence pour approcher le soutien familial en gérontologie sociale.

Quatrièmement, l'approche configurationnelle considère ensemble les aspects aussi bien positifs que négatifs des relations familiales, ceux-ci font partie intégrante des interdépendances familiales. Les interdépendances créées par les échanges génèrent du soutien et de la confiance mais elles sont aussi source de tensions. Le sentiment latent de redevabilité, suscité 
par les normes de réciprocité généralisée, « contraint» les individus à octroyer du soutien à ceux qui en ont le plus besoin au sein de la configuration familiale. Une telle pression normative, bien qu'elle garantisse le soutien au sein du réseau familial, peut générer du stress et des tensions, et cela d'autant plus si les ressources sont rares et sujettes à une forte compétition (Castrén \& Ketokivi, 2015; Sapin et al., 2008; Widmer \& Sapin, 2008; Widmer, 2016). Dans de telles situations, les demandes répétées d'aide des membres familiaux dans le besoin surchargent et épuisent les autres membres de la configuration qui se sentent obligés d'y répondre, accentuant encore davantage le stress et les tensions (Krause, 1997b; Offer, 2012). Outre le manque de ressources dans la famille, les échanges au sein de la configuration peuvent être déséquilibrés, certains disposant de plus de ressources que d'autres. En échange de leur soutien, les membres familiaux les plus avantagés peuvent être tentés d'exercer un certain contrôle sur les membres les moins dotés en faisant usage de pression, de chantage et parfois de violence pour inciter ces derniers à se conformer à leurs attentes (Offer, 2012; Widmer et al., 2004, 2009). Les tensions et les conflits que génèrent de tels rapports de force sont d'autant plus pesants que la rupture des liens familiaux est difficilement envisageable en raison des fortes interdépendances affectives qui sous-tendent ce type de configurations (Fingerman et al., 2004; Carr \& Moorman, 2011). Les membres qui en font partie sont en effet étroitement enchaînés les uns aux autres en raison des multiples ressources tant affectives que pratiques qu'ils se sont échangées au cours de leur vie (Castrén \& Ketokivi, 2015; Castrén, 2017; Widmer et al., 2008; Widmer, 2016).

Contraintes et affection, conflits et solidarité, pression et confiance ne s'opposent pas; ces diverses dimensions participent aux mêmes processus relationnels, faisant de l'ambivalence une caractéristique principale des configurations familiales (Castrén \& Ketokivi, 2015; Widmer, 2016). Dans la recherche en gérontologie sociale, la solidarité et l'ambivalence ont été largement étudiées mais seulement dans le cadre des relations dyadiques, intergénérationnelles (Bengtson, 2001; Lüscher \& Pillemer, 1998; Silverstein \& Bengtson, 1997). En considérant la famille comme un réseau de membres interconnectés par de multiples échanges, l'approche configurationnelle permet d'appréhender l'ambivalence au niveau «meso » de l'analyse. Nous pourrons en effet observer avec les outils statistiques de l'analyse de réseau comment les liens de soutien s'organisent au sein de 


\section{Problématique et hypothèses}

chaque configuration familiale et comment ils se combinent avec les relations de conflit (Widmer, 1999a; Widmer \& La Farga, 2000; Widmer, 2016). Ce faisant, nous dégagerons différents modes de conflit et de support reflétant le résultat de différentes stratégies collectives visant à gérer ou à négocier l'ambivalence familiale (Lüscher, 2000; Lüscher \& Pillemer, 1998; Widmer, 2016).

Cinquièmement, l'approche configurationnelle permet de mesurer le degré d'autonomie des individus au sein de leur configurations familiale. En gérontologie sociale, la théorie de la sélectivité socio-émotionnelle de Carstensen postule que l'individu a le « pouvoir» ou la « capacité » de gérer ses relations personnelles comme il le souhaite en vue de s'adapter au mieux au processus de fragilisation qui caractérise les dernières étapes de sa vie. Or, ce modèle ne tient pas compte du réseau d'interdépendances familiales dans lequel s'insère l'individu et qui le contraint dans ses choix et ses actions. Toutefois, l'individu peut disposer d'une certaine marge de manœuvre en fonction de la manière dont s'organisent les liens d'interdépendance au sein de son réseau et surtout de la position qu'il y occupe. S'il se situe au centre de sa configuration, jouant le rôle d'intermédiaire entre les différents membres de sa configuration qui n'ont pas ou peu de relations d'échange, il bénéficie dès lors d'une grande autonomie, voire d'un certain pouvoir, puisqu'il peut, étant central, contrôler le flux et la destination des échanges au sein de sa configuration familiale (Widmer, 1999a; Widmer \& La Farga, 2000; Widmer, 2016). Il peut même, s'il le souhaite, se désengager de certaines relations familiales qu'il juge trop fatiguantes ou peu gratifiantes sans qu'il y ait de conséquences sur le reste de sa configuration familiale. Si, au contraire, l'individu n'est pas central dans son réseau et qu'il est relié à des membres fortement connectés les uns aux autres, il n'a non seulement aucune prise sur les ressources qu'ils s'échangent mais plus encore il est soumis à un fort contrôle collectif qui le restreint drastiquement dans son autonomie. Ceci dit, un changement dans les liens d'interdépendance suite, par exemple, au départ d'un membre ou à l'arrivée d'un nouveau membre peut modifier le degré d'autonomie d'un individu en changeant sa position au sein de sa configuration familiale (Elias, 1991; Paulle et al., 2012). L'approche configurationnelle offre la possibilité de mesurer la position des individus dans leur configuration familiale et d'estimer leur degré d'autonomie relationnelle par rapport aux autres membres de leur réseau. La préservation de l' «autonomie» relationnelle est un enjeu central dans la vieillesse. Son maintien jusqu'à des âges 
très avancés est un objectif important pour de nombreux aînés qui sont de plus en plus amenés à demander de l'aide à leur entourage familial pour pouvoir faire face aux nombreuses pertes auxquelles ils sont confrontés dans la vieillesse (Cornwell, 2009a, 2009b, 2011).

Finalement, l'approche configurationnelle se centre sur les échanges de ressources qui sont nécessaires pour maintenir les liens d'interdépendance entre les membres significatifs de la famille. Or, le montant et la qualité des ressources dont dispose l'individu dans sa configuration familiale dépendent étroitement de sa position au sein de la société - notamment de son genre, de son âge et de son statut socio-économique - mais aussi de ses réserves démographiques et des transitions de vie qu'il a traversées au cours de sa vie comme le mariage, la parentalité ou encore des problèmes de santé. Il est probable en effet qu'un homme suisse, marié, appartenant à la classe d'âge des « jeunes-vieux », ayant un bon statut socio-économique et une bonne santé bénéficie de plus de ressources qu'une femme étrangère, veuve, malade, appartenant à la classe d'âge des " très-vieux » et affichant un bas statut socio-économique. Alors que le premier dispose d'un revenu élevé, d'une partenaire plus jeune et d'une bonne santé, la seconde n'a pas de partenaire, peu d'argent et une mauvaise santé. Entre ces deux cas extrêmes se déploie une variété de situations. Par leur appartenance multiple (genre, âge, classe sociale, etc.) et les diverses transitions de vie qu'ils ont vécues, les aînés présentent de fortes variations quant aux ressources qu'ils ont à disposition (Calasanti \& Kiecolt, 2012). Autrement dit, il est nécessaire de tenir compte de leur profil socio-démographique, de leur santé et de l'étendue de leur réservoir démographique pour appréhender l'état de leurs ressources dans la vieillesse. Seule une estimation du montant et de la qualité de ces ressources peut donner une idée de la " potentialité » des individus à entrer dans les échanges au sein de leur configuration familiale. La notion de «potentialité » est plus appropriée que celle de "pouvoir», car, dans l'approche configurationnelle, le pouvoir ne dépend pas des ressources propres des individus mais il se définit en fonction des ressources des autres membres de la configuration avec lesquels ils sont en lien (Elias, 1991; Johnson, 1988; Paulle et al., 2012). En somme, le pouvoir renvoie au différentiel de ressources entre l'individu focal et les membres de sa configuration. Quant à la notion de "potentialité », elle définit la capacité des individus à entrer dans les échanges au sein de la configuration familiale en fonction du montant et de la qualité de leurs ressources. On peut supposer que les aînés qui ont une forte « potentialité » 


\section{Problématique et hypothèses}

(bonnes ressources) s'engagent davantage dans leur configuration familiale et parviennent à mieux respecter la réciprocité des échanges que ceux qui ont une faible "potentialité » (faibles ressources). Il est même probable que cette «potentialité », appréhendée par l'état des ressources des individus, les influence dans leur conception de la famille significative, dans leur façon de percevoir l'organisation des liens de soutien et de conflit au sein de leur configuration familiale et de s'y positionner. Pour évaluer l'état des ressources des individus, nous nous appuierons sur leur profil socio-démographique, leur état de santé et l'étendue de leur réservoir démographique.

L'approche configurationnelle offre donc une perspective alternative dans l'analyse des relations familiales dans la vieillesse. Elle permet, en proposant un nouvel angle d'analyse, d'aller plus loin que les principaux modèles théoriques en gérontologie sociale présentés précédemment. En considérant la famille comme une configuration, à savoir un réseau de membres de la famille significatifs, liés entre eux par de multiples liens d'interdépendance, cette perspective propose des concepts et des outils d'analyse innovants pour observer les familles dans leur diversité et leur complexité. Bien que cette approche se soit révélée pertinente pour étudier les familles dans des populations diverses, on peut cependant se demander si elle est justifiée pour étudier les familles aux dernières étapes de la vie. Il se peut que les aînés, qui ont connu pour la plupart un parcours de vie relativement standard, aient une conception relativement homogène de la famille, se référant principalement à sa forme nucléaire, "normative », axée sur les liens de sang et d'alliance. De même, de nombreux travaux s'inspirant des modèles dominants de la gérontologie sociale ont souligné le soutien indéfectible de la famille aux dernières étapes de la vie. On peut dès lors se demander si les liens de soutien autour du parent vieillissant ne s'organisent pas tous de la même manière, produisant de fait le même type de capital social. Il se peut aussi que, sous l'effet de la sélectivité socio-émotionnelle, les relations familiales désignées comme significatives ne soient perçues par les aînés que comme positives, celles jugées conflictuelles ayant été totalement désinvesties, rendant le concept de l'ambivalence relativement peu pertinent aux dernières étapes de la vie.

En se basant sur des résultats récents en gérontologie sociale, nous montrerons dans les prochaines sections que ces diverses suppositions ne tiennent pas la route et que l'approche configurationnelle se justifie pleinement pour observer les familles dans la vieillesse. Pour ce faire, nous 
développerons les notions de «membres significatifs de la famille», de « capital social » et d' « ambivalence » aux dernières étapes de la vie puisqu'elles sont centrales dans l'approche configurationnelle. Nous mettrons d'abord en évidence que la significativité des membres familiaux est tout aussi importante dans la vieillesse qu'à d'autres étapes de la vie. Ensuite, nous soulignerons la pertinence de la notion de capital social et ses différents types pour aborder le soutien familial dans la vieillesse. Finalement, nous évoquerons les liens de soutien, de conflit et l'ambivalence au sein des configurations familiales. En reprenant les travaux sur l'ambivalence intergénérationnelle, nous montrerons que le soutien et le conflit sont intrinsèques aux relations familiales des personnes âgées, tout comme ils le sont à d'autres âges de la vie. A l'issue de ces différentes sections, plusieurs hypothèses de recherche seront posées, celles-ci orienteront le travail d'analyse et la suite de cet ouvrage.

\subsection{Les membres significatifs de la famille dans la vieillesse}

Cette section porte sur la notion de «membre significatif de la famille». Il s'agit de mettre en évidence sa pertinence dans la vieillesse bien que les courants dominants de la gérontologie l'aient peu considérée, se focalisant généralement soit sur la famille «nucléaire », définie «a priori » comme significative, soit sur l'étude des réseaux personnels. L'argumentation développée dans cette section suit plusieurs étapes. Dans un premier temps, nous montrons que les liens de sang ou d'alliance sont devenus plus aléatoires en raison de la complexification des trajectoires de vie familiale, générant chez certains individus une grande incertitude quant au soutien familial attendu. Ensuite, nous soulignons que, pour compenser cette incertitude, les individus tendent (inconsciemment) à davantage s'investir affectivement dans diverses relations personnelles, aussi bien familiales que non familiales. En nous appuyant sur ces deux processus - à savoir l'imprévisibilité des liens de sang et l'importance grandissante des relations personnalisées - nous mettons en lumière comment les individus ré-interprètent les liens familiaux pour définir « leur» famille significative, c'est-à-dire celle qui « importe ». Certaines données qualitatives sont assez édifiantes à ce propos; elles mettent en exergue le travail de «création» 


\section{Problématique et hypothèses}

que les individus mettent en œuvre pour construire leur famille significative, perçue comme plus fiable dans son soutien que la famille bio-légale. Nous terminons cette première section en soulignant que ces études, pour la plupart qualitatives, ne nous disent rien quant à la prépondérance de ce mécanisme au sein de la population âgée. Elles ne nous informent pas non plus quant à l'identité des membres familiaux qui composent les familles significatives dans la vieillesse et à la manière dont ils se combinent pour former diverses configurations familiales.

\subsubsection{Incertitude et significativité}

Bien que le réservoir de parenté offre un potentiel de support (Bengtson, 2001 ; Riley, 1983; Riley \& Riley, 1996), il ne suffit pas à garantir aux individus âgés le développement de relations significatives sur lesquelles ils peuvent compter en cas de problèmes. Même s'il est riche en réserves démographiques comme dans le cas des familles à structure multigénérationnelle, les relations qu'il propose ne sont pas toutes «mobilisables », et cela même si celles-ci se caractérisent par des liens de sang ou d'alliance (Connidis, 2010). Il peut s'agir, par exemple, de membres de la parenté éloignée comme des cousines ou des cousins avec lesquels l'individu n'a jamais lié de relation proche ou n'a pas pu maintenir le lien en raison des aléas du parcours de vie. Même les membres de la famille définis dans la littérature comme les «plus proches »-ceux que l'on qualifie de «primaires » (conjoint et enfants) - ne sont pas toujours considérés par les aînés comme émotionnellement proches (Cornwell et al., 2008; Pahl \& Spencer, 2004). Seuls ceux avec lesquels les contacts sont jugés fréquents s'associent à une forte proximité émotionnelle (Cornwell et al., 2008). Avoir un partenaire, par exemple, ne suffit pas à garantir la solidarité aux dernières étapes de la vie; son soutien dépendra de la qualité de la relation conjugale mais aussi de son état de santé (Antonucci et al., 2007). Il en est de même pour les relations intergénérationnelles qui s'avèrent très variables dans la vieillesse. Alors que certaines se caractérisent par une forte proximité émotionnelle et de fréquents échanges d'aide, d'autres se distinguent au contraire par l'absence de toute solidarité affective et pratique (Bengtson, 2001; Lawton et al., 1994a; Silverstein et al., 1994; Silverstein \& Bengtson, 1997). Les liens entre parents âgés et enfants adultes sont donc loin d'être tous empreints d'affection; ils sont au contraire pluriels et fort complexes. Même 
constat pour les relations entre grands-parents et petits-enfants; celles-ci sont aussi diverses. La proximité affective entre grands-parents et petitsenfants ne va pas de soi dans la vieillesse, elle est tributaire de la qualité de la relation qu'ils ont nouée au fil du temps (Lawton et al., 1994b; Mueller, Wilhelm, \& Elder, 2002; Silverstein \& Marenco, 2001). Compte tenu de ces divers résultats, le partenaire et les enfants ne peuvent plus être définis a priori comme les membres de la famille les plus significatifs dans la vieillesse du seul fait de leur existence et de leurs rôles socialement attendus, comme c'est encore le cas dans de nombreuses études en gérontologie sociale.

Comment expliquer une telle variabilité qui va à l'encontre de l'idée de « fiabilité » ou d' « indéfectibilité » des liens de sang? Dans la littérature, cette variabilité s'explique par la survenue d'événements dans le parcours de vie qui ont empêché ou interrompu le développement affectif des liens familiaux. L'absence de tout investissement affectif de part et d'autre de la relation peut sévèrement compromettre le lien familial - même intergénérationnel - à moyen-long terme (Silverstein \& Bengtson, 1997). Le divorce des pères est en ce sens fort révélateur de ce processus. Alors que les mères âgées divorcées ont pu maintenir au gré du temps le lien affectif avec leurs enfants en raison de leur engagement plus marqué dans la sphère familiale, il en est autrement pour les pères âgés divorcés (Campbell et al., 1999; Lawton et al., 1994b; Suitor et al., 2011; Van der Pas \& Van Tilburg, 2010). Les données montrent que, comparés aux pères mariés (non séparés), les pères divorcés ont non seulement moins de contacts avec leurs enfants adultes mais ils reçoivent aussi moins d'aide de leur part (Connidis, 2003 ; Pezzin, Pollak, \& Schone, 2008; Shapiro \& Cooney, 2007; Suitor et al., 2011). Plus encore, le risque de se désengager du lien intergénérationnel (faible proximité affective et peu de contacts et d'échanges) est dans la vieillesse cinq fois plus élevé chez les pères divorcés que chez les mères divorcées (Lawton et al., 1994b; Silverstein \& Bengtson, 1997). La garde unique largement attribuée aux mères lors du divorce, suivie d'une diminution drastique des contacts des pères non gardiens avec leurs enfants, ainsi que le remariage, plus fréquente chez les hommes, et l'éloignement géographique sont souvent avancés dans la littérature pour expliquer le désengagement des pères âgés divorcés (Lawton et al., 1994b; Shapiro \& Cooney, 2007; Silverstein \& Bengtson, 1997; Van der Pas \& Van Tilburg, 2010). Avec le remariage, les hommes tendent à s'investir dans d'autres relations familiales avec pour conséquence une réduction significative des 


\section{Problématique et hypothèses}

contacts avec leurs enfants biologiques ainsi qu'un certain désinvestissement affectif (Lawton et al., 1994b; Silverstein \& Bengtson, 1997). Bien que globalement la solidarité intergénérationnelle demeure vivace (voir le premier chapitre), il n'en reste pas moins que le désengagement affectif caractérise une part non négligeable des relations intergénérationnelles dans la société américaine (Bengtson, 2001; Silverstein \& Bengtson, 1997).

Le divorce des pères âgés représente l'explication la plus symptomatique de ce processus mais il n'est sans doute pas le seul. La mobilité géographique ainsi que le manque de temps des enfants adultes, pris par leurs exigences professionnelles et familiales, rendent plus compliqué le maintien des relations intergénérationnelles. Bien que la distance géographique et le manque de temps n'altèrent en rien le lien affectif ni même son potentiel de solidarité (Lowenstein et al., 2004; Silverstein \& Bengtson, 1997; Suitor et al., 2011), elles complexifient quelque peu l'entretien du lien. Les opportunités de rencontres et d'aides pratiques, qui contribuent à renforcer les interdépendances affectives, sont forcément limitées (Bengtson \& Roberts, 1991 ; Lawton et al., 1994b ; Lowenstein et al., 2004 ; Roberts \& Bengtson, 1990). Certes, face au déclin de santé du parent âgé, les enfants vivant à distance ou ayant peu de temps se mobiliseront, se sentant redevables vis-à-vis du parent âgé (cf. normes de réciprocité «généralisée ») mais la distance, le manque de temps et l'insertion dans d'autres relations d'interdépendance rendent difficile la mise en œuvre d'une telle mobilisation, générant des tensions et de l'ambivalence au sein de l'ensemble du réseau familial. En raison de la complexification des trajectoires de vie des parents et des enfants, les relations intergénérationnelles sont devenues plus compliquées, devant être entretenues de part et d'autre de la relation pour qu'elles demeurent solidaires et davantage «négociées » avec l'ensemble des membres du réseau familial dans lequel elles s'insèrent. Bien que relativement prégnantes dans l'absolu, les obligations familiales ne suffisent pas dans la pratique à garantir le maintien du lien et la solidarité familiale. Ce constat réfute l'affirmation souvent défendue dans les modèles dominants de la gérontologie sociale que la seule existence d'enfants adultes dans le réservoir de parenté des parents âgés garantit un soutien familial sans faille, immuable et indéfectible. Au contraire, les liens de sang peuvent être aléatoires, incertains et faillibles s'ils ne sont pas investis ou plus nourris affectivement.

Alors que la complexité des trajectoires de vie individuelles a engrangé une forte incertitude autour des liens de sang, réputés immuables, 
on assiste parallèlement à une plus grande importance des relations personnalisées - celles qui sont investies affectivement - quelle que soit leur nature (biologique, d'alliance ou autre) comme en témoigne la plus grande centralité des liens d'amitié dans la vie actuelle des individus de tout âge (Allan, 2001, 2008; Finch, 2007; Pahl \& Spencer, 2004). Face à l'instabilité de leur vie professionnelle et familiale, les individus comptent de plus en plus sur le soutien émotionnel et pratique de leurs amis. Ceuxci les accompagnent davantage dans les décisions importantes qu'ils sont amenés à prendre au cours de leur vie (divorce, changement professionnel, traitements médicaux, etc), et cela d'autant plus lorsque la famille fait défaut ou est déficiente (Allan, 2001, 2008; Merz \& Huxhold, 2010; Stevens \& Van Tilburg, 2011). Même si elles s'inscrivent au-delà des liens biologiques et conjugaux, certaines de ces relations amicales peuvent être perçues comme très significatives (Allan, 2001, 2008; Pahl \& Spencer, 2004; Smart, 2007). La plus forte prégnance de l'amitié a aussi été soulignée dans de nombreuses études sur les réseaux personnels dans la vieillesse (Fiori et al., 2008, 2006; Stevens \& Van Tilburg, 2011; Suanet, Van Tilburg, \& Broese Van Groenou, 2013). Par exemple, la comparaison de plusieurs cohortes de personnes âgées aux Pays-Bas montre que la proportion des relations non familiales, notamment amicales, dans les réseaux personnels est de plus en plus forte dans les cohortes d'âgés les plus récentes (Stevens \& Van Tilburg, 2011; Suanet et al., 2013). Bien que confrontées à la contraction de leur réseau personnel, ces dernières préservent au cœur même de leur réseau aussi bien des relations familiales que non familiales (amis notamment). Ces résultats se maintiennent même après avoir contrôlé l'effet de l'âge, du statut socio-économique et de la santé fonctionnelle (Stevens \& Van Tilburg, 2011; Suanet et al., 2013), facteurs qui influent sur la composition des réseaux personnels dans la vieillesse. De fait, la meilleure santé ou le meilleur statut socioéconomique qui caractérisent les cohortes les plus récentes n'expliquent pas - du moins, à elles-seules - le maintien des relations amicales au cœur même du réseau personnel des individus âgés (Stevens \& Van Tilburg, 2011; Suanet et al., 2013).

Ce résultat confirme la plus grande prégnance depuis les années 70 des relations personnalisées, ayant pour conséquence une diversification dans la composition du cœur même des réseaux personnels des personnes âgées. Alors que, pour certains, les membres familiaux demeurent toujours au centre de leur réseau personnel, pour d'autres, les amis constituent non 


\section{Problématique et hypothèses}

seulement les membres principaux de leur réseau personnel mais ils en occupent aussi le centre, côtoyant les membres familiaux les plus proches (Pahl \& Spencer, 2004). Ces résultats mettent en évidence le processus de «suffusion» proposé par Pahl et Spencer (2004). La présence d'amis au cœur du réseau personnel des personnes âgées, généralement centré dans la littérature sur les membres proches de la famille (cf. premier chapitre), révèle que les frontières entre famille et amis sont désormais perméables et que les rôles, qui jusqu'ici différenciaient les amis de la famille, sont devenus interchangeables (Allan, 2008; Pahl \& Spencer, 2004; Smart, 2007; Suanet et al., 2013). Au vu de ce constat, les critères du sang et d'alliance, qui distinguaient les relations familiales des relations non familiales, sont devenus pour certains auteurs totalement désuets. En somme, c'est le degré d'engagement émotionnel (« commitment») qui est important; celui-ci distingue les relations personnelles à fort engagement émotionnel («high commitment») de celles qui sont désinvesties («low commitment»), quelle que soit leur nature (Pahl \& Spencer, 2004).

Bien qu'amis et membres de la famille tendent à jouer les mêmes rôles dans les réseaux personnels, on peut néanmoins se demander s'ils sont perçus de manière égale comme faisant partie de la famille significative. En effet, ces divers auteurs négligent le fait que, même si la significativité des liens non familiaux devient de plus en plus importante, la famille «nucléaire», incarnant des valeurs familiales fortes d'unité et de soutien, demeure toujours, du moins pour une partie de la population, un modèle «idéal» fort prégnant (Allen et al., 2011). De fait, les liens de sang, même s'ils ne sont pas marqués par l'affectivité, sont particulièrement valorisés. On leur associe l'indéfectibilité du lien et le support inconditionnel en raison des fortes obligations familiales qui les sous-tendent. Et même si certains des membres de la famille «nucléaire» ne sont pas décrits comme affectivement proches, ils demeurent au cœur du réseau personnel, étant considérés par de nombreux aînés comme la principale source de support émotionnel et d'aide pratique (Allen et al., 2011; Pahl \& Spencer, 2004; Voorpostel, 2013). Entre l'imprévisibilité des liens de sang, la significativité des relations personnelles et le modèle familial «idéal » de la famille « nucléaire » axé sur les liens bio-légaux, on peut se demander à juste titre comment les individus âgés gèrent ces divers aspects pour construire leur famille significative. Dans la prochaine sous-section, nous mettrons en lumière certains mécanismes de réinterprétation des liens familiaux qui sont 
éloquents quant à la façon dont certains individus définissent leur famille significative.

\subsubsection{Une re-définition des liens familiaux et non familiaux}

Des données qualitatives montrent comment les individus de tous âges re-définissent des liens personnels, familiaux ou non familiaux, afin de créer leur famille significative (Allen et al., 2011; Braithwaite et al., 2010; Finch, 2007; Mac Rae, 1992). Ces mécanismes ne sont pas forcément « conscients» mais ils se révèlent au gré du discours des individus à qui l'on demande de définir «leur» famille. Par exemple, les personnes interrogées utilisent les « codes» de parenté, définis socialement comme proches tels que « frère » ou « fille», pour distinguer les relations qu'elles jugent importantes de celles qui ne le sont pas, et cela quelle qu'en soit la nature (biologique, d'alliance ou non) (Allen et al., 2011; Braithwaite et al., 2010; Mac Rae, 1992; Voorpostel, 2013). Elles associent un beau-frère ou un ami à un frère, («il est comme mon frère »), une belle-fille, une nièce, ou une aide soignante à une fille (« elle est comme ma fille») (Braithwaite et al., 2010; Karner, 1998; Mac Rae, 1992; Voorpostel, 2013). Ce mécanisme n'est pas sans rappeler le processus de re-évaluation des liens personnels au cours du parcours de vie mis en évidence dans le modèle du convoi et, plus spécifiquement, la « valorisation » de certains liens périphériques dans le réseau personnel (Antonucci, 2001; Kahn \& Antonucci, 1980; Van Tilburg, 1998; Van Tilburg \& Thomése, 2010). Évidemment ce re-codage ne concerne pas tous les liens personnalisés. Il peut s'agir, par exemple, d'amitiés qui se caractérisent par un fort investissement de part et d'autre de la relation ou d'ex-membres de la famille, comme l'ex-femme du fils ou l'ex-mari de la sœur, avec lesquels des liens affectifs ont été développés durant de longues années et que l'aîné souhaite préserver au sein de sa famille significative malgré le divorce de ses proches (Allan, 2001; Allen et al., 2011; Pahl \& Spencer, 2004; Voorpostel, 2013). Plus généralement, ce sont des relations de longue durée décrites comme affectivement proches et construites au fil des ans, au gré d'échanges multiples comme, par exemple, les amis d'enfance, des cousins éloignés avec lesquels on entretient des relations proches ou même des voisins de longue date (Allen et al., 2011; Braithwaite et al., 2010; Pahl \& Spencer, 2004; Voorpostel, 2013). 


\section{Problématique et hypothèses}

Les membres «re-définis » sont non seulement des relations de longue durée mais ils sont aussi actifs dans le «faire famille». Ils ont par exemple des contacts et des échanges fréquents avec l'individu interrogé, habitent généralement à proximité et participent à des activités communes comme à des fêtes de famille. Par ailleurs, ils sont présents dans les albums photos, sont intégrés dans les échanges de cadeaux, sont même évoqués dans les anecdotes familiales et parfois sont formellement désignés comme faisant partie de la famille par l'attribution du rôle de « parrain ou marraine » ou de « filleul(e)» (Allen et al., 2011; Braithwaite et al., 2010; Mac Rae, 1992; Morgan, 1996). C'est surtout le sens que revêtent ces pratiques pour les individus et la façon dont ils les interprètent qui contribuent à les qualifier de «familiales » et, plus encore, à définir ceux qui y participent comme membres à part entière de la famille (Allen et al., 2011; Braithwaite et al., 2010; Finch, 2007). Les termes assez éloquents de «parenté fictive (fictive kin) », de «parenté volontaire (voluntary kin)», ou encore de « familles de choix (families of choice) » sont utilisés dans la recherche pour désigner ceux qui, sans lien de sang ni d'alliance, sont considérés par les individus comme membres à part entière de leur famille (Allen et al., 2011; Braithwaite et al., 2010; Mac Rae, 1992; Voorpostel, 2013; Weeks, Heaphy, \& Donovan, 2001). Les exemples sans doute les plus frappants sont les relations qui peuvent se développer entre les individus âgés et le personnel soignant lors de soins à domicile ou en institution. Outre les liens d'amitié entre résidents qui peuvent être considérés par certains comme plus significatifs que les liens qu'ils entretiennent avec leur famille (Street, Burge, Quadagno, \& Barrett, 2007), le processus de soins offre un cadre particulièrement propice au développement de liens familiaux volontaires (Karner, 1998). En entrant dans l'intimité des personnes âgées par le biais d'actes de soins, usuellement assignés aux pratiques familiales, certains soignants développent des relations affectives avec les individus âgés dont ils ont la charge. En raison de cette forte implication et du temps partagé, aidants et aidés deviennent les uns pour les autres des membres significatifs de leur famille respective (Karner, 1998; Mac Rae, 1992). Ce faisant, soignants et personnes âgées entrent dans un réseau de soutien, soutenu par de fortes interdépendances émotionnelles, au point que certains aidants outrepassent largement leur mandat professionnel. Se sentant responsables des personnes qu'ils soignent, ils offrent leur temps, l'aide et le soutien que l'on attend habituellement des membres de la famille (visites le week end, visites à l'hôpital, etc.) (Karner, 1998). 
L'attribution d'un code de parenté, de sang si possible, n'est pas seulement une figure de style; elle permet d'une part de «légitimer» le lien aux yeux des autres mais elle signifie aussi que les individus désignés comme « frères » ou comme « filles » font « vraiment» partie de la famille qui « compte». Ce qui n'est pas sans conséquences dans la vie de tous les jours puisque ces membres «re-définis » ont non seulement les prérogatives mais aussi les responsabilités des membres usuels d'une famille bio-légale (Pahl \& Spencer, 2004). Même en l'absence d'un cadre statutaire clair, ces relations re-définies sont amenées à jouer une variété de rôles familiaux qui outrepassent leur fonction habituelle. En effet, elles ressemblent dans leur fonctionnement davantage à des relations familiales qu'amicales. Elles impliquent comme elles des obligations familiales, notamment de soutien, ce qui justifie pleinement leur inclusion dans la famille significative (Allen et al., 2011; Braithwaite et al., 2010; Mac Rae, 1992; Pahl \& Spencer, 2004; Voorpostel, 2013). Ce sont, par ailleurs, les aînés les plus attachés aux normes de solidarité familiale qui, sans en avoir pleinement conscience, recourent le plus à ce type de mécanismes. Ils incluent dans leur famille significative des liens non familiaux, cherchant à construire ou à renforcer leur réseau de soutien pour remédier à l'« instabilité » de leur famille bio-légale (Voorpostel, 2013). Ils perçoivent ces liens re-définis comme prometteurs d'un plus grand soutien que les relations familiales reposant uniquement sur un statut pré-défini (Allen et al., 2011; Braithwaite et al., 2010; Mac Rae, 1992; Voorpostel, 2013).

Cette re-définition des liens familiaux peut se révéler fort utile pour ceux qui ne peuvent pas ou qui ne peuvent plus compter sur les membres proches de leur famille. Elle leur permet d'acquérir des relations familiales fonctionnelles lorsque la famille de sang ou d'alliance est inexistante (décès), dysfonctionnelle (désengagement) ou inaccessible (manque de temps ou distance géographique) (Allen et al., 2011; Braithwaite et al., 2010; Mac Rae, 1992; Voorpostel, 2013; Voorpostel \& Van Der Lippe, 2007). Mais, cette ré-interprétation des liens familiaux n'est pas seulement l'apanage des individus dont la famille de sang est tronquée ou déficiente, elle est aussi active chez ceux dont les membres proches de la famille sont présents, accessibles et fonctionnels (Allen et al., 2011). Ces liens «re-définis » se mêlent, s'entremêlent aux autres membres familiaux, élargissant le réseau familial significatif et le renforçant dans son rôle de soutien (Allen et al., 2011; Braithwaite et al., 2010 ; Mac Rae, 1992; 


\section{Problématique et hypothèses}

Voorpostel, 2013). Ces membres « re-définis » font partie de la famille significative non seulement dans le discours et les pratiques familiales mais aussi dans les fonctions qu'ils doivent remplir et qui vont bien au-delà de celles de l'amitié (Allen et al., 2011; Braithwaite et al., 2010; Finch, 2007 ; Pahl \& Spencer, 2004).

Alors que l'on attribue symboliquement un lien de sang à des relations familiales éloignées ou non familiales pour marquer l'importance de la relation, la stratégie inverse existe aussi, à savoir présenter un membre du réservoir de parenté comme un «ami», et cette stratégie (inconsciente) relève des mêmes mécanismes de ré-interprétation. Dans ce cas, c'est la qualité de la relation et l'engagement affectif, caractéristiques des fortes amitiés, qui sont soulignés (Pahl \& Spencer, 2004). Il s'agit majoritairement de relations familiales qui ressemblent dans leur structure et leur fonctionnement à des relations d'amitié comme, par exemple, les liens entre frères et sœurs ou entre cousins. A cet égard, les relations fraternelles sont complexes et ne s'apparentent pas «a priori » à des relations de type affinitaire. Liens de sang, elles ne sont pas «choisies » (Widmer, 1999b). Imposées par le contexte familial dans lequel naît l'individu, celui-ci n'a pas d'autre choix que de vivre - ou du moins d'être en lien - avec sa fratrie durant l'enfance ou l'adolescence (Widmer, 1999b). Durant cette vie en commun, les frères et sœurs sont intégrés dans les liens d'interdépendance qui constituent la famille dite «bio-légale » et par conséquent ils doivent, à l'instar des autres membres de la configuration familiale, se soumettre aux obligations familiales qui régulent les relations au sein de la famille. Cependant, contrairement aux liens «parents-enfants » qui affichent des statuts différenciés, hiérarchisés (relations verticales), ces relations sont de type horizontal (même statut familial). Et même si certains ont plus de privilèges que d'autres en fonction de leur sexe et de leur ordre de naissance, les liens fraternels se caractérisent par des rapports plus égalitaires que ceux qui sous-tendent les liens intergénérationnels, ces derniers s'appuyant davantage sur une hiérarchie de statuts (Antonucci et al., 2007; Cicirelli, 1995; Connidis, 2010; Widmer, 1999b).

Bien que forcé, enfant, d'être en lien, il en est autrement à l'âge adulte. Chacun quitte le foyer parental avec ses normes et ses tensions pour poursuivre sa propre trajectoire de vie et s'investir dans de nouvelles relations d'interdépendance. Loin de l'espace commun et de ses règles, le maintien du lien fraternel à l'âge adulte - et qui plus est dans la vieillesse - est davantage le fruit d'un choix réciproque que celui d'obligations familiales 
(Cicirelli, 1995 ; Connidis, 2010; Eriksen \& Gerstel, 2002), ce qui le rend à cet égard comparable aux relations amicales. En raison de l'horizontalité de leurs liens, les relations fraternelles sont davantage soumises à la réciprocité «équilibrée » que les relations intergénérationnelles qui reposent sur la réciprocité « généralisée » (Antonucci et al., 2007; Campbell et al., 1999; Cicirelli, 1995; Eriksen \& Gerstel, 2002). Elles nécessitent dès lors d'être entretenues pour être maintenues (Voorpostel \& Van Der Lippe, 2007). La durabilité et la stabilité de tels liens dans la vieillesse ne dépendent pas de sentiments de redevabilité mais elles s'appuient plutôt sur des souvenirs, une histoire commune, des échanges de soutien, et surtout des affinités, des intérêts communs, des interactions positives et gratifiantes qui ont contribué à rendre la relation fraternelle «significative», marquée par l'affectivité et la solidarité (Carr \& Moorman, 2011; Cicirelli, 1995; Eriksen \& Gerstel, 2002; Suanet et al., 2013). Investies affectivement, les relations avec les frères, les sœurs, les cousin(e)s ou autres membres de la parenté éloignée peuvent se révéler particulièrement fortes dans la vieillesse (Connidis, 2010) au point de s'apparenter dans leur fonctionnement davantage à l'amitié qu'aux relations familiales (Antonucci et al., 2007; Pahl \& Spencer, 2004 ; Voorpostel \& Van Der Lippe, 2007). En effet, les affinités, les intérêts communs, les activités partagées, le soutien émotionnel et les échanges de confidences qui caractérisent certains liens familiaux de même génération font davantage état de fonctions que l'on attribue aux amis plutôt qu'à la famille (Pahl \& Spencer, 2004). Dans la vieillesse, les relations avec les frères, les sœurs ou les cousin(e)s répondent donc davantage à une logique d'électivité comme l'amitié qu'à une logique de sang (Cicirelli, 1995; Connidis, 2010; Voorpostel \& Van Der Lippe, 2007).

Toutefois, les membres de la fratrie ou de la parenté élargie ne sont pas tous re-définis comme « amis ». Il existe en effet une forte variabilité dans les relations fraternelles, celles-ci affichant divers degrés d'engagement et d'intimité affective, et cela même au sein d'une même fratrie. Certaines sont perçues comme chaleureuses et soutenantes alors que d'autres sont jugées distantes, indifférentes, voire même hostiles (Antonucci et al., 2007; Campbell et al., 1999; Cicirelli, 1995; Voorpostel \& Van Der Lippe, 2007). Pour certains auteurs, cette variabilité reflète le degré d'investissement différent des individus dans leurs relations avec les membres de leur fratrie au cours du parcours de vie (Campbell et al., 1999; Cicirelli, 1995; Connidis, 2010; Voorpostel \& Van Der Lippe, 2007). Selon la façon dont les liens fraternels sont investis au cours du temps, certains membres de la 


\section{Problématique et hypothèses}

fratrie sont amenés à jouer davantage de rôles que ceux formellement attendus alors que d'autres sont soit peu investis ou se désinvestissent avec le temps (Connidis, 2010; Finch, 2007; Pahl \& Spencer, 2004; Van Tilburg \& Thomése, 2010).

Par ailleurs, grâce au lien affectif privilégié, investi et engagé qui unit deux membres d'une même fratrie, des relations avec le partenaire et les enfants du frère ou de la sœur (les alliés) peuvent se développer et donner lieu à des échanges de soutien non négligeables (Campbell et al., 1999; Milardo, 2005, 2009; Pollet, Kuppens, \& Dunbar, 2006). Dans certains cas, les liens noués avec les membres de la famille de la sœur ou du frère deviennent aussi forts émotionnellement que le lien entretenu avec le frère ou la sœur, se construisant avec le temps autour d'activités et d'intérêts communs (Milardo, 2005, 2009; Pahl \& Spencer, 2004; Pollet et al., 2006). A l'instar des membres de la fratrie, les alliés peuvent aussi devenir des membres significatifs de la famille. Cette re-définition des liens familiaux a donc des répercussions sur la construction de l'ensemble de la famille significative. Il paraît clair que le code « ami » attribué à certains membres de la fratrie, et plus globalement à certains membres du réservoir de parenté, relève du mécanisme de sélection socio-émotionnelle, mis en lumière par Laura Carstensen (cf. premier chapitre). Certaines relations familiales sont donc, à l'image de l'amitié, choisies et investies émotionnellement, ce qui implique un travail relationnel actif, un certain engagement pour maintenir le lien au gré du temps; leur seule appartenance au réservoir de parenté ne suffit pas à les qualifier de « significatifs ».

Dans cette sous-section, nous avons mis en exergue que ni les critères du sang ou d'alliance ni l'appartenance au réservoir de parenté ne peuvent définir la famille significative (Allen et al., 2011; Braithwaite et al., 2010; Finch, 2007; Schmeeckle et al., 2006). Les liens familiaux sont sujet à re-définition, ne pouvant être considérés comme «allant de soi » dès lors qu'ils font partie du réservoir de parenté. Ce travail de réinterprétation, souvent inconscient, révèle que la famille «se fait» dans les discours, les pratiques et les interactions plus qu'elle n' « est» a priori. Ce mécanisme vise, pour ceux qui le déploient, à construire une famille significative axée sur des liens affectivement investis et porteurs de sens. L'incertitude générée par l'impermanence des liens bio-légaux et le besoin accru de soutien qui caractérise, chez certains, les dernières étapes de la vie expliquent l'émergence de ce mécanisme, entraînant l'insertion au sein de la famille significative d'une multitude de liens ayant en commun 
non pas le «sang », mais l'engagement (« commitment») (Allan, 2001, 2008; Pahl \& Spencer, 2004; Van Tilburg \& Thomése, 2010). Au vu de ces observations, se baser uniquement sur les indicateurs démographiques pour définir la famille est inadéquat pour approcher la famille significative dans la vieillesse. Ces indicateurs sont certes utiles pour mesurer l'état du réservoir de parenté mais ils ne permettent pas d'identifier les membres significatifs de la famille issus de ce travail de re-défintion. Bien que les études qualitatives ont mis en évidence ce travail de réinterprétation au sein de la famille, elles se sont essentiellement focalisées sur certains liens familiaux ou non familiaux (volontaires) et se sont cantonnées à une perspective dyadique. Elles n'ont pas considéré l'ensemble de la configuration familiale. Autrement dit, nous ne savons pas comment ces divers mécanismes de réinterprétation et de sélection se combinent les uns aux autres, et leurs conséquences sur la composition des configurations familiales. De même, nous ne savons pas non plus si tous les individus déploient de la même manière et à la même intensité ce genre de mécanismes. Des études sur les réseaux personnels et le parcours de vie nous donnent cependant quelques pistes.

\subsubsection{L'influence du parcours de vie}

Selon le modèle du convoi, les mécanismes de re-évaluation des liens personnels se mettent en place au gré des transitions de vie que les individus sont amenés à traverser au cours de leur parcours de vie, avec des conséquences sur la structure de leurs réseaux personnels. Les transitions de vie suscitent des changements de rôles, invitant les individus concernés à reévaluer leurs relations en fonction de leurs nouveaux besoins (Antonucci, 2001 ; Kahn \& Antonucci, 1980; Van Tilburg, 1998; Van Tilburg \& Thomése, 2010). Certaines transitions, notamment celles affectant le réservoir de parenté comme la perte du conjoint, s'associent à une réorganisation des liens, aboutissant généralement à une diversification des réseaux personnels dans la vieillesse (Cornwell, 2011; Silverstein \& Giarrusso, 2010). Bien que les enfants intensifient leurs contacts et leur soutien après le décès du conjoint, leur mobilisation tend à s'estomper avec le temps (Campbell et al., 1999; Silverstein et al., 2006; Van Tilburg, 1998). Afin de compenser à long terme la perte du conjoint, d'éviter la solitude et «libérés » d'un lien de forte dépendance affective et pratique, les aînés, veufs ou divorcés, tendent 


\section{Problématique et hypothèses}

à s'investir émotionnellement et pratiquement (temps, services, etc) dans d'autres liens affectifs (Cornwell et al., 2008; Cornwell, 2009b, 2011).

Certains ré-activent d'anciennes relations personnelles, familiales (frères ou sœurs, cousin(e)s, etc.) ou non familiales (amis) jusque-là laissées en berne, d'autres valorisent des relations périphériques ou rencontrent par le biais de diverses activités (volontariat) de nouveaux amis, voire un nouveau partenaire, ayant pour conséquence la diversification de leur réseau personnel qui devient dès lors moins centré sur la famille que celui des individus mariés (Antonucci, 2001; Campbell et al., 1999; Connidis, 2003; Ha, 2008; Shapiro \& Cooney, 2007). L'importance des amis s'accentue encore si les enfants sont absents, éloignés ou désengagés, comme dans le cas du divorce des pères. Des données montrent que les pères âgés divorcés - ainsi que les divorcé(e)s sans enfant - s'investissent essentiellement dans les relations amicales pour compenser l'absence de relations affectives dans leur réseau personnel tandis que les mères divorcées comptent non seulement sur le soutien de leurs enfants avec lesquels elles ont maintenu des liens après le divorce mais aussi sur celui des membres de leur fratrie ou de leur parenté éloignée (Campbell et al., 1999; Connidis, 2003 ; Pezzin et al., 2008; Shapiro \& Cooney, 2007). Par ailleurs, la remise en couple représente une opportunité non négligeable d'acquérir de nouveaux membres au sein du réseau personnel bien que les enfants et la fratrie du partenaire ne soient pas choisis ni par l'individu ni par la famille du partenaire (Ganong \& Coleman, 2006; Schmeeckle et al., 2006). Ceci dit, si les individus se sont investis dans les relations avec les enfants de leur conjoint lorsque ces derniers étaient petits, ceux-ci peuvent devenir dans la vieillesse des membres importants de leur réseau personnel, voire de leur configuration familiale (Ganong \& Coleman, 2006; Schmeeckle et al., 2006; Van der Pas \& Van Tilburg, 2010). Quant aux célibataires, sans enfant pour la plupart, ils développent tout au long de leur parcours de vie un réseau de relations personnelles variées, aussi bien familiales (fratrie, parenté éloignée, etc.) que non familiales (amis et voisins), leur apportant tout le soutien émotionnel et pratique dont ils ont besoin aux dernières étapes de leur vie (Dykstra \& Hagestad, 2007; Pudrovska et al., 2006; Schnettler \& Wöhler, 2014; Voorpostel, 2013). Ces données montrent l'importance du parcours de vie sur le travail de re-évaluation des liens au sein des réseaux personnels. Selon ces études, ce mécanisme est loin d'être marginal et se révèle même fort prégnant aux dernières étapes de la vie, celles-ci 
étant marquées par de nombreuses transitions de vie comme le veuvage, le déclin de la santé ou encore l'institutionnalisation.

Fort de ce constat, nous faisons l'hypothèse que ce travail de redéfinition des liens est aussi prépondérant dans le contexte familial et qu'il varie aussi en fonction du parcours de vie des individus. Alors que certains aînés ont un partenaire et des enfants avec lesquels ils ont développé des relations affectives solides, d'autres ont connu des parcours de vie plus chaotiques, marqués par des pertes telles que le divorce, le veuvage, l'infertilité ou encore la migration. Ils se retrouvent, par conséquent, aux dernières étapes de leur vie sans la présence (physique ou symbolique) d'un partenaire ou d'enfants, ceux-là même qui sont désignés socialement comme les principaux prestataires de soutien. Face à un soutien familial incertain, ils déploient souvent inconsciemment ces mécanismes de réinterprétation des liens familiaux qui leur permettent d'inclure dans leur famille significative une variété de membres, puisés aussi bien dans leur réservoir de parenté qu'en dehors, afin de construire une famille qui fasse sens à leurs yeux et qui soit prometteuse de soutien familial (Allen et al., 2011; Karner, 1998; Mac Rae, 1992; Voorpostel, 2013). Lidentité des membres significatifs de la famille dépendra donc du type de transitions que les aînés ont traversées, de l'état de leur réservoir de parenté, de la façon dont ils ont investi leurs relations personnelles, familiales, au cours de leur vie mais aussi de la prégnance normative de la famille dite «nucléaire ». En effet, la famille «nucléaire » demeure une valeur-phare aux dernières étapes de la vie. Dès lors, il se peut que les membres bio-légaux de la famille occupent une place importante dans la définition de la famille significative, même si ceux-ci ne sont pas perçus par les aînés comme émotionnellement proches. En plus du parcours de vie, du réservoir de parenté et de la prégnance du modèle de la famille «nucléaire », le profil socio-démographique des individus âgés est aussi un facteur important puisque certains d'entre eux sont plus enclins que d'autres, en raison des ressources dont ils disposent (financières, relationnelles, etc.), à développer une variété de relations personnelles, susceptibles d'être intégrées dans leur famille significative (cf. chapitre précédent).

Compte tenu de ces différents facteurs, il est probable que certains individus âgés sélectionnent une diversité de membres familiaux pour construire leur famille significative, allant bien au-delà des frontières de la famille "nucléaire ». Plus encore, nous faisons l'hypothèse que les différents membres significatifs désignés s'articulent de façon distincte, 


\section{Problématique et hypothèses}

formant une pluralité de configurations familiales, reflets de différentes définitions personnelles de la famille significative (De Carlo et al., 2014; Widmer, 1999a, 2006, 2016). Nous supposons que certaines personnes âgées privilégient d'abord les liens biologiques et d'alliance tels que le partenaire, les enfants et les petits-enfants alors que d'autres, au contraire, s'appuient davantage sur des relations d'affinité, positives et gratifiantes comme celles entretenues avec des membres de la fratrie, de la parenté éloignée ou encore des amis. D'autres encore combinent des liens «biolégaux » avec des liens plus affinitaires, voire « volontaires », donnant lieu à des configurations familiales fort diversifiées. Dès lors, nous nous positionnons contre le modèle du convoi qui postule que le cœur du réseau personnel, composé généralement des membres proches de la famille, reste stable et immuable au cours du temps, épargné de tout travail de ré-évaluation et de stratégies d'adaptation (inconscientes) telles que la valorisation, la ré-activation, la sélection et le désengagement des liens personnels (Antonucci, 2001; Kahn \& Antonucci, 1980; Van Tilburg, 1998; Van Tilburg \& Thomése, 2010). Aucunes données quantitatives n'existent à ce jour qui montrent la prépondérance de ces mécanismes sur les liens familiaux au sein de la population âgée et de leurs conséquences sur la composition des configurations familiales dans la vieillesse. L'approche configurationnelle et ses méthodes nous permettent de tester ces hypothèses puisque nous pouvons lister les membres de la famille qui sont considérés par les personnes âgées comme les plus significatifs et détecter les configurations familiales les plus fréquentes chez les âgés en procédant à des analyses de classification hiérarchique.

Nous avons souligné dans cette sous-section que la famille qui « compte » pour les individus ne correspond pas forcément au modèle « idéal» de la famille nucléaire. Sur la base de la littérature existante, nous avons mis en évidence deux tendances qui influencent les relations personnelles d'aujourd'hui. D'une part, les relations familiales - même «primaires »-et le soutien familial qui en découle sont devenus aléatoires et incertains en raison de la complexification des trajectoires de vie individuelles. D'autre part, les relations personnalisées, engagées et investies affectivement, sont particulièrement valorisées, quelle que soit leur nature. Des études qualitatives en gérontologie sociale montrent que ces deux tendances sont prégnantes dans la construction de la famille significative. En effet, pour compenser l'incertitude des liens familiaux bio-légaux, certains aînés recourent, souvent de manière inconsciente, à divers mécanismes de 
re-définition des liens familiaux pour construire une famille «significative » et y intègrent une variété de relations qu'ils ont investies et dont certaines s'inscrivent bien au-delà des critères bio-légaux " habituels ». Ces mécanismes permettent à certains individus âgés de « pérenniser» les liens qu'ils affectionnent et sur lesquels ils s'appuient au quotidien, ce qui contribue d'une certaine manière à les sécuriser face à l'instabilité et à la complexité des relations familiales actuelles (Allen et al., 2011; Braithwaite et al., 2010; Finch, 2007; Voorpostel, 2013). Ces mécanismes ne sont pas actionnés par tous les individus âgés mais ils peuvent s'avérer particulièrement nécessaires pour ceux qui ont un besoin accru de soutien pour faire face au stress et aux pertes qui caractérisent les dernières étapes de leur vie (Allen et al., 2011; Voorpostel, 2013). Il s'agit maintenant de savoir si la ré-interprétation des liens familiaux, qui donne lieu à une pluralité de configurations familiales, est un mécanisme « efficace» offrant, quels que soient les membres significatifs qui les composent, le potentiel de support - ou «capital social»- tant escompté. La prochaine section traite plus spécifiquement de ce sujet.

\subsection{Le capital social}

Une des questions cruciales, qui est depuis longtemps au centre des interrogations en gérontologie sociale, a trait à la capacité des réseaux personnels à assurer aux individus âgés le soutien dont ils ont besoin pour s'adapter au mieux aux aléas de leur vieillesse et maintenir leur bien-être (Antonucci, 2001; Antonucci et al., 2007; Cornwell et al., 2008, 2009; Moren-Cross $\&$ Lin, 2006). Cette question se pose aussi pour la famille; et cela d'autant plus qu'avec la contraction du réseau personnel aux dernières étapes de la vie, la famille est perçue, étant au cœur du réseau personnel, comme une source majeure de soutien, parfois la seule (Antonucci, 1990, 2001; Kahn \& Antonucci, 1980; Merz \& Huxhold, 2010; Silverstein et al., 2006). Alors que des études ont montré que divers types de réseaux personnels s'associent à des montants différents de soutien social (Litwin \& Landau, 2000), les conséquences de la diversification des contextes familiaux sur le soutien familial et surtout sur la façon dont il circule et se distribue au sein des configurations familiales ne sont jamais évoquées dans la recherche. 


\section{Problématique et hypothèses}

La gérontologie sociale s'est en effet peu questionnée sur les propriétés structurelles du soutien au sein des configurations familiales et sur les facteurs à même de les influencer. En d'autres termes, le capital social généré au sein des configurations familiales dans la vieillesse n'est jamais investigué. Pourtant, comme le montrent des études sur d'autres classes d'âges (Widmer, 2016; Widmer et al., 2013), les configurations familiales, définies comme des réseaux de liens d'interdépendance, produisent différents types de capital social selon la façon dont les liens de soutien s'organisent en leur sein. Dans cette sous-section, nous proposons de montrer la pertinence de la notion de «capital social» pour étudier le soutien familial dans la vieillesse. D'abord, nous le définissons et décrivons les mécanismes sous-jacents qui le produisent. Ensuite, nous en présentons les principaux types et nous nous interrogeons quant à l'influence de la composition des configurations familiales et à celle du profil des aînés sur son émergence.

\subsubsection{Le capital social et ses différents types}

Comme le suggèrent les modèles de la «solidarité intergénérationnelle » (Bengtson \& Roberts, 1991; Roberts \& Bengtson, 1990) ou du «support bank» (Antonucci, 1990, 2001; Antonucci et al., 2007; Antonucci \& Jackson, 1989), les obligations familiales, reposant uniquement sur le respect des rôles et des statuts au sein de la famille, ne suffisent pas à garantir le soutien entre les parents âgés et leurs enfants. Selon le modèle du «support bank » de Toni Antonucci, la solidarité entre parents âgés et enfants adultes est plus complexe; elle est tributaire de la norme de réciprocité " généralisée» qui régule les échanges de soutien entre parents et enfants tout au long du parcours de vie et qui, ce faisant, les rend fortement interdépendants aussi bien émotionnellement que pratiquement. Ceci dit, dans le modèle du «support bank », la norme de réciprocité " généralisée » est considérée uniquement dans une perspective dyadique. L'impact des normes de réciprocité dans le contexte plus large de la configuration familiale n'a jamais été étudié en gérontologie sociale. Ceci est cependant possible si l'on considère l'approche configurationnelle et que l'on intègre dans l'analyse la notion de « capital social » qu'elle propose. La notion de « capital social» permet en effet de saisir l'influence des normes de réciprocité sur l'organisation des liens de soutien au sein des configurations familiales. 
Le concept de « capital social» n'est pas nouveau et a été utilisé dans de nombreuses disciplines au point parfois d'en perdre sa signification première et sa valeur heuristique (Portes, 1998). L'un des premiers a en offrir une définition claire fut Pierre Bourdieu (Bourdieu, 1980, 1986). Généralement, le « capital social » renvoie à l'ensemble des ressources individuelles qui sont produites au sein d'un réseau durable de relations personnelles et auxquelles l'individu, en tant que membre du réseau, a accès ou peut y accéder en cas de besoin (Bourdieu, 1980, 1986; Moren-Cross \& Lin, 2006; Widmer, 2006, 2016). Parce que les ressources (dont le soutien) sont imbriquées au sein du réseau personnel, la notion de «capital social» se distingue de celle de «support social », à laquelle elle est pourtant souvent confondue (Bourdieu, 1980, 1986; Moren-Cross \& Lin, 2006). Ces ressources peuvent être de montants différents et de natures variées, non seulement économiques, pratiques, culturelles, mais aussi symboliques et affectives. Plus concrètement, il peut s'agir de soutien émotionnel, de services pratiques, de prêt d'argent mais aussi de compagnonnage, de reconnaissance ou encore d'affection. Quelle qu'en soit la nature, ces ressources sont échangées entre les différents membres du réseau personnel et ce sont ces multiples transferts, régulés par des normes d'échange (réciprocité), qui produisent avec le temps du capital social (Bourdieu, 1980, 1986). Il s'agit d'un long processus par lequel les échanges, reposant sur les normes de réciprocité et alimentés par les investissements personnels des membres du réseau (soutien, temps, etc.), transforment des relations occasionnelles ou peu investies en des liens émotionnellement engagés, interdépendants, soutenus par une reconnaissance et des sentiments d'obligations mutuelles (gratitude, affection, culpabilité, etc.) (Kadushin, 2012; Portes, 1998).

Ce processus de «transformation» ne se fait pas à la légère, il s'inscrit dans le temps, au gré d'échanges multiples et de reconnaissance. Ces deux éléments, "multiplexité des ressources » et "reconnaissance mutuelle», contribuent à l'émergence d'une "forte confiance» entre les différents membres du réseau personnel, celle-ci étant nécessaire à la stabilisation des échanges (Kadushin, 2012; Portes, 1998). L'individu, qui y prend part, a de nombreux avantages. Il peut non seulement profiter directement des ressources mises à disposition par les autres membres du réseau mais il peut aussi faire fructifier ses propres ressources en accédant, par exemple, à un nouvel emploi mieux payé grâce à l'aide de son réseau (Bourdieu, 1986; Portes, 1998). Par ce processus de «transformation» (Kadushin, 


\section{Problématique et hypothèses}

2012), le capital social comprend une dimension aussi bien « objective » que « subjective». En effet, les ressources que l'individu a à disposition au sein de son réseau personnel sont non seulement " actuelles » mais aussi « perçues » ou « potentielles », l'individu étant « certain » d'obtenir de son réseau personnel toute l'aide dont il aurait besoin en cas de nécessité, et cela dans n'importe quelles circonstances (Bourdieu, 1980, 1986; Portes, 1998).

Bien que tout réseau personnel produit du capital social, celui-ci varie dans sa forme et dans son volume. Si le réseau est de grande taille ou s'il est composé de membres pourvus de nombreuses ressources tant économiques, sociales que symboliques (un nom, une renommée, etc.), il produit un capital social de plus grand volume que celui généré au sein d'une petit réseau ou dont les membres ont peu de ressources (Bourdieu, 1980, 1986; Portes, 1998). Étant donné que les individus qui se connectent les uns aux autres participent généralement aux mêmes espaces socio-économiques et s'adonnent aux mêmes pratiques, l'homogénéité sociale étant l'une des bases du sentiment d'appartenance et de reconnaissance au sein du réseau personnel, un individu qui possède de nombreuses ressources a accès parce qu'il est relié à d'autres individus qui lui ressemblent - à un fort capital social qu'il peut maintenir et faire fructifier en entretenant activement les échanges (Bourdieu, 1980, 1986). A l'opposé, un individu qui possède peu de ressources socio-économiques a généralement peu de capital social. D'une part, il n'a pas les ressources nécessaires pour maintenir les échanges au sein de son réseau (réciprocité); il risque ainsi de perdre certaines de ses relations avec pour conséquence la réduction de son réseau (Antonucci et al., 2007; Broese Van Groenou \& Van Tilburg, 2007; Offer, 2012). D'autre part, il est connecté à des membres qui lui ressemblent (homogénéité sociale) et qui, comme lui, disposent de peu de moyens, ce qui réduit considérablement ses possibilités de faire fructifier ses propres ressources. Même si l'individu possède les capacités suffisantes, il ne peut que difficilement les développer au sein de son réseau personnel, ce dernier ne pouvant lui offrir les moyens et les opportunités dont il a besoin pour les faire fructifier (Portes, 1998).

Quels que soient le nombre et la nature des ressources mises à disposition au sein du réseau personnel, l'accès au capital social ne va pas de soi. Il ne suffit pas d'être entouré de personnes ayant de multiples ressources pour accéder au capital social, il faut que l'individu soit connecté et, si possible, 
«bien connecté » aux personnes qui forment le réseau. Autrement dit, l'accès au capital social dépend de l'engagement personnel de l'individu dans les échanges et de sa position au sein de son réseau (Kadushin, 2012). Si l'individu n'a aucune ou de très faibles connexions avec les membres de son réseau personnel, il ne peut pas prétendre au capital social qui y est produit. Il doit y investir du temps, de l'énergie et des ressources s'il souhaite maintenir à long terme le capital social dont il peut et pourra bénéficier au moment venu (Bourdieu, 1980, 1986; Portes, 1998). La position de l'individu au sein du réseau personnel influence aussi son accès au capital social. S'il se situe en périphérie, il n'a aucune prise sur le flux des ressources qui y circule alors que s'il y occupe une position centrale, il est au centre des échanges (Kadushin, 2012). Outre l'engagement et la position de l'individu dans son réseau, le capital social peut aussi varier en fonction de la manière dont s'organisent les liens d'interdépendance et les échanges au sein du réseau personnel (Kadushin, 2012). Autrement dit, les propriétés structurelles qui caractérisent les liens d'interdépendance et les échanges de soutien peuvent être très différentes d'un réseau à l'autre, donnant lieu à différents types de capital social (Bourdieu, 1980, 1986; Portes, 1998). Par conséquent, les individus ne bénéficient pas tous du même type de capital social. Deux types de capital social sont généralement distingués dans la littérature : le capital social de type chaîne et celui de type pont (Widmer, 2006, 2016).

Le capital social de type chaîne est particulièrement présent dans les réseaux personnels de petite taille dans lesquels la plupart des membres sont connectés entre eux au travers d'échanges de ressources multiples et réciproques, donnant lieu à une forte densité des liens (Coleman, 1988; Widmer, 2016, 2006). Ce sont des réseaux composés de membres qui se connaissent bien et qui entretiennent des relations depuis longtemps, fréquentant les mêmes endroits, pratiquant les mêmes activités et partageant les mêmes valeurs (forte homogénéité). Se suffisant à eux-mêmes dans leurs échanges multiples, ils interagissent peu avec l'extérieur du réseau (repli) (Coleman, 1988; Putnam, 2000). Parce qu'ils sont tous connectés directement les uns aux autres, c'est-à-dire interdépendants, aucun d'entre eux n'y occupe une position centrale (Widmer, 2006, 2016). Les normes de réciprocité "généralisée » régulent leurs relations, inscrivant les membres dans un long cycle d'échanges, renforçant encore davantage leurs liens d'interdépendance (Offer, 2012; Molm et al., 2007). Les membres du réseau développent entre eux des relations d'affection et de 


\section{Problématique et hypothèses}

confiance que 1'on peut qualifier de sécurisantes (Coleman, 1988; Cornwell, 2009b; Kadushin, 2002; Widmer, 2006, 2016), mais aussi de forts sentiments d'obligation, de redevabilité latente (gratitude) ainsi que la sensation diffuse qu'ils se doivent mutuellement quelque chose (attentes) (Coleman, 1988; Putnam, 2000). En raison des propriétés structurelles qui caractérisent ce type de réseaux, le capital social de type chaîne qui s'y développe promeut un soutien collectif (Coleman, 1988; Widmer, 2016). Étant tous interconnectés les uns aux autres, les membres du réseau peuvent coordonner leurs efforts pour offrir les ressources nécessaires aux membres qui en ont le plus besoin (Ashida \& Heaney, 2008; Cornwell et al., 2009; Cornwell, 2009b; Haines \& Hurlbert, 1992; Widmer, 2006, 2016). Dans les situations de «caregiving », typiques du grand âge, les membres du réseau personnel peuvent s'organiser et coordonner l'aide dont le parent âgé a besoin, ce qui est bénéfique pour ce dernier qui, fragilisé voire dépendant, nécessite de beaucoup de présence et d'attention, voire de l'aide constante d'une tierce personne (Cornwell, 2009a, 2009b, 2011). Il peut de fait être pris en charge par plusieurs membres de la famille qui peuvent, parce qu'ils sont fortement interconnectés, se coordonner et partager les tâches du caregiving. Les individus qui s'insèrent dans ce type de réseaux personnels ont donc un accès facile et rapide à l'ensemble des ressources collectives produites en son sein (Cornwell et al., 2008, 2009; Cornwell, 2009b; Haines \& Hurlbert, 1992).

Le capital social de type chaîne comprend aussi des aspects plus négatifs. D'une part, les individus dans ce type de réseaux, denses et peu ouverts vers l'extérieur ( « closure »), ont peu de choix dans les ressources proposées (Coleman, 1988; Cornwell, 2009a, 2009b; Granovetter, 1973). D'autre part, le soutien collectif s'accompagne de certaines exigences, les bénéficiaires devant répondre aux attentes des autres membres du réseau et se conformer aux normes en vigueur (Coleman, 1988; Cornwell, 2009b, 2011; Putnam, 2000; Widmer, 2016). Dès lors, le soutien collectif peut être perçu comme oppressif, vécu comme une tentative de contrôle, de surveillance ou d'entrave à l'autonomie individuelle et, par conséquent, il peut générer des tensions au sein du réseau (Cornwell, 2009a, 2009b, 2011; Silverstein, Chen, \& Heller, 1996). La supervision omniprésente de l'entourage et les conseils, même bien intentionnés, peuvent prendre la forme de reproches ou de contrôle comme lorsque les membres du réseau surveillent la prise de médicaments ou imposent une restriction alimentaire. 
Cette supervision ou ces conseils sont, par conséquent, perçus et vécus comme contraignants et intrusifs, pouvant engendrer du ressentiment et des tensions (Lewis \& Rook, 1999; Thoits, 2011; Tucker, 2002). Corrélée au sentiment de compétence, à l'estime de soi et au maintien de l'identité, l'autonomie est particulièrement valorisée et, qui plus est, dans la vieillesse. Mise à mal face au déclin de la santé, son maintien aux dernières étapes de la vie devient un enjeu central pour un grand nombre de personnes âgées (Cornwell, 2009a, 2011; Krause, 1997b; Pinquart \& Sörensen, 2000; Pyke \& Bengtson, 1996). Le soutien collectif peut donc, s'il est non désiré ou s'il est perçu comme excessif ou inapproprié, être ressenti comme une menace et avoir des effets néfastes sur l'estime de soi (Coyne et al., 1988; Krause, 1997a, 1997b; Pinquart \& Sörensen, 2000; Silverstein et al., 1996). Cependant, même si le soutien "effectif» s'accompagne de tensions, la « certitude»-inhérente au capital social de type chaîne - de pouvoir bénéficier d'une aide fiable et inconditionnelle favorise la santé et le bien-être (Krause, 1997a; Pinquart \& Sörensen, 2000). Le capital social de type chaîne se conjugue donc aussi bien avec obligations et contrôle qu'avec soutien, sécurité et confiance (Coleman, 1988; Putnam, 2000; Widmer, 2006, 2016).

Quant au deuxième type de capital social, celui de type pont, il se développe dans des réseaux personnels dont les propriétés structurelles se démarquent nettement de celles qui caractérisent les réseaux précédents. Il s'agit de réseaux personnels de grande taille et de faible densité. Ouverts vers l'extérieur, ces réseaux sont composés de membres qui proviennent de milieux variés, aussi bien familial, amical, professionnel qu'associatif, et qui ont des profils socio-démographiques divers (Cornwell et al., 2009; Widmer, 2006, 2016). Différents, les membres de ces réseaux ne se connaissent pas ou peu, ne partagent pas les mêmes intérêts, les mêmes valeurs et le même style de vie (Cornwell, 2009a, 2011). Par conséquent, ils sont relativement peu connectés les uns aux autres par des échanges multiples. Les liens qui caractérisent ce type de réseaux sont de nature plus dyadique que collective; les membres qui sont connectés sont davantage motivés par la satisfaction d'être ensemble que par un sentiment de redevabilité (De Carlo et al., 2014; Widmer, 2016). Dans ce type de réseaux, ce sont plutôt des normes de réciprocité « directe » et « équilibrée » qu' « indirecte » et "généralisée » qui y régulent les échanges. La faible densité des échanges, c'est-à-dire les faibles interactions entre les membres, crée des « trous » dans la structure du réseau, donnant l'opportunité à l'individu 


\section{Problématique et hypothèses}

d'y jouer le rôle d'intermédiaire et d'accéder au capital social de type pont (Burt, 2002; Cornwell et al., 2009; Cornwell, 2009b; Granovetter, 1973; Widmer, 2016). Être inséré dans ce type de réseaux et, qui plus est, d'y occuper une position centrale procure de nombreux avantages mais aussi des inconvénients.

Grâce à la nature composite de ces réseaux, les individus qui s'y insèrent peuvent compter sur une variété de ressources. S'ils ne sont pas satisfaits par l'aide reçue, ils peuvent se tourner vers d'autres sources de soutien, ce qui leur permet de maintenir une certaine autonomie au sein de leur réseau (Burt, 2002; Cornwell, 2009a, 2009b, 2011; Granovetter, 1973). Les relations qui caractérisent ce type de réseaux sont, étant de nature plutôt dyadique, généralement positives et gratifiantes. L'individu peut en effet se délester aisément des membres qu'il juge désagréables, ceux-ci n'étant pas imbriqués dans de multiples interconnexions, et éviter ainsi les tensions. Par ailleurs, occuper une position centrale au sein de ce type de réseaux procure un certain pouvoir. Connaissant le potentiel de ressources de chacun des membres de son réseau, l'individu peut, en jouant le rôle d'intermédiaire, contrôler le flux des ressources qui rendent les membres de son réseau interdépendants (Burt, 2002; Cornwell et al., 2009; Cornwell, 2009a, 2009b). Gestionnaire, il joue un rôle actif dans le maintien et la gestion de ses liens, rôle qui favorise le sentiment de contrôle, l'estime de soi et le bien-être (Berkman et al., 2000; Kadushin, 2002; Krause, 2006; Merz, Consedine, Schulze, \& Schuengel, 2009). Finalement, être l'intermédiaire de membres qui ne sont pas connectés signifie aussi être à l'abri de leur influence puisqu'ils ne peuvent pas, n'étant pas en lien, exercer un quelconque contrôle (Burt, 2002; Cornwell, 2009b; Granovetter, 1973). Bien qu'assurant une certaine autonomie individuelle, la faiblesse des liens d'interdépendance s'associe à un soutien incertain et instable (Cornwell, 2009b; Haines \& Hurlbert, 1992). Ce type de réseau n'étant pas soutenu par des normes de réciprocité « généralisée », la coopération entre les différents membres du réseau est difficile et le soutien collectif, peu probable. Plus dyadique que collectif, le soutien y est donc plus fragile. Par ailleurs, le capital social de type pont est exigeant et stressant (Burt, 2002; Cornwell, 2009b, 2009a). Il requiert de l'engagement de la part des individus centraux, ceux-ci doivent être actifs dans les échanges afin de maintenir sur le long terme l'ensemble de leurs relations. Et leur 
engagement est d'autant plus nécessaire que leur réseau personnel est instable en raison de sa faible densité (Burt, 2002; Cornwell, 2009b, 2009a). Par conséquent, ils se doivent d'être en bonne santé et avoir les facultés cognitives nécessaires pour mettre en lien et organiser des échanges entre des membres provenant de cercles de sociabilité différents, avec des normes et des attentes diverses (Cornwell, 2009b, 2009a). Si l'individu central perd ses facultés, par exemple lors de problèmes de santé, le réseau personnel se désagrège, n'étant plus soutenu par son pilier central (Widmer, 2016). Le capital social de type pont est donc synonyme d'accès à une diversité de ressources, d'échanges positifs et du maintien de l'autonomie relationnelle, mais il est exigeant et il est loin de garantir un soutien inconditionnel.

Dans cette partie, nous avons souligné que le capital social varie dans son volume et son type en fonction des propriétés structurelles qui caractérisent les réseaux personnels. Au vu de ces observations, nous pouvons nous demander si les configurations familiales dans la vieillesse présentent des propriétés structurelles distinctes, donnant lieu à du capital social de différents types et de différents volumes. Dans un grand pan de la gérontologie sociale, le soutien familial y est défini comme fiable et inconditionnel, se référant «a priori »- bien qu'il ne soit jamais nommé ainsi - au capital social de type chaîne. Celui-ci est produit au sein d'une famille «modèle" réunissant a priori des individus ayant des liens de sang ou d'alliance et qui sont étroitement connectés les uns aux autres au travers d'échanges multiples et dont les liens sont régis par des normes de réciprocité "généralisée » (Cornwell et al., 2008). Or, comme nous l'avons postulé précédemment, les aînés peuvent avoir des définitions plurielles de leur famille significative, en intégrant au sein de leur configuration familiale une diversité de membres familiaux. De fait, il est probable que le capital social au sein de ces diverses configurations varie plus que ne le suggère la recherche en gérontologie sociale. Dans les prochaines sections, nous nous interrogeons sur le lien possible entre l'identité des membres qui forment les configurations familiales dans la vieillesse et le type de capital social qu'elles génèrent. Nous nous questionnerons ensuite sur l'influence du profil socio-démographique des individus focaux sur le type de capital social produit au sein de leur configuration familiale. Ce faisant, nous proposerons quelques hypothèses qui guideront la suite de cet ouvrage. 


\section{Problématique et hypothèses}

\subsubsection{La composition des configurations familiales}

Dans cette sous-section, nous nous questionnons sur l'influence de la composition des configurations familiales sur la production du capital social et ses variations, ce qui, à notre connaissance, n'a jamais été étudié en gérontologie sociale. Il est pourtant probable que les aînés aient accès à différents types de capital social en fonction des membres qu'ils désignent comme faisant partie de leur famille significative. Sur la base de la littérature existante, nous mettons en exergue l'influence de certains membres de la famille sur les liens et les échanges au sein de la famille. D'abord, nous nous focalisons sur les membres de la famille «dits" primaires, à savoir le partenaire et les enfants, et nous mettons en évidence l'organisation différente des liens d'interdépendance au sein de la famille lorsqu'ils sont présents ou absents. Nous en déduisons ensuite les types de capital social que leur présence et leur absence favorisent. Puis, l'effet du sexe des membres de la famille sur les liens et les échanges familiaux est questionné, sachant que les femmes, quel que soit leur lien de parenté (épouse, fille, sœur, etc.), ont des rôles tournés vers le maintien du lien et des échanges au sein de la famille. En nous basant sur les observations récoltées, nous soulignons l'influence probable d'une présence majoritairement féminine dans la famille sur le type de capital social produit.

La recherche en gérontologie sociale montre que les conjoints dans le couple sont fortement interdépendants tant affectivement que pratiquement (Cornwell, 2009b, 2011). Les raisons qui expliquent un tel lien sont multiples : un engagement mutuel, une co-résidence, la pratique d'activités partagées, une histoire commune, des échanges de ressources multiples et des obligations d'aide mutuelles, plus prégnantes, par ailleurs, lorsqu'ils sont mariés (Carr \& Moorman, 2011; Marcussen, 2005 ; Pudrovska et al., 2006; Robles \& Kiecolt-Glaser, 2003). Les normes de réciprocité qui régissent leurs échanges sont " généralisées », ce qui, en inscrivant la dynamique des échanges dans le long terme, contribue à entretenir l'interdépendance des partenaires (Antonucci et al., 2007; Johnson, 1988). Cette interdépendance s'accentue encore davantage aux dernières étapes de la vie avec le déclin de la santé et surtout avec la contraction du réseau personnel, c'est-à-dire lorsque disparaissent peu à peu les membres du réseau. Les partenaires deviennent, dès lors, de plus en plus dépendants de l'un de l'autre et partagent davantage le même réseau de relations, composé généralement des membres proches de la famille tels que les enfants 
et les petits-enfants (Cornwell, 2009b, 2011; Kalmijn, 2003). Au cours de ce processus, le conjoint devient l'une des principales sources de soutien, parfois la seule. Au vu de ces résultats, la présence d'un partenaire semble davantage favoriser le développement du capital social de type chaîne, les liens et les échanges familiaux étant denses, soutenus par une réciprocité " généralisée », et la centralité des répondants étant plutôt faible.

Ces données portent généralement sur des relations conjugales de longue haleine, unissant des partenaires mariés depuis longtemps et ayant des enfants communs. Cependant, être en couple «non marié » s'associe dans la littérature à davantage de « liberté » dans la relation conjugale, c'est-à-dire à moins d'interdépendance et d'obligations d'aide mutuelle (Fingerman et al., 2004). Dans une telle situation, la présence du conjoint ne s'associe pas forcément à du capital social de type chaîne. Et même si les nouveaux partenaires se remarient, la remise en couple après un divorce ou un veuvage, plus fréquente chez les hommes âgés, n'a pas les mêmes conséquences sur l'organisation des liens au sein de la famille que la présence d'un partenaire de première union. Certes, si la remise en couple a eu lieu lorsque les enfants étaient petits et que la relation est qualifiée de bonne, les échanges entre « beaux-parents et beaux-enfants » peuvent être denses et réciproques, et cela notamment si le partenaire a peu d'échanges avec ses propres enfants (biologiques) (Schmeeckle et al., 2006). Plus généralement cependant, l'arrivée d'un nouveau partenaire peut déséquilibrer les échanges au sein de la configuration familiale et peut être source de tensions et d'ambivalence avec les autres membres de la famille, notamment avec les enfants, et cela d'autant plus si ces derniers dépendent de l'aide de leur parent biologique (Lüscher \& Pillemer, 1998; Schmeeckle et al., 2006). Rivalisant pour les mêmes ressources, tensions et conflits peuvent naître entre les enfants biologiques et le nouveau conjoint. Une telle situation se négocie ensuite entre les différents membres de la famille, avec plus ou moins de succès, en fonction des ressources et des alternatives à disposition. La prise de distance entre le nouveau partenaire et les autres membres de la famille pourrait être l'une des manières de gérer les tensions et l'ambivalence au sein de la famille (Lüscher \& Pillemer, 1998), ce qui rendrait les liens familiaux moins denses (Widmer, 1999a; Widmer \& La Farga, 2000). Par conséquent, une telle situation peut nuire au capital social de type chaîne tout en favorisant l'émergence du capital de type pont puisque le parent âgé (ego) est amené à jouer le rôle de médiateur entre son nouveau partenaire et les autres membres de sa famille. 


\section{Problématique et hypothèses}

La présence d'enfants (biologiques) et de petits-enfants contribue aussi à activer la vie familiale et à densifier les liens familiaux (Bickel \& Girardin, 2008; Cornwell, 2009b). La mise en couple des enfants et la présence des petits-enfants, voire des arrière-petits-enfants, renforcent les liens d'interdépendance au sein de la famille. L'entrée en parentalité des enfants adultes, notamment des filles, s'associe à un rapprochement entre les deux générations, rapprochement qui se veut à la fois émotionnel et pratique (Bucx, Van Wel, Knijn, \& Hagendoorn, 2008; Lüscher \& Pillemer, 1998). Devenues mères, les filles recherchent auprès de leur parents, surtout de leur mère, des conseils mais aussi du soutien émotionnel et souvent une aide pratique (garde d'enfants) (Lüscher \& Pillemer, 1998). Ce besoin d'aide et de soutien contribue non seulement à intensifier les contacts mais aussi à renforcer le lien émotionnel entre parents âgés et enfants adultes (Bickel \& Girardin, 2008; Bucx et al., 2008; Silverstein et al., 1994). Les pratiques communes autour des enfants et des petits-enfants, telles que les réunions de familles ou les fêtes, ainsi que les échanges de multiples ressources entre les membres des différentes générations (garde d'enfants, conseils, prêts d'argent, etc.) contribuent à consolider les liens d'interdépendance (Bengtson, 2001; Bucx et al., 2008; Silverstein et al., 1994; Silverstein \& Giarrusso, 2010). Ces échanges sont régulés par les normes de réciprocité "généralisée » qui sont particulièrement prégnantes dans les relations intergénérationnelles (Bowling, 1994; Johnson, 1988; Silverstein \& Bengtson, 1997; Silverstein et al., 2006). Suivant ces normes, les services rendus à la troisième génération (petits-enfants) pourront être réclamés plus tard par la première génération (grands-parents) à la deuxième génération (enfants) sous la forme d'aide et de soutien lorsque le déclin de la santé l'exigera (Antonucci et al., 2007). Ces normes s'associent à un fort engagement affectif mais aussi à de forts sentiments d'obligations d'aide mutuelle (redevabilité). Autrement dit, les enfants ressentent le devoir de soutenir leur parent âgé dans le besoin sans attendre le retour immédiat de l'aide qu'ils octroient et, étant fortement réticents à l'« abandonner ", certains sont prêts à effectuer des tâches particulièrement pénibles telles qu'effectuer des soins du corps ou assurer une présence auprès d'un malade agressif, et cela même sur une longue durée (Cornwell, 2009b, 2009a).

$\mathrm{Au}$ vu de ces différentes études, nous faisons l'hypothèse que la présence des enfants et des petits-enfants dans la configuration familiale favorise la densité et la réciprocité des échanges, contribuant à l'émergence du capital social de type chaîne plutôt que de celui de type pont. 
Ceci dit, cette hypothèse ne tient compte que du côté positif des interdépendances familiales, à savoir la promesse d'un soutien collectif. Or, les fortes interdépendances auxquelles s'associe la présence d'enfants et de petits-enfants s'accompagnent aussi de tensions, de conflits et d'ambivalence (Connidis \& McMullin, 2002; Lüscher, 2002; Lüscher \& Pillemer, 1998). Ces tensions peuvent devenir particulièrement importantes dans les familles «multigénérationnelles» dans lesquelles le nombre de bénéficiaires excède celui des prestataires, lorsque, par exemple, les enfants adultes doivent procurer de l'aide à leurs parents âgés, voire à leurs grandsparents, mais aussi à leurs propres enfants dépendants économiquement, voire à leurs petits-enfants. Dans de telles situations, le déséquilibre des échanges engendre de la fatigue et du stress chez les aidants (Bengtson, 2001; Johnson, 1988) et crée des tensions au sein des configurations familiales, et cela d'autant plus si les ressources sont rares (Connidis \& McMullin, 2002; Lüscher \& Pillemer, 1998). Au vu de ces éléments, il est possible que ces tensions mettent à mal la densité des échanges familiaux et, à la longue, altèrent le capital social de type chaîne au point d'en réduire le volume disponible.

Quant à l'absence de conjoint ou d'enfants dans la famille, la recherche en gérontologie sociale montre que les veufs, divorcés ou célibataires tendent à s'engager dans une variété de relations personnelles, ayant pour conséquence la diversification de leur réseau personnel (cf. section sur les membres significatifs). Pour compenser l'absence d'un conjoint ou d'enfants et renforcer leur réseau familial, certains d'entre eux procèdent à une re-évaluation de leurs relations personnelles et sélectionnent celles qu'ils ont investies affectivement et qui, à leurs yeux, "méritent » de faire partie de «leur» famille significative. Ce faisant, ils choisissent au sein de leur réseau personnel une diversité de membres dont certains proviennent de leur réservoir de parenté alors que d'autres n'ont ni lien de sang ni d'alliance. Leur insertion au sein des configurations familiales a des implications sur l'organisation des relations et des échanges.

En effet, des études portant sur d'autres classes d'âge révèlent que les configurations familiales dont la composition est diversifiée s'associent à des liens et des échanges moins denses que ceux dont les membres familiaux sont de même sang (De Carlo et al., 2014; Widmer, 2006, 2016). Ne se connaissant pas ou peu, les membres sélectionnés qui forment la même configuration familiale sont peu connectés entre eux au travers d'échanges multiples (Widmer, 2016, 2006; De Carlo et al., 2014). Même s'ils sont 


\section{Problématique et hypothèses}

définis par l'aîné comme très proches affectivement, les échanges qu'ils ont entre eux ne sont pas soumis à des normes de réciprocité "généralisée » qui renforcent les liens d'interdépendance au sein de la configuration familiale; ils répondent plutôt à une réciprocité «équilibrée », directe, de type dyadique. Dans ce type de configurations, les liens que l'individu âgé entretient avec les membres qu'il a choisis reposent davantage sur des affinités, des échanges positifs, des intérêts communs et la satisfaction d'être ensemble (Allen et al., 2011; Braithwaite et al., 2010; Pahl \& Spencer, 2004; Widmer, 2016) que sur le sentiment latent de redevabilité qui caractérise les relations intergénérationnelles (Bowling, 1994; Johnson, 1988; Silverstein \& Bengtson, 1997; Silverstein et al., 2006). En raison de la faible densité des liens, des trous se forment entre les membres de la configuration qui ne sont pas connectés, offrant la possibilité à l'individu âgé de se placer au centre des échanges et de jouer le rôle d'intermédiaire, lui assurant ainsi une grande autonomie (Cornwell, 2009b; De Carlo et al., 2014; Widmer, 2016, 2006). Cependant, moins tenus par le sentiment de redevabilité qui sous-tend les relations intergénérationnelles, le soutien promis par les membres familiaux dans ce type de configurations est fragile. Sans liens de sang ni d'alliance, ces membres se désengagent plus facilement lorsqu'il est question de procurer du soutien de plus grande ampleur («caregiving ») ou de plus longue durée comme dans les situations où l'aîné est sévèrement atteint dans sa santé (Cornwell, 2009b, 2009a; Dykstra \& Hagestad, 2007; Pinquart \& Sörensen, 2000). Alors que ces divers membres familiaux choisis offrent aux individus âgés sans conjoint ou sans enfant une « compensation » familiale, ils peinent cependant à offrir un soutien solide et de longue durée (Dykstra \& Hagestad, 2007).

$\mathrm{Au}$ vu de l'ensemble de ces observations, nous faisons l'hypothèse que l'absence de partenaire ou d'enfants dans la configuration familiale contribue, en raison de l'insertion de membres familiaux particuliers et de l'organisation spécifique des liens et des échanges qu'elle implique, au développement du capital social de type pont, avec le risque probable qu'il s'affaiblisse et disparaisse lorsque les tâches de soutien envers l'individu âgé s'avéreront trop lourdes. Cette hypothèse peut être remise en question si l'on considère le processus de "suffusion", proposé par Pahl et Spencer (2004). Ce processus rend compte de l'interchangeabilité des rôles entre la famille et les amis, ces derniers adoptant de plus en plus des comportements similaires à ceux des membres de la famille, ce qui n'est pas sans conséquence sur l'organisation de leurs liens. Par exemple, il se peut 
que des amis affectivement proches qui se connaissent depuis longtemps forment une même configuration familiale. Au gré d'échanges multiples, ils sont devenus avec le temps interdépendants au point de se considérer mutuellement comme des membres de la même famille significative. On peut, dès lors, imaginer que la configuration familiale à laquelle ils participent produise autant de capital social de type chaîne que celle qui inclut un partenaire ou des enfants.

Outre le lien de parenté, le sexe des membres de la famille est aussi à même d'influencer le type de capital social produit au sein de la configuration familiale. Nous avons souligné dans le chapitre précédent la prégnance des normes sociales qui attribuent aux femmes la responsabilité de la sphère familiale. Socialisées pour accomplir des tâches d'éducation et de soins, c'est à elles que revient la responsabilité du bien-être de l'ensemble des membres de la famille (Connidis \& McMullin, 2002; Silverstein et al., 2006; Willson et al., 2006). Devant se conformer au rôle qui leur incombe, les femmes sont davantage soumises aux pressions normatives de la solidarité familiale que les hommes et sont, par conséquent, plus impliquées dans le soutien familial même si, comme eux, elles occupent un emploi à plein temps (Connidis \& McMullin, 2002; Silverstein et al., 2006; Willson et al., 2006). Cette pression normative qui touche plus particulièrement les femmes a des conséquences sur l'organisation des liens et des échanges au sein de leur configuration familiale. Les données confirment en effet que les femmes sont plus actives dans le maintien des liens et des échanges (soutien) au sein de la famille que les hommes, et cela quel que soit leur rôle familial (épouse, fille, sœur, etc) (Ajrouch et al., 2005; Antonucci, 2001; Bowling, 1994; Silverstein et al., 2006).

Les « épouses », par exemple, tendent à relier leur conjoint aux autres membres de la famille et lui assurent soutien et soins lorsque sa santé décline (Ajrouch et al., 2005; Antonucci, 2001; Arber \& Cooper, 1999; Bowling, 1994). A l'instar des épouses, les filles adultes sont perçues dans la littérature comme d'importantes prestataires de soutien et de soins auprès du parent âgé (Bowling, 1994; Pinquart \& Sörensen, 2006; Silverstein et al., 2006; Suitor et al., 2011). Étant davantage socialisées aux obligations d'aide et de soins, elles se révèlent plus promptes que les fils à répondre aux besoins de leurs parents et font preuve à leur égard d'un plus grand attachement affectif (Pinquart \& Sörensen, 2006; Rossi \& Rossi, 1990; Silverstein et al., 2006; Walker et al., 1995). Le travail de soins (« caregiving ») est perçu comme «naturel » et attendu chez les femmes, qui sont 


\section{Problématique et hypothèses}

amenées à l'effectuer tout long de leur vie en tant qu'épouse, mère et fille, et «non naturel» chez les hommes à qui l'on excuse volontiers leur non implication (Bowling, 1994; Silverstein et al., 2006; Walker et al., 1995). Même lorsqu'ils sont « caregivers », les fils accomplissent une moins grande variété de tâches que les filles, y consacrent moins de temps et font davantage appel à une aide extérieure (Pinquart \& Sörensen, 2006). Ils se sentent, par ailleurs, moins responsables de leurs parents lorsqu'ils ont des sœurs alors que les filles se coordonnent entre sœurs pour accomplir les tâches de soins (Eriksen \& Gerstel, 2002; Szydlik, 2008; Willson et al., 2006). Les épouses des fils, les «belles-filles », jouent aussi un rôle important. Lorsqu'elles s'entendent bien avec leurs beaux-parents, elles favorisent les liens entre le fils, ses enfants et ses parents, ce qui semble être moins le cas des conjoints des filles, les « beaux-fils » (Fingerman, 2004). Quant aux partenaires des pères, les «belles-mères », elles encouragent davantage leur conjoint à entretenir des liens avec leurs enfants biologiques, voire à les renouer s'ils avaient été désinvestis, que les partenaires des mères, les « beaux-pères » (Schmeeckle et al., 2006). Finalement, les « sœurs » sont plus actives dans le maintien des relations fraternelles que les frères et elles constituent une source importante de soutien émotionnel au sein de la famille (Campbell et al., 1999). Au vu de ces différents résultats, nous faisons l'hypothèse que les configurations familiales majoritairement composées de femmes se caractérisent par des liens et des échanges plus denses et plus réciproques que celles qui sont constituées principalement d'hommes. Par conséquent, leur présence majoritaire favorise davantage l'émergence d'un capital social de type chaîne dans les configurations familiales que celle des hommes.

On peut cependant se questionner sur les conséquences du «surinvestissement » féminin sur l'organisation des liens au sein de la famille. A la fois « épouse », « fille », « belle-fille», « belle-mère » et « sœur », les femmes doivent accomplir une lourde charge de travail impliquant des responsabilités et des tâches diverses qui nécessitent du temps et de l'énergie. Cette multiplicité des rôles peut s'avérer particulièrement pesante si les femmes ont, en plus d'être actives professionnellement, plusieurs membres au sein de leur configuration qui dépendent directement de leur soutien tels que des parents âgés, un mari malade, des enfants non autonomes ou encore des enfants en bas âge (Ajrouch et al., 2005; Antonucci, 2001; Johnson \& Troll, 1996; Pinquart \& Sörensen, 2006). Il va sans dire que la charge de travail qui en découle génère chez les femmes de la fatigue et du stress 
et crée au sein de leur configuration familiale des tensions et du conflit (Ajrouch et al., 2005; Antonucci, 2001; Connidis, 2010; Connidis \& McMullin, 2002; Pinquart \& Sörensen, 2006). Par ailleurs, oscillant entre un rôle qui leur est socialement imposé et le désir d'autonomie, les femmes sont particulièrement vulnérables à l'ambivalence au sein de leur configuration familiale (Connidis \& McMullin, 2002; Willson et al., 2006). Prises entre deux feux, elles hésitent entre préserver leur autonomie avec un fort risque de sentiment de culpabilité ou de répondre aux sollicitations normatives avec le risque tout aussi grand de ressentir de la frustration (Connidis \& McMullin, 2002).

Afin de gérer au mieux l'ambivalence, les femmes sont appelées à négocier les solutions les plus adéquates avec tous les membres de leur configuration familiale. Cette négociation n'est possible que si elles disposent d'un certain nombre de ressources (Connidis, 2015; Connidis \& McMullin, 2002). Si elles ont un partenaire qui a un revenu confortable, elles peuvent réduire ou renoncer à leur activité professionnelle pour remplir les rôles qui leur sont socialement assignés. Elles peuvent même, si elles en ont les moyens économiques, trouver des substituts en employant une tierce personne pour remplir une partie de leurs rôles (Connidis, 2015). Dans les situations où il $\mathrm{y}$ a peu de ressources financières, elles doivent maintenir leur temps de travail tout en assurant à côté les tâches domestiques et de soins avec un risque certain de surcharge de travail (Connidis $\&$ McMullin, 2002). Cependant, quelle que soit l'option adoptée, elle implique des arrangements et des concessions qui peuvent donner lieu à des tensions au sein de la configuration familiale (Connidis \& McMullin, 2002). Frustrations, fatigue, tensions et ambivalence pourraient à la longue éroder le capital social de type chaîne généré au sein de ce type de configurations familiales. Le thème des conflits et de l'ambivalence sera traité de manière plus approfondie dans la prochaine section car ils résultent, tout comme le capital social, des liens d'interdépendance qui sous-tendent les configurations familiales.

Sur la base de la littérature en gérontologie sociale, nous avons mis en évidence dans cette sous-section que la présence d'un conjoint ou d'enfants a une influence sur l'organisation des liens au sein de la famille. De même, la présence de femmes dans la famille a un impact sur les liens et les échanges familiaux en raison de la plus grande prégnance des normes sociales qui les désignent comme responsables de la cohésion familiale. En nous appuyant sur ces différents constats, nous faisons l'hypothèse que 


\section{Problématique et hypothèses}

la composition des configurations familiales - c'est-à-dire l'identité des membres familiaux que les individus âgés désignent comme significatifs influence les propriétés structurelles qui les sous-tendent, en favorisant ou non le flux des échanges qui s'y produisent et, par conséquent, le capital social auquel ont accès les personnes âgées dans leur configuration familiale. Jusqu'à ce jour, la plupart des chercheurs en gérontologie sociale ne se sont jamais questionnés sur les propriétés structurelles du soutien familial, se contentant du postulat que la famille offre, coût que coût, un soutien fiable et inconditionnel, la famille étant définie «a priori » sous sa forme «nucléaire ». Or, cette perception du soutien familial est trop restrictive car la façon dont s'organise le soutien familial est différente selon les familles et les membres qui les constituent. Ceci dit, la composition des configurations familiales n'est pas le seul facteur susceptible d'influencer l'émergence du capital social, familial, dans la vieillesse. Le profil socio-démographique des individus âgés (ego) peut aussi constituer un facteur important.

\subsubsection{Le profil socio-démographique d'ego}

Le développement du capital social dans les configurations familiales dépend non seulement de leur composition mais aussi du profil sociodémographique d'ego. Le profil socio-démographique d'un individu renvoie aux statuts et aux rôles spécifiques qu'il joue au sein de sa famille et, plus généralement, au sein de la société. Ces rôles et statuts sont généralement associés à des ressources et à des compétences particulières qui favorisent, ou non, le développement des relations sociales et familiales (Krause, 2006). On peut donc s'interroger sur l'impact du profil sociodémographique des individus âgés sur la production du capital social au sein de leur configuration familiale. En nous appuyant sur la littérature gérontologique, nous mettons en évidence dans cette sous-section l'influence du genre, du statut socio-économique, de l'âge et de l'état de santé de l'individu âgé (ego) sur ses relations familiales et l'organisation du soutien au sein de sa famille. Sur la base de ces observations, nous discutons ensuite de l'impact probable de ces différents facteurs sur la production du capital social au sein des configurations familiales dans la vieillesse.

Concernant le genre, la socialisation, les rôles exercés dans la société et le parcours de vie différencié font que les hommes et les femmes ont 
une « sensibilité relationnelle » différente, influençant la façon dont ils entretiennent leurs relations non seulement sociales mais aussi familiales. Les femmes ont une plus grande propension que les hommes à développer des relations étroites, affectives, soutenantes avec des personnes variées qu'elles puisent aussi bien dans leur réservoir de parenté qu'en dehors (Antonucci et al., 1998, 2007; Carr \& Moorman, 2011; Kawachi $\&$ Berkman, 2001) alors que les hommes sont plus soumis au processus de sélectivité socio-émotionnelle lorsqu'ils vieillissent (Cornwell, 2011; Shaw et al., 2007). Ce processus se manifeste par une plus forte propension des hommes âgés à ne considérer que les membres primaires de leur famille, c'est-à-dire l'épouse et les enfants, comme importants, ceux-ci étant généralement perçus comme les seules sources de soutien émotionnel dont ils ont besoin (Cornwell, 2011; Shaw et al., 2007). De plus, le veuvage, qui touche davantage les femmes que les hommes, accentue encore cette tendance. En effet, les veufs, en majorité des femmes, s'investissent dans une diversité de relations alors que les mariés (ou en couple), surtout des hommes, restent fortement dépendants de leur conjoint et de leur réseau familial commun (enfants et petits-enfants) (Antonucci et al., 2007; Carr \& Moorman, 2011; Ha, 2008; Cornwell, 2011). Pour ces raisons, le réseau personnel des femmes âgées est généralement plus large et plus diversifié, avec des liens et des échanges moins denses que celui des hommes (Cornwell et al., 2009). Les femmes sont donc plus enclines que les hommes à jouer un rôle actif dans leur réseau en reliant entre eux les divers membres qui, n'appartenant pas aux mêmes cercles de sociabilité (famille et amis), ne sont pas ou peu connectés (Cornwell, 2011). Au vu de ce constat, nous faisons l'hypothèse que les femmes âgées tendent à insérer au sein de leur famille significative une plus grande variété de membres, avec lesquels elles développent un lien affectif, engagé et soutenant. Compte tenu de leur diversité, ces membres ne sont pas forcément connectés entre eux, laissant aux femmes âgées le privilège de jouer un rôle central, celui d'intermédiaire, au sein de leur configuration familiale. Les femmes âgées ont donc une plus grande probabilité que les hommes âgés de développer au sein de leur configuration familiale un capital social de type pont alors que les hommes, plus centrés sur les membres primaires de leur famille, ont plus accès au capital social de type chaîne.

Toutefois, cette hypothèse ne tient pas compte de la baisse des ressources - socio-économiques et santé - à laquelle les femmes doivent faire face dans la vieillesse et qui pourrait altérer le capital social de type pont 


\section{Problématique et hypothèses}

auquel elles ont accès. Avec le veuvage, les femmes âgées disposent en effet d'un moindre revenu, ce qui peut les pénaliser dans le maintien de leurs diverses relations familiales. De même, elles ont un risque plus grand que les hommes d'être atteintes dans leur santé fonctionnelle, ce qui affecte encore davantage leur capacité à maintenir la diversité de leurs relations familiales (Barer, 1994; Cornwell, 2011). Sans ressources économiques et sans une bonne santé, les femmes âgées ne peuvent plus garantir la réciprocité des échanges (dyadiques) avec l'ensemble des membres qui forment leur configuration familiale, ce qui rend les liens familiaux de plus en plus fragiles, ceux-ci n'étant pas soutenus par des normes de réciprocité « généralisée » (Antonucci et al., 2007; Cornwell, 2009a; Offer, 2012; Pinquart \& Sörensen, 2000). Parallèlement, certains membres de la famille peuvent se mobiliser autour du parent âgé, atteint dans sa santé, ce qui contribue à resserrer et à densifier les liens au sein de la configuration familiale (Cornwell, 2009a, 2011). Cette mobilisation est d'autant plus prononcée que le parent âgé est une femme car celle-ci reçoit plus de soutien de ses proches, notamment de ses enfants, que l'homme âgé, même si celui-ci est en plus mauvaise santé (Silverstein et al., 2002, 2006). En raison de leurs moindres ressources, les femmes âgées peuvent perdre leur capital social de type pont mais bénéficier en contrepartie d'un capital social de type chaîne. Il est aussi probable que la baisse importante de ressources des femmes âgées, déjà précarisées, ne leur permette plus de participer aux échanges au sein de leur réseau familial (Offer, 2012) et, ce faisant, affecte le volume de leur capital social, quelle qu'en soit la forme, au point même que ce dernier disparaisse. Ce risque est d'autant plus grand pour celles qui ne disposent que d'un faible réservoir de parenté. Avoir des ressources - et qui, plus est, socio-économiques - est donc primordial pour avoir accès au capital social.

En effet, les chercheurs s'accordent sur le fait que les individus âgés qui ont un statut socio-économique élevé ont un réseau personnel plus large et plus diversifié que ceux qui ont un statut socio-économique bas (Ajrouch et al., 2005; Cornwell et al., 2008; Fiori et al., 2006; Litwin, 2001). Ils peuvent, grâce à leurs compétences relationnelles, initier et maintenir une variété de relations au sein de leur réseau personnel (Ajrouch et al., 2005; Krause, 2006; Broese Van Groenou \& Van Tilburg, 2003). Moins axé sur la sphère familiale, leur réseau personnel se caractérise dès lors par des liens et des échanges de faible densité, créant des «trous » entre les différents membres. Diversité et faible densité permettent aux individus âgés de 
bénéficier d'une variété de ressources mais elles leur offrent aussi la possibilité d'occuper une position d'intermédiaire et, ce faisant, de superviser les échanges au sein de leur réseau personnel (Burt, 2002; Cornwell et al., 2009; Cornwell, 2009b). Quant aux individus âgés ayant un bas niveau socio-économique, ils affichent des réseaux personnels de petite taille, essentiellement centrés sur la famille, se caractérisant par une forte densité des échanges (Ajrouch et al., 2005; Cornwell, 2009b; Fiori et al., 2006; Litwin, 2001). Le besoin plus grand de soutien des individus âgés des milieux populaires, qui doivent faire face à davantage de risques comme des problèmes de santé ou des difficultés socio-économiques que les individus socialement avantagés, explique cette centration sur la famille (Krause, 2006; Pinquart \& Sörensen, 2000). En somme, ils n'ont pas d'autres alternatives que de dépendre de l'aide familiale, notamment de celle de leurs enfants (Antonucci, 2001; Krause, 1997b). Partant du principe que les configurations familiales ressemblent dans leur structure aux réseaux personnels, nous faisons l'hypothèse que les aînés issus des milieux populaires ont davantage accès au capital social de type chaîne, leurs configurations familiales reposant principalement, à l'instar de leur réseau personnel, sur des membres proches de la famille comme le conjoint et les enfants alors que les aînés provenant de milieux aisés ont davantage accès au capital social de type pont car leurs configurations familiales sont à l'image de leurs réseaux personnels, à savoir plus larges, plus diversifiées et moins denses que celles des milieux populaires.

Cependant, cette hypothèse néglige les effets de l'évolution des réseaux personnels, y compris familiaux, dans la vieillesse. Celle-ci pourrait avoir un impact sur la production du capital social au sein des configurations familiales des individus âgés ayant un statut socio-économique élevé. Selon la littérature scientifique, la contraction du réseau personnel avec l'âge et le déclin de la santé contribue à homogénéiser la structure du réseau personnel, celui-ci s'axant davantage sur les membres de la famille, quel que soit le statut socio-économique des individus âgés (Antonucci, 2001; Pinquart \& Sörensen, 2000; Van Tilburg, 1998). De plus, les individus âgés ayant un statut socio-économique élevé, majoritairement des hommes, ont une plus forte probabilité d'avoir un conjoint (Dykstra \& Hagestad, 2007; Pudrovska et al., 2006; Robles \& Kiecolt-Glaser, 2003) et une plus forte propension, avec l'âge avançant, de sélectionner les relations qui leur sont le plus gratifiantes, généralement le partenaire et les enfants (Carstensen, 
1991, 1992; Shaw et al., 2007). Or, la présence d'un partenaire et des enfants dans la famille contribue à y densifier les liens (Cornwell, 2011). Il est donc probable qu'avec l'avancée en âge, les individus d'un niveau socioéconomique élevé perdent leur capital social de type pont au profit d'un capital social de type chaîne.

Par ailleurs, la baisse des ressources (santé, économiques, etc.) ainsi que le besoin grandissant de soutien peuvent prétériter le volume du capital social produit au sein des configurations familiales dans les milieux populaires. Certaines études montrent que la solidarité dans les réseaux personnels est de mise lorsque les ressources, bien que peu nombreuses, existent. Cependant, quand les ressources manquent et que la demande d'aide se pérennise, la solidarité s'épuise (Antonucci, 2001; Krause, 1997b; Offer, 2012). Ceci est d'autant plus probable que les membres du réseau personnel auquel l'individu appartient présentent généralement les mêmes caractéristiques socio-économiques que lui (Bourdieu, 1980, 1986; Portes, 1998). A long terme, la précarité au sein du réseau personnel, la demande d'aide continuelle et le non retour des ressources octroyées affectent les relations au sein du réseau entraînant tensions, conflits et ambivalence, jusqu'à, parfois, la rupture définitive des liens, même familiaux (Krause, 1997b, 2006; Offer, 2012; Pinquart \& Sörensen, 2000). Nous faisons l'hypothèse qu'il en est de même dans les configurations familiales. Une précarité sévère peut compromettre le développement du capital social au sein des configurations familiales au point que certains individus âgés ne peuvent pas, ou plus, compter sur l'aide familiale.

Lâge et la santé de l'individu âgé (ego) sont aussi susceptibles d'influencer l'organisation des liens et des échanges au sein des configurations familiales. La littérature scientifique met en exergue l'importance de la santé en tant que ressource indispensable au maintien des liens dans les réseaux personnels et familiaux (Antonucci et al., 2007; Broese Van Groenou \& Van Tilburg, 2007; Carr \& Moorman, 2011; Cornwell, 2009a). Avec l'avancée en âge et le déclin de la santé qui lui est associé, les individus âgés perdent progressivement les capacités physiques et cognitives qui leur permettent de rester actifs dans les échanges et d'y maintenir la réciprocité directe, «équilibrée » (Antonucci et al., 2007; Broese Van Groenou \& Van Tilburg, 2007; Carr \& Moorman, 2011; Cornwell, 2009a). Cette norme d'échange est particulièrement en vigueur non seulement dans les relations "périphériques » mais aussi dans les liens personnalisés qui reposent sur des affinités et des intérêts partagés (Stevens \& Van Tilburg, 
2011 ; Suanet et al., 2013). Ne pouvant plus assurer la réciprocité «équilibrée » dans leurs échanges, certains se retirent et se désengagent de ce type de relations, jugées trop exigeantes (Antonucci et al., 2007; Broese Van Groenou \& Van Tilburg, 2007; Offer, 2012). Par ailleurs, les individus très âgés ou atteints dans leur santé ne parviennent plus à jouer le rôle d'intermédiaire au sein de leur réseau personnel, ni même - pour les plus atteints cognitivement - à y détecter les «trous », ceux-là même qui leur permettent de maintenir leur capital social de type pont et d'entretenir leur autonomie (Cornwell, 2009a, 2009b). De plus, sentant la fin proche, les très âgés activent le processus de sélectivité socio-émotionnelle en choisissant les membres de leur réseau personnel qui leur apportent le plus de satisfaction émotionnelle, tout en se désengageant des relations qu'ils jugent le moins gratifiantes (Carstensen, 1991, 1992; Shaw et al., 2007). Ils sélectionnent un nombre restreint de membres avec lesquels ils ont entretenus des liens forts d'interdépendance - comme le partenaire, les enfants, parfois, des membres de la fratrie - et desquels ils attendent un soutien inconditionnel, retour d'un investissement passé selon le principe du « support bank » (Antonucci, 2001; Antonucci et al., 2007). Parallèlement, les membres primaires de la famille, c'est-à-dire le conjoint et les enfants, se mobilisent afin d'apporter au parent très âgé ou atteint dans sa santé l'aide dont il a besoin (Cornwell, 2009b, 2009a; Silverstein et al., 2002, 2006; Van Tilburg, 1998). Cette mobilisation implique que les membres interagissent et coordonnent leurs efforts pour offrir le soutien nécessaire au parent âgé. Ce processus contribue donc à densifier les liens de soutien au sein de la configuration familiale (Broese Van Groenou \& Van Tilburg, 2007; Cornwell, 2009b, 2009a, 2011). Au vu de ces observations, nous faisons l'hypothèse que les individus très âgés ou atteints dans leur santé ont davantage accès, au sein de leur configuration familiale, à un capital social de type chaîne que de type pont alors que les plus jeunes ou en bonne santé parviennent davantage, ayant les capacités nécessaires, à maintenir leur capital social de type pont, synonyme d'autonomie.

Cette hypothèse postule «a priori » que la configuration familiale des plus âgés ou de ceux atteints dans leur santé comporte un partenaire ou des enfants, ceux-ci étant considérés dans la littérature comme les seuls en mesure de se mobiliser et d'offrir un solide soutien dans la grande vieillesse. Or, les individus très âgés ou atteints dans leur santé peuvent avoir des définitions variées de leur famille significative qui n'incluent pas forcément un partenaire ou des enfants. Il se peut en effet qu'ils aient maintenu des 


\section{Problématique et hypothèses}

liens affectifs profonds et engagés avec une diversité de membres significatifs au sein de leur configuration familiale et qu'ils accèdent ainsi, même à des âges très avancés ou sévèrement atteints dans leur santé, à un capital social de type pont. Toutefois, il est probable que le soutien proposé au sein de ce type de configurations soit plus fragile que celui généré dans les configurations composées d'un partenaire ou d'enfants. Outre le fait que certains des membres dans ce type de configurations soient aussi très âgés ou en mauvaise santé, ils ne sont pas tenus de se mobiliser et d'assurer des soins de longue durée, leurs liens ne reposant pas sur des normes de réciprocité "généralisée ». Par conséquent, ils tendent à se désengager plus facilement face à l'ampleur du soutien requis, par exemple, en situation de « caregiving ( (Cornwell, 2009a; Dykstra \& Hagestad, 2007). Nous faisons donc l'hypothèse alternative que les individus très âgés ou atteints dans leur santé ont un risque plus marqué que ceux qui sont jeunes et en bonne santé de n'avoir accès à aucun capital social dans leur configuration familiale, et cela d'autant plus si celle-ci ne comporte ni partenaire, ni enfants.

Sur la base de la littérature existante, nous avons mis en exergue l'influence du profil socio-démographique de l'individu âgé (ego) sur l'organisation des liens et des échanges au sein de sa configuration familiale. Partant de ces observations, nous avons fait l'hypothèse que le genre, le statut socio-économique, l'âge et la santé d'ego influencent le type de capital produit au sein de la configuration familiale. Nous avons souligné l'importance des ressources de l'individu âgé qui dépendent étroitement de sa position dans la société. Dès lors, nous avons postulé que les ainés qui ont des compétences relationnelles (femmes), des ressources socio-économiques (statut socio-économique élevé), des capacités physiques et cognitives ( «jeunes vieux» et en bonne santé) développent davantage un capital social de type pont que ceux qui ont peu de compétences relationnelles (hommes), peu de ressources socio-économiques (statut socio-économique bas), de faibles capacités physiques et cognitives (« très vieux » et en mauvaise santé). Ces derniers ont probablement davantage accès à un type de capital social de type chaîne. Nous avons aussi fait l'hypothèse que le manque sévère de ressources économiques, une mauvaise santé et la pauvreté du réservoir de parenté réduisent de manière drastique le volume du capital social produit au sein des configurations familiales. Grâce à l'approche configurationnelle, nous pouvons tester l'ensemble de ces hypothèses et évaluer la "potentialité» des individus âgés au sein de leur configuration familiale. Autrement dit, nous pouvons examiner 
la capacité des aînés à influer l'organisation des liens de soutien au sein de leur réseau familial et à y développer du capital social en considérant les ressources dont ils disposent.

Dans cette section, nous avons défini le concept de capital social, décrit son mécanisme de production, présenté ses différents types (chaîne ou pont) et les facteurs susceptibles d'expliquer leur émergence. Nous avons montré que la notion de capital social que propose la perspective configurationnelle est pertinente pour approcher le soutien familial dans la vieillesse; elle permet de l'aborder d'une façon plus large que ne le fait habituellement la gérontologie sociale. Dans la plupart des études gérontologiques, le soutien familial est d'abord perçu comme un «montant» de support que l'individu âgé échange soit avec un membre spécifique de la famille, soit avec l'ensemble du groupe « famille». Or, pour saisir toute la complexité du soutien familial dans la vieillesse, il est nécessaire de l'appréhender dans une perspective plus large, qui tient compte de l'ensemble des membres de la famille qui constituent le réseau familial et des normes de réciprocité qui structurent leurs échanges. En gérontologie sociale, le soutien familial n'est jamais considéré dans une perspective configurationnelle et conceptualisé en termes de « capital social». Pourtant, le simple montant du support échangé ne suffit pas à évaluer le soutien familial. Il faut tenir compte, pour le mesurer, de l'organisation de l'ensemble des échanges de soutien au sein de la configuration familiale. Certaines propriétés structurelles spécifiques, telles que la densité des liens de soutien, la réciprocité des échanges, l'engagement ou encore la centralité de l'individu âgé (ego), décrivent la façon dont les liens de soutien s'organisent au sein des configurations familiales et l'on peut, à partir de ces informations, déterminer le type et le volume du capital social qui y est produit. Alors que bon nombre de chercheurs en gérontologie sociale conçoivent le soutien familial comme inconditionnel et stable, la notion de capital social défie cette idée préconçue. Nous faisons en effet l'hypothèse que le capital social généré au sein des configurations familiales dans la vieillesse varie non seulement en fonction de l'identité des membres qui composent les différentes configurations familiales mais aussi en fonction du profil socio-démographique des aînés (ego). Les analyses permettront de tester l'ensemble de ces hypothèses et il est probable que les résultats obtenus donneront une image plus complexe du soutien familial dans la vieillesse que ne l'a fait jusqu'à présent la gérontologie sociale. 


\subsection{Les conflits et l'ambivalence structurelle}

Le capital social n'est pas le seul «produit» des liens d'interdépendance familiale, les tensions et les conflits n'y sont pas rares. Cette thématique a longtemps été négligée en gérontologie sociale; celle-ci a en effet tardé à concevoir le conflit comme faisant partie des dynamiques familiales, sans doute en raison de la forte prégnance du modèle théorique de la solidarité intergénérationnelle dans la recherche en gérontologie sociale (Bengtson \& Roberts, 1991; Roberts \& Bengtson, 1990). Reposant essentiellement sur le soutien et les diverses dimensions qui le sous-tendent, le modèle de la solidarité intergénérationnelle considère le conflit comme une dimension à part, davantage signe d'un dysfonctionnement qu'un trait intrinsèque des relations familiales. Critiquant ce modèle pour sa «non prise en compte » du conflit comme dimension à part entière des relations intergénérationnelles (Connidis, 2012, 2015; Connidis \& McMullin, 2002; Lüscher, 2000; Marshall \& Mueller, 2003), certains auteurs, comme Kurt Lüscher, ont proposé le concept d'ambivalence qui perçoit aussi bien le soutien que le conflit comme parties intégrantes des relations intergénérationnelles (Lüscher, 2000; Lüscher \& Hoff, 2013; Lüscher \& Pillemer, 1998). Ce concept permet de mieux saisir toutes les contradictions qui sous-tendent les liens «parents-enfants ». Depuis lors, l'ambivalence est souvent évoquée en gérontologie sociale pour étudier les relations interpersonnelles - majoritairement intergénérationnelles et parfois conjugales - mais elle reste cantonnée aux relations dyadiques. De telles études négligent en effet le contexte relationnel dans lequel s'insèrent ces différentes dyades (Connidis, 2012, 2015; Hillcoat-Nallétamby \& Phillips, 2011). Nous proposons dans cette section de sortir du carcan dyadique et d'explorer l'ambivalence dans l'ensemble des liens d'interdépendance qui constituent les configurations familiales. Sur la base de la littérature existante, nous nous interrogeons sur les mécanismes structurels qui favorisent l'ambivalence non seulement au niveau dyadique mais aussi au niveau configurationnel. Ensuite, nous présentons la typologie des quatre modes de conflit et de support qui explore l'ambivalence structurelle au sein des configurations familiales (Widmer, 2016). Finalement, nous nous questionnons quant aux conditions d'émergence de ces différents modes de conflit et de support dans les configurations familiales au cours de la vieillesse. 


\subsubsection{L'ambivalence intergénérationnelle}

Les mécanismes relationnels, structurels, qui favorisent l'émergence de l'ambivalence au sein des relations dyadiques font l'objet de cette soussection. L'ambivalence structurelle ne concerne pas toutes les relations interpersonnelles mais elle est intimement liée aux liens d'interdépendance, comme ceux qui relient les membres de la configuration familiale. Les relations intergénérationnelles représentent un bon exemple car cellesci sont perçues dans la littérature gérontologique comme particulièrement révélatrices de l'ambivalence structurelle dans la vieillesse (Connidis, 2015; Connidis \& McMullin, 2002; Lüscher, 2000; Lüscher \& Pillemer, 1998). En effet, régulées tout au long du parcours de vie par les normes de réciprocité "généralisée », les relations intergénérationnelles présentent, généralement, un fort lien d'interdépendance. En nous basant sur la littérature scientifique, nous montrons comment l'interdépendance, qui caractérise les relations intergénérationnelles, donne lieu à de l'ambivalence. Les mécanismes sous-jacents y sont expliqués car ils mettent en lumière le processus qui génère l'ambivalence structurelle au niveau plus large des configurations familiales.

Dans la littérature, l'ambivalence psychologique - c'est-à-dire la présence simultanée de sentiments ou d'émotions opposés - vis-à-vis d'une personne, d'une relation ou d'une situation n'est pas liée au hasard; elle s'inscrit dans le cadre de relations interpersonnelles particulières, tissées au fil du temps et affectivement proches, se caractérisant par une forte interdépendance affective et pratique (Carr \& Moorman, 2011; Fingerman et al., 2004; Willson et al., 2006). C'est particulièrement le cas des relations intergénérationnelles, mais elle peut aussi être ressentie vis-à-vis d'autres types de relations interpersonnelles ayant un lien fort d'interdépendance affective et pratique comme les relations conjugales ou même parfois certaines relations fraternelles, voire certaines amitiés (Connidis, 2012, 2015; Fingerman et al., 2004). Loin d'être passagers, ces sentiments mixtes que ressent l'individu expriment bel et bien l'ambivalence structurelle, c'est-à-dire les contradictions profondes, structurelles, qui caractérisent toutes relations affectives reposant sur des liens forts d'interdépendance (Connidis, 2015; Connidis \& McMullin, 2002; Lüscher, 2000; Lüscher \& Pillemer, 1998). L'une de ces contradictions est celle qui est à la base même des liens d'interdépendance à savoir, d'un côté, le besoin de l'autre et de ses ressources - créant nécessairement une dépendance à l'autre - et, de l'autre 


\section{Problématique et hypothèses}

côté, le besoin d'autonomie individuelle (Lüscher \& Pillemer, 1998; Masotti, 2016; Rappoport \& Lowenstein, 2007). Ces deux besoins, tous deux essentiels pour la construction et le renforcement de l'identité, co-existent dans chaque individu tout au long du parcours de vie (Lüscher \& Pillemer, 1998). Ces deux besoins s'opposent, et ce faisant, génèrent de l'ambivalence structurelle dans les relations. Celle-ci se manifeste par du soutien mais aussi par des tensions lors des échanges interpersonnels (Lüscher \& Pillemer, 1998). La satisfaction de ces deux besoins, contradictoires, est possible lorsque les échanges de ressources entre les protagonistes sont équilibrés. Tant que les deux interlocuteurs sont actifs dans les échanges - en donnant et en recevant - ils maintiennent tous deux leur autonomie tout en bénéficiant des ressources dont ils ont besoin. Si l'on considère les relations intergénérationnelles dans l'ensemble du parcours de vie, les échanges de ressources entre parents et enfants sont globalement équilibrés, même s'ils sont indirects et qu'ils s'inscrivent dans le temps. Dans de telles situations, l'ambivalence demeure latente même à des âges avancés (Connidis, 2015; Willson et al., 2006).

L'ambivalence structurelle devient manifeste dans les relations lors de certaines transitions de vie, telles que le veuvage, le divorce, la retraite, une baisse de revenu ou encore le déclin de la santé, qui, parce qu'elles s'associent à un changement de statut des individus et souvent à une diminution de leurs ressources, les vulnérabilisent, nécessitant un soutien accru de la part des autres membres de la famille pour pouvoir s'adapter au changements qui marquent leur nouvelle étape de vie (Antonucci, 2001 ; Connidis, 2015; Lüscher \& Pillemer, 1998; Willson et al., 2006). Cependant, en tel soutien implique une dépendance à l'autre, contrecarrant le maintien de l'autonomie si valorisée au sein des sociétés occidentales et qui, plus est, dans la population âgée (Cornwell, 2011; Masotti, 2016). Préserver son autonomie est en effet un objectif central pour les aînés, leur garantissant une image positive d'eux-mêmes et le maintien de leur identité face au processus de fragilisation auquel ils sont inéluctablement confrontés (Cornwell, 2009a, 2011; Krause, 1997b ; Pinquart \& Sörensen, 2000 ; Pyke \& Bengtson, 1996). Avoir des ressources, telles qu'un revenu décent, une bonne santé et un partenaire, facilite le maintien de l'autonomie jusqu'aux dernières étapes de la vie. Être marié, par exemple, assure à l'individu âgé un soutien quotidien qui le préserve de l'aide familiale à plus large échelle, notamment de celle de ses enfants (Willson et al., 2006). La perte de ces diverses ressources compromet sévèrement le maintien de l'autonomie, et 
cela d'autant plus lorsque cette perte est plurielle et concomitante comme chez les femmes, le veuvage ou le divorce s'associant souvent à une baisse importante de leur revenu (Willson et al., 2006).

Selon certaines transitions de vie, la perte de ressources peut être particulièrement importante, affectant à long terme la capacité des individus à soutenir l'échange au sein de la relation intergénérationnelle, ce qui a des répercussions négatives non seulement au niveau individuel (perte de l'estime de soi ou de l'identité) mais aussi au niveau relationnel. En effet, le manque de ressources empêche le parent âgé dans le besoin de jouer un rôle actif dans les échanges et d'occuper une position comparable à celle de son interlocuteur (Offer, 2012). En plus, l'incapacité à la réciprocité et le sentiment de redevabilité le placent dans une position d'infériorité face à ses enfants dont il dépend fortement, leur donnant ainsi un certain pouvoir. Cette position est d'autant plus difficile à accepter pour le parent âgé qu' elle résulte d'un changement de rôle et de pouvoir au sein des échanges intergénérationnels faisant suite à une diminution marquante de ses ressources (Suitor et al., 2011; Willson et al., 2006). Affirmer sa volonté et ses désirs lors de prises de décision ou de négociations devient de plus en plus difficile dans une relation marquée par la dépendance et le déséquilibre. Cette situation place donc le parent âgé dans une position de grande vulnérabilité face au bon vouloir de ses enfants, et celle-ci est d'autant plus prononcée si ce dernier a peu d'alternatives de soutien en dehors du contexte familial (Connidis, 2015; Masotti, 2016; Willson et al., 2006). Dans une telle situation, l'aide reçue peut s'accompagner de pressions ou de tentatives de contrôle de la part des enfants à l'égard du parent âgé (Lüscher \& Pillemer, 1998; Widmer, 2016). Celles-ci peuvent prendre la forme de critiques ou de reproches par rapport au comportement de santé ou à la manière de gérer l'argent, par exemple. Vécue comme un piège dont il est difficile de sortir, cette dépendance à l'aide familiale peut générer des sentiments de frustration et même parfois de la violence à l'égard de ceux dont on dépend et dont on se sent pourtant proche (Connidis \& McMullin, 2002; Lüscher \& Pillemer, 1998; Widmer, 2016; Willson et al., 2006).

La situation de dépendance n'affecte pas seulement l'autonomie du parent âgé dans le besoin, mais elle touche aussi celle du ou des enfants qui le soutiennent (Willson et al., 2006). En effet, ces transitions de vie difficiles exigent de la part des enfants une présence, un grand soutien, un certain sacrifice. Elles titillent les normes de réciprocité " généralisée » et suscitent chez les enfants un fort sentiment de redevabilité à l'égard du 


\section{Problématique et hypothèses}

parent dans le besoin (Lüscher \& Pillemer, 1998). Ce sentiment ne peut être que difficilement ignorer, au prix d'un fort sentiment de culpabilité (Connidis, 2015; Hillcoat-Nallétamby \& Phillips, 2011; Lüscher \& Pillemer, 1998; Rappoport \& Lowenstein, 2007). Contraignant, il les oblige à revoir leurs priorités, à remettre à plus tard leurs projets personnels, à restreindre en somme leur propre liberté, afin de permettre au parent âgé dans le besoin de s'adapter à sa nouvelle situation, et cela sans attendre de sa part un quelconque retour, du moins dans l'immédiat. Aider et soutenir le parent âgé octroient aux enfants prestataires une position privilégiée dans l'échange, leur donnant un certain pouvoir, mais l'aide et le soutien s'accompagnent aussi de fortes contraintes pouvant créer de part et d'autre de la relation intergénérationnelle des frustrations, des tensions et du conflit (Lüscher \& Pillemer, 1998; Willson et al., 2006). Soutenir son parent s'associe à des sentiments positifs dans les échanges intergénérationnels dits « équilibrés » alors que, quand ils sont déséquilibrés par le besoin accru et constant du parent âgé, l'ambivalence intergénérationnelle devient alors manifeste. C'est cette situation de déséquilibre qui, dépassant largement les situations d'aide « habituelles », génère une forte ambivalence (Lüscher \& Pillemer, 1998; Willson et al., 2006).

Dans la littérature scientifique, le déclin de la santé du parent âgé est, avec la précarité économique de ce dernier, l'une des situations chroniques les plus fréquentes dans la vieillesse (Krause, 1997a, 2006). S'enlisant dans le temps, le déclin sévère de la santé, qui nécessite une aide familiale constante et continue (caregiving), tend à épuiser les ressources des enfants « caregivers » et est par conséquent créateur de stress, de conflits et d'une forte ambivalence (Suitor et al., 2011; Willson et al., 2006). Même si le soutien émotionnel du parent âgé demeure une "monnaie d'échange » jusqu'à un âge avancé et même lorsque le corps est fortement fragilisé, l'échange n'en demeure pas moins déséquilibré si l'on tient compte de l'ensemble des ressources investies par les enfants adultes pour s'occuper de leurs parents en mauvaise santé. En effet, le caregiving - en termes de transport, de courses, de ménage, de compagnonnage, de gestion financière ou de démarche administrative - demande l'investissement de nombreuses ressources telles que le temps, l'affection, l'argent, l'énergie ainsi qu'une bonne santé physique et mentale (Gaugler \& Holmes, 2003 ; Pyke \& Bengtson, 1996; Willson et al., 2006). Bien que l'acte de soigner suscite des émotions positives chez le donneur de soins - ou plutôt chez la donneuse de soins puisqu'il s'agit surtout des « filles » (Silverstein et al., 2006) - 
comme l'affection et le sentiment d'être utile, il s'associe néanmoins à des tâches rébarbatives et fatiguantes, laissant peu de temps à la pratique d'activités extérieures, voire même à un emploi à plein temps (Lüscher \& Pillemer, 1998). Et, parce qu'il éloigne la donneuse de soins de la sphère publique, le caregiving peut être vécu par cette dernière comme oppressant, contribuant même à son isolement social (Lüscher \& Pillemer, 1998). Par conséquent, le caregiving s'associe souvent chez les caregivers à du stress, de la fatigue, de la dépression, une perte d'intimité, de la culpabilité et à la sensation d'être pris au piège (Gaugler \& Holmes, 2003; Suitor et al., 2011), sentiments qui perdurent même après l'institutionnalisation du parent âgé (Gaugler \& Holmes, 2003).

Même si les filles adultes peuvent se sentir satisfaites de remplir leur « devoir », répondant ainsi aux normes de la réciprocité " généralisée », et même si les parents âgés sont soulagés de récupérer leur investissement (« support bank »), le caregiving représente un tel déséquilibre dans les échanges qu'il entraîne de part et d'autre de la relation un profond malaise (Gaugler \& Holmes, 2003; Lüscher \& Pillemer, 1998; Willson et al., 2006). D'une part, les enfants adultes peuvent se sentir floués, conscients que les efforts et les ressources investies seront perdus à jamais et, d'autre part, les parents âgés culpabilisent, conscients de ne plus pouvoir soutenir l'échange et de devenir une charge de plus en plus lourde pour leurs enfants (Lüscher \& Pillemer, 1998; Offer, 2012; Willson et al., 2006). Malgré la fatigue et le stress que le "caregiving " génère, les enfants adultes persévèrent dans cette tâche car renoncer à s'occuper du parent âgé dans le besoin reviendrait à transgresser la norme de réciprocité " généralisée » et, ce faisant, à " trahir» le lien affectif qui la sous-tend (Lüscher $\&$ Pillemer, 1998). Le «caregiving » révèle clairement l'ambivalence intergénérationnelle qui sous-tend l'interdépendance entre parents âgés et enfants adultes dans la vieillesse. Il y a non seulement une forte solidarité, si l'on reprend les dimensions du modèle de Vern Bengtson telles que la proximité géographique, les contacts fréquents et l'aide intense, mais aussi du stress, des frustrations et des tensions de part et d'autre de la relation intergénérationnelle (Lüscher \& Pillemer, 1998; Willson et al., 2006).

Nous avons mis en exergue dans cette sous-section le lien entre l'ambivalence dite «structurelle » et l'interdépendance des relations intergénérationnelles dans la vieillesse. Nous avons souligné que l'ambivalence intergénérationnelle demeure latente si l'on considère l'ensemble du parcours de vie, les échanges entre parents et enfants étant relativement 


\section{Problématique et hypothèses}

équilibrés. Dans la vieillesse cependant, certaines transitions de vie, qui nécessitent un besoin accru de l'aide familiale, déséquilibrent les échanges et menacent l'autonomie de chacun générant de l'ambivalence structurelle. Autrement dit, solidarité et conflit, aide et contrôle, affection et frustration sont loin d'être antinomiques; ils participent au contraire à la même réalité familiale, complexe et ambivalente. Le concept d'ambivalence intergénérationnelle, proposé par Kurt Lüscher, va plus loin que celui de la solidarité intergénérationnelle en tenant compte simultanément des aspects aussi bien positifs que négatifs des relations entre parents et enfants dans la vieillesse. Il permet d'envisager les relations intergénérationnelles non plus comme des liens homogènes et positifs mais plutôt comme des relations complexes et diverses. Ceci dit, le concept d'ambivalence a surtout été utilisé en gérontologie sociale pour étudier les relations d'aide dyadique, intergénérationnelle pour la plupart, et a été rarement considéré dans une perspective plus large, configurationnelle. Pourtant, les configurations familiales, construites sur des liens d'interdépendance, génèrent aussi de l'ambivalence «structurelle» (Widmer, 2016).

\subsubsection{L'ambivalence dans les configurations familiales}

En considérant la famille comme une configuration de liens d'interdépendance reliant entre eux divers membres significatifs, toute configuration familiale est, par sa structure, amenée à produire de l'ambivalence (Widmer, 2016). En considérant l'ensemble des liens d'interdépendance qui constituent la configuration familiale, l'approche configurationnelle permet d'observer l'impact d'une transition de vie non seulement sur les échanges dyadiques mais aussi sur l'ensemble des échanges qui se produisent au sein de la configuration familiale (Widmer, 1999a, 2006, 2016; Widmer et al., 2013). Cette approche postule en effet que le déséquilibre créé au sein des dyades les plus centrales - intergénérationnelles, notamment - est susceptible d'avoir des répercussions sur l'ensemble des liens d'interdépendance dans lesquels s'insèrent ces dyades (Widmer et al., 2004, 2006, 2009; Widmer, 2016). Dans cette section, nous scrutons les mécanismes structurels qui suscitent l'émergence de l'ambivalence structurelle dans l'ensemble de la configuration familiale. Nous examinons ensuite la marge de manœuvre dont disposent les membres de la configuration pour gérer l'ambivalence à laquelle ils sont confrontés. 
Dans l'approche configurationnelle, le besoin accru de soutien d'un individu a un effet non seulement sur les relations d'aide qu'il entretient directement avec les autres membres de son réseau familial (échanges dyadiques), mais aussi sur l'ensemble des échanges qui se produisent au sein de la configuration. Avec la perte de ses ressources, l'individu voit sa capacité à la réciprocité diminuer, le plaçant dans une position d'infériorité dans chaque échange direct qu'il entretient avec les membres de sa configuration familiale. Ses ressources diminuées, il ne peut pas, ou plus, maintenir un rôle central au sein de sa configuration familiale puisque le maintien d'un capital social de type pont est particulièrement exigeant en termes d'énergie et de santé (Cornwell, 2009b, 2009a; Widmer, 2016). Ce changement de rôle et de position au sein de sa configuration familiale le rend plus vulnérable aux pressions et au contrôle des autres membres, ce qui peut créer des tensions avec ces derniers lorsque l'aide lui est octroyée. Par ailleurs, la dépendance à l'aide familiale perturbe, si elle est conséquente, l'équilibre des échanges au sein de l'ensemble de la configuration familiale (Connidis, 2003, 2015; Hillcoat-Nallétamby \& Phillips, 2011; Widmer, 2016). La mobilisation de ressources pour aider le parent âgé pénalise inévitablement les autres membres de la configuration familiale qui ne peuvent pas en bénéficier, ce qui peut générer du stress chez ces derniers (Connidis, 2010, 2012). Des tensions apparaissent, dès lors, lorsque l'un des membres - une fille, par exemple - investit du temps, de l'affection, de l'argent et de l'énergie pour répondre aux demandes du parent âgé et que, ce faisant, elle « néglige» les autres membres de la configuration, comme son partenaire et ses enfants (Connidis \& McMullin, 2002; Lüscher \& Pillemer, 1998; Widmer, 2016; Willson et al., 2006). Dans ce cas de figure, le flux des ressources au sein de la configuration familiale est déséquilibré et peut être vécu par les membres comme inégalitaire, générant des jalousies, des conflits et de l'ambivalence, et cela d'autant plus si ce déséquilibre perdure dans le temps ou si les ressources sont limitées ou si les membres familiaux sont nombreux à dépendre du soutien familial comme dans les familles où il $\mathrm{y}$ a des enfants, des adultes malades ou non autonomes économiquement (Hillcoat-Nallétamby \& Phillips, 2011; Sillars, Canary, \& Tafoya, 2004; Sprey, 1999; Widmer \& Sapin, 2008).

De plus, chaque membre de la famille significative n'est pas seulement imbriqué dans une configuration de liens d'interdépendance avec ses normes exigeantes et contraignantes, mais il est aussi engagé dans divers rôles sociaux (famille, amis et travail). A ce titre, chaque membre 


\section{Problématique et hypothèses}

de la configuration est confronté simultanément à diverses pressions normatives, des attentes contradictoires et difficilement conciliables, ce qui crée encore davantage d'ambivalence au sein de la configuration familiale (Connidis, 2015; Lüscher, 2000, 2002; Lüscher \& Hoff, 2013). Par exemple, l'organisation actuelle du travail n'est pas compatible avec les exigences des tâches domestiques et de soins. Cette double exigence, et surtout pour ceux qui ont des ressources limitées, pourrait générer davantage d'ambivalence dans les configurations familiales. Une fille adulte, par exemple, qui travaille et qui s'occupe de son parent âgé doit jongler entre les demandes contradictoires de son travail et de sa famille. Parfois, certaines filles sont amenées à réduire leur temps de travail professionnel pour remplir leur rôle de «caregiver» avec, pour conséquence, une perte de leur revenu, voire même un appauvrissement conséquent chez les femmes des milieux populaires (Wall, Aboim, Cunha, \& Vasconcelos, 2001). Une telle situation crée non seulement des tensions entre la fille adulte et son parent âgé, mais elle génère aussi des tensions et de l'ambivalence dans l'ensemble de la configuration familiale en raison des relations d'interdépendance qui les relient avec les autres membres de la famille (Connidis, 2003; Hillcoat-Nallétamby \& Phillips, 2011; Suitor et al., 2011; Widmer, 2016). Ainsi, une demande accrue de soutien, non routinière, met en lumière l'ambivalence structurelle au sein des configurations familiales; celle-ci est la résultante complexe de plusieurs facteurs aussi bien dyadiques (dépendance à l'autre), configurationnels (déséquilibre des échanges) que sociétaux (attentes sociales contradictoires) (Connidis, 2012, 2015; Connidis \& McMullin, 2002).

Le besoin accru d'un des membres et le déséquilibre qu'il génère appellent à une réorganisation des échanges au sein de la configuration familiale. La gestion et l'allocation des ressources doivent être ré-organisées afin de trouver un nouvel équilibre dans les échanges (Connidis, 2015; Hillcoat-Nallétamby \& Phillips, 2011; Widmer, 2016). Tous les membres de la configuration doivent revoir leurs attentes, ajuster leurs propres besoins ainsi que renoncer à certaines ressources pour aider la personne en situation de vulnérabilité (Connidis, 2003, 2012; Hillcoat-Nallétamby \& Phillips, 2011). Cette réorganisation nécessite des négociations non seulement avec le parent dans le besoin mais aussi avec tous les autres membres de la famille (Connidis, 2012; Connidis \& McMullin, 2002; Willson et al., 2006). Les enjeux de telles négociations, qui ne se passent pas sans tensions ni sans stress, sont aussi bien la répartition des ressources au sein 
du réseau que le maintien de l'autonomie de chacun et le respect des obligations familiales (aide) (Connidis, 2015; Connidis \& McMullin, 2002). Ne pouvant se désengager des relations familiales dont ils dépendent étroitement en raison des multiples ressources qu'ils s'échangent, les membres de la configuration familiale se doivent de négocier ensemble et de trouver des arrangements non seulement avec ceux dont ils dépendent mais aussi avec le reste de la famille, les amis, et le travail afin de gérer au mieux ces contradictions et l'ambivalence structurelle qu'elles produisent, et cela tout au long de leur vie (Connidis, 2012, 2015; Lüscher, 2002; Lüscher \& Pillemer, 1998; Willson et al., 2006). Par ailleurs, chaque individu dispose d'une certaine marge de manœuvre qui lui permet de négocier les solutions qui lui sont les plus adéquates afin de préserver son autonomie et de gérer au mieux l'ambivalence à laquelle il est confronté (Connidis, 2015; Connidis \& McMullin, 2002; Lüscher, 2002; Willson et al., 2006). Cette marge de manœuvre est variable selon les individus et donne lieu à différents types de gestion de l'ambivalence (Connidis, 2015; Connidis \& McMullin, 2002). Certains parviennent à s'affirmer et à atténuer les dépendances auxquelles ils sont soumis. Ce faisant, ils transforment leurs relations d'interdépendance et trouvent un nouvel équilibre au sein de leur configuration familiale. D'autres, par contre, n'y parviennent pas et s'enlisent dans les tensions et les conflits (Connidis, 2015; Connidis \& McMullin, 2002; Lüscher, 2000, 2002). Nous reviendrons sur ces différents modes de gestion de l'ambivalence dans la prochaine sous-section.

L'exploration de l'ambivalence au niveau configurationnel montre que les liens d'interdépendance qui sous-tendent les configurations familiales produisent non seulement du capital social mais aussi du contrôle, des tensions et des conflits (Widmer, 2016). Stimulée par les normes de réciprocité " généralisée ", la mobilisation familiale tend à concentrer l'ensemble des ressources de la configuration sur l'aide destinée au parent dans le besoin, ce qui tend à déstabiliser l'équilibre des échanges au sein de la configuration familiale. S'ensuivent des négociations entre les différents membres familiaux visant à retrouver l'équilibre, celui-ci étant nécessaire non seulement à la satisfaction des besoins de chacun mais aussi à la préservation de leur autonomie (Connidis, 2012, 2015; Connidis \& McMullin, 2002). Comme au niveau dyadique, l'ambivalence au niveau configurationnel ne constitue pas en soi un indicateur de dysfonctionnement mais elle est le "signe » d'un déséquilibre des échanges de ressources qui requiert dès lors un ré-équilibrage. Déséquilibre et ré-équilibrage génèrent 


\section{Problématique et hypothèses}

tous deux du stress et des tensions au sein de la configuration (Widmer, 2016). Pour approcher les différentes manières de gérer l'ambivalence, quelques auteurs ont proposé des typologies au niveau non seulement dyadique (Kurt Lüscher) mais aussi configurationnel (Eric Widmer). Ces modes rendent compte des résultats des négociations entre les différents membres familiaux pour gérer l'ambivalence structurelle.

\subsubsection{La typologie des modes de conflit et de support}

Les mécanismes qui sont à l'origine de l'ambivalence structurelle sont difficiles à appréhender de manière empirique. Il est difficile de mesurer l'ambivalence structurelle au sein des relations dyadiques et, plus encore, au sein des configurations familiales (Connidis, 2015; Lüscher, 2000; Lüscher \& Hoff, 2013; Widmer, 2016). Dans un grand nombre d'études en gérontologie sociale, l'ambivalence au sein des dyades est principalement approchée par sa dimension psychologique avec des mesures psychosociales et, plus rarement, par sa dimension structurelle avec des questions sur la qualité de la relation dyadique (Connidis, 2012, 2015). Ces diverses mesures ne présentent l'ambivalence que comme une caractéristique individuelle ou dyadique mais ne parviennent pas à rendre compte de l'ambivalence structurelle au sein des configurations familiales (HillcoatNallétamby \& Phillips, 2011; Widmer, 2016). Pour pallier à ce manque, nous utilisons la typologie des modes de conflit et de support pour appréhender l'ambivalence structurelle au sein des configurations familiales (Widmer, 2016). Cette typologie n'a jamais été utilisée en gérontologie sociale; elle représente pourtant un outil précieux pour explorer l'ambivalence structurelle dans la vieillesse et identifier les différentes manières de la gérer dans les configurations familiales. Comme cette typologie s'inspire de celle de Kurt Lüscher portant sur l'ambivalence intergénérationnelle (Lüscher, 2000, 2002; Lüscher \& Hoff, 2013), nous présentons, dans un premier temps, cette dernière et, dans un deuxième temps, celle des modes de conflit et de support au niveau configurationnel (Widmer, 2016). Il est en effet important d'identifier au niveau dyadique les logiques qui soustendent les quatre modes de gestion de l'ambivalence structurelle avant de passer ensuite au niveau configurationnel.

Afin de mieux comprendre comment les individus vivent l'ambivalence au quotidien et la négocient, Kurt Lüscher propose une typologie de 
l'ambivalence au sein des relations intergénérationnelles dans la vieillesse (Lüscher, 2000, 2002; Lüscher \& Hoff, 2013). Cette typologie repose sur deux dimensions fondamentales de l'ambivalence à savoir l'individu (autonomie) et la structure (interdépendance). Dans la première dimension, dite "personnelle», parents et enfants sont considérés comme des acteurs ayant des valeurs ou des projets qui leur sont propres et qui orientent leurs actions. Ils peuvent avoir les mêmes valeurs ou les mêmes projets familiaux ou, au contraire, aspirer à des valeurs ou à des projets différents, voire opposés. Dans le premier cas, on a une «convergence» et, dans le deuxième cas, une " divergence» sur la dimension "personnelle». Quant à la deuxième dimension, dite "structurelle », elle se réfère aux liens d'interdépendance entre parents et enfants et les normes qui les sous-tendent (Lüscher, 2000, 2002). Ces liens peuvent soit se maintenir au gré du temps (reproduction), soit se modifier sous l'effet de négociations ou d'actions individuelles (innovation) (Lüscher, 2000, 2002; Lüscher \& Hoff, 2013). En combinant ces deux dimensions, quatre types d'ambivalence intergénérationnelle sont possibles : la Solidarité, l'Émancipation, la Captivation et l'Atomisation. Ces quatre modes peuvent être interprétés comme différentes manières d'expérimenter l'ambivalence ou de la gérer au sein des relations intergénérationnelles (Lüscher, 2000, 2002; Lüscher \& Hoff, 2013). Ces modes montrent, en somme, comment les parents et les enfants gèrent leurs besoins d'autonomie et de soutien compte tenu du lien d'interdépendance qui les unit. Selon leur efficacité, les relations intergénérationnelles ne sont pas forcément perçues comme ambivalentes par leurs principaux protagonistes, ce qui ne signifie pas pour autant que l'ambivalence n'existe pas, mais plutôt qu'elle est gérée avec plus ou moins de succès (Connidis, 2015).

La Solidarité se manifeste lorsque les enfants et les parents partagent les mêmes valeurs et le même projet familial, celui de maintenir le "groupe familial », et tendent à préserver les liens intergénérationnels en se soutenant mutuellement. La solidarité et le consensus sont activement encouragés alors que les divergences et les tensions sont clairement évitées (Lüscher, 2000, 2002; Lüscher \& Hoff, 2013). Ce type de gestion de l'ambivalence se manifeste ensuite dans les relations intergénérationnelles par un niveau élevé de soutien et un faible niveau de conflit. L'Émancipation se caractérise par une volonté commune des parents et des enfants de maintenir entre eux les échanges mais aussi par le désir des uns et des autres de s'engager dans de nouvelles relations et d'affirmer 


\section{Problématique et hypothèses}

leur autonomie (Lüscher, 2000, 2002). Cet engagement à l'extérieur du lien intergénérationnel implique une remise en question de son organisation et exige certaines négociations de part et d'autre de la relation pour trouver une nouvelle façon d'être ensemble et d'échanger. Ce processus a pour conséquence de modifier progressivement la relation intergénérationnelle (Connidis, 2015; Lüscher \& Hoff, 2013). Dans ce type de gestion, le soutien est élevé mais il s'accompagne aussi de tensions car chacun négocie son autonomie face aux demandes et aux attentes de son interlocuteur (Lüscher, 2000, 2002; Lüscher \& Hoff, 2013). Dans les situations où prédomine la Captivation, parents et enfants ont des valeurs et des projets différents mais, étant soumis à de fortes pressions normatives, ils maintiennent néanmoins leurs liens (Lüscher, 2000, 2002). Généralement, le parent impose à l'enfant, qui a des aspirations en dehors du " groupe familial », le maintien du lien en s'appuyant davantage sur des obligations morales que sur des valeurs familiales telles que la solidarité ou l'empathie. En somme, enfants et parents se sentent obligés de rester ensemble même s'ils ne s'entendent pas. La Captivation se traduit dans les relations intergénérationnelles par un faible soutien et de fortes tensions, alimentées par une pression normative constante (Lüscher, 2002; Lüscher \& Hoff, 2013). Finalement, l'Atomisation se caractérise, d'une part, par un faible engagement des parents et des enfants aux échanges intergénérationnels, et d'autre part, par des différences personnelles quant aux valeurs et aux projets familiaux (Lüscher, 2000, 2002). Ni les pressions normatives, ni même une histoire relationnelle commune ne suffisent à maintenir actifs les liens entre parents et enfants (Lüscher, 2000, 2002). Les tensions entre parents âgés et enfants adultes se règlent le plus souvent par un désinvestissement émotionnel et la séparation (Lüscher, 2000, 2002). Désengagé, le lien intergénérationnel ne produit ni soutien, ni conflit.

L'identification de ces différents modes au niveau empirique repose sur le soutien et le conflit, et surtout sur la façon dont ils se combinent. Soutien et conflit sont, en quelque sorte, les conséquences «mesurables» des différentes gestions de l'ambivalence intergénérationnelle et doivent donc être considérés ensemble lors de la mesure (Connidis, 2015; Lüscher \& Hoff, 2013). La typologie de Kurt Lüscher peut être considérée comme l'un des premiers modèles théoriques en gérontologie sociale à avoir modélisé ensemble le soutien et le conflit dans les relations intergénérationnelles. Par ailleurs, cette typologie a montré toute sa pertinence sur le terrain puisqu'elle a été testée empiriquement dans plusieurs études 
portant sur les relations intergénérationnelles dans des situations particulièrement créatrices d'ambivalence structurelle dans la vieillesse telles que le caregiving ou la garde intensive des petits-enfants (Letiecq, Bailey, \& Dahlen, 2008; Lüscher \& Hoff, 2013; Rappoport \& Lowenstein, 2007). Cependant, elle reste limitée à l'étude des relations dyadiques et ne peut pas être utilisée, en l'état, à l'observation de l'ambivalence structurelle au sein des configurations familiales des personnes âgées.

Afin d'examiner comment l'ambivalence structurelle est négociée au sein des différentes configurations familiales, nous utilisons dans cet ouvrage la typologie des modes de conflit et de support (Widmer, 2016). Cette typologie représente une version «adaptée » ou «transposée » de la typologie de Kurt Lüscher pour explorer l'ambivalence structurelle à un contexte relationnel plus large que la dyade. Il s'agit de quatre modes de conflit et de support qui combinent de manière distincte les densités de soutien et de conflit mesurées au sein des configurations familiales. Ces quatre modes, dont les noms sont presque identiques à la version dyadique, reflètent au niveau configurationnel les différentes gestions de l'ambivalence structurelle dégagées par Kurt Lüscher (Widmer, 2016). Cette typologie a été testée dans des études portant sur les configurations familiales de jeunes adultes mais elle n'a jamais été utilisée pour explorer l'ambivalence structurelle dans les configurations familiales des personnes âgées (Widmer, 2016). Son application en gérontologie sociale est d'autant plus intéressante que la densité de conflit - et, qui plus est, associée à celle de soutien - est rarement considérée dans les études portant sur les réseaux personnels dans la vieillesse (Connidis, 2015). Dans la présentation de ces quatre modes de conflit et de support, nous mettons en exergue à la fois les logiques qui les sous-tendent et les propriétés structurelles qui les caractérisent (densités de soutien et de conflit) car ce sont ces propriétés-là qui révèlent comment les membres de la configuration familiale gèrent entre eux l'ambivalence à laquelle ils sont confrontés.

Le premier mode de conflit et de support, la Solidarité, se caractérise par des liens denses de soutien et une faible densité de conflits au sein de la configuration familiale (Widmer, 2016). La forte densité du soutien révèle une volonté commune de l'ensemble des membres de la configuration familiale de s'engager activement dans les liens familiaux pour y préserver l'équilibre et la cohésion familiale. Ils s'investissent dans les échanges familiaux - non pas parce qu'ils se sentent «obligés »- mais plutôt parce qu'ils aspirent, tous, au maintien d'interactions positives et 


\section{Problématique et hypothèses}

à l'entretien de la qualité du lien familial. Autrement dit, une volonté commune les pousse à entretenir la solidarité. Parce que le consensus est recherché par l'ensemble des membres de la configuration familiale, les divergences personnelles et les tensions sont rapidement traitées ou soigneusement évitées. On peut imaginer que, lorsque l'un des membres requiert du soutien, les autres s'empressent de l'aider en lui fournissant les ressources dont il a besoin et, ce faisant, ils affirment les valeurs communes de solidarité qui les animent. L'engagement dont font preuve les différents membres de la configuration exige qu'ils aient tous des ressources disponibles pour maintenir l'équilibre des échanges et la satisfaction de leurs besoins (Hillcoat-Nallétamby \& Phillips, 2011; Offer, 2012).

L'Ambivalence se distingue des autres modes par de fortes densités de soutien et de conflit (Widmer, 2016). Autrement dit, les échanges de soutien sont soutenus entre les différents membres de la configuration mais ils sont teintés de fortes tensions. Le soutien collectif - caractéristique du capital social de type chaîne - s'accompagne souvent par du contrôle et des contraintes normatives, notamment dans les configurations familiales reposant sur des liens forts d'interdépendance et sur des normes de réciprocité " généralisée ». Ce contrôle et ces contraintes peuvent être perçus par les membres de la configuration comme des menaces à leur autonomie et générer du conflit (Coleman, 1988; Cornwell, 2011; Putnam, 2000). Dans un tel contexte, le besoin accru d'un des membres qui implique une ré-organisation des échanges de soutien au sein de la configuration, doit être négociée par l'ensemble des membres, soucieux de trouver des solutions pratiques qui répondent à la fois aux besoins d'un des leurs, tout en préservant leur propre autonomie (Connidis, 2015; Connidis \& McMullin, 2002). Tous sont donc appelés à négocier et à s'adapter à cette nouvelle ré-organisation des échanges. Bien que source de tensions et de conflits, ces négociations entre les différents membres ne remettent pas en question le soutien échangé mais visent à trouver de nouvelles façons d'aider tout en préservant l'autonomie de chacun (Connidis \& McMullin, 2002; Hillcoat-Nallétamby \& Phillips, 2011; Widmer, 2016). Par conséquent, bien que gérée par les membres de la configuration, l'ambivalence structurelle, mesurée à ce moment précis, demeure importante.

D'autres configurations familiales affichent une forte densité de tensions alors que les liens de soutien sont faibles (Widmer, 2016). Le mode de conflit et de support qui les caractérise renvoie à la Captivation. Comme précédemment, les membres de la configuration sont reliés par de forts 
liens d'interdépendance et, en raison des normes de réciprocité "généralisée » et du sentiment de redevabilité qu'ils ressentent, ils se sentent contraints de maintenir les liens familiaux, même s'ils ne s'entendent pas ou s'ils aspirent à plus d'autonomie (Widmer, 2016). Les fortes tensions qui gangrènent ce type de configurations familiales peinent à être canalisées car elles s'associent généralement à un manque de ressources disponibles ou à un nombre important de membres dépendants de l'aide familiale (Sillars et al., 2004; Sprey, 1999). Par conséquent, les membres deviennent rivaux, combattant pour obtenir le peu de ressources à disposition (Widmer, 2016). Dans un tel contexte, on peut imaginer qu'un besoin accru de soutien d'un des membres déstabilise encore davantage l'organisation des liens de soutien au sein de la configuration familiale et épuise, s'il perdure, les dernières ressources encore disponibles tout en aggravant encore davantage les tensions. Les ressources étant limitées, leur attribution au sein du réseau repose principalement sur le statut familial (père, mari, enfant, etc), répondant donc à une logique statutaire plutôt qu'à une logique de solidarité (Lüscher, 2002). Fortement dépendants les uns des autres et n'ayant aucune autre alternative en dehors de leur famille, les membres de la configuration entretiennent entre eux des contacts, certes réguliers, peu soutenants mais particulièrement conflictuels (Widmer, 2016).

Finalement, le mode de conflit et de support Atomisation se démarque des trois autres modes par de faibles densités tant de soutien que de conflit. L'absence de liens de soutien et de conflits s'explique par la faiblesse des échanges entre les différents membres de la configuration familiale. Ceux-ci ne sont que très faiblement connectés entre eux. Ce manque d'interconnexions indique que les liens affectifs ont été désinvestis ou qu'ils ont disparu. Il semble en effet que les individus qui expérimentent l'Atomisation dans leur configuration familiale ne disposent pas, ou plus, de relations familiales de longue durée, sur lesquelles ils peuvent compter en cas de besoin, et celles-ci ne sont pas compensées par d'autres liens significatifs. Dans de telles situations, le soutien est faible puisque les liens d'interdépendance, généralement activés par les échanges de ressources, sont lâches, libérant les membres de toute obligation de solidarité. Parce que les membres significatifs ne sont pas dépendants les uns des autres, les conflits sont aussi rares. S'ils émergent, ils sont traités et réglés par le désengagement des liens et la séparation. Au vu de ces éléments, l'Atomisation s'accompagne pour ceux qui l'expérimentent au sein de 


\section{Problématique et hypothèses}

leur configuration familiale d'un fort risque de détachement affectif et de solitude (Widmer, 2016).

Nous avons présenté dans cette sous-section la typologie des modes de conflit et de support (Widmer, 2016) qui est une version « revisitée » de la typologie des modes de gestion de l'ambivalence intergénérationnelle (Lüscher, 2000, 2002). Elle vise à explorer les différentes manières de gérer l'ambivalence structurelle au niveau configurationnel. Cette typologie, axée sur différentes combinaisons des densités de soutien et de conflit, présente quatre modes de conflit et de support : la Solidarité, l'Ambivalence, la Captivation et l'Atomisation. Ces modes révèlent que les membres des configurations familiales développent différentes façons de gérer l'ambivalence structurelle inhérente aux liens d'interdépendance dans lesquels ils s'insèrent. Les deux dimensions fondamentales de l'ambivalence (structure et individu), sur lesquelles repose la typologie de Kurt Lüscher, sont aussi apparentes au niveau configurationnel. En effet, les quatre modes de conflit et du support varient en fonction du degré d'interdépendance entre les différents membres de la configuration familiale (structure) et du degré d'investissement personnel des membres au sein de leur configuration (individu).

Si l'on considère la première dimension, on constate que, lorsque le degré d'interdépendance est élevé et que le sentiment de redevabilité est fort (Ambivalence et Captivation), les tensions sont non seulement exacerbées, l'autonomie des membres étant menacée, mais aussi difficiles à canaliser alors que, lorsque le degré d'interdépendance est faible ou inexistant (Solidarité et Atomisation), les tensions sont rares, les membres pouvant se délester facilement des relations négatives, n'étant pas soumis à de fortes pressions normatives (Carr \& Moorman, 2011; Fingerman et al., 2004; Widmer, 2016). Ceci dit, le faible degré d'interdépendance au sein des configurations familiales s'associe aussi à un soutien plus fragile et plus aléatoire. Quant au degré d'investissement personnel des membres dans leur configuration - deuxième dimension - il influence aussi la façon dont l'ambivalence structurelle est gérée au niveau configurationnel. Certains membres sont plus motivés à s'engager dans les échanges familiaux que d'autres, ce qui influence la densité des liens de soutien au sein de leur configuration. Le soutien s'avère en effet plus dense lorsque l'engagement des membres est important (Solidarité et Ambivalence) que lorsqu'il est faible (Captivation et Atomisation). Comme cette typologie n'a jamais été utilisée pour étudier l'ambivalence structurelle au sein des configurations 
familiales dans la vieillesse, la prévalence de ces différents modes de conflit et de soutien au sein de la population âgée est inconnue. Les différents facteurs ou conditions qui favorisent leur émergence dans les configurations familiales des personnes âgées restent encore à explorer.

\subsubsection{Les conditions d'émergence des modes de conflit et de support}

La typologie des modes de conflit et de support s'est révélée pertinente pour observer l'ambivalence structurelle dans les configurations familiales de jeunes adultes (Widmer, 2016); elle n'a cependant jamais été appliquée dans la vieillesse. A ce jour, nous ne connaissons pas les facteurs qui conditionnent l'émergence de ces quatre modes dans les configurations familiales des aînés. Dans cette sous-section, nous nous intéressons principalement à deux facteurs qui sont susceptibles d'influencer leur développement : la composition des configurations familiales et les ressources dont disposent les individus âgés au sein de leur réseau familial. L'identité des membres significatifs de la famille et le profil socio-démographique des individus âgés (ego) ont, selon nos hypothèses, un impact sur l'organisation des liens d'interdépendance au sein des configurations familiales et, par conséquent, sur le volume et le type de capital social qu'elles produisent. Il est donc fort probable que ces deux facteurs influencent aussi la façon dont l'ambivalence structurelle est gérée au sein des configurations familiales des personnes âgées.

Les configurations familiales peuvent être fort diverses dans la vieillesse. Certains individus âgés peuvent avoir une conception «normative » de la famille, intégrant dans leur configuration familiale essentiellement des membres primaires de la famille tels que leur partenaire et leurs enfants, voir leurs petits-enfants. D'autres, au contraire, peuvent mentionner comme membres significatifs de leur famille une diversité de membres dont certains s'inscrivent bien au-delà des liens de sang ou d'alliance. Nous avons vu que les configurations familiales composées de liens intergénérationnels ou d'alliance présentent un degré élevé d'interdépendance qui s'accompagne souvent de fortes pressions normatives (Widmer, 2016). Celles-ci, en limitant l'autonomie de leurs membres, génèrent des tensions qui sont difficiles à gérer dans un contexte de forte interdépendance (Connidis \& McMullin, 2002; Hillcoat-Nallétamby \& Phillips, 2011; Widmer, 2016). Rompre le lien conflictuel entraînerait un déséquilibre dans 


\section{Problématique et hypothèses}

l'ensemble des liens d'interdépendance. Ne pouvant fuir leurs obligations familiales, les membres de ce type de configurations familiales doivent négocier entre eux pour préserver leur autonomie tout en maintenant les échanges de ressources dont ils ont tous besoin. Par ailleurs, ces négociations, qui sont nécessaires, sont aussi source de stress et de tensions. Dès lors, nous faisons l'hypothèse que la présence du partenaire, des enfants et des petits-enfants au sein des configurations familiales s'associe à des modes de conflit et de support marqués par les tensions et les conflits, à savoir l'Ambivalence et la Captivation. Quant aux configurations familiales exemptes du partenaire, des enfants et des petits-enfants, elles se caractérisent par un moindre degré d'interdépendance. Venant de milieux divers, les membres qui les composent sont moins connectés les uns aux autres par de multiples échanges que ceux qui constituent les configurations précédentes. Le maintien des liens au sein de ce type de configurations repose davantage sur le désir d'être ensemble que sur des obligations familiales. N'étant pas soudées par de fortes pressions normatives, les relations jugées inappropriées, insatisfaisantes ou conflictuelles peuvent être facilement rompues. Même si la rupture implique quelques désagréments émotionnels, voire pratiques, elle n'a aucune conséquence sur les autres relations au sein de la configuration. Par conséquent, la rupture devient plus facile et plus probable en cas de litige (Carr \& Moorman, 2011; Fingerman et al., 2004; Litwak, 1985). Compte tenu du faible degré d'interdépendance qui caractérise ce type de configurations familiales, nous faisons l'hypothèse que l'absence du partenaire, des enfants et des petits-enfants favorise une gestion plus « sereine» de l'ambivalence structurelle, avec l'émergence des modes de conflit et de support Solidarité ou Atomisation qui sont exempts de fortes tensions.

Le deuxième facteur, qui peut influencer la gestion de l'ambivalence structurelle dans les configurations familiales, a trait aux ressources des aînés (ego), telles que le revenu, l'énergie, la santé, l'affection ou encore les réserves démographiques. Associées au profil socio-démographique et au parcours de vie, elles sont à même d'influer l'organisation des liens d'interdépendance et la production du capital social au sein des configurations familiales. Il est, par conséquent, probable qu'elles aient aussi un impact sur le type de modes de conflit et de support qui s'y développe (Connidis, 2015; Connidis \& McMullin, 2002; Lüscher \& Pillemer, 1998). La littérature scientifique montre qu'avoir des ressources favorise 
le maintien de l'autonomie des individus âgés au sein de leur configuration familiale. Ces derniers peuvent recourir à diverses aides alternatives en dehors du contexte familial et, ce faisant, ils atténuent l'ambivalence structurelle au sein de leur configuration, en réduisant leur dépendance à l'aide familiale, notamment à celle de leurs enfants (Connidis, 2015; Connidis \& McMullin, 2002). Par ailleurs, avoir certaines ressources leur permet de préserver leur capacité à la réciprocité et de s'engager activement dans les négociations et les échanges familiaux (Cornwell, 2011; Offer, 2012). En s'investissant, les aînés peuvent même jouer un rôle central dans la distribution des ressources au sein de leur configuration familiale, y maintenir la stabilité des échanges et influencer l'organisation des liens d'interdépendance qui la caractérise (Connidis \& McMullin, 2002). Fort de ce constat, nous faisons l'hypothèse que les individus âgés qui ont des ressources développent une gestion de l'ambivalence structurelle axée sur la promotion du soutien au sein de leur configuration familiale, telle que celle qui caractérise les modes de conflit et de support Solidarité et Ambivalence. A l'opposé, le manque de ressources affecte l'autonomie au sein de la configuration familiale. La littérature scientifique montre que les aînés sans aucune ressource dépendent presque exclusivement du soutien familial comme en témoignent les individus âgés des milieux populaires qui reçoivent plus de soutien de leur famille que les autres (Antonucci, 2001). Plus encore, l'absence de ressources les entrave dans leur participation aux échanges et aux négociations avec les membres de leur configuration familiale. Ils ne sont donc pas en mesure de stimuler les liens de soutien dont ils dépendent. Lorsqu'un événement critique survient, leur non engagement dans les échanges et leur forte dépendance à l'aide familiale accentuent encore davantage l'ambivalence structurelle au sein de leur configuration familiale (Connidis, 2015; Connidis \& McMullin, 2002). Nous faisons donc l'hypothèse que les individus âgés qui ont peu de ressources peinent à stimuler le soutien au sein de leur configuration familiale pour gérer l'ambivalence à laquelle ils sont confrontés. Ils ont, par conséquent, un risque plus élevé de déployer au sein de leur configuration familiale des modes de conflit et de support Captivation et Atomisation qui se distinguent des autres par la faiblesse du soutien.

En combinant ces deux facteurs, « composition» et « ressources », nous pouvons identifier les conditions qui expliquent le mieux l'émergence de chacun de ces quatre modes de conflit et de support. Nous faisons l'hypothèse que la Solidarité est plus probable lorsque l'individu âgé a 


\section{Problématique et hypothèses}

des ressources importantes et qu'il désigne comme membres significatifs de sa famille une diversité de membres qui s'inscrivent bien au-delà des liens de sang et d'alliance. L'absence de liens conjugaux ou intergénérationnels indique que le maintien des liens de soutien répond davantage à une envie commune d'entretenir des interactions positives qu'à des pressions normatives, ce qui expliquerait l'absence de tensions dans ce type de configurations familiales. Par ailleurs, pour maintenir la densité du soutien dans sa configuration hétéroclite, l'aîné a non seulement la volonté mais doit aussi avoir les ressources nécessaires pour activer les échanges de soutien, et cela d'autant plus que ces derniers sont directs entre ego et les autres membres de sa configuration. Plus dyadiques que collectifs, ces échanges sont plus fragiles, n'étant pas soutenus par des normes de réciprocité « généralisée ». En revanche, l'Ambivalence est plus probable lorsque l'individu âgé a des ressources et est intégré dans une configuration familiale reposant sur des liens conjugaux ou intergénérationnels. Les membres sont étroitement interconnectés et reliés par des normes de réciprocité «généralisée ». De fait, les liens de soutien y sont denses et collectifs mais ils s'associent aussi, en raison du degré élevé d'interdépendance, à de fortes pressions normatives et du contrôle, susceptibles d'engendrer de fortes tensions (Coleman, 1988; Putnam, 2000; Rappoport \& Lowenstein, 2007; Widmer, 2016). En d'autres termes, le besoin accru du parent âgé active l'organisation du soutien collectif et, ce faisant, génère des tensions. Quant à la personne âgée, elle peut, ayant les ressources nécessaires, s'engager et participer aux échanges au sein de sa configuration. Plus encore, elle peut intervenir dans les négociations afin d'y défendre son autonomie et y influencer le flux des ressources. La forte densité du soutien qui caractérise ce mode indique que l'individu âgé n'est pas le seul à disposer d'un certain nombre de ressources, les membres de sa configuration en sont probablement tous aussi pourvus, du moins de manière suffisante pour soutenir les échanges.

A l'inverse, lorsque les ressources manquent au sein des configurations familiales majoritairement composées d'enfants et de petits-enfants, nous faisons l'hypothèse que le mode de conflit et de support Captivation s'y développe davantage. Dans ce type de configurations familiales, les membres significatifs de la famille sont fortement dépendants les uns des autres par les ressources qu'ils s'échangent. L'interdépendance y est élevée et les pressions normatives qui en résultent suscitent du stress et des tensions. Cependant, contrairement aux situations où le mode de conflit et 
de support Ambivalence prédomine, les tensions sont exacerbées soit par le manque chronique de ressources, soit par le nombre important de membres dépendants de l'aide familiale. La présence de membres de plusieurs générations dans la même configuration familiale s'associe en effet à une variété de besoins et, selon le parcours de vie de chacun, ceux-ci peuvent être relativement importants. Il peut s'agir d'un conjoint malade, d'enfants adultes en difficulté (chômage, divorce, etc.), de petits-enfants scolarisés ou économiquement dépendants. Dans une telle situation, les besoins et les attentes sont grands et ne peuvent que difficilement être comblés, et cela d'autant plus si la charge du soutien repose sur un nombre restreint de membres tels qu'un enfant adulte, la fille généralement. Comme les ressources sont limitées, elles ne peuvent pas contenter tous les membres; elles sont allouées à ceux qui en ont le plus besoin ou qui, par leur statut familial, sont prioritaires. Une telle distribution peut être perçue par les autres membres de la configuration comme inégalitaire, créant au sein de ce type de configurations de la jalousie, des frustrations et des tensions (Hillcoat-Nallétamby \& Phillips, 2011; Widmer, 2016). Quant à l'individu âgé qui a peu de ressources, il peine à stimuler les liens de soutien au sein de sa configuration familiale puisqu'il ne peut plus participer activement aux échanges et aux négociations. Dans un tel contexte, le soutien devient difficile et s'épuise, laissant place au stress et aux tensions, résultat d'une gestion difficile de l'ambivalence structurelle.

Quant à l'Atomisation, nous faisons l'hypothèse qu'elle se développe dans des configurations familiales d'individus âgés précarisés. Celles-ci n'intègrent ni partenaire, ni enfants, ni petits-enfants; elles sont composées d'un nombre restreint de membres significatifs qui, provenant de divers cercles de sociabilité, ne sont pas ou peu connectés entre eux. N'étant pas tenus par de fortes obligations familiales, les différents membres peinent à offrir un soutien solide et efficace. Plus encore, l'individu âgé sans ressources perd sa capacité à la réciprocité, ce qui prétérite grandement ses échanges avec les autres membres de sa configuration. Les échanges dans ce type de configurations familiales étant majoritairement dyadiques requièrent une participation active et engagée de la part des différents protagonistes pour être maintenus (Offer, 2012). Lorsque la personne âgée est en difficulté et ne parvient plus à participer aux échanges, le soutien octroyé s'essouffle rapidement. Les membres de la configuration se désengagent peu à peu, et cela d'autant plus si l'aide demandée par la personne âgée est importante (Offer, 2012; Widmer \& Sapin, 2008; Widmer, 2016). Dans un 


\section{Problématique et hypothèses}

tel contexte, les relations significatives perdues, désengagées, ne peuvent être que difficilement remplacées car les aînés qui ont peu de ressources éprouvent des difficultés à s'engager dans de nouvelles relations. Toutefois, les tensions et les conflits sont rares. Non soutenues par une fort sentiment de redevabilité, les relations dans ce type de configurations familiales se rompent facilement si elles ne sont plus gratifiantes ou si elles sont jugées conflictuelles (Widmer \& Sapin, 2008; Offer, 2012). Dans cette situation, la précarité sous toutes ses formes pourrait expliquer l'absence de soutien et de conflit qui caractérise l'Atomisation.

Les individus âgés sont donc loin d'être égaux pour faire face à l'ambivalence structurelle au sein de leur configuration familiale. Parmi les personnes âgées les plus vulnérables se trouvent les femmes. Celles-ci ont non seulement un risque plus élevé d'y être confrontées mais elles ont aussi plus de difficultés à la gérer (Connidis \& McMullin, 2002; Willson et al., 2006). Comme le montre la littérature gérontologique, les femmes âgées sont plus amenées que les hommes à traverser certaines transitions de vie négatives - comme le veuvage ou encore le déclin de la santé fonctionnelle - et à subir une diminution drastique de leurs ressources (Arber, Davidson, \& Ginn, 2003 ; Connidis \& McMullin, 2002 ; Wanner, SauvainDugerdil, Guilley, \& Hussy, 2005; Willson et al., 2006). Par ailleurs, ces pertes de ressources sont souvent concomitantes. Le veuvage par exemple, comme le divorce d'ailleurs, s'accompagne chez les femmes âgées par une baisse de revenu plus conséquente que chez les hommes (Arber et al., 2003; Moen, 1996; Wanner et al., 2005; Willson et al., 2006). Certes, la perte du partenaire chez les hommes peut aussi avoir des conséquences tout aussi importantes. Le veuvage et surtout le divorce peuvent déboucher sur le désengagement des liens familiaux et l'isolement social. Ceci dit, la probabilité plus forte des hommes âgés à se remettre en couple montre que les ressources qu'ils ont à disposition leur permettent de compenser rapidement un tel manque. De plus, les femmes ont un risque plus grand que les hommes d'être atteintes dans leur santé fonctionnelle au point, parfois, de nécessiter de la présence active et continue de leurs proches (Arber et al., 2003; Moen, 1996; Wanner et al., 2005; Willson et al., 2006). Vulnérabilisées par les transitions de vie qu'elles sont amenées à traverser aux dernières étapes de leur vie, les femmes âgées ont non seulement une probabilité plus grande que les hommes de dépendre du soutien familial (Arber et al., 2003; Moen, 1996; Wanner et al., 2005; Willson et al., 2006) et 
d'être ainsi confrontées à l'ambivalence structurelle au sein de leur configuration familiale, mais elles sont aussi plus démunies que les hommes pour y faire face et la gérer (Connidis \& McMullin, 2002; Willson et al., 2006). Sans ressources, elles peinent à maintenir un rôle actif et engagé au sein de leur configuration familiale et perdent ainsi le capital social pont dont elles bénéficiaient jusque-là. Au vu de ce constat, nous posons l'hypothèse que les femmes ont un risque plus grand que les hommes d'expérimenter la Captivation ou l'Atomisation au sein de leur configuration familiale en raison de leur plus grande probabilité d'être en situation de précarité.

Nous avons mis en exergue dans cette sous-section deux facteursclé qui sont les plus à même d'expliquer les variations dans la gestion de l'ambivalence structurelle au sein des configurations familiales dans la vieillesse. Il s'agit de la composition des configurations et de l'état des ressources des personnes âgées. Ces deux facteurs sont susceptibles d'influencer les deux dimensions fondamentales de la typologie des quatre modes de conflit et de support, à savoir le degré d'interdépendance (structure) et l'engagement des individus dans les échanges (individu). La présence - ou l'absence - des liens intergénérationnels et conjugaux dans la composition des configurations familiales détermine leur degré d'interdépendance et plus il est élevé, plus les tensions y sont fortes. Quant aux ressources dont dispose l'individu, leur influence est double. D'une part, elles atténuent la dépendance de l'individu âgé à l'aide familiale et réduisent ainsi l'ambivalence structurelle au sein de sa configuration familiale. D'autre part, elles permettent à l'individu âgé de jouer un rôle actif et engagé au sein de son réseau familial. Il peut en effet participer aux négociations familiales et surtout alimenter le flux et les échanges de soutien au sein de sa configuration. Selon ces hypothèses, les individus âgés sont donc inégaux face à la gestion de l'ambivalence structurelle au sein de leurs configurations familiales. Cette gestion dépend en effet de leur parcours de vie et des transitions qu'ils ont traversées (composition) ainsi que de leur position dans la structure sociale (ressources). Grâce aux outils de l'analyse de réseau que propose l'approche configurationnelle, nous pouvons construire les quatre modes de conflit et de support en nous basant sur les densités du soutien et du conflit au sein des configurations familiales et tester l'influence de ces différents facteurs sur l'émergence de chacun d'entre eux. 


\section{Problématique et hypothèses}

La dernière partie de ce chapitre s'est surtout centrée sur l'ambivalence structurelle au sein des configurations familiales. Nous avons d'abord mis en évidence la pertinence du concept de l'ambivalence intergénérationnelle, proposé par Kurt Lüscher, qui considère «ensemble » les aspects positifs (soutien) et négatifs (conflits) des relations intergénérationnelles dans la vieillesse. Nous avons décrit les mécanismes structurels avancés dans la littérature en gérontologie sociale pour expliquer l'ambivalence intergénérationnelle. Nous avons notamment souligné l'effet déclencheur du besoin accru de soutien du parent âgé qui, tout en activant les normes de réciprocité " généralisée », menace l'autonomie du parent bénéficiaire comme celle, d'ailleurs, de l'enfant prestataire. En remettant en question le modèle de la solidarité intergénérationnelle de Vern Bengtson, le concept de l'ambivalence intergénérationnelle constitue une avancée dans la connaissance des dynamiques familiales aux dernières étapes de la vie.

Cependant, ce concept a surtout été utilisé en gérontologie sociale pour étudier les relations dyadiques. Autrement dit, l'impact du besoin accru de soutien de l'individu âgé sur l'ensemble de la configuration familiale n'a jamais été étudié. Pourtant, un tel événement peut avoir un effet sur l'ensemble des liens d'interdépendance qui sous-tendent les configurations familiales et générer des tensions et de l'ambivalence qui vont bien au-delà des dyades. Pour étudier l'ambivalence structurelle au niveau configurationnel, nous avons proposé d'utiliser la typologie des modes de conflit et de support (Widmer, 2016) qui permet - en modélisant les densités de soutien et de conflit - d'identifier différentes manières de vivre et de gérer l'ambivalence structurelle au niveau configurationnel. Ce faisant, nous avons postulé que ces différents modes de gestion - Solidarité, Ambivalence, Captivation et Atomisation - varient selon les individus âgés, non seulement en fonction de la composition de leur configuration familiale mais aussi en fonction des ressources qu'ils ont à disposition. De telles hypothèses nous permettent d'explorer les inégalités sociales face à la gestion de l'ambivalence structurelle à laquelle les individus âgés sont confrontés au sein de leur configuration familiale; les plus démunis ayant un fort risque de manquer de soutien familial, d'être aux prises avec de forts conflits familiaux, voire même d'être isolés socialement. Avant de passer aux méthodes, une synthèse de la problématique énoncée dans ce chapitre s'impose ainsi qu'une formulation plus formelle des différentes hypothèses. 


\subsection{Résumé et hypothèses}

D'abord, nous avons mis en évidence la complexification des trajectoires de vie des individus âgés ainsi que celle de leurs proches qui rendent le soutien familial plus compliqué et plus aléatoire. Cette situation crée de l'incertitude chez les individus âgés quant au soutien familial dû ou attendu. Des données qualitatives montrent que, pour pallier à un tel flou, certains individus âgés intègrent au sein de leur famille significative une variété de membres familiaux avec lesquels ils entretiennent des liens actifs et sur lesquels ils pensent pouvoir compter en cas de besoin. Les études quantitatives portant sur des populations plus jeunes montrent que ces membres significatifs de la famille sont fort variés. Ils dépassent largement les frontières de la famille bio-légale et ils forment, en s'articulant les uns aux autres, différentes configurations familiales, signes d'une pluralité de définitions personnelles de la famille significative. A ce jour, nous ne connaissons pas l'identité des membres significatifs de la famille dans la vieillesse et encore moins la façon dont ils s'articulent entre eux pour donner lieu à différentes configurations familiales. Les deux premières hypothèses concernent les membres significatifs de la famille et la diversité des configurations familiales dans la vieillesse.

\section{H1.}

Nous faisons l'hypothèse qu'à l'instar des populations plus jeunes, les individus âgés mentionnent une variété de membres significatifs de la famille. Face à leur besoin grandissant de soutien familial et à l'incertitude qu'ils en bénéficieront un jour, certains d'entre eux désignent comme membres significatifs de la famille des individus qui s'inscrivent bien au-delà des frontières de la famille «nucléaire».

\section{H2.}

Nous faisons l'hypothèse que les différents membres significatifs sélectionnés s'articulent de façon distincte donnant lieu à une diversité de configurations familiales. Selon leur profil socio-démographique, les transitions de vie qu'ils ont traversées, l'état de leur réservoir de parenté et la façon dont ils ont investi certaines relations au cours de leur vie, certains individus âgés privilégient plutôt des liens de sang et d'alliance alors 
que d'autres s'appuient plutôt sur des critères affinitaires (amitié, etc) pour définir leur famille significative.

Se pose ensuite la question sur la « capacité » de ces différentes configurations familiales à fournir le soutien familial attendu. Pour répondre à cette interrogation, nous avons proposé d'utiliser le concept de capital social. Pour rappel, le capital social renvoie à l'ensemble des ressources individuelles produites au sein des réseaux personnels, mobilisables en cas de besoin. En considérant la famille comme une configuration de liens d'interdépendance reliant entre eux les différents membres significatifs de la famille, l'approche configurationnelle permet, en utilisant les outils de l'analyse de réseaux, de mesurer le capital social généré au sein de chaque configuration familiale. Dans la littérature, le capital social n'est pas uniforme, il varie dans son volume et son type selon la façon dont les liens et les échanges de ressources s'organisent au sein des réseaux personnels. Deux types de capital social sont généralement distingués : le capital social de type chaîne et celui de type pont. Le premier émerge dans les réseaux denses et réciproques alors que le deuxième se développe dans des réseaux peu denses. Dans ces derniers, les individus tendent à relier les membres non connectés et, ce faisant, ils y occupent une position centrale. Le capital social de type chaîne est prometteur d'un solide soutien mais menace l'autonomie des individus alors que celui de type pont la favorise mais le soutien y est fragile. Le capital social au sein de la famille n'a jamais été au cœur des investigations de la recherche en gérontologie sociale, sans doute parce qu'elle a peu considéré la diversité des contextes familiaux et qu'elle a toujours perçu « a priori » le soutien familial comme solide, uniforme et inconditionnel en raison des fortes normes de réciprocité "généralisée » qui sous-tendent les liens de sang et d'alliance. Dans cet ouvrage, nous remettons en question cette idée "préconçue » en faisant l'hypothèse que le capital social produit dans les configurations familiales est fort variable et qu'il dépend, d'une part, de l'identité des membres qui forment les différentes configurations familiales et, d'autre part, du profil sociodémographique des individus âgés (ego). Les trois hypothèses suivantes portent sur le capital social :

\section{H3.}

Nous faisons l'hypothèse que les individus âgés qui définissent leur famille significative en citant leur partenaire, leurs enfants et leurs petits-enfants ont davantage accès à un capital social de type chaîne. Celui-ci est favorisé 
par la forte densité des connexions et la réciprocité des échanges familiaux qui caractérisent les liens conjugaux et intergénérationnels. Pris dans la toile florissante de ces échanges, l'individu âgé n'y est point central. A l'opposé, les individus âgés qui mentionnent dans leur configuration familiale des membres de leur fratrie, de leur parenté éloignée ou encore des amis - c'est-à-dire des liens " affinitaires »- ont davantage accès à du capital social de type pont. Dans de telles configurations familiales, les individus jouent le rôle d'intermédiaire entre les différents membres qui ne sont pas connectés. Les faibles densité et réciprocité des liens au sein de ce type de configurations familiales permettent à l'individu âgé d'être central dans son réseau et de préserver son autonomie.

\section{H4.}

Outre les liens de parenté, nous faisons l'hypothèse que les configurations familiales composées majoritairement de femmes génèrent davantage de capital social de type chaîne que les configurations formées principalement d'hommes. Poussées par les normes sociales qui leur attribuent le rôle de " gardienne des liens », les femmes - qu'elles soient " épouses », « filles », « belles-filles » ou encore « sœurs »- encouragent davantage que les hommes les échanges de soutien entre les différents membres de leur famille, favorisant ainsi la densité et la réciprocité des liens au sein de leur configuration familiale.

\section{H5.}

Nous faisons l'hypothèse que le profil socio-démographique de l'individu âgé a aussi un impact sur le volume et le type de capital social auquel il a accès au sein de sa configuration familiale. Parce que les femmes, les individus ayant un statut socio-économique élevé, les «jeunes-vieux» et ceux qui sont en bonne santé ont les ressources à la fois relationnelles, économiques et physiques d'initier et de maintenir des liens avec une grande variété de membres familiaux, ils développent au sein de leur configuration familiale davantage de capital social de type pont que ceux qui ont moins de ressources. Ceux-ci - les hommes, les individus ayant un statut socio-économique bas, les «très-vieux » et les personnes en mauvaise santé - ont au contraire davantage accès à un capital social de type chaîne, du moins lorsqu'ils disposent encore de suffisamment de ressources (démographiques, financières, santé, etc.) pour l'entretenir. 


\section{Problématique et hypothèses}

Les configurations familiales, en tant que réseaux de liens d'interdépendance, ne produisent pas que du capital social mais elles génèrent aussi du conflit et de l'ambivalence structurelle. D'un côté, le besoin accru de soutien de l'individu âgé stimule les normes de réciprocité "généralisée » au sein de la configuration familiale et active les liens de soutien. D'un autre côté, la dépendance à l'aide familiale suscite des tensions parce qu'elle limite l'autonomie de chacun et déséquilibre les échanges au sein de la configuration familiale. En gérontologie sociale, l'ambivalence structurelle a été rarement étudiée au niveau configurationnel; celle-ci a surtout été observée au niveau dyadique. Afin de l'explorer au sein des configurations familiales, nous proposons d'utiliser la typologie des modes de conflit et de support (Widmer, 2016), inspirée de celle de Kurt Lüscher (Lüscher, 2000, 2002; Lüscher \& Hoff, 2013), qui rend compte de différentes manières de gérer ou de négocier l'ambivalence structurelle au niveau configurationnel. En combinant les densités de soutien et de conflit - conséquences « observables» des gestions de l'ambivalence - cette typologie produit quatre modes distincts : la Solidarité [fort soutien-peu de conflit], l'Ambivalence [fort soutien-fort conflit], la Captivation [peu de soutien-fort conflit] et finalement l'Atomisation [peu de soutien-peu de conflit]. Nous faisons l'hypothèse que l'émergence de ces différents modes n'est pas aléatoire mais qu'elle dépend de deux principaux facteurs : la composition des configurations familiales et les différentes ressources (revenu, santé, réserves démographiques, etc.) dont disposent les individus âgés aux dernières étapes de leur vie et qui varient non seulement en fonction de leur parcours de vie mais aussi en fonction de leur profil socio-démographique (genre, statut socio-économique et classe d'âge). Nous proposons quatre hypothèses à tester :

\section{H6.}

Considérant le premier facteur, nous faisons l'hypothèse que les configurations familiales qui sont majoritairement composées de liens intergénérationnels et conjugaux s'associent, en raison de la forte interdépendance qui les caractérise, à une gestion de l'ambivalence structurelle davantage marquée par les conflits que les configurations familiales formées par des liens familiaux de type " affinitaire », se distinguant des autres par un moindre degré d'interdépendance. Par conséquent, les configurations familiales qui intègrent en leur sein un partenaire, des enfants ou des petits-enfants développent davantage l'Ambivalence ou la Captivation que les configurations 
familiales qui sont constituées de membres de la fratrie, de la parenté éloignée ou encore d'amis, celles-ci s'associant davantage à la Solidarité ou à l'Atomisation.

H7.

Quant au deuxième facteur, nous faisons l'hypothèse que les ressources des individus âgés - en termes de revenu, de santé ou de réserves démographiques - influent sur la façon dont ils gèrent l'ambivalence à laquelle ils sont confrontés. Parce qu'elles permettent aux individus âgés de s'engager dans les échanges de soutien, avoir des ressources s'associe à la promotion du soutien au sein de leur configuration alors qu'en manquer réduit leur capacité à participer aux échanges avec le risque, à moyen-long terme, d'un affaiblissement du soutien au sein du réseau familial. Ce risque est d'autant plus grand que les autres membres de la configuration familiale sont aussi démunis. Par conséquent, nous faisons l'hypothèse que les personnes âgées qui ont des ressources ont une probabilité plus grande de développer au sein de leur configuration familiale un mode axé sur le soutien, comme la Solidarité ou l'Ambivalence, alors que celles qui n'ont pas de ressources développent davantage la Captivation ou l'Atomisation, se caractérisant par de faibles densités de soutien.

H8.

Nous faisons l'hypothèse que l'interrelation de ces deux facteurs - composition des configurations familiales et ressources de l'individu âgé (ego) explique l'émergence de chacun de ces modes. D'une part, nous émettons l'hypothèse que les configurations familiales composées de liens intergénérationnels et conjugaux développent l'Ambivalence lorsque l'individu âgé a suffisamment de ressources pour alimenter les échanges et la Captivation lorsque ce dernier a peu de ressources. D'autre part, nous faisons l'hypothèse que les configurations familiales axées sur des liens de type " affinitaire » s'associent à la Solidarité lorsque l'individu âgé a les ressources suffisantes pour s'engager activement dans les échanges familiaux et à l'Atomisation lorsque ce dernier en est dépourvu.

H9.

Finalement, nous faisons l'hypothèse que les femmes âgées - parce qu'elles ont un risque plus grand que les hommes d'être confrontées à des 


\section{Problématique et hypothèses}

pertes concomitantes de ressources (baisse de revenu, veuvage, atteintes à la santé fonctionnelle, etc.) aux dernières étapes de leur vie - ont davantage de peine que les hommes à gérer l'ambivalence à laquelle elles sont confrontées au sein de leur famille. Sans ressources, les femmes âgées ne parviennent pas à s'engager activement dans les échanges de soutien au sein de leur configuration familiale. Par conséquent, nous faisons l'hypothèse que les femmes déploient davantage que les hommes des modes de conflit et de support se caractérisant par une faible densité de soutien, tels que la Captivation ou l'Atomisation, et cela d'autant plus si les membres de leur configuration familiale sont aussi en situation de précarité.

Pour conclure, les trois points centraux de l'approche configurationnelle présentés dans ce chapitre, à savoir les «membres significatifs de la famille », le « capital social» et les « conflits et ambivalence » ainsi que les hypothèses s'y rapportant, défient l'image « lisse », « stable » et « soutenante » de la famille généralement présentée dans les modèles dominants de la gérontologie sociale. Ces divers aspects remettent en question la définition normative de la famille usuellement adoptée dans un grand pan de la recherche gérontologique. Selon la perspective configurationnelle, la famille est loin d'être un groupe de personnes unies par des liens de sang et d'alliance, et prometteur d'un soutien sans faille. La famille ou plutôt « les» familles sont au contraire des réseaux complexes de liens d'interdépendance et qui, selon les propriétés structurelles qui les caractérisent, produisent différentes formes de capital social mais aussi du conflit et de l'ambivalence. Trop focalisés sur la problématique du support social, les principaux courants de la gérontologie sociale ont négligé l'exploration de la famille dans sa diversité et sa complexité.

En définissant la famille selon le modèle « idéal» de la famille nucléaire, de nombreux chercheurs en gérontologie sociale ne se questionnent pas sur les définitions personnelles de la famille. Pourtant, à l'instar des populations plus jeunes, il est probable que les individus âgés conçoivent leur famille significative en y intégrant une diversité de membres qui vont bien au-delà des critères bio-légaux habituels. Et, ce sont ces « familles-là » que nous souhaitons investiguer dans cet ouvrage car ce sont elles qui font sens pour les individus âgés. De plus, le soutien familial n'a jamais été davantage exploré, qualifié « a priori » comme inconditionnel et indéfectible. Or, la notion de capital social nuance la perception formatée du soutien familial dans les modèles dominants de la gérontologie sociale, car le capital social varie dans sa forme et son volume, et est loin d'être aussi « uniforme» 
que ne le supposent généralement la plupart des travaux en gérontologie sociale. Finalement, étudier les conflits et l'ambivalence structurelle au niveau configurationnel et sortir du cadre dyadique usuellement utilisé en gérontologie sociale rend mieux compte de toute la complexité des liens d'interdépendance et de l'ambivalence structurelle qu'ils génèrent. Aux dernières étapes de la vie, les individus âgés y sont pleinement confrontés et doivent la gérer avec l'ensemble des membres de leur configuration familiale. Selon nos hypothèses, il y a de fortes inégalités sociales parmi les individus âgés et leurs familles, tous ne sont pas suffisamment armés pour négocier et gérer au mieux l'ambivalence structurelle qui caractérise leurs relations familiales. Certains d'entre eux pourraient même être confrontés à de forts conflits familiaux ou risquer l'isolement social. Avant de tester l'ensemble de ces hypothèses et de passer aux résultats, nous présentons dans le prochain chapitre les données «VLV », les mesures utilisées ainsi que les différents types d'analyses effectuées dans cet ouvrage. 



\section{Données et méthodes}

Ce chapitre est consacré à la présentation des données, des méthodes et des mesures utilisées dans ce travail. Nous présentons d'abord l'étude « Vivre/Leben/Vivere (VLV) » qui s'est déroulée entre 2011 et 2012 et qui a permis la récolte de nombreuses données sur les conditions de vie et de santé des individus âgés en Suisse. Ensuite, nous décrivons l'échantillon genevois sur lequel portent les analyses. Nous passons après aux « Mesures », section dans laquelle nous introduisons la méthode « Family Network Method (FNM)» développée par le prof. Eric Widmer. Cette méthode permet, d'une part, de recenser les différents membres significatifs de la famille et, d'autre part, de saisir les liens d'interdépendance qui relient entre eux les différents membres significatifs cités, et sur la base desquels sont calculés les différents indices du capital social. Puis, nous décrivons la démarche adoptée pour construire de manière empirique les quatre modes de conflit et de support qui renvoient aux manières distinctes de gérer l'ambivalence au sein des configurations familiales. Sont aussi présentées dans cette section les différentes variables utilisées pour approcher les facteurs explicatifs tels que le réservoir de parenté, le genre, le statut socio-économique, l'âge et la santé. Finalement, nous concluons ce chapitre par une section "Analyses », dans laquelle nous introduisons les principales analyses réalisées pour mener à bien ce travail telles que l'analyse de classification hiérarchique, l'analyse de variance (ANOVA), la régression linéaire et l'analyse des correspondances multiples (ACM).

\subsection{Les données}

Dans cette première section, nous présentons l'étude «VLV » («Vivre/Leben/Vivere »), ses objectifs et son déroulement. Nous y détaillons entre autres la procédure d'approche qui a été adoptée pour rencontrer les individus de l'étude ainsi que celle utilisée pour aborder 
les personnes qui, en raison de problèmes cognitifs, n'ont pas pu suivre une procédure «normale ». Puis, nous introduisons le matériel utilisé et les questions posées. Après avoir présenté l'étude et les données, nous passons à l'échantillon genevois sur lequel portent essentiellement les analyses. Afin de mieux saisir les caractéristiques de l'échantillon genevois, nous présentons l'évolution de la population âgée à Genève depuis 1979, date à laquelle une première étude transversale a été menée pour examiner les conditions de santé et de vie de la population âgée résidant dans ce canton. Ce faisant, nous ferons mention des changements socio-historiques de 1979 à 2011 qui ont influencé l'évolution de la structure de la population âgée dans ce canton. Puis, nous consacrons une sous-section à la politique de la vieillesse à Genève afin de décrire le dispositif d'aides destiné aux personnes âgées dépendantes vivant à Genève et évaluer les attentes du canton vis-à-vis de leur entourage familial. Nous détaillons ensuite plus spécifiquement l'échantillon d'analyse et ses principales caractéristiques.

\subsubsection{L'étude «VLV»}

L'étude «VLV » («Vivre / Leben/ Vivere ») est une vaste enquête interdisciplinaire portant sur les conditions de vie et de santé des personnes âgées de 65 ans et plus vivant en Suisse (Ludwig, Cavalli, \& Oris, 2014; Oris et al., 2016). Elle a été menée dans cinq cantons suisses : Genève, Valais, Berne, Bâle et Tessin. Dans chacune de ces régions, un échantillon représentatif de la population âgée de 65 ans et plus, vivant à domicile ou en institution, a été sélectionné de manière aléatoire. Stratifié selon le genre et l'âge, chaque échantillon comprend six groupes d'âge : les 65-69 ans, 70-74 ans, 75-79 ans, 80-84 ans, 85-89 ans et 90 ans et plus. Au total, un échantillon global de 3635 participants, représentatif de la population âgée suisse, a été récolté. L'objectif principal de l'étude visait à décrire la population âgée en Suisse et à mieux connaître ses besoins et les difficultés auxquelles elle est confrontée au quotidien. Les données récoltées donnent un aperçu précis des ressources - matérielles, financières, sociales, familiales ou encore psychologiques - dont disposent les individus âgés en Suisse pour faire face aux aléas de leur vieillesse et permettent ainsi de détecter les inégalités en termes de santé, de moyens socio-économiques, de 
relations sociales et familiales au sein de la population âgée suisse. Interdisciplinaire, cette enquête a été réalisée avec la collaboration de plusieurs équipes situées à Genève, à Lausanne, à Berne et à Lugano, et travaillant dans différents champs de la gérontologie aussi bien médical, psychologique, démographique que sociologique. Soutenue financièrement par le Fonds national suisse de la recherche scientifique, elle a été supervisée par le Centre Interfacultaire de Gérontologie et d'Études des Vulnérabilités ( CIGEV ») à Genève et a été dirigée par son directeur, le prof. Michel Oris. L'étude «VLV » constitue le troisième volet d'une série d'enquêtes transversales réalisées en 1979 et en 1994/5 menées dans les cantons de Genève et du Valais par le CIG (Centre Interfacultaire de Gérontologie) sous la direction du prof. Christian Lalive d'Épinay.

Pour mener à bien l'étude "VLV », une dizaine d'enquêteurs ont été engagés et formés dans chaque canton pour procéder aux entretiens « faceà-face ». Au cours de la formation, la procédure d'approche et le matériel utilisé, à savoir le questionnaire auto-administré, le calendrier de vie et le questionnaire standardisé «face-à-face », leur étaient présentés. Tout au long de la phase " enquête », ils étaient encadrés par des responsables de terrain qui distribuaient, contrôlaient et réceptionnaient leur travail. L'enquête s'est déroulée en suivant une procédure d'approche en trois étapes. Dans un premier temps, une lettre, qui présentait l'étude «VLV » et annonçait le téléphone d'un enquêteur, était envoyée aux personnes de l'échantillon. Dans un deuxième temps, l'enquêteur téléphonait aux personnes sélectionnées pour expliquer les objectifs de l'enquête, répondre aux questions éventuelles et demander si elles étaient d'accord de participer à l'étude. L'accord donné, l'enquêteur fixait un rendez-vous avec la personne âgée pour réaliser l'entretien « face-à-face » et lui faisait parvenir, par la poste, un questionnaire auto-administré et un calendrier de vie pour qu'elle les complète avant l'entretien. Dans un troisième temps, l'enquêteur se rendait à domicile ou en institution pour rencontrer la personne âgée et procéder à l'interview. Lors de l'entrevue, l'enquêteur " contrôlait » avec le participant si ce dernier avait bien rempli le questionnaire autoadministré et le calendrier de vie. Si tel n'était pas le cas, il l'aidait à les compléter ou, si nécessaire, à clarifier certaines informations. Finalement, l'enquêteur menait l'entretien «face-à-face » en saisissant directement les réponses sur un ordinateur portable ( Computer Assisted Personal Interview (CAPI) ») (Nicolet \& Oris, 2016). Afin d'avoir une population 


\section{Données et méthodes}

d'enquête qui soit la plus représentative de la population âgée suisse, une procédure d'approche alternative, "proxy», a été élaborée visant les personnes âgées ayant des difficultés cognitives (par exemple, des problèmes d'orientation dans le temps et l'espace) et qui, de ce fait, n'étaient pas en mesure de répondre seules aux questionnaires ou de participer à un entretien. Lorsque l'enquêteur repérait au cours du premier contact (téléphone) des problèmes cognitifs chez son interlocuteur, il lui demandait s'il avait dans son entourage proche une personne qui serait disposée à répondre à quelques questions le concernant. Les proches désignés («proxys») - pour la plupart, le partenaire, un enfant ou un soignant dans un établissement médicalisé - répondaient, lors d'un entretien «face-à-face », à une série de questions descriptives concernant la santé, l'entourage et les activités de la personne âgée concernée. Les questions plus subjectives du questionnaire principal, notamment celles concernant le réseau familial et les membres significatifs de la famille, n'étaient pas posées. Sur l'ensemble de l'échantillon, 555 " proches » ont été questionnés selon la procédure "proxy » (Nicolet \& Oris, 2016).

Pour les personnes sans troubles cognitifs, les questions posées portaient sur de nombreux thèmes comme les conditions de vie (logement, ressources économiques, etc.), le réservoir de parenté (partenaire, expartenaires, enfants, petits-enfants et fratrie), les relations familiales, amicales, la participation sociale (activités), la santé tant physique, fonctionnelle, psychologique que cognitive, les croyances et les valeurs. Toutes ces questions avaient pour objectif d'identifier l'ensemble des ressources économiques, démographiques, sociales, familiales, psychologiques et de santé dont disposent aujourd'hui les individus âgés en Suisse pour maintenir aussi longtemps que possible leur bien-être et leur autonomie. Environ un tiers de ces questions ont été reprises des enquêtes transversales précédentes afin de pouvoir comparer les résultats dans le temps. Par ailleurs, une majorité des indicateurs utilisés font référence à des mesures validées internationalement, permettant la confrontation des résultats "VLV » à ceux d'autres études internationales. Quant aux questions sur le « réseau familial», elles étaient posées au cours de l'entretien «face-à-face », mode de récolte le plus adéquat compte tenu de la complexité des questions « réseau » (Widmer et al., 2013). Les données «VLV » sont riches, interdisciplinaires et complexes; elles donnent une image complète, actuelle, de la diversité de la population âgée suisse. 


\subsubsection{L'échantillon genevois et son contexte socio-historique}

Les résultats dans cet ouvrage portent essentiellement sur les données genevoises; le choix de se centrer exclusivement sur un seul échantillon s'explique, en partie, par les questions de recherche. L'objectif principal de ce travail est de dégager différentes configurations familiales dans la vieillesse, reflétant des manières distinctes de définir la famille significative, et de mettre à jour les liens d'interdépendance positifs et négatifs qui les sous-tendent. Il ne s'agit pas de produire des résultats représentatifs sur les relations familiales dans la population âgée suisse ou de procéder à une analyse comparative entre cantons, mais plutôt de mettre le doigt sur certains processus relationnels qui interviennent au sein des configurations familiales. Bien que ce choix soit restrictif d'un point de vue statistique puisque l'effectif est petit, il permet néanmoins de se focaliser sur les différents processus relationnels qui sont au centre de ce travail, tout en neutralisant les effets probables des contextes socio-politico-économiques qui varient en fonction des cantons sondés et qui rendent plus complexe l'interprétation des résultats. L'analyse de l'impact des différents contextes cantonaux sur les configurations familiales en Suisse est un projet intéressant et nécessaire, mais cette analyse n'entre pas dans le cadre de ce travail.

Afin de mieux connaître les caractéristiques de l'échantillon genevois, il est nécessaire de décrire la population âgée à Genève et l'évolution qu'elle a suivie depuis 30 ans en fonction des multiples changements sociohistoriques qui ont marqué le 20e siècle. Comme nous l'avons indiqué précédemment, l'étude «VLV》 (2011) est le troisième volet de deux études transversales, l'une en 1979 et l'autre en 1994. La comparaison des résultats de ces trois enquêtes permet de décrire l'évolution sur 30 ans de la population âgée à Genève. Pour ce faire, nous nous appuyons sur le travail de recherche de Marie Baeriswyl (Baeriswyl, 2016) qui a dressé un portrait de la population âgée ${ }^{1}$ à ces trois époques en mettant en exergue les changements socio-historiques qui ont contribué à modeler la population âgée actuelle (2011) vivant à Genève.

1 Pour permettre la comparaison entre ces trois enquêtes, seules les personnes aptes de 65 à 94 ans vivant à domicile ont été considérées; les proxys et les personnes âgées en institution ont donc été écartées des analyses. 
Parmi ces changements socio-historiques, les progrès scientifiques, notamment médico-sociaux, ont marqué les pays industrialisés au cours du 20e siècle et ont contribué à allonger l'espérance de vie des personnes âgées en Suisse ${ }^{2}$. A Genève, ils ont entraîné une modification importante de la structure de la population genevoise des 65 ans et plus; en 1979, la part des âgés (65 ans et plus) de la population résidant à Genève est de $13.2 \%$ (2.8\% ont 80 ans et plus) alors qu'elle atteint $16.0 \%$ en 2011 (4,6\% ont 80 ans et plus $)^{3}$. Outre l'augmentation des âgés en Suisse et à Genève, les avancées dans le domaine médical ont permis d'améliorer leur santé. Si l'on considère le processus de fragilisation ${ }^{4}$, la comparaison des données sur 30 ans montre que ce processus est moins prononcé en 2011 que lors des années d'enquête précédentes. L'indice moyen de fragilité parmi les âgés résidant à Genève atteint 1.67 en 1979 et baisse significativement à 1.34 en 2011. En moyenne, ce sont les femmes qui sont les plus fragiles, quels que soient leur âge et la date de l'enquête. En 2011, elles affichent un score de fragilité de 1.43 alors que celui des hommes est de $1.02^{5}$. Le même constat peut être dressé pour les atteintes fonctionnelles; elles touchent une proportion moindre d'aînés et apparaissent plus tardivement que précédemment (vers 80 ans). A Genève, en 1979, ils sont $14 \%$ des répondants ${ }^{6}$ à avoir une mauvaise santé fonctionnelle alors qu'ils ne sont plus que $6 \%$ en 2011. Comme pour la fragilité, la proportion des femmes souffrant de limites fonctionnelles est globalement plus élevée que celle des hommes, augmentant surtout de manière marquée aux âges avancés, et cela quelle que soit la date de l'enquête. En 2011, elles étaient $9 \%$ à déclarer des difficultés fonctionnelles contre $5 \%$ des hommes. Pour ce qui

2 A la date de l'enquête VLV (2011), l'espérance de vie en Suisse est de 80.3 ans pour les hommes (72.3 ans en 1980) et de 84.7 ans pour les femmes (78.8 ans en 1980) (Source : OFS, Evolution des données démographiques, 2016).

3 Source : Office cantonal de la statistique, Population résidente totale selon le groupe d'âges quinquennal de 1970 à 2015, février 2016.

4 Pour une définition du processus de fragilisation, se référer à la section «Lâge et la santé » du chapitre « Facteurs explicatifs ».

5 Les analyses selon le sexe comprennent les résultats pour Genève et le Valais.

6 Il s'agit, pour rappel, uniquement de personnes aptes à participer à l'enquête et vivant à domicile. 
est de l'institutionnalisation des personnes âgées dans des EMS (Établissements médico-sociaux $)^{7}$, les chiffres de l'Office cantonal de la statistique montrent qu'en 2011, huit personnes sur dix ont 80 ans et plus et que $74 \%$ des résidents en EMS sont des femmes ${ }^{8}$.

Une meilleure santé générale et une espérance de vie plus longue ont des conséquences sur le réservoir de parenté des individus âgés. De 1979 à 2011 , la proportion des âgés qui ont au moins un enfant vivant est passé de $65 \%$ à $83 \%$; celle ayant au moins un petit-enfant de $41 \%$ à $67 \%$ et finalement celle ayant au moins une sœur ou un frère vivant de $68 \%$ à $72 \%$. Si l'on considère maintenant le statut matrimonial, la proportion des personnes mariées a augmenté de 1979 à 2011, passant de 49\% à 55\%. Il s'agit surtout d'hommes, quelle que soit la date de l'enquête. En 2011, les chiffres en disent long sur la surreprésentation masculine parmi les «mariés» : 76\% des hommes sont mariés contre $46 \%$ des femmes et leur situation matrimoniale reste stable, quel que soit leur âge. Quant au veuvage, la proportion des veuf(e)s a diminué : ils sont passés de $32 \%$ en 1979 à $21 \%$ en 2011. Contrairement au mariage, le veuvage de 1979 à 2011 se conjugue d'abord au féminin. En 2011, 32\% des femmes sont veuves contre seulement 8\% des hommes et le risque de perdre l'époux augmente rapidement en fonction de l'âge. A partir de 80 ans, le veuvage concerne plus de la moitié des femmes en 2011. Pour ce qui est des divorcés, ils sont minoritaires et leur proportion a légèrement augmenté en 30 ans, passant de $8 \%$ à $16 \%{ }^{9}$ et touche principalement les jeunes cohortes. Quant aux célibataires, leur proportion est faible. Ils représentent $8 \%$ des aînés genevois en 2011 alors qu'ils étaient $11 \%$ en 1979.

Outre les progrès en matière de médecine et d'hygiène, la démocratisation du système scolaire au cours du 20e siècle en Suisse a eu un impact important sur la population âgée genevoise actuelle. En 30 ans, le niveau d'instruction a augmenté de manière marquante au point que la part des personnes âgées ayant uniquement une formation obligatoire s'est fortement

7 Les établissements médico-sociaux renvoient aux institutions de long séjour pour les personnes âgées : ils offrent des prestations médico-sociales, de soins ainsi que des animations (Masotti, 2016).

8 Source : Office Cantonal de la Statistique (OCSTAT), Informations statistiques. Les établissements de santé à Genève : Résultats 2011, Mai, 2013.

9 Selon les statistiques, le divorce concerne plus d'un mariage sur deux à Genève en 2006 (Source : OCSTAT, Communications statistiques : Mariages et divorces. Evolution selon quelques déterminants socio-démographiques, 2008). 
réduite à Genève, devenant minoritaire en 2011 (16\%) alors qu'elle était largement majoritaire en 1979 (55\%). En 1979, 73\% des femmes âgées et $58 \%$ des hommes avaient uniquement une formation obligatoire. Ce n'est plus le cas que de $26 \%$ de femmes et $12 \%$ des hommes en 2011 . Bien que le niveau d'instruction se soit amélioré entre 1979 et 2011, les différences de genre restent importantes en 2011, particulièrement parmi les cohortes les plus anciennes. Ce résultat s'explique par la lenteur de l'insertion des femmes dans le système de formation post-obligatoire, notamment gymnasial, en Suisse (en 1970) et par la reconnaissance tardive du principe de l'égalité d'instruction dans la Constitution (1981). Jusqu'alors, le rôle de l'école visait avant tout à faire des filles de futures ménagères (Baeriswyl, 2016). Au delà du système de formation, la structure même de l'économie suisse s'est transformée au cours du 20e siècle avec les Trente Glorieuses et la croissance économique qui les caractérise : le secteur tertiaire s'est implanté, supplantant peu à peu le secteur primaire. Cette transformation de la structure économique se reflète en 2011 dans la part importante des employés et cadres (dernier emploi) parmi les individus âgés à Genève alors que celle des petits indépendants (dont les agriculteurs) est faible. De même, l'insertion massive des femmes sur le marché du travail dans les années 1960-1970 transparaît dans la structure de la population âgée en 2011. Alors qu'en 1979, et même en 1994, une grande part des femmes étaient professionnellement inactives au moment de l'âge de la retraite ( $52 \%$ en 1979 et $49 \%$ en 1994), les « inactives » ne constituent plus qu'une petite part en $2011(10 \%)^{10}$. Toutefois, l'insertion croissante des femmes sur le marché de l'emploi ne s'accompagne pas pour autant d'égalité. En effet, en 2011, les derniers emplois féminins sont majoritairement des postes d'employées alors qu'en fin de carrière, les hommes occupent davantage des postes supérieurs (cadres) (Baeriswyl, 2016).

La croissance économique mais aussi l'instauration progressive d'un système de prévoyance à trois piliers ${ }^{11}$ au cours du $20 \mathrm{e}$ siècle ont permis

10 Cette proportion peut être sous-évaluée puisque nous demandions aux enquêté(e)s de mentionner leur dernier emploi sans qu'ils précisent leur âge. Il se peut qu'entre le dernier emploi et l'âge de la retraite, il y ait eu une certaine période d' « inactivité » professionnelle.

11 Le premier pilier renvoie à l'Assurance vieillesse et survivant (AVS). Mise en vigueur en 1948, elle est régie par la Confédération et vise à assurer un montant existentiel minimum aux personnes de 65 ans et plus vivant en Suisse, indépendamment de toute activité professionnelle passée. Puis, l'AVS fut complétée par un deuxième pilier en 
d'améliorer la situation financière des personnes âgées en Suisse depuis 1979. Il n'en demeure pas moins qu'à Genève, une faible proportion d'aînés vit toujours en 2011 sous le seuil de la pauvreté (15\% contre 33\% en 1979) et qu'un certain nombre d'entre eux connaît la précarité (36\% contre $32 \%$ en 1979). Il s'agit surtout de ceux qui appartiennent aux cohortes les plus anciennes et qui n'ont pas pu participer au système des trois piliers. Par ailleurs, la situation financière des femmes en 2011 est plus difficile que celle des hommes, notamment parmi les plus âgées : 25\% des femmes peuvent être qualifiées de pauvres contre $17 \%$ des hommes. Les femmes sont particulièrement désavantagées concernant la prévoyance professionnelle (le deuxième pilier), celle-ci étant liée à la trajectoire professionnelle. Les cotisations au deuxième pilier s'effectuant seulement à partir d'un certain salaire, il exclut de fait les bas salaires, les trajectoires professionnelles discontinues ou les temps partiels - caractéristiques majeures de l'emploi féminin en Suisse (Baeriswyl, 2016; Kuehni et al., 2013). En 2011, par exemple, elle sont 57\% à toucher le deuxième pilier contre $72 \%$ des hommes. Quant au troisième pilier, les femmes ayant des revenus moindres que les hommes sont moins nombreuses à épargner pour leur retraite (Kuehni et al., 2013). Ainsi, malgré la croissance économique, la démocratisation du système de formation et le système de prévoyance en trois piliers, il reste une partie de la population âgée en Suisse et à Genève - des femmes pour la plupart - qui connaît en 2011 une certaine précarité socio-économique.

Finalement, la montée de l'individualisme au cours du 20e siècle a bouleversé les valeurs et les modes de vie en Suisse et plus particulièrement à Genève, en promouvant l'autonomie, le choix individuel, l'épanouissement personnel mais aussi l'égalité. Les âgés genevois sont plus nombreux en 2011 qu'en 1979 à préconiser l'indépendance des enfants adultes vis-àvis de leurs parents ( $83 \%$ vs $64 \%)$ et nettement moins nombreux à penser que les femmes doivent rester au foyer (13\% vs $58 \%)$. Les femmes sont plus nombreuses à rejeter une telle opinion : en 2011, seules $14 \%$ acceptent cette affirmation contre $21 \%$ des hommes. A noter que ce sont surtout les cohortes les plus récentes (les 65-74 ans) qui sont porteuses d'idées plus

1985 (obligatoire), celui de la LPP (prévoyance professionnelle), ayant pour objectif de maintenir le niveau de vie des personnes salariées après la retraite. Finalement, un troisième pilier (non obligatoire) complète les deux précédents piliers (épargne privée) (Baeriswyl, 2016; Kuehni et al., 2013). 
égalitaires et individualistes. Devenues adultes pendant les Trente Glorieuses, ces cohortes ont connu les changements des années 60-70 et les femmes ont pu profiter pleinement de leur droit de vote (1971), contrairement aux cohortes plus anciennes. Hormis le changement dans les valeurs et les opinions parmi les jeunes cohortes en 2011, l'individualisme montant a aussi bouleversé leurs pratiques relationnelles. En 2011, l'ami - lien choisi et personnalisé - occupe une place de plus en plus importante dans les réseaux personnels de la population âgée à Genève. Cette progression est la plus marquante entre 1979 et 1994. Ils sont, en 1979, 63\% à déclarer au moins un ami proche alors qu'en 1994, ils sont $81 \%$ et, en $2011,84 \%$. Les femmes sont, à ce titre, les plus enclines à l'amitié : en $2011,83 \%$ d'entre elles ont un ami proche contre $76 \%$ des hommes. Cet accent plus prononcé pour l'amitié influence la participation sociale des aînés genevois ; ceux-ci s'avèrent en effet plus enclins à la sociabilité amicale (visites entre amis) que ceux dans d'autres cantons comme le Valais.

En somme, les progrès dans la médecine, la démocratisation du système scolaire, la croissance économique et l'instauration d'un système de prévoyance ont permis à la population âgée de Genève d'améliorer ses conditions de vie et de santé (Baeriswyl, 2016). Cependant, il n'en demeure pas moins qu'une part non négligeable de personnes âgées résidant à Genève se trouvent dans des situations difficiles en termes de santé (limites fonctionnelles), de réserves démographiques (veuvage) ou encore de pauvreté. Par ailleurs, la description de l'évolution de l'échantillon genevois sur 30 ans met aussi en exergue que, malgré les changements socio-culturels qui se sont produits au cours de ce laps de temps, les inégalités de genre sont toujours présentes dans le canton de Genève en 2011 (Baeriswyl, 2016).

\subsubsection{Genève et sa politique de la vieillesse}

L'autonomie des personnes âgées, par le biais du maintien à domicile, constitue l'une des priorités des politiques de la vieillesse de la Confédération suisse. Le maintien à domicile renvoie aux différentes "pratiques, sociales et sanitaires qui peuvent être mises en place pour permettre à une personne de demeurer dans son lieu de vie habituel le plus longtemps possible, malgré l'existence de déficits physiques ou cognitifs » (Masotti, 2016, p.7). Le «maintien à domicile» fait généralement référence à une 
combinaison de prestations de soins médicaux ou paramédicaux, d'aides pratiques (ménage) mais aussi d'encadrement social : transports, repas à domicile, consultations sociales, foyers et hôpitaux de jour. Ce dispositif est perçu par le politique comme le garant d'une certaine qualité de vie et, surtout, de l' « autonomie » individuelle des personnes qui sont, de fait, reconnues dans leur capacité de décider et de prendre soin d'elles-mêmes (Masotti, 2016). Rester à la maison est aussi souhaité par les personnes âgées, le domicile étant perçu comme le lieu de repères et de liberté (Masotti, 2016). Ainsi, l'institutionnalisation intervient en dernier recours lorsque toutes les aides proposées à domicile ne suffisent plus pour assurer l'autonomie des personnes âgées, atteintes dans leur santé. Celles qui entrent en institution sont dès lors très âgées ou atteintes sévèrement dans leur santé. Par ailleurs, le maintien à domicile permet aux autorités cantonales de réduire les coûts des dépenses publiques liés à l'institutionnalisation des personnes âgées (EMS). Son développement est donc largement encouragé par les politiques cantonales, notamment à Genève (Conseil d'Etat de Genève, 2012).

En Suisse, ce sont surtout des associations à but non lucratif qui se chargent des soins à domicile (environ $80 \%$ ), viennent ensuite les organismes privés (15\%) et les infirmiers indépendants (5\%) (Masotti, 2016). Leur poids respectif dans le réseau d'aide et de soins à domicile varie fortement selon les cantons (Lucas, 2010, 2011). A noter que l'assurancemaladie de base (LAMal) prend en charge les prestations de soins mais les aides pratiques (ménage) sont à la charge des bénéficiaires sauf s'ils bénéficient d'assurances privées (complémentaires) ou de prestations complémentaires à l'AVS. Depuis 2011, avec le nouveau régime fédéral de financement des soins, les bénéficiaires doivent davantage contribuer aux frais des prestations qu'ils reçoivent. Certains cantons, comme le Tessin ou le Valais, prennent en charge ces frais. Outre les associations, les familles sont aussi sollicitées pour garantir le maintien à domicile des parents âgés. En effet, un tel dispositif implique la participation active des proches aidants, généralement des membres primaires de la famille, afin de compléter l'aide formelle (Masotti, 2016). Ces derniers doivent souvent être présents, coopérer, coordonner, informer le personnel soignant et apporter leur soutien émotionnel au parent âgé (Masotti, 2016). Comme pour les autres acteurs du réseau d'aide, la contribution des proches aidants dans le maintien à domicile du parent âgé peut aussi changer en fonction des 
cantons, selon ce qui est usuellement «attendu » dans les pratiques cantonales (Lucas, 2011). Encouragé par la Confédération dans sa politique de la vieillesse, la mise en place du maintien à domicile relève cependant des cantons et des communes en raison du fédéralisme de la Suisse (Lucas, 2010, 2011). Par conséquent, la façon dont les gouvernements cantonaux l'instaurent au sein de leur territoire fluctue d'un canton à l'autre avec des conséquences différentes sur les individus âgés et leurs proches (Lucas, 2011). Dans son étude portant sur les politiques traitant des démences séniles (Alzheimer), Lucas (2011) distingue trois modèles de politiques cantonales de la vieillesse : le modèle du «partenariat» (exemple : Genève), de la «responsabilisation » (exemple : Thurgovie) et du « secours » (exemple : Valais).

Genève - francophone, urbaine, protestante et de culture politique plutôt de centre-gauche - se catégorise parmi les politiques du «partenariat» (Lucas, 2011). Le gouvernement genevois vise en effet à favoriser le partenariat entre les différents intervenants de l'aide à domicile, à savoir les diverses associations en charge du maintien à domicile mais aussi les proches aidants et la personne dépendante. Ainsi, la personne âgée dépendante et les proches sont considérées comme des partenaires de soins pouvant orienter les dispositifs d'aide en fonction de leurs besoins. L'État genevois tend aussi à promouvoir la diversification des prestations, notamment par le biais d'incitations financières, afin qu'elles répondent à tous les besoins et que leur accès soit garanti à tous (Lucas, 2010, 2011). En 2011, environ $4 \%$ de la population résidant à Genève fait recours à ce type de prestations, $45 \%$ d'entre eux ont 80 ans et plus ${ }^{12}$. Le maintien à domicile à Genève repose donc sur un réseau privé, dense, diversifié et spécialisé, majoritairement composé d'associations que l'État subventionne tout en les invitant à se développer. Partenaires, leur relation implique un contrat de prestations, le contrôle des services dispensés et des négociations sur les modalités de réalisation qui s'appliquent ensuite sur l'ensemble du canton. A noter que le bénévolat et les aides privées payantes sont relativement peu présentes à Genève, contrairement à d'autres cantons (Lucas, 2011).

La Thurgovie - canton germanophone, protestant, composé de plusieurs petites villes, affichant politiquement une droite conservatrice renvoie plutôt au modèle de la «responsabilisation ». L'État thurgovien

12 Source : Office cantonal de la statistique (OCSTAT) : Informations statistiques.

Statistique de l'aide et des soins à domicile (Spitex), résultats 2011. 
s'investit peu financièrement et encourage le bénévolat et le développement de structures privées, payantes (Lucas, 2010, 2011). Celles-ci peuvent offrir aux personnes âgées, qui ont les moyens financiers, des services très diversifiés, flexibles et spécialisés (Masotti, 2016). Bien qu'il ne les subventionne pas, l'État cherche à responsabiliser les professionnels par l'information, la transmission de bonnes pratiques mais aussi par le contrôle de la qualité de leurs prestations (Lucas, 2010). Dans ce type de modèle, il revient aux personnes âgées et à leurs proches de prévoir et d'assumer, avec leurs propres ressources, le déclin de la santé et l'entrée en dépendance (Lucas, 2010, 2011). Seules les personnes âgées qui ont les moyens financiers peuvent accéder à des prestations de soins et d'aides à domicile soit par le biais de ces organismes privés, soit par le biais d'auxiliaires de vie (personnel domestique privé a priori non formé aux soins). Pour les autres, ce sont les proches aidants, c'est-à-dire les membres de la famille, qui doivent subvenir aux besoins d'aide et de soutien de la personne âgée dépendante (Lucas, 2011). C'est seulement en dernier recours, lorsque les aides familiales et privées sont épuisées, que l'État intervient (Lucas, 2010).

Le Valais - bilingue, catholique, rural et politiquement conservateur se catégorise plutôt dans le modèle du « secours ». Dans ce cas de figure, l'État (canton) souhaite porter secours aux plus démunis et soutenir leur famille : les ayants-droit sont définis en fonction de critères précis tels que le revenu et la présence de problèmes spécifiques (Lucas, 2010). Occupant une place dominante dans l'organisation du réseau d'aide, l'État est actif dans la planification sanitaire (hôpitaux et homes), il délègue cependant sa gestion concrète aux régions et repose surtout sur les services de proximité, c'est-à-dire sur des réseaux de soins peu diversifiés et peu flexibles, et les solidarités informelles telles que la famille et la communauté (les voisins, les amis, etc) (Lucas, 2010, 2011). Au Tessin, dont la politique de la vieillesse peut aussi être catégorisée dans ce type de modèle, les services d'aide à domicile interviennent de manière subsidiaire, seulement si les proches ne sont pas à même d'apporter l'aide requise (Masotti, 2016). Il est en effet attendu que les «aidants naturels » - les enfants et, plus précisément, les filles - s'occupent de leurs parents âgés (Lucas, 2010). Dans le modèle politique du « secours », l'individu âgé dépendant qui reçoit de l'aide à domicile n'est pas un partenaire mais un «assisté » ayant des difficultés, tout comme sa famille, bien que largement sollicitée (Lucas, 2010, 2011). Le Tessin, comme le Valais, octroie des allocations directes 
aux proches aidants mais ces contributions «symboliques » ne compensent pas les pertes de revenu liées à une diminution ou à un retrait de l'activité professionnelle (Masotti, 2016). Dans un tel modèle politique, les proches aidants sont contraints de subvenir eux-mêmes aux besoins de leur parent dépendant, les services d'aide à domicile ne répondant pas à tous les besoins, ou de faire appel à des aides privées payantes s'ils en ont les moyens ou s'ils sont au bénéfice de subsides (bas revenu) (Masotti, 2016).

Les politiques de la vieillesse sont donc diverses au sein de la Suisse et leur influence sur les conditions de vie des âgés et de leurs proches peut être très différente (Lucas, 2011). En effet, selon le modèle en vigueur, les membres de la famille sont sollicités de manière différente au point que, dans certains cantons, ils prennent en charge une grande partie des aides et des soins à domicile. Au vu de ces observations, on peut faire l'hypothèse que les contextes cantonaux, par le modèle de politique qu'ils appliquent, ont un impact non seulement sur la façon dont les individus conçoivent leur famille significative mais aussi sur la manière dont s'organisent les liens d'interdépendance au sein de leur configuration familiale. En d'autres termes, les échanges de soutien tout comme les tensions au sein des familles peuvent varier dans leur intensité en fonction des cantons. Dans les cantons où les aides étatiques sont faibles ou inadéquates, le maintien à domicile du parent âgé dépendant est synonyme de fortes contraintes pour les proches aidants, les filles notamment. De telles contraintes sont à même de créer des tensions au sein des configurations familiales. Ces contraintes sont d'autant plus prononcées si les filles ont des ressources limitées en termes de moyens financiers, de réserves démographiques (fratrie), d'énergie et de temps ou si elles sont soumises à d'autres responsabilités économiques, professionnelles et familiales. Comparés aux autres types de modèles, celui du "partenariat» en vigueur à Genève semble le plus tenir compte des besoins des proches aidants en offrant aux personnes âgées à domicile une palette de services et de prestations. Ces divers services "permettraient » aux parents âgés de s'affranchir des interdépendances familiales et de renforcer leur autonomie individuelle, et de limiter ainsi les tensions intrafamiliales (Lucas, 2010). Ceci dit, au-delà du discours politique axé sur l'autonomie individuelle, le dispositif «maintien à domicile » requiert la présence de la famille ainsi que sa participation active afin que l'aide professionnelle se réalise de manière adéquate. Ainsi, même à Genève, où les services d'aide à domicile sont bien développés, le degré d'engagement des proches aidants (familiaux) dans le maintien à 
domicile du parent âgé peut avoir un impact sur la façon dont ce dernier perçoit sa famille significative mais aussi sur la manière dont il perçoit les liens d'interdépendance qui la caractérisent.

\subsubsection{Description de l'échantillon genevois}

L'échantillon genevois comprend au départ 704 individus âgés ${ }^{13}$. Parmi les 704, 126 participants ont été retirés de l'échantillon en raison de leurs difficultés cognitives, ceux-ci n'étant pas capables de répondre seuls aux questions, notamment à celles portant sur le réseau familial. Nous avons également exclu 15 personnes des analyses : bien qu'aptes, elles n'ont répondu à aucune des questions portant sur le réseau familial. Finalement, 563 individus âgés, vivant à domicile et en institution, composent l'échantillon d'analyse. Le tableau 4.1 présente les principales caractéristiques de l'échantillon utilisé dans les analyses $(\mathrm{n}=563)$ et celles de l'échantillon total, c'est-à-dire celui de départ $(\mathrm{n}=704)$.

L'échantillon d'analyse comporte $49 \%$ de femmes, $40 \%$ ont entre 65-74 ans, $35 \%$ ont entre $75-84$ ans et $25 \%$ ont entre 85 ans et plus. Au niveau de la formation, $23 \%$ des répondants ont un niveau d'éducation élevé ${ }^{14}, 61 \%$ ont un niveau d'éducation moyen et $16 \%$ ont un niveau d'éducation faible. Quant au revenu, 14\% déclarent un revenu élevé, 48\% un revenu moyen et $38 \%$ un revenu bas. A noter que $34 \%$ sont d'origine étrangère (nés étrangers, naturalisés ou non). Pour ce qui est du statut conjugal, $55 \%$ sont mariés, $22 \%$ sont veufs, $12 \%$ sont divorcés, $7 \%$ sont célibataires et $4 \%$ sont cohabitants. Ils sont $61 \%$ à avoir un partenaire (marié ou non, cohabitant ou non), $82 \%$ ont au moins un enfant (vivant), $69 \%$ ont des petits-enfants et $68 \%$ ont au moins un membre de leur fratrie encore vivant au moment de l'entretien. Par ailleurs, $40 \%$ d'entre eux vivent seuls (non indiqué dans le tableau). Du point de vue de la santé, une grande majorité d'entre eux sont robustes (75\%), c'est-à-dire qu'ils sont en mesure d'effectuer, sans l'aide d'une tierce personne, les huit activités

13 Précisons que le taux de réponses à Genève est de 36\%. Bien que faible, ce résultat correspond pourtant aux taux de réponses (moyens) obtenus généralement dans les enquêtes portant sur les personnes âgées en Suisse (De Luca \& Peracchi, 2005).

14 Se référer à la sous-section des « Mesures » (point 4.2.4 facteurs explicatifs) pour avoir le détail des modalités et du codage. 
Tab. 4.1: Statistiques descriptives de l'échantillon genevois $(\mathrm{N}=563)$.

\begin{tabular}{|c|c|c|}
\hline \multirow{3}{*}{ Genre } & \multicolumn{2}{|c|}{ Échantillon de Genève (\%) } \\
\hline & \multirow[t]{2}{*}{ Analyse $(\mathrm{n}=563)$} & \multirow[t]{2}{*}{ Total $(n=704)$} \\
\hline & & \\
\hline Féminin & 49 & 50 \\
\hline Masculin & 51 & 50 \\
\hline \multicolumn{3}{|l|}{$\hat{A g e}$} \\
\hline $65-74$ ans & 40 & 33 \\
\hline $75-84$ ans & 35 & 33 \\
\hline$\geq 85$ ans & 25 & 34 \\
\hline \multicolumn{3}{|l|}{$\dot{E}$ ducation } \\
\hline Bas & 16 & 21 \\
\hline Moyen & 61 & 58 \\
\hline Élevé & 23 & 21 \\
\hline \multicolumn{3}{|c|}{ Revenu ajusté à la taille du ménage } \\
\hline Bas & 38 & 41 \\
\hline Moyen & 48 & 46 \\
\hline Élevé & 14 & 13 \\
\hline \multicolumn{3}{|l|}{ Citoyenneté } \\
\hline Né(e) Suisse & 66 & $-\dagger$ \\
\hline Né(e) étranger(ère) & 34 & $-\dagger$ \\
\hline \multicolumn{3}{|l|}{ Statut conjugal } \\
\hline Marié(e) & 55 & 58 \\
\hline Cohabitant(e) & 4 & 3 \\
\hline Veuf(ve) & 22 & 22 \\
\hline Divorcé(e) & 12 & 11 \\
\hline Célibataire & 7 & 6 \\
\hline \multicolumn{3}{|l|}{ Réserves démographiques } \\
\hline A un partenaire & 61 & 62 \\
\hline A un/des enfant(s) & 82 & 84 \\
\hline A un/des petit-enfant(s) & 69 & 72 \\
\hline $\mathrm{A}$ un/des frère(s)/sœur(s) & 68 & 67 \\
\hline \multicolumn{3}{|l|}{ Santé fonctionnelle } \\
\hline Robustesse AVQs & 75 & 64 \\
\hline Difficultés AVQs & 16 & 16 \\
\hline Dépendance AVQs & 9 & 20 \\
\hline \multicolumn{3}{|l|}{ Institutionnalisation } \\
\hline Non institutionnalisé(e) & 93 & 85 \\
\hline Institutionnalisé(e) & 7 & 15 \\
\hline
\end{tabular}

Notes : 15 participants $(\mathrm{N}=578)$ n'ont pas répondu aux questions portant sur le réseau familial; ceux-ci ont été exclus des analyses. †La question de la naturalisation n'a pas été posée aux proxys. 
de la vie quotidienne (AVQs) $)^{15}$. Une faible minorité (16\%) déclare avoir des difficultés à réaliser seule une ou plusieurs des huit activités de la vie quotidienne. En tout, 9\% des personnes âgées de l'échantillon d'analyse sont considérées comme dépendantes sur les AVQs; elles ont besoin de l'aide d'une tierce personne pour accomplir l'une ou plusieurs de ces activités. Par ailleurs, une faible minorité (7\%) des répondants sont institutionnalisés, vivant dans des établissements médico-sociaux ou dans des appartements sécurisés.

La comparaison des deux échantillons - celui de l'analyse versus celui de départ - montre que globalement l'échantillon d'analyse reflète dans les grandes lignes l'échantillon total, à l'exception de l'âge, de la santé, du placement en institution et, dans une moindre mesure, du niveau d'éducation. Après l'exclusion des personnes présentant des difficultés cognitives, l'échantillon d'analyse inclut davantage de personnes âgées de moins de 85 ans $(75 \%$ vs $66 \%)$ et en bonne santé fonctionnelle (75\% vs 64\%) que l'échantillon total. Par ailleurs, la proportion de personnes institutionnalisées est nettement plus faible dans l'échantillon d'analyse (7\%) que dans l'échantillon total (15\%). A noter que les individus ayant un niveau d'éducation moyen à élevé sont davantage représentés dans l'échantillon d'analyse que dans l'échantillon de départ ( $84 \%$ vs $79 \%$ ). L'âge relativement jeune, les bonnes conditions de santé et le bon niveau d'éducation des répondants qui caractérisent l'échantillon d'analyse s'expliquent par le retrait de l'échantillon de départ des personnes ayant des problèmes cognitifs, celles-ci étant généralement plus âgées, en plus mauvaise santé ou affichant un niveau d'éducation plus bas - majoritairement des femmes (Oris et al., 2016).

La sous-représentation dans l'échantillon des «très-vieux » et de ceux qui sont le plus atteints dans leur santé a nécessairement des implications sur les résultats, les effets d' «âge» sur les configurations familiales ne pouvant être estimés à leur juste valeur. En d'autres termes, l'impact du déclin de la santé et celui de la sélectivité socio-émotionnelle risquent d'être sousévalués en raison de la faible proportion des «très-vieux » et de ceux qui

15 Les huit activités de la vie quotidienne (AVQ) considérées sont : se laver, s'habiller et se déshabiller, manger et couper les aliments, se coucher et se lever, se déplacer d'une pièce à l'autre (Katz, Ford, Moskowitz, Jackson, \& Jaffe, 1963), se mouvoir comme monter ou descendre un escalier, se déplacer à l'extérieur du logement, et parcourir à pied 200 mètres au moins (Rosow \& Breslau, 1966). 
sont en mauvaise santé dans l'échantillon d'analyse. Ce biais est d'autant plus marqué que les «très-vieux », qui sont à la fois « aptes » et d'accord de participer à un entretien, peuvent présenter un profil particulier, celui de « survivant», qui les différencie des autres individus du même âge (même cohorte). Ils peuvent en effet se distinguer de ces derniers par un meilleur état de santé général ou encore par le recours à des mécanismes d'adaptation efficaces (Lalive d'Epinay \& Spini, 2008). En raison de ces effets de sélection dans le grand âge, il est possible que les « très-vieux » de l'échantillon d'analyse - ou ceux qui ont des difficultés fonctionnelles - ne se différencient pas des autres répondants plus jeunes ou en meilleure santé quant à leur « engagement» actif ou à leur autonomie au sein de leur configuration familiale ou ne se montrent pas plus sélectifs dans leurs relations familiales que les plus jeunes ou que ceux qui sont en meilleur santé (sélectivité socio-émotionnelle). Ceci dit, même si les questions sur le réseau familial avaient été posées à l'ensemble de l'échantillon total, y compris aux individus âgés ayant des problèmes cognitifs, la question de la qualité des données récoltées se serait posée. En effet, des études portant sur des populations plus jeunes montrent que les individus ayant des déficiences intellectuelles peinent à percevoir l'ensemble des relations au sein de leur réseau familial, notamment celles avec lesquelles ils n'ont pas de liens directs (Widmer, Kempf-Constantin, \& Carminati, 2010). Ces résultats se vérifient aussi chez les individus âgés ayant des atteintes cognitives : ils ne perçoivent pas les «trous » structurels au sein de leur réseau personnel (Cornwell, 2009a). Décrire son réseau personnel et s'y situer nécessitent de bonnes facultés cognitives. La prise en compte des personnes âgées ayant des difficultés cognitives dans les analyses aurait donc comporter d'autres biais.

\subsection{Les mesures}

Nous introduisons dans cette section les questions relatives au réseau familial, posées aux participants lors de l'entretien «face-à-face ». Celles-ci sont issues de la méthode «Family Network Method (FNM)», développée à la fin des années 90 par le prof. Eric Widmer et ses collègues (Widmer \& La Farga, 2000; Widmer, 2016; Widmer et al., 2013). Cette méthode vise à approcher la famille dans une perspective configurationnelle et à dépasser 
les limites des outils statistiques classiques qui se contentent de recenser les réserves démographiques. Cette méthode comprend deux parties. La première sert à identifier l'ensemble des membres significatifs de la famille qui forment les configurations familiales et la deuxième, à dégager l'organisation particulière des liens d'interdépendance positifs et négatifs qui caractérise chaque configuration et à en identifier les propriétés structurelles (Widmer, 2006; Widmer \& La Farga, 2000; Widmer et al., 2013). Nous présentons également les indices de l'analyse de réseaux utilisés dans les analyses pour approcher ces différentes propriétés structurelles et pour déterminer le type le capital social produit dans chaque configuration familiale. Puis, nous expliquons comment nous avons construit empiriquement la typologie des quatre modes de conflit et de support, évoquée précédemment. Nous terminons cette section par la présentation des variables utilisées pour mesurer les différents facteurs explicatifs mentionnés dans les chapitres théoriques.

\subsubsection{Les membres significatifs de la famille}

Les indicateurs démographiques axés sur des critères de sang ou d'alliance ne permettent pas d'identifier les membres significatifs de la famille (Firth, Hubert, \& Forge, 1970; Levin, 1993 ; Mac Rae, 1992; Widmer, 2016). Pour y parvenir, la « Family Network Method» propose que ce soit les répondants eux-mêmes qui énumèrent au moment de l'entretien les membres de leur famille qu'ils considèrent comme significatifs. Pour ce faire, un générateur de noms est utilisé, c'est-à-dire que les enquêteurs demandent aux participants lors de l'entretien de nommer par le prénom les personnes qui sont, selon eux, des membres significatifs de leur famille. La question est : « Je vais vous demander de me citer les prénoms des personnes que vous considérez ACTUELLEMENT comme des membres significatifs (importants) de votre FAMILLE? Par membres significatifs (importants) de votre famille, nous entendons des personnes qui ont joué un rôle, soit positif (qui vous ont aidé, soutenu, défendu), soit négatif (qui vous ont mis en colère) DURANT L'ANNÉE EN COURS. Limitez-vous à un maximum de 5 personnes ». Les majuscules dans l'énoncé de la question figurent telles quelles dans le questionnaire afin d'attirer l'attention des enquêteurs sur ces points importants. 


\section{Données et méthodes}

Comme le révèle la question, nous demandons aux enquêtés de lister uniquement les membres « significatifs » de leur famille et non les membres familiaux avec qui ils interagissent le plus (Widmer, 2006, 2016; Widmer et al., 2013). L'objectif est d'identifier au sein de la famille les personnes qui sont les plus importantes pour les individus questionnés, à savoir celles auxquelles ils sont le plus émotionnellement attachés, quelle que soit la fréquence de leurs interactions. La méthode «Family Network» insiste sur le terme « famille » sans pour autant donner une définition de la notion de «famille» afin de permettre aux répondants de donner leur propre conception de la famille (Widmer, 2006, 2016; Widmer et al., 2013). Lors de l'entretien, les enquêteurs ont en effet pour consigne de ne pas intervenir et de laisser les participants lister les personnes qu'ils considèrent comme faisant partie de leur famille significative, ce pour autant que ces dernières soient encore « en vie » et fassent référence à des êtres « animés ». De fait, une telle perspective autorise l'inclusion des animaux de compagnie dans la famille significative, si ceux-ci sont mentionnés comme membres significatifs de la famille. Par ailleurs, le terme «significatif» qualifie les membres de la famille qui ont joué un rôle soit «positif», soit «négatif» durant l'année en cours (Widmer, 2006, 2016; Widmer et al., 2013). Certains membres de la famille sont source soit d'émotions positives, soit négatives, soit les deux à la fois, ce qui ne les rend pas moins « significatifs » (Fingerman et al., 2004; Widmer, 1999a; Widmer \& La Farga, 2000). Cette consigne permet donc d'identifier les membres de la famille qui sont considérés comme significatifs non seulement parce qu'ils sont aimés et appréciés mais aussi parce qu'ils sont à l'origine d'énervements, de jalousies, de soucis et de tensions (Widmer, 1999a; Widmer \& La Farga, 2000; Widmer, 2016). Finalement, en raison du caractère dynamique des configurations familiales (Elias, 1991; Quintaneiro, 2005; Widmer, 2016), l'énoncé de la question précise aux répondants qu'il s'agit de lister les membres de leur famille qu'ils considèrent « actuellement » comme significatifs et non dix ans auparavant. Centrés sur leur configuration familiale actuelle, il est plus facile pour les répondants de décrire le plus précisément possible leurs liens d'interdépendance.

Une fois avoir obtenu la liste des membres significatifs de la famille, les enquêteurs questionnent les participants sur le profil sociodémographique de chaque personne citée (Widmer, 2006, 2016; Widmer et al., 2013). Il est ainsi demandé aux répondants l'âge, le sexe [ $1=$ femme, 
$2=$ homme], le niveau d'éducation [ $1=$ aucune scolarité achevée ou scolarité obligatoire, $2=$ apprentissage ou école professionnelle, $3=$ maturité ou école normale, $4=$ formation ou école professionnelle supérieure, $5=$ université ou école polytechnique], et le lieu de vie de chaque membre significatif de la famille mentionné. Ensuite, les enquêteurs interrogent les participants sur leur lien de parenté avec les personnes listées. Les enquêteurs ont pour consigne de noter avec précision le lien de parenté en le décortiquant scrupuleusement, tel que la «fille de ma fille» au lieu de petite-fille ou la «partenaire de mon fils » au lieu de ma belle-fille (Widmer, 2006, 2016). Une telle décomposition des liens de parenté permet non seulement de pallier à l'imprécision de la langue française - qui, bien souvent, renvoie au même terme des liens de parenté distincts - mais aussi de repérer le processus de « chaining », mécanisme sous-jacent du développement des interdépendances, qui met en lien des individus reliés par un ou plusieurs membres communs. Une fille, par exemple, met en lien son conjoint («partenaire de la fille») et son fils (« fils de la fille») avec le parent âgé. Finalement, l'enquêteur questionne le répondant sur la qualité de ses relations avec chacun des membres de la famille cités. Il lui est notamment demandé depuis combien d'années il les connaît, la fréquence de leurs contacts en termes de rencontres, téléphones ou e-mails [ $1=$ jamais ou presque, $2=$ moins souvent, $3=$ une ou deux fois par mois, $4=$ une ou deux fois par semaine, $5=$ tous les jours ou presque], et le degré de confiance qu'il leur portent $[1=$ pas de confiance du tout, $2=$ peu de confiance, $3=$ une certaine confiance, $4=$ une grande confiance, $5=$ une confiance absolue].

Cette technique de "free-listing », particulièrement utilisée en psychologie sociale, laisse les participants définir, eux-mêmes, leur propre famille significative et représente une technique utile pour étudier les définitions « personnelles » de la famille et sortir du carcan bio-légal (Levin \& Trost, 1992; Levin, 1993; Widmer, 2016; Widmer et al., 2013). Elle permet par ailleurs de produire des réseaux ego-centriques, c'est-à-dire axés sur les liens personnels de l'individu étudié (ego) (Kadushin, 2012; Widmer, 1999a; Widmer et al., 2013). Cette technique est depuis longtemps utilisée dans la recherche sur la famille (Widmer, 1999a; Widmer et al., 2013) mais elle est aussi depuis peu utilisée en gérontologie sociale pour construire les réseaux personnels des individus âgés, jugée plus neutre que l'approche «classique » axée sur les rôles sociaux (Cornwell et al., 2008, 
2009). En effet, l'approche « classique », privilégiée dans les études se référant au modèle du convoi, tend à inférer un degré de proximité affective aux membres du réseau personnel en fonction du rôle qu'ils jouent par rapport à l'individu interviewé (Cornwell et al., 2008, 2009). Cependant, la technique de «free-listing » n'a jamais été utilisée en gérontologie sociale pour construire plus spécifiquement des réseaux familiaux. Pourtant, elle a d'ores et déjà montré son efficacité dans plusieurs études visant à identifier les membres significatifs de la famille dans d'autres classes d'âge (Levin \& Trost, 1992; Levin, 1993; Widmer \& La Farga, 2000; Widmer, 2016). Nous l'utilisons dans cet ouvrage pour mettre en exergue les principaux membres significatifs de la famille dans la vieillesse.

\subsubsection{Le capital social}

Après avoir listé l'ensemble des membres significatifs de la famille, la méthode «Family Network Method (FNM)» propose une série de questions visant à détecter les relations d'interdépendance entre les différents membres significatifs de la famille. Pour ce faire, l'enquêteur demande au répondant de préciser non seulement toutes les relations qu'il entretient avec chacun des membres significatifs qu'il a cités, mais aussi toutes les relations qui relient entre eux tous les membres mentionnés (Widmer \& La Farga, 2000; Widmer, 2006; Widmer et al., 2013). Quatre types de liens ont été considérés lors de l'entretien : le soutien émotionnel, l'aide pratique, l'influence et les conflits. Ces questions permettent de donner une vision globale de la façon dont s'organisent les liens d'interdépendance au sein de la configuration familiale, et cela aussi bien sur des dimensions positives que négatives (Widmer, 2016; Widmer et al., 2013). Ces questions mettent en exergue les propriétés structurelles de chaque configuration et, par conséquent, le type de capital social qui y est produit. Pour mesurer le capital social, nous nous sommes centrés essentiellement sur le soutien émotionnel. Celui-ci est moins limité par la distance géographique ou par la santé physique que ne l'est l'aide instrumentale. De plus, dans le domaine familial, l'émotionnel tend à colorer l'ensemble des échanges aussi bien affectifs, instrumentaux que financiers. En ce sens, un prêt d'argent peut être davantage perçu comme un signe d'amour que comme une transaction. C'est pour ces raisons que nous avons privilégié les liens de soutien émotionnel pour mesurer le capital social. 
Dans l'étude «VLV », le soutien émotionnel est défini en termes d'accompagnement ou de réconfort moral dans des situations difficiles. Pour le mesurer, la question suivante est posée aux participants : "Qui donnerait $d u$ soutien émotionnel à X lors de problèmes légers (par exemple : lorsque $X$ est triste ou bien lorsque $X$ a eu une journée difficile, qui peut l'aider, le consoler)? ». " $\mathrm{X}$ » correspond à chaque membre de la configuration familiale du répondant - considéré l'un après l'autre lors de l'entretien - y compris le répondant (Widmer, 2006, 2016; Widmer et al., 2013). Dans l'énoncé de la question, le «conditionnel » est utilisé - «Qui donnerait...?»-afin de saisir la perception que les individus ont des relations de soutien émotionnel au sein de leur configuration familiale. On s'intéresse dans cette question à la représentation et non aux relations effectives entre les membres de la configuration familiale. La méthode «Family Network » repose plus sur une approche socio-cognitive que descriptive des liens d'interdépendance (Widmer \& La Farga, 2000). Il est évident que la perception d'un individu des liens au sein de sa configuration ne traduit pas avec exactitude la réalité; cette perception est en effet tributaire de ses sentiments (satisfaction) vis-à-vis de ses relations et du support qu'il reçoit des membres de sa famille (Kadushin, 2012; Krackhardt, 1987). De plus, la perception du répondant peut se révéler fort différente de celle des autres membres de sa configuration (Krackhardt, 1987; Widmer \& La Farga, 2000). Ceci dit, comme le relève Krackhardt (1987), les schémas cognitifs des structures sociales (représentation) se forment au gré des expériences relationnelles (bonnes ou mauvaises) - dans notre cas, familiales - des individus. Ces schémas renvoient plus à une «moyenne» d'expériences familiales qu'à des exemples de relations précises variant selon les moments et les circonstances (Kadushin, 2012). Ces schémas cognitifs s'avèrent pertinents pour appréhender le «capital social » puisque celui-ci est difficilement saisissable en utilisant des indicateurs mesurant les échanges effectifs à un moment précis dans le temps. Le capital social se construisant au gré du temps et des échanges, seule une représentation «globale» des échanges familiaux permet de détecter si les configurations familiales produisent du capital social et d'en déterminer le type.

Après avoir passé en revue l'ensemble des liens entre les différents membres de la configuration familiale, la méthode "Family Network» permet de construire, pour chaque personne interviewée, la matrice sociométrique de soutien émotionnel. Celle-ci indique quels membres, y 


\section{Données et méthodes}

compris le répondant, donnent du soutien aux autres membres de la configuration et quels membres, y compris le répondant, reçoivent du soutien des autres membres de la configuration familiale (Widmer, 2016; Widmer et al., 2013). Sur la base de ces matrices, nous avons calculé plusieurs indices d'analyse de réseaux, ceux usuellement considérés dans les études traitant des réseaux personnels (ego-centriques) (Berkman et al., 2000; Broese Van Groenou \& Van Tilburg, 2007; Hanneman \& Riddle, 2005; Moren-Cross \& Lin, 2006; Scott, 2000). Ces divers indices mesurent les propriétés structurelles qui caractérisent les configurations familiales; ils signalent, selon leur score, le volume et le type de capital social que génère chaque configuration familiale (Widmer, 2006, 2016; Widmer et al., 2013). Nous avons calculé ces indices pour chaque répondant de l'échantillon (Scott, 2000; Widmer et al., 2013). Ces calculs ont été effectués sous R (R Core Team, 2015). Nous avons considéré six indicateurs du capital social dans cet ouvrage : la taille, la réciprocité, la densité, le degré rentrant (In-degree), le degré sortant (Out-degree) et la centralité du répondant.

Les trois premiers, la taille, la réciprocité et la densité, mesurent le capital social de type « chaîne ». La taille correspond au nombre de membres familiaux cités comme significatifs par les répondants (Widmer, 2006). Il s'agit du nombre de membres significatifs qui composent la configuration familiale. Cet indicateur varie de 0 à $5 ; 5$ étant le nombre maximum possible de membres cités comme significatifs. Dans l'échantillon genevois, la taille moyenne des configurations familiales est de «3.39» $(\mathrm{SD}=1.73)$. La réciprocité indique jusqu'à quel point les liens de soutien émotionnel entre les différents membres de la configuration familiale sont réciproques (Berkman et al., 2000; Broese Van Groenou \& Van Tilburg, 2007; MorenCross \& Lin, 2006; Rao \& Bandyopadhyay, 1987). Elle mesure la tendance des dyades à établir des échanges mutuels de soutien (Rao \& Bandyopadhyay, 1987). Plus précisément, cet indice se rapporte au nombre de liens réciproques (soutien) sur le nombre de dyades connectées au sein de l'ensemble de la configuration familiale. Cet indicateur varie de 0 à $1 ; 1$ indiquant que toutes les dyades connectées au sein de la configuration familiale ont des échanges de soutien réciproques. La moyenne de réciprocité dans l'ensemble des configurations familiales de l'échantillon atteint « 0.39 » $(\mathrm{SD}=0.33)$. Dans la littérature, la réciprocité est considérée comme un indicateur d'équilibre entre ce qui est donné et reçu dans l'ensemble du réseau familial; elle en garantit la cohésion et la stabilité (Broese Van Groenou \& Van Tilburg, 2007; Offer, 2012; Rao \& Bandyopadhyay, 1987). 
A l'inverse, la non réciprocité indique un déséquilibre dans les relations dyadiques au sein du réseau (Broese Van Groenou \& Van Tilburg, 2007).

Quant à la densité, elle indique le degré de connexion entre les différents membres du réseau (Berkman et al., 2000; Broese Van Groenou \& Van Tilburg, 2007; Cornwell et al., 2008, 2009; Moren-Cross \& Lin, 2006). Elle mesure plus précisément jusqu'à quel point les membres de la configuration sont interconnectés les uns aux autres au travers d'échanges de soutien émotionnel. Une densité élevée signale que les membres du réseau familial sont tous, ou presque tous, connectés les uns aux autres par des relations de soutien. Pour calculer cet indice, on divise le nombre de liens de soutien déclarés par les répondants sur les liens potentiels, à savoir le nombre de paires possibles entre les différent membres de la configuration familiale, y compris celles comprenant les répondants (Broese Van Groenou \& Van Tilburg, 2007; Hanneman \& Riddle, 2005; MorenCross \& Lin, 2006). Dans le calcul, on considère les liens «directs» puisque le soutien donné à l'individu « $\mathrm{A}$ » par l'individu « $\mathrm{B}$ » peut être différent du soutien offert par « $\mathrm{B}$ » $\mathrm{à}$ « $\mathrm{A}$ ». Cet indice varie de 0 à $1 ; 1$ indiquant que tous les membres de la famille cités comme significatifs sont interconnectés. La densité moyenne de l'ensemble des configurations familiales de l'échantillon est de «0.37» $(\mathrm{SD}=0.29)$. Dans la littérature, la densité s'associe à la cohésion et à la stabilité du réseau puisque les membres d'un réseau qui sont fortement interconnectés interagissent généralement depuis longtemps, ainsi qu'à un meilleur accès aux ressources du réseau telles que l'information et le soutien (Broese Van Groenou \& Van Tilburg, 2007; Kadushin, 2012; Moren-Cross \& Lin, 2006). Ces trois premiers indices vont de pair : plus le réseau est petit, plus les membres tendent à être interconnectés (forte densité) au travers d'échanges réciproques (forte réciprocité). Si la plupart de ces conditions sont réunies, le capital social produit est de type « chaîne ».

Alors que la taille, la réciprocité et la densité ont trait à l'organisation des échanges de soutien au sein des configurations familiales, le degré rentrant (In-degree), le degré sortant (Out-degree) et la centralité du répondant renseignent davantage sur la situation d'ego (le répondant) au sein de sa configuration familiale et sont généralement utilisés pour mesurer le capital social de type «pont». Les deux premiers indicateurs, le degré rentrant (In-degree) et le degré sortant (Out-degree), évaluent l'importance d'ego dans les échanges au sein de son réseau personnel (Kadushin, 2012). Ils indiquent si le répondant a des liens directs, réciproques ou non, avec 
les autres membres de son réseau (Broese Van Groenou \& Van Tilburg, 2007; Moren-Cross \& Lin, 2006).

Le degré rentrant (In-degree) du répondant se réfère au nombre de membres significatifs que le répondant soutient (directement) au sein de sa configuration familiale (Widmer, 2006). Il informe de l'importance du répondant en tant que source de soutien au sein de son réseau familial. Un degré rentrant (In-degree) élevé indique que le répondant se perçoit comme un pourvoyeur de soutien très actif au sein de sa configuration familiale. Cet indice varie de 0 à $5 ; 5$ indiquant que le répondant estime qu'il soutient tous les membres significatifs de sa configuration familiale. Le degré rentrant (In-degree) moyen de l'ensemble des répondants au sein de leur configuration familiale est de «2.11» $(\mathrm{SD}=1.67)$. Le degré sortant (Out-degree) du répondant renvoie au nombre de membres significatifs qui soutiennent (directement) le répondant au sein de sa configuration familiale (Widmer, 2006). Cet indice mesure la capacité du répondant à mobiliser du soutien au sein de sa configuration familiale. Un degré sortant (Out-degree) élevé révèle que le répondant se perçoit comme capable d'activer un grand nombre de liens de soutien, s'il en a besoin, au sein de sa configuration familiale. Il varie de 0 à $5 ; 5$ indiquant que le répondant estime qu'il est soutenu par l'ensemble des membres significatifs de sa configuration familiale. Le degré sortant (Out-degree) moyen de l'ensemble des participants de l'échantillon est de «1.50» $(\mathrm{SD}=1.35)$. Tant le degré rentrant (Indegree) que le degré sortant (Out-degree) évaluent le degré d'engagement du répondant dans les échanges de soutien avec les membres significatifs de sa configuration familiale. L'individu âgé se perçoit comme jouant un rôle particulièrement actif au sein de son réseau si ces deux indices sont grands.

Finalement, l'indice de centralité (d'intermédiarité) - «betweenness centrality $\gg-\mathrm{du}$ répondant indique dans quelle mesure ce dernier joue un rôle d'intermédiaire dans les échanges de soutien entre les différents membres significatifs de sa configuration (Cornwell et al., 2009; Cornwell, 2009a, 2009b; Widmer, 2006). Dans l'analyse de réseaux, il mesure l'autonomie structurelle des individus au sein de leur réseau personnel (Kadushin, 2012; Scott, 2000), problématique des plus fondamentales lorsqu'on touche à la vieillesse (Cornwell, 2009a, 2009b, 2011). Plus clairement, l'indice de centralité se rapporte à la proportion de relations dans lesquelles le répondant occupe une position intermédiaire entre les différents membres de sa configuration qui, sans lui, ne seraient pas connectés 
(Hanneman \& Riddle, 2005; Widmer, 2006). Le répondant est alors considéré comme central s'il se perçoit comme étant l'intermédiaire de toutes, ou presque toutes, les connexions qui relient les membres de sa configuration familiale (Widmer, 2006). La centralité du répondant tend à être d'autant plus forte lorsque son réseau est grand, peu dense et faiblement réciproque. Cet indice varie de 0 à $1 ; 1$ indiquant que tous les membres de la configuration familiale passent par le répondant pour se connecter les uns aux autres. La centralité moyenne de l'ensemble des participants genevois est de « $0.15 »(\mathrm{SD}=0.22)$. Généralement, ces trois derniers indices indiquent, s'ils sont élevés, que le répondant non seulement joue un rôle important au sein de sa configuration mais qu'il y occupe aussi une position centrale, connectant entre eux les membres qui ne sont pas directement en lien. Si tel est le cas, le répondant dispose donc d'un fort capital social de type « pont».

\subsubsection{Les modes de conflit et de support}

Cette partie porte sur la construction des quatre modes de conflit et de support qui traduisent des façons distinctes de gérer l'ambivalence au niveau du réseau familial. Deux questions du "Family Network Method» (Widmer et al., 2013) ont été utilisées. La première concerne les échanges de soutien émotionnel au sein de la configuration familiale (cf. plus haut) et la deuxième a trait aux tensions au sein de la configuration et est posée aux répondants de la manière suivante : "Chaque famille a ses conflits et ses tensions, à votre avis, qui met souvent $X[c .-\grave{a}-d$., le répondant et chaque membre de la famille cité comme significatif, et considérél'un après l'autre] en colère (par exemple : qui l'énerve souvent ou le fâche?) ». Plus que de graves conflits, l'objectif est de repérer l'agacement et les tensions que produisent certaines relations familiales (Widmer, 2016; Widmer et al., 2013). Comme pour le soutien émotionnel, les répondants doivent mentionner non seulement les tensions et les conflits entre eux et les différents membres qu'ils ont listés mais aussi toutes les tensions et les conflits entre ces derniers (Widmer, 2016; Widmer et al., 2013).

En nous basant sur ces deux questions, nous avons mesuré les densités du soutien émotionnel et les densités du conflit dans l'ensemble des configurations familiales. Nous avons ensuite fixé un seuil de 33\%, qui renvoie 
à la médiane de l'indicateur de densité de soutien émotionnel, pour distinguer les configurations familiales à forte densité de soutien de celles à faible densité. Autrement dit, les configurations familiales, dans lesquelles au moins 33\% des relations sont jugées soutenantes, sont définies comme ayant une forte densité de soutien. Quant aux conflits, la médiane est trop basse pour dichotomiser la densité des relations conflictuelles puisqu'elle est seulement de 0.03 ; ce résultat révèle, plus que l'absence totale de conflits, la réticence des individus à faire mention des tensions au sein de leur famille, et cela d'autant plus lorsqu'ils sont âgés (Fingerman \& Birditt, 2003). Par conséquent, nous avons opté pour un seuil de $10 \%$, ce qui signifie que les configurations familiales, dans lesquelles au moins une relation mentionnée sur dix est conflictuelle, sont considérées comme ayant une forte densité de conflit. Le croisement des deux densités dichotomisées (forte versus faible) de soutien et de conflit permet d'obtenir les quatre modes de conflit et de support, présentés dans le chapitre «Problématique et hypothèses $»$.

Les densités de soutien et de conflit des quatre modes de conflit et de support varient d'un mode à l'autre. La Solidarité se caractérise par une forte densité de soutien et par une faible densité de conflit. En moyenne, $58 \%$ des liens mentionnés sont associés à du soutien et seules $2 \%$ des connexions sont jugées conflictuelles. Quant à l'Ambivalence, elle allie une forte densité de soutien à une forte densité de conflit. En moyenne, $57 \%$ des relations sont jugées positives (soutien) et 33\% sont considérées comme conflictuelles. Dans les situations de Captivation, la densité de conflit est aussi élevée que celle du soutien puisqu'en moyenne, $20 \%$ des relations sont évaluées conflictuelles et $21 \%$ des connexions sont perçues comme positives. La Captivation se distingue nettement de l'Ambivalence; cette dernière se caractérise par une forte densité de soutien même si les tensions sont importantes, ce qui n'est pas le cas de la Captivation. Finalement, l'Atomisation diffère des trois autres par ses faibles densités tant au niveau du soutien que du conflit. En moyenne, seulement $11 \%$ des liens sont perçus comme positifs et seulement $2 \%$ renvoient à des tensions. En cumulant les cas où dominent la Solidarité ou l'Ambivalence, plus de la moitié des configurations familiales de l'échantillon (54\%) affichent une forte densité de soutien. Bien que plus faible, le conflit caractérise une proportion importante des relations familiales (32\%) comme le montre le cumul des configurations familiales dans lesquelles prévalent l'Ambivalence ou la Captivation. Ces différents modes, qui combinent 
densités de conflit et de soutien, représentent les résultats de différentes manières de gérer l'ambivalence structurelle au niveau des configurations familiales. Si l'on considère leur distribution dans l'échantillon genevois, l'Atomisation est le mode le plus fréquent (37\%), viennent ensuite la Solidarité (31\%), l'Ambivalence (23\%), et, en dernier lieu, la Captivation (9\%).

\subsubsection{Les facteurs explicatifs}

Dans cette sous-section sont exposés les différents facteurs explicatifs utilisés dans les analyses. Le réservoir de parenté, le genre, le statut socioéconomique, l'âge et la santé ont été sélectionnés en fonction de leur pertinence dans la littérature gérontologique. Ils sont généralement mis en avant dans la recherche pour expliquer les principales variations dans les relations personnelles et familiales dans la vieillesse (cf. chapitre « Facteurs explicatifs des relations familiales en gérontologie sociale »). Jusqu'à maintenant, ces différents facteurs n'ont jamais été associés en gérontologie sociale à des configurations familiales, à des types de capital social au sein de réseaux familiaux ni même aux modes de conflit et de support identifiés précédemment.

Le premier facteur considéré est le « réservoir de parenté » qui renvoie à l'existence de différents membres de la famille. Il mesure l'ensemble des réserves démographiques dont dispose l'individu âgé au moment de l'entretien. Bien que ces réserves ne soient pas toutes mobilisables, le réservoir de parenté offre un potentiel relationnel susceptible d'influencer la façon dont les aînés perçoivent leur famille significative. Pour le mesurer, nous avons utilisé les indicateurs démographiques classiques qui recensent l'existence des membres familiaux jugés comme les plus importants dans la littérature en gérontologie sociale. Lors de l'enquête, nous avons en effet demandé aux répondants s'ils avaient un partenaire, cohabitant ou non [ $1=\mathrm{a}$ un partenaire, $0=$ n'a pas de partenaire], s'ils avaient au moins un enfant vivant [ $1=\mathrm{a}$ au moins un enfant vivant, $0=$ n'a pas d'enfant vivant $]$, s'ils avaient au moins un petit-enfant $[1=\mathrm{a}$ au moins un petit-enfant vivant, $0=$ n'a pas de petits-enfants] et s'ils avaient au moins une sœur ou un frère encore vivant $[1=\mathrm{a}$ au moins une sœur ou un frère vivant, $0=$ n'a pas ou plus de sœur ou de frère vivant]. Nous avons également pris en compte dans certaines analyses le statut conjugal $[1=$ célibataire, $2=$ marié (cohabitant 
ou non), $3=$ cohabitant non marié, $4=$ veuf, $5=$ divorcé $]$ et dans d'autres, le statut matrimonial $[1=$ célibataire, $2=$ marié, $3=$ veuf, $4=$ divorcé $]$. En tant que mesures de la trajectoire familiale, le statut conjugal et le statut matrimonial sont étroitement associés au réservoir de parenté, notamment à la présence d'un partenaire ou d'enfants.

En raison de leur position sociale distincte dans la société et de leur parcours de vie différenciés, les femmes et les hommes ainsi que les riches et les pauvres n'ont pas accès aux mêmes opportunités professionnelles et relationnelles au cours de leur parcours de vie. Par conséquent, à l'orée de la vieillesse, ils ne bénéficient pas tous des mêmes réserves démographiques, des mêmes ressources relationnelles (amis) et socio-économiques pour affronter les aléas de leur vieillesse (Arber et al., 2003; Dannefer, 2003; Moen, 1996; Wanner et al., 2005). Pour approcher le profil socio-démographique des répondants, nous avons sélectionné les variables indépendantes suivantes : le «sexe» $[1=$ homme, $0=$ femme $]$, le «niveau d'éducation » $[1=$ bas (primaire et secondaire inférieur), $2=$ moyen (apprentissage, secondaire supérieur et école technique ou professionnelle supérieure), $3=$ élevé (Université et EPF) , et le « revenu » [1= bas (moins de 1200 à $3599 \mathrm{Chf}$ ), $2=$ moyen (de 3600 à $7199 \mathrm{Chf}$ ), $3=$ élevé (de 7200 à $15000 \mathrm{Chf}$ et plus)]. Nous avons ajusté le revenu à la taille du ménage en attribuant la valeur 1 si le répondant habite seul, additionnée de 0.5 pour chaque cohabitant supplémentaire (Atkinson, Rainwater, \& Smeeding, 1995; Gabriel, Oris, Studer, \& Baeriswyl, 2015). Nous avons également considéré la « citoyenneté » $[1=$ né étranger, $0=$ né suisse $]$. Être un immigré âgé en Suisse, notamment d'origine du sud de l'Europe, peut s'associer à un risque plus grand d'être au bas de l'échelle sociale que des personnes nées en Suisse (Bolzman, Poncioni-Derigo, Vial, \& Fibbi, 2004). Genre, niveau d'éducation, revenu, citoyenneté sont donc de précieux indicateurs des ressources dont disposent les répondants au moment de l'entretien.

Comme les autres variables socio-démographiques, l'«âge »s'associe à des ressources différentes, notamment à la santé. Nous avons considéré trois classes d'âge distinctes [ $1=65-74$ ans, $2=75-84$ ans, $3=85$ ans et plus]; elles correspondent à la catégorisation usuellement faite dans la littérature gérontologique qui distingue les jeunes-vieux (« young-old»), des vieux-vieux (les « old-old») et des très-vieux (les « oldest-old») (Suzman 
\& Riley, 1985). Il est communément admis dans la littérature gérontologique qu'en moyenne la santé décline au fur et à mesure que l'on vieillit (cf. chapitre «Facteurs explicatifs des relations familiales en gérontologie sociale »). Par conséquent, les « jeunes-vieux » ont généralement une meilleure santé que les «très-vieux ».

Pour mesurer la "santé », nous avons considéré principalement sa dimension fonctionnelle. Celle-ci est importante à plusieurs titres. Lorsqu'elle est mauvaise, elle réduit de manière drastique la capacité relationnelle des individus âgés, ceux-ci ne pouvant plus soutenir la réciprocité dans les échanges avec les membres de leur famille. Plus encore, elle implique un besoin accru de soutien de l'aîné et, par conséquent, une diminution importante de son autonomie, situation critique tant pour le parent âgé que pour les membres de sa famille. Pour mesurer la santé fonctionnelle, nous avons demandé aux répondants dans quelle mesure ils pouvaient accomplir seuls cinq activités de base de la vie quotidienne (AVQs) - se laver, s'habiller et se déshabiller, manger et couper les aliments, se coucher et se lever, se déplacer d'une pièce à l'autre (Katz et al., 1963) - et trois activités liées à la mobilité - monter ou descendre un escalier, se déplacer à l'extérieur du logement, et parcourir à pied 200 mètres au moins (Rosow \& Breslau, 1966). Le coefficient de fiabilité (alpha de Cronbach) calculé sur l'ensemble de ces mesures atteint 0,91 . Dans certaines analyses, nous avons distingué les répondants qui effectuent seuls l'ensemble de ces activités de ceux qui éprouvent des difficultés à réaliser seuls une ou plusieurs de ces activités ou qui nécessitent l'aide d'une autre personne pour les réaliser $[0=$ accomplit seul, sans difficulté majeure, $1=$ a des difficultés sur une ou plusieurs activités] et, dans d'autres analyses, nous avons considéré la variable de santé fonctionnelle en trois catégories [1= Robustesse AVQs (capable d'accomplir seul les huit activités), $2=$ Difficulté AVQs (a des difficultés à effectuer seul une ou plusieurs de ces activités), et $3=$ Dépendance AVQs (a besoin de l'aide d'une tierce personne pour accomplir une ou plusieurs de ces activités)]. En plus de la santé fonctionnelle, nous avons également pris en compte le «placement institutionnel» au sein d'établissements médico-sociaux ou en appartements sécurisés [ $1=$ institutionnalisé, $0=$ non institutionnalisé]. Age, santé et placement en institution signalent à quelle étape du processus de fragilisation se situent les répondants au moment de l'enquête, processus qui est susceptible d'influencer leurs relations familiales. 


\subsection{Les analyses}

Cette section est consacrée à la description des analyses effectuées au cours de ce travail et dont les résultats sont exposés dans le prochain chapitre. Seules les analyses qui sont centrales dans cette recherche comme l'analyse de classification hiérarchique, les analyses de variance (ANOVA), les régressions linéaires ainsi que l'analyse des correspondances multiples (ACM) sont présentées. Ces procédures sont parfois compliquées et nécessitent quelques explications. Toutefois, nous n'abordons pas dans cette section les analyses plus simples qui ne requièrent pas d'explications poussées; celles-ci sont introduites au fur et à mesure de la présentation des résultats au cours du prochain chapitre.

\subsubsection{L'analyse de classification hiérarchique}

Dans cette sous-section, nous expliquons les principales étapes de l'analyse de classification hiérarchique que nous avons réalisée pour identifier les différentes configurations familiales dans l'échantillon genevois. La procédure est la même que celle utilisée dans de nombreux travaux se référant à l'approche configurationnelle (Widmer, 2006, 2016). La première étape de l'analyse consiste à lister les membres significatifs de la famille les plus souvent mentionnés par les répondants lors de l'enquête. En tout, nous comptons 1'906 mentions produites par les répondants et pas moins de 97 différents termes de parenté ont été utilisés. Sur la base de cette liste, nous avons retenu les 14 termes les plus mentionnés. Ces derniers constituent $85 \%$ de l'ensemble des 1'906 mentions et ont été cités par au moins $5 \%$ des répondants. Quant aux autres termes, ils ont été regroupés sous une catégorie " autres ». C'est sur ces 14 termes les plus cités et la catégorie « autres » que nous nous sommes basés pour créer une typologie de configurations familiales.

Nous avons d'abord appliqué une analyse factorielle exploratoire sur l'ensemble des 14 termes de parenté et la catégorie "autres » afin d'en extraire les facteurs principaux (Widmer, 2016, 2006). Le processus d'extraction, qui comporte une analyse en composantes principales avec une rotation varimax, permet de réduire toute l'information contenue dans l'ensemble des variables (termes de parenté) à un nombre restreint de facteurs. 
Cette procédure vise à limiter le nombre de variables pour ne garder que l'essence de l'information, ce qui facilite ensuite la mise en œuvre de l'analyse de classification hiérarchique, celle-ci se faisant plus difficilement lorsque le nombre de variables est grand. Conformément à la pratique standard de l'analyse factorielle (Tabachnick \& Fidell, 1996), nous avons sélectionné les facteurs ayant une valeur propre supérieure à 1 . Les six facteurs retenus expliquent $55 \%$ de la variance de l'ensemble de nos données (termes de parenté). Suivant certaines procédures statistiques exploratoires (Lebart, Morineau, \& Piron, 2002), nous avons utilisé les scores factoriels obtenus sur les six facteurs - à savoir ceux des 14 termes de parenté et de la catégorie « autres »-dans l'analyse de classification hiérarchique.

Dans la deuxième étape de la procédure, nous avons effectué l'analyse de classification hiérarchique qui permet de construire des typologies d'objets ou des profils d'individus. Elle vise en somme à regrouper des objets, des personnes ou encore des variables qui présentent des caractéristiques communes et à distinguer ceux qui affichent au contraire des caractéristiques différentes (Everitt, Landau, Leese, \& Stahl, 2011; Lebart et al., 2002; Studer, 2013). Pour calculer les similarités ou les dissimilarités entre deux objets, plusieurs mesures sont possibles telles que les coefficients de corrélation ou des mesures de distance. Le calcul deux à deux des similarités ou des dissimilarités entre les différents objets produit une matrice de similarités ou de dissimilarités qui sert ensuite de base pour l'analyse de classification hiérarchique (Studer, 2013). Parmi les différentes mesures possibles, nous avons utilisé la distance euclidienne qui calcule la distance géographique la plus courte entre deux objets, ceux-ci pouvant être représentés par des points dans un espace multidimensionnel contenant autant de dimensions qu'il y a de composantes (variables internes) (Vachon, Beaulieu-Prévost, Ouellette, \& Achille, 2005). Les objets les plus proches géographiquement dans cet espace renvoient par conséquent aux objets les plus similaires sur plusieurs composantes ou caractéristiques (Studer, 2013; Vachon et al., 2005).

Une méthode d'agrégation est ensuite utilisée afin de créer des groupes (clusters) qui contiennent des objets similaires sur plusieurs caractéristiques. Autrement dit, elle permet de regrouper un objet ou un groupe à d'autres groupes. Plusieurs méthodes d'agrégation, utilisant différents algorithmes, sont possibles. Nous avons opté pour la méthode de Ward qui s'associe particulièrement bien aux distances euclidiennes (Everitt et al., 2011; Lebart et al., 2002; Shalizi, 2009; Studer, 2013). 
Celle-ci produit, selon certains auteurs, des résultats plus solides que ceux obtenus par d'autres méthodes car elle reflète davantage la structure « naturelle » des données que des critères arbitraires (Dieng, 2014; Shalizi, 2009; Studer, 2013; Vachon et al., 2005). La méthode de Ward repose sur le calcul de la variance intra-groupe, agrégeant dans le même groupe des objets qui affichent une faible variance entre eux. Idéalement, les groupes obtenus forment des ensembles homogènes, affichant une faible variance intra-groupe, et se distinguent des autres par une forte variance intergroupes (Dieng, 2014; Everitt et al., 2011; Shalizi, 2009; Studer, 2013). Comme son nom l'indique, «hiérarchique », la procédure d'agrégation se fait par étapes, la première consiste à unir, paire par paire, les objets les plus similaires, ensuite, d'y associer par étapes successives les objets qui ont des caractéristiques communes. Ce faisant, des petits groupes se forment, s'agrandissant au fur et à mesure des étapes, jusqu'à n'en former plus qu'un en dernière étape (Dieng, 2014; Everitt et al., 2011; Shalizi, 2009; Studer, 2013). Cette procédure est visible sous la forme d'un dendrogramme qui représente graphiquement les étapes successives de regroupements.

La difficulté réside à s'arrêter à la bonne étape du processus afin de trouver le bon nombre de groupes qui décrit le mieux la structure des données (Dieng, 2014; Everitt et al., 2011; Shalizi, 2009; Studer, 2013). Il s'agit, lors de cette étape cruciale, de choisir la meilleure solution. L'analyse de classification hiérarchique propose toujours des résultats, qu'ils soient pertinents ou non d'un point de vue sociologique. Et, c'est au chercheur de décider laquelle des solutions proposées est la meilleure (Shalizi, 2009; Studer, 2013; Vachon et al., 2005). Opter pour un petit nombre de groupes implique une trop forte simplification des données avec le risque de noyer des types pourtant bien distincts alors que choisir un nombre trop élevé de groupes donne du poids à des informations qui sont, somme tout, peu importantes et sans valeur explicative (Shalizi, 2009). Les bons groupes devraient se différencier clairement sur les caractéristiques utilisées dans l'analyse (variables), facilitant de fait leur interprétation (Shalizi, 2009; Studer, 2013; Vachon et al., 2005). Les analyses de variance (ANOVA) sur les caractéristiques étudiées - les termes de parenté, dans notre cas - permettent de confirmer, si les résultats sont significatifs, que les groupes formés se différencient significativement les uns des autres sur ces caractéristiques (Vachon et al., 2005). Cela dit, comme le souligne Shalizi (2009), il n'est pas nécessaire qu'ils se distinguent sur tout pour qu'ils soient pertinents. De même, une bonne solution devrait 
permettre aux groupes obtenus de se distinguer les uns des autres en fonction de variables externes, c'est-à-dire en fonction de variables n'ayant pas été utilisées dans l'analyse de classification hiérarchique (Shalizi, 2009; Vachon et al., 2005). Ces variables peuvent être « dépendantes», variant significativement selon les groupes obtenus, ou « indépendantes », expliquant significativement les différences entre les groupes. Finalement, les groupes obtenus devraient s'appuyer, pour qu'ils soient solides, sur des hypothèses théoriques fondées. Il faut que ces groupes fassent sens d'un point de vue sociologique et qu'ils puissent être interprétés au regard de la problématique théorique proposée (Dieng, 2014; Shalizi, 2009; Studer, 2013; Vachon et al., 2005).

Au-delà de leur pouvoir interprétatif, certains critères «purement» statistiques permettent d'aiguiller le chercheur quant au nombre de groupes à choisir. La taille de l'échantillon, par exemple, est importante. En général, le nombre de groupes est proportionnel à la taille de l'échantillon. Un grand nombre de groupes est plus approprié lorsque l'échantillon est grand que lorsqu'il est petit, et cela d'autant plus que chaque groupe doit être composé d'un nombre suffisant d'individus pour procéder à des analyses statistiques complexes (Vachon et al., 2005). L'application d'autres algorithmes d'agglomération permet de détecter les solutions les plus stables et de choisir le bon nombre de groupes, du moins celui qui semble correspondre le plus à la structure des données (Shalizi, 2009; Studer, 2013; Vachon et al., 2005). Il existe aussi des mesures statistiques de validation, telles que l' « Average Silhouette Width (ASW)» et la «Calinski-Harabasz $(\mathrm{CH}) »$, qui indiquent parmi plusieurs solutions celle qui affiche statistiquement le meilleur nombre de groupes (Dieng, 2014; Everitt et al., 2011; Studer, 2013). L'ASW teste, en somme, la cohérence des assignations. Si cette mesure est élevée, elle indique que les groupes sont fortement homogènes (variance intra-groupe) et que les distances entre eux sont élevées (variance inter-groupes) et, si au contraire elle est petite, elle révèle que l'homogénéité intra-groupe est faible et que les groupes ne sont pas suffisamment distincts les uns des autres (Studer, 2013). Cet indice varie de -1 à 1 . Lorsqu'il se situe entre 0.71 et 1 , la structure des données et forte; de 0.51 à 0.70 , la structure est « raisonnable »; de 0.26 à 0.50 , la structure est faible; et de -1 à 0.25 , il n'y a aucune structure dans les données (Studer, 2013). Quant à la mesure «Calinski-Harabasz $(\mathrm{CH}) »$, reconnue dans la 


\section{Données et méthodes}

littérature comme étant efficace, elle repose sur la statistique $\mathrm{F}$ de l'analyse de variance (ANOVA) et varie de 0 à l'infini, la valeur la plus élevée indiquant la meilleure solution (Dieng, 2014; Studer, 2013).

Dans cette recherche, plusieurs solutions ont été testées, allant de 4 à 8 groupes distincts. Bien que les mesures de validité ASW (Average Silhouette Width) et $\mathrm{CH}$ (Calinski-Harabasz) indiquent que la meilleure solution est celle à 7 groupes $(\mathrm{CH}=208.41$; $\mathrm{ASW}=0.30)$, nous avons retenu la solution à 6 groupes $(\mathrm{CH}=189.47$; $\mathrm{ASW}=0.26)$. A noter que l'amélioration de la mesure de validité ASW est relativement légère, passant de 0.26 pour la solution à 6 groupes à 0.30 pour celle à 7 groupes et retombant ensuite à 0.28 pour celle à 8 groupes. Ces deux dernières solutions n'affichent pas une meilleure structure des données que celle à 6 groupes puisque leur ASW ne dépasse pas les 0.50. Parce que l'amélioration n'est pas en soi substantielle, nous avons privilégié l'interprétabilité sociologique des résultats. Les groupes obtenus se distinguent clairement les uns des autres et leur différence dans leur composition fait pleinement écho aux hypothèses et à la problématique théorique qui les sous-tend. De plus, la taille de l'échantillon étant limitée, il était plus judicieux de sélectionner une solution avec un nombre relativement restreint de groupes, garantissant des effectifs suffisants et qui fassent sens. Nous avons donc opté pour une solution qui répond au critère d'interprétabilité des résultats tout en s'approchant de la solution «idéale » d'un point de vue statistique (Everitt et al., 2011; Shalizi, 2009; Widmer, 2006). Ces différentes analyses ont été effectuées sous R (R Core Team, 2015). Les six configurations familiales obtenues et leurs caractéristiques sont présentées dans le prochain chapitre.

\subsubsection{L'analyse de variance (ANOVA) et la régression linéaire}

Après avoir dégagé six configurations familiales, nous nous sommes intéressés au capital social et à ses différentes formes. Une des questions de recherche de cet ouvrage est de savoir si les ainés ont accès au sein de leur configuration familiale à du capital social, et si oui sous quelle forme et à quel volume. Pour répondre à cette question, nous avons calculé sur la base des liens de soutien émotionnel six indices du capital social : la taille, la réciprocité, la densité, le degré rentrant (In-degree), le degré sortant (Out-degree) et la centralité du répondant. Selon les scores obtenus 
sur ces indicateurs, nous avons pu déterminer le type et le volume de capital social produit au sein de chaque configuration familiale. Ensuite, nous avons cherché à identifier les principaux facteurs à même de les influencer. Plusieurs facteurs ont été testés comme la composition des configurations familiales, le genre, le niveau d'éducation, la citoyenneté, la classe d'âge, la santé fonctionnelle, le placement en institution ainsi que le réservoir de parenté, et plus spécifiquement l'existence d'un partenaire, d'au moins un enfant, d'au moins un petit-enfant et d'au moins un frère ou une sœur. Au cours de cette investigation, deux types d'analyse ont été effectués : des analyses de variance («one-way ANOVA ») et des régressions linéaires multivariées.

Les analyses de variance (« one-way ANOVA ») permettent d'observer si les six indices du capital social varient selon les différents facteurs sélectionnés. On peut, avec ce type d'analyses, comparer les scores moyens de chaque indice sur les différentes modalités des variables explicatives. Si ces scores sont différents d'une modalité à l'autre, on peut en conclure que le facteur testé s'associe, selon les indices mesurés, soit au capital social de type chaîne, soit au capital social de type pont. Et, si ces scores moyens ne varient pas, le facteur examiné a dès lors peu d'impact sur la production de capital social au sein des configurations familiales. Le test de Fisher (F-test) et le test de Kruskal-Wallis, version non paramétrique de l'ANOVA destinée aux variables «non normalement » distribuées, signalent si les éventuelles variations observées sont significatives, c'est-à-dire si elles sont dues aux facteurs testés et non au hasard de l'échantillonnage (Widmer, 2006, 2016). Pour les facteurs ayant plus de deux modalités, nous avons procédé au test de Duncan (analyse post hoc) qui indique, au moyen de lettres de l'alphabet distinctes, quelles modalités se différencient significativement les unes des autres.

Après avoir examiné, séparément, l'effet de chaque facteur sur les indices du capital social par le biais d'analyses de variance (ANOVA), nous avons procédé à des analyses de régressions linéaires multivariées. Celles-ci permettent, contrairement aux ANOVA, de tester ensemble, dans le même modèle, tous les facteurs et de déterminer ceux qui expliquent le mieux les variations des différents indices du capital social. Autrement dit, les estimations obtenues dans ce type d'analyses tiennent compte de l'effet des autres variables. Pour ce faire, nous avons procédé en deux temps. D'abord, nous avons inséré dans un premier modèle les six configurations familiales pour tester l'effet de leur composition sur les divers indices du 
capital social. Les résultats obtenus à ce stade ne sont pas différents de ceux produits par l'analyse de variance (ANOVA). Ensuite, nous avons ajouté dans un deuxième modèle, en plus des configurations familiales, le genre, le niveau d'éducation, la citoyenneté, la classe d'âge, la santé fonctionnelle, le placement en institution ainsi que le réservoir de parenté (statut conjugal, enfants, petits-enfants et fratrie). Ce deuxième modèle vise à tester si les résultats du premier modèle, c'est-à-dire les effets des configurations familiales sur les indices du capital social, se maintiennent, s'atténuent ou disparaissent lorsqu'on y introduit d'autres facteurs explicatifs. Cette procédure permet de comparer le poids explicatif des configurations familiales par rapport à celui des autres facteurs dans la production du capital social dans la vieillesse. Si les effets se maintiennent, on peut en déduire qu'ils sont solides puisqu'ils subsistent, quels que soient le profil sociodémographique des répondants et l'état de leur réservoir de parenté. S'ils disparaissent, d'autres facteurs dans le deuxième modèle expliquent davantage les variations du capital social. L'inclusion du réservoir de parenté dans l'analyse permet, par exemple, de contrôler que les effets des configurations familiales sur le capital social sont « réels » et qu'ils ne sont pas simplement dus à la seule existence de certains membres familiaux comme le partenaire ou les enfants, jugés dans la littérature en gérontologie sociale comme importants.

Concernant la méthode, nous avons opté dans ces divers modèles de régressions linéaires pour la variante « contrast deviation» (Chambers \& Hastie, 1992). Dans les modèles « classiques" de régression, on choisit pour chaque facteur une modalité de référence (fixée à 0.00 ) à laquelle l'on compare les autres modalités. Dans la variante que nous avons choisie (« contrast deviation »), on compare pour chaque facteur les scores de toutes les modalités à leur moyenne générale et non à une seule modalité de référence, ce qui facilite l'interprétation des résultats. Pour estimer la significativité des modèles, le chi-carré («Chi-square ») et le test de Fisher (F-test) ont été utilisés, tout comme le R-carré ajusté («adjusted R-square ») qui informe quant au montant de la variance expliquée pour chaque modèle. Par ailleurs, nous avons procédé à un test de significativité pour déterminer si la différence de variances expliquées entre le premier et le deuxième modèle est significative. Ajoutons à cela que ces modèles, comme les analyses de variance précédentes, examinent le «lien » entre le capital social et différents facteurs. Bien que le terme «facteur» soit évoqué, on ne peut pas «a priori» interpréter les résultats en termes de 
causalité en raison de la nature transversale des données. Il est difficile pour certains d'entre eux d'avancer avec certitude le sens de la causalité. La santé, par exemple, peut avoir un effet sur le capital social tout comme le capital social peut avoir un impact sur la santé. Toutefois, pour d'autres facteurs comme le genre ou la composition des configurations familiales, le sens de la causalité ne fait aucun doute; nous interpréterons, dans ces caslà, les résultats en termes de causalité. Toutes ces analyses ont été effectuées sous R (R Core Team, 2015).

\subsubsection{L'analyse des correspondances multiples (ACM)}

Afin d'explorer les conditions d'émergence des quatre modes de conflit et de support, nous avons procédé à une analyse des correspondances multiples (ACM). Cette méthode permet de considérer ensemble, dans la même analyse, les liens entre les quatre modes de conflit et de support, qui sont pour rappel les reflets de gestions différentes de l'ambivalence au sein de la famille, et les divers « facteurs » qui forment les conditions structurelles de leur émergence. Elle donne, en somme, un aperçu global de l'influence concomitante de différents facteurs sur l'émergence des quatre modes de conflit et de support. Les facteurs que nous avons retenus pour cette analyse sont les configurations familiales, le genre, le revenu, la citoyenneté, la santé fonctionnelle et le réservoir de parenté (avoir un partenaire, avoir au moins un enfant et avoir au moins un frère ou une sœur vivant). La classe d'âge n'a pas été insérée dans l'analyse afin d'éviter toute multicolinéarité, la variable "âge » étant corrélée avec celle de la santé fonctionnelle dans la vieillesse $(r=0,409, \mathrm{p}=0,000)$. L'examen simultané de ces variables met en exergue comment ces différents facteurs - dans leurs interrelations - forment des « conditions-type » qui favorisent, ou non, le développement des quatre modes de conflit et de support.

L'analyse des correspondances multiples (ACM), multivariée et non linéaire, est utilisée pour observer les relations entre différentes variables catégorielles (Abdi \& Valentin, 2007). Méthode avant tout descriptive et exploratoire, l'ACM permet de détecter les structures sous-jacentes d'un ensemble d'observations regroupées en variables catégorielles. En offrant une vue d'ensemble de leurs structures, elle permet de mieux comprendre comment s'organisent les relations entre les différentes modalités des 


\section{Données et méthodes}

variables analysées (Abdi \& Valentin, 2007; Avolio et al., 2013). Concrètement, cette méthode décompose la matrice des correspondances dans sa structure de base, en examinant les relations entre les lignes et les colonnes de tableaux de contingence (à deux ou plusieurs entrées) sans distinguer les variables dépendantes et indépendantes (Avolio et al., 2013). Ce faisant, elle réduit l'information à un nombre restreint de dimensions principales (deux ou trois dimensions, par exemple) qui sont généralement suffisantes pour synthétiser les informations les plus importantes contenues dans les tableaux de contingence. L'information produite par les autres dimensions peut être considérée comme marginale (Desbois, 2008). Il s'agit ensuite de définir les dimensions dégagées par l'analyse. Grâce aux mesures de discrimination que l'ACM produit pour toutes les variables et leurs modalités, il est possible d'interpréter les dimensions retenues. Mesures de corrélation entre les variables ou leurs modalités et les dimensions, les mesures de discrimination indiquent non seulement les variables qui expliquent le mieux les dimensions sélectionnées mais aussi leur poids explicatif(contribution) (Abdi \& Valentin, 2007; Avolio et al., 2013). Plus ces mesures sont fortes, plus la variable et ses modalités expliquent la dimension concernée (Avolio et al., 2013; Hoffman \& De Leeuw, 1992). Dans l'analyse effectuée, toutes les variables utilisées dans l'ACM sont considérées comme des variables actives, c'est-à-dire que toutes ont contribué à définir les principales dimensions.

Par ailleurs, cette méthode permet de projeter sur un graphique les différentes modalités des variables analysées autour des principales dimensions (axes), ce qui rend l'interprétation des résultats plus aisée. Les variables qui expliquent le mieux les dimensions, c'est-à-dire celles qui ont des mesures de discrimination les plus plus élevées, se repèrent facilement sur le graphique. Ce sont en effet les variables dont les modalités s'écartent le plus les unes des autres sur la dimension (axe) analysée qui l'expliquent le plus fortement. Autrement dit, la position des modalités sur le graphique (coordonnées) permet d'interpréter les dimensions sélectionnées (Avolio et al., 2013; Hoffman \& De Leeuw, 1992). Plus encore, les distances sur le graphique entre les modalités des différentes variables donnent un aperçu des associations entre elles et, ce faisant, signalent des profils de réponses (Abdi \& Valentin, 2007; Avolio et al., 2013; Desbois, 2008; Hoffman \& De Leeuw, 1992). En effet, les modalités qui sont proches sur le graphique signifient qu'elles apparaissent ensemble dans les observations. Ainsi, les 
individus qui ont sélectionné l'une de ces modalités ont une forte probabilité d'avoir choisi les autres modalités à proximité, ce qui traduit chez ces différents individus un même profil de réponses. A l'inverse, les modalités qui sont, au contraire, distantes les unes des autres dans le même espace indiquent que les individus qui les ont choisies affichent des profils de réponses différents (Abdi \& Valentin, 2007; Hoffman \& De Leeuw, 1992). En d'autres termes, sur la base de différentes variables explicatives, l'ACM permet d'identifier des « profils-types » ou des « conditions-types » auxquelles s'associent des réponses spécifiques. Afin de faciliter l'identification des différents groupes de modalités sur le graphique, une rotation des principales dimensions (axes) a été effectuée. Cette opération affine la représentation graphique en améliorant le placement des différentes modalités de variables le long des axes, ce qui en facilite l'interprétation (Saracco, Chavent, \& Kuentz, 2010). Lors de cette opération, le pourcentage de la variance expliquée est redistribué entre les axes, ce qui, en somme, n'affecte en rien la totalité de la variance expliquée par les deux dimensions (Saracco et al., 2010). Ces analyses (ACM) ont été réalisées dans R (Husson, Josse, Le, \& Mazet, 2014).

Nous avons présenté dans ce chapitre l'étude «VLV», ses objectifs et ses procédures d'enquête. Ce faisant, nous avons décrit les caractéristiques de l'échantillon genevois, sur lequel portent les analyses, et expliqué les raisons qui nous ont poussés à nous centrer exclusivement sur les données genevoises. Dans la section « Mesures », nous avons introduit la méthode «Family Network Method (FNM)» qui permet à la fois d'identifier les membres significatifs de la famille et de dégager les liens d'interdépendance qui existent entre eux, base sur laquelle les diverses mesures du capital social ont été calculées. Celles-ci servent à distinguer le capital social de type chaîne de celui de type pont. Nous avons ensuite introduit les facteurs explicatifs et leurs mesures. Pour clore ce chapitre, nous avons exposé les analyses utilisées pour identifier les configurations familiales dans la population âgée (Analyse de classification hiérarchique), celles réalisées ensuite pour expliquer les variations du capital social en termes de volume et de types (ANOVA et Régressions), et, finalement, celles pour explorer les conditions d'émergence des différents modes de conflit et de support (Analyse des correspondances multiples). 



\section{Membres significatifs de la famille et configurations familiales}

Dans le chapitre «Problématique et hypothèses », nous avons évoqué certains mécanismes de re-définition ou de ré-interprétation des liens familiaux qui ont été mis en exergue dans certaines études portant sur des données qualitatives. Selon les hypothèses que nous faisons, ces mécanismes pourraient être source de variabilité quant à l'identité des membres significatifs de la famille et donner lieu à différentes configurations familiales aux dernières étapes de la vie. Ce chapitre a trait à la composition des familles significatives au sein de l'échantillon genevois. Dans un premier temps, nous présentons les résultats concernant les membres significatifs de la famille les plus mentionnés par les âgés. Dans un deuxième temps, nous introduisons les différentes configurations familiales obtenues suite à une analyse de classification hiérarchique. Celles-ci incarnent des définitions personnelles distinctes de la famille significative dans la vieillesse. Les résultats de ce chapitre permettent d'en connaître un peu plus sur la diversité des membres significatifs de la famille chez les personnes âgées à Genève et de détecter les principales configurations familiales qui prévalent dans ce canton.

\subsection{Les membres significatifs de la famille}

Afin d'identifier les membres significatifs de la famille dans la vieillesse, nous procédons par étapes. Il s'agit d'abord de donner un aperçu global du réservoir de parenté au moment de l'entretien afin d'évaluer l'état des réserves démographiques des personnes âgées interviewées. Ensuite, nous nous centrons sur les membres de la famille que ces personnes désignent comme significatifs. Il est question en premier lieu du nombre de membres de la famille cités comme significatifs par les âgés et ensuite nous nous intéressons à l'identité de ceux qui sont le plus mentionnés. Cette première 
section se termine sur le pourcentage d'individus âgés qui ne désignent pas comme significatifs les membres primaires de leur famille, ceux-là même qui sont perçus « a priori » comme «proches » en gérontologie sociale. Une sous-section conclusive met en exergue les principaux résultats de cette première série d'analyses.

\subsubsection{L'état du réservoir de parenté}

Le réservoir de parenté est une donnée importante lorsqu'on s'intéresse aux relations familiales et à leur diversité dans la vieillesse. Il renvoie à l'ensemble des réserves démographiques dont disposent les individus âgés aux dernières étapes de leur vie et il est probable qu'il influence la façon dont les individus âgés définissent leur famille significative en offrant, ou non, une diversité d'opportunités relationnelles. Dans le deuxième chapitre de cet ouvrage, nous avons souligné les nombreux changements sociodémographiques qui ont contribué à diversifier la structure familiale des individus âgés. Plus encore, nous avons mis en exergue que, selon le parcours de vie des individus âgés et celui de leurs proches, le montant et la qualité des réserves démographiques non seulement évoluent rapidement mais varient aussi selon les individus. Certains sont insérés dans des structures familiales multigénérationnelles ayant accès à une diversité de réserves démographiques alors que d'autres, célibataires et sans enfant, n'ont au contraire qu'un nombre limité de possibilités relationnelles. Au vu de ce constat, il est nécessaire de scruter le réservoir de parenté des individus âgés que nous avons interviewés pour connaître précisément l'état de leurs réserves démographiques au moment de l'enquête.

Le tableau 5.1 présente le réservoir de parenté des participants de l'étude. Les indicateurs démographiques exposés dans ce tableau mesurent essentiellement l'existence des membres de la famille de sang ou d'alliance qui sont perçus « a priori » comme « les plus proches» dans la littérature en gérontologie sociale. Ces indicateurs ne suffisent pas à décrire toute l'étendue du réservoir de parenté puisque les répondants n'ont pas été questionnés sur d'autres types de liens de parenté, jugés sans doute comme «moins proches », comme les membres de la belle-famille et leurs alliés (beaux-frères, neveux, etc.) ou ceux de la parenté éloignée tels que les cousins et leurs alliés. Bien que limités, ces indicateurs donnent d'ores et déjà 
Tab. 5.1: Réservoir de parenté (\%)

\begin{tabular}{|c|c|c|c|}
\hline & & $\%$ & (n) \\
\hline A un conjoint & oui & 61 & $(345)$ \\
\hline A des enfants & oui & 82 & $(461)$ \\
\hline \multirow[t]{4}{*}{ Nombre d'enfants : } & 0 & 18 & $(102)$ \\
\hline & 1 & 21 & $(119)$ \\
\hline & 2 & 40 & $(222)$ \\
\hline & 3 et plus & 21 & $(120)$ \\
\hline A des petits-enfants & oui & 69 & $(389)$ \\
\hline \multirow[t]{4}{*}{ Nombre de petits-enfants : } & 0 & 31 & $(173)$ \\
\hline & 1 & 8 & (43) \\
\hline & 2 & 20 & $(115)$ \\
\hline & 3 et plus & 41 & $(231)$ \\
\hline A des arrière-petits-enfants : & oui & 29 & (71) \\
\hline \multirow[t]{4}{*}{ Nombre arrière-petits-enfants : } & 0 & 71 & $(173)$ \\
\hline & 1 & 9 & (23) \\
\hline & 2 & 8 & (19) \\
\hline & 3 et plus & 12 & (29) \\
\hline A des frères et sœurs : & oui & 68 & $(378)$ \\
\hline \multirow[t]{4}{*}{ Nombre de frères et sœurs : } & 0 & 32 & $(178)$ \\
\hline & 1 & 30 & $(164)$ \\
\hline & 2 & 17 & $(96)$ \\
\hline & 3 et plus & 21 & $(118)$ \\
\hline A sa mère et/ou père : & oui & 4 & $(25)$ \\
\hline
\end{tabular}

un bon aperçu de la diversité des réserves démographiques à disposition des individus âgés de l'échantillon.

En tout, $61 \%$ des répondants ont un partenaire et la plupart, mariés (98\%), affirment n'avoir jamais eu d'autre partenaire (70\%). Les participants, qui déclarent un conjoint, sont pour la plupart des hommes (64\%) si bien que, sur l'ensemble des hommes de l'échantillon, 77\% sont en couple (contre $45 \%$ des femmes). L'espérance de vie plus longue des femmes et la remise en couple plus fréquente chez les hommes expliquent ces différences de genre. Outre le partenaire, $82 \%$ des répondants ont au moins un enfant vivant et $61 \%$ en déclarent plus d'un. Il sont aussi $69 \%$ à avoir des petits-enfants - $41 \%$ en rapportent au moins trois - et $29 \%$ déclarent des arrière-petits-enfants. Quant à la fratrie, une majorité d'entre 
eux $(68 \%)$ ont au moins une sœur ou un frère vivant lors de l'interview, $38 \%$ en rapportent au moins deux. Finalement, seuls $4 \%$ ont encore au moins un parent vivant (mère ou père). Quant à ceux qui ont peu ou pas de réserves démographiques, ils sont relativement peu nombreux. Ils sont $18 \%$ sans enfant vivant, $39 \%$ sans partenaire et $32 \%$ sans fratrie. Ils ne sont en tout que $3 \%(n=19)$ à n'avoir aucune réserve démographique (ni partenaire, ni enfant, ni fratrie). Ces divers résultats montrent que, globalement, les répondants ont pour la plupart un réservoir de parenté fourni.

\subsubsection{Le nombre et l'identité des membres significatifs de la famille}

Ayant tous, en majorité, de bonnes réserves démographiques, on peut néanmoins se demander si les individus interviewés investissent affectivement les mêmes relations familiales ou si au contraire, et conformément à l'hypothèse « $\mathrm{H} 1$ », ils désignent comme significatifs des membres familiaux très différents. Avant de présenter l'identité des membres de la famille qui sont le plus cités comme significatifs par les personnes âgées, nous considérons le «nombre » de membres significatifs de la famille cités par les individus âgés au moment de l'entretien. Le tableau 5.2 présente la distribution des personnes interviewées (\%) selon le nombre de membres de leur famille qu'elles considèrent comme significatifs.

Sachant que les répondants ne pouvaient pas citer plus de cinq membres significatifs, on aurait pu penser que la grande majorité d'entre eux en mentionnent au moins cinq. Or, comme le révèle le tableau 5.2, c'est

Tab. 5.2: Nombre des membres cités comme significatifs (\%)

\begin{tabular}{ccc}
\hline & $\%$ & $(\mathrm{n})$ \\
\hline 0 & 11 & $(62)$ \\
1 & 7 & $(41)$ \\
2 & 10 & $(54)$ \\
3 & 16 & $(91)$ \\
4 & 16 & $(91)$ \\
5 & 40 & $(224)$ \\
\hline Total & 100 & $(563)$ \\
\hline
\end{tabular}


loin d'être le cas puisque $60 \%$ des participants en citent moins de cinq. Plus encore, si l'on considère la dispersion des effectifs le long de l'échelle proposée, on constate une certaine diversité dans le nombre de membres cités comme significatifs. En effet, $40 \%$ des répondants mentionnent cinq membres, $16 \%$ quatre, $16 \%$ trois, $10 \%$ deux, $7 \%$ un seul et finalement $11 \%$ n'en mentionnent aucun (moyenne $=3.39$, écart-type $=1.729$ ). Ainsi, les aînés se différencient quant il s'agit d'estimer le nombre de membres de leur famille qui comptent vraiment pour eux, ce qui atteste déjà d'une certaine diversité dans la construction des familles significatives.

Un autre résultat est à souligner : au moins une personne sur dix de l'échantillon (11\%) se perçoit dans un relatif isolement, ne citant aucune relation familiale significative. En comparant ces individus $(n=62)$ à ceux qui mentionnent au moins un membre significatif, ils se distinguent clairement par leur statut matrimonial puisque $40 \%$ d'entre eux sont soit célibataires, soit divorcés (contre $20 \%$, Chi2 $=14.459$, à $p .=0.001$ ). Ils sont significativement moins nombreux à avoir un partenaire (44\% vs $64 \%$, Chi $2=9.231$ à $p .=0.002)$ ou avoir un enfant $(61 \%$ vs $84 \%$, Chi $2=19.916$ à $p .=0.000)$. Plus encore, la pauvreté de leur réservoir de parenté s'associe à un faible statut socio-économique. Plus de la moitié d'entre eux rapportent un bas revenu ( $54 \%$ vs $37 \%$ ) alors que $4 \%$ seulement ont un revenu élevé (vs $15 \%$, Chi $2=7.620$, à $p$. $=0.02$ ). Ces analyses mettent en exergue l'importance du réservoir de parenté sur la perception de la famille significative. Lorsque les individus âgés ont de nombreuses réserves démographiques au sein de leur réservoir démographique, ils développent un nombre varié de relations familiales significatives; certains mentionnent au moins une ou deux relations alors que d'autres en citent davantage. Lorsque le réservoir démographique est au contraire peu fourni, il restreint de manière drastique les opportunités relationnelles et celles-ci, en l'absence de ressources socio-économiques, peinent à être compensées en dehors du réseau familial puisque les aînés dont le statut socio-économique est bas ont des réseaux personnels restreints et relativement pauvres en amitiés (cf. chapitre « Facteurs explicatifs »).

Se distinguant sur le nombre de membres mentionnés comme significatifs, on peut se demander si les aînés diffèrent aussi quant à l'identité de ceux qu'ils ont cités. Pour rappel, les répondants étaient libres lors de l'entretien de mentionner toutes les personnes qu'ils considéraient comme 
Tab. 5.3: Distribution des 14 termes familiaux les plus cités

\begin{tabular}{lcccc}
\hline Termes de parenté & $\begin{array}{c}\text { N citations } \\
\text { par terme }\end{array}$ & $\begin{array}{c}\text { \% des répondants } \\
\text { citant le terme }(\mathrm{n}=501)\end{array}$ & $\begin{array}{c}\text { \% des termes sur } \\
1906 \text { cités }\end{array}$ & Cum. \% \\
\hline Fils & 355 & 71 & 19 & 19 \\
Fille & 351 & 70 & 18 & 37 \\
Partenaire & 232 & 46 & 12 & 49 \\
Sœur & 108 & 22 & 6 & 55 \\
Amie (féminine) & 105 & 21 & 6 & 60 \\
Frère & 83 & 17 & 4 & 65 \\
Ami (masculin) & 71 & 14 & 4 & 68 \\
Fille de la fille & 64 & 13 & 3 & 72 \\
Fille du fils & 62 & 12 & 3 & 75 \\
Fils du fils & 48 & 10 & 3 & 78 \\
Partenaire du fils & 45 & 9 & 2 & 80 \\
Fils de la fille & 38 & 8 & 2 & 82 \\
Partenaire de la fille & 36 & 7 & 2 & 84 \\
Cousine (féminine) & 24 & 5 & 1 & 85 \\
Autres termes & 284 & 57 & 15 & 100 \\
\hline
\end{tabular}

faisant partie de leur famille significative puisqu'aucune définition préalable de la famille ne leur était imposée afin d'avoir leur propre conception de la famille significative. Le tableau 5.3 montre « qui » sont les membres de la famille les plus fréquemment mentionnés comme significatifs dans la vieillesse. En tout, 97 termes de parenté ont été cités par les répondants et nous avons gardé pour les analyses les 14 termes les plus mentionnés. Le tableau 5.3 présente leur distribution (effectif) ainsi que les pourcentages des répondants citant chacun des 14 termes et les pourcentages de chacun des termes cités sur le nombre total de citations.

Une large majorité des répondants (70\%) mentionnent un ou des enfants (fils ou filles) comme membres significatifs de leur famille, et $46 \%$ un partenaire bien qu'ils soient plus nombreux (61\%) à avoir un partenaire au moment de l'entretien. La fratrie est aussi largement citée, venant même avant les petits-enfants, qu'ils soient les enfants d'une ou de plusieurs filles ou ceux d'un ou de plusieurs fils. Ce sont d'abord les sœurs qui sont le plus mentionnées (22\%), puis, les frères (17\%). Étonnement, les ami(e)s occupent une place privilégiée dans la liste des membres significatifs de la famille les plus cités, égalant celle de la fratrie. Comme les 
sœurs et les frères, les ami(e)s viennent avant les petits-enfants, la bellefamille (conjoints des enfants) et la parenté éloignée. Les amies sont citées, comme les sœurs, par $21 \%$ des répondants et les amis, par 14\%. En considérant ensemble les amis et les amies, 19\% des répondants incluent au moins un(e) ami(e) dans leur famille significative. Ensuite, viennent les petitsenfants, notamment les petites-filles (12-13\%) et les petits-fils (10-8\%). Ils sont suivis de près par les beaux-enfants, avec d'abord les belles-filles (épouses des fils) (9\%) et ensuite les beaux-fils (époux des filles) (7\%). Finalement, ce sont les cousines qui clôturent la liste des membres significatifs de la famille les plus cités (5\%). Quant aux autres types de membres, ils sont regroupés sous la mention " autres termes »; ceux-ci se réfèrent à une variété de membres familiaux unis soit par le sang (neveux, nièces, petits-cousins, etc.) soit par l'alliance (partenaires des frères ou des sœurs, famille du partenaire, etc.).

Ces résultats montrent que les membres de la famille "nucléaire » occupent la première place des membres de la famille significative. La famille "nucléaire » a une influence normative importante; elle demeure toujours, pour un certain nombre d'âgés, le modèle « idéal» de la famille qui « compte ». Ceci dit, bien que les membres de la famille «nucléaire» soient perçus comme importants, ils ne sont pas les seuls. Les membres de la fratrie et plus encore les amis se situent aussi en haut de la liste des membres significatifs les plus cités. Ils se positionnent même avant les petits-enfants et les beaux-enfants (conjoints des enfants). Ceci confirme l'importance des liens affinitaires dans la construction de la famille significative. En effet, les amis ne sont pas seulement une composante importante des réseaux personnels, ils sont aussi fort présents dans la définition de la famille qui « compte», et cela même s'ils s'inscrivent en dehors du réservoir de parenté. Ces 14 termes cités révèlent donc une grande diversité des membres de la famille significative. Nombreux sont issus du réservoir de parenté comme le partenaire, les enfants, les petits-enfants, la fratrie, la belle-famille et la parenté éloignée mais d'autres membres, comme les amis, n'y appartiennent pas. Autrement dit, même si l'impact des réserves démographiques est indéniable, il n'en demeure pas moins que certains membres de la famille significative s'inscrivent bien au-delà des liens de sang et de l'alliance. Ce résultat vérifie l'hypothèse « $\mathrm{H} 1$ » quant à la variété des membres significatifs de la famille (cf. chapitre «Problématique et hypothèses »). 


\subsubsection{Les membres de la famille non cités}

Alors que les aînés puisent des membres significatifs de leur famille dans leur réservoir de parenté, on peut se demander si la seule présence de certains membres familiaux dans le réservoir de parenté assure automatiquement leur mention dans la famille significative. La question se pose notamment en ce qui concerne le partenaire et les enfants qui sont socialement définis comme a priori «très proches ». Comme le montre le tableau 5.3, ils sont effectivement les plus cités par les répondants comme des membres significatifs de la famille. Ceci dit, il se peut que leur forte mention soit en partie due à la pression normative qui incite certains individus à désigner les membres primaires de leur famille comme significatifs. Cette pression normative étant telle, on peut en effet s'interroger s'ils sont automatiquement cités comme membres significatifs de la famille dès lors qu'ils existent, et cela même si, dans certaines familles, ces relations sont en réalité peu investies d'un point de vue affectif. Le tableau 5.4 répond à cette question. Il présente le pourcentage de répondants qui ont un partenaire, un fils, une fille ou un membre de leur fratrie mais qui ne les désignent pas comme membres significatifs de leur famille.

Ces résultats sont sans ambiguïté : l'existence du partenaire et des enfants dans le réservoir de parenté ni même celle des membres de la fratrie ne garantissent leur mention « automatique » dans la liste des membres significatifs de la famille. Deux-tiers des répondants qui ont au moins un frère ou une sœur et $33 \%$ qui ont un partenaire ne les mentionnent pas comme membres significatifs de leur famille. Plus étonnant encore, 20\%

Tab. 5.4: Pourcentages des personnes citant ou ne citant pas un membre existant de leur famille comme significatif

\begin{tabular}{lccccc}
\hline & $\begin{array}{c}\text { Partenaire } \\
\%(\mathrm{n})\end{array}$ & $\begin{array}{c}\text { Enfant } \\
\%(\mathrm{n})\end{array}$ & $\begin{array}{c}\text { Fille } \\
\%(\mathrm{n})\end{array}$ & $\begin{array}{c}\text { Fils } \\
\%(\mathrm{n})\end{array}$ & $\begin{array}{c}\text { Frère/sœur } \\
\%(\mathrm{n})\end{array}$ \\
\hline Ne cite pas & 33 & 20 & 23 & 26 & 64 \\
\multirow{2}{*}{ Cite } & $(113)$ & $(93)$ & $(78)$ & $(94)$ & $(243)$ \\
& 67 & 80 & 77 & 74 & 36 \\
\hline Total & $(232)$ & $(368)$ & $(261)$ & $(265)$ & $(135)$ \\
& 100 & 100 & 100 & 100 & 100 \\
& $(345)$ & $(461)$ & $(339)$ & $(359)$ & $(378)$ \\
\hline
\end{tabular}


des participants qui ont au moins un enfant ne le désignent pas non plus comme faisant partie de leur famille significative. Les fils ne sont d'ailleurs pas moins mentionnés que les filles puisque $23 \%$ des participants qui ont au moins une fille et $26 \%$ qui ont au moins un fils ne les citent pas comme membres significatifs de leur famille. Même les liens de sang, qualifiés de "proches » dans la littérature, peuvent donc être considérés comme « non significatifs », signe sans doute d'un certain désengagement affectif. Par ailleurs, lorsque les enfants sont nombreux, une certaine sélection s'opère : $75 \%$ des répondants qui ont deux enfants les citent les deux comme membres significatifs de leur famille alors que seuls $61 \%$ de ceux qui en ont trois et $46 \%$ qui en ont quatre les mentionnent « tous » comme faisant partie de leur famille significative. En raison de la limite imposée de cinq membres significatifs, les parents âgés sont amenés à « choisir » certains enfants plutôt que d'autres, délaissant ces derniers au profit d'autres liens familiaux. Une telle sélection se manifeste aussi lorsqu'il s'agit des membres de la fratrie, seuls certains sont désignés comme significatifs alors que d'autres sont mis à l'écart. Ces résultats remettent en question la primauté « exclusive» des liens de sang sur d'autres types de liens lorsque l'on considère la famille significative, et cela même chez les personnes âgées.

\subsubsection{Conclusion sur les membres significatifs de la famille}

Cette première série de résultats met en évidence plusieurs points importants. Premièrement, le réservoir de parenté influence la façon dont les âgés définissent leur famille significative en leur offrant une diversité d'opportunités relationnelles qu'ils peuvent, ou non, développer en relations familiales significatives. La plupart des membres familiaux cités comme significatifs proviennent en effet du réservoir de parenté. Son influence est d'autant plus marquante lorsque les réserves démographiques qu'il offre sont faibles. En effet, la pauvreté du réservoir de parenté réduit drastiquement les possibilités de développer des relations familiales significatives, et cela d'autant plus si les personnes âgées ont peu d'alternatives en dehors du réservoir de parenté en raison notamment de leur bas statut socioéconomique. Il s'avère donc que les mécanismes de ré-interprétation, qui permettent de compenser la faiblesse du réservoir de parenté, ne peuvent pas être mis en place par tout le monde puisque ces mécanismes requièrent 
certaines ressources socio-économiques. Sans moyens économiques, les aînés qui ont un faible réservoir de parenté sont donc particulièrement à risque d'être socialement isolés.

Deuxièmement, la famille "nucléaire », axée sur les liens de sang et d'alliance, reposant sur la réciprocité « généralisée » et prometteuse d'une solidarité sans faille, demeure un modèle familial fort prégnant, comme le montre la place des membres familiaux «primaires » dans la liste des membres significatifs de la famille. Cependant, le partenaire et les enfants ne sont pas « toujours » considérés comme des membres significatifs de la famille malgré la prégnance du modèle de la famille «nucléaire ». Il se peut qu'en raison de la complexification des parcours de vie des uns et des autres, ces liens primaires aient pu être désinvestis au cours du temps, désengagés affectivement et ne constituent plus des relations familiales significatives. Comme d'autres membres du réservoir de parenté, le partenaire et les enfants peuvent être sujets à une ré-évaluation de la part des âgés, ce qui remet pleinement en question l'indéfectibilité des liens primaires, avancée dans de nombreuses études en gérontologie sociale.

Troisièmement, la prégnance des liens affinitaires dans la liste des membres significatifs de la famille, tels que les membres de la fratrie et plus encore les amis, montre l'importance de l'investissement affectif dans la définition de la famille qui « compte». Les amis, par exemple, qui n'appartiennent pas au réservoir de parenté peuvent être re-définis, en raison de l'engagement affectif qui les caractérise, comme des membres à part entière de la famille significative. Et, compte tenu de la forte proportion des répondants qui citent des amis comme significatifs, nombreux sont ceux qui intègrent un ou deux amis au sein de leur famille significative, les plaçant aux côtés des autres membres familiaux issus de leur réservoir de parenté. Plus que des substituts, les amis contribuent à élargir la famille significative en la renforçant dans son potentiel de support. Alors que certaines études en gérontologie sociale montrent leur plus forte prégnance dans les réseaux personnels (Stevens \& Van Tilburg, 2011; Suanet et al., 2013; Van Tilburg \& Thomése, 2010), les résultats du tableau 5.3 montrent qu'ils peuvent être considérés non seulement comme des membres proches du réseau personnel mais plus encore comme des membres de la famille significative. 
Ceci dit, la part des ami(e)s considéré(e)s comme des membres significatifs de la famille varie en fonction des cantons : elle atteint $19 \%$ à Genève, $16 \%$ à Bâle, $12 \%$ dans le Valais, $7 \%$ à Berne et seulement $2 \%$ au Tessin. La prépondérance plus marquée des amis dans la famille significative à Genève, comme à Bâle, pourrait s'expliquer par le contexte urbain et les opportunités plus grandes de s'engager dans des activités sociales et de rencontrer des amis, la participation sociale et les liens d'amitié allant de pair (Baeriswyl, 2016). A Genève, les aînés participent davantage aux activités culturelles (spectacles, cours, etc.) et sont plus engagés dans le domaine associatif et le bénévolat que dans d'autres cantons. Par conséquent, ils sont plus nombreux à avoir au moins un ami proche (84\% contre $76 \%$ en Valais) et à être actifs dans la sociabilité amicale (visites). Outre l'offre plus grande d'activités sociales, la dimension culturelle peut expliquer ces différences : en Valais, par exemple, la participation sociale des personnes âgées est plus traditionnelle, axée sur la famille, l'église et la communauté (fêtes de village) alors qu'à Genève, la sociabilité amicale et les activités visant l'épanouissement personnel sont plus importantes (Baeriswyl, 2016). Rencontrer des amis par le biais d'activités sociales est non seulement plus facile mais aussi plus valorisé dans le contexte urbain, ce qui a des implications claires sur les définitions personnelles de la famille à $\mathrm{Ge}-$ nève : les aînés genevois intègrent davantage d'amis au sein de leur famille significative que ceux qui vivent dans d'autres cantons.

Ces premiers résultats soulignent la pluralité des membres significatifs de la famille, fruit à la fois de la diversité des réservoirs de parenté et de la mise en place de mécanismes relationnels comme le désengagement, l'investissement et la sélection, qui permettent aux aînés de se modeler, sur la base des réserves démographiques qu'ils ont à disposition mais aussi au-delà, une famille qui fait « sens ». L'activation de ces divers mécanismes donne lieu à une diversité de membres significatifs de la famille dans la vieillesse, ce qui vérifie la première hypothèse (« $\mathrm{H} 1 »)$. Ces résultats confirment qu'à l'instar des populations plus jeunes, la famille qui « compte » chez les personnes âgées ne peut pas être appréhendée que par le simple recensement des réserves démographiques qui composent leur réservoir de parenté. 


\subsection{La composition des configurations familiales}

La question qui se pose dès lors est de savoir comment ces divers membres significatifs se combinent les uns aux autres pour former différentes configurations familiales. L'approche configurationnelle, utilisée dans des études portant sur des populations plus jeunes, met en évidence différentes logiques de construction, amenant à une diversité de configurations familiales. Nous faisons l'hypothèse qu'il en est de même chez les personnes âgées («H2») (cf. chapitre « Problématique et hypothèses »). Nous proposons dans cette section de dégager les différentes logiques qui sous-tendent la construction des configurations familiales dans la vieillesse et d'observer si elles génèrent, à l'instar des populations plus jeunes, une pluralité de configurations familiales. Pour ce faire, nous décrivons les six configurations familiales identifiées lors de l'analyse de classification hiérarchique en mettant en exergue leur logique particulière. Ensuite, nous présentons les différents facteurs qui expliquent leur pluralité et les caractéristiques socio-démographiques et relationnelles des membres qui les composent.

\subsubsection{Une diversité de compositions}

Le tableau 5.5 présente les résultats de l'analyse de classification hiérarchique. Six configurations familiales distinctes ont été identifiées; elles reflètent des définitions différentes de la famille significative chez les personnes âgées. L'analyse de variance (ANOVA) effectuée met en exergue leurs différentes composantes. Dans le tableau 5.5 figurent le nombre moyen et l'écart-type des citations pour chaque terme de parenté par configurations familiales (cf. les colonnes). Ces scores indiquent le nombre de fois que chaque terme a été en moyenne cité au sein de chaque configuration. Pour les termes renvoyant à une seule personne comme le partenaire - il ne peut a priori en avoir qu'un - ce nombre correspond au pourcentage de répondants mentionnant ce terme (Widmer, 2006). Ces divers scores révèlent en somme le poids de chaque lien de parenté pour chaque configuration; plus il est élevé et dépasse la moyenne totale, plus il la définit. La combinaison des termes de parenté ayant le plus de poids dans chaque configuration familiale contribue à déterminer sa spécificité et à en définir le profil particulier. La distribution des termes cités selon les 
différentes colonnes indiquent que nous avons six configurations familiales bien distinctes qui reflètent différentes manières de définir la « famille significative $»$.

La configuration familiale de type «Conjugal » - première colonne se focalise sur le partenaire et les enfants du répondant. Environ $63 \%$ des répondants appartenant à cette configuration familiale citent leur partenaire alors qu'ils ne sont que $41 \%$ à le faire dans l'ensemble de l'échantillon. Les enfants - filles ou fils - sont aussi fort présents dans cette configuration. Le fils affiche un score moyen de 0.96 et la fille de 0.92 , surpassant largement leurs scores totaux sur l'ensemble de l'échantillon ( 0.63 et 0.62 ). A noter que les enfants cités ne sont les enfants biologiques du partenaire actuel (cité) que dans 58\% des cas. Sinon, ils sont les enfants d'ex-partenaires soit divorcés, soit décédés. Les aînés dans cette configuration familiale suivent une logique de type " normative » pour définir leur famille significative, citant leurs enfants et leur partenaire actuel. Ils reproduisent ainsi le modèle idéal de la famille «nucléaire ». Cette configuration familiale est la plus fréquente $(39 \%)$ de l'échantillon.

Dans la deuxième colonne du tableau se dessine la configuration «Fils». Celle-ci se centre sur le fils ${ }^{1}$ du répondant, sa belle-fille (partenaire $\mathrm{du}$ fils) et ses petits-enfants (enfants du fils). La moyenne de citations du fils dans cette configuration familiale est la plus élevée parmi les six configurations. Elle atteint 1.35 contre 0.63 dans l'ensemble de l'échantillon. Il en est de même pour la partenaire du fils. Comparé aux autres configurations, le score moyen atteint par la belle-fille est dans cette configuration le plus haut (1.05). Viennent ensuite les enfants du fils, d'abord la fille du fils (0.37) et ensuite le fils du fils (0.19). Leurs scores moyens, bien que nettement plus faibles, excèdent ceux obtenus dans les autres configurations. La logique qui sous-tend cette configuration est celle du sang et de la descendance puisque l'accent porte sur le fils, la belle-fille et les petits-enfants. Nous rendons le lecteur attentif à l'importance de la belle-fille qui est mentionnée bien avant les petits-enfants. Le couple «fils et belle-fille» semble indissociable, comme si la mention $\mathrm{du}$ « fils » appelle « forcément» celle

1 Nous utilisons le singulier pour les termes de parenté cités afin de ne pas alourdir le texte, mais il va de soi que les individus interviewés ont pu citer plusieurs fils et plusieurs belles-filles, etc.; et il en est de même dans les autres configurations présentées. 
Membres significatifs et configurations

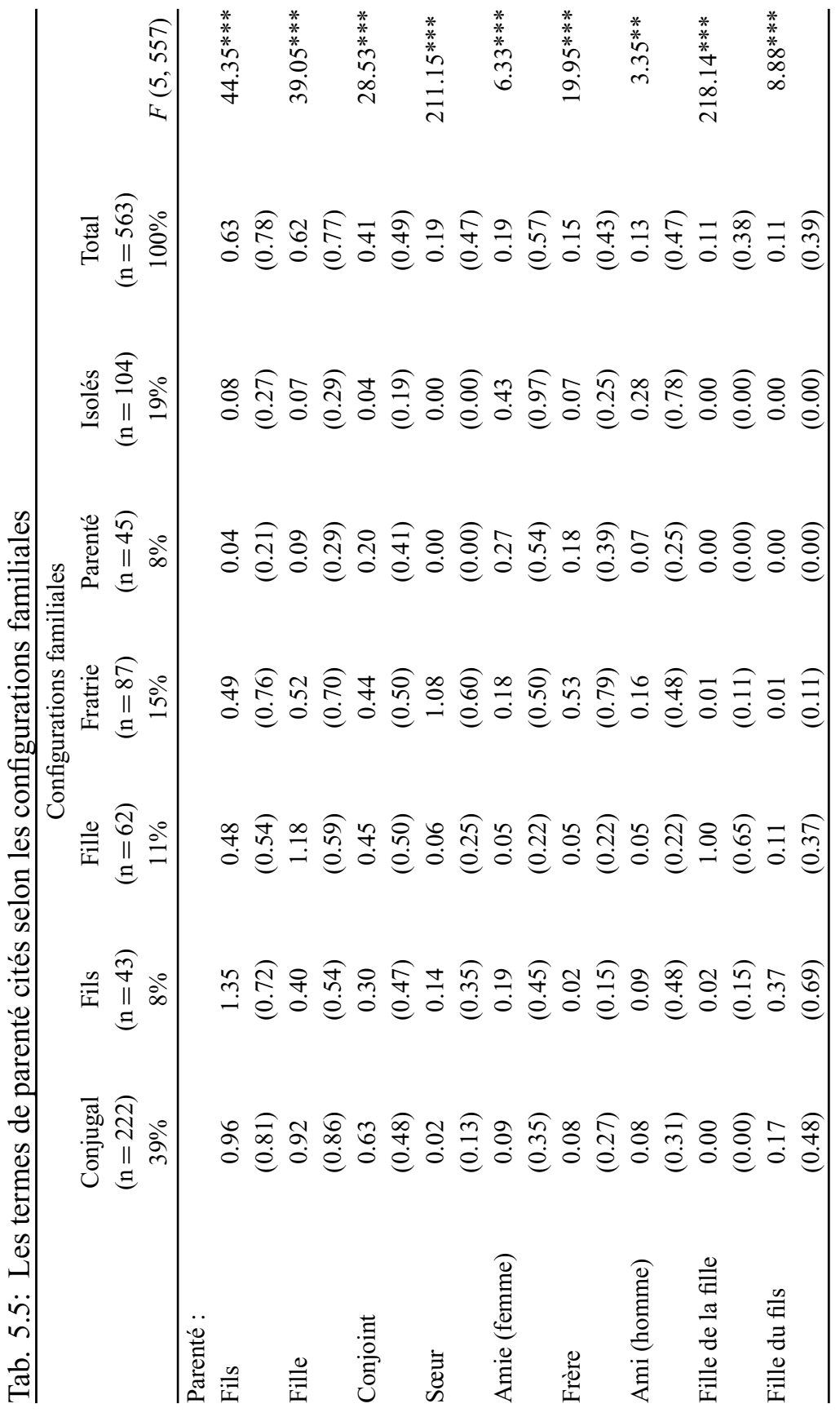


La composition des configurations familiales

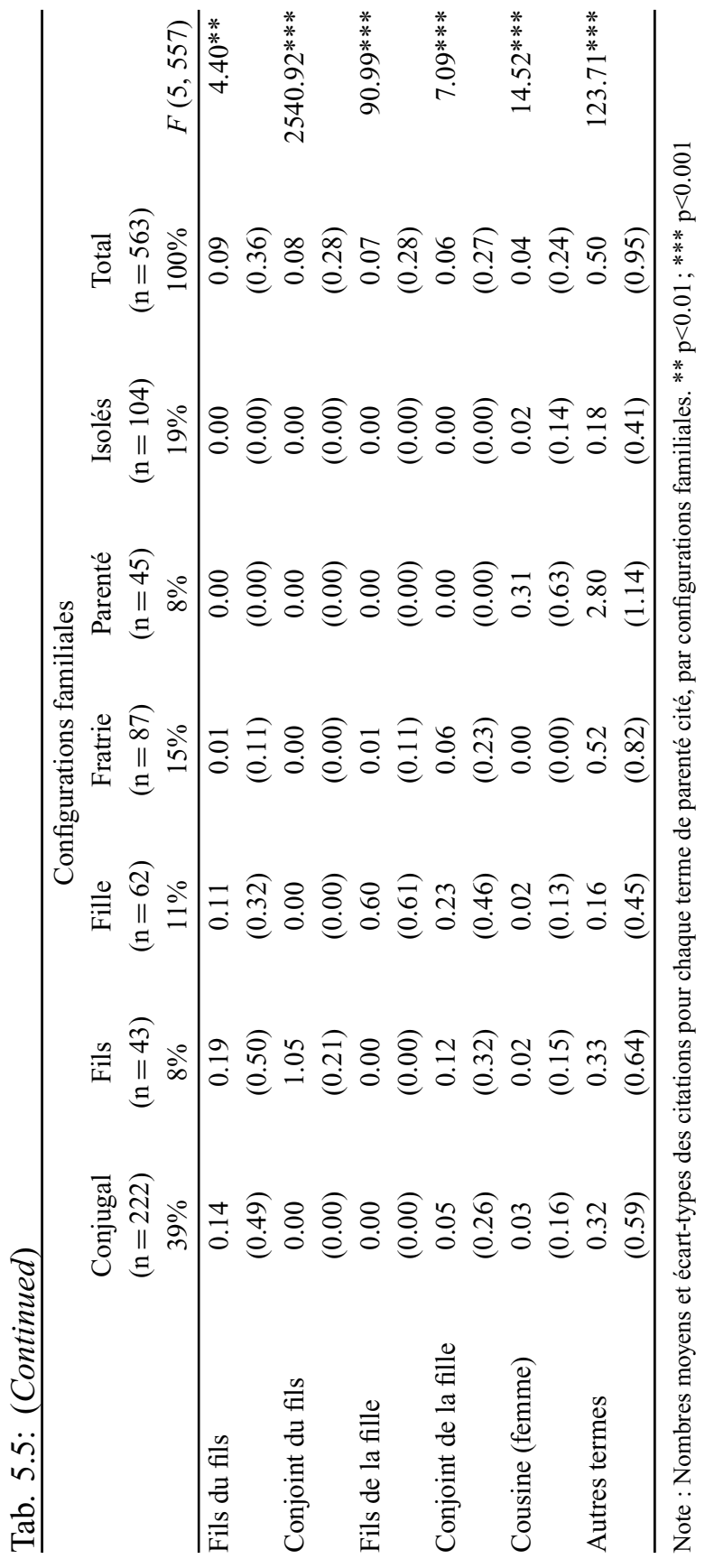


de la belle-fille et ensuite celle des petits-enfants. Comparée à la configuration précédente, la configuration «Fils» est relativement peu fréquente (8\%) dans l'échantillon.

La configuration «Fille» se compose de la fille du répondant, de ses petits-enfants (enfants de la fille) et, dans une moindre mesure, de son gendre (partenaire de la fille). C'est dans cette configuration que la fille est en moyenne la plus citée. Elle affiche un score moyen de 1.18 qui dépasse largement le score moyen total (0.63). C'est, ensuite, la fille de la fille qui est en moyenne la plus mentionnée; son score de 1.00 est significativement plus élevé dans cette configuration que dans toutes les autres. Le fils de la fille est aussi souvent cité, atteignant un score moyen de 0.60 , alors qu'il n'est que de 0.07 dans l'ensemble de l'échantillon. Et finalement, c'est le partenaire de la fille qui affiche un score moyen de 0.23 , dépassant largement ceux dans les autres configurations. Contrairement à la configuration précédente, «Fils», dans laquelle la belle-fille est fortement citée (1.05), le partenaire de la fille (gendre) est relativement peu mentionné (0.23). Par contre, la fille de la fille est davantage mentionnée dans cette configuration que la fille du fils dans la configuration précédente, faisant de la configuration «Fille » un réseau familial particulièrement féminin. Comme pour la configuration précédente, la logique sous-jacente dans cette configuration repose sur les liens de sang et la descendance.

Bien que la logique dans ces deux configurations «Fils» et «Fille» soit similaire, la combinaison différente des membres familiaux qui les composent font d'elles des configurations familiales très distinctes. Alors que, dans la configuration familiale de type «Fils», la composante-clé est le couple « fils » et sa "partenaire », dans la configuration familiale de type «Fille», la fille et ses enfants (filles) en constituent la composante dominante. La première citation - fils ou fille - n'est donc pas vaine, elle influence nécessairement toutes les autres mentions qui lui sont directement ou indirectement liées, faisant du « chaining » une logique de sélection de type «structurel» dans la construction de la famille significative (Widmer \& Jallinoja, 2008; Widmer, 2016). Étonnamment, ce principe donne lieu à une combinaison différente selon le sexe de l'enfant mentionné. Lorsque le fils est mentionné, sa partenaire est aussi souvent citée alors que leurs enfants le sont moins et, lorsque la fille est désignée comme significative, son partenaire est peu cité tandis que les petit-enfants, surtout les petitesfilles, sont davantage mentionnés. Il semblerait que la mention de la fille, à qui il incombe socialement un rôle de donneuse de soins au sein de la 
famille, suffise en somme à garantir la présence et le potentiel de soutien que l'on attend dans la vieillesse alors que celle du fils nécessite celle de sa partenaire pour offrir le même potentiel de soutien. Ces différentes combinaisons de composition peuvent paraître insignifiantes, mais elles peuvent avoir un impact différent sur l'organisation des liens d'interdépendance au sein de ces deux types de configurations familiales. La configuration familiale «Fille» est légèrement plus fréquente (11\%) que la configuration «Fils» $(8 \%)$.

Ce sont les membres de la fratrie du répondant qui dominent la configuration «Fratrie ». La sœur est particulièrement mentionnée; elle atteint un score moyen de 1.08, surpassant largement la moyenne totale (0.19). Quant au frère, il est en moyenne nettement plus cité dans la configuration «Fratrie » que dans les autres configurations. Il affiche un score moyen de 0.53 alors que ce score atteint 0.15 dans l'ensemble de l'échantillon. A noter que l'ami masculin est aussi plus cité comme membre significatif de la famille que dans le reste de l'échantillon ( 0.16 vs 0.13$)$. Le ou la partenaire du répondant est relativement bien cité(e); son score moyen de 0.44 dépasse légèrement celui de l'échantillon total (0.41). Les « autres termes » de parenté sont aussi en moyenne bien mentionnés. Ils atteignent un score moyen de 0.52 , dépassant de peu le score moyen total $(0.50)$. Un rapide recensement des « autres termes » montre que les répondants dans cette configuration familiale tendent à mentionner les partenaires des membres de leur fratrie, surtout les époux des sœurs et, parfois, leur propre mère alors que leurs nièces et neveux sont peu mentionnés. Par rapport aux configurations précédentes, ce sont les liens horizontaux, plutôt de type affinitaire, qui sont privilégiés lorsque les individus âgés définissent leur famille significative. Cette configuration familiale est relativement fréquente puisqu'elle constitue $15 \%$ de l'ensemble des réseaux familiaux de l'échantillon.

Les répondants dont la configuration est de type "Parenté » citent divers membres de leur parenté, de sang ou d'alliance, comme membres significatifs de leur famille comme les cousines qui atteignent un score moyen de 0.31, score excédant largement ceux obtenus dans les autres configurations familiales (0.04). C'est aussi la configuration familiale qui atteint le score moyen le plus haut (2.80) sur les citations des "autres termes ». Ce score dépasse largement celui obtenu sur l'ensemble de l'échantillon (0.50). A nouveau, un recensement des termes de parenté « autres » montre que ce sont surtout les enfants, les sœurs ou les frères 
du partenaire bien que ce dernier soit peu mentionné - seuls 58\% des répondants appartenant à cette configuration ont encore un partenaire vivant - mais aussi les enfants ou les partenaires des sœurs ou des frères qui, eux aussi, sont peu cités - seuls 52\% d'entre eux ont encore de la fratrie ainsi que la parenté éloignée (cousins et alliés) ou encore des filleuls (enfants d'amis proches). Comme dans la configuration «Fratrie», les liens horizontaux, affinitaires, puisés dans la parenté élargie ou la belle-famille dominent dans cette conception de la famille qui « compte ». Cette configuration familiale est peu fréquente puisqu'elle ne représente que $8 \%$ des configurations familiales au sein de l'échantillon genevois.

Quant à la configuration familiale «Isolés», elle regroupe les répondants qui ne citent qu'un nombre restreint d'amis proches comme faisant partie de leur famille significative ou qui ne citent personne. Les aînés qui déclarent n'avoir aucun membre significatif de la famille représentent $60 \%$ des répondants au sein de cette configuration. Lorsque certains membres significatifs sont cités, c'est l'amie qui est la plus listée. Elle affiche un score moyen de 0.43 , surpassant celui de l'ensemble de l'échantillon (0.19). Dans une moindre mesure, l'ami (masculin) est aussi plus cité dans cette configuration que dans les autres. Il atteint un score moyen de 0.28 qui dépasse largement le score moyen total (0.13). Lorsqu'ils le peuvent, les aînés dans ce type de configurations investissent des liens familiaux « volontaires », c'est-à-dire des amis proches sans lien de sang ni d'alliance, pour construire leur famille significative. Cette configuration familiale est l'une des plus fréquentes dans l'échantillon puisqu'elle représente 19\% de l'ensemble des réseaux familiaux; elle se situe après la configuration familiale « Conjugal» $(39 \%)$.

Ces six configurations familiales mettent en exergue la pluralité des conceptions de la famille significative au sein de la population âgée. Certains ont une conception normative de la famille, d'autres privilégient les liens de sang et leur descendance alors que d'autres encore préfèrent les liens familiaux de type affinitaire puisés soit au sein de leur réservoir de parenté, soit en dehors. En somme, les logiques qui poussent les personnes âgées à sélectionner et à combiner les différents membres significatifs qu'elles ont cités les conduit à construire des familles significatives fort distinctes. Ces résultats vérifient l'hypothèse (« $\mathrm{H} 2 »)$ selon laquelle les différents membres cités comme significatifs s'articulent de façon distincte pour donner lieu à une diversité de configurations familiales. La question qui se pose dès lors a trait aux facteurs qui expliquent une telle diversité. 


\subsubsection{Les facteurs explicatifs de la composition}

Plusieurs facteurs sont susceptibles d'influencer ces diverses conceptions de la famille. Le profil socio-démographique des individus (genre, âge et statut socio-économique), la santé, le statut matrimonial et les réserves démographiques sont perçus dans la littérature gérontologique comme des facteurs déterminants de la composition et de la taille des réseaux personnels. Alors que leurs effets sur les relations personnelles dans la vieillesse ont été largement documentés, on ne connaît pas à ce jour leur influence sur la façon dont les aînés conçoivent leur famille significative. Pour répondre à cette question, nous avons réalisé des analyses de régressions logistiques pour chacune des six configurations familiales afin d'identifier les facteurs qui les déterminent. Pour chaque configuration familiale, nous avons procédé en deux étapes. Nous avons inséré dans un premier modèle les variables explicatives qui mesurent le profil socio-démographique (sexe, âge, revenu et citoyenneté), la santé fonctionnelle et le statut matrimonial. Ensuite, nous avons ajouté dans un deuxième modèle les réserves démographiques (avoir un partenaire, des enfants et des membres de la fratrie) afin de tester si la construction des familles significatives est contrainte ou non par le réservoir de parenté.

Dans ces analyses, nous avons opté pour la variante « contrast deviation » dans les modèles de régression (Chambers \& Hastie, 1992). Pour chaque variable indépendante (non dichotomique), les scores obtenus de chaque modalité sont comparés à la moyenne générale de toutes les modalités alors que, dans une régression classique, les scores obtenus de chaque modalité sont rapportés à une modalité de référence, et cela pour chaque variable indépendante. Par ailleurs, ces modèles différent les uns des autres pour des raisons de faisabilité et de logique. Pour les trois premières configurations analysées («Conjugal», «Fils» et «Fille»), les divorcés et les célibataires sont regroupés sous une seule modalité, leur effectif étant trop faible dans ces trois types de configurations familiales. La variable «a des enfants » n'a pas été insérée dans les analyses consacrées aux types de configurations familiales «Fils» et «Fille» puisque seuls les individus âgés ayant des enfants peuvent appartenir à ces deux types de configurations familiales. De même, la variable «a des frères/sœurs » n'a pas été considérée dans les analyses sur le type de configuration "Fratrie » car seuls les aînés ayant au moins une sœur ou un frère peuvent construire de telles configurations familiales. Le tableau 5.6 présente les résultats des 
analyses de régressions logistiques pour les configurations familiales de types «Conjugal», «Fils» et «Fille» et le tableau 5.7, ceux pour les configurations familiales de types «Fratrie », «Parenté » et «Isolés». Toutes ces analyses ont été effectuées dans R (R Core Team, 2015).

Dans les deux premières colonnes du tableau 5.6 figurent les résultats des analyses de régression logistique sur les configurations familiales de type «Conjugal ». On relève dans le premier modèle que ce sont surtout les individus âgés qui ont un revenu élevé $(\mathrm{OR}=1.60, p .<0.05)$ et qui sont « mariés » qui reproduisent le modèle idéal de la famille «nucléaire » en mentionnant leur partenaire et leurs enfants comme membres significatifs de leur famille. En effet, les aînés «mariés » ont 2 fois plus de chances $(\mathrm{OR}=2.00, p .<0.001)$ d'appartenir à ce type de configurations familiales que les «non mariés». Dans le deuxième modèle, l'effet de la modalité « être marié », bien que perdant légèrement de sa puissance, demeure à la limite de la significativité $(\mathrm{OR}=1.75, p .<0.10)$ lorsque l'on introduit la variable «partenaire ». Ce résultat montre que l'engagement que représente le mariage, et cela au-delà de la seule présence du partenaire, influence ce type de conception de la famille significative. Outre l'aspect «normatif» du mariage, ceux qui ont des enfants ont 8 fois plus de chances $(\mathrm{OR}=8.34$, $p .<0.001)$ d'appartenir à ce type de configurations familiales. L'absence de fratrie s'associe aussi à ce type de configurations familiales puisque les individus âgés sans frères ni sœurs ont presque 2 fois plus de chances $(\mathrm{OR}=1.82[1 / 0.55], p .<0.01)$ de mentionner leur conjoint et leurs enfants comme membres significatifs de la famille. Autrement dit, avoir au moins un enfant, un partenaire « marié » - signe d'un engagement affectif sérieux et stable - avoir un revenu élevé et n'avoir aucun frère ou sœur favorisent les configurations familiales de type «Conjugal». Globalement, ce sont donc des individus privilégiés qui adoptent ce type de conception de la famille.

Quant aux configurations familiales de type «Fils», dans le premier modèle, ce sont surtout les veufs $(\mathrm{OR}=2.01, p .<0.05)$ et ceux d'origine suisse $(\mathrm{OR}=3.57$ [1/0.28], $p .<0.05)$ qui s'associent à ce type de configurations familiales. La perte de significativité du veuvage dans le deuxième modèle s'explique sans doute par l'insertion de la variable «a un partenaire » dans l'analyse; la corrélation de ces deux variables atténue l'effet propre du veuvage. L'insertion des réserves démographiques dans le deuxième modèle n'apporte aucune amélioration substantielle à l'analyse (Chi-2(block) : 3.24, $p$. =ns). Les aînés qui appartiennent à ce type de configurations familiales sont aussi relativement avantagés : socialement, 
La composition des configurations familiales

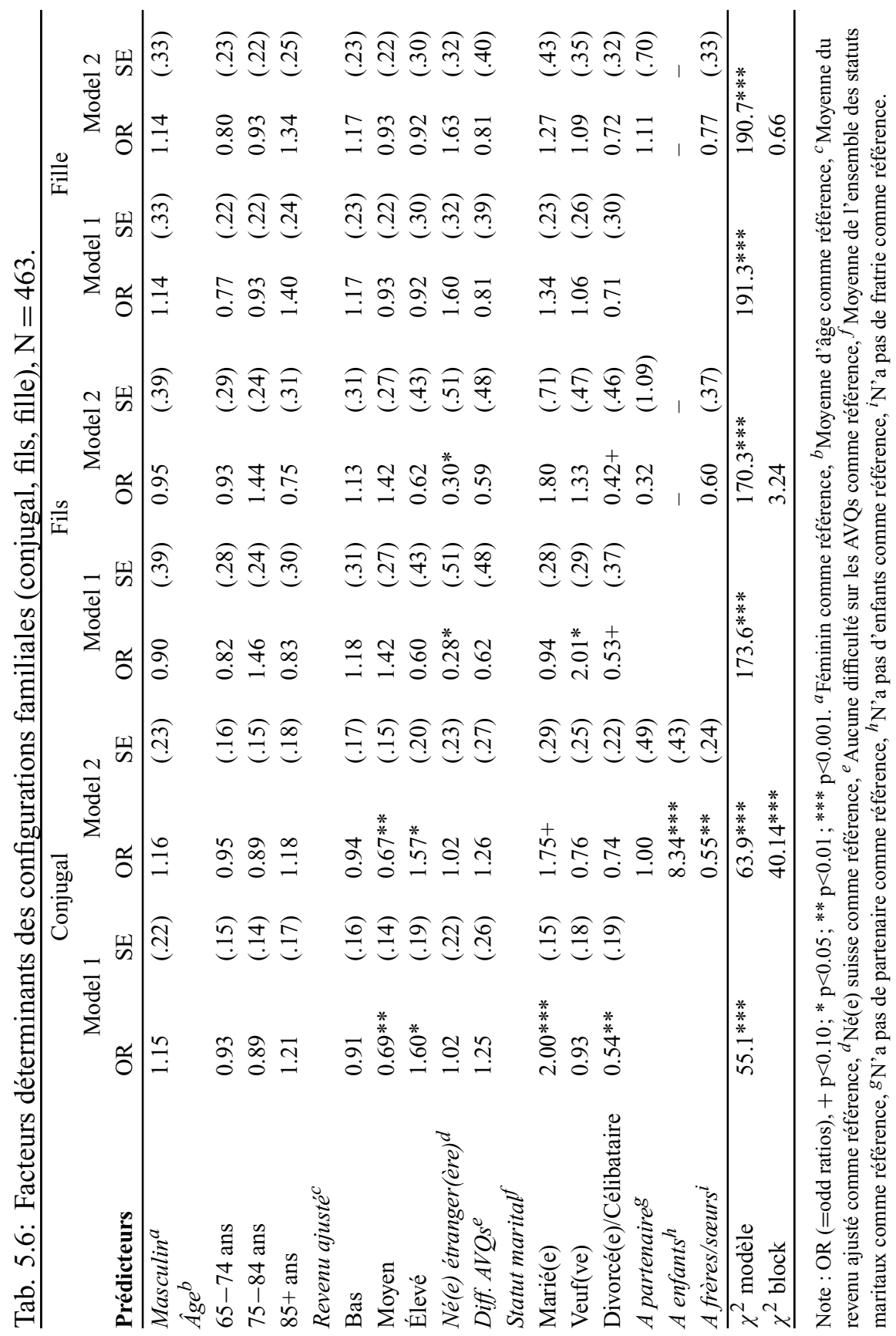


d'une part, puisqu'ils sont pour la plupart d'origine suisse et, démographiquement, d'autre part, puisqu'ils ont des enfants au sein de leur réservoir de parenté. Ceux-ci leur offrent des opportunités relationnelles directes et indirectes (beaux-enfants, petits-enfants, etc.). Veufs, pour la plupart, ils s'appuient sur les liens de sang et la descendance pour construire leur famille significative. Pour ce qui est de la configuration familiale de type «Fille », aucun effet significatif n'apparaît ni dans le premier ni dans le deuxième modèle. Aucun des facteurs testés ne s'associe à ce type de configurations familiales. Il se peut que les facteurs sélectionnés n'aient effectivement aucun impact ou que certains effets, opposés, s'annulent.

Dans le tableau 5.7 sont présentés les résultats d'analyse de régression logistique pour les configurations familiales «Fratrie», «Parenté » et «Isolés ». Concernant les configurations familiales «Fratrie», ce sont surtout les célibataires qui mentionnent leurs frères ou sœurs comme membres significatifs de leur famille. Ils ont en effet 3 fois plus de chances d'appartenir à ce type de configurations que les autres $(\mathrm{OR}=3.40 ; p .<0.001)$ alors que les "mariés » $(\mathrm{OR}=0.65 ; p .<0.05)$ - et même les "divorcés " $(\mathrm{OR}=0.59 ; p .<0.10)$ - ont peu de chances de s'y insérer. Les « jeunesvieux » tendent aussi davantage que les plus vieux à appartenir à ce type de configurations $(\mathrm{OR}=1.40 ; p .<0.10)$. Dans le deuxième modèle, le fait d'être "célibataire » s'associe toujours de manière significative à cette configuration familiale $(\mathrm{OR}=2.95 ; p .<0.01)$, et cela malgré l'insertion des variables « a un partenaire » ou «a des enfants ». D'ailleurs, l'insertion des réserves démographiques dans le deuxième modèle n'améliore pas l'analyse (Chi-2(block) : 2.45, $p$. =ns). Ce n'est donc pas l'absence du conjoint ni même celle d'enfants qui expliquent la propension des célibataires à mentionner certain(e)s de leurs sœurs ou frères comme membres significatifs de leur famille. L'explication réside sans doute dans l'investissement affectif dont font preuve les célibataires auprès de certains membres de leur fratrie. N'ayant jamais été mariés et sans enfant, les célibataires renvoient, surtout à ces âges-là, à des individus qui ont suivi des parcours de vie hors normes (carrière professionnelle chez les femmes, homosexualité, etc.) et qui les ont poussés, dès les premières étapes de leur vie, à rechercher des alternatives relationnelles en dehors du mariage et de la parentalité (Connidis, 2010). Certains ont donc développé tout au long de leur vie des liens affectifs avec certain(e)s de leurs sœurs ou frères avec qui ils entretiennent encore aujourd'hui des relations privilégiées qu'ils qualifient de significatives. 
Comme pour la configuration précédente, ce sont les célibataires $(\mathrm{OR}=2.61 ; p .<0.01)$ qui ont le plus de chances de développer des configurations familiales de type «Parenté» alors que les « mariés » ont significativement peu de risques de s'y insérer $(\mathrm{OR}=0.42 ; p .<0.01)$. Contrairement à l'analyse précédente ( Fratrie »), l'insertion des réserves démographiques (avoir un partenaire, avoir des enfants, avoir des frères/sœurs) dans le deuxième modèle améliore de manière significative l'analyse (Chi2 (block) $=49.43, p .<0.001)$. Ce deuxième modèle confirme que les «mariés » $(\mathrm{OR}=0.36 ; p .<0.05)$ ne développent pas ce type de configurations familiales. Plus encore, il montre que ce sont surtout ceux qui n'ont pas d'enfants $(\mathrm{OR}=16.67[1 / 0.06] ; p .<0.001)$ et ceux qui ont un partenaire (non marié) $(\mathrm{OR}=4.30 ; p .<0.05)$ qui puisent dans leur famille élargie ou d'alliance (familles des membres de la fratrie ou famille du partenaire) des membres significatifs de leur famille. Il s'agit donc d'individus qui ne sont pas mariés (célibataires/veufs/divorcés), bien qu'ayant un partenaire, et ils sont majoritairement sans enfant. Ce sont par ailleurs des individus ayant un bon statut socio-économique $(\mathrm{OR}=1.92[1 / 0.52] ; p .<0.05)$ et surtout des femmes $(\mathrm{OR}=2.33[1 / 0.43] ; p .<0.05)$ qui appartiennent à ce type de configurations familiales. Ce résultat reflète la propension des femmes à intégrer au sein de leur famille significative une plus grande diversité de membres familiaux. Elles sélectionnent les membres de leur famille significative au sein de leur parenté élargie, au sein de la famille de leurs frères ou sœurs mais aussi au sein de la famille de leur conjoint sans pour autant le mentionner comme membre significatif. Elles font donc preuve d'un grand travail de ré-évaluation en re-définissant des membres de la parenté éloignée comme des liens familiaux significatifs.

Quant aux configurations de type "Isolés », l'analyse révèle que les «mariés" ont moins de risque d'appartenir à ce type de configurations familiales que les non mariés $(\mathrm{OR}=0.52 ; p .<0.01)$. Ce sont surtout les individus âgés ayant un bas revenu $(\mathrm{OR}=1.70 ; p .<0.05)$ qui appartiennent à ce type de configurations alors que ceux qui sont socioéconomiquement privilégiés sont peu concernés par ce type de configurations $(\mathrm{OR}=0.39 ; p$. $<0.05)$. La santé a aussi un léger impact : les personnes âgées qui ont des difficultés fonctionnelles tendent à se retrouver davantage dans ce type de configurations familiales que les autres $(\mathrm{OR}=1.75 ; p .<0.10)$. L'insertion des réserves démographiques dans le deuxième modèle contribue à améliorer les résultats de manière substantielle $($ Chi-2(block $\left.)=16.59^{* * *}, p .<0.001\right)$. Ce deuxième modèle montre 
que ce sont surtout l'absence du partenaire $(\mathrm{OR}=3.85[1 / 0.26] ; p .<0.05)$ et le manque d'enfants $(\mathrm{OR}=3.23[1 / 0.31] ; p .<0.001)$ qui favorisent l'émergence de ce type de configurations familiales. Par ailleurs, l'effet significatif du statut socio-économique se confirme dans le deuxième modèle puisque les individus qui ont un revenu élevé ont un risque moindre que les autres $(\mathrm{OR}=0.40 ; p .<0.05)$ d'appartenir à ce type de configurations familiales, quel que soit l'état de leur réservoir de parenté. L'âge a aussi une légère influence, les « jeunes-vieux » étant plus enclins à appartenir à ce type de configurations que les plus vieux $(\mathrm{OR}=1.49 ; p .<0.10)$.

Les individus, qui se retrouvent dans ce type de configurations familiales, cumulent de nombreux désavantages. Ils sont en majorité non mariés (veufs/divorcés/célibataires), sans partenaire ou sans enfant. Certains parviennent à compenser la pauvreté de leur réservoir de parenté en intégrant dans leur famille significative des amis de longue date, d'autres par contre n'y parviennent pas. Parmi les aînés qui font partie de ce type de configurations, $60 \%$ ne citent aucun membre significatif. La difficulté de certains à se créer une famille significative s'explique non seulement par la pauvreté du réservoir de parenté, qui restreint les opportunités de développer des relations significatives, mais aussi par le manque de ressources socioéconomiques et la mauvaise santé. Comme nous l'avons mentionné plus haut (cf. 5.2), un faible statut socio-économique (peu de compétences relationnelles, peu de moyens financiers, etc.) et une mauvaise santé s'associent à un réseau personnel plus petit et moins diversifié, ce qui fragilise encore davantage les aînés sans partenaire et sans enfants. Ceux-ci ont peu d'alternatives relationnelles en dehors de leur réservoir de parenté ni l'énergie pour compenser l'absence du partenaire et le manque d'enfants.

Ces analyses montrent que les diverses logiques qui sous-tendent la construction de la famille significative dépendent en partie du parcours de vie des aînés. Certains individus ont suivi une trajectoire de vie continue, attendue, alors que d'autres sont sortis des sentiers battus. Certains, par exemple, ont accumulé des avantages démographiques (mariage ou remise en couple) et socio-économiques (succès professionnel, etc.). D'autres au contraire ont perdu de tels avantages au gré de transitions de vie négatives, connaissant le veuvage, le divorce, le décès d'enfants ou encore une perte de revenu. D'autres encore n'ont jamais acquis de tels avantages; ils ne se sont jamais mariés ou n'ont jamais eu d'enfants (infertilité, etc.). Ainsi, ces diverses trajectoires de vie influencent la manière dont les â̂nés construisent leur famille significative. Toutefois, le parcours de vie 


\section{La composition des configurations familiales}

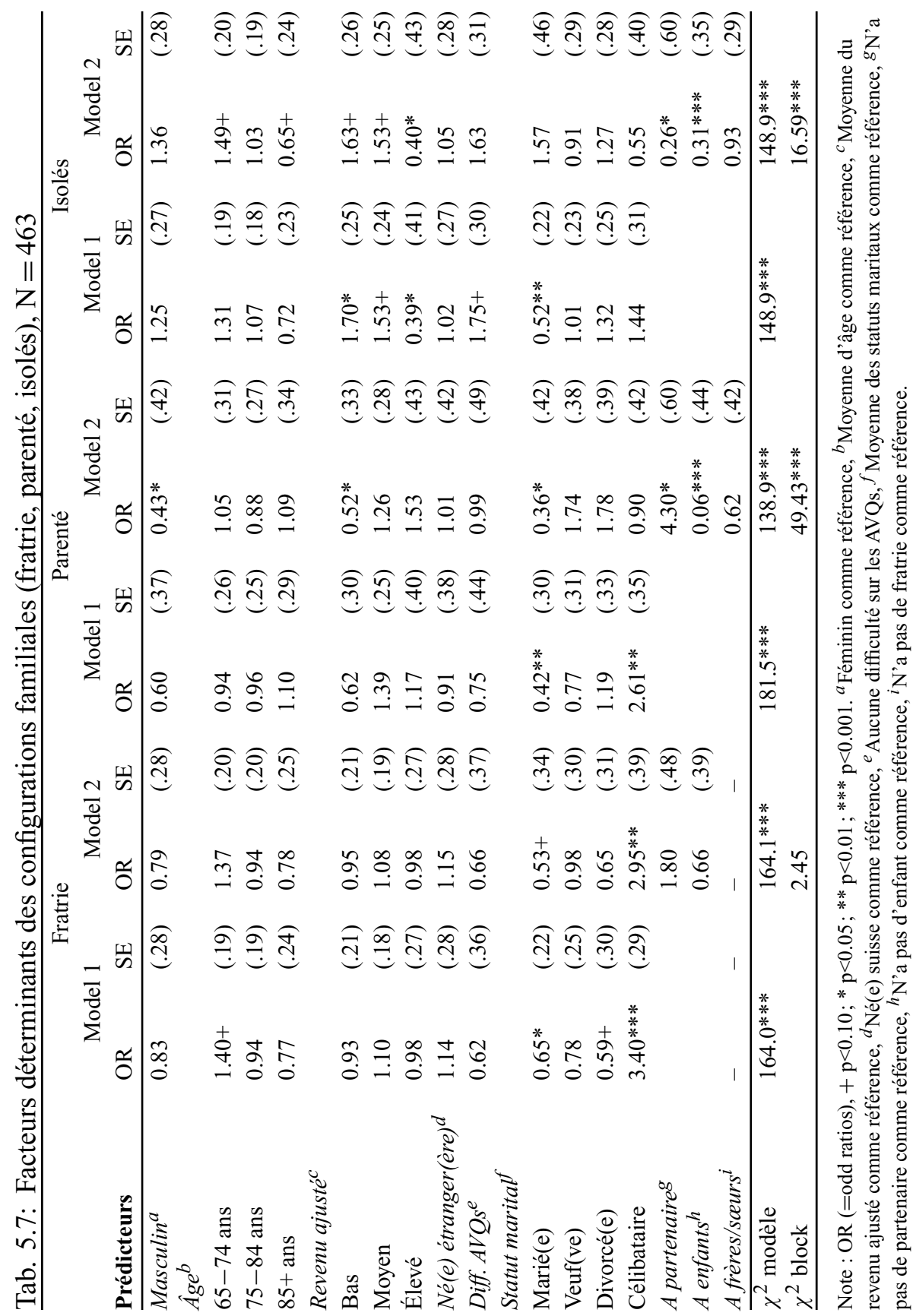


ne constitue pas le seul facteur. La position des individus dans la société (genre, statut socio-économique, âge, citoyenneté) influe aussi sur le développement de leur configuration familiale. Grâce aux ressources qu'ils ont à disposition (moyens financiers, réseau personnel diversifié, bonne santé et énergie) et leur «potentialité » relationnelle (cf. chapitre " Problématique et hypothèses »), certains aînés, notamment les femmes et les individus ayant un statut socio-économique élevé, parviennent à mettre en place des mécanismes d'adaptation qui leur permettent de développer des familles significatives en s'investissant dans une variété de relations personnelles qui vont bien au-delà de la famille «nucléaire », et cela malgré les pertes qu'ils ont pu connaître et la faiblesse de leur réservoir de parenté. Ces résultats confirment l'hypothèse « $\mathrm{H} 2$ », selon laquelle la diversité des configurations familiales s'inscrit dans le parcours de vie des individus âgés et leur profil socio-démographique.

\subsubsection{Le profil des membres des configurations}

Après avoir identifié les facteurs explicatifs de la diversité des configurations familiales, nous nous intéressons aux caractéristiques sociodémographiques (sexe, âge, niveau d'éducation et lieu d'habitation) des membres qui composent les différentes configurations familiales ainsi que la qualité des relations que le répondant entretient avec eux en termes de durée de la relation, de la fréquence des contacts et du degré de confiance qu'il porte aux membres de sa configuration. Le profil des membres cités constitue un indicateur précieux quant à la diversité et à la qualité des ressources disponibles au sein de ces six configurations familiales. Avoir, par exemple, une majorité de femmes au sein de son réseau familial est synonyme d'un potentiel d'aide plus facile à mobiliser en raison des rôles de soins et d'aide qui sont socialement attribués à la gent féminine. De même, avoir une configuration composée essentiellement de membres familiaux qui ont un statut socio-économique élevé est davantage prometteur de ressources diverses (financières, informatives, etc.) qu'un réseau constitué uniquement de membres ayant un bas statut socio-économique. Vivre dans le même canton que les membres cités, entretenir avec eux des contacts réguliers et avoir en eux une confiance absolue offrent des conditions favorables aux échanges et peuvent procurer un sentiment de sécurité chez l'aîné, sachant qu'il peut compter sur eux en cas de besoin. Finalement, 
l'âge et la durée de la relation renseignent si les membres qui composent la configuration familiale sont de même génération. Bien que la présence de petits-enfants, signe de continuité familiale, soit importante pour de nombreuses personnes âgées, les petits-enfants peinent à répondre aux besoins de partage et de pratique d'activités communes. A la fois compagnons et confidents, les membres de même génération ou ceux qui entretiennent avec le répondant une relation de longue durée apportent des ressources de grande qualité, s'associant au maintien du bien-être dans la vieillesse. Ceci dit, ces ressources sont fragiles, pouvant disparaître à tout moment en raison de l'âge avancé des prestataires.

Pour explorer les profils des membres qui composent les six configurations familiales, nous avons calculé, pour chaque configuration familiale, le nombre moyen de femmes parmi les membres cités, la moyenne d'âge des membres cités, le nombre moyen de membres cités ayant passé la maturité (niveau d'éducation élevé), le nombre moyen de membres vivant dans le canton de Genève, la durée moyenne des relations entre le répondant et les membres cités, le nombre moyen de membres avec qui le répondant entretient des contacts réguliers (au moins une fois par semaine) et le nombre moyen de membres en qui le répondant a une confiance absolue. Pour la configuration familiale «Isolés», seuls les répondants ayant cité au moins 1 membre significatif $(n=42)$ ont été considérés dans l'analyse. Nous avons ensuite pondéré l'ensemble de ces indices par la taille des configurations familiales - c'est-à-dire le nombre des membres cités comme significatifs - afin de les rendre comparables. Ces indices varient donc de 0 (aucun membre cité ne présente la caractéristique étudiée) à 1 (tous les membres cités présentent la caractéristique étudiée). Nous avons ensuite procédé à une analyse de variance (ANOVA), complétée par le test de Duncan qui indique, par des lettres de l'alphabet différentes, quelles configurations se distinguent significativement les unes des autres. Le tableau 5.8 présente ces différents résultats.

Ces résultats indiquent que ces six configurations familiales diffèrent quant au profil socio-démographique et relationnel des membres qui les constituent. A l'exception du genre des membres, majoritairement féminins, et la confiance absolue que leur portent les répondants, ces six configurations familiales réunissent des membres qui se distinguent sur plusieurs points. Concernant la configuration familiale «Conjugal», nous avons montré précédemment que les répondants qui ont une telle conception de la famille sont privilégiés tant démographiquement (partenaire 
Membres significatifs et configurations

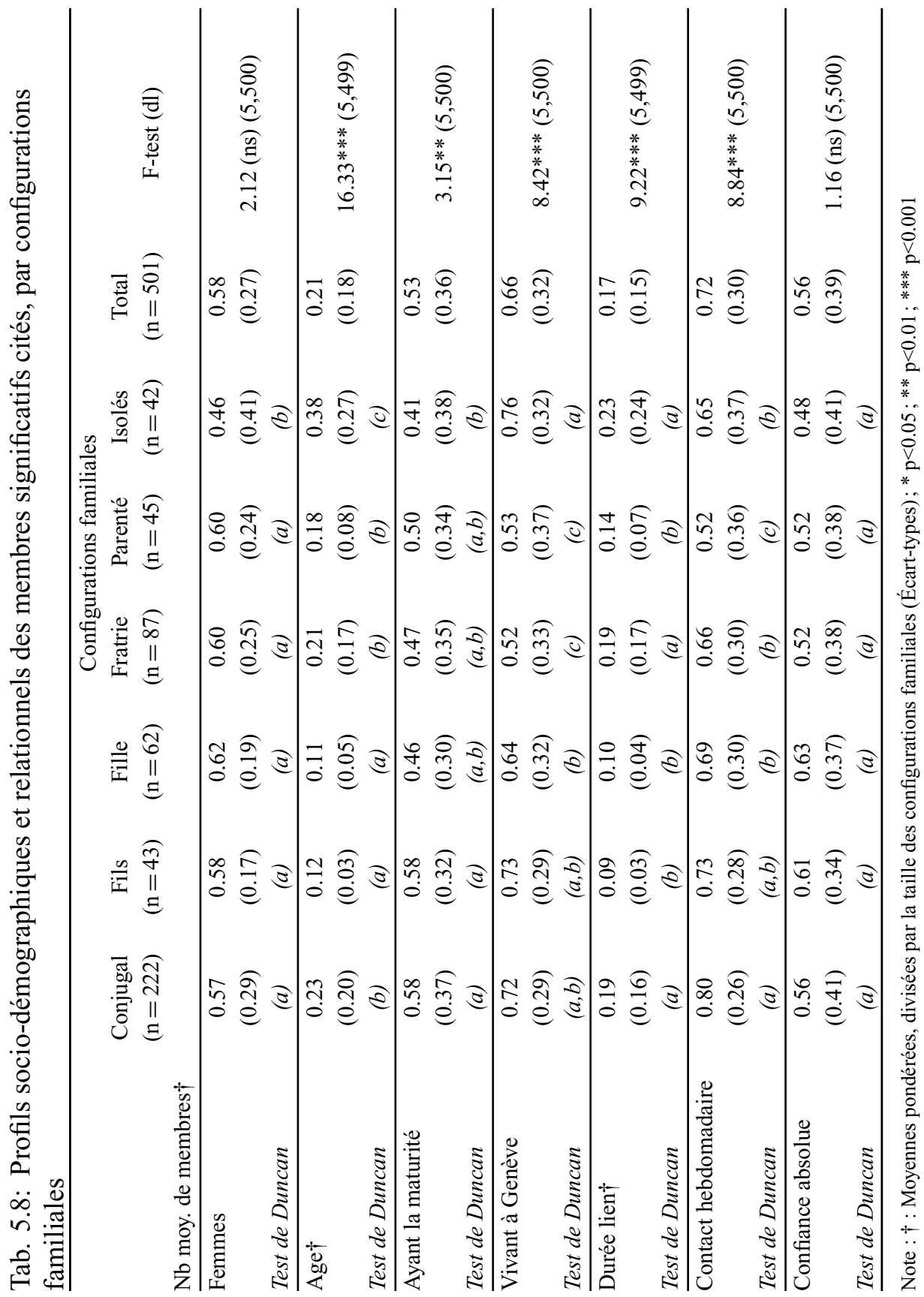


marié et enfants) qu'économiquement (statut socio-économique élevé). Les membres qu'ils citent comme significatifs sont en moyenne plus nombreux à avoir un niveau d'éducation élevé (maturité) $(\mathrm{M}=0.58$ vs Mtot $=0.53$ ) et à vivre à Genève que dans l'ensemble de l'échantillon $(\mathrm{M}=0.72$ vs $\mathrm{Mtot}=0.66)$. Le partenaire étant mentionné, l'âge moyen est relativement élevé $(\mathrm{M}=0.23 \mathrm{vs} \mathrm{Mtot}=0.21)$ et les relations avec le répondant sont globalement de longue durée $(\mathrm{M}=0.19$ vs $\mathrm{Mtot}=0.17)$. La présence du partenaire est «a priori » synonyme de compagnonnage et de partage au quotidien. Plus encore, le parent âgé a des contacts réguliers avec la plupart des membres qu'il cite $(\mathrm{M}=0.80 \mathrm{vs} \mathrm{Mtot}=0.72)$. La présence du partenaire, certes, explique un tel score mais les enfants cités semblent aussi actifs dans le maintien des liens. Les membres dans ce type de configurations offrent donc des conditions favorables aux échanges : un statut socio-économique élevé, une proximité géographique et des contacts fréquents.

Quant aux configurations familiales de type «Fils», les répondants sont veufs mais relativement avantagés puisqu'ils sont d'origine suisse et ont une descendance (enfants et petits-enfants). Les membres qu'ils insèrent au sein de leur configuration ont, dans l'ensemble, un niveau d'éducation élevé (maturité) $(\mathrm{M}=0.58$ vs $\mathrm{M}=0.53)$ et vivent en moyenne à proximité (Genève) $(\mathrm{M}=0.73$ vs $\mathrm{Mtot}=0.66)$. Par ailleurs, un bon nombre d'entre eux entretiennent avec les répondants des contacts fréquents (hebdomadaires) $(\mathrm{M}=0.73$ vs $\mathrm{Mtot}=0.72)$. Ils affichent un âge moyen plus bas que les membres des autres configurations $(\mathrm{M}=0.12$ vs Mtot $=0.21)$ et une durée moyenne de relations plus courte $(M=0.09$ vs Mtot $=0.17$ ), ce qui s'explique par l'inclusion des petits-enfants dans ce type de configurations. La présence de membres de différentes générations représente pour l'individu âgé un signe positif de continuité et de stabilité familiale. Cependant, l'absence de membres de même génération signale le manque de ressources d'une autre qualité comme le compagnonnage ou les échanges de confidences. Ceci dit, comme dans les configurations «Conjugal», les conditions sont «a priori » favorables aux échanges : un statut socio-économique élevé, une proximité géographique et des contacts fréquents avec le répondant.

La configuration familiale de type «Fille» inclut aussi des petitsenfants. Par conséquent, l'âge moyen des membres est moins élevé $(\mathrm{M}=0.11$ vs $\mathrm{Mtot}=0.21)$ et la durée moyenne des liens est plus courte $(\mathrm{M}=0.10$ vs $\mathrm{Mtot}=0.17)$ que dans le reste de l'échantillon. Comme 


\section{Membres significatifs et configurations}

dans la configuration précédente, le répondant ne dispose pas «a priori » d'un compagnon ou d'un confident avec lequel il peut partager certaines activités ou des confidences. Cependant, les résultats précédents étant peu concluants (cf. modèles des régressions logistiques), on ne sait pas si le partenaire du répondant est décédé (veufs), inexistant (divorcés ou célibataires) ou s'il n'est simplement pas mentionné. Ces analyses révèlent cependant - et cela, contrairement à la configuration précédente - que les ressources offertes par les membres mentionnés sont de moins bonne qualité : le niveau d'éducation est en moyenne plus bas $(\mathrm{M}=0.46$ vs Mtot $=0.53)$ que dans le reste de l'échantillon, les membres sont en moyenne moins nombreux à vivre à Genève $(\mathrm{M}=0.64 \mathrm{vs}$ Mtot $=0.66)$ et à entretenir des contacts hebdomadaires avec les répondants $(\mathrm{M}=0.69 \mathrm{vs}$ Mtot $=0.72$ ) que dans l'échantillon total.

Pour ce qui est des configurations familiales de type "Fratrie», les répondants sont généralement célibataires, ayant vécu pour la plupart une trajectoire de vie hors normes (sans mariage, ni enfants), et se sont particulièrement investis au cours de leur vie dans l'une ou l'autre de leurs relations fraternelles. Les membres de cette configuration affichent en moyenne des relations de plus longue durée que dans l'ensemble de l'échantillon $(M=0.19$ vs Mtot $=0.17)$. Il s'agit dans ce type de configurations de relations affectives axées sur une histoire commune et, étant définies comme significatives, elles sont sans doute source de partage et de compagnonnage. Cependant, les membres cités ont en moyenne un niveau d'éducation plus bas $(\mathrm{M}=0.47$ vs $M$ tot $=0.53)$, sont en moyenne géographiquement plus éloignés $(\mathrm{M}=0.52 \mathrm{vs} \mathrm{Mtot}=0.66)$ et leurs relations sont en moyenne moins rythmées par des contacts réguliers $(\mathrm{M}=0.66$ vs Mtot $=0.72$ ) que dans le reste de l'échantillon.

En ce qui concerne les configurations de type "Parenté », les analyses précédentes ont montré qu'il s'agit d'aînés en couple (non mariés) mais sans enfant, des femmes pour la plupart et des individus ayant un statut socio-économique élevé qui appartiennent à ce type de configurations familiales. Les membres de la famille élargie ou d'alliance qui sont cités comme significatifs ressemblent sur de nombreux points à ceux de la configuration «Fratrie » : il sont moyennement âgés $(M=0.18$ vs Mtot $=0.21)$, ont en moyenne un niveau d'éducation plus bas $(\mathrm{M}=0.50 \mathrm{vs}$ Mtot $=0.53)$, habitent en moyenne plus à l'extérieur de Genève $(M=0.53$ vs Mtot $=0.66)$ et ont en moyenne des contacts moins fréquents avec le répondant $(\mathrm{M}=0.52$ vs $\mathrm{Mtot}=0.72)$ que dans le reste de l'échantillon. Ils 
se distinguent cependant des membres de la configuration " Fratrie» par des relations en moyenne de plus courte durée $(M=0.14$ vs Mtot $=0.17)$. Les liens de parenté acquis par l'intermédiaire du partenaire ou de la fratrie (belles-familles) expliquent la durée plus courte des relations au sein de cette configuration. La provenance multiple des membres sélectionnés offre «a priori » une grande diversité de ressources potentielles. Cependant, le nombre de membres avec qui les répondants entretiennent en moyenne des contacts réguliers est particulièrement bas au sein de cette configuration.

Quant aux configurations familiales de type «Isolés», les analyses précédentes de régression ont mis en évidence la pauvreté du réservoir de parenté et la précarité socio-économique des répondants appartenant à ce type de configurations. Cependant, une minorité d'entre eux parvient à compenser la pauvreté de leur réservoir de parenté en mentionnant un nombre restreint d'amis proches comme membres significatifs de la famille. En moyenne, les amis cités ont un niveau d'éducation plus bas $(\mathrm{M}=0.41$ vs $M$ tot $=0.53)$ et sont plus nombreux à vivre à Genève $(\mathrm{M}=0.76$ vs Mtot $=0.66)$ que dans le reste de l'échantillon. Par ailleurs, ils sont significativement plus âgés $(\mathrm{M}=0.38$ vs $\mathrm{Mtot}=0.21)$ et entretiennent des relations en moyenne de plus longue durée avec les répondants $(\mathrm{M}=0.23$ vs Mtot $=0.17)$ que ceux mentionnés dans l'ensemble de l'échantillon. Ces résultats montrent que les amis désignés comme membres significatifs de la famille ne sont pas issus de nouvelles rencontres. Il s'agit d'amitiés de longue durée, soutenues par des relations affectives fortes et alimentées de nombreux échanges au fil du temps. Les ressources potentielles que ces membres sont susceptibles d'apporter sont de grande qualité, telles que le compagnonnage et les échanges de confidences. Cependant, à l'instar des membres des configurations «Fratrie » et «Parenté», ils sont en moyenne moins nombreux à entretenir des contacts réguliers avec les répondants que ceux de l'échantillon total $(M=0.65$ vs Mtot $=0.72)$. Il est probable qu'étant aussi âgés, le processus de fragilisation qui touche les répondants affecte aussi l'énergie et la santé des amis proches qui tendent par conséquent à diminuer le rythme de leurs contacts.

L'analyse du profil socio-démographique et relationnel des membres des configurations familiales montre que ceux-ci sont, parce qu'ils affichent des liens de parenté distincts, significativement différents tant dans leur profil que dans la qualité de leurs relations qu'ils entretiennent avec les répondants. Ces résultats révèlent que les configurations familiales composées de membres familiaux de type « horizontal » et «affinitaire», tels 


\section{Membres significatifs et configurations}

que les frères ou sœurs, les cousins ou encore les amis, réunissent des membres de même génération avec qui les répondants entretiennent des relations de longue durée. L'inclusion de ces membres dans la famille significative n'est pas le «fruit» du hasard, mais s'explique par la qualité des relations, celles-ci s'étant construites et surtout maintenues au fil du temps au travers d'échanges et de partages. Cependant, en raison de leur âge avancé et, pour certains, de l'éloignement géographique, les contacts sont moins fréquents que dans d'autres configurations. Quant aux trois configurations familiales axées sur les liens intergénérationnels, les résultats « opposent» les configurations de types « Conjugal» et «Fils» à celles de type «Fille». Les deux premiers types semblent offrir des conditions favorables aux échanges telles que des ressources socio-économiques, une proximité géographique et des contacts fréquents alors que les configurations de type «Fille» y sont moins propices (statut socio-économique plus faible, éloignement géographique des membres et surtout moins de contacts fréquents). Cette analyse montre que la composition distincte de ces six configurations familiales s'associe à des différences en termes de qualité des ressources et de contextes d'échanges.

\subsubsection{Conclusion sur la composition des configurations familiales}

Cette exploration des configurations familiales dans la vieillesse met en évidence plusieurs points importants. D'abord, les divers membres significatifs identifiés dans la première partie de ce chapitre se combinent les uns aux autres pour former une pluralité de configurations familiales. Leur développement n'est pas aléatoire et suit des logiques qui leur sont propres. Certaines de ces configurations familiales se calquent sur le modèle idéal de la famille «nucléaire», dans lesquelles le partenaire marié et les enfants occupent une place privilégiée. D'autres configurations reposent sur des critères de sang et de la descendance; ce sont principalement les enfants et les petits-enfants qui les composent. Dans d'autres encore dominent les relations horizontales, affinitaires, basées sur le partage de valeurs et d'activités communes. Conformément à cette logique, ce sont les membres de la fratrie, de la parenté élargie ou encore les amis proches qui en constituent les principales composantes. 
La composition des configurations familiales et les logiques qui les sous-tendent sont en partie influencées par le parcours de vie. Les transitions familiales, telles que le mariage, la parentalité, la grand-parentalité, le divorce ou encore le veuvage, ont un impact sur la façon dont les individus construisent leur famille significative. Certaines de ces transitions enrichissent le réservoir de parenté comme le mariage, le remariage, la parentalité et la grand-parentalité, offrant de multiples opportunités relationnelles sur lesquelles les individus âgés s'appuient pour construire leur famille significative alors que d'autres transitions, telles que le veuvage, le divorce, l'infertilité ou la perte d'enfants, restreignent les réserves démographiques invitant les personnes âgées à déployer des mécanismes de re-définition familiale pour «compenser » la pauvreté de leur réservoir de parenté. Les ressources à disposition, telles que les moyens financiers, les compétences relationnelles, un réseau personnel fourni ou encore une bonne santé, sont aussi déterminantes dans la construction des configurations familiales. Ces ressources dépendent de la position des individus dans la société mais aussi de leurs expériences relationnelles passées. Diverses, elles offrent une certaine marge de manœuvre aux individus qui en disposent. Ils peuvent plus aisément que les autres s'investir dans des relations qu'ils puisent aussi bien dans leur réservoir de parenté qu'à l'extérieur pour construire une famille qui fait sens. Quant à ceux qui n'ont ni réserves démographiques, ni ressources socio-économiques, ils peinent à déployer des mécanismes d'adaptation et se retrouvent sans relation familiale significative aux dernières étapes de leur vie.

L'insertion de membres familiaux différents au sein des configurations familiales implique des individus aux profils socio-démographiques et relationnels distincts, porteurs de ressources de qualité variée. L'analyse distingue les configurations familiales qui réunissent des membres de différentes générations de celles qui regroupent des membres de même génération. Les premières se caractérisent, à l'exception des configurations familiales de type "Fille », par des membres ayant en moyenne un niveau d'éducation élevé, vivant à Genève et entretenant des contacts réguliers avec le parent âgé. Ces configurations familiales sont en somme rassurantes, symbolisant la continuité et la stabilité familiale. Quant aux configurations qui intègrent des membres de même génération, elles offrent des relations de qualité, reposant sur l'échange de confidences et le compagnonnage. Cependant, elles s'associent à de moins bonnes conditions d'échanges que les configurations précédentes puisqu'elles regroupent des 
membres âgés, ayant en moyenne un niveau d'éducation plus bas, vivant davantage à l'extérieur de Genève et qui sont moins enclins aux contacts hebdomadaires.

Ces résultats montrent qu'à l'instar des plus jeunes, les aînés construisent une diversité de configurations familiales selon leur parcours de vie et leurs ressources. Ils vérifient donc l'hypothèse « $\mathrm{H} 2$ » selon laquelle les membres significatifs cités s'articulent de façon distincte et forment une pluralité de configurations familiales en fonction du profil socio-démographique des aînés et de leur parcours de vie. Ces observations mettent en évidence que la famille significative des personnes âgées est loin d'être stable contrairement aux conclusions de certaines études s'appuyant sur le modèle du convoi. Au contraire, la famille significative évolue en fonction du parcours de vie des individus âgés et des événements qu'ils sont amenés à expérimenter. L'absence de partenaire et d'enfants, réserves démographiques considérées par la gérontologie sociale comme fondamentales, représentent certes une sérieuse contrainte dans la construction de la famille significative. Cependant, ce manque peut être surmonté et dépassé par ceux qui sont en mesure, grâce aux ressources dont ils disposent et aux expériences relationnelles qu'ils ont acquises, de re-définir les liens qui les entourent en relations familiales significatives. 


\section{Capital social et ambivalence au sein des configurations familiales}

Après avoir exploré la composition des configurations familiales, ce sixième chapitre se centre sur le capital social, les conflits et l'ambivalence qui sont produits par les liens d'interdépendance qui sous-tendent les configurations familiales. Nous observons d'abord les variations du capital social en fonction de la composition des configurations familiales et du profil socio-démographique des répondants par le biais d'analyses de variance (ANOVA) et de régressions linéaires. Pour illustrer ces résultats, nous présentons des exemples de configurations familiales, tirés de l'échantillon genevois, qui reflètent les six types de configurations familiales précédemment identifiés et le type de capital social qu'ils génèrent. Nous abordons ensuite les conflits et l'ambivalence. Grâce à l'analyse des correspondances multiples (ACM), nous examinons comment les différents modes de conflit et de support, reflets de gestions diverses de l'ambivalence structurelle, s'associent à des configurations familiales distinctes et à des conditions sociales particulières. Finalement, nous agrémentons cette dernière partie par une présentation de situations empiriques qui illustrent la façon dont ces différents modes se mettent en place selon les types de configurations familiales et les ressources dont disposent les individus à cette étape-là de la vie.

\subsection{Le capital social}

Dans le chapitre précédent, nous avons mis en exergue six types de configurations familiales qui se distinguent les uns des autres par l'identité des membres qui les composent et la façon dont ces derniers se combinent les uns aux autres. Nous avons montré que la construction de ces différentes configurations familiales dépend du profil socio-démographique et du parcours de vie des individus âgés. Nous avons aussi souligné que 
les membres qui composent ces différents types de configurations familiales ne se différencient pas seulement par leur lien de parenté mais aussi par leur profil socio-démographique et relationnel. Par leur statut socio-économique élevé, leur proximité géographique avec l'aîné et leurs contacts fréquents, certains créent un contexte favorable aux échanges de soutien au sein de la configuration familiale alors que d'autres, ayant un faible statut socio-économique, vivant à distance ou ayant des contacts peu fréquents avec l'aîné, n'offrent pas a priori les conditions suffisantes pour favoriser les échanges de soutien au sein de la famille. Bien qu'ils soient en soi instructifs, les indicateurs de proximité géographique, de fréquence des contacts ou même le montant de l'aide échangée sont trop limités pour saisir le soutien familial dans toute sa complexité. Il ne suffit pas en effet d'être entouré de personnes d'un niveau d'éducation élevé, vivant à proximité et avec lesquelles on entretient des contacts réguliers pour bénéficier d'un solide soutien. Selon l'approche configurationnelle, il faut être connecté aux autres membres de la configuration familiale et impliqué dans les échanges familiaux pour avoir accès au soutien familial. Nous proposons dans cet ouvrage d'utiliser le concept de capital social pour approcher le potentiel de soutien au sein des configurations familiales; les indices utilisés pour le mesurer permettent d'aller plus loin que les indicateurs factuels (proximité géographique, fréquence des contacts, etc.) généralement privilégiés dans la recherche en gérontologie sociale.

L'approche configurationnelle met à disposition des outils statistiques pour repérer la façon dont les échanges s'organisent au sein des configurations familiales mais aussi pour détecter la position des individus âgés dans leur configuration. Les scores obtenus sur ces divers indices permettent d'identifier le type de capital social produit dans ces différentes configurations (Widmer, 2006, 2016). Nous avons sélectionné six indices qui mesurent différentes propriétés structurelles des réseaux personnels : la taille, la réciprocité, la densité, le degré rentrant (In-degree), le degré sortant (Out-degree) et la centralité du répondant (cf. Chapitre «Données et méthodes »). Les trois premiers permettent d'approcher le capital social de type chaîne, prometteur d'un soutien familial collectif et solide, alors que les trois derniers mettent plutôt en évidence le capital social de type pont, garant de l'autonomie structurelle des individus au sein de leur configuration familiale. Dans cette section, nous examinons, à l'aide de ces indices, les propriétés structurelles des six types de configurations familiales précédemment dégagés afin d'identifier le type de capital social qu'ils 
produisent. Nous observons comment le capital social varie dans sa forme et son volume selon les configurations familiales et nous cherchons à identifier les facteurs qui expliquent le mieux ces variations. D'abord, nous nous interrogeons sur l'influence de la composition des configurations familiales (hypothèses « $\mathrm{H} 3$ » et « $\mathrm{H} 4 »)$, sur celle du profil socio-démographique des aînés («H5 ») mais aussi sur l'impact du réservoir de parenté comme variable de contrôle. L'analyse se fera en deux temps. Dans un premier temps, nous considérons séparément les différents facteurs en effectuant une analyse de variance (ANOVA) et, dans un deuxième temps, nous les intégrons ensemble dans des analyses de régression linéaire. Et, finalement, nous présentons des exemples de configurations familiales qui illustrent les variations du capital social au sein des configurations familiales dans la vieillesse.

\subsubsection{Le capital social dans les configurations familiales}

Dans cette sous-section, nous examinons l'effet de la composition des configurations familiales sur le capital social. Nous avons fait l'hypothèse dans le chapitre «Problématique et hypothèses » que le type de liens de parenté (liens intergénérationnels vs affinitaires) ( «H3») ainsi que le sexe des membres significatifs de la famille (« $\mathrm{H} 4 »)$ s'associent à différents types de capital social. Pour tester ces hypothèses, nous avons procédé à des analyses de variance (ANOVA). Le tableau 6.1 affiche les scores moyens des indices du capital social selon les types de configurations familiales. Le test de Duncan indique, par des lettres de l'alphabet différentes, les scores moyens qui diffèrent significativement les uns des autres selon les types de configurations familiales. Dans le tableau 6.1, ce sont les modalités en colonne qui doivent être comparées les unes aux autres (et non en ligne). Dans les grandes lignes, les résultats révèlent que le capital social varie significativement selon les types de configurations familiales.

Les configurations familiales de type «Conjugal», qui se composent $\mathrm{du}$ partenaire et des enfants du répondant, affichent une taille plutôt moyenne $(\mathrm{M}=3.50$ vs $\mathrm{Mtot}=3.39)$ avec des liens de soutien denses $(\mathrm{M}=0.46 \mathrm{vs}$ Mtot $=0.37)$ et réciproques $(\mathrm{M}=0.45 \mathrm{vs}$ Mtot $=0.39)$. Elles font partie des configurations les plus denses et les plus réciproques de l'échantillon. Quant à l'engagement de l'aîné dans son réseau familial, on observe que le nombre moyen de membres que le répondant soutient (Degré rentrant (In-degree) $: \mathrm{M}=2.26 \mathrm{vs} \mathrm{Mtot}=2.11$ ) et le nombre moyen 


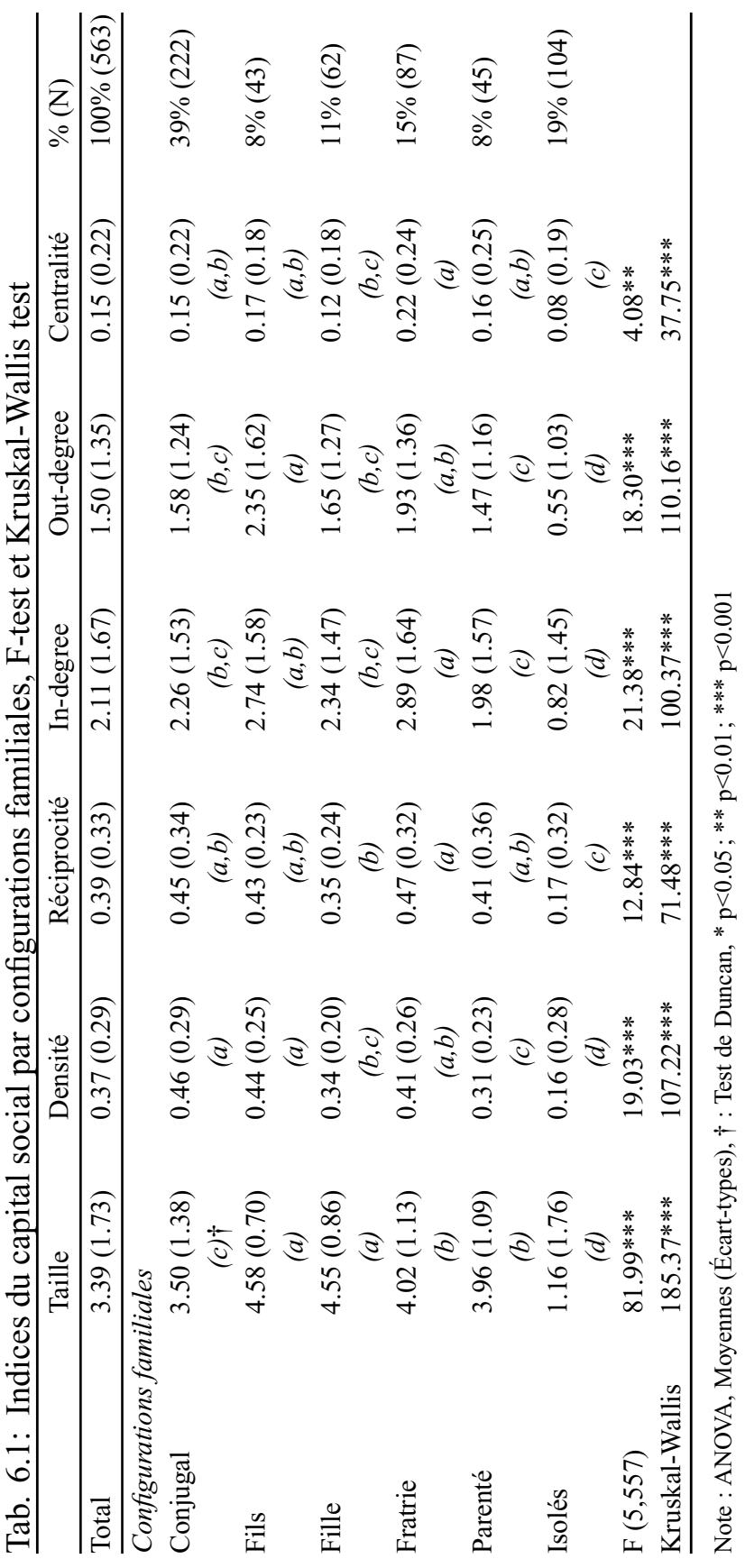


des membres qui le soutiennent (Degré sortant (Out-degree) : $\mathrm{M}=1.58$ vs Mtot $=1.50$ ) ne dépassent que légèrement la moyenne de l'échantillon. L'aîné dans ce type de configurations familiales est donc moyennement actif dans les échanges mais aussi moyennement central, son score sur la centralité n'excédant pas le score moyen total $(M=0.15$ vs $M$ tot $=0.15)$. Les propriétés structurelles de ce type de configurations, telles que les fortes densité et réciprocité des échanges de soutien, révèlent que les configurations familiales de type «Conjugal» produisent du capital social de type chaîne. Conformément à l'hypothèse « $\mathrm{H} 3$ », la présence du partenaire et des enfants dans la configuration densifie les liens d'interdépendance et de soutien. La réciprocité "généralisée », qui caractérise généralement les liens conjugaux et intergénérationnels, a contribué à tisser des liens forts d'interdépendance entre les divers membres de la configuration. A souligner cependant que le partenaire cité comme membre significatif est dans $58 \%$ des cas issu d'un remariage et n'est pas le parent biologique des enfants cités par le répondant. Par conséquent, la remise en couple ou plutôt le remariage - puisqu'il s'agit surtout de répondants mariés dans ce type de configurations - n'entraîne pas une plus grande centralité du répondant et ne favorise pas plus l'émergence du capital social de type pont que le premier mariage. L'individu âgé n'est pas plus amené à servir d'intermédiaire entre ses enfants et son partenaire que lors d'une première union. L'engagement que représente le «mariage » à cette étape de la vie semble suffire à consolider les liens d'interdépendance entre les différents membres de la famille significative, qu'ils soient liés ou non entre eux par des liens de sang.

Les configurations familiales de type «Fils», qui incluent le fils, la femme du fils (la belle-fille) et les enfants du fils, se caractérisent en moyenne par une grande taille $(\mathrm{M}=4.58 \mathrm{vs} \mathrm{Mtot}=3.39)$, une forte densité $(\mathrm{M}=0.44$ vs Mtot $=0.37)$ et une forte réciprocité des liens de soutien $(\mathrm{M}=0.43$ vs $\mathrm{Mtot}=0.39)$. Par ailleurs, le répondant dans ce type de configurations est particulièrement actif : il donne du soutien à un grand nombre de membres de sa configuration (Degré rentrant: $\mathrm{M}=2.74 \mathrm{vs}$ Mtot $=2.11$ ) et en reçoit aussi de la part d'un grand nombre (Degré sortant : $\mathrm{M}=2.35$ vs Mtot $=1.50)$. Le faible écart entre les membres soutenus et soutenants indique que le répondant est actif autant dans la provision du soutien que dans sa mobilisation, ce qui est loin d'être le cas dans la plupart des autres configurations. En moyenne, en effet, on note un décalage entre membres 


\section{Capital social et ambivalence}

aidés et aidants, les premiers étant perçus par les répondants comme nettement plus nombreux que les deuxièmes (Degré rentrant $=2.11$ vs Degré sortant $=1.50)$. Outre son engagement, le répondant se perçoit comme moyennement central au sein des échanges, sa centralité excédant légèrement celle de l'échantillon $(\mathrm{M}=0.17 \mathrm{vs} \mathrm{Mtot}=0.15)$. Au vu des fortes densité et réciprocité des liens de soutien, les configurations familiales «Fils» produisent, à l'instar des configurations « Conjugal», du capital social de type chaîne. Comme nous l'avons postulé (« $\mathrm{H} 3 »)$, la présence des enfants et celle des petits-enfants renforcent les échanges de soutien au sein de la configuration familiale, ceux-ci sont en effet perçus comme denses et réciproques. Les pratiques communes comme les réunions ou les fêtes de famille mais, surtout, les normes de réciprocité "généralisée », qui sont particulièrement prégnantes dans les liens intergénérationnels, contribuent à consolider les liens d'interdépendance entre les différentes générations. Cependant, contrairement aux configurations « Conjugal», le répondant se démarque par son engagement particulièrement actif au sein de sa configuration familiale. Il se peut que la présence de petits-enfants stimule son engagement et qu'il soit davantage sollicité dans les échanges de soutien que dans les configurations de type "Conjugal».

Quant aux configurations familiales de type «Fille», elles reposent aussi sur des liens intergénérationnels mais elles affichent une composition différente des configurations de type «Fils». Ce sont la fille du répondant et les enfants de sa fille, majoritairement des petites-filles, qui constituent ce type de configurations. Les configurations de type «Fille» ont une taille aussi grande que celle des configurations précédentes $(M=4.55$ vs Mtot = 3.39). Toutefois, elles se distinguent de ces dernières par leurs faibles scores de densité $(M=0.34$ vs $M t o t=0.37)$ et de réciprocité $(\mathrm{M}=0.35$ vs $\mathrm{Mtot}=0.39)$. Le répondant donne du soutien à un nombre élevé de membres de sa configuration $(\mathrm{M}=2.34 \mathrm{vs} \mathrm{Mtot}=2.11)$ alors qu'il n'en reçoit que d'un nombre restreint $(\mathrm{M}=1.65$ vs Mtot $=1.50)$. Contrairement aux configurations de type «Fils», le répondant est principalement actif dans un rôle de prestataire de soutien. Plus encore, il ne se perçoit pas au centre des échanges puisque sa centralité est l'une des plus basses de l'échantillon $(\mathrm{M}=0.12 \mathrm{vs} \mathrm{Mtot}=0.15)$. Contre toutes attentes, les configurations familiales de type «Fille» génèrent en moyenne un faible volume de capital social, que ce soit de type chaîne ou de type pont. Elles se différencient donc des configurations familiales de type «Fils» qui se composent aussi de liens intergénérationnels. Dans les configurations « Fille», 
la présence des enfants et des petits-enfants, dont les échanges sont soutenus par des normes de réciprocité « généralisée», ne contribue pas à activer la densité et la réciprocité des liens de soutien contrairement à l'hypothèse «H3 ». Ces résultats sont d'autant plus surprenants que les configurations familiales de type «Fille» sont constituées majoritairement de femmes, de filles et de petites-filles. Les femmes - et qui, plus est, dans leur rôle de «Fille »-sont décrites dans la littérature comme responsables des liens et perçues comme les principales prestataires de soutien dans la famille. Dans de telles circonstances, elles devraient soutenir les échanges familiaux et contribuer à la production du capital social de type chaîne («H4 »). Or, les faibles densité et réciprocité qui caractérisent cette configuration réfutent cette hypothèse.

Ceci dit, nous avons aussi suggéré dans une hypothèse alternative que les femmes sont surchargées par les tâches et les responsabilités qui leur incombent, devant jouer une multitude de rôles de soutien au sein de la famille. Et, cette situation peut être à la longue créatrice de tensions, de conflits et d'ambivalence au sein de la famille. La composition différente des configurations familiales «Fils» et «Fille» vérifie cette hypothèse. Dans la configuration de type «Fils», la mention du fils va de pair avec celle de la femme du fils (belle-fille). Et, comme le montrent les fortes densité et réciprocité des échanges, la femme du fils est aussi active dans la configuration familiale que les autres membres significatifs de la famille. Alors que le fils peut compter sur son épouse pour soutenir les échanges au sein de la configuration, la fille dans la configuration « Fille» est seule, sans partenaire pour l'épauler. Certes, les petits-enfants sont mentionnés mais ils sont plus bénéficiaires de soutien que prestataires comme le montre l'écart entre les indices degré rentrant et degré sortant. Dans une telle situation, la fille, surchargée, ne peut pas répondre adéquatement à toutes les attentes des autres membres de la famille, ce qui expliquerait la faiblesse du capital social généré au sein de ce type de configurations. Une gestion difficile de l'ambivalence structurelle pourrait être à l'origine de ce faible capital social, comme nous le montrons plus loin.

Les configurations familiales de type «Fratrie», composées majoritairement des membres de la fratrie, de sœurs notamment, sont plutôt de taille moyenne $(M=4.02$ vs $M t o t=3.39)$, denses $(M=0.41$ vs $M t o t=0.37)$ et particulièrement réciproques $(\mathrm{M}=0.47 \mathrm{vs} M t o t=0.39)$. C'est surtout l'engagement actif dont fait preuve le répondant dans ce type de configurations familiales qui rend celles-ci particulièrement intéressantes. Ce 
dernier se démarque des autres répondants par le nombre élevé de membres qu'il soutient (Degré rentrant: $\mathrm{M}=2.89 \mathrm{vs} \mathrm{Mtot}=2.11$ ). Et, bien qu'il soit aussi soutenu par plusieurs membres de sa configuration (Degré sortant : $\mathrm{M}=1.93 \mathrm{vs} \mathrm{Mtot}=1.50$ ), ce qui expliquerait la forte réciprocité qui caractérise cette configuration, l'écart important entre ces deux scores met en exergue le rôle très actif du répondant en tant que prestataire de soutien au sein de sa configuration familiale. Cet engagement prononcé se reflète aussi dans la position centrale qu'il occupe au sein des échanges; son score de centralité est le plus élevé de tout l'échantillon $(\mathrm{M}=0.22 \mathrm{vs}$ $M$ tot $=0.15$ ). Intermédiaire, il jouit d'une grande autonomie structurelle. Il peut ainsi gérer à sa guise le flux des échanges entre les différents membres de sa configuration. Au vu des propriétés structurelles qui la caractérisent, la configuration familiale de type «Fratrie » offre aux répondants qui s'y insèrent un capital social de type pont. Il s'agit surtout de célibataires qui appartiennent à ce type de configurations. Sans enfant et sans partenaire, ils se sont investis tout au long de leur vie dans des relations avec certains membres de leur fratrie. Ils ont développé avec eux des relations affectives, dyadiques, chaleureuses, motivées par un plaisir commun d'être ensemble. Bien qu'étant des liens de sang, les membres de la fratrie sont à l'âge adulte des interlocuteurs « choisis » avec qui l'on maintient une relation positive, équilibrée et gratifiante. N'étant pas axé sur des normes de réciprocité «généralisée » et un fort sentiment de redevabilité, le lien fraternel requiert des protagonistes une certaine volonté de l'entretenir, un engagement actif. Conformément aux hypothèses («H3»), les configurations familiales composées de liens affinitaires produisent du capital social de type pont, n'étant pas soutenues par des liens forts d'interdépendance.

Les configurations familiales de type «Parenté» sont composées aussi de liens affinitaires mais elles se démarquent des configurations de type «Fratrie » par leur composition particulièrement diversifiée, comprenant majoritairement des membres issus de la famille de la fratrie, de la famille du partenaire ou encore de la parenté élargie. L'analyse révèle qu'elles sont plutôt de taille moyenne $(\mathrm{M}=3.96$ vs $\mathrm{Mtot}=3.39)$, avec une faible densité $(\mathrm{M}=0.31$ vs $\mathrm{Mtot}=0.37)$ mais une réciprocité relativement importante $(M=0.41$ vs $M$ tot $=0.39)$. Le répondant est peu actif dans les échanges, comparé aux autres répondants de l'échantillon. Ses faibles scores sur les indices degré rentrant $(\mathrm{M}=1.98 \mathrm{vs} \mathrm{Mtot}=2.11)$ et degré sortant $(\mathrm{M}=1.47$ vs $\mathrm{Mtot}=1.50)$ signalent en effet que le répondant ne 
donne du soutien qu'à un nombre restreint de membres de sa configuration tout comme il n'en reçoit que d'un petit nombre. Ceci dit, il se décrit comme moyennement central $(\mathrm{M}=0.16$ vs $\mathrm{Mtot}=0.15)$. Au vu de ces résultats, on peut conclure que les répondants dans ce type de configurations familiales bénéficient d'un faible capital social aussi bien de type chaîne que de type pont. Ceci dit, les scores moyens sur la réciprocité des échanges et sur la centralité du répondant laissent à penser qu'un engagement accru de sa part pourrait le conduire à davantage de capital social de type pont. Ces résultats suggèrent toutefois que la forte diversité des membres qui constituent ce type de configurations familiales rend le rôle d'intermédiaire particulièrement difficile; un tel rôle est exigeant et requiert une bonne santé aussi bien physique que cognitive. Ces résultats mettent en évidence que les répondants peinent, en raison sans doute de leur âge, à préserver leur position centrale dans des configurations qui sont fort diversifiées et à y entretenir un capital social de type pont. Autrement dit, les configurations familiales composées de liens affinitaires, parce qu'elles sont exigeantes en termes d'entretien des liens, ne produisent pas toujours du capital social de type pont, ce qui réfute d'une certaine manière l'hypothèse « $\mathrm{H} 3$ ».

Les configurations familiales de type "Isolés» se distinguent des autres sur de nombreux aspects. Il s'agit généralement d'individus âgés ayant un faible réservoir de parenté qui ne désignent soit aucun membre significatif de leur famille, soit qu'un nombre très limité d'amis comme faisant partie de leur famille significative. Par conséquent, ces configurations sont de petite taille $(\mathrm{M}=1.16$ vs $\mathrm{Mtot}=3.39)$, de faibles densité $(M=0.16$ vs $M$ tot $=0.37)$ et réciprocité $(M=0.17$ vs $M t o t=0.39)$. Le répondant y est très peu actif; il ne donne du soutien qu'à un nombre très limité de membres (Degré rentrant $=0.82$ vs Mtot $=2.11$ ) et ceux-ci ne le soutiennent pas davantage (Degré sortant $=0.55$ vs Mtot $=1.50$ ). Son score sur la centralité est le plus faible de tout l'échantillon $(\mathrm{M}=0.08$ vs Mtot $=0.15$ ). Cette analyse montre que ce type de configurations familiales ne produit qu'un très faible, voire aucun, capital social. Bien que les membres cités soient des amis proches de longue date, de même génération et habitant à proximité, ils ne permettent pas de garantir au répondant âgé un solide soutien. Ils ne sont pas ou peu connectés entre eux, ce qui rend toute coordination de l'aide impossible. Par ailleurs, l'âge avancé des amis proches et les problèmes de santé qu'ils rencontrent fragilisent grandement les liens au point que ceux-ci sont amenés à progressivement disparaître. Alors que les amis favorisent l'émergence du capital social de type pont 
dans les populations plus jeunes, il n'en est rien dans la vieillesse puisque ceux-ci s'associent à un faible capital social lorsqu'ils sont majoritaires dans la configuration familiale. Étant parmi les membres significatifs de la famille les plus cités, les amis se combinent généralement à d'autres types de membres familiaux dans l'ensemble des configurations familiales, contribuant ainsi à « renforcer» les liens de soutien. Or, lorsqu'ils en constituent la composante principale comme dans les configurations de type «Isolés », ils sont plutôt synonymes d'instabilité et de fragilité du soutien. A nouveau, ce résultat nuance quelque peu l'hypothèse « $\mathrm{H} 3$ » puisque les configurations familiales composées de liens affinitaires comme les amis ne produisent pas toujours du capital social de type pont.

Ces analyses montrent que la composition des configurations familiales a un impact sur le volume et le type de capital social qu'elles génèrent. Cependant, ses effets ne vont pas toujours dans le sens attendu. Premièrement, les mentions du partenaire, des enfants et des petits-enfants comme membres significatifs de la famille s'associent à l'émergence du capital social de type chaîne, comme le montrent les résultats concernant les configurations familiales de type «Conjugal » et «Fils». Interconnectés les uns aux autres, partenaire, enfants et petits-enfants sont prometteurs d'un soutien collectif important et efficace, pouvant être mobilisés rapidement. Deuxièmement, l'absence de partenaire, d'enfants et de petits-enfants dans les configurations familiales se corrèle soit à du capital social de type pont, soit à un faible capital social. Dans les configurations de type «Fratrie», les individus âgés, actifs prestataires de soutien, tendent à jouer le rôle d'intermédiaire entre les membres de leur configuration familiale qui ne sont pas connectés. Occupant une position centrale dans les échanges, ils disposent d'un capital social de type pont. L'analyse montre cependant que le capital social de type pont est fragile, exigeant des individus des compétences tant physiques que cognitives qui s'émoussent avec l'âge. Pour preuve, une composition trop diversifiée, comme celle des configurations de type « $\mathrm{Pa}$ renté », rend difficile le maintien du capital social de type pont en raison du nombre plus important de «trous» structurels qu'elle implique. Connecter un grand nombre de membres familiaux qui ne sont pas du tout en lien demande de l'énergie, ce qui peut s'avérer particulièrement ardu dans la vieillesse. De même, il devient de plus en plus compliqué de rester connecté avec l'ensemble de ses amis et de maintenir avec tous des liens de soutien actifs et équilibrés (réciprocité). Par conséquent, le soutien dyadique s'évapore au fur et à mesure que progresse le processus de fragilisation. 
Troisièmement, la présence exclusive de femmes au sein de la configuration familiale ne garantit pas «forcément» l'émergence du capital social de type chaîne, comme en témoignent les résultats concernant les configurations familiales de type «Fille». Ces dernières s'associent à un faible volume de capital social, quel que soit son type. L'hypothèse alternative semble se vérifier : les femmes, surchargées par les tâches et les responsabilités familiales qui leur sont dévolues, ne parviennent pas à remplir, « seules », leur rôle de « kinkeeper » et de « caregiver », entraînant au sein de la configuration familiale des tensions et un certain désengagement relationnel.

Ces premiers résultats mettent en évidence que l'identité des membres de la famille que les aînés désignent comme significatifs a des conséquences sur le capital social auquel ils accèdent. Cependant, ces résultats ne vérifient que partiellement les hypothèses « $\mathrm{H} 3$ » et « $\mathrm{H} 4$ ». En effet, les liens intergénérationnels et conjugaux ne produisent pas «toujours » du capital social de type chaîne puisque les configurations de type "Fille» s'associent à un faible capital social. De même, les liens affinitaires ne génèrent pas systématiquement du capital social de type «pont». Certaines configurations s'y associent alors que d'autres se conjuguent à un faible capital social. Et, finalement, contrairement à l'hypothèse « $\mathrm{H} 4$ », la composition majoritairement féminine des configurations familiales n'engendre pas du capital social de type chaîne.

\subsubsection{Le capital social selon le profil des répondants}

Outre la composition des configurations familiales, nous avons fait l'hypothèse que le profil socio-démographique des aînés influence le volume et le type de capital social au sein de leur configuration familiale. Pour tester cette hypothèse («H5»), nous avons procédé à des analyses de variance (ANOVA) sur l'ensemble des indices du capital social selon le profil sociodémographique et de santé des répondants. Nous avons considéré leur sexe, leur niveau d'éducation, leur citoyenneté, leur classe d'âge, leur santé fonctionnelle et s'ils sont placés ou non en institution au moment de l'entretien. Dans le tableau 6.2 figurent les moyennes des indices du capital social en fonction de ces différentes variables. Les résultats présentés dans ce tableau mettent en évidence l'impact du profil des répondants sur les propriétés structurelles des configurations familiales et, par conséquent, sur le type de capital social qu'elles produisent. 


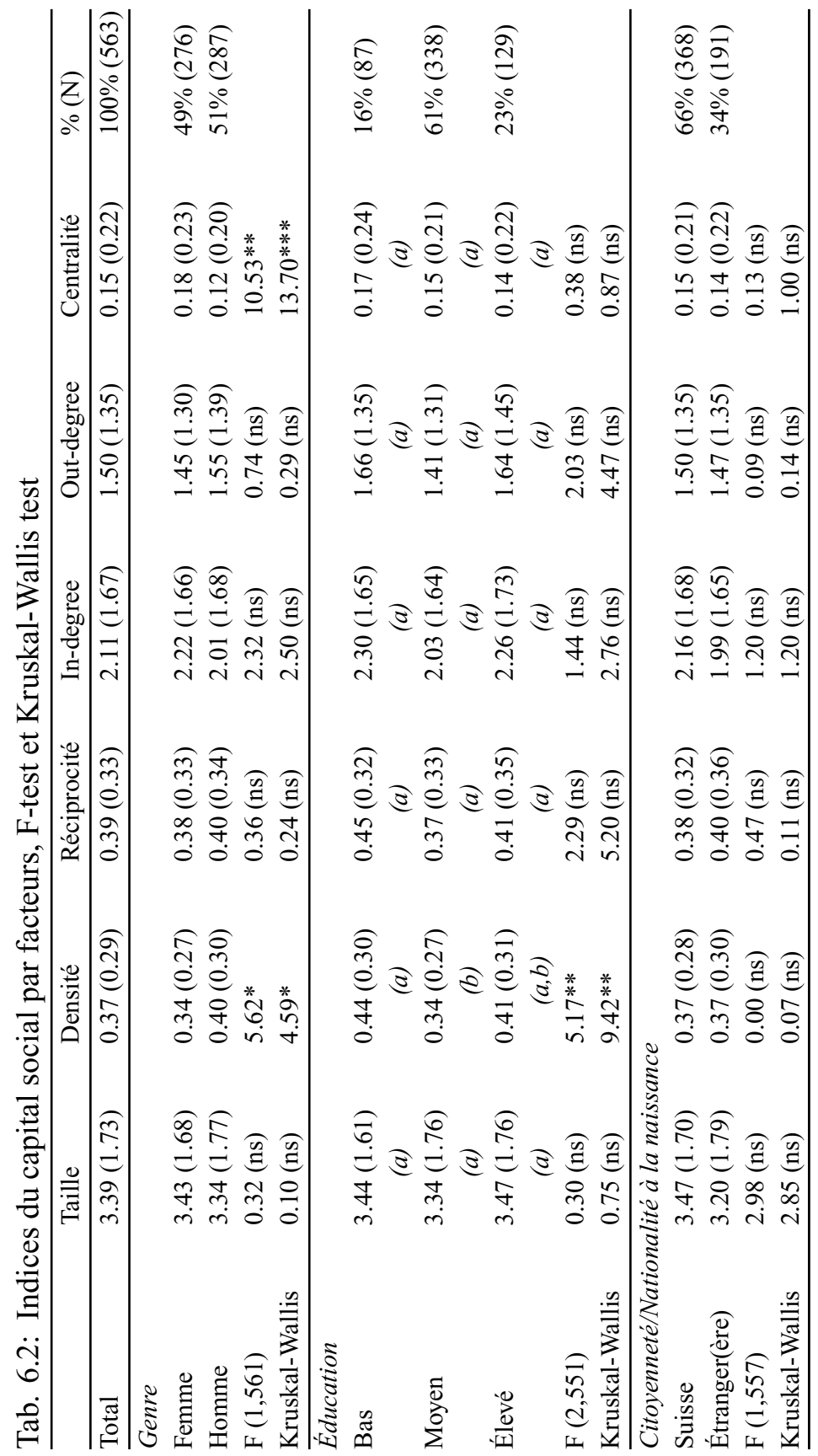


Le capital social

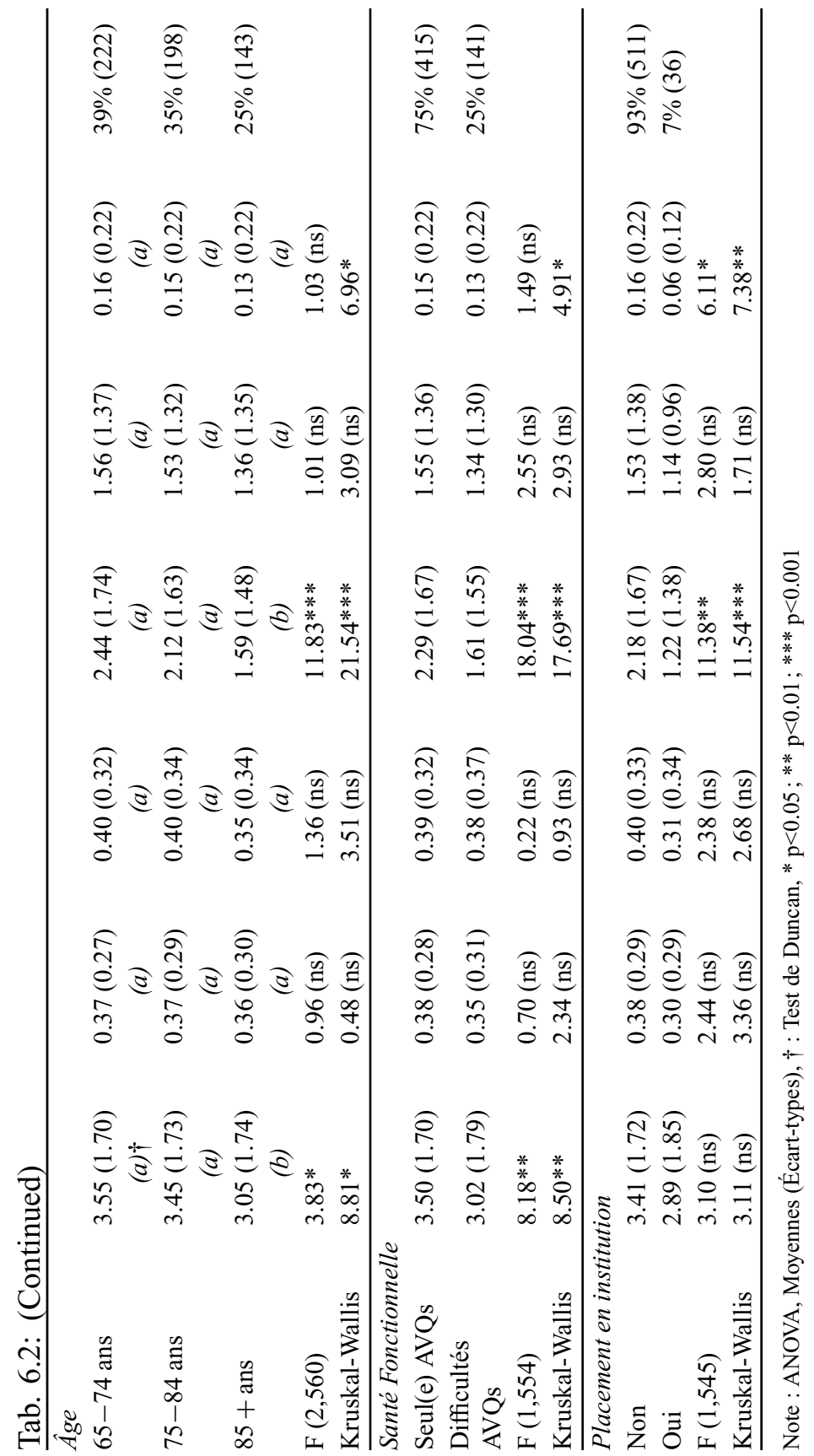


Les analyses montrent que les hommes évaluent les liens de soutien au sein de leur configuration familiale comme plus denses que les femmes $(\mathrm{Mh}=0.40 \mathrm{vs} \mathrm{Mf}=0.34)$. Celles-ci, à l'inverse, se perçoivent davantage au centre de leur configuration familiale que les hommes $(\mathrm{Mf}=0.18$ vs $\mathrm{Mh}=0.12$ ). Conformément aux hypothèses, les hommes bénéficient davantage que les femmes du capital social de type chaîne alors que celles-ci ont davantage accès au capital social de type pont. La propension des femmes à inclure au sein de leur famille significative une plus grande variété de membres explique sans doute un tel résultat. Quant aux hommes, leur tendance à être plus sélectifs avec l'âge, à se centrer davantage sur les membres primaires de leur famille - la partenaire et les enfants - pourrait expliquer la densification des liens de soutien au sein de leur configuration familiale et leur accès privilégié au capital social de type chaîne. Dès lors, ces données ne vérifient pas l'hypothèse alternative que nous avons posée, selon laquelle la baisse plus prononcée des ressources chez les femmes dans la vieillesse en termes de santé, de revenu et de partenaire (veuvage) entraîne une mobilisation accrue des membres de la configuration favorisant le développement du capital social de type chaîne chez les femmes.

Concernant le statut socio-économique, les aînés ayant un faible niveau d'éducation déclarent, d'une part, des liens de soutien plus denses que ceux qui ont un niveau d'éducation moyen $($ Mbas $=0.44$ vs Mmy $=0.34)$ et, d'autre part, des liens aussi denses que ceux qui ont un niveau d'éducation élevé $(\mathrm{Mbas}=0.44$ vs $\mathrm{Mht}=0.41)$. Ces derniers n'ont pas une configuration de plus grande taille ni même une meilleure centralité que les autres. Ils n'ont donc pas plus accès au capital social de type pont que les moins privilégiés. Ils ont presque autant accès au capital social de type chaîne que ceux qui sont issus des milieux populaires, résultat qui va à l'encontre de l'hypothèse « $\mathrm{H} 5$ ». Deux processus distincts peuvent expliquer ces résultats. Pour ce qui est des individus issus des milieux populaires, le besoin plus marqué de soutien dû à des risques plus grands d'avoir des problèmes économiques ou de santé et surtout l'absence d'aides alternatives qui caractérisent ces milieux s'accompagnent, dans la littérature, d'une solidarité accrue au sein de la famille. Un tel processus génère, conformément aux hypothèses, du capital social de type chaîne. Quant aux individus socialement privilégiés, la présence d'un partenaire, plus fréquente chez les individus dont le statut socio-économique est élevé, explique probablement la densité des liens de soutien au sein de leur configuration familiale et leur accès au capital social de type chaîne. 
Associés l'un à l'autre dans la vieillesse, âge et santé ont un impact similaire sur l'ensemble des propriétés structurelles des configurations familiales. D'abord, les «très-vieux » et ceux qui sont atteints dans leur santé fonctionnelle ont des configurations plus petites que celles des «jeunesvieux » $(\mathrm{Mtv}=3.05 \mathrm{vs} \mathrm{Mjv}=3.55)$ ou de ceux qui sont en bonne santé $(\mathrm{Mms}=3.02 \mathrm{vs} \mathrm{Mbs}=3.50)$. Ensuite, ils donnent du soutien à un nombre de membres plus restreint que ceux qui sont plus jeunes $(\mathrm{Mtv}=1.59 \mathrm{vs}$ $\mathrm{Mjv}=2.44)$ ou en meilleure santé $(\mathrm{Mms}=1.61 \mathrm{vs} \mathrm{Mbs}=2.29)$ et se déclarent moins centraux que les plus jeunes $(\mathrm{Mtv}=0.13 \mathrm{vs} \mathrm{Mjv}=0.16)$ ou ceux en meilleure santé $(\mathrm{Mms}=0.13 \mathrm{vs} \mathrm{Mbs}=0.15)$. Cet effet est toutefois léger puisque le test de Fisher et le test de Duncan ne signalent aucune différence significative. L'effet du placement en institution sur la centralité est par contre plus tranché. L'institutionnalisation s'accompagne d'une perte marquée de l'autonomie structurelle $(\mathrm{Mins}=0.06 \mathrm{vs}$ Mdom $=0.16)$ et de l'engagement, le nombre de membres significatifs que les répondants institutionnalisés soutiennent étant plus petit que ceux qui ne sont pas institutionnalisés (Mins $=1.22$ vs Mdom $=2.18$ ).

Conformément à l'hypothèse « $\mathrm{H} 5$ », ces résultats montrent qu'être un « jeune-vieux » ou avoir une bonne santé sont des ressources nécessaires pour rester actifs dans les échanges familiaux et y jouer un rôle central, condition "sine qua non » à un accès privilégié au capital social de type pont et, ce faisant, au maintien de l'autonomie au sein de la configuration familiale. Parallèlement, ces résultats soulignent la difficulté des individus très âgés ou en mauvaise santé à maintenir leurs relations non seulement amicales mais aussi familiales. Le processus de fragilisation contribue à affecter la capacité relationnelle des aînés qui ne parviennent plus à s'engager activement dans les échanges, ce qui expliquerait la taille plus petite de leur configuration familiale, le nombre plus restreint des membres à qui ils octroient du soutien et la moindre centralité des individus très âgés ou limités dans leur santé et, plus encore, de ceux qui sont institutionnalisés. Il se peut aussi que la réduction des liens familiaux ne soit pas seulement subie mais qu'elle soit en partie volontaire, signe du processus de la sélectivité socio-émotionnelle. Conscients de leur fin proche, les «très-vieux » ou ceux atteints dans leur santé sélectionnent un nombre limité de membres significatifs de leur famille, avec lesquels ils maintiennent des liens affectifs gratifiants. Cette centration, volontaire ou non, sur un nombre limité de membres de la famille ne s'accompagne ni de capital social de type pont, l'engagement et la centralité étant faibles, ni de capital social de 
type chaîne, les liens de soutien étant peu denses. Il n'y a donc pas de mobilisation accrue des membres significatifs de la famille, ce qui réfute l'hypothèse « $\mathrm{H} 5$ ».

Il se peut que les membres sélectionnés volontairement par le biais de la sélectivité socio-émotionnelle ne correspondent pas aux membres primaires de la famille, c'est-à-dire au partenaire et aux enfants, et qu'ils se réfèrent plutôt à des membres diversifiés qui sont peu connectés entre eux, ce qui expliquerait le faible volume du capital social de type chaîne. Par ailleurs, un effet lié à la composition de l'échantillon peut expliquer un tel résultat : les «très-vieux » et les individus en mauvaise santé qui participent à l'étude (aptes) font partie des « survivants ». Ils se distinguent des aînés de même cohorte ou de même santé par des ressources aussi bien physiques que mentales qui leur permettent de participer à une telle enquête malgré leur grand âge ou leurs atteintes fonctionnelles. En d'autres termes, leurs conditions de santé sont, comparées à celles des individus de leur âge, relativement bonnes, ne justifiant pas «a priori » une mobilisation accrue des membres de leur famille significative. De plus, le réseau d'aide à domicile à Genève, qui propose une diversité de prestations d'aide et de soins, pourrait aussi expliquer l'absence de mobilisation de la famille significative en répondant aux besoins de soutien des parents très âgés ou atteints dans leur santé fonctionnelle. Au vu de ces différents éléments, on peut en conclure que le processus de fragilisation, en affectant les ressources et la capacité relationnelle, s'associe à un faible volume de capital social, quel que soit le type.

En résumé, le profil socio-démographique des répondants et les ressources qui y sont associées ont un impact majeur sur les relations familiales dans la vieillesse. Alors que de nombreuses études en gérontologie sociale ont montré leur influence de manière globale, les résultats de ce travail vont plus loin en mettant en évidence l'importance des ressources des individus âgés en termes de santé, d'énergie ou de réserves démographiques (partenaire) sur l'organisation des liens de soutien au sein de leur configuration familiale. Ces résultats vérifient partiellement l'hypothèse « H5 ». Ils confirment certes que les femmes, les « jeunes-vieux» et les individus en bonne santé ont davantage accès au capital social de type pont que les hommes, les «très-vieux » et les personnes en mauvaise santé. De même, et conformément à l'hypothèse, les hommes et les aînés issus des milieux populaires ont davantage accès au capital social de type chaîne que les femmes et les aînés des classes moyennes. Cependant, les âgés qui ont 
un statut socio-économique élevé ont autant accès au capital social de type chaîne que ceux qui sont moins privilégiés. Et, les «très-vieux » et les individus atteints dans leur santé ne développent pas plus de capital social de type chaîne que les plus jeunes ou que ceux qui sont en meilleure santé. Ces derniers résultats vont donc à l'encontre de l'hypothèse « $\mathrm{H} 5$ ».

Globalement, ces données attestent que les diverses ressources dont disposent les femmes, les «jeunes-vieux » et ceux en bonne santé les aident à maintenir leur capacité relationnelle ou leur «potentialité » au sein de leur configuration familiale. Ils peuvent en effet s'engager activement dans les échanges de soutien et y préserver leur position centrale. Ce faisant, ils maintiennent leur capital social de type pont, garant de leur autonomie relationnelle. Cependant, le processus de fragilisation a des effets délétères sur les liens de soutien au sein de la configuration familiale. Il affecte les ressources des aînés et nuit à leur capacité relationnelle, limitant leur engagement et leur centralité. Et, contrairement à l'hypothèse « H5 », la diminution du capital social de type pont n'est pas compensée par une mobilisation accrue de la famille significative, synonyme de capital social de type chaîne. En conséquence, les ultimes étapes de la vie s'associent en moyenne à un faible capital social, sauf pour les individus ayant un statut socio-économique élevé et les hommes qui ont une probabilité plus grande de bénéficier de la présence d'une partenaire même à des âges avancés, et celle-ci est généralement propice au développement du capital social de type chaîne.

\subsubsection{Le capital social selon le réservoir de parenté}

Après avoir mis en lumière l'importance du profil socio-démographique des aînés sur le capital social, nous nous intéressons dans cette sous-section à l'influence du réservoir de parenté sur le développement du capital social au sein de la famille. Avoir des membres significatifs dans sa famille favorise l'émergence du capital social mais nous ne connaissons pas l'effet propre du réservoir de parenté. Il se peut que la seule existence de réserves démographiques suffise à produire du capital social au sein des configurations familiales. Plus précisément, nous examinons si le fait d'avoir un partenaire, des enfants, des petits-enfants, des frères et des sœurs dans son réservoir de parenté a un impact sur le type de capital social dans les configurations familiales, et cela même si ces personnes ne sont pas désignées comme membres significatifs de la famille. Pour approcher le réservoir 
de parenté, nous avons considéré plusieurs variables : le statut conjugal (marié, cohabitant, veuf, divorcé et célibataire), avoir au moins un enfant, avoir au moins un petit-enfant et avoir au moins un frère ou une sœur. Le tableau 6.3 présente les résultats de l'analyse de variance (ANOVA); ils révèlent que l'existence du partenaire, des enfants, de petits-enfants, de frères ou de sœurs influence la production du capital social au sein des configurations familiales, qu'ils soient perçus ou non par les répondants comme des membres significatifs de leur famille.

Comme l'indique ce tableau, les répondants mariés sont ceux qui ont les configurations familiales de plus grande taille $(\mathrm{Mm}=3.63$ vs Mtot $=3.39)$ et les plus denses $(\mathrm{Mm}=0.41$ vs Mtot $=0.37)$. Les mariés sont parmi les plus actifs : ils donnent du soutien à un nombre élevé de membres au sein de leur configuration familiale $(\mathrm{Mm}=2.35 \mathrm{vs}$ Mtot $=2.11)$; ils reçoivent aussi du soutien d'un nombre de membres plus élevé que la moyenne de l'échantillon $(\mathrm{Mm}=1.66 \mathrm{vs}$ Mtot $=1.50)$. Les cohabitants présentent aussi des configurations de grande taille $(\mathrm{Mcoh}=3.50$ vs Mtot $=3.39)$ mais les liens de soutien y sont moins denses $($ Mcoh $=0.31$ vs Mtot $=0.37$ ). Ils sont particulièrement engagés dans la provision de soutien au sein de leur configuration $(\mathrm{Mcoh}=2.60 \mathrm{vs}$ Mtot $=2.11)$ mais ils en reçoivent relativement peu en retour $(\mathrm{Mcoh}=1.20 \mathrm{vs} \mathrm{Mtot}=1.50)$. Les veufs affichent des scores moyens sur tous les indices : ils ont une configuration familiale de taille moyenne $(\mathrm{Mvf}=3.27 \mathrm{vs}$ Mtot $=3.39)$ et une densité moyenne $(\mathrm{Mvf}=0.37 \mathrm{vs} \mathrm{Mtot}=0.37)$. Ils donnent du soutien à un nombre moyen de membres $(\mathrm{Mvf}=1.95 \mathrm{vs} \mathrm{Mtot}=2.11)$ et en reçoivent d'un nombre tout aussi moyen ( $\mathrm{Mvf}=1.45 \mathrm{vs}$ Mtot $=1.50)$. Quant aux divorcés, leurs configurations sont plus petites que la moyenne (Mdiv $=3.16$ vs Mtot $=3.39)$ et sont de faible densité $($ Mdiv $=0.29$ vs Mtot $=0.37)$. Ils sont moyennement actifs dans les échanges, leurs scores sur les indices degré rentrant $(\mathrm{Mdiv}=1.90 \mathrm{vs} \mathrm{Mtot}=2.11)$ et degré sortant $(\mathrm{Mdiv}=1.41$ vs Mtot $=1.50$ ) sont légèrement en dessous des moyennes de l'échantillon. Finalement, les célibataires affichent des scores bas sur l'ensemble des indices considérés. Leurs configurations familiales sont petites $(\mathrm{Mcel}=2.50$ vs Mtot $=3.39$ ) et peu denses $($ Mcel $=0.30$ vs Mtot $=0.37$ ). Par ailleurs, les membres auxquels ils offrent du soutien $($ Mcel $=1.37$ vs Mtot $=2.11)$ et ceux desquels ils en reçoivent $(\mathrm{Mcel}=1.00 \mathrm{vs}$ Mtot $=1.50)$ sont peu nombreux.

Avoir un partenaire «marié » dans son réservoir de parenté offre donc un potentiel plus grand de relations familiales significatives et favorise, 


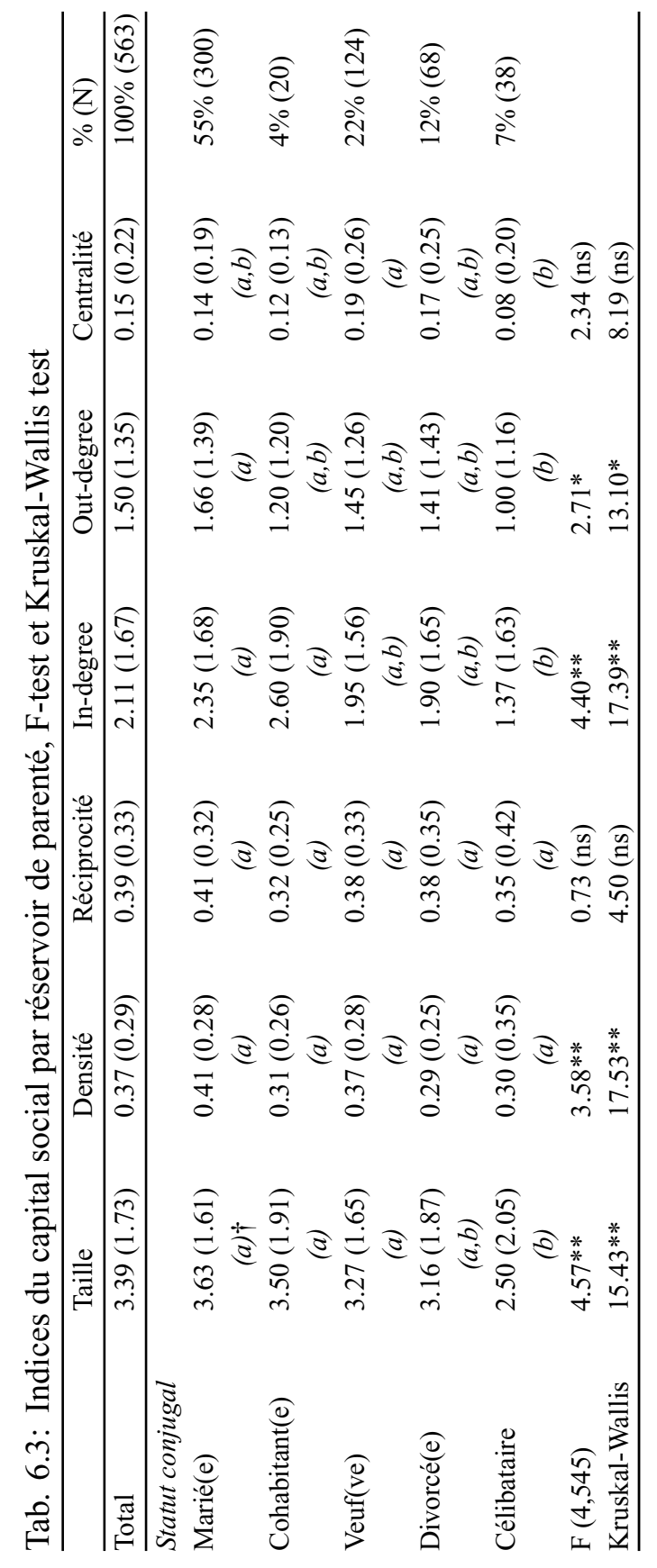




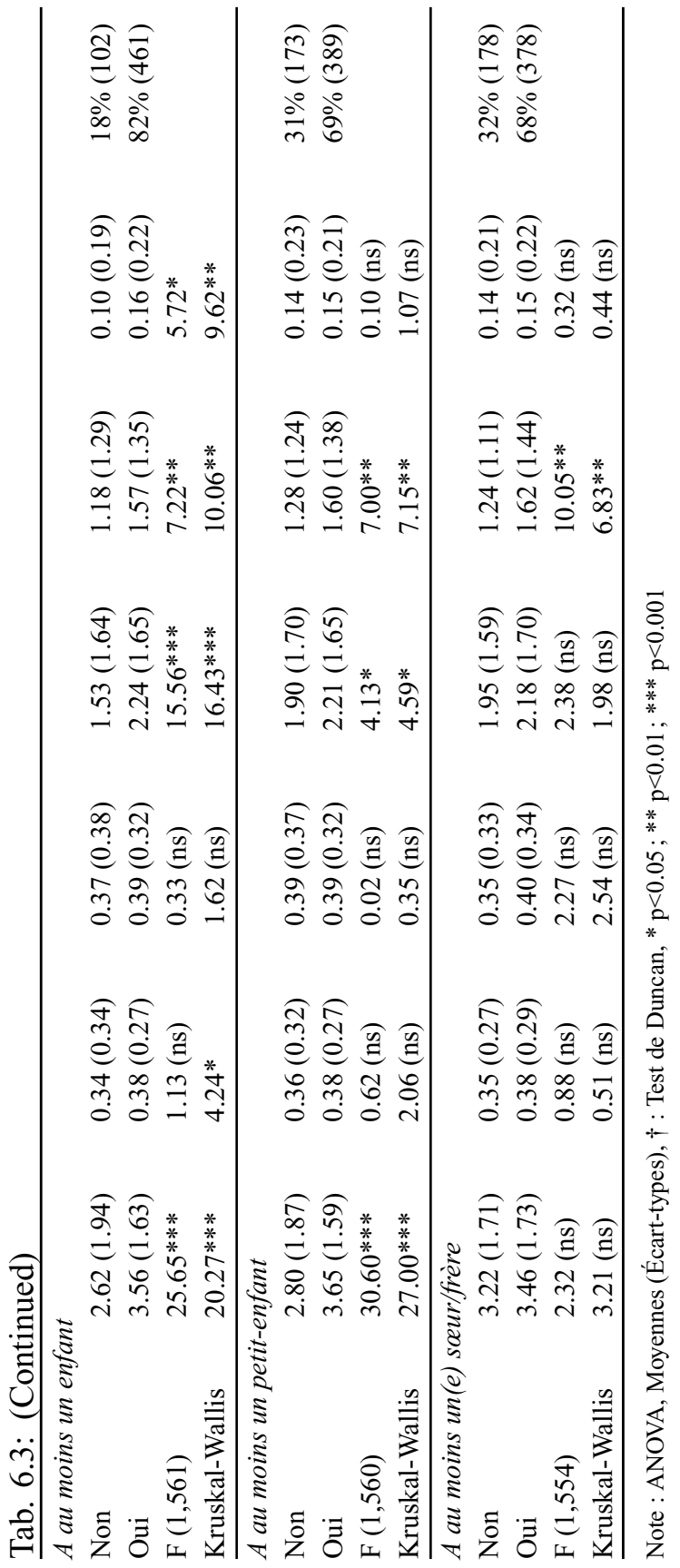


en ce sens, la production de capital social au sein des configurations familiales. Le partenaire marié, par la stabilité et l'engagement qu'il induit, s'associe à davantage d'échanges équilibrés entre le répondant et les membres de sa configuration et à une plus forte densité des liens de soutien. Vivre avec un partenaire non marié conduit à une faible densité de soutien et à un certain déséquilibre dans les échanges avec le reste des membres de la configuration, ceux-ci étant moins disposés à rendre le soutien qu'ils reçoivent du répondant. En somme, la présence d'un partenaire promeut l'émergence du capital social de type chaîne seulement si celui-ci s'inscrit dans une relation maritale, sinon le capital social produit est plutôt faible. L'absence de partenaire parmi les réserves démographiques réduit le potentiel de développer des relations familiales significatives. La situation la plus flagrante est celle des célibataires qui, sans partenaire et sans enfant, ont un réservoir de parenté peu fourni, ce qui tend à limiter le nombre de membres de la famille qu'ils perçoivent comme significatifs et avec lesquels ils échangent activement du soutien. L'absence de partenaire dans le réservoir de parenté conduit donc «a priori » à un faible capital social, quel que soit son type.

Outre l'existence d'un partenaire, avoir des enfants et des petitsenfants dans son réservoir de parenté a aussi un impact sur le capital social généré au sein des configurations familiales. En effet, les répondants qui ont des enfants ont des configurations familiales plus grandes $($ Menf = 3.56 vs Mpasenf =2.62), des liens de soutien légèrement plus denses que la moyenne de l'échantillon $(\mathrm{Menf}=0.38 \mathrm{vs}$ Mpasenf $=0.34)$, sont plus actifs dans les échanges tant dans la provision de soutien $(\mathrm{Menf}=2.24 \mathrm{vs}$ Mpasenf $=1.53)$ que dans sa mobilisation $(\mathrm{Menf}=1.57$ vs Mpasenf $=1.18)$ et sont plus centraux $(\mathrm{Menf}=0.16 \mathrm{vs}$ Mpasenf $=0.10)$ que ceux qui n'ont pas d'enfants. Ainsi, la présence d'enfants dans le réservoir de parenté offre des possibilités de développer des relations familiales significatives et favorise l'émergence du capital social au sein des configurations familiales. Il en est de même pour les petits-enfants puisque les grands-parents affichent des configurations familiales de plus grande taille (Mptenf $=3.65$ vs Mpasptenf $=2.80$ ) et sont plus actifs dans les échanges de soutien que les non grands-parents. Ils donnent en effet plus de soutien (Mptenf $=2.21$ vs Mpasptenf $=1.90)$ et en reçoivent plus au sein de leur réseau familial $($ Mptenf $=1.60 \mathrm{vs}$ Mpasptenf $=1.28)$ que ceux qui n'ont pas de petits-enfants. Avoir des frères et des sœurs au sein du réservoir de parenté constitue pour les répondants une source significative de soutien 
supplémentaire (Degré sortant $:$ Mfratrie = 1.62 vs Mpasfratrie =1.24) et, en ce sens, peut favoriser la production de capital social au sein de leur configuration familiale.

En résumé, avoir un partenaire marié, des enfants, des petits-enfants, des frères et des sœurs au sein du réservoir de parenté favorise la formation du capital social au sein des configurations familiales. De telles réserves démographiques offrent aux aînés d'importantes opportunités relationnelles; ceux-ci peuvent puiser au sein de leur réservoir de parenté les membres de leur famille qu'ils affectionnent et avec lesquels ils échangent du soutien depuis longtemps. S'il est riche et varié, le réservoir de parenté constitue une sorte de «terreau » favorable à l'émergence de capital social alors que, si les réserves démographiques qu'il contient sont insuffisantes, il s'associe à un faible capital social. Toutefois, nous avons vu précédemment que certaines personnes âgées parviennent, même si elles ont un réservoir de parenté peu fourni, à se façonner une famille significative productrice de capital social en citant une diversité de membres significatifs qui vont bien au-delà des liens de sang et de l'alliance. Souvent les plus dotées en compétences relationnelles ou en moyens socio-économiques, elles intègrent au sein de leur famille significative des relations sélectionnées en dehors de leur réservoir de parenté. La question qui se pose dès lors est de savoir si ces familles significatives ont plus d'impact sur la production de capital social que le réservoir de parenté per se; si elles sont en mesure, en somme, de générer du capital social malgré la pauvreté du réservoir de parenté.

\subsubsection{Les facteurs déterminants du capital social}

Pour répondre à cette question, nous avons procédé à des analyses de régression linéaire sur les indices du capital social en considérant dans l'analyse l'ensemble des facteurs examinés, à savoir les configurations familiales, le profil socio-démographique et de santé des répondants ainsi que leur réservoir de parenté. Les analyses de variance (ANOVA) précédemment effectuées révèlent que tous ces facteurs, étudiés séparément, ont des effets significatifs sur l'émergence du capital social dans les configurations familiales. L'analyse de régression permet de les confronter et de déterminer ceux qui ont le plus de poids dans le développement du capital social dans les familles. Nous avons procédé en deux étapes. Dans un premier modèle, nous avons d'abord considéré les effets de la composition des configurations familiales sur le capital social (hypothèses « $\mathrm{H} 3 »$ et « $\mathrm{H} 4 »)$. 
Ensuite, dans un deuxième modèle, nous avons ajouté aux configurations familiales, le genre, le niveau d'éducation, la citoyenneté, la classe d'âge, la santé fonctionnelle, le placement en institution (hypothèse « H5 ») et le réservoir de parenté (variable de contrôle) afin d'observer si l'adjonction de ces facteurs module ou annule l'effet des configurations familiales sur le capital social. Nous testons ainsi l'impact de la composition des configurations familiales sur le capital social en tenant compte des effets des autres facteurs. Dans ces analyses, nous avons privilégié la variante « contrast deviation » (Chambers \& Hastie, 1992) qui permet de comparer, pour chaque variable à plus de deux catégories, le score d'une modalité à la moyenne des modalités, et non à une modalité de référence. Dans les deux tableaux suivants figurent les résultats de l'analyse. Le tableau 6.4 a trait aux indices mesurant le capital social de type «chaîne »- la taille, la densité et la réciprocité - et le tableau 6.5 concerne les indices évaluant le capital social de type «pont»- le degré rentrant, le degré sortant et la centralité. Les résultats du premier modèle, où seul l'effet des configurations familiales est testé, sont similaires à ceux obtenus dans les analyses bivariées (ANOVA). Dans le deuxième modèle, les résultats affichés ne diffèrent pas de ceux du premier modèle même après l'inclusion des autres facteurs dans l'analyse. Nous allons en détailler les principaux résultats.

Les configurations familiales de type «Conjugal» se caractérisent par une taille significativement plus petite $(\beta=-0.24, \mathrm{p}<.05)$, une densité $(\beta=0.11, \mathrm{p}<.001)$ et une réciprocité $(\beta=0.07, \mathrm{p}<.05)$ significativement plus fortes que la moyenne générale, celle-ci étant la référence. Les répondants appartenant à cette configuration ne sont cependant pas plus actifs ni plus centraux que la moyenne de l'échantillon. Ce résultat montre que, conformément à l'hypothèse « $\mathrm{H} 3$ », la présence du partenaire s'associe à une plus forte densité des liens de soutien, générant du capital social de type chaîne au sein de la configuration familiale «Conjugal», et cela quels que soient le profil socio-démographique, la santé fonctionnelle et le réservoir de parenté du répondant. Ceci dit, avoir un partenaire marié ou cohabitant dans son réservoir de parenté n'est pas, en soi, prometteur de capital social de type chaîne puisqu'il n'a pas d'effet «propre» sur aucun des indices présentés dans ce tableau. Par conséquent, le partenaire doit être mentionné comme membre significatif de la famille pour qu'il puisse être associé au capital social de type chaîne.

Quant aux configurations familiales de type «Fils», elles sont significativement plus grandes $(\beta=0.76, \mathrm{p}<.001)$ et plus denses $(\beta=0.12$, 


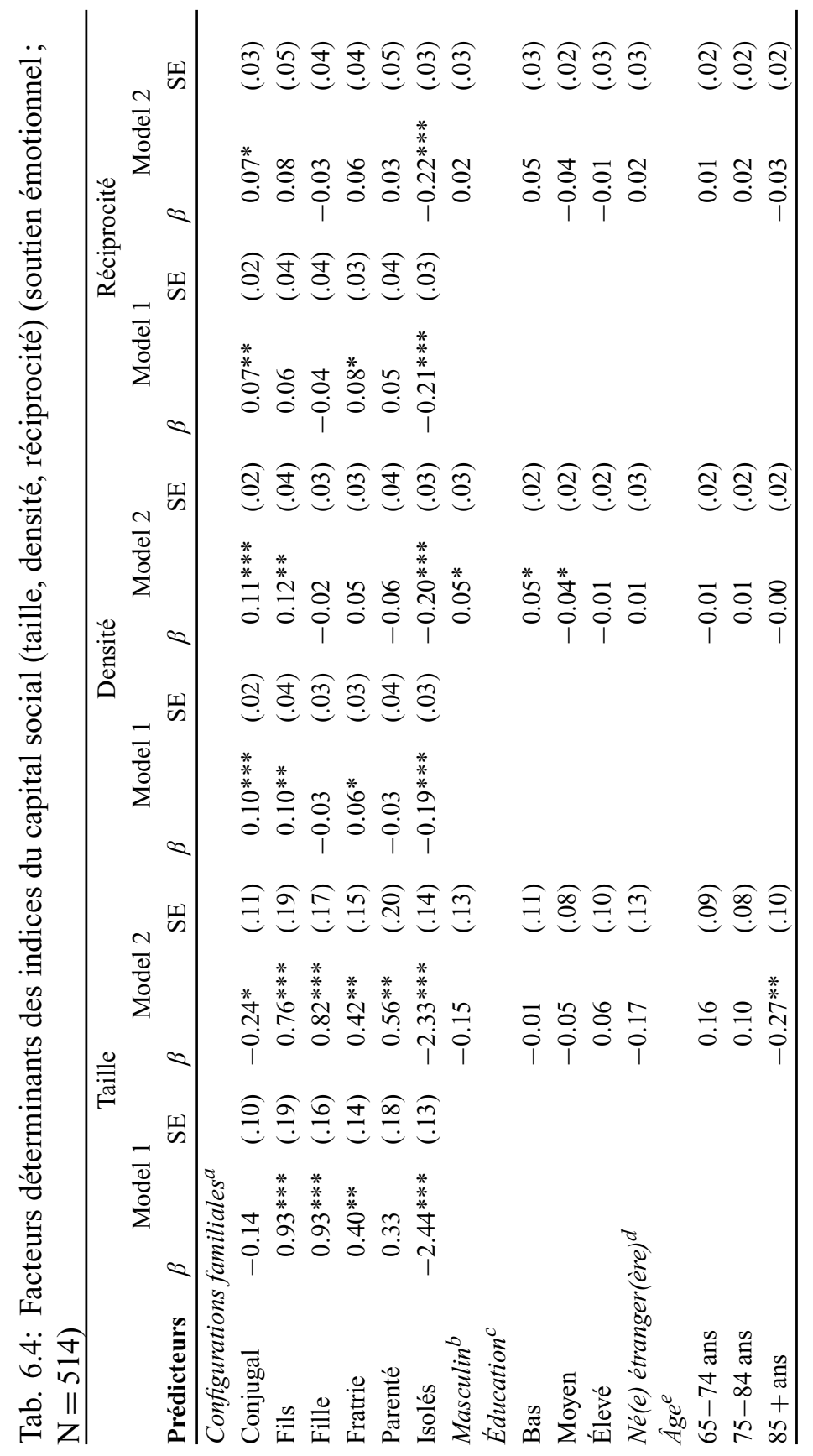




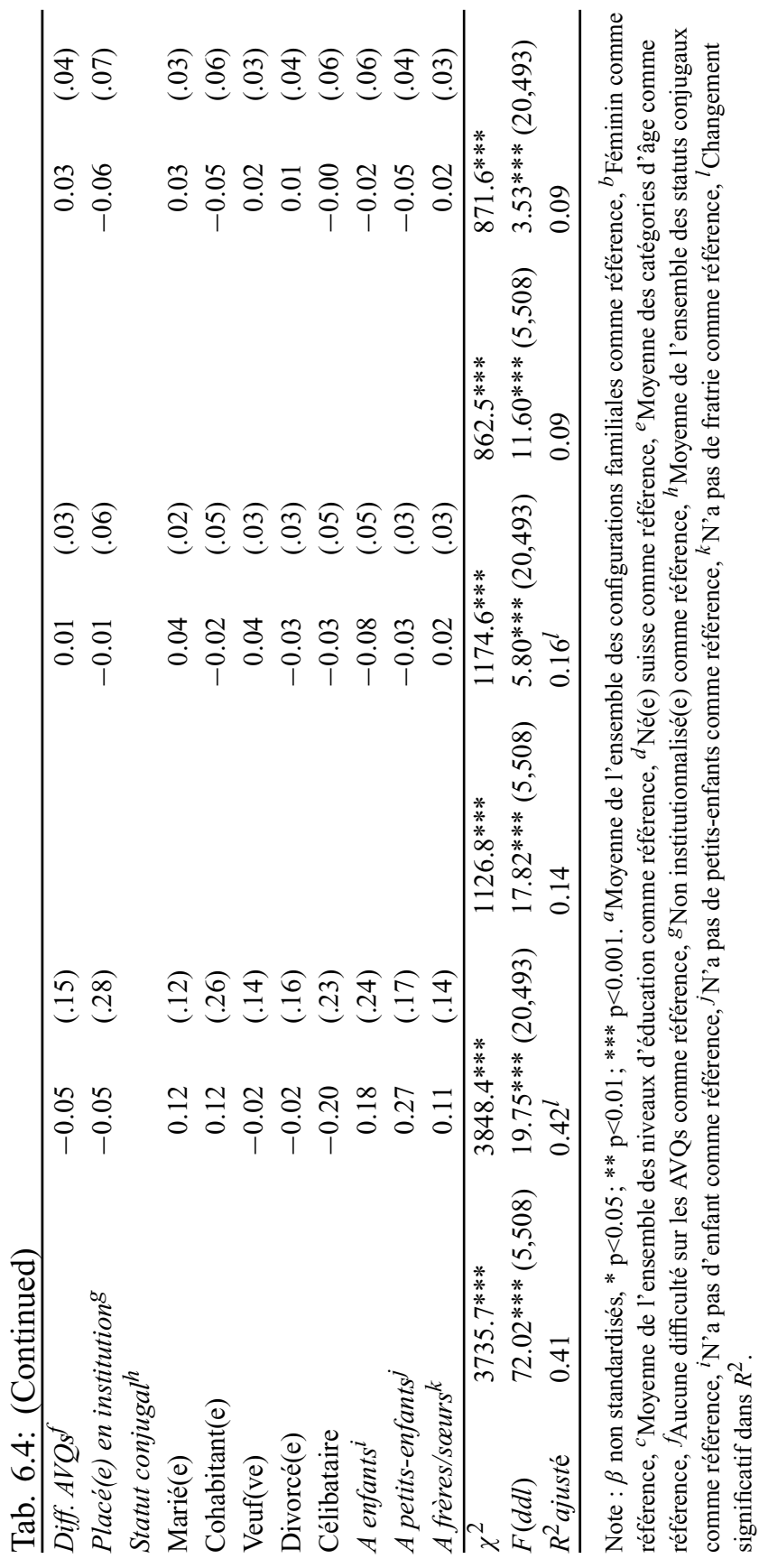




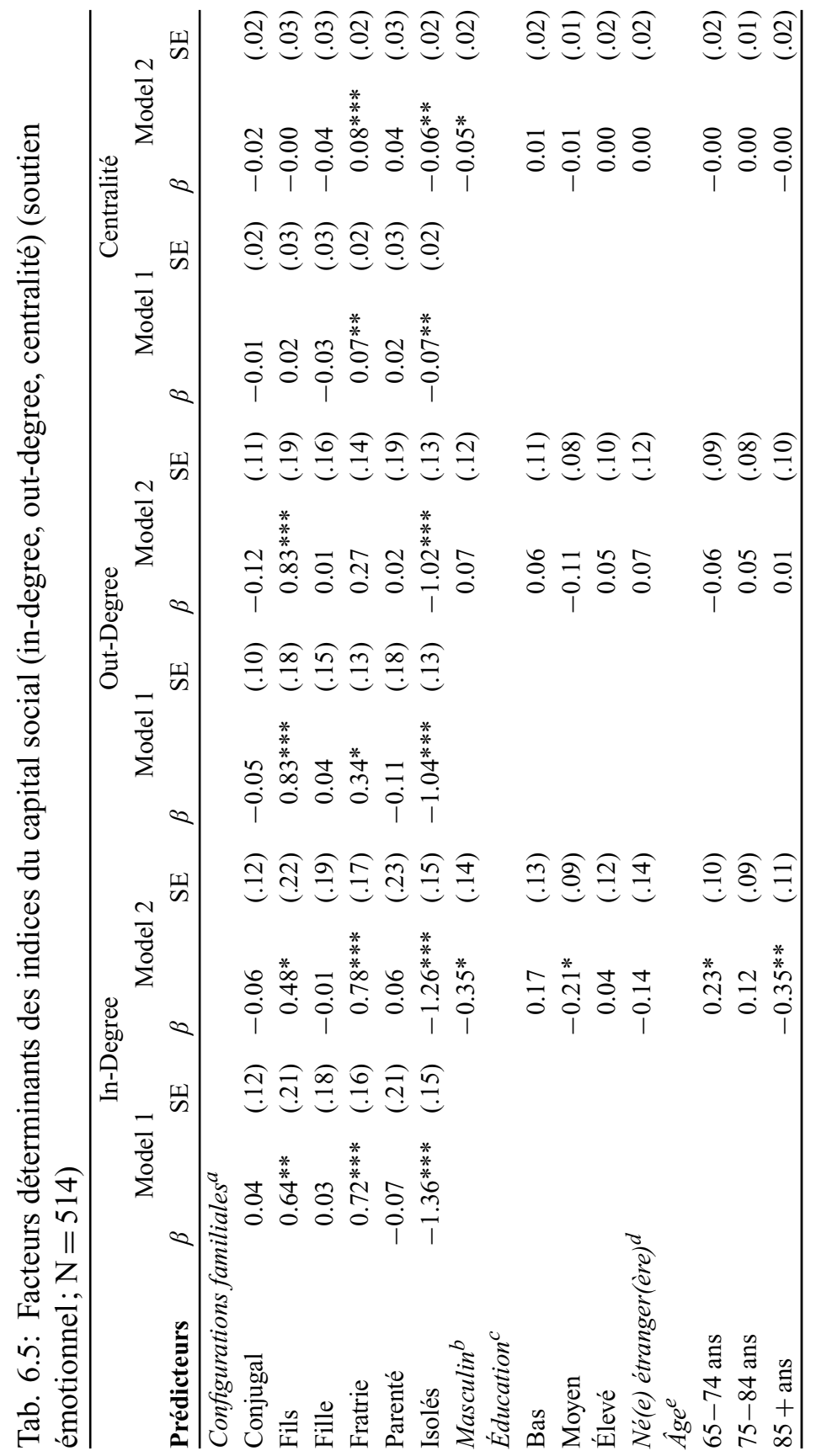




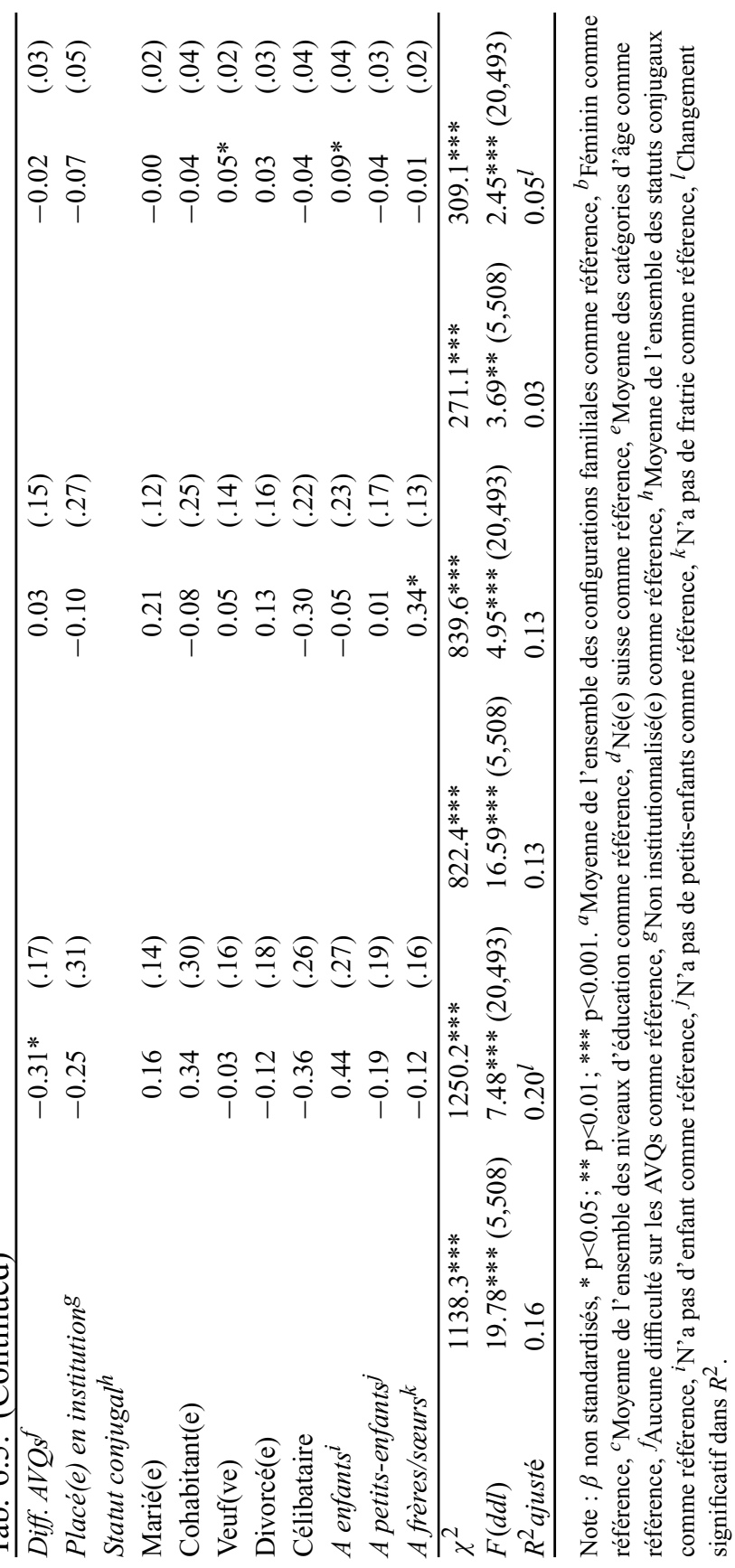


$\mathrm{p}<.01)$ que la moyenne générale. Les répondants qui s'y insèrent y sont aussi significativement plus engagés et plus actifs. Non seulement ils octroient du soutien à un nombre plus grand de membres $(\beta=0.48, \mathrm{p}<.05)$ mais ils en reçoivent aussi de la part d'un plus grand nombre $(\beta=0.83$, $\mathrm{p}<.001)$. La présence d'enfants et de petits-enfants dans la configuration familiale «Fils » contribue à stimuler les échanges, y compris ceux impliquant le parent âgé, donnant lieu à une densification des liens de soutien et, conformément à l'hypothèse « $\mathrm{H} 3$ », à l'émergence du capital social de type chaîne, et cela même après l'inclusion des autres facteurs dans le modèle. L'analyse montre aussi que la seule existence de descendance (petits-enfants) dans le réservoir de parenté n'a pas d'effet "propre » sur les échanges de soutien au sein de la configuration familiale. Avoir des enfants dans le réservoir de parenté s'associe toutefois à une meilleure centralité du répondant au sein de la configuration familiale $(\beta=0.09, \mathrm{p}<.05)$, qu'ils aient été ou non cités comme membres significatifs de la famille. Les enfants et les petits-enfants doivent être, en somme, mentionnés comme significatifs pour activer les échanges de soutien et créer du capital social de type chaîne au sein de ce type de configurations familiales.

Ceci dit, même si les enfants et les petits-enfants sont désignés comme faisant partie de la famille significative, ils ne sont pas toujours synonymes de capital social de type chaîne, comme en témoignent les résultats de la configuration familiale de type «Fille». De taille plus grande que la moyenne $(\beta=0.82, \mathrm{p}<.001)$, les configurations familiales de type «Fille» affichent une densité et une réciprocité qui ne se distinguent pas de la moyenne générale. De même, les aînés qui s'y insèrent ne sont significativement pas plus actifs ni plus centraux que le reste de l'échantillon. Ils ne sont donc pas aussi engagés dans les échanges que ceux qui participent aux configurations de type " Fils». Contrairement à l'hypothèse « $\mathrm{H} 3$ », ce type de configurations familiales, bien que composées d'enfants et de petits-enfants, ne produit pas de capital social de type chaîne mais seulement un faible volume de capital social, et cela quels que soient le profil socio-démographique, la santé et le réservoir de parenté du répondant. Plus encore, ce résultat va aussi à l'encontre de l'hypothèse « $\mathrm{H} 4$ », selon laquelle une présence majoritairement féminine est davantage prometteuse de capital social de type chaîne que de type pont. Ce qui n'est pas le cas dans ce type de configurations.

L'analyse de régression montre que les variations du capital social dans les configurations familiales de type «Fils» et «Fille» ne sont pas dues au 
profil du répondant mais bien à la combinaison différente des membres cités qui composent ces deux configurations familiales. Dans la première, le fils est épaulé par son épouse pour maintenir les liens de soutien au sein de la configuration familiale alors que, dans la deuxième, la fille est « seule » pour gérer les échanges de soutien. Nous avons fait l'hypothèse alternative que les filles, surchargées par leur rôle de "donneuse de soins » à l'ensemble des membres de la famille, peinent à entretenir, seules, les liens de soutien au sein de leur famille, ayant pour conséquence des effets négatifs sur le production du capital social au sein de leur configuration familiale. Cette hypothèse alternative se vérifie dans les analyses de régression même en tenant compte des effets des autres facteurs.

Concernant les configurations familiales de type «Fratrie», elles sont significativement plus grandes que la moyenne générale $(\beta=0.42, \mathrm{p}<.01)$ mais leur densité et leur réciprocité ne s'en démarquent pas significativement. Les répondants sont plus actifs dans la provision du soutien $(\beta=$ $0.78, \mathrm{p}<.001)$ et plus centraux que les autres répondants de l'échantillon $(\beta=0.08, \mathrm{p}<.001)$. La mention de sœurs ou de frères comme membres significatifs de la famille favorise donc le développement du capital social de type pont, et cela quels que soient le profil des répondants et leur réservoir de parenté. Ce résultat confirme que la présence majoritaire de liens affinitaires dans les configurations familiales s'associe à davantage de capital social de type pont que de type chaîne («H3»). Notons, par ailleurs, que l'existence de frères ou de sœurs dans le réservoir de parenté s'associe à un nombre plus élevé de membres soutenants $(\beta=0.34, \mathrm{p}<.05)$, et cela quelle que soit la composition des configurations familiales. Or, dans la configuration «Fratrie », c'est l'inverse. C'est le répondant qui donne plus de soutien qu'il n'en reçoit, signe de l'investissement particulièrement important du répondant dans ce type de configurations familiales et c'est cet investissement particulier qui explique la forte centralité de l'aîné dans les configurations familiales de type «Fratrie».

A l'exception de la taille qui est significativement plus grande que celle des autres $(\beta=0.56, \mathrm{p}<.01)$, les configurations familiales de type «Parenté » présentent des scores sur les indices du capital social qui ne se différencient pas des scores moyens de l'échantillon total. Contrairement à l'hypothèse « $\mathrm{H} 3$ », la variété des membres significatifs qui composent ce type de configurations n'est pas synonyme d'une plus grande centralité du répondant, ni même d'un plus grand investissement de ce dernier, et donne plutôt lieu à un faible capital social aussi bien de type chaîne que 


\section{Capital social et ambivalence}

de type pont. Autrement dit, il est difficile dans la vieillesse de jouer le rôle d'intermédiaire au sein de ce type de configurations familiales et de préserver le capital social de type pont lorsque les membres significatifs qui les constituent sont trop variés. Ces résultats subsistent même après la prise en compte du profil socio-démographique des répondants dans le deuxième modèle. Même si les aînés ont a priori les ressources ou les compétences nécessaires pour entretenir les échanges familiaux comme les femmes, les «jeunes-vieux » ou encore ceux en bonne santé, ils éprouvent autant de difficultés que les autres à connecter les membres trop disparates de leur configuration familiale. C'est la raison pour laquelle le capital social produit au sein de ce type de configurations familiales est faible.

Finalement, les configurations familiales de type «Isolés » affichent des scores négatifs sur l'ensemble des indices considérés. En effet, ces configurations se démarquent des autres par leur plus petite taille $(\beta=$ $-2.33, \mathrm{p}<.001)$, leur plus faibles densité $(\beta=-0.20, \mathrm{p}<.001)$ et réciprocité $(\beta=-0.22, \mathrm{p}<.001)$, l'engagement médiocre du répondant tant dans la provision du soutien $(\beta=-1.26, \mathrm{p}<.001)$ que dans sa mobilisation $(\beta=-1.02, \mathrm{p}<.001)$ et son manque de centralité $(\beta=-0.06, \mathrm{p}<.001)$. La construction de la famille significative autour de quelques amis ne génère aucun capital social, quel qu'il soit, et cela même après avoir contrôlé l'effet des autres facteurs. A nouveau, ce résultat nuance quelque peu l'hypothèse « $\mathrm{H} 3$ » qui postule que la présence de liens affinitaires dans les configurations familiales promeut le capital social de type pont. Ceci ne se vérifie pas lorsqu'il s'agit de quelques amis qui, aussi âgés que le répondant, subissent comme lui le processus inéluctable de la fragilisation. Par conséquent, les liens amicaux dans la vieillesse, même avec des amis très proches, sont particulièrement fragiles et amenés à disparaître avec le temps.

Au vu de l'ensemble de ces résultats, nous pouvons en déduire que la composition des configurations familiales a bel et bien un impact important sur leurs propriétés structurelles, quels que soient le profil et le réservoir de parenté des répondants. Ainsi, l'identité des membres de la famille qui sont mentionnés comme significatifs et la façon dont ils s'articulent entre eux influencent de manière significative l'organisation des liens d'interdépendance au sein des configurations familiales et déterminent le type et le volume du capital social qu'elles produisent. Bien que ces analyses soulignent le poids de la composition des configurations familiales dans 
la production du capital social, elles montrent aussi que le profil sociodémographique des aînés influe sur certaines propriétés structurelles des configurations familiales, quelle que soit la composition de ces dernières.

Les hommes, par exemple, ont des configurations familiales plus denses $(\beta=0.05, \mathrm{p}<.05)$, soutiennent un nombre plus limité de membres $(\beta=-0.35, \mathrm{p}<.05)$ et occupent une position moins centrale dans leur configuration $(\beta=-0.05, \mathrm{p}<.05)$ que les femmes, et cela indépendamment de la composition de leur configuration familiale. Conformément à l'hypothèse «H5 », les analyses soulignent la propension des hommes au capital social de type chaîne et celle des femmes au capital social de type pont. L'analyse de régression met en exergue l'effet "propre » du genre en le confrontant au statut conjugal comme variable de contrôle. Effectivement, l'effet significatif du veuvage sur la centralité - les veuf(ves) étant plus centraux dans leur configuration que les autres $(\beta=0.05, \mathrm{p}<.05)$ - ne contribue pas à réduire l'impact significatif du genre sur la centralité. Ce n'est donc pas seulement le veuvage plus fréquent des femmes et la composition de leur configuration familiale qui expliquent leur plus forte tendance au capital social de type pont. Elles développent du capital social de type pont parce qu'elles ont, en raison des rôles familiaux qui leur incombent, une propension plus forte que les hommes à créer du lien avec une diversité de membres familiaux. Concernant le niveau d'éducation, les analyses de régression vérifient à nouveau l'hypothèse « $\mathrm{H} 5$ » : les répondants qui ont un faible niveau d'éducation jugent en moyenne leurs liens de soutien plus denses que les autres $(\beta=0.05, \mathrm{p}<.05)$, et cela indépendamment de la composition de leur configuration familiale. Ils ont donc davantage accès au capital social de type chaîne que de type pont. Quant à ceux qui ont un niveau d'éducation élevé, ils affichent - et cela contrairement à l'hypothèse « H5 » - un meilleur score sur la densité des liens que ceux qui ont un niveau d'éducation moyen. En effet, les aînés qui ont un niveau intermédiaire se distinguent des autres par le nombre plus limité de membres qu'ils soutiennent $(\beta=-0.21, \mathrm{p}<.05)$ et la plus faible densité de leurs liens de soutien $(\beta=-0.04, \mathrm{p}<.05)$. La mention du partenaire dans la configuration familiale, plus fréquente chez les individus ayant un statut socio-économique élevé, pourrait expliquer ce résultat.

Pour ce qui est de l'âge, les « jeunes-vieux » se différencient des autres classes d'âge par le nombre plus grand de membres auxquels ils apportent $\mathrm{du}$ soutien $(\beta=0.23, \mathrm{p}<.05)$. Parallèlement, les «très-vieux » ont des configurations familiales plus petites $(\beta=-0.27, \mathrm{p}<.01)$ et soutiennent 
un nombre plus restreint de membres au sein de leur configuration familiale $(\beta=-0.35, \mathrm{p}<.01)$, quels que soient leur état de santé fonctionnelle, leur sexe, leur niveau d'éducation et la composition de leur configuration familiale. L'analyse de régression confirme les effets «propres » de l'âge en les contrôlant par l'influence de la santé fonctionnelle. Avec l'avancée en âge et le processus de fragilisation qui l'accompagne, les " très-vieux » ne parviennent plus à être actifs au sein de leur configuration familiale. Ils sélectionnent dès lors un nombre restreint de membres significatifs de leur famille, ceux qu'ils estiment source de satisfaction émotionnelle et auxquels ils peuvent encore apporter un peu de soutien (sélectivité socio-émotionnelle). Ceci dit, et contrairement à l'hypothèse « $\mathrm{H} 5$ », cette centration sur les membres «les plus significatifs » de la famille ne génère pas de capital social de type chaîne, les liens de soutien n'étant pas plus denses chez les «très-vieux » que chez les plus jeunes. Quant aux problèmes de santé fonctionnelle, ils affectent la capacité des répondants à offrir leur soutien puisque les répondants en mauvaise santé soutiennent moins de membres de leur famille que les autres $(\beta=-0.31, \mathrm{p}<.05)$, quels que soient leur âge, leur sexe, leur niveau d'éducation et la composition de leur configuration familiale. Comme pour le grand âge, la mauvaise santé ne s'associe pas à une densification des liens de soutien au sein de la configuration familiale, quelle que soit la composition de cette dernière. Ces résultats montrent que le processus de fragilisation empêche les « trèsvieux » et ceux atteints dans leur santé de jouer un rôle engagé et central au sein de leur configuration, ce qui compromet leur accès au capital social de type pont au sein de leur famille. Ces analyses révèlent aussi qu'il n'y a pas, contrairement à l'hypothèse « $\mathrm{H} 5$ », une mobilisation accrue des membres significatifs de la famille avec le processus de fragilisation. Les «trèsvieux » comme ceux en mauvaise santé n'ont pas plus accès au capital social de type chaîne que les "jeunes-vieux » ou ceux en bonne santé.

Plusieurs raisons peuvent expliquer l'absence de mobilisation accrue de la famille autour des «très-vieux » ou de ceux qui ont des problèmes de santé. D'une part, le processus de la sélectivité socio-émotionnelle qui pousse les individus très âgés, conscients de leur fin proche, à ne choisir qu'un nombre restreint de membres significatifs de leur famille n'aboutit pas forcément à la sélection de personnes qui sont connectées les unes aux autres; elles ne sont donc pas en mesure de se coordonner et de garantir une aide collective. D'autre part, les très âgés ou ceux en mauvaise santé qui ont accepté de participer à l'étude malgré les difficultés qu'ils rencontrent 
peuvent se caractériser par un profil spécifique, celui de « survivant», qui les distingue des autres du même âge et de même état de santé. Ce profil particulier qui les caractérise - ils ont par exemple un bon moral, sont optimistes, sont volontaires ou font preuve d'une grande autonomie, etc. - peut expliquer qu'ils n'aient pas «a priori » besoin d'une mobilisation accrue de leur famille, même s'ils sont moins actifs dans les échanges familiaux. Ce résultat peut aussi être spécifique à Genève, où les services de maintien à domicile sont bien développés. Les besoins des «très-vieux » ou de ceux en incapacité étant devancés par les professionnels, la famille ne se mobilise dès lors pas davantage. Globalement, les analyses de régression confirment l'effet significatif du profil socio-démographique des répondants sur le capital social, et cela indépendamment de la composition de leur configuration familiale. Afin de donner du corps à ces différents résultats, nous proposons dans la sous-section suivante quelques illustrations de configurations familiales.

\subsubsection{Illustrations de types de capital social}

Afin de mieux saisir le lien entre la composition des configurations familiales, le profil des répondants et le capital social, nous avons sélectionné dans l'échantillon six exemples de configurations familiales qui illustrent bien comment l'organisation des liens de soutien varie d'une configuration à l'autre et donne lieu à différents types de capital social. La figure 6.1 présente ces six cas distincts ${ }^{1}$. Les pointes de flèches désignent les membres significatifs, y compris le répondant, qui sont source de soutien. Dans chaque exemple, nous exposons le profil du répondant, l'état de son réservoir de parenté et les propriétés structurelles qui caractérisent sa configuration familiale et le type de capital social qu'elle génère.

Dans le premier exemple (a), il s'agit du graphe d'une configuration familiale de type «Conjugal» (voir 6.1(a)) d'un homme âgé de 86 ans, sans atteintes fonctionnelles. Il est marié, a deux enfants et quatre petits-enfants mais ne déclare aucun frère ou aucune sœur vivant. Il cite comme membres significatifs de sa famille, son fils, sa fille et son épouse (Taille $=3$ vs

1 Les tracés des réseaux ont été effectués sous $\mathrm{R}$ ( $\mathrm{R}$ Core Team, 2015) à l'aide du package spnet (Rousseaux, Deville \& Ritschard, 2014). 
Mtot $=3.39)$. On remarque que les liens de soutien au sein de cette configuration familiale sont extrêmement denses (Densité $=0.67$ vs Mtot $=0.37$ ), tous les membres sont en effet interconnectés et les échanges sont moyennement réciproques (Réciprocité $=0.33$ vs Mtot $=0.39$ ). Cet homme se décrit comme relativement actif dans les échanges avec les membres de sa famille significative. Il déclare y soutenir deux membres (Degré rentrant $=2$ vs Mtot $=2.11$ ) et être soutenu par l'ensemble des membres de sa configuration familiale (Degré sortant $=3 \mathrm{vs}$ Mtot $=1.50$ ). Étant moyennement actif, la centralité de ce répondant dépasse légèrement la moyenne de l'échantillon (Centralité $=0.17$ vs Mtot $=0.15$ ). Au vu de la petite taille de sa configuration familiale et de la forte densité des liens de soutien qui la caractérise, il a davantage accès à un capital social de type chaîne que de type pont. La mention du partenaire ainsi que celle de ses enfants s'associent à une forte densité des liens, traduisant des échanges de soutien de longue date. Reliés entre eux par des normes de réciprocité " généralisée », la partenaire et les enfants proposent au répondant un soutien collectif et solide.

Le deuxième graphe (b) représente une configuration familiale de type «Fils» (voir 6.1(b)). Il s'agit de la configuration d'un homme âgé de 72 ans, en bonne santé (fonctionnelle). Il est marié, a deux enfants, deux petits-enfants et trois frères ou sœurs. Il mentionne son fils, la partenaire de son fils, sa fille, les deux filles de son fils comme membres significatifs de sa famille (Taille $=5 \mathrm{vs}$ Mtot $=3.39$ ). Bien que marié, il ne mentionne pas son épouse comme faisant partie de sa famille significative. Le graphe montre que la fille, le fils, la belle-fille et les petits-enfants sont fortement interconnectés les uns aux autres au travers d'échanges de soutien mutuels. Les normes de réciprocité "généralisée», qui sous-tendent les liens intergénérationnels et qui plus est à trois générations, contribuent à tisser des liens de soutien fort denses, prometteurs d'un soutien collectif et fiable. La densité et la réciprocité y sont donc fortes, atteignant 0.73 pour la densité $(v s \mathrm{Mtot}=0.37)$ et 0.57 pour la réciprocité $(\mathrm{vs} M t o t=0.39)$. Le répondant participe aussi activement aux échanges puisque non seulement il soutient une majorité des membres de sa configuration (Degré rentrant $=4$ vs Mtot $=2.11$ ) mais il est surtout soutenu par tous sans exception (Degré sortant $=5 \mathrm{vs}$ Mtot $=1.50$ ). Tout aussi actif que ces derniers, il se perçoit comme central au sein de sa configuration familiale (Centralité $=0.23$ vs Mtot $=0.15$ ). Baignant dans des liens de soutien denses et réciproques, le répondant bénéficie donc d'un fort capital social de type chaîne, bien qu'il parvienne, en étant actif, à préserver sa centralité et, ce faisant, son autonomie structurelle. 
Fig. 6.1: Capital social selon les six configurations familiales.

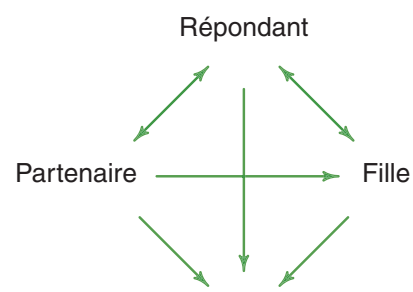

Fils

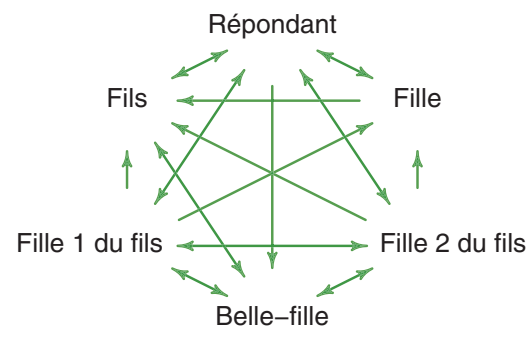

(b) Fils. Taille $=5$; Densité $=0.73$; Réciprocité $=0.57$; In-degree $=4$; Out-degree $=5$; Centralité $=0.23$.

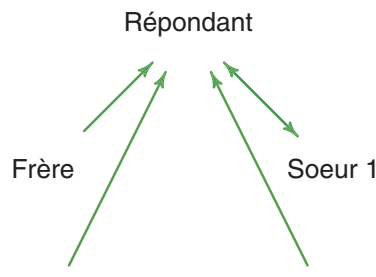

Soeur 2

Fils

(d) Fratrie. Taille $=4$; Densité $=0.25$; Réciprocité $=0.25$; In-degree $=4$; Outdegree $=1$; Centralité $=0.25$.

Répondant

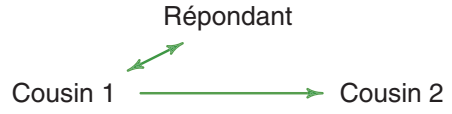

Neveu 1

Neveu 2

Neveu 3

(e) Parenté. Taille $=5$; Densité $=0.13$; Réciprocité $=0.33$; In-degree $=1$; Outdegree $=1$; Centralité $=0.00$. (f) Isolés. Taille $=1$; Densité $=0.00$;

Réciprocité $=0.00$; In-degree $=0$;

Out-degree $=0$; Centralité $=0.00$. 
Le graphe (c) dépeint les liens de soutien d'une configuration familiale de type "Fille» (voir 6.1(c)). Le répondant est veuf, âgé de 72 ans et en bonne santé (fonctionnelle). Il n'a pas de partenaire mais il a une fille, quatre petits-enfants et un membre de sa fratrie encore vivant. Il cite comme membres significatifs de sa famille sa fille, les trois filles et le fils de sa fille. Contrairement à l'exemple précédent dans lequel la partenaire du fils (belle-fille) fait partie de la liste des membres significatifs de la famille, le répondant ne mentionne pas le partenaire de sa fille. Bien que la taille (Taille $=5$ vs Mtot $=3.39$ ) soit identique à l'exemple précédent («Fils»), les échanges de soutien s'organisent différemment. Dans cet exemple, la densité (Densité $=0.27$ vs Mtot $=0.37$ ) et la réciprocité (Réciprocité $=0.14$ vs Mtot $=0.39)$ sont faibles, se différenciant clairement des résultats de l'exemple précédent. Quant à l'engagement du répondant, il est plutôt ténu, ce dernier soutenant seulement sa fille et l'une de ses petites-filles (Degré rentrant $=2$ vs Mtot $=2.11$ ) tout en ne recevant du soutien que de sa fille (Degré sortant $=1$ vs Mtot $=1.50$ ). Peu engagé, cet homme âgé n'est pas central dans les échanges de soutien au sein de sa configuration familiale (Centralité $=0.00$ vs Mtot $=0.15$ ). C'est plutôt sa fille qui occupe une position centrale au sein des échanges, soutenant émotionnellement l'ensemble des membres de la configuration. Elle est en effet sollicitée par l'ensemble des membres de la famille et peine, en étant seule - le beau-fils n'étant pas mentionné comme significatif -, à stimuler les échanges entre les différents membres. Dans cet exemple précis, la mention de la fille et des petits-enfants comme membres significatifs de la famille ne suffit pas à garantir au répondant un soutien collectif. Au vu de ses propriétés structurelles, cette configuration familiale ne produit qu'un faible volume de capital social, aussi bien de type chaîne que de type pont.

Le graphe (d) illustre l'organisation des liens de soutien dans une configuration familiale de type "Fratrie » (voir 6.1(d)) d'un homme de 78 ans, sans atteinte fonctionnelle. Il est divorcé et remarié depuis 2005. Il a trois enfants de son premier mariage, sept petits-enfants, quatre arrière-petits-enfants et cinq frères ou sœurs. Son réservoir de parenté est donc particulièrement vaste. Lors de l'entretien, il cite son fils aîné, deux de ses sœurs et son frère comme membres significatifs de sa famille. Il ne mentionne ni sa partenaire actuelle, ni ses deux autres enfants, ni ses descendants. Sa configuration, relativement grande (Taille $=4$ vs Mtot $=3.39$ ), se caractérise par de faibles scores sur la densité $($ Densité $=0.25$ vs Mtot $=0.37$ ) et la réciprocité $($ Réciprocité $=0.25 \mathrm{vs}$ 
Mtot $=0.39)$. Le graphe révèle que les membres cités ne sont pas connectés entre eux. Alors qu'il ne reçoit du soutien que de sa sœur (Degré sortant $=1$ vs Mtot $=1.50)$, le répondant se décrit comme un actif prestataire de soutien au sein de sa configuration puisqu'il en donne à tous les membres qu'il mentionne (Degré rentrant $=4$ vs Mtot $=2.11$ ). Au centre des échanges, le répondant relie les différents membres de sa configuration par le soutien qu'il procure. Occupant une position centrale au sein de son réseau, son score sur la centralité dépasse largement la moyenne totale (Centralité $=0.25$ vs Mtot $=0.15$ ). Au vu des propriétés structurelles de sa configuration, le répondant bénéficie d'un capital social de type pont. Cette configuration familiale représente bien le processus de ré-évaluation des liens familiaux lors de la construction de la famille significative. Malgré son riche réservoir de parenté, comprenant aussi bien une partenaire que des enfants et des petits-enfants, cet homme remarié sélectionne comme membres significatifs de sa famille l'un de ses trois enfants et trois membres de sa fratrie. Ce «choix » dans la composition de sa configuration a des implications directes sur l'organisation des liens de soutien en son sein. Bien que cet homme préserve son autonomie en occupant une place centrale dans les échanges, le soutien sur lequel il peut compter est plutôt fragile, étant dyadique plutôt que collectif.

Dans le cinquième graphe (e) figurent les échanges de soutien d'une configuration familiale de type «Parenté » (voir 6.1(e)). Le répondant est une femme âgée de 92 ans, divorcée et sans enfant. Elle n'a pas de partenaire ni de frères ou sœurs vivants. Elle vit seule et a des problèmes de mobilité. Elle cite comme membres significatifs de sa famille les trois enfants de son seul frère décédé, une cousine et un cousin. Sa configuration est de grande taille (Taille $=5$ vs Mtot $=3.39$ ), peu dense $($ Densité $=0.13$ vs Mtot $=0.37$ ) et légèrement réciproque (Réciprocité $=0.33$ vs Mtot $=0.39)$. Le graphe met en évidence la faiblesse des connexions entre les différents membres significatifs qu'elle a listés. La répondante est peu active dans les échanges; elle ne donne du soutien qu'à son cousin (Degré rentrant $=1 \mathrm{vs}$ Mtot $=2.11)$ qui, en retour, lui apporte le sien $(D e-$ gré sortant $=1$ vs Mtot $=1.50)$. On note qu'elle n'est pas du tout centrale au sein de sa configuration familiale (Centralité $=0$ vs Mtot $=0.15) . \mathrm{Vu}$ les propriétés structurelles qu'elle affiche, cette configuration familiale ne produit qu'un faible capital social, quel que soit son type. Bien que les membres de cette configuration familiale proviennent tous du réservoir de 
parenté de la répondante, ils n'en sont pas moins divers, se caractérisant par des profils générationnels distincts. Cette diversité explique sans doute les « trous » structurels entre les cousins, que l'on suppose de même génération que la répondante, et les neveux, plus jeunes. En raison de son âge avancé et de ses problèmes de santé, la répondante ne parvient pas à combler les « trous » et à jouer le rôle d'intermédiaire, rôle qui lui permettrait pourtant d'accéder au capital social de type pont. Cet exemple reflète bien la difficulté de maintenir un tel capital lorsque le processus de fragilisation affecte peu à peu les capacités physiques et cognitives des individus âgés.

Finalement, le dernier graphe (f) (voir 6.1(f)) représente une configuration familiale de type "Isolés». Il s'agit d'une femme âgée de 96 ans, veuve et sans partenaire. Elle a un fils, deux petits-enfants, un arrièrepetit-enfant et un frère ou une sœur. Sévèrement atteinte dans sa santé fonctionnelle, elle vit en institution. Elle mentionne comme membre significatif de sa famille seulement une amie, plus jeune ( 78 ans), qu'elle connaît depuis longtemps et avec laquelle elle n'échange aucun soutien émotionnel. A l'exception de la taille (Taille $=1$ vs Mtot $=3.39$ ), tous les autres indicateurs du capital social affichent 0 . Cette répondante ne bénéficie donc d'aucun capital social, quel qu'il soit. A nouveau, cet exemple reflète assez bien le mécanisme de re-définition des liens familiaux qui conduit à une ré-évaluation des relations affectives significatives. Bien qu'elle ait un fils, des petits-enfants et un frère ou une sœur, elle ne les mentionne pas comme membres significatifs de sa famille et cite au contraire une amie, avec laquelle pourtant elle n'échange pas ou plus de soutien émotionnel. Très âgée, en mauvaise santé et placée en institution, elle ne semble plus en mesure de s'investir dans une vie relationnelle. Le maintien du lien, même significatif, devient difficile et, n'étant pas soutenu par de fortes obligations familiales, un tel lien s'amenuise.

\subsubsection{Conclusion sur le capital social}

Le soutien familial est souvent perçu et défini dans les modèles dominants de la gérontologie sociale comme relativement homogène, fiable et inconditionnel. Or, l'exploration du « capital social » au sein des configurations familiales atteste du contraire et révèle que le soutien est divers tant dans sa forme que dans son volume. Certaines configurations familiales produisent du capital social de type chaîne, d'autres du capital social de type pont. 
Dans certaines familles, le capital social produit est volumineux alors que, dans d'autres, il est faible, voire inexistant. Conformément aux hypothèses (cf. Chapitre "Problématique et hypothèses »), les résultats présentés vérifient que les variations du capital social dépendent, en grande partie, de la composition des configurations familiales. L'identité des membres de la famille significative et leur combinaison spécifique ont bel et bien des implications sur l'organisation des liens de soutien au sein des configurations familiales et donnent lieu à différents types et volumes du capital social. Outre l'identité des membres de la configuration familiale, le profil sociodémographique et la santé du répondant ont aussi, comme nous l'avons postulé, un impact sur le type et le volume du capital social auquel il a accès.

Conformément à l'hypothèse « $\mathrm{H} 3$ », la présence du conjoint, des enfants et des petits-enfants dans la configuration familiale renforce généralement les liens d'interdépendance au sein des configurations familiales. Soutenus par des normes de réciprocité " généralisée », différée et indirecte, les échanges de soutien contribuent à densifier les liens entre les différents membres et favorisent par conséquent l'émergence du capital social de type chaîne, garantissant à l'individu âgé un soutien familial collectif, solide et fiable. Toutefois, la présence de filles et de petites-filles conduit à un faible volume de capital social, ce qui réfute l'hypothèse susmentionnée. Bien que désignées dans la littérature gérontologique comme des prestataires « majeures » du soutien familial, les filles peinent, lorsqu'elles sont 《 seules » dans la configuration, à remplir leur rôle de «kinkeeper» et à stimuler les liens de soutien, ce qui aboutit à la longue à l'affaiblissement du capital social dans les familles. Un tel résultat va aussi à l'encontre de l'hypothèse « $\mathrm{H} 4$ » selon laquelle les membres féminins promeuvent, en raison des rôles familiaux qui leur reviennent, le capital social de type chaîne au sein de leur configuration familiale. Ce résultat remet en question le postulat des modèles dominants de la gérontologie sociale qui allie la présence de membres primaires de la famille - et qui, plus est, féminins - à l'infaillibilité du soutien familial.

Les résultats montrent aussi que, conformément à l'hypothèse « $\mathrm{H} 3$ », les configurations familiales composées majoritairement de liens affinitaires s'associent à une organisation différente des liens de soutien au sein des configurations familiales. Dans ce type de configurations, les membres qui les composent sont moins connectés les uns aux autres et entretiennent 
avec le parent âgé des échanges de soutien dyadiques, reposant davantage sur des normes de réciprocité " équilibrée» que "généralisée ». Ce type de configurations permet aux individus âgés d'y jouer un rôle central et engagé, leur donnant accès au capital social de type pont, garant de leur autonomie structurelle. Les résultats ayant trait aux configurations de type «Fratrie» - les liens fraternels étant à l'âge adulte davantage choisis qu'imposés par des obligations familiales - vérifient en partie cette hypothèse. Toutefois, l'analyse souligne la difficulté des aînés d'entretenir ce type de capital social dans la vieillesse. En effet, lorsque la composition de la famille significative est trop diversifiée, comme dans les configurations de type "Parenté », ou fragile et incertaine, comme dans celles de type "Isolés», le rôle d'intermédiaire devient difficile, les individus âgés n'ayant plus l'énergie et les compétences physiques et mentales pour l'exercer. Les résultats montrent que, dans ces types de configurations, le capital social de type pont se fragilise et disparait.

L'hypothèse « $\mathrm{H} 5$ » est en partie vérifiée puisque les résultats confirment que le profil socio-démographique des répondants influence le flux des échanges au sein des configurations familiales et, par conséquent, détermine le capital social auquel ils ont accès. Les ressources auxquelles s'associe le profil socio-démographique comme le revenu, la santé, l'énergie ou encore la capacité relationnelle, ont une influence sur le type et le volume du capital social au sein des configurations familiales. Les femmes et les «jeunes-vieux», par exemple, sont plus engagés et plus centraux au sein de leurs échanges familiaux et ont, de ce fait, davantage accès au capital social de type pont que les hommes et les plus vieux. Le rôle familial des femmes de « kinkeeper » et l'énergie des « jeunes-vieux » peuvent expliquer leur propension plus grande au capital social de type pont. De même, les individus issus des milieux populaires, qui sont généralement plus dépendants de l'aide familiale que les autres, déclarent des liens de soutien plus denses, accédant dès lors à davantage de capital social de type chaîne. Cependant, et contrairement à l'hypothèse « H5 », les individus âgés ayant un statut socio-économique élevé, s'associant généralement au capital social de type pont, ont plus accès au capital social de type chaîne dans la vieillesse que les répondants des classes moyennes. Ce résultat peut s'expliquer par la probabilité plus forte des personnes âgées socialement privilégiées d'être encore mariées à des âges avancés, le mariage étant une condition favorable au maintien du capital social de type chaîne dans la vieillesse. 
Le processus de fragilisation a aussi un impact important sur les échanges de soutien au sein des configurations familiales. Il réduit la capacité relationnelle des « très-vieux » ou de ceux qui sont atteints dans leur santé et limite ainsi leur accès au capital social de type pont. Cependant, et contrairement à l'hypothèse « $\mathrm{H} 5$ », ce processus ne s'accompagne pas d'un meilleur accès au capital social de type chaîne, les liens n'étant pas plus denses dans leurs configurations que dans celles des plus jeunes ou de ceux en meilleure santé. Les raisons qui peuvent expliquer ce résultat sont multiples. Soit, suivant le processus de sélectivité socio-émotionnelle, l'individu très âgé a progressivement réduit le nombre de membres de sa configuration pour ne garder que ceux qui lui apportent une certaine satisfaction émotionnelle et ceux-ci, pouvant être de profils divers, ne sont pas forcément connectés les uns aux autres. Soit la mobilisation familiale n'a pas lieu d'être en raison du profil de « survivant» qui caractérise les « trèsvieux » ou ceux en mauvaise santé qui ont participé à l'enquête; ceux-ci peuvent faire preuve d'une certaine autonomie vis-à-vis de l'aide familiale (voir le chapitre «Données et méthodes»). De plus, les services de maintien à domicile à Genève, diversifiés dans les prestations qu'ils offrent, pourvoient à de nombreux besoins des « très-vieux » ou de ceux qui ont une mauvaise santé, allégeant ainsi les charges de soutien des membres de la famille. Ces diverses raisons peuvent expliquer la faiblesse du capital social dans les familles aux ultimes étapes de la vie.

Finalement, le réservoir de parenté n'a pas d'effet important sur le développement du capital social dans les configurations familiales. Certes, s'il est riche, il offre une pluralité de possibilités relationnelles alors que s'il est pauvre, il limite le potentiel relationnel. Cependant, les résultats des analyses de régression montrent que c'est plutôt la désignation des membres de la famille comme "significatifs » qui influence le développement du capital social au sein des configurations familiales bien plus que leur seule existence dans le réservoir de parenté.

L'approche configurationnelle utilisée pour mesurer le capital social dans les configurations familiales offre de nouvelles perspectives d'analyse et apporte un nouveau regard sur les liens de soutien dans la vieillesse. Elle montre que le soutien est bien plus complexe que ne le supposent la plupart des travaux en gérontologie sociale. Il ne peut être appréhendé qu'avec le seul montant de soutien échangé entre l'individu âgé et sa famille, considérée comme un groupe homogène, ou comme une ressource octroyée au sein des relations intergénérationnelles. Les résultats exposés dans cette 
section confirment que la notion de capital social, proposée par l'approche configurationnelle, est pertinente pour élargir l'analyse des liens de soutien à l'ensemble de la configuration familiale et en saisir ainsi toute la complexité et la diversité. Le capital social n'est pas la « seule production » observable au sein des configurations familiales, les tensions et les conflits peuvent aussi être examinés au niveau de l'ensemble du réseau familial. La perspective configurationnelle permet en effet d'observer comment les conflits se diffusent au sein des configurations familiales et s'articulent avec les liens de soutien pour former des modes de conflit et de support distincts, révélateurs des gestions différentes de l'ambivalence structurelle au niveau familial.

\subsection{Les conflits et l'ambivalence}

Les six configurations familiales dégagées dans le chapitre précédent produisent différents volumes et types de capital social, mais elles peuvent aussi être source de conflit et d'ambivalence structurelle. L'interdépendance, fondement de toute configuration, suscite des frustrations, des tensions et de l'ambivalence. Intrinsèque aux relations d'interdépendance, l'ambivalence doit être gérée au sein des configurations familiales par les différents membres qui les composent. On peut dès lors se questionner sur la façon dont les aînés la vivent et la gèrent au quotidien avec les membres de leur configuration familiale. Nous avons montré dans le chapitre «Problématique et hypothèses » que la typologie des modes de conflit et de support (Solidarité, Ambivalence, Captivation et Atomisation) est la plus adéquate pour répondre à cette question. Ces quatre modes reflètent différentes manières de négocier l'ambivalence structurelle au niveau configurationnel, avec des conséquences distinctes sur les relations au sein des configurations familiales. Il est nécessaire d'en savoir plus sur les facteurs qui expliquent leur émergence afin de détecter les inégalités sociales dans la gestion de l'ambivalence au sein des familles dans la vieillesse. Selon les hypothèses formulées précédemment, deux principaux facteurs jouent un rôle sur leur développement : la composition des configurations familiales (« $\mathrm{H} 6 »)$ et les ressources à disposition des aînés 
(«H7 ») (cf. chapitre « Problématique et hypothèses »). Pour tester ces hypothèses, nous avons procédé à une analyse des correspondances multiples (ACM) afin d'examiner les effets concomitants de la composition des configurations familiales et les ressources des personnes âgées sur l'émergence de ces différents modes de gestion de l'ambivalence. Pour illustrer ces résultats, nous présentons à la fin de cette section quatre situations concrètes qui traduisent bien ces différents modes.

\subsubsection{Les effets de composition}

Avant d'observer ensemble les effets des deux facteurs susmentionnés, nous examinons d'abord l'influence de la composition des configurations familiales sur les modes de conflit et de support sans prendre en compte l'impact des ressources individuelles. La question est de savoir si le degré d'interdépendance, qui varie en fonction de la composition des configurations familiales selon l'hypothèse « H6», influe sur les modes de conflit et de support présents dans les différentes configurations familiales identifiées. Dans le tableau (6.6) figure la distribution des quatre modes de conflit et de support selon les six types de configurations familiales. Ce premier résultat offre déjà quelques éléments de réponse.

Ce tableau montre que le mode de gestion Ambivalence est plus fréquent parmi les configurations familiales composées du partenaire, des enfants, voire des petits-enfants : $31 \%$ des configurations « Conjugal» et $30 \%$ des configurations «Fils» développent l'Ambivalence contre $11 \%$ des

Tab. 6.6: Modes de conflit et de support selon les types de configurations familiales $(\mathrm{N}=563)$

\begin{tabular}{lccccccc}
\hline & $\begin{array}{c}\text { Conjugal } \\
(\mathrm{n}=222)\end{array}$ & $\begin{array}{c}\text { Fils } \\
(\mathrm{n}=43)\end{array}$ & $\begin{array}{c}\text { Fille } \\
(\mathrm{n}=62)\end{array}$ & $\begin{array}{c}\text { Fratrie } \\
(\mathrm{n}=87)\end{array}$ & $\begin{array}{c}\text { Parenté } \\
(\mathrm{n}=45)\end{array}$ & $\begin{array}{c}\text { Isolés } \\
(\mathrm{n}=104)\end{array}$ & $\begin{array}{c}\text { Total } \\
(\mathrm{n}=563)\end{array}$ \\
\hline Ambivalence & 31 & 30 & 24 & 24 & 11 & 4 & 23 \\
Solidarité & 36 & 30 & 26 & 37 & 33 & 19 & 31 \\
Captivation & 11 & 10 & 13 & 9 & 11 & 1 & 9 \\
Atomisation & 22 & 30 & 37 & 30 & 45 & 76 & 37 \\
\hline Total & 100 & 100 & 100 & 100 & 100 & 100 & 100 \\
$\chi^{2}$ & & \multicolumn{10}{l}{$104.44^{* * *}$} & & & \\
\hline
\end{tabular}

Note : Table de contingence, pourcentages, $* * *$ p. $<0.001$ 
configurations «Parenté » et $4 \%$ des configurations «Isolés ». On observe la tendance inverse pour l'Atomisation : 76\% des configurations «Isolés » et $45 \%$ des configurations « Parenté » déploient ce mode contre $22 \%$ des configurations « Conjugal» ou 30\% des configurations «Fils». Ce premier résultat atteste que le mode de gestion Ambivalence, qui se manifeste dans les configurations à la fois par du soutien et du conflit, est surtout présent dans les familles où les liens d'interdépendance sont forts comme dans celles composées de liens intergénérationnels ou conjugaux. A l'inverse, l'absence de tels liens donne davantage lieu au mode de gestion Atomisation qui se traduit à la fois par peu de conflit et peu de soutien au sein des configurations. Quant aux modes de gestion Solidarité et Captivation, les résultats sont moins tranchés que précédemment. Ils se répartissent de manière plus égale selon les six types de configurations familiales. La Solidarité, qui se manifeste par du soutien et peu de conflit dans les réseaux familiaux, tend à être plus fréquente dans les configurations "Fratrie » (37\%) que dans les configurations «Fille» $(26 \%)$ ou «Isolés» $(19 \%)$. Quant à la Captivation, qui se révèle dans les familles par du conflit et peu de soutien, elle est légèrement plus fréquente dans les configurations «Fille» $(13 \%)$ que dans les configurations « Fratrie » $(9 \%)$ et «Isolés» $(1 \%)$. Ceci dit, ces résultats traduisent seulement de légères tendances qu'il faut davantage investiguer. Pour aller plus loin, nous avons procédé à une analyse de variance (ANOVA) sur les densités de soutien et de conflit afin de mieux connaître l'organisation des liens positifs et négatifs au sein de ces diverses configurations familiales. Ces analyses nous renseignent sur la manière dont est gérée l'ambivalence structurelle dans chacune de ces six configurations familiales. Les résultats de cette analyse figurent dans le tableau suivant (6.7).

Tab. 6.7: Densités de support et de conflit selon les types de configurations familiales $(\mathrm{N}=563)$

\begin{tabular}{lccccccccc}
\hline & $\begin{array}{l}\text { Conjugal } \\
(\mathrm{n}=222)(\mathrm{n}=43)\end{array}$ & Fils & Fille & Fratrie Parenté & Isolés & Total & $(\mathrm{n}=87)(\mathrm{n}=45)(\mathrm{n}=104)(\mathrm{n}=563)$ & $\begin{array}{c}\mathrm{F} \\
(5,557)\end{array}$ & $\begin{array}{c}\text { Kruskal } \\
\text {-Wallis }\end{array}$ \\
\hline $\begin{array}{l}\text { Densité } \\
\text { de soutien } \\
\begin{array}{l}\text { Densité } \\
\text { de conflit }\end{array}\end{array}$ & 0.46 & 0.44 & 0.34 & 0.41 & 0.31 & 0.16 & 0.37 & $19.03 * * * 107.22 * * *$ \\
\hline
\end{tabular}

Note : ANOVA, moyennes, ***p. $<0.001$ 
L'analyse de variance révèle la co-présence de conflit et de soutien au sein des configurations de types «Conjugal» et «Fils», leurs scores moyens dépassant ceux de l'ensemble de l'échantillon. Le conflit caractérise aussi les configurations familiales de type «Fille»; il atteint le même score que celui du type «Fils». Ceci dit, le faible soutien des configurations «Fille» les distingue des précédentes (« Conjugal» et «Fils»). Ces résultats confirment les tendances soulignées précédemment : la Captivation se développe davantage dans les configurations familiales de type «Fille» alors que l'Ambivalence est plus présente dans les configurations de types «Fils» et «Conjugal». La plus forte densité de conflit qui distingue ces trois configurations familiales des trois autres montre que la gestion de l'ambivalence est plus conflictuelle dans les configurations composées de liens intergénérationnels et conjugaux que dans celles reposant exclusivement sur des liens affinitaires. La forte interdépendance qui sous-tend ce type de liens rend en effet la gestion de l'ambivalence plus difficile, les relations conflictuelles ne pouvant être aisément rompues.

Quant aux relations fraternelles, elles s'associent à une plus faible densité de conflit que les liens intergénérationnels et conjugaux mais elles présentent la même densité de soutien. Ce résultat souligne la propension des configurations de type «Fratrie » à développer la Solidarité. Contrairement aux configurations précédentes, les liens fraternels ne sont pas soutenus dans la vieillesse par un fort sentiment d'obligation mais plutôt par une envie d'être ensemble qui se traduit dans les configurations de type "Fratrie » par un fort engagement individuel. L'investissement dont font preuve les aînés auprès de leur fratrie contribue non seulement à stimuler les échanges de soutien au sein de leur configuration mais leur offre aussi une certaine centralité qui leur permet de se détacher des liens qu'ils jugent conflictuels sans déstabiliser le réseau familial. Bien que composées aussi de liens affinitaires, les configurations familiales de type «Parenté » ou «Isolés» affichent, certes, peu de conflit mais aussi peu de soutien. Ces résultats confirment leur tendance plus marquée à développer l'Atomisation. Comme pour les configurations «Fratrie », la faible interdépendance qui caractérise les liens familiaux « volontaires» explique l'absence de conflit, toute relation jugée négative pouvant être facilement rompue. Cependant, et contrairement au type "Fratrie », les aînés dans ces deux types de configurations ne parviennent pas à entretenir les échanges de soutien, étant peu connectés et peu engagés au sein de leur configuration familiale, ce qui expliquerait leur plus forte propension à l'Atomisation. 
En résumé, les configurations familiales construites sur des liens intergénérationnels et conjugaux développent davantage des modes de gestion de l'ambivalence axés sur le conflit, tels que l'Ambivalence et la Captivation, que celles composées principalement de liens affinitaires. La forte interdépendance qui caractérise les liens intergénérationnels ou conjugaux ainsi que les obligations familiales qui les sous-tendent contraignent les membres de la configuration à gérer ensemble les tensions et l'ambivalence, de tels liens ne pouvant être que difficilement rompus. A l'inverse, la faible interdépendance et l'absence de fortes obligations familiales, qui caractérisent les configurations familiales constituées de liens affinitaires, rendent plus facile la rupture des liens conflictuels. Par conséquent, ces types de configurations développent des modes de conflit et de support exempts de conflit, tels que la Solidarité et l'Atomisation. Ces résultats vérifient l'hypothèse « $\mathrm{H} 6$ » : la composition des configurations familiales influence le mode de conflit et de support qui s'y déploie.

\subsubsection{La prise en compte des ressources dans l'analyse}

La composition des configurations familiales n'est pas le seul facteur qui détermine les modes de gestion de l'ambivalence structurelle. Les ressources dont disposent les individus, telles que le revenu, la santé ou encore le réservoir de parenté, peuvent aussi avoir une influence sur l'émergence de ces différents modes au sein des familles (hypothèse « $\mathrm{H} 7 »)$ ). Plus encore, il est probable que ces deux facteurs se combinent et forment, dans leur interrelation, les conditions favorables à l'émergence des différents modes de conflit et de support (hypothèse « H8 »). Pour tester ces hypothèses, nous avons effectué une analyse des correspondances multiples (ACM) qui considère ensemble, dans la même analyse, la composition des configurations familiales et les ressources des aînés au moment de l'enquête. Plus concrètement, l'ACM permet de repérer sur un graphe des « conditions-types » auxquelles s'associent des réponses spécifiques, à savoir les modes de conflit et de support que nous avons dégagés.

L'ACM procède par étapes (cf. " Données et méthodes »). Il est d'abord nécessaire d'interpréter les principales dimensions - axes sur le graphe - qui sont produites par l'analyse. Ces principales dimensions synthétisent les informations générées par les tableaux de contingence des variables étudiées. On sélectionne généralement les deux (ou trois) 
premières dimensions; ce sont elles qui expliquent le plus de variance puisqu'elles contiennent l'essentiel de l'information synthétisée. Nous n'avons gardé que les deux premières, celles-ci suffisent à identifier les conditions d'émergence des modes de conflit et de support. Pour interpréter ces deux principales dimensions, nous nous référons aux mesures de discrimination des variables, qui renvoient aux degrés de corrélation de chaque variable avec chaque dimension (cf. Tableau 6.8), et les coordonnées des modalités de chaque variable sur le graphe (cf. Figure 6.2). En effet, plus les mesures de discrimination des variables sont élevées, plus elles expliquent les dimensions auxquelles elles sont corrélées (Avolio et al., 2013; Hoffman \& De Leeuw, 1992) et, sur le graphe, plus les modalités des variables s'écartent les unes des autres le long de la dimension (axe), plus elles l'expliquent (Avolio et al., 2013; Hoffman \& De Leeuw, 1992). Dans le tableau 6.9 figurent les coordonnées des modalités de chaque variable sur les deux axes du graphe.

Le tableau 6.8 présente les mesures de discrimination pour chaque variable sur les deux premières dimensions. Si l'on considère la première dimension, ces mesures sont les plus fortes pour les variables suivantes : le « genre », le « revenu », « avoir un partenaire », la « santé fonctionnelle » et, dans une moindre mesure, «avoir un frère ou une soeur ». Cette première dimension explique $11 \%$ de la variance totale, à savoir l'ensemble de la variation mesurée dans l'ensemble des données analysées. Pour ce qui est

Tab. 6.8: Mesures de discrimination des variables selon les deux premières dimensions après rotation $(\mathrm{N}=476)$

\begin{tabular}{lcc}
\hline Variables actives : & $\begin{array}{c}\text { Dimension 1 } \\
\text { poids }\end{array}$ & $\begin{array}{c}\text { Dimension 2 } \\
\text { poids }\end{array}$ \\
\hline Genre & 0.26 & 0.01 \\
Revenu & 0.33 & 0.03 \\
Citoyenneté & 0.01 & 0.01 \\
A un partenaire & 0.49 & 0.04 \\
A au moins un enfant & 0.02 & 0.58 \\
A au moins un frère/sœur & 0.12 & 0.08 \\
Configurations familiales & 0.24 & 0.75 \\
Modes de conflit et de support & 0.11 & 0.19 \\
Santé fonctionnelle & 0.31 & 0.00 \\
\hline \% de la variance expliquée & $11.09 \%$ & $9.90 \%$ \\
\hline
\end{tabular}


Tab. 6.9: Coordonnées des modalités des variables sur les deux premières dimensions après rotation $(\mathrm{N}=476)$

\begin{tabular}{|c|c|c|c|}
\hline \multirow{2}{*}{ Variables actives } & & \multicolumn{2}{|c|}{ Coordonnées } \\
\hline & & Dimension 1 & Dimension 2 \\
\hline \multicolumn{4}{|l|}{$\begin{array}{l}\text { Profil socio- } \\
\text { démographique : }\end{array}$} \\
\hline \multirow[t]{2}{*}{ Genre } & Femme & 0.55 & -0.08 \\
\hline & Homme & -0.48 & 0.07 \\
\hline \multirow[t]{3}{*}{ Revenu } & Bas & 0.57 & 0.02 \\
\hline & Moyen & -0.09 & -0.12 \\
\hline & Élevé & -1.18 & 0.36 \\
\hline \multirow[t]{2}{*}{ Citoyenneté } & Suisse (Né Suisse) & -0.08 & 0.07 \\
\hline & Étrangère (Né Étranger) & 0.17 & -0.14 \\
\hline \multicolumn{4}{|l|}{$\begin{array}{l}\text { Réservoir } \\
\text { démographique: }\end{array}$} \\
\hline \multirow[t]{2}{*}{ Partenaire } & A un partenaire (A Conj) & -0.54 & 0.15 \\
\hline & N'a pas de partenaire (NoConj) & 0.90 & -0.25 \\
\hline \multirow[t]{2}{*}{ Enfants } & A un/des enfants (A Enfant) & -0.06 & 0.36 \\
\hline & Aucun enfant (NoEnfant) & 0.29 & -1.60 \\
\hline \multirow[t]{2}{*}{ Fratrie } & $\mathrm{A}$ des frères/sœurs (A frère/sœur) & -0.24 & -0.20 \\
\hline & $\begin{array}{l}\text { N'a pas de frère/sœur } \\
\text { (Nofrère/sœur) }\end{array}$ & 0.51 & 0.43 \\
\hline \multicolumn{4}{|l|}{$\begin{array}{l}\text { Santé } \\
\text { fonctionnelle: }\end{array}$} \\
\hline \multirow[t]{3}{*}{ États } & Indépendant s/AVQs (Indépendant) & -0.31 & -0.02 \\
\hline & En difficulté s/AVQs (En difficulté) & 0.82 & -0.02 \\
\hline & Dépendant s/AVQs (Dépendant) & 1.25 & 0.18 \\
\hline \multicolumn{4}{|l|}{$\begin{array}{l}\text { Configurations } \\
\text { familiales: }\end{array}$} \\
\hline \multirow[t]{6}{*}{ Types } & Conjugal & -0.25 & 0.64 \\
\hline & Fils & 0.51 & 0.99 \\
\hline & Fille & 0.21 & 0.82 \\
\hline & Fratrie & -0.66 & -0.99 \\
\hline & Parenté & 0.02 & -1.43 \\
\hline & Isolés & 0.82 & -0.79 \\
\hline \multicolumn{4}{|l|}{ Conflit et support } \\
\hline \multirow[t]{4}{*}{ Modes } & Solidarité & -0.21 & -0.09 \\
\hline & Ambivalence & -0.38 & 0.58 \\
\hline & Captivation & 0.10 & 0.58 \\
\hline & Atomisation & 0.42 & -0.47 \\
\hline
\end{tabular}


Fig. 6.2: Projection des deux premières dimensions de l'analyse des correspondances multiples après rotation.

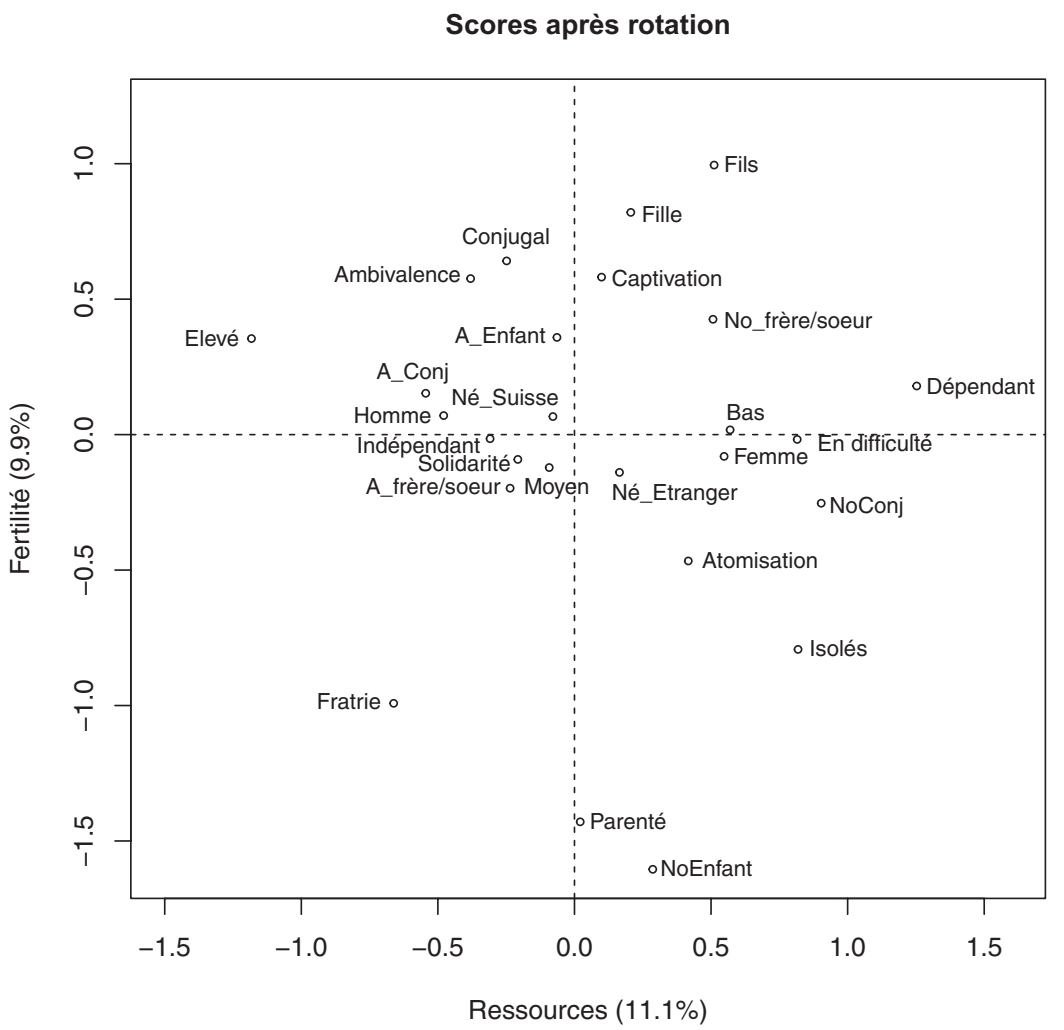

de la deuxième dimension, ce sont les variables « avoir des enfants » et les « configurations familiales » qui affichent des mesures de discrimination les plus fortes. Cette deuxième dimension explique $10 \%$ de la variance totale. A noter que la « citoyenneté » (« né suisse » versus «né étranger») a un faible pouvoir discriminant sur les deux dimensions. Les aînés ne différent pas dans leur profil de réponses en fonction de leur citoyenneté. Venant pour la plupart des pays européens limitrophes, les personnes âgées d'origine étrangère diffèrent peu des personnes âgées suisses en termes de ressources (revenu, réservoir de parenté et santé) et de composition de leur configuration familiale. Quant aux modes de conflit et de support, 
ils présentent un score légèrement plus fort sur la deuxième dimension. $\mathrm{Au}$ vu de ces observations, nous en déduisons que la première dimension distingue les individus âgés en fonction des différentes ressources qu'ils ont à disposition et la deuxième, en fonction de leur statut parental et de la composition de leur configuration familiale (avec ou sans enfant). Fort de ce constat, nous nommons la première dimension « Ressources » et la deuxième, «Fertilité ».

La figure 6.2 représente graphiquement les deux dimensions (axes) autour desquelles se positionnent les différentes modalités des variables étudiées. L'observation de leur position sur les axes permet de vérifier l'interprétation de ces deux dimensions. Si l'on considère l'axe horizontal (première dimension), les modalités des variables « ressources» s'y répartissent distinctement, ce qui confirme le pouvoir discriminant de ces variables. Les modalités positives des variables « ressources» se positionnent du côté négatif de l'axe horizontal (à gauche du 0). On y trouve en effet les modalités « revenu élevé», «homme», et «né suisse» qui renvoient à une position sociale privilégiée. De même, les modalités «a conjoint», «a enfant» et «a frère/sœur», qui se référent à la richesse du réservoir de parenté, se situent aussi du côté négatif de l'axe horizontal, tout comme la modalité « indépendant» qui indique une bonne santé fonctionnelle. A l'inverse, les modalités reflétant une position défavorable dans la société (« revenu bas », « femme », et « né étranger»), un faible réservoir de parenté (« no conjoint », « no enfant», « no frère/sœur ») ou une mauvaise santé fonctionnelle (« en difficulté » et « dépendant») se situent sur le côté positif de l'axe horizontal (à droite du 0 ). Quant à la deuxième dimension, on constate que les deux modalités «a enfant» versus «no enfant» font preuve d'une forte dispersion le long de l'axe vertical (deuxième dimension), attestant du poids de la variable « avoir au moins un enfant vivant» dans l'interprétation de cette deuxième dimension. On observe en effet sur le graphe que la modalité «a enfant» et les configurations familiales composées d'enfants - les types «Conjugal », «Fils» et «Fille»-se projettent sur la partie positive de l'axe vertical (au-dessus de 0 ) alors que la modalité «no enfant» ainsi que les configurations familiales exemptes d'enfants les types «Fratrie », «Parenté» et «Isolés»-se situent sur le côté négatif de l'axe vertical (au-dessous de 0). La dispersion de ces différentes modalités sur les deux axes confirme l'interprétation de ces deux dimensions : la première renvoie bel et bien aux « Ressources » alors que la deuxième, à la « Fertilité ». 
Dès lors que les deux principales dimensions sont définies, nous pouvons dégager les conditions d'émergence des modes de conflit et de support. Pour ce faire, nous localisons sur le graphe ces quatre modes de gestion de l'ambivalence et nous repérons les variables qui leur sont proches spatialement. La figure 6.2 indique que ces quatre modes sont projetés, chacun, dans un cadran du graphique. L'Ambivalence se place du côté négatif de l'axe horizontal, correspondant à la première dimension « Ressources », et du côté positif de l'axe vertical, se référant à la dimension «Fertilité ». L'Ambivalence est spatialement très proche du type de configurations familiales «Conjugal» et de la modalité «a enfant». Elle se situe aussi dans la même zone que les modalités « a conjoint», «homme», «né suisse », « indépendant» et « revenu élevé». Quant à la Captivation, elle se situe sur les côtés positifs des deux axes, horizontal et vertical. La proximité entre l'Ambivalence et la Captivation sur l'axe vertical, c'est-à-dire sur la dimension « Fertilité », montre que toutes deux s'associent au fait d'avoir des enfants. Cependant, contrairement à l'Ambivalence, la Captivation se distancie de la modalité «avoir un conjoint», se rapprochant des configurations familiales qui se caractérisent par l'absence du partenaire tout en y intégrant des enfants et des petits-enfants, telles que les types «Fils» et «Fille». Par ailleurs, sa position du côté positif de l'axe horizontal indique qu'elle s'associe aux modalités mesurant un manque de ressources à la fois démographiques («no conjoint», « no frère/sœur»), socio-économiques («femme», «bas revenu », « né étranger») et de santé (« en difficulté » et « dépendant»).

Quant à la Solidarité, elle se positionne du côté négatif de l'axe horizontal « Ressources» et du côté négatif de l'axe vertical «Fertilité ». La Solidarité est très proche des modalités «a frère/sœur», « indépendant », " né suisse », " revenu moyen », « homme », " a conjoint» et, dans une moindre mesure, «revenu élevé ». Se situant du côté négatif de l'axe vertical qui s'explique principalement par la modalité «no enfant», la Solidarité s'associe davantage à l'absence d'enfant qu'à leur existence. De fait, elle se place dans le même cadran du graphique que les configurations familiales de type «Fratrie». Pour ce qui est de l'Atomisation, elle se situe du côté positif de l'axe horizontal «Ressources » et du côté négatif de l'axe vertical «Fertilité». Sa position sur l'axe vertical place l'Atomisation dans la même zone que la modalité «no enfant». L'Atomisation s'associe donc à l'absence d'enfant et aux configurations familiales qui en sont exemptes, telles 
que les configurations familiales de type «Parenté» ou «Isolés». Se positionnant sur le côté positif de l'axe horizontal «Ressources », l'Atomisation est aussi proche des modalités de variables mesurant un manque de ressources telles que «femme », "né étranger», «bas revenu», «no conjoint» et «no frère/sœur» mais aussi de celles décrivant un mauvais état de santé fonctionnelle, " en difficulté » voire même « dépendant».

Dans le tableau 6.10 sont récapitulés ces différents résultats. Il y est précisé les modalités de variables auxquelles s'associent les quatre modes de conflit et de support. Autrement dit, ce tableau expose les conditions d'émergence de ces quatre modes de gestion de l'ambivalence au niveau configurationnel. L'émergence de ces quatre modes de conflit et de support au sein des configurations familiales n'est pas aléatoire mais répond à diverses conditions, combinant à la fois la composition des configurations familiales et les ressources des individus âgés, ce qui vérifie l'hypothèse « $\mathrm{H} 8 »$.

L'Ambivalence est plus probable dans les configurations familiales de type «Conjugal», qui intègrent le partenaire et les enfants, et lorsque l'aîné bénéficie de diverses ressources. Il s'agit généralement d'individus privilégiés socialement - des hommes, ayant un revenu élevé et suisses - et disposant d'une bonne santé fonctionnelle. Par ailleurs, ils ont un riche réservoir de parenté comprenant un partenaire, des enfants et des membres de la fratrie. Les tensions qui caractérisent ce mode de gestion s'expliquent par la forte interdépendance qui sous-tend les liens intergénérationnels et conjugaux qui composent les configurations familiales «Conjugal». La rupture des liens n'étant pas envisageable, les tensions et l'ambivalence

Tab. 6.10: Conditions d'émergence des modes de conflit et de support

\begin{tabular}{lllll}
\hline & Ambivalence & Solidarité & Captivation & Atomisation \\
\hline Configurations familiales & Conjugal & Fratrie & Fille ou Fils & Isolés ou Parenté \\
A un conjoint & Oui & Oui & Non & Non \\
A des enfants & Oui & Non & Oui & Non \\
A des frères/sœurs & Oui & Oui & Non & Non \\
Genre & Homme & Homme & Femme & Femme \\
Revenu & Élevé & Moyen & Bas & Bas \\
Citoyenneté & Suisse & Suisse & Étranger & Étranger \\
Santé fonctionnelle & Indépendant & Indépendant & En difficulté & En difficulté \\
& & & ou Dépendant & ou Dépendant \\
\hline
\end{tabular}


doivent être gérées entre les différents membres de la configuration familiale. Les ressources dont disposent l'individu âgé et les membres de sa configuration jouent un rôle primordial. Celles-ci permettent au parent âgé et aux membres de la famille de négocier et de trouver des arrangements pour que chacun puisse conserver son autonomie sans affecter les liens de soutien qui demeurent vivaces, étant alimentés par les investissements de chacun. C'est donc par le biais de négociations que les ainés et les membres de leur configuration gèrent l'ambivalence structurelle. Les tensions restent cependant importantes mais elles ne sont pas le signe d'un dysfonctionnement mais plutôt celui de négociations animées pour retrouver un équilibre dans les échanges de soutien au sein de la configuration familiale, tout en préservant l'autonomie de chacun.

Quant à la Solidarité, elle se développe davantage dans les configurations familiales de type "Fratrie » que dans les autres. Composées de liens fraternels « choisis», les relations sont davantage maintenues dans ce type de configurations par une envie commune d'être ensemble que par des sentiments d'obligation. Par conséquent, partageant les mêmes envies, les mêmes aspirations et des intérêts communs, les conflits et les tensions entre les différents membres y sont plutôt rares. Comme pour l'Ambivalence, ce sont surtout des individus âgés pourvus de ressources qui développent la Solidarité au sein de leur configuration familiale. Ce sont en effet des hommes, suisses, qui ont un revenu moyen et qui ont une bonne santé fonctionnelle. Bien que sans enfant, ils ont un bon réservoir de parenté incluant un partenaire et des membres de la fratrie. De telles ressources leur sont nécessaires pour entretenir les échanges au sein de leur configuration familiale. Elles sont d'autant plus importantes que les aînés dans les configurations «Fratrie » y occupent une position centrale et sont particulièrement investis dans les liens de soutien avec les autres membres de leur configuration. Jouant le rôle d'intermédiaire dans les échanges, ils peuvent, autonomes et grâce aux ressources dont ils disposent, alimenter et contrôler le flux de soutien au sein de leur configuration tout en se désengageant des relations qui ne leur apportent plus de satisfaction. C'est par cet engagement énergique qu'ils gèrent l'ambivalence; ils préservent leur autonomie tout en entretenant activement le soutien au sein de leur configuration familiale.

La Captivation s'associe aux configurations familiales composées d'enfants et de petits-enfants, telles que les types «Fille» et «Fils», et touche surtout les individus âgés ayant peu de ressources comme les 
femmes, ceux qui sont sans partenaire (veufs ou divorcés), les aînés d'origine étrangère, ceux qui ont un bas revenu ou encore les individus dont la santé fonctionnelle est mauvaise. Les tensions sont loin d'être rares et s'expliquent par le fort degré d'interdépendance qui caractérise les liens intergénérationnels dans ce type de configurations familiales. Comme pour les configurations de type "Conjugal», les relations conflictuelles ne peuvent pas être rompues en raison du réseau complexe de liens d'interdépendance et des obligations familiales qui les sous-tendent. Cependant, et contrairement aux configurations familiales de type «Conjugal», les ressources manquent et ne suffisent pas à combler tous les besoins des membres de la configuration familiale. Sans ressources, l'aîné ne peut pas s'engager dans les échanges mais plus encore il dépend de l'aide familiale, ce qui tend à déséquilibrer encore davantage les liens de soutien au sein de sa configuration. Les membres prestataires, les enfants généralement, se sentent obligés de répondre aux besoins de soutien du parent âgé et, surchargés, ils perdent peu à peu leur autonomie. Dans un tel contexte, l'ambivalence est difficilement gérable et négociable, le manque de ressources rendant les arrangements difficiles voire impossibles. Dès lors, les liens de soutien s'affaiblissent peu à peu, grevés par les frustrations et les tensions.

Finalement, l'Atomisation se développe dans les configurations familiales composées de liens familiaux affinitaires comme ceux entretenus avec des membres de la parenté élargie ou avec des amis ("Parenté » ou «Isolés »). Comme pour la Solidarité, les liens « choisis» sont généralement positifs, gratifiants et les conflits y sont rares. Cependant, contrairement à la Solidarité, l'Atomisation concerne plutôt des individus âgés ayant peu de ressources. Il s'agit généralement de femmes, d'individus d'origine étrangère, ayant un bas revenu, ayant une mauvaise santé fonctionnelle et disposant d'un faible réservoir de parenté. Ces aînés n'ont en effet ni partenaire, ni enfants, ni frères ou sœurs vivants. Compte tenu de la composition de ce type de configurations, le maintien des liens ne dépend pas d'obligations familiales mais plutôt d'échanges engagés, équilibrés et réciproques visant une satisfaction commune. Or, avec le manque de ressources, cet investissement devient de plus en plus difficile. Les individus âgés dans cette situation ne peuvent pas ou plus s'engager avec les membres de leur configuration et se retirent peu à peu des échanges, ayant perdu leur capacité à la réciprocité avec la baisse de leurs ressources. 
N'étant plus activement entretenus par les individus âgés, les liens de soutien se fragilisent au point que les membres de la configuration, n'étant tenus par aucune obligation familiale, préfèrent se désengager lorsque les besoins de soutien du parent âgé se font plus saillants et, ce faisant, ils préservent ainsi leur autonomie. L'ambivalence structurelle est donc gérée par le désengagement tant de l'individu âgé que par celui des membres de sa configuration familiale.

L'analyse révèle aussi des inégalités de genre quant à la gestion de l'ambivalence structurelle. Les femmes sont en effet plus enclines que les hommes à être confrontées à la Captivation lorsque leur configuration familiale est composée de liens intergénérationnels ou à l'Atomisation lorsque les membres familiaux qu'elles citent comme significatifs sont majoritairement de type affinitaire. Ce résultat peut surprendre puisque les femmes sont perçues dans la littérature scientifique comme ayant une propension plus marquée que les hommes à entretenir les liens de soutien au sein de leur famille en raison de leur rôle de "kinkeeper» (cf. chapitre « Facteurs explicatifs »). Les femmes âgées, par ailleurs, sont décrites dans de nombreux travaux gérontologiques comme davantage entourées et soutenues par leur entourage. Toutefois, aux dernières étapes de la vie, certaines d'entre elles sont plus vulnérables, soumises à davantage de pertes que les hommes. En moyenne, elles sont en effet plus souvent veuves, ont un revenu plus bas et sont davantage atteintes dans leur santé fonctionnelle que les hommes. Ces événements, souvent concomitants, non seulement déséquilibrent les échanges familiaux et accentuent l'ambivalence à laquelle les femmes âgées sont confrontées mais ils restreignent aussi les ressources dont elles disposent pour y faire face. Certaines d'entre elles n'ont donc plus suffisamment de ressources pour soutenir activement les échanges, pour maintenir leur centralité au sein de leur configuration familiale et pour préserver le capital social de type pont dont elles bénéficient généralement. Les résultats de cette analyse vérifient l'hypothèse « H9 », selon laquelle certaines femmes âgées ont un risque plus grand que les hommes de se trouver totalement démunies face à la gestion de l'ambivalence structurelle au sein de leur configuration familiale.

Ces résultats attestent que l'émergence de ces quatre modes de conflit et de support varie non seulement en fonction de la composition des configurations familiales mais aussi en fonction des ressources dont les 
individus âgés disposent aux dernières étapes de leur vie, telles que le revenu, la santé et les réserves démographiques. Ces résultats vérifient les hypothèses « $\mathrm{H} 6$ » et « $\mathrm{H} 7$ » (cf. chapitre « Problématique et hypothèses »). Plus encore, l'interrelation de la composition des configurations familiales et des ressources des individus âgés donne lieu à différentes gestions de l'ambivalence structurelle qui sont très inégales quant aux conséquences qu'elles produisent au sein des familles en termes de soutien et de conflit, ce qui confirme l'hypothèse «H8 ». Les individus âgés ne sont donc pas tous égaux face à la gestion de l'ambivalence au niveau configurationnel. A cet égard, l'analyse met particulièrement en évidence le cas des femmes âgées. Celles-ci ont un risque plus grand que les hommes d'être totalement démunies face à la gestion de l'ambivalence structurelle au sein de leur configuration familiale, résultat qui vérifie l'hypothèse « $\mathrm{H} 9$ ».

\subsubsection{Illustrations de modes de conflit et de support}

Afin de mieux mettre en lumière cette inégalité, nous proposons dans la sous-section suivante de présenter les situations de quatre individus âgés, deux hommes et deux femmes, qui illustrent bien les conditions d'émergence de ces quatre modes de conflit et de support. Ces quatre cas dépeignent les gestions différentes de l'ambivalence structurelle en fonction de la composition des configurations familiales et des ressources dont disposent les aînés et les membres de leur configuration. Ces exemples sont assez éloquents quant à l'influence de la position sociale et du parcours de vie sur la qualité des ressources aux dernières étapes de la vie et l'impact de ces dernières sur la gestion de l'ambivalence structurelle au niveau familial. Pour chaque situation, nous présentons d'abord le profil de l'individu âgé et son parcours de vie. Nous décrivons ensuite les liens de soutien et de conflit entre les différents membres de sa configuration. Et, sur la base de ces diverses informations, nous mettons en exergue la façon dont l'individu âgé et les membres de sa famille gèrent l'ambivalence à laquelle ils sont confrontés. Le premier cas présenté est celui d'Henri dont les relations familiales peuvent être qualifiées d'ambivalentes. Ensuite, est décrite la situation de Marie, en prise avec la Captivation au sein de sa famille. Puis, nous exposons l'exemple de Roberto qui est actif dans la Solidarité et, finalement, le cas de Simone qui illustre particulièrement bien l'Atomisation. 


\section{Ambivalence}

Henri a depuis peu dépassé le cap des nonante ans et, malgré son âge avancé, il ne souffre d'aucune incapacité fonctionnelle. Marié, il vit avec son épouse dans une grande maison dont il est le propriétaire. Il dispose d'un revenu élevé, ayant fait carrière en tant que fonctionnaire dans une organisation internationale à Genève. Après un premier mariage sans enfant, Henri a épousé sa femme actuelle avec qui il a eu deux fils qui vivent à Genève. Son fils aîné, marié, a trois enfants alors que son fils cadet est célibataire et sans enfant. Lors de l'entretien, Henri cite comme membres significatifs de sa famille, son épouse et ses deux fils. Il ne mentionne pas ses petits-enfants, ni l'épouse de son fils, ni sa sœur, pourtant encore en vie. $\mathrm{Au}$ vu de sa composition, sa configuration familiale est de type «Conjugal ». Lors de l'entretien, Henri décrit les relations de soutien au sein de sa configuration comme assez denses et réciproques. Comme le révèle la figure $6.3^{2}$, l'épouse d'Henri est centrale dans la dynamique des échanges de soutien au sein de la famille. En effet, elle donne ${ }^{3}$ du soutien à tous les membres de la famille bien qu'elle n'en reçoive en retour que de la part d'Henri. Quant à Henri, il échange du soutien avec son épouse et son fils cadet qui, célibataire et sans charge de famille, s'investit davantage dans la relation avec ses parents que son frère aîné. Ce dernier est moins intégré dans les échanges puisqu'il ne donne aucun soutien et n'en reçoit que de sa mère. Concernant les relations de conflit, Henri se perçoit comme la principale source de tensions, comme le montre la figure 6.3. Il énerve ${ }^{4}$ en effet tous les membres de sa configuration alors que seul son fils aîné l'énerve en retour. Quant aux autres membres de la famille, ils n'entretiennent aucun lien de conflit entre eux, ce qui souligne d'autant plus la position centrale d'Henri dans les relations de conflit et d'ambivalence au sein de sa configuration familiale.

Henri est un homme socialement privilégié; il est marié, il a un bon revenu et est indépendant du point de vue de sa santé fonctionnelle. Il a les

Les tracés des réseaux ont été effectués sous $\mathrm{R}$ ( $\mathrm{R}$ Core Team, 2015) à l'aide du package spnet (Rousseaux et al., 2014).

3 Les pointes de flèches désignent les membres de la famille qui sont source de soutien, c'est-à-dire ceux qui donnent du soutien.

4 Les pointes de flèches se dirigent vers les membres de la famille qui sont source de tensions, ceux qui énervent ou qui agacent. 
Fig. 6.3: L'Ambivalence dans la configuration familiale d'Henri.

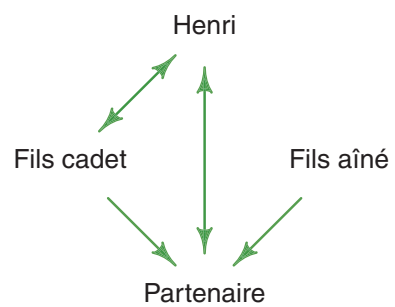

(a) La dynamique du soutien émotionnel dans la famille d'Henri; densité $=0.50$; réciprocité $=0.50$

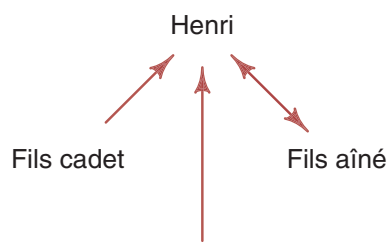

Partenaire

(b) La dynamique du conflit dans la famille d'Henri; densité $=0.33$; réciprocité $=0.33$

ressources suffisantes pour maintenir les échanges au sein de sa configuration familiale et y jouer un rôle actif. Une de ses ressources majeures est sans nul doute son épouse, plus jeune. Elle joue un rôle central dans la dynamique du soutien au sein de la famille, dont bénéficie Henri, puisqu'elle procure du soutien à chacun de ses membres. Ce faisant, elle assure la cohésion familiale en reliant ensemble tous les membres de la configuration grâce au soutien qu'elle déploie. Les ressources dont dispose Henri lui permettent non seulement de maintenir les échanges de soutien - son épouse y veille - mais aussi de négocier activement au sein de sa configuration familiale, ce qui pourrait expliquer sa position centrale dans la dynamique du conflit et sa relation tendue avec son fils aîné. Bien que très âgé, il a en effet encore du poids dans les décisions et les négociations, ce qui peut créer certaines frictions au sein de sa configuration. Bien que source de tensions, ces négociations sont nécessaires pour trouver des arrangements et gérer au mieux l'ambivalence structurelle au sein de la configuration familiale. Le cas d'Henri est une bonne illustration des conditions d'émergence de l'Ambivalence qui se manifeste par de fortes densités de soutien et de conflit au sein de la configuration familiale.

\section{Solidarité}

D'origine italienne, Roberto est venu en Suisse, à la fin de son adolescence, pour travailler comme maçon afin de subvenir aux besoins économiques de sa famille restée en Italie. Depuis peu à la retraite, il reçoit une faible pension et habite seul dans un petit appartement qu'il loue. Ayant construit 
Fig. 6.4: La Solidarité dans la configuration familiale de Roberto.

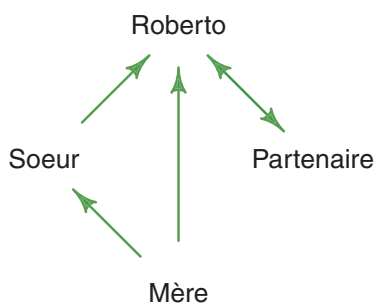

(a) La dynamique du soutien émotionnel dans la famille de Roberto; densité $=0.42$; réciprocité $=0.25$
Roberto

Soeur Partenaire

Mère

(b) La dynamique du conflit dans la famille de Roberto ; densité $=0.00$; réciprocité $=0.00$

une maison dans son pays d'origine, il espère s'y installer dans un avenir proche. Sa pension lui permettra d'y vivre confortablement. Bien qu'il ait exercé un métier exigeant physiquement, Roberto ne souffre pas de problèmes de santé. Roberto n'a jamais été marié et n'a pas d'enfant. Il a cependant une partenaire de longue date, avec qui il ne vit pas. Il a toujours sa mère et quatre frères et sœurs qui résident en Italie. Bien que sans enfant, Roberto dispose d'un riche réservoir de parenté incluant une partenaire, des frères et sœurs, et sa mère. Comme membres significatifs de sa famille, il cite, en premier lieu, sa mère, sa sœur cadette et, finalement, sa partenaire. Sa configuration familiale appartient dès lors au type "Fratrie». Concernant les liens de soutien au sein de sa famille, la figure 6.4 montre que Roberto est particulièrement engagé dans les échanges : il donne du soutien à tous les membres de sa configuration bien qu'il n'en reçoive que de sa partenaire. Quant à sa sœur, elle ne donne du soutien qu'à sa mère. L'engagement de Roberto est tel qu'il occupe une position centrale au sein de sa configuration familiale, reliant sa partenaire à sa mère et à sa sœur qui, sans lui, ne seraient pas connectées. Grâce à son engagement dans sa famille, la densité de soutien est élevée mais la réciprocité relativement faible. Quant au conflit, Roberto ne signale aucune tension entre les membres de sa configuration familiale.

L'investissement affectif dont fait preuve Roberto dans sa configuration familiale reflète le fort engagement qu'il a eu tout au long de sa vie pour sa famille. Son parcours de vie met en évidence sa volonté de préserver sa famille d'origine et d'y promouvoir la cohésion. Pour mener à bien 
son projet, Roberto a investi la plupart de ses ressources, comme de l'argent, du temps et de l'énergie, dans le maintien des liens avec sa famille restée en Italie. Adolescent, il est venu en Suisse pour soutenir financièrement les siens et a maintenu, au fil des ans, un contact régulier avec sa fratrie. Il a même acheté une maison en Italie pour y passer sa retraite et se rapprocher des membres de sa famille. Motivé par son projet familial et ayant les ressources nécessaires - homme en bonne santé et ayant certains avantages matériels (maison et pension) - il participe activement aux échanges de soutien au sein de sa configuration familiale au point même d'y jouer un rôle central. Sa position d'intermédiaire lui permet de soutenir activement les liens de soutien au sein de sa famille, tout en tenant à l'écart sa partenaire du reste de sa configuration afin d'éviter l'émergence de jalousies et de tensions. Autrement dit, Roberto, qui aspire au maintien de la cohésion familiale, gère l'ambivalence au sein de sa configuration familiale en s'investissant de manière active avec les autres membres de sa famille, comme l'exprime la forte densité de soutien au sein de sa configuration, tout en contrôlant efficacement le développement de liens potentiellement conflictuels comme en témoigne la faible densité de conflit au sein de sa famille. L'histoire de Roberto illustre bien l'émergence de la Solidarité au sein de configurations familiales.

\section{Captivation}

Marie, la septantaine, est veuve et n'a pas de partenaire. Elle vit seule dans un petit appartement qu'elle loue à Genève. Sans formation et ayant perdu son mari alors qu'elle était encore jeune, elle a toujours vécu de petits boulots jusqu'à la retraite pour subvenir aux besoins de ses deux filles. De ce fait, elle touche une faible pension. Souffrant de rhumatisme, elle peine à monter et à descendre les escaliers et reçoit régulièrement la visite d'une infirmière. Marie a un riche réservoir de parenté puisque sa fille aînée a une fille et un fils ainsi qu'un petit-fils. Marie est donc arrière-grand-mère. Sa fille cadette, par contre, n'a pas d'enfant. Outre sa descendance, elle a encore des frères ou des sœurs vivants. Lors de l'interview, Marie mentionne ses filles et sa descendance comme faisant partie de sa famille significative citant, en premier lieu, son arrière-petit-fils, son petit-fils, sa petite-fille, sa fille cadette et, finalement, sa fille aînée. Reposant sur des liens intergénérationnels, la configuration familiale de Marie appartient au type «Fille». Marie rapporte que les liens de soutien au sein de sa famille sont relativement peu denses et peu réciproques. Comme le montre la figure 6.5, 
Fig. 6.5: La Captivation dans la configuration familiale de Marie.

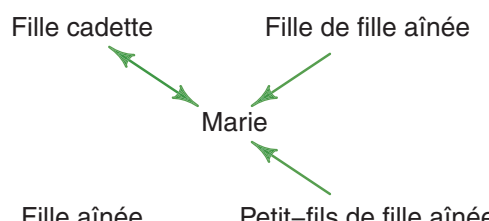

Fils de fille aînée

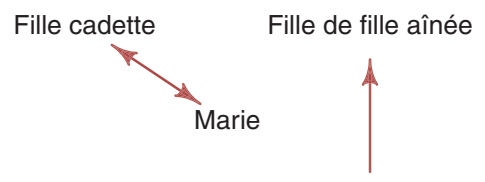

Fille aînée

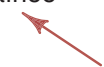

Fils de fille aînée

\begin{abstract}
(a) La dynamique du soutien émotionnel dans la famille de Marie; densité $=0.13$; réciprocité $=0.33$
\end{abstract}

\begin{abstract}
(b) La dynamique du conflit dans la famille de Marie; densité $=0.13$; réciprocité $=0.33$
\end{abstract}

Marie se considère comme la personne la plus active au sein de son réseau familial en procurant du soutien à sa fille cadette, à sa petite-fille ainsi qu'à son arrière-petit-fils. Cependant, seule sa fille cadette lui rend le soutien qu'elle pourvoit. Marie est centrale puisqu'elle relie ensemble les autres membres de sa configuration familiale par le soutien qu'elle distribue. Toutefois, même si elle est active, elle ne parvient pas à connecter tous les membres : deux d'entre eux - sa fille aînée et son petit-fils - sont isolés puisque ni l'un ni l'autre ne donne ni ne reçoit du soutien au sein de la configuration familiale. Quant aux tensions (cf. 6.5), elles restent confinées au niveau dyadique, intergénérationnel. Selon Marie, sa fille aînée agace son propre fils (le petit-fils de Marie). De même, la petite-fille de Marie énerve son propre enfant (arrière-petit-fils de Marie). Quant à la troisième relation intergénérationnelle conflictuelle, elle implique Marie et sa fille cadette. Dans ce cas, la tension est réciproque, chacune énervant l'autre. Ceci dit, comme toutes les deux s'échangent également du soutien, cette relation peut être qualifiée d'ambivalente. Bien que ces tensions ne se répandent pas dans l'ensemble de la configuration par le biais de multiples connexions, elles n'en demeurent pas moins importantes, la densité du conflit mesurée étant relativement élevée.

Les tensions et le faible soutien qui caractérisent cette configuration familiale peuvent s'expliquer à la fois par les liens intergénérationnels qui la composent et par le manque de ressources qui touche Marie et sa famille, fruit d'un parcours de vie chaotique. Devenue veuve tôt dans son parcours de vie, sans formation professionnelle, Marie a dû subvenir seule 


\section{Capital social et ambivalence}

aux besoins économiques de sa famille en cumulant des jobs à bas revenus. Le stress occasionné par sa condition de veuve avec des enfants et le manque chronique de ressources, lié à son instabilité professionnelle, ont contribué à réduire la capacité de Marie à promouvoir les échanges de soutien au sein de sa famille. En somme, elle n'a pas pu construire au sein de sa configuration un solide capital social. Son veuvage prématuré a donc eu un impact important non seulement sur sa trajectoire de vie mais aussi sur l'organisation des liens de soutien au sein de sa famille. Aujourd'hui, elle semble en payer le prix puisqu'elle ne reçoit du soutien que de sa fille cadette. Ayant peu de ressources, Marie ne peut pas compter sur le soutien des membres de sa configuration familiale, et cela d'autant plus que ses deux filles, divorcées et professionnellement inactives, sont aussi dans une situation difficile. Marie mentionne même qu'elle aide financièrement certains membres de sa famille, situation peu aisée pour cette dernière qui reçoit une faible pension. Dans le cas de Marie, l'ambivalence est particulièrement forte, générée à la fois par son propre besoin de soutien mais aussi par les demandes d'aide financière de ses enfants et petits-enfants auxquelles elle se sent «obligée» de répondre. En raison de la forte interdépendance qui sous-tend cette configuration et le manque chronique de ressources, les relations sont tendues et peu soutenantes. La gestion de l'ambivalence structurelle par le biais de négociations ou d'arrangements semble impossible, chaque membre cherchant à «survivre » comme il le peut. La configuration familiale de Marie représente un bon exemple de Captivation comme mode de conflit et de support.

\section{Atomisation}

Suisse d'origine, Simone a franchi depuis peu le cap du quatrième âge et touche actuellement une faible pension. Sans formation professionnelle, elle a toujours travaillé à plein temps comme vendeuse dans divers magasins et a acheté un kiosque à journaux une quinzaine d'années avant sa retraite. Simone a quelques difficultés à se déplacer et a été placée en institution quelques mois avant l'interview. Simone est célibataire, elle n'a pas de partenaire et n'a pas d'enfants. Elle a une sœur plus âgée et une nièce avec lesquelles elle a des contacts hebdomadaires (téléphones). Sa nièce lui fait parfois les courses. Elle a aussi quelques amis qui lui téléphonent ou lui rendent visite régulièrement. Pourtant, Simone ne cite personne comme 
Fig. 6.6: L'Atomisation dans la configuration familiale de Simone.

Simone

(a) La dynamique du soutien émotionnel dans la famille de Simone; densité $=0.00$; réciprocité $=0.00$
Simone

(b) La dynamique du conflit dans la famille de Simone; densité $=0.00$; réciprocité $=0.00$

membre significatif de sa famille lors de l'entretien (Figure 6.6). Par conséquent, sa configuration familiale est de type "Isolés» et l'Atomisation reflète clairement l'absence de tout lien significatif au sein de sa famille.

L'analyse du parcours de vie de Simone, par le biais du calendrier de vie et les étapes marquantes de sa vie qu'elle a évoquées au cours de l'entretien, montre que des événements durant l'enfance ont pu l'empêcher de s'investir dans des relations significatives. Autrement dit, l'absence de membres significatifs dans sa configuration familiale ne semble pas être le résultat d'un choix personnel. Simone a perdu sa mère lorsqu'elle était enfant et a été ballottée d'une institution à l'autre, son père ne pouvant pas s'occuper d'elle. Aujourd'hui, Simone semble avoir des difficultés à développer des relations significatives avec les autres. Durant l'entretien, Simone signale en effet qu'elle fait rarement confiance aux autres. De plus, elle se montre très réticente à évoquer ses relations affectives, refusant de répondre aux questions concernant sa vie amoureuse (cf. remarques de l'enquêteur dans le feuillet d'entretien). Son placement dans plusieurs institutions durant l'enfance pourrait en partie expliquer ces difficultés (Dumaret, Coppel-Batsch, \& Couraud, 1997). Cet événement a eu, semblet-il, des répercussions tout au long de son parcours de vie et, plus encore, aux dernières étapes de sa vie puisqu'elle ne perçoit aucun membre significatif dans sa famille, ce qui la prive de tout soutien émotionnel mais la protège aussi des conflits et de l'ambivalence. Son «non engagement» dans des relations significatives et l'Atomisation qu'il produit peuvent être interprétés comme une gestion "par évitement» de l'ambivalence structurelle.

Les cas d'Henri, de Roberto, de Marie et de Simone illustrent bien comment l'interrelation entre la composition des configurations familiales 


\section{Capital social et ambivalence}

et les ressources à disposition influe sur l'émergence des quatre modes de conflit et de support au sein des configurations familiales, conformément à l'hypothèse « $\mathrm{H} 8$ ». Ces exemples montrent à quel point le parcours de vie des individus et les événements qui le ponctuent ont un impact non seulement sur la composition des configurations familiales dans la vieillesse mais aussi sur le montant et la qualité des ressources dont disposent les aînés pour faire face à l'ambivalence au sein de leur configuration familiale. A ce titre, les situations exposées révèlent de fortes inégalités entre les individus dans la gestion de l'ambivalence et dans ses conséquences en termes de soutien et de conflit dans les relations familiales.

Henri, par exemple, a une épouse plus jeune que lui. Elle constitue pour lui une ressource indispensable non seulement pour maintenir des liens de soutien au sein de sa configuration familiale mais aussi pour lui garantir une certaine autonomie par rapport à l'aide de ses enfants. Ce faisant, il peut négocier plus facilement les tensions et l'ambivalence inhérentes aux relations intergénérationnelles. En revanche, le manque de ressources engendré par un parcours de vie difficile, comme celui de Marie, peut avoir un effet délétère sur les échanges de soutien au sein de la famille et conduire à de fortes tensions dans la vieillesse. Celles-ci sont encore plus prononcées lorsque cette précarité touche aussi les autres membres de la configuration comme les filles de Marie qui connaissent des problèmes financiers dus au divorce et à l'insécurité économique. Les tensions et le stress généré par une telle situation sont difficilement gérables, et cela d'autant plus que la rupture des liens intergénérationnels n'est pas envisageable. Le parcours de vie de Roberto, orienté par son désir de conserver les liens avec sa famille d'origine vivant en Italie, explique son engagement actuel au sein de sa configuration familiale. Les ressources qu'il a accumulées au cours de sa vie lui permettent d'alimenter les liens de soutien au sein de sa famille et d'y jouer le rôle d'intermédiaire. Quant au «non engagement» de Simone dans des relations familiales, il s'explique aussi par le parcours de vie difficile qu'elle a connu, marqué dès son plus jeune âge par la perte de liens significatifs due au décès de sa mère et à l'abandon de son père. En conséquence de son «non engagement», elle n'a pas enrichi son réservoir de parenté puisqu'elle ne s'est jamais mariée et n'a pas eu d'enfants. Ce faisant, elle s'est certes préservée de déceptions affectives mais elle se retrouve seule, sans soutien, aux dernières étapes de sa vie. Ces différents cas soulignent que la gestion inégale de l'ambivalence structurelle dans la vieillesse dépend des ressources accumulées au cours du parcours de vie 
et des transitions de vie traversées telles que le mariage, la parentalité ou la grand-parentalité. Ces quatre différents modes de conflit et de support s'inscrivent donc dans le parcours de vie des individus âgés et dans celui des membres de leur famille.

\subsubsection{Conclusion sur les conflits et l'ambivalence}

L'ambivalence structurelle n'est pas seulement dyadique, intergénérationnelle, mais elle s'inscrit aussi dans l'ensemble des liens d'interdépendance qui sous-tendent les configurations familiales. L'étude de sa gestion au quotidien doit donc sortir du cadre dyadique et prendre en compte un niveau plus large de l'analyse, celui de la configuration familiale. Suivant la perspective configurationnelle, nous avons calculé les densités de soutien et de conflit dans chaque configuration et nous les avons combinées pour former quatre modes de conflit et de support : l'Ambivalence, la Solidarité, la Captivation et l'Atomisation. Ces différents modes reflètent les différentes gestions de l'ambivalence structurelle au niveau configurationnel (Widmer, 2016). Nous avons ensuite cherché à en identifier les conditions d'émergence afin de mettre à jour les inégalités sociales quant à la gestion de l'ambivalence structurelle dans la vieillesse. Les hypothèses précédemment faites portent principalement sur deux facteurs, la composition des configurations familiales (« H6 ») et les ressources des aînés (« H7 »). Les résultats obtenus confirment l'impact de ces deux facteurs sur l'émergence des quatre modes de conflit et de support au sein des configurations familiales dans la vieillesse.

En effet, conformément à l'hypothèse « $\mathrm{H} 6$ », les configurations familiales basées sur des liens intergénérationnels ou conjugaux s'associent, en raison du fort degré d'interdépendance qui les caractérise, à l'Ambivalence ou à la Captivation, modes de gestion donnant lieu à de fortes tensions alors que celles composées de liens affinitaires développent plutôt des modes de gestion peu conflictuels tels que la Solidarité ou l'Atomisation. Dans la première situation, l'ambivalence se doit d'être négociée entre les différents membres de la configuration familiale qui, tenus par des liens de dépendance et soumis à de fortes pressions normatives, sont particulièrement soucieux de défendre leur autonomie. Ces négociations sont souvent source de stress et de tensions entre les différents membres de la configuration. A l'inverse, les tensions sont faibles dans les configurations familiales 
composées de liens affinitaires, la rupture étant plus facile dans ce type de configurations. Les membres, étant moins liés par de fortes obligations familiales, se sentent moins dépendants les uns des autres et peuvent, lorsque leur autonomie est menacée, se délester des relations trop contraignantes. Autrement dit, ils réduisent l'ambivalence structurelle en se désengageant de leurs relations qu'ils jugent trop exigeantes ou conflictuelles. Ensuite, les ressources des individus âgés, en termes de revenu, de santé et de réserves démographiques, ont aussi un impact sur la façon dont l'ambivalence est gérée au sein de leur configuration familiale. Elles permettent aux aînés de préserver leur capacité à la réciprocité, de s'engager activement dans les échanges de soutien et d'y stimuler le flux au sein de leur famille. Ce résultat vérifie donc l'hypothèse « $\mathrm{H} 7$ », selon laquelle les individus âgés ayant des ressources connaissent dans leur configuration des modes axés sur le soutien comme l'Ambivalence ou la Solidarité alors que ceux qui n'ont pas de ressources, c'est-à-dire ceux qui ont un bas revenu, une mauvaise santé ou qui n'ont pas de partenaire, ont une gestion de l'ambivalence difficile, exempte de soutien, expérimentant plutôt la Captivation ou l'Atomisation au sein de leur configuration familiale.

Les analyses attestent aussi l'effet concomitant de ces deux facteurs sur l'émergence des quatre modes de conflit et de support. Conformément à l'hypothèse « $\mathrm{H} 8$ », les configurations familiales composées de liens intergénérationnels ou conjugaux s'associent à l'Ambivalence lorsque le parent âgé a des ressources et à la Captivation lorsqu'il n'en a pas. La satisfaction des demandes de soutien au sein des configurations composées d'enfants et de petits-enfants est difficile et source de tensions. Les ressources permettent aux individus âgés de soutenir les échanges de soutien au sein de leur famille et de participer aux négociations avec les membres de leur configuration. Lorsque les ressources sont insuffisantes dans la famille, l'ambivalence est difficilement gérable : sans ressources, le soutien s'épuise, laissant place à la frustration et aux tensions générées par des attentes d'aide qui ne peuvent pas être satisfaites. Dans les configurations familiales axées sur des liens familiaux affinitaires, la Solidarité se développe lorsque l'individu âgé a les ressources suffisantes pour s'engager dans sa configuration familiale et y maintenir activement les échanges. Sans ressources, de tels liens sont difficiles à préserver et peuvent disparaître. Étant peu alimentés et surtout n'étant pas soutenus par des obligations d'aide, les liens de soutien s'essoufflent rapidement 
et laissent place à l'Atomisation. Ces résultats confirment que l'interrelation de ces deux conditions - composition et ressources - donne lieu à différentes gestions de l'ambivalence structurelle qui, selon les processus adoptés (négociation, investissement ou désengagement), sont très inégales quant aux conséquences qu'elles produisent en termes de soutien et de conflit dans les relations familiales. Par conséquent, certains parents âgés sont, selon leur profil socio-démographique et les ressources qu'ils ont à disposition, plus à risque que d'autres d'être confrontés à de sévères conflits familiaux, voire à de la violence, et à l'isolement social. Les aînés ne sont donc pas tous égaux face à l'ambivalence structurelle dans la vieillesse.

Un des exemples les plus flagrants d'inégalité est celui qui prévaut entre hommes et femmes dans la vieillesse. Les résultats vérifient en effet l'hypothèse des inégalités de genre quant à la gestion de l'ambivalence structurelle («H9»). Les femmes âgées sont plus enclines que les hommes à expérimenter au sein de leur configuration la Captivation ou l'Atomisation, modes se caractérisant par une faible densité de soutien. Confrontées dans la vieillesse à davantage de pertes de ressources que les hommes (santé, revenu et partenaire), les femmes ont un risque plus grand de dépendre de leurs proches tout en éprouvant des difficultés à maintenir la réciprocité et les échanges au sein de leur configuration familiale. En raison de leur position sociale désavantagée et de leur espérance de vie plus longue, elles cumulent aux dernières étapes de leur vie de nombreux désavantages qui les placent dans une situation de grande précarité. Ceux-ci contribuent, par un effet cumulatif, à fragiliser les interdépendances familiales dans lesquelles elles s'insèrent. Le processus d'accumulation des (dés)avantages au cours du parcours de vie, qui contribue aux inégalités dans la vieillesse, se confirme aussi dans ces données lorsqu'il est question du genre (Dannefer, 2003). Ainsi, aux dernières étapes de la vie, hommes et femmes sont armé(e)s de manière inégale face à l'ambivalence structurelle qu'ils ou elles doivent gérer au sein de leur configuration familiale.

L'analyse des conditions structurelles - composition et ressources met en évidence les inégalités sociales quant à l'ambivalence et à sa gestion au sein des configurations familiales. La contraction du réseau personnel sur les membres de la famille aux dernières étapes de la vie ne s'accompagne pas d'un nivellement des différences sociales et de l'uniformisation des pratiques familiales. Au contraire, les individus âgés baignent dans des contextes familiaux pluriels, contrastés et marqués par les inégalités sociales. Par ailleurs, les membres significatifs de la famille ne sont pas 
forcément ceux qui sont le plus source de satisfaction émotionnelle, ce qui contredit le modèle de la sélectivité socio-émotionnelle (Carstensen, 1991, 1992). Dans la sphère familiale, la sélection n'est pas un processus qui s'opère librement, elle est contrainte par les interdépendances affectives et pratiques dans lesquelles les individus âgés s'insèrent et qui sont porteuses de tensions et d'ambivalence. Seuls ceux dont les configurations familiales reposent sur un faible degré d'interdépendance peuvent maintenir les relations familiales qui leur sont le plus gratifiantes et se délester des relations conflictuelles mais ce processus exige de l'individu qu'il ait suffisamment de ressources pour maintenir les liens. En l'absence de ces ressources, le choix est contraint, se restreignant à un nombre limité de membres. En somme, les relations familiales «significatives" aux dernières étapes de la vie ne sont pas toujours source de satisfaction émotionnelle; elles se conjuguent aussi avec du conflit, de l'ambivalence et, parfois, du désengagement familial et ce risque est d'autant plus grand en situation de précarité socio-économique. 


\section{Discussion et conclusion}

Dans le premier chapitre de cet ouvrage, nous avons présenté les principaux modèles théoriques qui ont guidé pendant des décennies la recherche en gérontologie sociale sur les relations sociales et familiales. Nous avons souligné que la plupart de ces modèles ont été élaborés pour étudier le support social et son impact sur le bien-être dans la vieillesse. Influencé par ces modèles, un grand pan de la recherche gérontologique a pendant longtemps considéré la famille - et la considère toujours - comme une ressource indispensable et nécessaire, sans laquelle il serait difficile pour l'individu âgé de faire face aux aléas de la vieillesse. S'inspirant de ces modèles dominants, bon nombre de chercheurs anglo-saxons se sont peu questionnés sur « la» famille en tant que telle et ses dynamiques. Centrés sur le soutien, ils n'ont jamais - ou rarement - remis en question la définition de la famille, s'appuyant généralement sur le modèle de la famille nucléaire. En partant de cette définition pré-établie, ces chercheurs ont mesuré « la» famille dans leurs études en recensant les membres familiaux qu'ils considèrent comme les plus importants et, ce faisant, distinguent les individus âgés qui ont «a priori » du soutien familial - c'est-à-dire ceux qui ont un partenaire et des enfants - de ceux qui n'en ont pas, n'ayant ni partenaire ni enfant. Or, cette conception manichéenne est aujourd'hui par trop simpliste pour approcher la famille dans sa pluralité, celle-ci se construisant, dans bien des cas, audelà des liens de sang et d'alliance (Allan, 2001; Smart, 2007; Widmer, 2016). Trop focalisés sur le support social, ces nombreux chercheurs en gérontologie sociale ont tardé à considérer la complexité des formes familiales contemporaines et peinent aujourd'hui à changer d'angle d'analyse et à innover dans leurs approches et leurs méthodes (Silverstein \& Giarrusso, 2010).

Cet ouvrage vise à pallier ce manque. Pour ce faire, nous sommes partis « des » familles et de leurs dynamiques relationnelles plutôt que du soutien pour aller plus loin que les travaux généralement présentés en gérontologie sociale. Nous avons adopté l'approche configurationnelle qui considère la famille au plus près de sa réalité à savoir plurielle, dynamique et complexe. Cette approche définit la famille comme une configuration, c'est-à-dire un réseau complexe d'individus qui, par le biais d'échanges de 


\section{Discussion et conclusion}

ressources multiples, sont interdépendants les uns des autres. Cette perspective se démarque des modèles dominants de la gérontologie sociale sur plusieurs points. D'abord, les membres importants de la famille ne sont pas définis a priori par le chercheur en fonction des rôles de soutien qu'ils sont censés remplir mais ce sont les individus âgés, eux-mêmes, qui désignent les membres significatifs de leur famille, ceux qui comptent vraiment pour eux. Ensuite, ce sont ces liens d'interdépendance, aussi bien positifs que négatifs, qui définissent et délimitent les familles et non une définition «normative » qui uniformise la famille. Dans la plupart des travaux en gérontologie sociale, les liens d'interdépendance qui relient les membres de la famille ne sont jamais étudiés comme si ces derniers étaient totalement indépendants les uns des autres. Les échanges de soutien sont, certes, considérés mais seulement entre l'individu âgé et les "autres », par exemple, entre le parent âgé et l'un de ses enfants ou entre l'individu âgé et un «type » de membres comme les frères et sœurs, ou encore entre le parent âgé et la famille dans son ensemble. Pourtant, comme nous l'avons mis en évidence dans ce travail, l'analyse des liens d'interdépendance entre les différents membres du réseau familial ouvre de nouvelles perspectives de recherche. En tenant compte par exemple de l'organisation particulière du soutien et du conflit dans chaque configuration familiale, il est possible d'y mesurer le capital social, d'en identifier le type et le volume mais aussi de dégager les différentes façons dont les aînés et les membres de leur configuration gèrent les tensions et l'ambivalence au niveau configurationnel. Ni les définitions personnelles de la famille, ni le capital social, ni l'ambivalence structurelle au sein des configurations familiales n'ont jamais fait l'objet d'investigation en gérontologie sociale, du moins dans des études quantitatives. La démarche que nous avons adoptée ainsi que les résultats que nous avons obtenus sont originaux et innovants. Nous allons reprendre ces derniers et en souligner la pertinence pour la recherche en gérontologie sociale.

\section{H1 : Une variété de membres significatifs de la famille}

La première question qui a guidé ce travail a trait à l'identité des membres significatifs de la famille dans la vieillesse. Des données qualitatives ont montré que, face à l'impermanence des liens de sang et à l'incertitude du soutien attendu, certains aînés déploient des mécanismes de re-définition des liens familiaux visant à se modeler une famille qui fait « sens » (Allen et al., 2011; Braithwaite et al., 2010; Mac Rae, 1992; Pahl \& Spencer, 
2004). Ce faisant, certains y intègrent des liens qu'ils ont investis affectivement et qui sortent du cadre de la famille nucléaire. Selon la littérature, ces mécanismes souvent inconscients permettent aux individus âgés qui y recourent de renforcer leur réseau familial en y associant des membres qu'ils perçoivent comme significatifs et sur lesquels ils peuvent compter en cas de nécessité (Allen et al., 2011; Braithwaite et al., 2010; Mac Rae, 1992; Pahl \& Spencer, 2004). Alors que les données qualitatives font état de ce processus, aucune étude quantitative en gérontologie sociale n'en mesure vraiment la portée ni la fréquence. Dès lors, nous avons fait l'hypothèse qu'à l'instar des populations plus jeunes (Widmer, 2006, 2016; Widmer \& Jallinoja, 2008), les individus âgés désignent comme membres significatifs de la famille une variété de personnes qui s'inscrivent bien au-delà des liens de sang et d'alliance ( $« \mathrm{H} 1 »)$.

Les analyses vérifient cette première hypothèse (« $\mathrm{H} 1 »)$ puisqu'elles révèlent que les individus âgés mentionnent comme membres significatifs de leur famille une variété de personnes, puisées aussi bien dans leur réservoir de parenté qu'en dehors. De fait, ils ne s'appuient pas exclusivement sur des critères statutaires pour définir leur famille significative et font preuve d'une certaine sélection lorsqu'il s'agit de désigner les membres importants de leur famille. Le partenaire et les enfants occupent, certes, le haut de la liste des membres significatifs de la famille les plus cités. Le sang et l'alliance demeurent, de prime abord, des principes forts de définition de la famille au sein de la population âgée, mais ils ne sont pas les seuls. Les frères ou les sœurs et, plus encore, les ami(e)s sont souvent cité(e)s comme membres significatifs de la famille; ils se situent même bien avant les petits-enfants et les beaux-enfants. Par conséquent, les relations dites affinitaires comme les liens fraternels, qui relèvent à l'âge adulte davantage du choix que de l'obligation, sont tout aussi prégnantes dans la construction de la famille significative que les liens intergénérationnels. La significativité du lien est importante et qui, plus est, aux dernières étapes de la vie comme le postule le modèle de la sélectivité socio-émotionnelle (Carstensen, 1991, 1992). Ce processus de sélection s'opère même parmi les liens de sang les plus proches puisque les parents âgés ne mentionnent pas « tous » leurs enfants comme significatifs, ils en choisissent certains parmi d'autres et, parfois, une minorité certes, ils ne les désignent même pas comme faisant partie de leur famille significative. La seule existence d'enfants ne garantit pas leur inclusion dans le contexte familial significatif. Les liens de sang, même parmi les plus proches, ne sont pas tous 


\section{Discussion et conclusion}

investis de la même manière : certains sont maintenus par un attachement affectif profond alors que d'autres sont moins investis, et même parfois désengagés.

La famille n'est pas une entité figée, elle se développe en fonction des réserves démographiques des aînés, desquelles ils puisent les relations familiales qu'ils jugent significatives, mais la famille évolue aussi en fonction des rencontres que les individus font au cours de leur parcours de vie. Selon le contexte et les circonstances, ces rencontres peuvent donner lieu à des relations investies, durables, qui peuvent être ensuite intégrées au sein de leur famille significative. C'est particulièrement le cas à Genève qui, parce qu'elle offre une grande palette d'activités sociales, favorise les rencontres et la sociabilité amicale (Baeriswyl, 2016). Les aînés genevois sont en effet $84 \%$ à déclarer avoir au moins un ami proche contre $76 \%$ en Valais (Baeriswyl, 2016). L'accent marqué pour l'amitié à Genève se reporte sur la composition de la famille significative. En effet, comparée à d'autres cantons, Genève affiche la part la plus forte d'ami(e)s considéré(e)s comme des membres significatifs de la famille. Ces résultats remettent en question la pertinence des indicateurs démographiques classiques, axés sur des rôles familiaux pré-définis comme le partenaire, les enfants ou les petitsenfants, et auxquels recourent de nombreux chercheurs en gérontologie sociale pour approcher la famille des personnes âgées. Bien qu'ils soient utiles pour donner un aperçu du réservoir de parenté, ils ne suffisent pas à saisir toute la complexité des liens familiaux significatifs d'aujourd'hui. Les résultats de cette recherche montrent que c'est seulement en demandant aux individus âgés d'énumérer « eux-mêmes » les membres de leur famille significative que l'on appréhende les familles dans la vieillesse et non en se contentant d'une définition préalable qui désigne a priori « qui » fait partie de la famille.

\section{H2 : Une composition plurielle des configurations familiales}

Après avoir identifié les membres significatifs de la famille dans la vieillesse, la deuxième étape de l'analyse a consisté à repérer comment ces derniers s'articulent entre eux pour former une pluralité de configurations familiales, reflets de différentes conceptions de la famille significative. Selon le modèle du convoi, les réseaux personnels évoluent dans leur structure en fonction du parcours de vie des individus, des transitions de vie qu'ils traversent mais aussi en fonction de leur profil socio-démographique. Les individus sont amenés, tout au long de leur parcours de vie, à re-évaluer leur 
réseau personnel afin de s'entourer de personnes qui les aident à s'adapter aux transitions de vie qu'ils traversent. En fonction de leur parcours de vie et de leur profil socio-démographique, les individus présentent dès lors des réseaux personnels fort divers dans leur structure (Antonucci, 2001; Kahn \& Antonucci, 1980; Van Tilburg, 1998; Van Tilburg \& Thomése, 2010). Dans le modèle du convoi et dans les études qui s'y réfèrent, le cœur du réseau personnel, composé généralement des membres «proches» de la famille, demeure stable tout au long du parcours de vie, n'étant pas soumis a priori aux mécanismes de ré-évaluation des relations personnelles. Sur la base de ces travaux, nous avons fait l'hypothèse que les aînés construisent aussi leur famille significative en fonction de leur parcours de vie et de leur profil socio-démographique et que, de ce fait, ils présentent des configurations familiales fort diverses ( $« \mathrm{H} 2 »)$. En posant cette hypothèse, nous nous démarquons du modèle du convoi en supposant la mise en œuvre de mécanismes de ré-évaluation au cœur même du réseau personnel, à savoir au sein même de la famille « dite » proche, supposée stable et homogène.

L'analyse de classification hiérarchique a produit six configurations familiales, traduisant des conceptions distinctes de la famille significative, ce qui vérifie l'hypothèse « $\mathrm{H} 2$ ». A l'instar d'autres groupes d'âge (Widmer, 2016), les aînés développent une diversité de configurations familiales : certains privilégient une définition «normative » de la famille («Conjugal »), d'autres s'axent plutôt sur les liens de sang et la descendance («Fils» et «Fille $)$ alors que d'autres encore y intègrent majoritairement des liens affinitaires («Fratrie », «Parenté » et «Isolés»). La pluralité de ces configurations familiales révèle que les logiques qui sous-tendent la construction des familles significatives sont diverses et ne s'appuient pas exclusivement sur des critères statutaires, même dans la vieillesse.

La configuration familiale «Conjugal» est constituée du partenaire et des enfants du répondant, faisant ainsi écho au modèle «idéal» de la famille «nucléaire». Cette configuration est particulièrement présente chez les individus mariés et ayant un statut socio-économique élevé. Par ailleurs, les membres de la famille qui composent cette configuration sont aussi nombreux à bénéficier d'un statut socio-économique élevé, affichant généralement un niveau de formation supérieur. Ils habitent, pour la plupart, à Genève et ont des contacts réguliers avec le parent âgé. Les configurations «Fils» et «Fille» se construisent autour des enfants et des petits-enfants du répondant. Bien que la logique qui les sous-tend soit similaire, elles présentent pourtant des compositions distinctes. Les configurations «Fils» 


\section{Discussion et conclusion}

sont composées du fils, de l'épouse du fils et de leurs enfants. Ceux-ci ont, pour la plupart, un niveau d'éducation supérieur, sont nombreux à vivre à Genève et à entretenir des contacts réguliers avec l'aîné. Ce sont surtout des veuf(ve)s ou des individus d'origine suisse qui présentent ce type de configurations. Quant aux configurations de type «Fille», elles sont principalement constituées de la fille et des enfants de la fille, majoritairement des petites-filles. Le partenaire de la fille y est rarement mentionné. Les membres de la famille cités sont moins nombreux que les membres des configurations «Fils» à avoir atteint un niveau de formation élevé, à vivre à Genève et à entretenir des contacts réguliers avec le parent âgé. Quant au profil des répondants adoptant ce type de configurations familiales, les résultats produits par les analyses de régression sont à cet égard peu concluants.

Quant aux trois autres configurations familiales, elles se composent de liens affinitaires et, même si elles reposent sur la même logique, elles sont différentes dans leur composition. Les configurations de type «Fratrie » intègrent majoritairement des membres de la fratrie des répondants. Ce sont principalement des célibataires, sans enfants, qui développent ce type de configurations. Plus que l'absence d'enfant en tant que telle, c'est l'engagement affectif dont ils font preuve qui explique la composition de ces configurations. Sans charge de famille, ils se sont investis tout au long de leur vie dans le maintien de liens significatifs avec certains membres de leur fratrie. Les membres qui forment ce type de configurations sont moins nombreux à vivre à Genève et à entretenir des contacts réguliers avec l'aîné que ceux cités dans les configurations de types «Conjugal» ou «Fils». Quant aux configurations de type «Parenté », elles sont composées d'une diversité de membres de la famille, puisés surtout dans le réservoir de parenté, tels que des membres de la parenté élargie ou ceux de la famille du partenaire. Les répondants qui ont ce type de configurations familiales ont généralement un partenaire, sont «non mariés » (veufs, divorcés ou célibataires) et sans enfants. Ils sont de sexe féminin et issus des classes moyennes ou supérieures. Contrairement à la configuration «Fratrie», c'est l'absence d'enfant qui explique l'investissement plus marqué des répondants dans la parenté élargie ou dans la famille du partenaire. Les membres cités vivent plutôt à l'extérieur de Genève et ont peu de contacts réguliers avec l'individu âgé. Finalement, la configuration de type "Isolés» se caractérise soit par l'absence de membres cités soit par la mention d'un nombre limité d'amis. Il s'agit surtout de répondants «non mariés », sans partenaire, sans 
enfants, et ayant un statut socio-économique bas qui développent ce type de configurations. Lorsqu'ils sont cités, les membres de cette configuration sont des amis de longue date, de même génération, vivant à Genève. Ils sont en moyenne aussi nombreux à entretenir des contacts réguliers avec l'individu âgé que ceux qui forment les configurations de type "Fratrie».

Conformément à l'hypothèse « $\mathrm{H} 2$ », ces résultats montrent que le parcours de vie des individus âgés ainsi que celui de leurs "proches" ont un impact sur la composition des configurations familiales en influençant le contenu de leurs réserves démographiques. Certaines transitions de vie comme le mariage, la parentalité ou la grand-parentalité renflouent le réservoir de parenté alors que d'autres transitions, telles que le veuvage ou le divorce, le limitent. Selon l'état de leurs réserves démographiques, certains aînés ont donc plus d'opportunités de développer des relations familiales significatives que d'autres. Cependant, l'état du réservoir de parenté n'est pas le seul facteur à influencer la composition des configurations familiales. L'investissement personnel, affectif, dont font preuve certains aînés, révèle que la composition des configurations familiales n'est pas qu'une question d'opportunités relationnelles. Les célibataires en constituent un bon exemple. Les liens fraternels qui composent leur configuration familiale, généralement de type « Fratrie », reflètent l'engagement sélectif des célibataires dans le maintien de liens affectifs significatifs avec certains membres de leur fratrie. Les femmes aussi, sans enfants, sont actives dans l'entretien des relations significatives avec certains membres de leur parenté élargie ou ceux de leur belle-famille. Quant aux personnes qui n'ont ni partenaire ni enfant, certaines d'entre elles s'investissent dans des liens affectifs en dehors du réservoir de parenté, intégrant des relations amicales de longue date au sein de leur configuration familiale. Ceci dit, cette capacité d'investissement n'est pas donnée à tout le monde; elle dépend du profil socio-démographique des individus âgés et des ressources qu'ils ont à disposition. Les aînés qui ont un statut socio-économique élevé, des hommes pour la plupart, ont les ressources nécessaires, telles que le revenu, des compétences relationnelles et un réseau personnel diversifié, pour retrouver une partenaire et se remarier lorsqu'ils sont veufs ou divorcés. Quant aux femmes, elles ont été socialisées au maintien des liens au sein de la famille et en dehors; elles ont donc une plus forte propension que les hommes à s'investir affectivement dans une variété de relations aussi bien familiales que non familiales. A l'inverse, un manque sévère de ressources aussi bien financières, démographiques que relationnelles prétérite 


\section{Discussion et conclusion}

tout élan d'investissement personnel. C'est notamment le cas des individus âgés qui ne mentionnent personne comme membre significatif de leur famille. La sélection de liens familiaux significatifs n'est donc pas possible pour tous; elle exige certaines ressources, démographiques certes, mais aussi socio-économiques. Le maintien du lien, qui plus est choisi, requiert la réciprocité des échanges et celle-ci nécessite un certain nombre de ressources. L'absence de toutes ressources, notamment financières, rompt la réciprocité des échanges et nuit ainsi au maintien de relations significatives même au sein de la famille (Offer, 2012).

La diversité des configurations familiales, mise à jour dans cet ouvrage, montre que les mécanismes de ré-évaluation des relations personnelles au cours du parcours de vie, qui contribuent à diversifier la structure des réseaux personnels dans la vieillesse, sont aussi actifs au cœur même des réseaux personnels, c'est-à-dire au sein même de la famille. Contrairement à ce que suggère le modèle du convoi, les relations familiales significatives ne sont ni homogènes ni stables au cours du parcours de vie; elles sont aussi soumises aux mêmes mécanismes de ré-évaluation. Les transitions de vie, positives ou négatives, invitent les individus qui les traversent à re-définir sans cesse ce que signifie, pour eux, la famille significative. Par des processus de sélection, d'engagement ou de désengagement, les individus façonnent ainsi leur famille qui «compte» avec les diverses ressources dont ils disposent. Celle-ci évolue donc en fonction du parcours de vie de l'individu et de ses besoins (soutien). La pluralité des configurations familiales atteste non seulement de la vigueur de ces processus mais aussi de la variabilité des trajectoires de vie individuelles et de la disparité des ressources dans la vieillesse. La définition pré-définie et homogène de la famille reposant sur des critères bio-légaux est désormais dépassée. Il est donc temps que la recherche scientifique considère les définitions personnelles de la famille qui vont bien au-delà des liens de sang ou d'alliance, usuellement utilisés pour appréhender la famille en gérontologie sociale.

Alors que la pluralité des familles significatives est généralement négligée en gérontologie sociale, le droit suisse en tient compte comme l'atteste le nouveau droit de la protection des adultes du Code civil suisse ${ }^{5}$ entré en vigueur en 2013. En effet, toute personne, encore capable de discernement, peut désigner volontairement un individu de son choix, appartenant ou non à son réservoir de parenté, qui la représentera lorsqu'elle

https ://www.admin.ch/opc/fr/classified-compilation/19070042/index.html\#a378 
perdra sa capacité de discernement. Il existe dans le nouveau droit deux mesures personnelles anticipées. La première, le «mandat pour cause d'inaptitude », permet à la personne de charger un individu de son choix de certaines tâches d'assistance personnelle, de représentation (actes juridiques) et de gestion (biens, revenus, frais courants et courrier) (Guillod, 2012). La deuxième mesure, les « directives anticipées », concerne le domaine médical, notamment tout ce qui a trait aux traitements médicaux, à l'hospitalisation ou à l'institutionnalisation. Comme précédemment, toute personne peut exprimer ses souhaits dans des directives anticipées quant aux traitements médicaux désirés et désigner « un représentant thérapeutique », faisant partie ou non de son réservoir de parenté, qui la représentera dans toutes les décisions médicales lorsqu'elle ne sera plus en mesure de décider tout seule. Si ces mesures personnelles anticipées répondent de manière adéquate aux besoins de la personne, l'État ne peut pas instituer une mesure de protection; celui-ci intervient seulement si ces conditions ne sont pas remplies (curatelle).

En l'absence d'un «mandat pour cause d'inaptitude», le nouveau droit désigne le conjoint ou le partenaire enregistré comme représentant légal de la personne incapable de discernement (art. $374 \mathrm{CC}$ ). L'ouverture au partenariat enregistré permet la reconnaissance des couples de même sexe. Ceci dit, un concubin ne détient pas légalement un pouvoir de représentation pour les questions de gestion courante sauf s'il a été désigné par la personne dans un mandat pour cause d'inaptitude (Guillod, 2012). Au niveau médical, s'il n'y a ni directives anticipées ni mandat pour cause d'inaptitude, le nouveau droit (art. 378 CC) désigne d'abord le curateur, s'il y a une curatelle de représentation, qui a la tâche de représenter la personne dans le domaine médical. S'il n'y a pas de curateur, le nouveau droit établit un «ordre de priorité légale parmi les proches de la personne incapable de discernement qui lui fournissent une assistance personnelle régulière » (Guillod, 2012, p. 153). Il s'agit, en premier lieu, du conjoint ou du partenaire enregistré s'il fait ménage commun avec la personne ou s'il lui fournit une assistance personnelle régulière, notamment en cas de placement en institution. Le concubin vient en deuxième position, seulement s'il vit avec la personne et qu'il lui fournit une assistance personnelle régulière. Aussi bien le ménage commun que l'assistance régulière sont requis pour que le concubin puisse être désigné comme représentant légal pour les questions médicales. Viennent ensuite les descendants à condition qu'ils fournissent à la personne une assistance personnelle régulière. Sans 


\section{Discussion et conclusion}

descendants, ce sont la mère et le père et, en dernier lieu, les membres de la fratrie à condition que tous fournissent à la personne une assistance personnelle régulière (Guillod, 2012). Si ceux-ci sont absents ou refusent cette charge, l'État nomme un curateur.

Le nouveau droit de protection des adultes tend donc à reconnaître les définitions personnelles de la famille significative en permettant aux personnes de désigner "elles-mêmes » les membres qu'elles perçoivent comme suffisamment " proches », quel que soit leur lien de parenté, pour les représenter légalement lorsqu'elles auront perdu leur capacité de discernement. Ceci dit, lorsqu'aucun individu n'a été désigné au préalable, le nouveau droit détermine les représentants légaux en se basant à la fois sur des critères classiques d'alliance (conjoint ou partenaire enregistré) et de sang (descendants, ascendants ou fratrie) mais aussi en tenant compte, pour les décisions médicales, des pratiques familiales (vie commune pour les concubins) et de l'investissement pratique et affectif des membres familiaux auprès de la personne concernée puisqu'il exige qu'il y ait une assistance personnelle régulière. Ainsi, dans le domaine médical, le seul critère de l'alliance ou du sang ne suffit plus pour désigner les représentants " légaux » des personnes âgées ayant perdu leur capacité de discernement. Il en est cependant autrement pour la gestion du patrimoine. Seul le conjoint légal, marié ou enregistré, peut être institué comme représentant légal si aucune mesure anticipée n'existe. En son absence ou en cas d'incapacité, c'est l'État qui se charge de nommer un curateur.

\section{H3 : Capital social et Composition diversifiée}

Après avoir identifié différentes configurations familiales, nous nous sommes questionnés sur le capital social qu'elles produisent. Le capital social renvoie à l'ensemble des ressources individuelles générées au sein d'un réseau durable de relations personnelles; tout membre du réseau y a accès et peut le mobiliser en cas de besoin (Bourdieu, 1980, 1986). En considérant les familles comme des configurations, à savoir des réseaux de membres significatifs connectés entre eux par des liens d'interdépendance, celles-ci créent du capital social. Or, un tel capital social, d'ordre « familial », n'a jamais fait l'objet de travaux en gérontologie sociale. La notion de «support bank» proposée par Toni Antonucci et son équipe (Antonucci, 2001; Antonucci et al., 2007; Silverstein et al., 2002) est certes très proche mais elle ne caractérise que les liens dyadiques, intergénérationnels. Selon le concept du «support bank», les parents âgés qui ont investi tout au 
long de leur vie de nombreuses ressources dans l'éducation et les soins de leurs enfants - et de leurs petit-enfants - sont certains, en retour de leur investissement, de pouvoir compter sur le soutien de leurs enfants en cas de besoin. Ces fortes attentes vis-à-vis de l'aide des enfants s'expliquent par les normes de réciprocité " généralisée » qui sont particulièrement prégnantes dans les relations intergénérationnelles tout au long du parcours de vie. Ces normes se manifestent par de fortes obligations d'aide et un sentiment de redevabilité à l'égard de l'autre. Les enfants adultes se doivent de rendre à leurs parents âgés tout le soutien qu'ils ont reçu au cours de leur vie. Par leur forte prégnance, de telles normes créent un potentiel de support sur lequel s'appuient les parents dans la vieillesse. Le concept de capital social repose sur la même idée mais va plus loin en considérant le potentiel de soutien à l'échelle du réseau familial; il permet, en somme, d'observer les effets des normes de réciprocité sur l'ensemble des liens familiaux et sortir ainsi du carcan dyadique.

La littérature distingue deux types de capital social : celui de type chaîne, s'associant à une forte densité et à une forte réciprocité des liens de soutien au sein du réseau familial, et celui de type pont qui se caractérise par la centralité et l'engagement de l'individu dans son réseau. Selon l'hypothèse « $\mathrm{H} 3$ », le type de capital social varie en fonction de la composition des configurations familiales. Nous avons en effet postulé que les configurations familiales composées de liens intergénérationnels ou conjugaux produisent du capital social de type chaîne en raison des normes de réciprocité " généralisée » qui les caractérisent et qui renforcent les liens d'interdépendance entre les différents membres du réseau familial. Se manifestant par le biais de fortes obligations familiales, ces normes d'échanges convient les membres de la configuration à s'entraider sous peine d'être en prise avec un fort sentiment de culpabilité si elles sont transgressées. S'inscrivant dans le long terme, ces échanges de soutien procurent ainsi un sentiment diffus de redevabilité qui favorise la solidarité familiale et contribue à resserrer les liens d'interdépendance au sein de la configuration familiale. En raison des fortes connexions qui relient l'ensemble des membres du réseau familial, ce type de capital social est prometteur d'un soutien collectif. Quant aux configurations familiales constituées de liens de type affinitaire, nous avons émis l'hypothèse qu'elles génèrent plutôt du capital social de type pont. Comme les membres dans ce type de configurations proviennent de divers cercles familiaux ou amicaux, les liens de soutien qui les relient sont plus lâches et clairsemés. 


\section{Discussion et conclusion}

N'étant pas soumis à de fortes obligations d'aide, les liens affinitaires s'entretiennent par une envie partagée d'être en lien. Le maintien de ce type de relations exige dès lors un certain investissement personnel de la part de l'individu (ego) et repose plutôt sur une réciprocité « équilibrée » entre l'individu et chacun des membres de sa configuration. Ces normes d'échanges sont plus directes, moins différées dans le temps et plus exigeantes du point de vue de l'équilibre des ressources échangées que celles qui sous-tendent les liens intergénérationnels ou conjugaux. Le soutien obtenu est davantage dyadique que collectif, et de ce fait plus fragile. Le tableau 6.11 résume les principaux résultats concernant le capital social et ses variations selon la composition des configurations familiales.

Les résultats vérifient partiellement l'hypothèse « $\mathrm{H} 3$ ». A l'exception des configurations familiales de type "Fille», dont les résultats seront discutés dans la prochaine section, les liens intergénérationnels et conjugaux génèrent du capital social de type chaîne dans les configurations de types «Conjugal» et «Fils». L'insertion du partenaire et des enfants dans la configuration familiale de type «Conjugal» s'associe à une forte densité et à une forte réciprocité des liens de soutien. Tous les membres y sont étroitement connectés par des échanges mutuels de soutien, laissant peu de place à l'autonomie individuelle comme le signale la faible centralité de

Tab. 6.11: Capital social selon les configurations familiales.

\begin{tabular}{|c|c|c|}
\hline $\begin{array}{l}\text { Configurations } \\
\text { familiales }\end{array}$ & Propriétés structurelles & $\begin{array}{l}\text { Types de capital } \\
\text { social }\end{array}$ \\
\hline Conjugal & $\begin{array}{l}\text { Forte densité, forte réciprocité, faible } \\
\text { engagement, faible centralité }\end{array}$ & Chaîne \\
\hline Fils & $\begin{array}{l}\text { Forte densité, réciprocité moyenne, fort } \\
\text { engagement, centralité moyenne }\end{array}$ & Chaîne \\
\hline Fille & $\begin{array}{l}\text { Faible densité, faible réciprocité, faible } \\
\text { engagement, faible centralité }\end{array}$ & Faible \\
\hline Fratrie & $\begin{array}{l}\text { Densité moyenne, réciprocité moyenne, } \\
\text { fort engagement, forte centralité }\end{array}$ & Pont \\
\hline Parenté & $\begin{array}{l}\text { Faible densité, réciprocité moyenne, } \\
\text { faible engagement, centralité moyenne }\end{array}$ & Faible \\
\hline Isolés & $\begin{array}{l}\text { Très faible densité, très faible } \\
\text { réciprocité, très faible engagement, très } \\
\text { faible centralité }\end{array}$ & Très faible à inexistant \\
\hline
\end{tabular}


l'aîné dans ce type de configurations. Sous le couvert de la solidarité familiale, les normes de réciprocité "généralisée », qui sous-tendent les liens intergénérationnels et conjugaux au cours du temps, renforcent les liens d'interdépendance entre les différents membres significatifs de la famille et contribuent ainsi à produire du capital social de type chaîne dans ce type de configurations familiales.

Il en est de même lorsque les petits-enfants sont cités comme membres significatifs de la famille, comme en témoigne la forte densité des configurations familiales de type «Fils». Leur présence densifie les liens de soutien entre les différents membres de la configuration, en encourageant le partage d'activités communes et en renforçant les échanges de diverses ressources aussi bien émotionnelles que pratiques (garde des enfants, devoirs, etc.) (Bengtson, 2001; Bucx et al., 2008; Silverstein \& Giarrusso, 2010). Dans ce type de configurations, les normes de réciprocité " généralisée » sont particulièrement prégnantes. Soumis à ce type de normes, les échanges sont à la fois directs et indirects : le soutien circule non seulement entre le parent âgé et ses enfants, mais passe aussi par l'aide octroyée aux petits-enfants. Mais, plus encore, la mention des petits-enfants stimule l'investissement du parent âgé dans les échanges de soutien puisque ce dernier rapporte soutenir un grand nombre de membres au sein de sa configuration. Son rôle actif dans les échanges de soutien contribue à consolider son «support bank» et à lui assurer une aide dans le futur comme en témoigne le nombre de membres sur lesquels il déclare pouvoir compter en cas de besoin. De plus, par son engagement actif, le parent âgé occupe une position moyennement centrale au sein des échanges, il parvient donc à préserver une certaine autonomie relationnelle au sein de sa famille, tout en bénéficiant d'un capital social de type " chaîne ». Ainsi, à l'instar de la configuration de type «Conjugal», la configuration de type «Fils» garantit au parent âgé qui s'y insère un soutien familial fiable, solide et collectif.

Contrairement aux configurations précédentes, qui sont composées de membres familiaux fortement reliés les uns aux autres, les configurations formées de liens affinitaires («Fratrie», «Parenté», et «Isolés») rassemblent des membres provenant de cercles familiaux et amicaux divers et sont, de ce fait, peu connectés entre eux. Plus que d'être soutenus par de fortes obligations familiales ou un sentiment de redevabilité, les liens affinitaires sont davantage entretenus par des intérêts communs, une envie d'être ensemble, de partager et d'échanger. Conformément à l'hypothèse « H3 », 


\section{Discussion et conclusion}

les résultats confirment que la composition particulière de ce type de configurations familiales conduit à une organisation plus distendue des liens d'interdépendance et produit davantage de capital social de type "pont» que dans les configurations précédentes. Toutefois, et contrairement à l'hypothèse « $\mathrm{H} 3$ », les configurations familiales basées majoritairement sur des liens affinitaires ne génèrent pas toutes du capital social de type pont; certaines d'entre elles s'associent à un faible capital social, voire à son inexistence.

Dans les configurations de type «Fratrie », l'individu âgé est particulièrement engagé dans la provision de soutien au sein de sa configuration. Il se présente même comme la principale source de soutien auprès des membres de sa configuration qui, sans lui, ne seraient pas connectés. Il occupe donc la position centrale au sein des échanges de soutien. A noter que les frères et, plus encore, les sœurs sont reconnus dans la littérature comme une source importante de soutien émotionnel dans la vieillesse (Campbell et al., 1999; Connidis, 2010; Eriksen \& Gerstel, 2002). Hormis l'engagement particulier de l'individu âgé, les échanges de soutien au sein de sa configuration familiale sont moyennement denses et réciproques. En raison des propriétés structurelles qui le distinguent, ce type de configurations familiales offre à l'aîné un accès privilégié au capital social de type pont, prometteur d'une grande autonomie relationnelle. De fait, il bénéficie d'une certaine marge de manœuvre; il peut s'investir davantage dans certaines relations sans éveiller la jalousie des autres membres et se désinvestir de celles qui ne lui paraissent plus gratifiantes. Toutefois, une telle autonomie relationnelle est exigeante puisque l'individu âgé doit avoir de l'énergie et une bonne santé pour maintenir constant son engagement et préserver sa centralité au sein de son réseau familial. Par ailleurs, le soutien auquel il a accès est, contrairement aux configurations précédentes, plus dyadique que collectif et, de ce fait, plus fragile et instable.

Quant aux configurations familiales de type "Parenté », elles sont composées d'une diversité de membres, puisés généralement dans le réservoir de parenté mais aussi en dehors. Il peut s'agir des membres de la parenté éloignée, de la famille du partenaire ou encore de quelques amis proches. Parce qu'ils proviennent de différents cercles familiaux, ils sont peu connectés entre eux au travers d'échanges de soutien. Les liens y sont, par conséquent, peu denses bien que moyennement réciproques. L'individu âgé est peu actif dans ce type de configurations; il ne donne ni ne reçoit du soutien d'un grand nombre de membres. Il se perçoit, néanmoins, comme 
moyennement central au sein de son réseau, faisant le lien entre certains membres de sa configuration. Compte tenu de ses propriétés structurelles, ce type de configurations familiales produit un faible capital social. Ceci dit, la diversité des membres qui le composent et la centralité moyenne de l'aîné laissent à penser que, si ce dernier s'investissait davantage dans les échanges de soutien, ce type de configurations pourrait générer du capital social de type pont. Cependant, la forte diversité des membres qui caractérise cette configuration exige de l'individu des capacités à la fois physiques et cognitives pour repérer les « trous » structurels et y jouer le rôle d'intermédiaire (Cornwell, 2009b, 2009a). Avec l'âge et le déclin de la santé, de telles capacités s'amenuisent. L'aîné perd donc peu à peu l'accès au capital social de type pont, ce type de configurations devenant trop exigeant avec le temps.

Les configurations familiales de type "Isolés» se caractérisent par l'absence de membres cités ou par la présence d'un nombre restreint d'amis. La faiblesse des scores sur l'ensemble des indices du capital social montre qu'en moyenne, les individus âgés dans ce type de configurations ne bénéficient d'aucun capital social. Alors qu'à d'autres étapes du parcours de vie, les configurations familiales composées d'amis produisent du capital social de type pont (Widmer, 2006, 2016), elles ne génèrent aucun capital social aux dernières étapes de la vie. Ce résultat peut s'expliquer par la nature affinitaire des liens amicaux. Choisis, ceux-ci sont principalement motivés par une envie commune d'être ensemble. Mais, les liens avec les amis, même les plus proches, sont exigeants; ils reposent sur des normes de réciprocité « équilibrée » et doivent être activement entretenus de part et d'autre de la relation. Lorsque les liens avec les amis peinent à être entretenus en raison de problèmes de santé ou lorsque les échanges sont perçus comme déséquilibrés ou physiquement trop contraignants (visites, par exemple), ils se distendent au fil du temps et se rompent. Par ailleurs, le soutien, principalement dyadique dans ce type de configurations, est fragile s'il n'est plus alimenté par l'individu âgé et ses amis. Autrement dit, avec l'avancée en âge et le déclin de leur santé, les aînés, comme leurs amis d'ailleurs, ne parviennent plus à s'investir activement dans les relations amicales et peinent à répondre aux exigences de la réciprocité « équilibrée ». Ils perdent dès lors le capital social de type pont auquel ils avaient accès plus jeunes. Ainsi, la mention d'amis comme principaux « substituts » familiaux dans les configurations de type «Isolés» rend la personne 


\section{Discussion et conclusion}

âgée particulièrement vulnérable; celle-ci risque de ne bénéficier d'aucun soutien en cas de besoin.

La façon dont les individus âgés définissent « leur » famille significative a un impact sur le capital social auquel ils ont accès dans la vieillesse. Cet impact est même plus important que le fait d'avoir des enfants, des petits-enfants, des frères ou des sœurs dans son réservoir de parenté. En effet, l'influence de la composition des configurations familiales sur le capital social reste significative même après avoir inclus dans l'analyse ces diverses réserves démographiques. Or, une majorité de recherches en gérontologie sociale s'appuie toujours sur ces indicateurs démographiques pour estimer le montant et la qualité du soutien familial potentiel. Systématiquement, ces études opposent les personnes âgées ayant un partenaire et des enfants à celles qui n'en ont pas, les premières disposant « a priori » d'un grand soutien familial alors que les dernières n'en ont aucun. Or, approcher le soutien familial de cette manière est trop restrictif puisque l'on ne tient pas compte de la diversité des familles et du potentiel de soutien qu'elles offrent. Dans cet ouvrage, nous avons mis en évidence les différents types et volumes de capital social générés dans les configurations familiales dans la vieillesse. Certaines produisent du capital social de type chaîne, prometteur d'un soutien fiable et collectif alors que d'autres créent du capital social de type pont, garant de l'autonomie relationnelle des individus âgés. D'autres encore ne procurent à l'aîné qu'un faible capital social. En ne se reposant que sur l'état du réservoir de parenté pour évaluer le soutien potentiel, un grand pan de la recherche gérontologique sous-estime la diversité et la complexité du soutien familial auquel accèdent les individus âgés aux dernières étapes de leur vie.

\section{H4 : Capital social et Composition genrée}

Outre le lien de parenté des membres de la famille significative, nous nous sommes interrogés sur l'effet de leur sexe sur la production du capital social au sein des configurations familiales. Comme nous l'avons précédemment relevé, il incombe aux femmes non seulement d'éduquer et de prendre soin des membres de la famille mais aussi de maintenir les liens familiaux interet intragénérationnels afin d'assurer la stabilité et la cohésion familiale (Bowling, 1994; Pinquart \& Sörensen, 2000; Rossi \& Rossi, 1990; Silverstein et al., 2006). Garantes des liens au sein de la famille, elles s'engagent plus que les hommes dans les échanges de soutien et sont plus promptes, ayant été davantage socialisées aux obligations familiales, à répondre aux 
besoins d'aide des membres familiaux qui le sollicitent (Antonucci et al., 2007; Rossi \& Rossi, 1990; Silverstein \& Bengtson, 1997; Silverstein et al., 2006). C'est particulièrement le cas des épouses mais aussi celui des filles adultes qui sont perçues dans la littérature comme les principales prestataires de soins auprès du parenté âgé (Pinquart \& Sörensen, 2006; Silverstein et al., 2006; Suitor et al., 2011; Walker et al., 1995). Au vu de ces différentes observations, nous avons fait l'hypothèse que la présence majoritaire de membres familiaux féminins au sein des configurations familiales, de filles notamment, favorise les échanges de soutien entre les différents membres et contribue ainsi au développement du capital social de type chaîne (« $\mathrm{H} 4 »)$.

Contre toutes attentes, les résultats réfutent cette hypothèse ( « $\mathrm{H} 4 »)$ : les configurations familiales de type «Fille», à composante majoritairement féminine, génèrent moins de capital social chaîne que les configurations familiales de type «Fils». Elles ne produisent, pour ainsi dire, qu'un faible capital social de type chaîne, les liens de soutien étant peu denses et faiblement réciproques alors qu'elles comprennent en moyenne le même nombre de membres significatifs que les configurations familiales de type «Fils». Bien que les répondants dans les configurations de type «Fille» soient moyennement actifs dans la provision du soutien, ils ne bénéficient du soutien que d'un nombre limité de membres. Par ailleurs, ils n'affichent qu'une faible centralité et n'ont donc pas accès au capital social de type pont. En moyenne, les configurations familiales de type «Fille» présentent donc des propriétés structurelles opposées à celles des configurations de type «Fils», ces dernières se caractérisant par des liens de soutien denses et réciproques dans lesquels les répondants, actifs et engagés, reçoivent du soutien autant qu'ils n'en donnent. Comment expliquer que ces deux types de configurations, «Fille » et «Fils», axés tous deux sur les liens de sang et la descendance, donnent lieu à de telles différences?

L'explication se trouve dans la composition, somme toute distincte, de ces deux types de configurations familiales. La partenaire du fils (bellefille) est une composante importante dans les configurations familiales de type «Fils»; elle est même mentionnée juste après le fils et bien avant les petits-enfants. L'analyse des propriétés structurelles dans ce type de configurations révèle que la belle-fille, comme les autres membres d'ailleurs, participe activement aux échanges de soutien et contribue à entretenir la densité et la réciprocité des liens, et à générer ainsi du capital social de type 


\section{Discussion et conclusion}

chaîne. La belle-fille joue donc un rôle crucial : elle permet, lorsqu'elle entretient des relations de bonne qualité avec ses beaux-parents, de maintenir un lien positif entre, d'un côté, le fils et ses enfants et, de l'autre côté, le parent âgé (Fingerman, 2004). Dans les configurations «Fille», la mention de la fille n'appelle pas celle de son partenaire (beau-fils), qui est peu cité, mais plutôt celle des petites-filles. Celles-ci sont, d'ailleurs, bien davantage citées dans les configurations de type «Fille» que dans les configurations de type «Fils ». L'insertion du beau-fils ne semble pas nécessaire, contrairement à celle de la belle-fille, puisque les répondants peuvent compter sur leur fille - et, dans une moindre mesure, sur leur petite-fille - pour obtenir le soutien qu'ils attendent. La fille, désignée socialement pour remplir le rôle d'aidante et offrir du soutien à l'ensemble des membres de la famille (Silverstein et al., 2006; Walker et al., 1995), est, de prime abord, perçue comme largement suffisante pour jouer un tel rôle, ne nécessitant point l'aide de son partenaire (beau-fils).

La composition distincte de ces deux types de configurations a des effets différents sur l'organisation de leurs liens d'interdépendance et l'équilibre de leurs échanges. Dans la configuration de type «Fils», c'est le couple « fils et belle-fille» qui supervise les échanges; il favorise la densité des liens et inclut le parent âgé dans le flux des échanges de soutien. Dans la configuration de type «Fille», la fille est « seule » pour stimuler les échanges et ne parvient pas à y intégrer pleinement le parent âgé. L'insertion des petits-enfants dans la configuration de type "Fille» n'accroît pas la densité des liens de soutien ni même la réciprocité. Leur présence implique, certes, un engagement plus marqué de l'individu âgé, celui-ci donne du soutien mais il n'en reçoit pas plus en retour. Quant à la centralité des répondants, elle est faible dans la configuration «Fille» car c'est la fille qui joue le rôle d'intermédiaire, connectant les petits-enfants aux grand-parents et distribuant le soutien au sein de la configuration familiale. La forte centralité de la fille explique la faiblesse du capital social généré au sein de ce type de configurations familiales. Son rôle comme principale prestataire de soutien peut s'avérer lourd si elle doit répondre, seule, aux besoins du parent âgé ainsi qu'à ceux de ses enfants, ce qui peut à la longue éroder les liens de soutien, tout en nuisant à l'autonomie relationnelle du parent âgé qui peine à trouver sa place dans ce type de configurations. Alors que le fils dans les configurations «Fils» s'appuie sur l'aide de sa partenaire pour entretenir l'équilibre des échanges au sein de sa configuration, la 
fille, surchargée, ne peut compter que sur elle-même pour assurer le maintien des liens familiaux. La difficulté des femmes à remplir la multitude des rôles familiaux qui leur incombent a été soulignée dans la littérature gérontologique (Ajrouch et al., 2005; Antonucci, 2001; Pinquart \& Sörensen, 2006; Silverstein et al., 2006). Les femmes, à la fois "épouse", « mère » et « fille », se doivent d'accomplir de nombreuses tâches comme l'éducation, le ménage, les soins ou encore le "caregiving » auprès des parents âgés, tout en menant de front une activité professionnelle, ce qui constitue une situation stressante pour un grand nombre d'entre elles, et cela d'autant plus si elles sont seules à porter une telle charge de travail (Ajrouch et al., 2005; Antonucci, 2001; Pinquart \& Sörensen, 2006; Silverstein et al., 2006). Les attentes multiples à l'égard des femmes sont telles qu'elles génèrent au sein des relations familiales des tensions et de l'ambivalence (Connidis \& McMullin, 2002; Lüscher \& Pillemer, 1998), mettant à mal la densité et la réciprocité des liens de soutien au sein des configurations familiales. Autrement dit, les rôles familiaux que l'on attribue aux femmes, et plus particulièrement aux filles adultes, ont, par la lourdeur des tâches qu'ils impliquent, des conséquences inattendues sur le capital social au sein des configurations familiales dans la vieillesse.

Dans la littérature gérontologique, la présence des femmes dans la famille rime généralement avec cohésion et soutien familial. Certes, la surcharge de travail des femmes « caregivers », épouses ou filles, est reconnue dans la littérature mais seuls ses effets sur la santé de ces dernières en termes de stress, fatigue et même dépression ont été l'objet d'études en gérontologie sociale. En revanche, il n'y a pas de données qui montrent les effets pervers du sur-investissement féminin sur l'organisation des liens de soutien au sein de l'ensemble du réseau familial. Les mesures du capital social dans les configurations familiales de type «Fille» et de type «Fils» montrent clairement de fortes différences dans la production du capital social entre ces deux types de configurations, bien que tous deux soient axés sur des liens de sang et la descendance. Ces résultats révèlent que les attentes normatives trop lourdes qui pèsent sur les femmes et la surcharge de travail qui en découle ont des effets négatifs marqués, inattendus, sur l'ensemble des échanges de soutien au sein des configurations familiales. Et même si, aujourd'hui, les fils semblent plus enclins à s'engager dans les échanges de soutien au sein de la famille et à y jouer un rôle actif, ils ne le font pas seuls, contrairement aux filles, puisqu'ils bénéficient de l'aide précieuse de leur partenaire qui les seconde dans l'activation des échanges. 


\section{Discussion et conclusion}

Le sexe des membres qui composent les configurations familiales a donc bel et bien un effet sur le type de capital social qu'elles génèrent, mais pas dans le sens de l'hypothèse « $\mathrm{H} 4$ » proposée.

\section{H5 : Capital social et Profil socio-démographique}

La littérature en gérontologie sociale a souligné l'influence du profil sociodémographique et de santé des individus âgés sur les relations sociales et familiales. Parce qu'il renvoie à des rôles sociaux et qu'il s'associe à diverses ressources telles que les moyens financiers, les compétences relationnelles ou encore l'énergie, le profil socio-démographique et de santé des individus a généralement un impact sur leur réseau personnel et familial. Les femmes, par exemple, ont une propension plus marquée à développer des liens affectifs avec une variété de personnes, aussi bien dans la sphère familiale qu'en dehors (Ajrouch et al., 2005; Antonucci et al., 2007; Carr \& Moorman, 2011; Kawachi \& Berkman, 2001) alors que les hommes se replient davantage sur leur partenaire et leurs enfants, le processus de sélectivité socio-émotionnelle dans la vieillesse étant plus prononcé chez les hommes que chez les femmes (Cornwell, 2011; Shaw et al., 2007). Les aînés dont le statut socio-économique est élevé disposent de réseaux personnels plus larges et plus diversifiés que ceux qui sont issus des milieux populaires (Cornwell et al., 2008; Fiori et al., 2006; Litwin, 2001 ; Broese Van Groenou \& Van Tilburg, 2003). De même, les « jeunesvieux » ou les aînés en bonne santé ont plus d'énergie pour s'engager dans de nouvelles relations que les « très-vieux » ou les individus âgés en mauvaise santé, ceux-ci aspirant davantage au repli familial (Antonucci et al., 2007; Carr \& Moorman, 2011; Cornwell, 2009a; Broese Van Groenou \& Van Tilburg, 2007). D'ailleurs, ces derniers ont un besoin accru du soutien familial, ce qui accentue d'autant plus leur centration sur le partenaire et les enfants, lorsqu'ils existent, ceux-ci étant considérés dans la littérature comme les principaux prestataires de soutien dans la vieillesse (Cornwell et al., 2008; Cornwell, 2009a, 2009b, 2011). Partant de ce constat, nous avons fait l'hypothèse (« $\mathrm{H} 5 »)$ que le profil socio-démographique et de santé des individus âgés a un impact sur le type et le volume de capital social produit au sein de leur configuration familiale. Nous avons postulé que les femmes, les individus issus de milieux privilégiés, les «jeunesvieux » ou ceux en bonne santé ont, en raison de leurs ressources (finances, compétences, énergie, etc.), plus accès au capital social de type pont que les hommes, les individus issus des milieux populaires, les «très-vieux » 
Tab. 6.12: Capital social selon le profil socio-démographique.

\begin{tabular}{|c|c|c|c|}
\hline \multicolumn{2}{|c|}{ Profil socio-démographique } & \multirow{2}{*}{$\begin{array}{l}\text { Propriétés } \\
\text { structurelles } \\
\begin{array}{l}\text { Densité faible, engagement fort, } \\
\text { centralité forte }\end{array}\end{array}$} & \multirow{2}{*}{$\begin{array}{l}\text { Types de capital } \\
\text { social }\end{array}$} \\
\hline Genre & Femme & & \\
\hline & Homme & $\begin{array}{l}\text { Densité forte, engagement faible, } \\
\text { centralité faible }\end{array}$ & Chaîne \\
\hline \multirow[t]{3}{*}{ Éducation } & Bas & $\begin{array}{l}\text { Densité forte, engagement } \\
\text { moyen, centralité moyenne }\end{array}$ & Chaîne \\
\hline & Moyen & $\begin{array}{l}\text { Densité faible, engagement } \\
\text { faible, centralité moyenne }\end{array}$ & Faible \\
\hline & Élevé & $\begin{array}{l}\text { Densité moyenne-forte, } \\
\text { engagement moyen, centralité } \\
\text { moyenne }\end{array}$ & Chaîne faible \\
\hline \multirow[t]{3}{*}{ Âge } & Jeune-vieux & $\begin{array}{l}\text { Densité moyenne, engagement } \\
\text { fort, centralité moyenne }\end{array}$ & Pont \\
\hline & Vieux-vieux & $\begin{array}{l}\text { Densité moyenne, engagement } \\
\text { moyen-fort, centralité moyenne }\end{array}$ & Pont faible \\
\hline & Très-vieux & $\begin{array}{l}\text { Petite taille, densité moyenne, } \\
\text { engagement faible, centralité } \\
\text { moyenne }\end{array}$ & Faible \\
\hline \multirow[t]{2}{*}{ Santé } & $\begin{array}{l}\text { Sans difficultés } \\
\text { fonctionnelles }\end{array}$ & $\begin{array}{l}\text { Densité moyenne, engagement } \\
\text { fort, centralité moyenne }\end{array}$ & Pont \\
\hline & $\begin{array}{l}\text { Avec difficultés } \\
\text { fonctionnelles }\end{array}$ & $\begin{array}{l}\text { Petite taille, densité moyenne, } \\
\text { engagement faible, centralité } \\
\text { moyenne }\end{array}$ & Faible \\
\hline
\end{tabular}

ou ceux en mauvaise santé; ceux-ci bénéficient davantage du capital social de type chaîne. Dans le tableau 6.12 figurent les principaux résultats (ANOVA et régressions) quant aux variations du capital social selon le profil socio-démographique et de santé des individus âgés.

Les résultats vérifient partiellement l'hypothèse « $\mathrm{H} 5$ » : le profil socio-démographique et de santé des individus âgés influence bel et bien le type de capital social auquel ces derniers ont accès mais certains résultats ne vont pas dans le sens attendu. Conformément à l'hypothèse « H5 », les hommes sont moins actifs que les femmes dans la provision du soutien et décrivent les liens de soutien au sein de leur configuration familiale comme plus denses. Ils ont donc davantage accès au capital social de type chaîne que les femmes. Celles-ci se perçoivent non seulement comme plus 


\section{Discussion et conclusion}

actives mais aussi comme plus centrales que les hommes, quels que soient leur âge, leur niveau d'éducation, leur état de santé, leur statut conjugal (veuve ou non), l'état de leur réservoir de parenté et la composition de leur configuration familiale. Plus engagées et plus centrales dans les liens de soutien, elles bénéficient davantage de capital social de type pont que les hommes. Le rôle relationnel des femmes au sein de la famille, en tant que «kinkeeper», se manifeste au sein même de leur configuration familiale puisqu'elles parviennent à y maintenir un rôle central et à y préserver leur autonomie relationnelle. Quant au capital social de type chaîne auquel accèdent les hommes, il peut s'expliquer par leur plus grande probabilité d'être encore en couple dans la vieillesse, la présence de l'épouse s'associant à l'émergence de ce type de capital social (Cornwell, 2011). L'homme bénéficie ainsi de la présence de son épouse qui se charge d'activer les liens de soutien autour de lui.

Le statut socio-économique des aînés influence également le type de capital social produit au sein de leur configuration familiale, mais les résultats sont plus nuancés que ceux postulés. Conformément à l'hypothèse «H5», les personnes ayant un bas niveau d'éducation décrivent les liens de soutien au sein de leur configuration comme plus denses que ceux qui ont un niveau d'éducation moyen ou supérieur. Les membres de la famille se mobilisent davantage autour du parent âgé qui a peu de ressources, notamment financières (Antonucci, 2001), ce qui a un impact sur l'organisation du soutien au sein de l'ensemble de la configuration familiale, quelle que soit sa composition. De fait, la plus grande dépendance du parent âgé au soutien familial s'associe, dans les milieux populaires, à l'émergence du capital social de type chaîne. Ceci dit, il est important de rappeler que, parmi les individus âgés n'ayant cité aucun membre significatif de la famille, un grand nombre d'entre eux sont dans une situation socio-économique difficile. La précarité, en effet, ne s'associe pas «automatiquement» au capital social de type chaîne et peut, au contraire, donner lieu à l'absence totale de capital social. Le besoin plus grand de soutien du parent âgé dans les milieux populaires doit, en somme, demeurer «gérable » par les autres membres de la configuration familiale qui se mobilisent. Si ceux-ci disposent de peu de ressources comme de l'affection, du temps, de l'argent ou encore d'une bonne santé, le soutien peut vite s'épuiser et disparaître, et cela d'autant plus si des solutions alternatives, payantes, s'avèrent impossibles en raison de moyens financiers 
limités (Offer, 2012). De plus, et contrairement à l'hypothèse «H5», les individus âgés qui ont un statut socio-économique élevé n'ont pas plus accès au capital social de type pont que les aînés moins dotés; ils ne se perçoivent pas plus engagés ni même plus centraux que ces derniers. Ils ont même davantage accès au capital social de type chaîne que les personnes âgées des classes moyennes, les liens de soutien au sein de leur configuration familiale y sont en effet plus denses. La plus forte propension des aînés les mieux dotés à être mariés ou remariés pourrait expliquer la densité plus forte des liens de soutien au sein de leur configuration familiale.

Lâge et la santé des individus âgés ont également un impact sur le capital social au sein de leur configuration familiale. D'abord, la taille des configurations familiales se réduit significativement au grand âge. Le nombre plus limité de membres cités chez les «très-vieux » et chez les individus en mauvaise santé fonctionnelle révèle que l'avancée en âge et le processus de fragilisation poussent les aînés à être plus sélectifs dans leur réseau familial. La difficulté de maintenir les échanges avec tous les membres de la famille peut en partie expliquer la taille réduite des configurations familiales. Seuls ceux avec lesquels les personnes âgées peuvent encore échanger quelques ressources comme du soutien affectif sont maintenus au sein de la famille significative. Quant aux autres liens familiaux, ils sont désinvestis par les aînés qui n'ont plus la force de s'engager de la même manière avec tous les membres de leur famille. On peut aussi interpréter ce résultat comme la manifestation du processus de sélectivité socio-émotionnelle au niveau familial. Selon le modèle de la sélectivité socio-émotionnelle, les «très vieux », conscients de leur fin imminente, tendent à sélectionner au sein de leur réseau personnel un nombre limité de personnes, généralement des membres «proches » de la famille comme le partenaire et les enfants; et ces personnes choisies sont, selon ce modèle, source d'émotions positives et de soutien à même de procurer aux aînés qui les sélectionnent le bien-être dont ils ont besoin jusqu'à la fin de leur vie (Carstensen, 1991, 1992; Shaw et al., 2007). Dans la littérature scientifique, ce processus a été mis en exergue dans les réseaux plus larges, personnels, mais jamais dans le contexte familial. Les résultats que nous avons obtenus révèlent que ce processus de sélectivité socio-émotionnelle est aussi à l'œuvre au sein même de la famille significative puisque les « très-vieux » ou ceux qui ont des limitations fonctionnelles se montrent nettement plus sélectifs que les plus jeunes ou que ceux qui ont une bonne 


\section{Discussion et conclusion}

santé fonctionnelle puisque le nombre de membres de la famille qu'ils désignent comme significatifs est statistiquement plus petit que le nombre des membres de la famille cités par les « jeunes-vieux», par les « vieux-vieux» ou par ceux qui n'ont aucune atteinte fonctionnelle.

Contre toute attente, cette sélection d'un nombre limité de membres significatifs de la famille ne s'accompagne pas d'une densification des liens de soutien au sein des configurations familiales ni d'une perte de centralité de l'aîné. L'hypothèse d'une mobilisation familiale accrue autour des « très-vieux » ou des personnes ayant une mauvaise santé fonctionnelle ne se vérifie donc pas, quel que soit le type de configuration familiale. Même au sein des configurations plus petites des «très-vieux » ou de celles des personnes souffrant de limitations fonctionnelles, les membres sélectionnés ne sont pas plus interconnectés entre eux que dans les configurations de plus grande taille. Il n'y a pas de densité ni de réciprocité plus fortes dans les configurations des « très vieux » ou dans celles des personnes atteintes dans leur santé fonctionnelle que dans celles des plus jeunes ou que dans celles des aînés qui ont une bonne santé. Plusieurs explications peuvent être avancées. Il se peut, d'une part, que les membres sélectionnés ne soient pas tous des membres primaires de la famille, c'est-à-dire le partenaire et les enfants, comme le postule la théorie de la sélectivité socio-émotionnelle, ce qui pourrait expliquer l'absence de densification des liens de soutien. Il est possible, d'autre part, que les « très vieux » ou que ceux qui sont atteints dans leur santé fonctionnelle n'aient pas besoin d'un soutien accru de la part des membres significatifs de leur famille. Ils peuvent compter sur les ressources qu'ils ont à disposition, telles qu'une bonne santé générale, un bon moral ou la capacité à déployer des mécanismes d'adaptation efficaces, ressources qui leur ont permis d'arriver jusqu'au grand âge. En effet, les très âgés qui ont pu participer à l'enquête et répondre seuls aux questionnaires ont un profil particulier, celui de «survivant», ce qui expliquerait leur moindre dépendance au soutien familial. Par ailleurs, cette absence de mobilisation familiale peut aussi être liée au contexte genevois. Dans ce canton, le réseau d'aide et de soins à domicile offre une palette de prestations qui contribue à répondre à un nombre important de besoins. Un tel dispositif peut contribuer à libérer les membres de la famille de leurs obligations de soutien auprès du parent âgé. La mobilisation accrue des membres significatifs de la configuration n'ayant pas lieu, les «trèsvieux » et ceux en mauvaise santé ne bénéficient pas plus de capital social 
de type chaîne que les plus jeunes ou que ceux qui sont en meilleure santé, ce qui va à l'encontre de l'hypothèse « $\mathrm{H} 5$ ».

De plus, la capacité des aînés à s'engager dans les échanges de soutien et à jouer un rôle actif au sein de leur configuration familiale est atténuée par l'avancée en âge et le processus de fragilisation. Les «très-vieux » ainsi que ceux qui ont des difficultés fonctionnelles sont nettement moins actifs dans la provision du soutien que les plus jeunes ou que ceux qui sont en bonne santé fonctionnelle; ils ont donc moins accès au capital social de type pont que ces derniers. Ainsi, les «très-vieux » ou ceux en mauvaise santé fonctionnelle n'ont pas plus accès au capital social de type pont que de type chaîne; ils disposent donc d'un faible capital social. A l'inverse, les «jeunes-vieux » et les individus en bonne santé fonctionnelle se démarquent clairement par leur plus grand engagement dans les liens de soutien au sein de leur configuration familiale. Ils disposent des ressources nécessaires, en termes de capacités physiques et cognitives, pour rester actifs au sein de leur configuration familiale. Par conséquent, ils ont davantage accès au capital social de type pont que les «très-vieux » ou que les personnes en mauvaise santé fonctionnelle, ce qui confirme en partie l'hypothèse « H5 ».

Alors que les effets du profil socio-démographique des individus sur le réseau personnel ont été largement soulignés dans la littérature gérontologique, son impact sur l'organisation des liens de soutien au sein des familles n'a jamais été exploré. Les résultats de ce travail mettent en exergue l'importance des ressources aussi bien socio-économiques, relationnelles, physiques que démographiques (partenaire) sur la dynamique des échanges familiaux. Ces ressources, dont la qualité et la quantité dépendent étroitement du profil socio-démographique des aînés, ont un impact important sur l'organisation des liens de soutien et sur le type de capital social produit au sein des configurations familiales. Les aînés, qui ont de l'énergie, des capacités physiques ou cognitives comme les «jeunesvieux » ou les personnes ayant une bonne santé fonctionnelle, parviennent à rester actifs dans les échanges de soutien au sein de leur configuration familiale. Il en est de même pour les femmes qui, en tant que «kinkeeper », ont développé des compétences relationnelles qui leur permettent de maintenir les liens de soutien au sein de leur famille. En raison des ressources dont ils disposent, les « jeunes-vieux », les personnes âgées en bonne santé et les femmes ont un accès privilégié au capital social de type pont au sein de leur famille. Les hommes et les individus âgés ayant un bon 


\section{Discussion et conclusion}

statut socio-économique bénéficient du capital social de type chaîne en raison de leur plus forte probabilité d'avoir un(e) partenaire à leurs côtés, sa présence représente une ressource démographique indispensable, contribuant à renforcer les liens d'interdépendance au sein des configurations familiales. Quant aux personnes âgées issues des milieux populaires, elles accèdent, pour la plupart, au capital social de type chaîne si les membres de leur configuration familiale ont suffisamment de ressources pour garantir le soutien collectif dont elles ont besoin. Cependant, lorsque les aînés ont peu ou aucune ressource, leur capacité à la réciprocité en est fortement réduite, prétéritant de fait tout accès au capital social, quel qu'il soit. Ainsi, le grand âge, l'absence de partenaire, une mauvaise santé (fonctionnelle) et la précarité socio-économique représentent, et cela d'autant plus s'ils sont concomitants, des situations à risque pour les individus âgés qui ne parviennent pas ou plus à maintenir les relations au sein de leur configuration familiale et à accéder au capital social. La « capacité » relationnelle ou la " potentialité » des individus âgés à entrer en relation est non seulement déterminante pour maintenir la vie sociale, amicale, mais aussi pour entretenir les liens de soutien avec les plus intimes, ceux-là même qui forment la famille significative. La composition des configurations familiales et les ressources individuelles sont donc importantes pour maintenir l'accès au capital social au sein des familles mais elles sont aussi déterminantes pour la gestion des conflits et de l'ambivalence structurelle au sein des relations familiales.

\section{H6 : Ambivalence et Composition diversifiée}

Les configurations familiales ne créent pas que du capital social, elles génèrent des conflits et de l'ambivalence en raison des liens d'interdépendance qui les caractérisent. Le concept d'ambivalence a surtout été utilisé dans le cadre des relations intergénérationnelles qui, soutenues par des normes de réciprocité « généralisée », présentent un fort degré d'interdépendance (Connidis, 2015; Lüscher, 2002; Lüscher \& Pillemer, 1998; Willson et al., 2006). L'ambivalence survient lorsque le besoin de soutien du parent âgé, ou celui de l'enfant adulte, s'accroît et perturbe l'équilibre des échanges intergénérationnels. Le besoin accru de soutien familial, l'obligation d'aider et le désir de préserver son autonomie placent les protagonistes dans un dilemme permanent, dilemme qui est amené à perdurer dans la vieillesse avec le processus de fragilisation. C'est de ce dilemme, entre besoin de soutien familial et besoin d'autonomie relationnelle, que 
naît l'ambivalence intergénérationnelle. D'un côté, l'autonomie du parent âgé peut être menacée par le soutien familial qu'il reçoit et dont il a besoin. En devenant dépendant du soutien de ses enfants, il peut être soumis au contrôle et à la surveillance de ces derniers. De l'autre coté, c'est l'autonomie des enfants adultes qui est limitée. Mus par un fort sentiment de redevabilité, ces derniers sont contraints d'aider le parent âgé et, ce faisant, ils se doivent de revoir, voire renoncer, à certains de leurs projets personnels. Dès lors, l'ambivalence se manifeste au sein de la relation intergénérationnelle à la fois par du soutien mais aussi par de l'agacement et des tensions. Inévitable dans le contexte spécifique de la vieillesse, l'ambivalence intergénérationnelle se doit être gérée au sein de la relation par le parent âgé et l'enfant adulte (Connidis, 2015; Lüscher, 2000, 2002; Willson et al., 2006).

L'ambivalence, cependant, n'est pas seulement une propriété dyadique, intergénérationnelle, mais elle est inhérente à l'ensemble des liens d'interdépendance. Elle peut donc se développer dans le contexte plus large des configurations familiales. Le besoin accru de soutien du parent âgé déséquilibre non seulement les relations dyadiques, intergénérationnelles, mais aussi l'ensemble des liens d'interdépendance qui sous-tendent les configurations familiales (Connidis, 2015; Hillcoat-Nallétamby \& Phillips, 2011; Widmer, 2016). Dans une telle situation, l'ambivalence structurelle se doit d'être gérée non seulement par le parent âgé et les membres dont il dépend directement mais elle doit être aussi négociée avec l'ensemble des membres de la configuration familiale puisque tous, étant étroitement dépendants les uns des autres, pâtissent du déséquilibre des liens de soutien au sein du réseau familial, pouvant le percevoir comme particulièrement inégalitaire. Certains se sentent floués, n'obtenant pas le soutien dont ils ont besoin, alors que les autres se sentent menacés dans leur autonomie, devant satisfaire les besoins de tous. L'ambivalence structurelle et sa gestion n'ont jamais été explorées au niveau configurationnel dans la recherche gérontologique, du moins de manière quantitative. Considérant la dyade intergénérationnelle comme l'une des relations les plus importantes du point de vue du soutien dans la vieillesse, la majorité des études en gérontologie sociale ont rarement pris en compte l'influence des autres dyades auxquelles la relation «parent-enfant» est pourtant étroitement liée. Dans l'approche configurationnelle, l'ambivalence et sa gestion ne concernent pas uniquement le parent âgé et l'enfant adulte mais elles 
doivent être considérées à un niveau plus large, celui des configurations familiales.

Pour observer comment les aînés et les membres de leur configuration gèrent l'ambivalence structurelle, nous avons utilisé la typologie des quatre modes de conflit et de support proposée par Eric Widmer, à savoir l'Ambivalence, la Solidarité, la Captivation et l'Atomisation (Widmer, 2016). Il s'agit d'une version de la typologie de Kurt Lüscher adaptée à la perspective configurationnelle. Ces quatre modes combinent de manière distincte la présence, ou l'absence, du soutien et du conflit (densités) au sein des configurations familiales. L'Ambivalence allie la présence du soutien et du conflit alors que la Solidarité se caractérise par du soutien sans conflit. Quant à la Captivation, elle se distingue par du conflit sans soutien et l'Atomisation n'affiche ni soutien ni conflit. Ces quatre modes peuvent être interprétés comme le reflet de gestions différentes de l'ambivalence structurelle au niveau configurationnel. Selon les mécanismes adoptés par les membres de la configuration tels que la négociation, l'investissement ou le désengagement, ces quatre modes de gestion de l'ambivalence structurelle donnent lieu à des conséquences relationnelles distinctes, soit du soutien, soit du conflit, soit les deux à la fois, soit aucun des deux. Appliquée et validée auprès de jeunes adultes (Widmer, 2016), cette typologie n'a jamais été utilisée dans une population de personnes âgées. Afin de mieux connaître les conditions d'émergence de ces quatre modes dans la vieillesse, nous avons fait plusieurs hypothèses sur les facteurs susceptibles de favoriser leur apparition. Dans un premier temps, nous avons postulé qu'ils dépendent de la composition des configurations familiales (« $\mathrm{H} 6 »)$. Nous avons émis l'hypothèse que les modes de gestion « conflictuels » de l'ambivalence structurelle, c'est-à-dire l'Ambivalence et la Captivation, se développent davantage dans les configurations familiales reposant sur des liens intergénérationnels ou conjugaux, se caractérisant par un fort degré d'interdépendance, alors que ceux qui sont peu conflictuels, comme la Solidarité et l'Atomisation, émergent plutôt dans des configurations familiales composées de liens de type affinitaire, se distinguant des autres par un faible degré d'interdépendance.

Cette première hypothèse ( « H6 ») est vérifiée par les analyses : la composition des configurations familiales a un impact sur l'émergence de ces différents modes de conflit et de support. Les résultats montrent que la gestion de l'ambivalence structurelle est plus conflictuelle lorsque les configurations familiales sont composées d'enfants et de petits-enfants que 
lorsqu'elles en sont exemptes. L'Ambivalence et la Captivation sont en effet plus prégnantes dans les configurations familiales composées de liens intergénérationnels ou conjugaux alors que la Solidarité et l'Atomisation se développent davantage dans les configurations familiales constituées majoritairement de liens de type affinitaire. Le fort degré d'interdépendance qui caractérise les configurations familiales composées de liens intergénérationnels rend toute rupture difficile, voire impossible, et « force» les membres de la configuration à discuter, concéder et négocier pour trouver des solutions afin d'assurer le soutien tout en préservant l'autonomie relationnelle de chacun. Parfois, de telles négociations aboutissent à des arrangements, non sans tensions, ce qui permet de garantir le soutien et d'atténuer l'ambivalence. Parfois, cependant, elles n'aboutissent pas et s'enlisent dans le conflit. A l'opposé, les configurations familiales sans enfant reposent principalement sur des membres «choisis», tels que les membres de la fratrie, de la parenté éloignée ou des amis, qui sont intégrés au sein de la famille significative pour la qualité des ressources et la satisfaction émotionnelle qu'ils apportent. N'étant pas tenus par des normes de réciprocité "généralisée », les membres au sein de ce type de configurations sont peu connectés entre eux par de multiples échanges et affichent par conséquent un faible degré d'interdépendance. Point d'ancrage dans sa configuration familiale, l'individu âgé dispose d'une certaine autonomie relationnelle. Il peut se désengager des liens qu'il perçoit comme déséquilibrés, conflictuels ou intrusifs, tout en ne préservant que ceux qu'il estime comme satisfaisants. Par conséquent, les tensions et les conflits y sont rares, ceux-ci se gérant par le désengagement et la rupture. Conformément à l'hypothèse « $\mathrm{H} 6$ », la composition des configurations familiales joue donc un rôle central dans la gestion de l'ambivalence structurelle au sein des configurations familiales.

Les tensions font donc partie intégrante des relations dans les familles significatives composées d'enfants et de petits-enfants. Un tel résultat nuance quelque peu le modèle de la sélectivité socio-émotionnelle (Carstensen, 1991, 1992; Shaw et al., 2007). Il est postulé dans ce modèle théorique que les individus âgés tendent, avec l'âge avançant et l'approche de la mort, à ne sélectionner que les membres de leur réseau personnel qui leur procurent le plus d'émotions positives et de satisfaction émotionnelle tout en se désengageant des liens qu'ils estiment peu gratifiants, source d'émotions négatives et de tensions. L'analyse révèle cependant que les 


\section{Discussion et conclusion}

membres sélectionnés comme « significatifs » ne sont pas toujours prometteurs d'émotions positives et de satisfaction. Bien qu'il y ait une certaine sélection socio-émotionnelle, celle-ci ne s'opère pas sans contraintes structurelles. Imbriqué dans des liens complexes d'interdépendance, l'individu n'est pas entièrement libre dans ses choix relationnels, il désigne généralement ceux dont il dépend étroitement pour le soutien dont il a besoin - ce qui est particulièrement le cas dans les situations de "caregiving 》- mais aussi des membres affiliés, tels que les partenaires des enfants (les beauxenfants), qui participent eux aussi aux échanges de soutien au sein de la configuration familiale. Ceux-ci peuvent même représenter une source importante d'aide pour le parent âgé sans pour autant que ce dernier y soit fortement attaché. Dans certains cas, l'aîné peut ne pas les apprécier et se sentir agacé par leur présence. Ces liens d'interdépendance, bien que source de capital social, n'en sont donc pas moins générateurs de frustration et de tensions. La sélection socio-émotionnelle est moins contrainte lorsque l'individu âgé construit sa famille significative en y intégrant essentiellement des liens de type affinitaire. Les liens d'interdépendance étant plus lâches, l'aîné peut gérer ses relations significatives en se désengageant de celles qu'il estime conflictuelles tout en ne préservant que celles qui lui apportent de fortes émotions positives. En omettant les liens d'interdépendance dans lesquels le parent âgé évolue, le modèle de la sélectivité socio-émotionnelle donne trop de poids à l'individu et à son autonomie supposée. Or, les résultats de ce travail montrent clairement que cette autonomie relationnelle est limitée si l'on tient compte des liens complexes d'interdépendance dans lesquels s'insèrent de nombreux individus âgés.

\section{H7 : Ambivalence et Ressources}

La position des individus âgés dans la société, mesurée par leur profil socio-démographique et de santé, et plus précisément par le genre, le statut socio-économique, l'âge et la santé fonctionnelle, influence de manière déterminante la production du capital social au sein des configurations familiales. Cette influence s'explique par les différentes ressources aussi bien financières, relationnelles, démographiques, physiques que cognitives qui varient en termes de qualité et de quantité en fonction du profil sociodémographique et de santé des individus. De telles ressources permettent aux individus âgés de s'engager dans les échanges de soutien au sein de leur configuration familiale et, en ce sens, contribuent à promouvoir le capital social. Leur absence, et qui plus est cumulée, s'associe au contraire 
à un faible capital social, quel qu'il soit. Jouant un rôle prépondérant dans la production du capital social au sein des configurations familiales, nous avons postulé qu'elles ont aussi un impact sur la façon dont les aînés et les membres de leur famille gèrent ensemble l'ambivalence structurelle. Plus précisément, nous avons fait l'hypothèse (« H7 ») qu'avoir des ressources s'associe à des modes de gestion de l'ambivalence axés sur le soutien - l'Ambivalence et la Solidarité - alors qu'en manquer rime plutôt avec des modes reposant sur un faible soutien, tels que la Captivation et 1'Atomisation.

Les résultats vérifient l'hypothèse « $\mathrm{H} 7$ » : la gestion de l'ambivalence structurelle au niveau configurationnel dépend des ressources à disposition de l'individu âgé mais aussi de celles des membres de sa configuration familiale. Conformément à cette hypothèse, les modes de conflit et de support axés sur le soutien - l'Ambivalence et la Solidarité - se développent davantage lorsque l'individu âgé détient un certain nombre de ressources, telles qu'un bon revenu, une bonne santé et la présence d'un partenaire, alors que les modes de support et de conflit exempts de soutien - la Captivation et l'Atomisation - se manifestent plutôt lorsque le parent âgé dispose de peu de ressources comme un bas revenu, une mauvaise santé ou n'a aucun partenaire. Avoir des ressources permet à l'aîné de maintenir la réciprocité des échanges et de participer à l'organisation des liens de soutien au sein de sa configuration familiale. Par ailleurs, avoir des ressources signifie avoir plus de poids dans les négociations et une dépendance moindre à l'aide familiale, et donc plus d'autonomie vis-à-vis des autres membres de la configuration. Les analyses confirment que, dans les modes Ambivalence et Solidarité, l'individu âgé a les ressources nécessaires, telles qu'avoir un partenaire, des moyens financiers, de l'énergie ou une bonne santé, pour activer les échanges de soutien au sein de sa configuration et y affirmer son autonomie. A l'inverse, dans les modes de gestion exempts de soutien tels que la Captivation et l'Atomisation, l'individu âgé a peu de ressources. Il peine à assurer la réciprocité des échanges et ne parvient pas à stimuler les liens de soutien au sein de sa configuration familiale. De plus, sa précarité accroît sa dépendance à l'aide familiale, ce qui déséquilibre encore davantage les liens de soutien au sein de la configuration. Et, ce déséquilibre est d'autant plus prononcé lorsque les autres membres de la famille ont aussi peu de ressources (peu de temps, une mauvaise santé ou de faibles revenus) ou lorsqu'ils sont nombreux à dépendre de l'aide familiale. Le stress engendré par une telle situation 


\section{Discussion et conclusion}

peut générer de fortes tensions qui sont difficilement gérables et négociables lorsque les ressources manquent. N'étant pas ou peu alimenté, le soutien s'épuise au point même qu'à la longue il disparaît, laissant la place soit aux conflits, comme dans le cas de la Captivation, soit au désengagement, comme dans le cas de l'Atomisation. Conformément à l'hypothèse « $\mathrm{H} 7$ », les ressources constituent un deuxième facteur explicatif important dans les variations de gestion de l'ambivalence structurelle au niveau configurationnel.

Tenir compte du profil socio-démographique et de santé des aînés et des ressources auxquelles il renvoie montre que les individus âgés ne sont pas tous égaux face à l'ambivalence structurelle au sein de leur famille. Tous ne sont pas armés de la même manière pour y faire face et la gérer de manière satisfaisante au sein de leur configuration familiale. Ceux qui ont des ressources parviennent à gérer l'ambivalence structurelle en stimulant le soutien au sein de leur configuration familiale tout en affirmant leur autonomie, soit par le biais de négociations (Ambivalence) soit par un fort engagement au sein de leur configuration familiale (Solidarité). Quant à ceux qui n'ont pas de ressources, ils ne parviennent pas à participer aux échanges de soutien au sein de leur configuration, pire encore leur dépendance à l'aide familiale y déséquilibre les liens de soutien, créant de fortes tensions et des conflits (Captivation). Ceux-ci, par ailleurs, s'avèrent difficilement gérables dans des configurations familiales au sein desquelles les membres ont eux-mêmes peu de ressources. La forte dépendance à l'aide familiale peut même pousser les autres membres à se désengager, la demande de soutien étant trop lourde pour les membres qui n'ont pas ou peu de ressources (Atomisation). Ces inégalités sociales dans la gestion de l'ambivalence structurelle au sein des familles ont été peu explorées en gérontologie sociale, du moins empiriquement. Les résultats obtenus montrent que les inégalités sociales transparaissent dans les liens les plus intimes et les plus significatifs; elles ont en effet un impact important sur les échanges au sein des configurations familiales. L'ambivalence et sa gestion ne peuvent se comprendre qu'en prenant en compte non seulement le niveau « meso » (configurations familiales) mais aussi le niveau macro (position sociale) puisque ces deux niveaux ont une influence importante sur la façon dont les aînés gèrent l'ambivalence structurelle au sein de leur famille au cours de la vieillesse. 


\section{H8 : Ambivalence et Interrelation composition/ressources}

Parce que la composition des configurations familiales et les ressources des individus âgés influencent la gestion de l'ambivalence structurelle au niveau configurationnel, nous nous sommes questionnés quant à leur effet conjoint. Nous avons fait l'hypothèse que l'interrelation entre composition et ressources offre les conditions d'émergence de chacun des quatre modes de conflit et de support ( H8 »). Nous avons en effet postulé que les configurations familiales composées du partenaire, des enfants et des petits-enfants s'associent au mode Ambivalence lorsque le parent âgé possède de nombreuses ressources et à la Captivation lorsqu'il n'en a pas. Quant aux configurations constituées principalement de membres familiaux de type affinitaire, sélectionnés au sein du réservoir de parenté ou en dehors, nous avons émis l'hypothèse qu'elles donnent lieu à la Solidarité lorsque l'aîné a des ressources et à l'Atomisation lorsqu'il en est dépourvu.

L'analyse des correspondances multiples vérifie l'hypothèse « $\mathrm{H} 8$ » portant sur l'effet concomitant de ces deux facteurs. Ceux-ci offrent dans leur interrelation les conditions d'émergence spécifiques pour le développement de chacun de ces quatre modes. L'Ambivalence, alliant le soutien et le conflit (densités), se développe dans les configurations familiales composées de liens intergénérationnels ou conjugaux et lorsque le parent âgé a des ressources telles qu'avoir un partenaire, une bonne santé fonctionnelle et un bon revenu. De telles ressources lui permettent de soutenir les échanges de soutien mais aussi de négocier pour trouver des arrangements pratiques, voire même de recourir à des aides à l'extérieur de la famille (recours à des professionnels, etc.); de tels arrangements garantissent le soutien aux membres de la famille qui en ont besoin tout en préservant l'autonomie de chacun. Ces négociations sont nécessaires puisque les liens intergénérationnels ou conjugaux ne peuvent être que difficilement rompus en raison de la forte interdépendance qui les sous-tend. Ce processus de négociations n'est pas exempt de discussions houleuses ou de tensions, puisqu'il s'agit pour chaque membre de la configuration de défendre son autonomie. Les ressources de l'individu âgé, tout comme celles des membres de la famille, permettent cependant de trouver des arrangements qui, en satisfaisant la plupart des membres, contribuent à y maintenir des liens de soutien. Par contre, lorsque l'individu âgé et les membres de sa configuration ont peu de ressources, les négociations sont laborieuses et peinent à aboutir à des solutions pratiques adéquates. Elles s'enlisent peu à peu dans le conflit, conduisant à la Captivation. C'est notamment le cas dans 


\section{Discussion et conclusion}

les familles à faible revenu et dans lesquelles se trouve un grand nombre de membres dans le besoin. Dans de telles situations, le déséquilibre des échanges est particulièrement marqué et il est difficile pour les membres de la configuration familiale de satisfaire à toutes les demandes d'aide tout en préservant une certaine autonomie. Tenu par de fortes pressions normatives, le parent âgé, tout comme les membres de sa configuration, ne peut pas se désinvestir des relations qu'il considère comme peu gratifiantes ou trop demandeuses. Prises au piège, les personnes qui appartiennent à ce type de configurations se sentent flouées et frustrées. Le soutien, n'étant pas ou peu nourri d'échanges de ressources, tend à s'épuiser et ne laisse place qu'aux ressentiments et aux tensions. La gestion de l'ambivalence structurelle est dans ces situations fort difficile, marquée par le conflit et l'absence de soutien.

Quant à la Solidarité, elle se développe dans les configurations familiales composées de liens de type affinitaire et lorsque l'individu possède un certain nombre de ressources. Dans ce type de configurations, les liens sont maintenus davantage par une volonté commune d'être ensemble que par de fortes obligations familiales. N'étant pas soudées par des liens forts d'interdépendance, les relations familiales au sein de ce type de configurations reposent sur l'engagement individuel; chaque membre y investit de multiples ressources afin d'y préserver la solidarité et l'harmonie. Lorsqu'émergent des tensions, l'individu âgé peut, au même titre que les autres membres de la configuration, se détacher des liens familiaux qu'il juge problématiques, intrusifs ou conflictuels sans craindre de déséquilibrer les échanges au sein de la configuration, les membres étant peu connectés entre eux. Tout en se dégageant de certains liens, l'aîné peut en parallèle s'investir activement dans ceux qu'il estime gratifiants et soutenants. En tant que gestion active de l'ambivalence structurelle, la Solidarité permet à l'aîné de maintenir son autonomie tout en s'assurant des liens de soutien de qualité. Ceci dit, ce mode de conflit et de support a un prix : il requiert de la part du parent âgé un certain nombre de ressources physiques, cognitives mais aussi financières pour activer les liens de soutien au sein de sa configuration familiale. Lorsque de telles ressources viennent à manquer, l'Atomisation prend place progressivement. Sans ressources, il devient difficile pour l'aîné de maintenir la réciprocité des échanges avec chacun des membres de sa configuration. Ne pouvant plus s'investir activement dans les échanges, l'individu vieillissant se désengage peu à peu des liens de soutien au sein de sa configuration. Il en est de même pour les membres de 
sa famille qui rechignent à prendre en charge, seuls et sans contrepartie, le parent âgé dont les besoins deviennent de plus en plus grands. Au cours de ce processus, le parent âgé évite certes les tensions et l'ambivalence que le déséquilibre des échanges pourrait susciter mais il doit cependant faire face à l'absence de soutien, celui-ci s'essouffle en effet rapidement et disparaît, n'étant plus alimenté par des échanges de ressources réciproques.

La prise en compte de l'effet conjoint de ces deux facteurs révèle que le conflit familial et l'ambivalence structurelle ne sont pas le fruit d'incompatibilités individuelles mais plutôt la résultante d'un processus social qui évolue et qui varie en fonction de contraintes normatives et des ressources à disposition. Sa gestion ne dépend pas uniquement de deux individualités - le parent âgé bénéficiaire et l'enfant adulte prestataire - mais elle s'inscrit dans des structures plus larges : la configuration (composition), qui dépend du parcours de vie, et la société, marquée par des inégalités. En tenant compte de leur interrelation, on peut mettre en lumière la diversité des situations familiales dans la vieillesse et les différentes façons d'y gérer l'ambivalence structurelle. Ce faisant, on peut identifier les aînés qui ont le plus de difficultés à gérer l'ambivalence au sein de leur configuration familiale, ceux-là même qui sont le plus à risque d'être confrontés à de sévères conflits familiaux, voire à de la violence, et à l'isolement social. Les dernières étapes du parcours de vie sont donc loin d'être marquées par la standardisation, le nivellement des différences et la stabilité des pratiques familiales; elles se caractérisent au contraire par la pluralité et les inégalités sociales.

D'un point de vue politique, l'ambivalence structurelle constitue pour les pouvoirs publics un perpétuel défi qu'ils doivent relever. Certains auteurs, comme Connidis (2015), soulignent qu'il advient à l'État non seulement d'aider financièrement les familles les plus démunies mais aussi de développer des alternatives d'aide efficaces pour les familles qui ont la charge de personnes dépendantes - comme des enfants, de jeunes adultes ou des personnes âgées - afin de les soulager dans leurs tâches d'aide et de soins. De telles mesures permettraient de réduire les inégalités sociales face à l'ambivalence structurelle et d'enrayer ainsi les risques de violence et de désengagement familial. A Genève, le dispositif de maintien à domicile, mis en place par des associations et soutenu par le canton, répond à ces exigences en offrant une palette de soins et de services diversifiés. Ce dispositif permet aux parents âgés dans le besoin de préserver leur autonomie relationnelle vis-à-vis des membres de leur famille et, par 


\section{Discussion et conclusion}

conséquent, de réduire l'ambivalence structurelle au sein de leur configuration familiale. Même si ces services soulagent grandement les familles, ils s'appuient cependant sur la participation des proches (aidants) pour qu'ils soient efficaces. Autrement dit, le parent très âgé ou en incapacité fonctionnelle qui recourt à ce type de services n'est pas totalement affranchi des interdépendances familiales. A ce titre, il est aussi amené à expérimenter l'ambivalence structurelle au sein de sa configuration familiale. Cette ambivalence est d'autant plus manifeste lorsque les familles, qui ont peu de ressources (démographiques, économiques, physiques ou encore temporelles), se sentent sous pression, devant s'organiser, trouver des arrangement avec les différents intervenants professionnels et investir leurs faibles ressources (temps, argent, énergie, etc.) dans le soutien du parent âgé. Ces familles encourent un risque accru non seulement de développer la Captivation ou l'Atomisation en leur sein mais aussi, dans certains cas, de se paupériser davantage (Wall et al., 2001).

\section{H9 : Ambivalence et Genre}

Après avoir mis en exergue les inégalités sociales dans la gestion de l'ambivalence structurelle au sein des configurations familiales, nous avons évoqué les inégalités de genre, tout aussi marquantes. La recherche en gérontologie sociale a souligné la plus grande probabilité des femmes âgées de connaître le veuvage, une baisse de revenu et le déclin de la santé fonctionnelle en raison de leur position sociale désavantagée et de leur espérance de vie plus longue (Arber et al., 2003; Moen, 1996; Wanner et al., 2005; Willson et al., 2006). Quant aux hommes âgés, un grand nombre d'entre eux disposent toujours, même à des âges avancés, d'une partenaire, d'un bon revenu et souffrent moins d'incapacités fonctionnelles que les femmes du même âge. En perdant leurs ressources dans la vieillesse, les femmes âgées ont un risque plus grand que les hommes de dépendre totalement de l'aide familiale, ne pouvant plus s'engager dans les liens de soutien et participer activement aux échanges au sein de leur configuration familiale. Autrement dit, elles perdent l'autonomie relationnelle dont elles bénéficiaient plus jeunes. Au vu de ce constat, nous avons fait l'hypothèse que les femmes âgées, plus démunies, peinent davantage que les hommes à gérer l'ambivalence structurelle au sein de leur configuration familiale. Plus précisément, nous avons postulé que les femmes âgées connaissent davantage que les hommes des modes de gestion de l'ambivalence exempts 
de soutien, à savoir la Captivation, si leur configuration familiale se compose de liens intergénérationnels ou conjugaux, ou l'Atomisation, si leur configuration repose sur des liens de type affinitaire (« H9 »).

Les résultats de l'analyse des correspondances multiples confirment les différences de genre dans la gestion de l'ambivalence structurelle. Conformément à l'hypothèse « $\mathrm{H} 9$ », les femmes âgées ont un risque plus grand que les hommes de développer la Captivation lorsqu'elles s'insèrent dans des configurations familiales composées de liens intergénérationnels et l'Atomisation lorsque leur configuration est majoritairement constituée de liens affinitaires. Les femmes âgées qui cumulent toutes les pertes aussi bien démographiques (veuvage), financières que physiques (atteintes fonctionnelles), ne parviennent pas à rester actives dans les échanges de soutien et à préserver leur position centrale au sein de leur configuration familiale, quelle qu'en soit la composition. Le manque de ressources et le besoin accru du soutien familial créent un fort déséquilibre dans les échanges entre les différents membres de la configuration familiale, générant de l'ambivalence structurelle en son sein. Lorsque la configuration comporte des relations intergénérationnelles, le désengagement n'est pas envisageable, les liens étant soumis à de fortes obligations d'aide. La dépendance des mères âgées au soutien familial génère de fortes tensions, et cela d'autant plus si les ressources sont rares ou mobilisées principalement autour des petits-enfants. Surchargés ou sans ressources, les enfants adultes - les filles, notamment - se sentent obligés d'aider leur mère âgée sous peine de ressentir de la culpabilité s'ils ne le font pas. Obligations familiales et déséquilibre dans les échanges contribuent à l'épuisement progressif du soutien et à l'émergence de nombreuses frustrations et tensions qui deviennent avec le temps difficilement gérables. Lorsque la configuration familiale repose sur des liens affinitaires, les femmes sans grandes ressources et les membres de leur famille significative gèrent l'ambivalence structurelle en se détachant les uns des autres (Atomisation), les liens qui les relient n'étant pas soutenus par de fortes obligations familiales. Aux dernières étapes de la vie, hommes et femmes abordent donc l'ambivalence structurelle au sein de leur configuration familiale de manière très inégale, les premiers, accompagnés d'une épouse souvent plus jeune, d'un bon revenu et d'une bonne santé, et les dernières, moins chanceuses, totalement démunies de toutes ressources.

Ces résultats sont paradoxaux si l'on tient compte de la place privilégiée de la femme au sein de la famille. Nous avons relevé dans le 


\section{Discussion et conclusion}

deuxième chapitre que la centration des femmes sur la sphère familiale leur assure de nombreux avantages dans la vieillesse. Certaines études en gérontologie sociale soulignent, par exemple, que les mères âgées ont davantage de contacts avec leurs enfants adultes et reçoivent plus de soutien de leur part que les pères (Barer, 1994; Kawachi \& Berkman, 2001; Parrott \& Bengtson, 1999; Suitor et al., 2011). De même, les enfants adultes se sentent plus proches affectivement de leur mère que de leur père (Antonucci et al., 1998; Lawton et al., 1994b; Pyke \& Bengtson, 1996; Silverstein \& Bengtson, 1997; Silverstein et al., 2006). Pour ce qui est des liens affinitaires, les femmes âgées ont plus d'échanges de soutien avec les membres de leur fratrie et avec leurs amis que les hommes du même âge (Campbell et al., 1999; Eriksen \& Gerstel, 2002; Voorpostel \& Van Der Lippe, 2007). Ces diverses études cependant sont ciblées sur les liens dyadiques et ne prennent pas en compte le niveau configurationnel, ni même la position sociale et le parcours de vie plus désavantagés des femmes. Certes, ces dernières entretiennent en moyenne des liens affectifs privilégiés avec l'ensemble des membres significatifs de leur famille, mais ce n'est pas le cas de toutes les femmes, comme le révèlent les résultats de l'analyse des correspondances multiples. Les désavantages que certaines femmes âgées ont accumulés au cours de leur vie tels qu'une vie professionnelle entrecoupée, peu qualifiée ou inexistante, le veuvage ou encore le déclin de leur santé fonctionnelle, les placent dans une situation particulièrement précaire lorsqu'elles doivent gérer l'ambivalence structurelle au sein de leur configuration familiale. Les pertes sévères que certaines d'entre elles ont cumulées tout au long de leur vie ont peu à peu fragilisé et érodé les liens de soutien qui sous-tendent leur configuration familiale. Le processus d'accumulation des (dés)avantages au cours du parcours de vie qui contribue aux inégalités de classe et de genre dans la vieillesse (Dannefer, 2003) a un effet discriminant tout aussi déterminant sur les relations de soutien au sein des configurations familiales et sur la gestion de l'ambivalence structurelle aux dernières étapes de la vie. En somme, les femmes âgées des milieux populaires, pauvres, veuves et en mauvaise santé, constituent une part de la population âgée particulièrement vulnérable, susceptible d'être davantage confrontée aux conflits familiaux et à l'isolement social que les autres, et qui par conséquent devrait être au centre de toutes les attentions des pouvoirs publics. 
En conclusion, l'approche configurationnelle permet d'étudier les familles dans toute leur diversité et leur complexité. Les liens d'interdépendance positifs (soutien) et négatifs (tensions) qui connectent entre eux les différents membres significatifs des configurations familiales ne sont jamais considérés dans la plupart des recherches en gérontologie sociale. Pourtant, les étudier ouvre de nouvelles perspectives d'analyse en permettant aux chercheurs d'observer les familles autrement que par le biais du support social, problématique dominante en gérontologie sociale. S'intéressant peu aux dynamiques familiales, les modèles dominants de la gérontologie sociale se sont contentés de définir la famille en s'appuyant sur sa forme nucléaire, stable et uniforme, qui, parce qu'elle est soutenue par de fortes obligations familiales d'aide, est prometteuse d'un soutien collectif. En nous basant sur les liens d'interdépendance, nous avons fait le constat que les familles dans la vieillesse sont loin de correspondre à ce modèle; elles sont à fois diverses et complexes tant dans leur composition que dans l'organisation des liens d'interdépendance qui les sous-tendent. En tant que configurations familiales, elles produisent en effet différents types de capital social et l'ambivalence structurelle qu'elles génèrent est gérée de manière distincte en fonction de leur composition et de la qualité des ressources dont disposent les membres qui les constituent. En adoptant une perspective configurationnelle, cet ouvrage remet en question certaines idées reçues sur la famille des personnes âgées, idées qui sont encore en vigueur dans de nombreuses études en gérontologie, et porte un regard critique sur les modèles théoriques qui ont orienté - et qui orientent toujours - la gérontologie sociale ces dernières décennies.

Toutefois, ce travail comporte certaines limites. L'une d'elles a trait à la collecte des données sur le réseau familial lors de l'enquête «VLV ». Il était demandé aux participants de ne citer pas plus de cinq membres significatifs. Cette limite était nécessaire afin de réduire le temps de passation du questionnaire qui comportait de nombreuses questions mais aussi d'alléger la pénibilité des questions portant sur le réseau familial. Celles-ci, répétitives, sont ennuyeuses tant pour les enquêteurs que pour les participants. Lorsque les mentions demandées sont nombreuses, les données obtenues risquent d'être de moins bonne qualité; elles produisent davantage de valeurs manquantes ou aberrantes (Merluzzi \& Burt, 2013; Widmer et al., 2013). Ce risque est d'autant plus grand si l'on considère l'âge des personnes interviewées. Par ailleurs, les enquêteurs sont tentés de réduire, de leur propre chef, le nombre de citations afin d'accélérer la passation des 


\section{Discussion et conclusion}

questions, biaisant dès le départ les réponses des participants. Un tel biais est connu et largement répandu dans les enquêtes portant sur les réseaux personnels (Cornwell et al., 2009; Marsden, 2003; Widmer et al., 2013).

Malgré sa nécessité, cette limite a pu compromettre la diversité des membres cités. Comme l'ont montré certaines études (Widmer, 2016), la prise en compte d'un plus grand nombre de mentions permet d'avoir une plus grande diversité de membres significatifs et, par conséquent, une plus large palette de configurations familiales. Contraints de faire un choix, il est probable que les participants mentionnent d'abord les membres de la famille «attendus », c'est-à-dire ceux qui renvoient à la famille normative, nucléaire, au détriment d'autres membres, tout aussi significatifs mais moins conventionnels. Cependant, les résultats que nous avons obtenus vont à l'encontre de cette hypothèse. Le partenaire et les enfants sont, certes, souvent mentionnés mais ils sont loin d'être les seuls. En effet, même avec un générateur de noms limité à cinq, nous avons recensé une variété de membres qui dépassent largement les frontières de la famille nucléaire et nous avons identifié une pluralité de configurations familiales. D'ailleurs, certains chercheurs relèvent que les enquêtés citent en moyenne entre quatre à sept personnes lorsqu'il est question de nommer les relations les plus proches de leur réseau personnel comme les intimes ( « intimate social contacts ») ou les confidents (Cornwell et al., 2008, 2009; Fingerman $\&$ Birditt, 2003). L'accent mis sur la significativité des membres du réseau personnel encourage les individus à ne mentionner qu'un nombre restreint de membres et les plus significatifs sont généralement cités en premier lorsqu'il s'agit de les lister (D'Andrade, 1995). Autrement dit, la restriction à cinq mentions n'affecte pas la pertinence des données; elle permet au contraire de déceler les membres de la famille qui sont les plus significatifs. Il serait néanmoins intéressant dans de futures recherches de permettre aux aînés de lister les membres de leur famille significative sans en limiter le nombre. Pour ce faire, il serait nécessaire de réduire le nombre de questions de l'ensemble du questionnaire afin de consacrer suffisamment de temps aux questions portant sur le réseau familial.

Une autre limite a trait à la «Family Network Method» (FNM) que nous avons utilisée pour récolter les données sur les réseaux familiaux. Cette méthode vise à lister les membres significatifs de la famille et à décrire les liens d'interdépendance qui sous-tendent les configurations familiales mais elle ne permet pas de saisir les raisons qui motivent les répondants à désigner certains individus comme membres significatifs de 
leur famille. En somme, nous ne connaissons pas les motifs qui poussent les participants à inclure dans leur famille significative certaines personnes plutôt que d'autres. Seuls des entretiens approfondis peuvent mettre en lumière ces critères de choix, qui sans nul doute varient selon le profil et le parcours de vie des individus âgés (Castrén \& Ketokivi, 2015; Wall et al., 2008). Comme l'ont suggéré Castrén et Ketokivi (2015), compléter la «FNM» par des entretiens approfondis permettrait de saisir le « sens » que donnent les individus âgés à leur configuration familiale et les raisons qui expliquent leurs choix tout en ayant une description détaillée des liens d'interdépendance et des contraintes structurelles dans lesquels ils s'insèrent et dont ils n'ont pas forcément conscience. Il serait par ailleurs intéressant de considérer cette combinaison «FNM » et entretiens dans une perspective longitudinale. Il s'agirait dès lors de rencontrer des aînés à plusieurs reprises dans un temps donné, de récolter à chaque fois des informations sur leur réseau familial par le biais de la «FNM» et de procéder ensuite à des entretiens approfondis. Un tel design d'enquête permettrait de mieux comprendre les mécanismes de re-définition ou de ré-interprétation des liens familiaux mis en place par les personnes âgées; ceux-ci sont particulièrement dignes d'intérêt puisqu'ils contribuent à l'évolution et à la pluralité des configurations familiales dans la vieillesse.

Une troisième limite tient à la «mise à l'écart » des individus âgés jugés inaptes à répondre aux questions sur le réseau familial en raison de problèmes cognitifs. Leur absence a pour conséquence la sous-représentation dans l'échantillon des "très-vieux » et des individus âgés en mauvaise santé. Une telle absence contribue à donner plus de poids aux «survivants ", à savoir aux aînés qui ont suffisamment de ressources pour répondre seuls aux questions de l'enquête malgré leur grand âge ou leur mauvaise santé. Et, ce sont ces ressources qui les distinguent des autres individus de même âge ou de mêmes conditions de santé et qui font d'eux des « survivants ». Cet effet de sélection qui conduit à la sur-représentation des « survivants » dans l'échantillon tend à atténuer l'impact négatif de l'avancée en âge et du déclin de la santé sur les résultats. L'absence de mobilisation des membres significatifs de la famille chez les «très-vieux » ou chez les personnes ayant des limites fonctionnelles pourrait s'expliquer par de tels effets. Ceci dit, questionner des personnes âgées ayant des atteintes cognitives sur leur réseau familial pose d'autres problèmes. Diverses études ont souligné la difficulté des individus ayant des atteintes 


\section{Discussion et conclusion}

cognitives à percevoir et à décrire les liens d'interdépendance qui soustendent leur réseau personnel ou familial (Cornwell, 2009a; Widmer et al., 2010). Pour l'heure, les questions sur le réseau familial, telles qu'elles sont posées dans l'enquête «VLV », ne sont pas adaptées à une population cognitivement fragile. Il serait pourtant fascinant d'en connaître un peu plus sur la façon dont les personnes âgées souffrant de problèmes cognitifs conçoivent leur famille significative et comment elles s'intègrent dans un réseau complexe de liens d'interdépendance. On ne sait pas en effet si ces personnes affichent aussi une diversité de définitions personnelles de la famille, si elles ont un accès au capital social et si elles baignent dans des relations familiales soutenantes ou grevées par les tensions. Il serait donc intéressant à l'avenir de développer un instrument de mesure qui soit approprié à des individus âgés présentant des problèmes cognitifs afin d'en connaître un peu plus sur la perception qu'ils ont de leur famille significative et sur la façon dont ils se positionnent au sein de leur réseau familial.

Une autre limite relève de l'objectif même de ce travail qui nous a amenés à centrer les analyses sur certains facteurs plutôt que sur d'autres. Notre objectif n'était pas de donner une image représentative des configurations familiales en Suisse mais plutôt de mieux comprendre les processus qui sous-tendent la construction des familles significatives dans la vieillesse et leurs conséquences en termes de capital social et d'ambivalence dans les familles d'individus âgés. Pour ce faire, nous nous sommes concentrés uniquement sur le canton de Genève et nous avons mis en exergue les effets du genre, du statut socio-économique, de l'âge et de la santé. Or, en privilégiant le canton de Genève, nous avons négligé l'impact des contextes socio-politico-économiques des cantons. Les cantons présentent des contextes variés qui sont à même d'influencer les définitions personnelles de la famille, l'organisation des liens de soutien et la gestion de l'ambivalence structurelle au sein des configurations familiales. Les effets des cantons sont multiples et complexes, pouvant se manifester sur plusieurs dimensions : socio-économique, culturelle et politique. Ils méritent dès lors une analyse approfondie, basée sur une comparaison intercantonale avec, en filigrane, une recherche poussée sur l'éventail des services de maintien à domicile proposés par chaque canton ou encore sur les mesures politiques prises à l'échelle cantonale à l'égard des personnes âgées.

Un autre facteur qui n'a pas été traité dans cet ouvrage est celui de l'immigration. L'échantillon principal sur lequel portent les analyses contient 
un tiers de personnes d'origine étrangère, naturalisées ou non. Les analyses incluant cette variable donnent peu de résultats, sans doute parce que les individus "nés étrangers » dans l'échantillon principal diffèrent peu des Suisses d'origine; nombreux d'entre eux sont en effet nés français. Il serait intéressant dans une analyse future de prendre en compte le sur-échantillon d'immigrés, espagnols et portugais, vivant à Genève et de comparer leurs réponses à celles des Suisses d'origine. On peut en effet se demander si le parcours de vie des migrants a un impact sur la façon dont ceux-ci construisent leur famille significative dans la vieillesse. Si tel est le cas, leurs configurations familiales peuvent s'avérer fort distinctes de celles des Suisses tant dans leur composition que dans le capital social et l'ambivalence qu'elles produisent.

Nous avons privilégié dans cet ouvrage l'étude des facteurs qui influencent la composition des configurations familiales, le capital social et la manière dont les aînés gèrent l'ambivalence structurelle au sein de leurs relations familiales. Cependant, nous n'avons pas considéré les effets inverses, c'est-à-dire l'impact de la composition des configurations familiales et celui des liens d'interdépendance sur la santé physique, la satisfaction de vie ou le bien-être subjectif des aînés. Il est probable que le nombre et l'identité des membres significatifs des configurations familiales et les types de capital social qu'elles génèrent s'associent à différents niveaux de santé physique, à divers degrés de satisfaction de vie ou de bien-être subjectif chez les individus âgés. De même, on peut s'interroger sur l'impact des quatre modes de gestion de l'ambivalence structurelle au niveau configurationnel, qui allient de manière distincte le soutien et le conflit, sur la santé physique et mentale des personnes âgées. Ces quatre modes ont probablement des effets très contrastés. Cet ouvrage représente, en somme, une première étape dans l'exploration des configurations familiales dans la vieillesse mais il est nécessaire de pousser plus loin l'analyse et de s'intéresser, dans de futures recherches, aux conséquences de la composition différenciée des configurations familiales, des différents types de capital social et des modes de gestion de l'ambivalence structurelle sur la santé et le bien-être des individus âgés.

Finalement, la nature transversale des données «VLV » constitue une autre limite dans ce travail de recherche. Les analyses (ANOVA, Régressions ou ACM) que nous avons effectuées révèlent des associations entre la composition des configurations familiales, le capital social, les modes de gestion de l'ambivalence structurelle et diverses variables telles que la 


\section{Discussion et conclusion}

santé. Cependant, il est difficile sur la base de ces résultats d'en déduire le sens de la causalité. Par exemple, l'association entre santé et capital social peut signifier que la santé a un impact sur le capital social ou que le capital social influence la santé. Seules des données longitudinales permettent de clarifier le sens de l'impact. Avec de telles données, il est ainsi possible de vérifier si la composition des configurations familiales, le capital social et les modes de gestion de l'ambivalence structurelle ont des effets sur la santé physique et mentale des personnes âgées. Les données longitudinales permettent aussi d'observer comment les configurations familiales évoluent au cours du temps et d'étudier l'effet de certaines transitions de vie, comme le veuvage ou l'entrée en EMS, sur la composition des configurations familiales. On peut aussi examiner les conséquences de ces diverses transitions de vie sur la production du capital social au sein des familles ainsi que sur la gestion de l'ambivalence structurelle au niveau configurationnel. De fait, les données longitudinales élargissent le champ des analyses possibles et sont plus que nécessaires pour mieux comprendre les mécanismes qui sous-tendent la construction des familles significatives dans la vieillesse et leurs effets non seulement au niveau « meso » (liens familiaux) mais aussi au niveau individuel (santé et bien-être).

Appliquer l'approche configurationnelle dans des enquêtes comparatives ou longitudinales, développer des outils de récolte des données « réseaux » plus inclusifs et combiner des données quantitatives avec des entretiens qualitatifs représentent, dans le futur, d'excellentes perspectives de recherche pour améliorer les connaissances sur les relations familiales dans la vieillesse. En changeant d'angle d'analyse, l'approche configurationnelle permet d'élargir les perspectives de recherche sur les relations et les dynamiques familiales aux dernières étapes de la vie; celles-ci ont été, jusqu'à présent, relativement peu investiguées et rarement considérées comme le centre des analyses dans les études s'appuyant sur les modèles dominants de la gérontologie sociale. 


\section{Table des figures}

Figure 6.1: Capital social selon les six configurations familiales ......309

Figure 6.2: Projection des deux premières dimensions de l'analyse des correspondances multiples après rotation ..................323

Figure 6.3: L'Ambivalence dans la configuration familiale d'Henri ..332

Figure 6.4: La Solidarité dans la configuration familiale

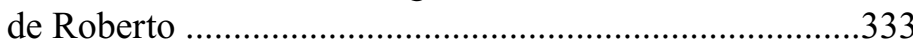

Figure 6.5: La Captivation dans la configuration familiale de Marie

Figure 6.6: L'Atomisation dans la configuration familiale de Simone 



\section{Liste des tableaux}

Tableau 4.1: $\quad$ Statistiques descriptives de l'échantillon genevois ......214 Tableau 5.1: $\quad$ Réservoir de parenté (\%) ...........................................243

Tableau 5.2: $\quad$ Nombre des membres cités comme significatifs (\%)...244

Tableau 5.3: Distribution des 14 termes familiaux les plus cités .....246

Tableau 5.4: Nombre de personnes ne citant pas un membre existant de leur famille (\%) .........................................248

Tableau 5.5: Les termes de parenté cités selon les configurations familiales

Tableau 5.6: Facteurs déterminants des configurations familiales (conjugal, fils, fille). .261

Tableau 5.7: Facteurs déterminants des configurations familiales (fratrie, parenté, isolés)

Tableau 5.8: Profils des membres significatifs cités, par configurations familiales

Tableau 6.1: Indices du capital social par configurations familiales 278

Tableau 6.2: Indices du capital social par facteurs ..........................286

Tableau 6.2: Indices du capital social par facteurs ..........................287

Tableau 6.3: Indices du capital social par réservoir de parenté ........293

Tableau 6.3: Indices du capital social par réservoir de parenté ........294

Tableau 6.4: Facteurs déterminants du capital social (taille, densité, réciprocité).....................................................298

Tableau 6.4: Facteurs déterminants du capital social (taille, densité, réciprocité).

Tableau 6.5: Facteurs déterminants du capital social (in-degree, out-degree, centralité) 300

Tableau 6.5: Facteurs déterminants du capital social (in-degree, out-degree, centralité)

Tableau 6.6: Modes de conflit et de support selon les types de configurations familiales

Tableau 6.7: Densités de support et de conflit selon les types de configurations familiales

Tableau 6.8: $\quad$ Mesures de discrimination des variables selon les deux premières dimensions après rotation 
Tableau 6.9: Coordonnées des modalités des variables sur les deux premières dimensions après rotation.............................322

Tableau 6.10: Conditions d'émergence des modes de conflit et de

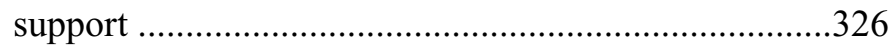

Tableau 6.11: Capital social selon les configurations familiales .........354

Tableau 6.12: Capital social selon le profil socio-démographique ......363 


\section{Références}

Abdi, H., \& Valentin, D. (2007). Multiple correspondence analysis. Encyclopedia of measurement and statistics, 2(4), 651-657.

Ajrouch, K. J., Blandon, A. Y., \& Antonucci, T. C. (2005). Social networks among men and women: The effects of age and socioeconomic status. The Journals of Gerontology, Series B : Psychological Sciences and Social Sciences, 60B(6), S311-S317.

Allan, G. (2001). Personal relationships in late modernity. Personal Relationships, 8(3), 325-339.

Allan, G. (2008). Flexibility, friendship, and family. Personal relationships, 15(1), 1-16.

Allen, K. R., Blieszner, R., \& Roberto, K. A. (2011). Perspectives on extended family and fictive kin in the later years strategies and meanings of kin reinterpretation. Journal of Family Issues, 32(9), 1156-1177.

Antonucci, T. C. (1990). Social supports and social relationships. In R. H. Binstock \& L. K. George (Eds.), Handbook of aging and the social sciences (3rd éd., pp. 205-226). San Diego, CA : Academic Press.

Antonucci, T. C. (2001). Social relations : An examination of social networks, social support, and sense of control. In J. E. Birren \& K. W. Shaie (Eds.), Handbook of the psychology of aging (pp. 427-453). San Diego, CA : Academic Press.

Antonucci, T. C., \& Akiyama, H. (1995). Convoys of social relations : Family and friendships within a life span context. In R. Blieszner \& V. H. Bedford (Eds.), Handbook of aging and the family (pp. 355371). Westport, CT : Greenwood Press.

Antonucci, T. C., Akiyama, H., \& Lansford, J. E. (1998). Negative effects of close social relations. Family Relations : An Interdisciplinary Journal of Applied Family Studies, 47(4), 379-384.

Antonucci, T. C., Akiyama, H., \& Sherman, A. M. (2007). Social network, support, and integration. In J. E. Birren (Ed.), Encyclopedia of gerontology (2nd éd., pp. 531-541). New York, NY : Elsevier. 


\section{Références}

Antonucci, T. C., \& Jackson, J. S. (1989). Successful aging and life course reciprocity. In A. Warnes (Ed.), Human aging and later life : Multidisciplinary perspectives (pp. 83-95). London, UK : Hodder \& Soughton.

Arber, S., \& Cooper, H. (1999). Gender differences in health in later life : The new paradox? Social science \& Medicine, 48(1), 61-76.

Arber, S., Davidson, K., \& Ginn, J. (2003). Changing approaches to gender and later life. In S. Arber, K. Davidson, \& J. Ginn (Eds.), Gender and ageing : Changing roles and relationships. (p. 1-14). Maidenhead, UK : Open University Press.

Armi, F., Guilley, E., \& Lalive d'Epinay, C. (2008). Entraide et recours aux services. In C. Lalive d'Épinay \& D. Spini (Eds.), Les années fragiles : La vie au-delà de quatre-vingts ans (pp. 171-208). Québec : Les Presses de Université Laval.

Ashida, S., \& Heaney, C. A. (2008). Differential associations of social support and social connectedness with structural features of social networks and the health status of older adults. Journal of Aging and Health, 20(7), 872-893.

Atkinson, A. B., Rainwater, L., \& Smeeding, T. M. (1995). Income distribution in OECD countries : Evidence from the Luxembourg income study (LIS). Paris : OECD.

Attias-Donfut, C. (1991). Générations et âges de la vie. Paris : Presses universitaires de France.

Avolio, M., Montagnoli, S., Marino, M., Basso, D., Furia, G., Ricciardi, W., \& de Belvis, A. (2013). Factors influencing quality of life for disabled and nondisabled elderly population : The results of a multiple correspondence analysis. Current Gerontology and Geriatrics Research, 2013, 1-6.

Baeriswyl, M. (2016). Participations et rôles sociaux à l'âge de la retraite: Une analyse des évolutions et enjeux autour de la participation sociale et des rapports sociaux de sexe (Thèse de doctorat non publiée). Université de Genève, Genève.

Baltes, P. B., \& Baltes, M. M. (1990). Psychological perspectives on successful aging : The model of selective optimization with compensation. In P. B. Baltes \& M. M. Baltes (Eds.), Successful aging : Perspectives from the behavioral sciences (Vol. 1, pp. 1-34). New York, NY : Cambridge University Press. 
Baltes, P. B., \& Smith, J. (2003). New frontiers in the future of aging : From successful aging of the young old to the dilemmas of the fourth age. Gerontology, 49(2), 123-135.

Barer, B. M. (1994). Men and women aging differently. The International Journal of Aging and Human Development, 38(1), 29-40.

Bax, M. (1978). Figuration analysis : A better perspective for networker; with an illustration from Ireland. Anthropological Quarterly, 51(4), 221-230.

Bengtson, V. L. (2001). Beyond the nuclear family : The increasing importance of multigenerational bonds. Journal of Marriage and Family, 63(1), 1-16.

Bengtson, V. L., Giarrusso, R., Mabry, J. . B., \& Silverstein, M. (2002). Solidarity, conflict, and ambivalence : Complementary or competing perspectives on intergenerational relationships? Journal of Marriage and Family, 64(3), 568-576.

Bengtson, V. L., \& Roberts, R. E. (1991). Intergenerational solidarity in aging families : An example of formal theory construction. Journal of Marriage and the Family, 53(4), 856-870.

Bengtson, V. L., Rosenthal, C. J., \& Burton, L. M. (1990). Families and aging : Diversity and heterogeneity. In R. H. Binstock \& L. K. George (Eds.), The handbook of aging and the social sciences (3rd éd., pp. 263-287). San Diego, CA : Academic Press.

Bengtson, V. L., Rosenthal, C. J., \& Burton, L. M. (1995). Paradoxes of families and aging. In R. H. Binstock \& L. K. George (Eds.), Handbook of aging and the social sciences (4th éd., pp. 253-282). San Diego, CA : Academic Press.

Berkman, L. F., Glass, T., Brissette, I., \& Seeman, T. E. (2000). From social integration to health : Durkheim in the new millennium. Social science \& Medicine, 51(6), 843-857.

Bickel, J.-F., \& Girardin, M. (2008). Vie familiale et relationnelle. In C. Lalive d'Epinay \& D. Spini (Eds.), Les années fragiles : La vie audelà de quatre-vingts ans (pp. 171-208). Québec : Les Presses de Université Laval.

Blau, P. M. (1964). Exchange and power in social life. New York, NY : John Wiley \& Sons.

Bolzman, C., Poncioni-Derigo, R., Vial, M., \& Fibbi, R. (2004). Older labour migrants' well being in Europe : The case of switzerland. Ageing and Society, 24(3), 411-429. 


\section{Références}

Bott, E. (1955). Urban families : Conjugal roles and social networks. Human Relations, 8(4), 345-384.

Bourdieu, P. (1980). Le capital social. Actes de la Recherche en Sciences Sociales, 31, 2-3.

Bourdieu, P. (1986). The forms of capital. In J. G. Richardson (Ed.), Handbook of theory and research for the sociology of education (pp. 241-258). New York, NY : Greenwood Press.

Bowling, A. (1994). Social networks and social support among older people and implications for emotional well-being and psychiatric morbidity. International Review of Psychiatry, 6(1), 41-58.

Braithwaite, D. O., Bach, W. B., Baxter, L. A., DiVerniero, R., Hammonds, J. R., Hosek, A. M., ... Wolf, B. M. (2010). Constructing family : A typology of voluntary kin. Journal of Social and Personal Relationships, 27(3), 388-407.

Broese Van Groenou, M. I., \& Van Tilburg, T. (2003). Network size and support in old age : Differentials by socio-economic status in childhood and adulthood. Ageing \& Society, 23(5), 625-645.

Broese Van Groenou, M. I., \& Van Tilburg, T. (2007). Network analysis. In J. E. Birren (Ed.), Encyclopedia of gerontology : Age, aging, and the aged (2nd éd., pp. 242-250). San Diego, CA : Academic Press.

Bucx, F., Van Wel, F. W., Knijn, T., \& Hagendoorn, L. (2008). Intergenerational contact and the life course status of young adult children. Journal of Marriage and Family, 70(1), 144-156.

Burt, R. (2002). The social capital of structural holes. In M. F. Guillén, R. Collins, P. England, \& M. Meyer (Eds.), The new economic sociology : Developments in an emerging field (pp. 148-189). New York, NY : Russel Sage Foundation.

Calasanti, T., \& Kiecolt, K. J. (2012). Intersectionality and aging families. In R. Blieszner \& V. H. Bedford (Eds.), Handbook on families and aging (2nd éd., pp. 263-286). Santa-Barbara, CA : Praeger / ABCCLIO.

Campbell, L. D., Connidis, I. A., \& Davies, L. (1999). Sibling ties in later life : A social network analysis. Journal of Family Issues, 20(1), 114-148.

Carr, D., \& Moorman, S. M. (2011). Social relations and aging. In R. A. Settersten \& J. L. Angel (Eds.), Handbook of sociology of aging (pp. 145-160). New York, NY : Springer. 
Carstensen, L. L. (1991). Selectivity theory : Social activity in a life-span context. In K. W. Schaie \& M. P. Lawton (Eds.), Annual review of gerontology and geriatrics (1st éd., Vol. 11, pp. 195-217). New York, NY : Springer.

Carstensen, L. L. (1992). Social and emotional patterns in adulthood : Support for socioemotional selectivity theory. Psychology and Aging, 7(3), 331-338.

Carstensen, L. L., Pasupathi, M., Mayr, U., \& Nesselroade, J. R. (2000). Emotional experience in everyday life across the adult life span. Journal of Personality and Social Psychology, 79(4), 644-655.

Castrén, A.-M. (2008). Post-divorce family configurations. In E. D. Widmer \& R. Jallinoja (Eds.), Beyond the nuclear family : Families in a configurational perspective (pp. 233-254). Bern : Peter Lang.

Castrén, A.-M. (2017). Rebuilding the family : Continuity and change in family membership and relationship closeness in post-separation situations. In V. esnuitytè, D. Lück, \& E. D. Widmer (Eds.), Family continuity and change : Contemporary european perspectives (p. 165186). London, UK : Palgrave Macmillan.

Castrén, A.-M., \& Ketokivi, K. (2015). Studying the complex dynamics of family relationships : A figurational approach. Sociological Research Online, 20(1), 108-121.

Castrén, A.-M., \& Maillochon, F. (2009). Who chooses the wedding guests, the couple or the family? Individual preferences and relational constraints in France and Finland. European Societies, 11(3), 369-389.

Cavalli, S. (2007). Modèle de parcours de vie et individualisation. Gérontologie et Société, 30(123), 55-69.

Chambers, J. M., \& Hastie, T. J. (1992). Statistical models. In J. M. Chambers \& T. J. Hastie (Eds.), Statistical models in $S$ (p. 13-44). Pacific Grove, CA : Wadsworth \& Brooks/Cole Advanced Books \& Software.

Cicirelli, V. G. (1995). Sibling relationships across the life span. New York, NY : Plenum Press.

Clarke, E. J., Preston, M., Raksin, J., \& Bengtson, V. L. (1999). Types of conflicts and tensions between older parents and adult children. The Gerontologist, 39(3), 261-270.

Coenen-Huther, J., Kellerhals, J., Von Allmen, M., Hagmann, H.-M., Jeannerat, F. C., \& Widmer, E. D. (1994). Les réseaux de solidarité dans la famille. Lausanne : Réalités sociales. 


\section{Références}

Cohen, S. (2004). Social relationships and health. American Psychologist, $59(8), 676-684$.

Coleman, J. S. (1988). Social capital in the creation of human capital. The American Journal of Sociology, 94, S95-S120.

Connidis, I. A. (2003). Divorce and union dissolution : Reverberations over three generations. Canadian Journal on Aging / La Revue Canadienne $d u$ Vieillissement, 22(4), 353-368.

Connidis, I. A. (2010). Family ties and aging (2nd éd.). Thousand Oaks, CA : Pin Forge Press / Sage.

Connidis, I. A. (2012). Theoretical directions for studying family ties and aging. In R. Blieszner \& V. H. Bedford (Eds.), Handbook on families and aging (2nd éd., pp. 35-60). Santa-Barbara, CA : Praeger / ABC-CLIO.

Connidis, I. A. (2015). Exploring ambivalence in family ties : Progress and prospects. Journal of Marriage and Family, 77(1), 77-95.

Connidis, I. A., \& McMullin, J. A. (2002). Sociological ambivalence and family ties : A critical perspective. Journal of Marriage and Family, 64(3), 558-567.

Conseil d'Etat de Genève, . (2012). Rapport du Conseil d'Etat au Grand Conseil sur la politique publique cantonale en faveur des personnes agées en perte d'autonomie (RD952). Genève : Atar Roto Presse.

Cornwell, B. (2009a). Good health and the bridging of structural holes. Social Networks, 31(1), 92-103.

Cornwell, B. (2009b). Network bridging potential in later life : Life-course experiences and social network position. Journal of Aging and Health, 21(1), 129-154.

Cornwell, B. (2011). Independence through social networks : Bridging potential among older women and men. The Journals of Gerontology, Series B : Psychological Sciences and Social Sciences, 66B(6), 782794.

Cornwell, B., Laumann, E. O., \& Schumm, L. P. (2008). The social connectedness of older adults : A national profile. American Sociological Review, 73(2), 185-203.

Cornwell, B., Schumm, L. P., Laumann, E. O., \& Graber, J. (2009). Social networks in the NSHAP study : Rationale, measurement, and preliminary findings. The Journals of Gerontology, Series B : Psychological Sciences and Social Sciences, 64B(S1), i47-i55. 
Coyne, J. C., Wortman, C. B., \& Lehman, D. R. (1988). The other side of support : Emotional overinvolvement and miscarried helping. In B. H. Gottlieb (Ed.), Marshaling social support : Formats, processes, and effects (pp. 305-330). Thousand Oaks, CA : Sage.

Cropanzano, R., \& Mitchell, M. S. (2005). Social exchange theory : An interdisciplinary review. Journal of Management, 31(6), 874-900.

D'Andrade, R. G. (1995). The development of cognitive anthropology. Cambridge, UK : Cambridge University Press.

Dannefer, D. (2003). Cumulative advantage/disadvantage and the life course : Cross-fertilizing age and social science theory. The Journals of Gerontology, Series B : Psychological Sciences and Social Sciences, $58 B(6)$, S327-S337.

De Carlo, I., Aeby, G., \& Widmer, E. (2014). La variété des configurations familiales après une recomposition : choix et contraintes. Swiss Journal of Sociology, 40(1), 9-27.

De Jong Gierveld, J., Broese van Groenou, M., Hoogendoorn, A. W., \& Smit, J. H. (2009). Quality of marriages in later life and emotional and social loneliness. The Journals of Gerontology, Series B : Psychological Sciences and Social Sciences, 64B(4), 497-506.

De Luca, G., \& Peracchi, F. (2005). Survey participation in the first wave of SHARE. In A. Börsch-Supan \& H. Jürges (Eds.), The survey of health, ageing and retirement in europe - Methodology (pp. 88-104). Mannheim : MEA.

Desbois, D. (2008). L'analyse des correspondances multiples à la "hollandaise" : Introduction à l'analyse d'homogénéité. Modulad, 38, 194-244.

Dieng, A. (2014). Capital social, configurations familiales et statut d'activité en Afrique subsaharienne: Quels liens et quelles implications économiques et sociales pour les femmes sénégalaises? (Thèse de doctorat non publiée). Université de Genève, Genève.

Ducret, A. (2011). Le concept de "configuration" et ses implications empiriques : Elias avec et contre weber. SociologieS [En ligne], La recherche en actes, Régimes d'explication en sociologie., 1-12.

Dumaret, A.-C., Coppel-Batsch, M., \& Couraud, S. (1997). Adult outcome of children reared for long-term periods in foster families. Child abuse \& Neglect, 21(10), 911-927.

Duvoux, N. (2011). Configuration. In S. Paugam (Ed.), Les 100 mots de la sociologie (pp. 52-54). Paris : Presses universitaires de France. 


\section{Références}

Dykstra, P. A., \& Hagestad, G. O. (2007). Childlessness and parenthood in two centuries : Different roads-different maps? Journal of Family Issues, 28(11), 1518-1532.

Elder, G. H. (1991). Lives and social change. In W. Heinz (Ed.), Theoretical advances in life course research (Vol. 1, pp. 58-86). Weinheim : Deutscher Studien Verlag.

Elder, G. H., \& Johnson, M. K. (2003). The life course and aging : Challenges, lessons, and new directions. In R. A. Settersten (Ed.), Invitation to the life course: Toward new understandings of later life (pp. 49-81). Amityville, NY : Baywood Publishing.

Elias, N. (1991). Qu'est-ce que la sociologie? La Tour-d'Aigues : Editions de l'Aube.

Eriksen, S., \& Gerstel, N. (2002). A labor of love or labor itself : Care work among adult brothers and sisters. Journal of Family Issues, 23(7), 836-856.

Everitt, B. S., Landau, S., Leese, M., \& Stahl, D. (2011). Cluster analysis (5th éd.). Chichester, UK : John Wiley \& Sons.

Finch, J. (2007). Displaying families. Sociology, 41(1), 65-81.

Finch, J., \& Mason, J. (1993). Negotiating family responsibilities. London, UK : Routledge.

Fingerman, K. L. (2004). The role of offspring and in-laws in grandparents'ties to their grandchildren. Journal of Family Issues, 25(8), $1026-1049$.

Fingerman, K. L., \& Birditt, K. S. (2003). Do age differences in close and problematic family ties reflect the pool of available relatives? The Journals of Gerontology, Series B : Psychological Sciences and Social Sciences, 58B(2), P80-P87.

Fingerman, K. L., Hay, E. L., \& Birditt, K. S. (2004). The best of ties, the worst of ties: Close, problematic, and ambivalent social relationships. Journal of Marriage and Family, 66(3), 792-808.

Fiori, K. L., Antonucci, T. C., \& Akiyama, H. (2008). Profiles of social relations among older adults : A cross-cultural approach. Ageing and Society, 28(2), 203-231.

Fiori, K. L., Antonucci, T. C., \& Cortina, K. S. (2006). Social network typologies and mental health among older adults. The Journals of Gerontology, Series B : Psychological Sciences and Social Sciences, $61 B(1), \mathrm{P} 25-\mathrm{P} 32$. 
Firth, R., Hubert, J., \& Forge, A. (1970). Families and their relatives : Kinship in a middle-class sector of London. An anthropological study. London, UK : Routledge.

Foa, E. B., \& Foa, U. G. (1980). Resource theory : Interpersonal behavior as exchange. In K. J. Gergen, M. S. Greenberg, \& R. H. Willis (Eds.), Social exchange: Advances in theory and research (pp. 77-94). Boston, MA : Springer.

Freeman, L. C. (2004). The development of social network analysis : A study in the sociology of science. Vancouvers, BC : Empirical press.

Freund, A. M., Li, K. Z. H., \& Baltes, P. B. (1999). Successful development and aging: The role of selection, optimization, and compensation. In J. Brandtstaedter \& R. M. Lerner (Eds.), Action \& self-development : Theory and research through the life span (Vol. 11, pp. 401-434). Thousand Oaks, CA : Sage.

Gabriel, R., Oris, M., Studer, M., \& Baeriswyl, M. (2015). The persistence of social stratification? A life course perspective on poverty in old-age in switzerland. Swiss Journal of Sociology, 41(3), 465-487.

Ganong, L. H., \& Coleman, M. (2006). Obligations to stepparents acquired in later life : Relationship quality and acuity of needs. The Journals of Gerontology, Series B : Psychological Sciences and Social Sciences, $61 B(2)$, S80-S88.

Gaugler, J. E., \& Holmes, H. H. (2003). Families' experiences of long-term care placement : Adaptation and intervention. Clinical Psychologist, 7(1), 32-43.

Giarrusso, R., Feng, D., Silverstein, M., \& Bengtson, V. L. (2001). Grandparent-adult grandchild affection and consensus : Crossgenerational and cross-ethnic comparisons. Journal of Family Issues, 22(4), 456-477.

Giudici, F., \& Widmer, E. D. (2017). Gendered occupational shifts in the transition to parenthood: The influence of personal networks. Sociology, 51(2), 429-449.

Gouldner, A. W. (1960). The norm of reciprocity : A preliminary statement. American Sociological Review, 25(2), 161-178.

Granovetter, M. S. (1973). The strength of weak ties. American Journal of Sociology, 78(6), 1360-1380. 


\section{Références}

Guilley, E., Armi, F., \& Ghisletta, P. (2008). Indépendance, fragilité et dépendance : Trois statuts de santé. In C. Lalive d'Épinay \& D. Spini (Eds.), Les années fragiles : La vie au-delà de quatre-vingts ans (pp. 101-122). Québec : Les Presses de Université Laval.

Guilley, E., Armi, F., Ghisletta, P., \& Lalive d'Epinay, C. (2008). Trajectoires de vies, transitions de santé. In C. Lalive d'Épinay \& D. Spini (Eds.), Les années fragiles: La vie au-delà de quatre-vingts ans (pp. 123-147). Québec : Les Presses de Université Laval.

Guilley, E., Guillet, L.-A., \& Girardin, M. (2008). Does the gender paradox persist in the last years of very long lives? In H. T. Benninghouse \& A. G. Rosset (Eds.), Women and aging : New research (pp. 615-625). Hauppauge, NY : Nova Science Publishers.

Guilley, E., \& Lalive d'Epinay, C. (2008). Is the final stage of a long life one of long-term ADL-dependence? In E. Guilley \& C. Lalive d'Épinay (Eds.), The closing chapters of long lives: Results from the 10-year swilsoo study on the oldest old (pp. 37-44). Hauppauge, NY : Nova Science Publishers.

Guillod, O. (2012). Droit des personnes (4th éd.). Basel : Helbing Lichtenhahn Verlag.

Ha, J.-H. (2008). Changes in support from confidants, children, and friends following widowhood. Journal of Marriage and Family, 70(2), 306318.

Haines, V. A., \& Hurlbert, J. S. (1992). Network range and health. Journal of Health and Social behavior, 33(3), 254-266.

Hanneman, R. A., \& Riddle, M. (2005). Introduction to social network methods. Riverside, CA : University of California.

Hauri, R. (2011). Christmas celebration, an annual family gathering. In R. Jallinoja \& E. D. Widmer (Eds.), Families and kinship in contemporary europe: Rules and practices of relatedness (pp. 45-62). London, UK : Palgrave Macmillan.

Heinz, W. R. (2001). Work and the life course : A cosmopolitan-local perspective. In V. W. Marshall, W. R. Heinz, H. Krüger, \& A. Verma (Eds.), Restructuring work and the life course (pp. 3-22). Toronto, Canada : University of Toronto Press.

Hillcoat-Nallétamby, S., \& Phillips, J. E. (2011). Sociological ambivalence revisited. Sociology, 45(2), 202-217. 
Hoffman, D. L., \& De Leeuw, J. (1992). Interpreting multiple correspondence analysis as a multidimensional scaling method. Marketing Letters, 3(3), 259-272.

House, J. S. (2001). Social isolation kills, but how and why? Psychosomatic Medicine, 63(2), 273-274.

Husson, F., Josse, J., Le, S., \& Mazet, J. (2014). Factominer : Multivariate exploratory data analysis and data mining with $\mathrm{r}$ [Manuel de logiciel]. http :/CRAN.R-project.org/package=FactoMineR (R package version 1.26)

Jang, Y., Haley, W. E., Small, B. J., \& Mortimer, J. A. (2002). The role of mastery and social resources in the associations between disability and depression in later life. The Gerontologist, 42(6), 807-813.

Johnson, C. L. (1988). Relationships among family members and friends in later life. In R. M. Milardo (Ed.), Families and social networks (New perspectives on family) (pp. 168-189). Thousand Oaks, CA : Sage.

Johnson, C. L., \& Troll, L. (1996). Family structure and the timing of transitions from 70 to 103 years of age. Journal of Marriage and Family, 58(1), 178-187.

Johnson, C. L., \& Troll, L. E. (1994). Constraints and facilitators to friendships in late late life. The Gerontologist, 34(1), 79-87.

Jonas, N., \& Le pape, M.-C. (2008). My own relatives or my partner's relatives? A configurational approach of kinship prioritzation. In E. D. Widmer \& R. Jallinoja (Eds.), Beyond the nuclear family : Families in a configurational perspective (pp. 157-178). Bern : Peter Lang.

Kadushin, C. (2002). The motivational foundation of social networks. Social Networks, 24(1), 77-91.

Kadushin, C. (2012). Understanding social networks : Theories, concepts, and findings. New York, NY : Oxford University Press.

Kahn, R. L., \& Antonucci, T. C. (1980). Convoys over the life course : Attachment, roles, and social support. In P. B. Baltes \& O. G. Brim (Eds.), Life-span development and behavior (Vol. 3, pp. 253-286). New York : Academic Press.

Kalmijn, M. (2003). Shared friendship networks and the life course : An analysis of survey data on married and cohabiting couples. Social Networks, 25(3), 231-249. 


\section{Références}

Karner, T. X. (1998). Professional caring : Homecare workers as fictive kin. Journal of Aging Studies, 12(1), 69-82.

Katz, S., Ford, A. B., Moskowitz, R. W., Jackson, B. A., \& Jaffe, M. W. (1963). Studies of illness in the aged. The index of ADL : A standardized measure of biological and psychosocial function. Journal of the American Medical Association, 185(12), 914-919.

Kawachi, I., \& Berkman, L. F. (2001). Social ties and mental health. Journal of Urban Health, 78(3), 458-467.

Kearns, J. N., \& Leonard, K. E. (2004). Social networks, structural interdependence, and marital quality over the transition to marriage : A prospective analysis. Journal of Family Psychology, 18(2), 383-395.

Kelley, H. H., \& Thibaut, J. W. (1978). Interpersonal relations : A theory of interdependence. New York, NY : John Wiley \& Sons.

Klein Ikkink, K., \& Van Tilburg, T. G. (1999). Broken ties : Reciprocity and other factors affecting the termination of older adults' relationships. Social Networks, 21(2), 131-146.

Kohli, M., \& Meyer, J. W. (1986). Social structure and social construction of life stages. Human Developement, 29(3), 145-149.

Krackhardt, D. (1987). Cognitive social structures. Social Networks, 9(2), $109-134$.

Krause, N. (1997a). Anticipated support, received support, and economic stress among older adults. The Journals of Gerontology, Series B : Psychological Sciences and Social Sciences, 52B(6), P284-P293.

Krause, N. (1997b). Received support, anticipated support, social class, and mortality. Research on Aging, 19(4), 387-422.

Krause, N. (2006). Social relationships in late life. In R. H. Binstock, L. K. George, S. J. Cutler, J. Hendricks, \& J. H. Schulz (Eds.), Handbook of aging and the social sciences (6th éd., pp. 181-200). Cambridge, MA : Academic Press.

Krüger, H., \& Levy, R. (2001). Linking life courses, work, and the family : Theorizing a not so visible nexus between women and men. Canadian Journal of Sociology / Cahiers Canadiens de Sociologie, 26(2), 145166.

Kuehni, M., Rosende, M., \& Schoeni, C. (2013). Maintien en emploi et inégalités de sexe. Lien Social et Politiques, 69, 197-213.

Lalive d'Epinay, C., Bickel, J.-F., Maystre, C., \& Vollenwyder, N. (2000). Vieillesses au fil du temps. 1979-1994: Une révolution tranquille. Lausanne : Réalités Sociales. 
Lalive d'Epinay, C., \& Spini, D. (Eds.). (2008). Les années fragiles : La vie au-delà de quatre-vingts ans. Québec : Les Presses de Université Laval.

Lawton, L., Silverstein, M., \& Bengtson, V. L. (1994a). Affection, social contact, and geographic distance between adult children and their parents. Journal of Marriage and Family, 56(1), 57-68.

Lawton, L., Silverstein, M., \& Bengtson, V. L. (1994b). Solidarity between generations in families. In V. L. Bengtson \& R. A. Harootyan (Eds.), Intergenerational linkages : Hidden connections in American society (1st éd., pp. 19-42). New York, NY : Springer.

Lebart, L., Morineau, A., \& Piron, M. (2002). Statistique exploratoire multidimensionnelle (3rd éd.). Paris : Dunod.

Le Bihan, B., \& Martin, C. (2008). Caring for dependent elderly parents and family configurations. In E. D. Widmer \& R. Jallinoja (Eds.), Beyond the nuclear family : Families in a configurational perspective (pp. 59-76). Bern : Peter Lang.

Lefrançois, R., Leclerc, G., Hamel, S., \& Gaulin, P. (2000). Stressful life events and psychological distress of the very old : Does social support have a moderating effect? Archives of Gerontology and Geriatrics, 31(3), 243-255.

Letiecq, B. L., Bailey, S. J., \& Dahlen, P. (2008). Ambivalence and coping among custodial grandparents. In B. Hayslip \& P. Kaminski (Eds.), Parenting the custodial grandchild: Implications for clinical practice (pp. 3-16). New York, NY : Springer.

Levin, I. (1993). Family as mapped realities. Journal of Family Issues, 14(1), 82-91.

Levin, I., \& Trost, J. (1992). Understanding the concept of family. Family Relations, 41(3), 348-351.

Levy, R. (2013). Regulating life courses : National regimes of gendered trajectories. In R. Levy \& E. D. Widmer (Eds.), Gendered life courses between standardization and individualization : A European approach applied to Switzerland (pp. 225-242). Zürich : LIT Verlag.

Levy, R., Gauthier, J.-A., \& Widmer, E. D. (2006). Entre contraintes institutionnelle et domestique : Les parcours de vie masculins et féminins en Suisse. The Canadian Journal of Sociology / Revue Canadienne de Sociologie, 31(4), 461-489. 


\section{Références}

Levy, R., Widmer, E. D., \& Kellerhals, J. (2002). Modern family or modernized family traditionalism? Master status and the gender order in Switzerland. Electronic Journal of Sociology, 6(4), 1-42.

Lewis, M. A., \& Rook, K. S. (1999). Social control in personal relationships : Impact on health behaviors and psychological distress. Health Psychology, 18(1), 63-71.

Litwak, E. (1985). Helping the elderly : The complementary roles of informal networks and formal systems. New York, NY : Guilford Press.

Litwin, H. (2001). Social network type and morale in old age. The Gerontologist, 41(4), 516-524.

Litwin, H., \& Landau, R. (2000). Social network type and social support among the old-old. Journal of Aging Studies, 14(2), 213-228.

Lowenstein, A. (2007). Solidarity-conflict and ambivalence : Testing two conceptual frameworks and their impact on quality of life for older family members. The Journals of Gerontology, Series B : Psychological Sciences and Social Sciences, 62B(2), S100-S107.

Lowenstein, A., \& Katz, R. (2010). Family and age in a global perspective. In D. Dannefer \& C. Phillipson (Eds.), The SAGE handbook of social gerontology (pp. 190-201). London, UK : Sage.

Lowenstein, A., Katz, R., \& Daatland, S. O. (2004). Filial norms and intergenerational support in Europe and Israel : A comparative perspective. In M. Silverstein \& K. W. Schaie (Eds.), Annual review of gerontology and geriatrics (1st éd., Vol. 24, pp. 200-223). New York, NY : Springer.

Lüscher, K. (2000). Ambivalence : A key concept for the study of intergenerational relations. In S. Trnka (Ed.), Family issues between gender and generations : Seminar report (pp. 11-25). Luxembourg : Office for Official Publications of the European Communities.

Lüscher, K. (2002). Intergenerational ambivalence : Further steps in theory and research. Journal of Marriage and Family, 64(3), 585-593.

Lüscher, K., \& Hoff, A. (2013). Intergenerational ambivalence : Beyond solidarity and conflict. In I. Albert \& D. Ferring (Eds.), Intergenerational relations : European perspectives in family and society (pp. 39-63). Bristol, UK : Policy Press.

Lüscher, K., \& Pillemer, K. (1998). Intergenerational ambivalence : A new approach to the study of parent-child relations in later life. Journal of Marriage and Family, 60(2), 413-425. 
Lucas, B. (2010). Politiques du care et gouvernement de la vie : Une typologie des pratiques locales en Suisse. Swiss Political Science Review, 16(1), 109-146.

Lucas, B. (2011). Trois gouvernements des démences : Les biopolitiques à l'épreuve des réseaux d'action publique locaux. Revue Internationale de Politique Comparée, 18(4), 61-75.

Ludwig, C., Cavalli, S., \& Oris, M. (2014). "Vivre/Leben/Vivere" : An interdisciplinary survey addressing progress and inequalities of aging over the past 30 years in Switzerland. Archives of Gerontology and Geriatrics, 59(2), 240-248.

Mac Rae, H. (1992). Fictive kin as a component of the social networks of older people. Research on Aging, 14(2), 226-247.

Manning, W. D., \& Brown, S. L. (2011). The demography of unions among older Americans, 1980-present : A family change approach. In R. A. Settersten \& J. L. Angel (Eds.), Handbook of sociology of aging (pp. 193-210). New York, NY : Springer.

Marcussen, K. (2005). Explaining differences in mental health between married and cohabiting individuals. Social Psychology Quarterly, 68(3), 239-257.

Marsden, P. V. (2003). Interviewer effects in measuring network size using a single name generator. Social Networks, 25(1), 1-16.

Marshall, V. W., Matthews, S. H., \& Rosenthal, C. J. (1993). The elusiveness of family life : A challenge for the sociology of aging. In G. L. Maddox \& M. P. Lawton (Eds.), Annual review of gerontology and geriatrics (1st éd., Vol. 13, pp. 39-72). New York, NY : Springer.

Marshall, V. W., \& Mueller, M. M. (2003). Theoretical roots of the lifecourse perspective. In W. R. Heinz \& V. W. Marshall (Eds.), Social dynamics of the life course (pp. 3-32). New York, NY : New York : Aldine de Gruyter.

Masotti, B. (2016). Faire l'épreuve de l'aide à domicile au grand âge: Une étude qualitative et quantitative des services formels au Tessin (Thèse de doctorat non publiée). Université de Genève, Genève.

Merluzzi, J., \& Burt, R. S. (2013). How many names are enough? Identifying network effects with the least set of listed contacts. Social Networks, 35(3), 331-337. 


\section{Références}

Merz, E.-M., Consedine, N. S., Schulze, H.-J., \& Schuengel, C. (2009). Wellbeing of adult children and ageing parents : Associations with intergenerational support and relationship quality. Ageing and Society, 29(5), 783-802.

Merz, E.-M., \& Huxhold, O. (2010). Wellbeing depends on social relationship characteristics : Comparing different types and providers of support to older adults. Ageing and Society, 30(5), 843-857.

Milardo, R. M. (2005). Generative uncle and nephew relationships. Journal of Marriage and Family, 67(5), 1226-1236.

Milardo, R. M. (2007). Conjugal roles and social networks. In G. Ritzer (Ed.), The Blackwell encyclopedia of sociology (pp. 672-674). Oxford, UK : Wiley-Blackwell.

Milardo, R. M. (2009). The forgotten kin : Aunts and uncles. Cambridge, UK : Cambridge University Press.

Moen, P. (1996). Gender, age, and the life course. In R. H. Binstock, L. K. George, V. W. Marshall, G. C. Myers, \& J. H. Schulz (Eds.), Handbook of aging and the social sciences (4th éd., pp. 171-187). San Diego, CA : Academic Press.

Moen, P. (2001). The gendered life course. In R. H. Binstock \& L. K. George (Eds.), Handbook of aging and the social sciences (5th éd., pp. 179-1996). San Diego, CA : Academic Press.

Molm, L. D., Collett, J. L., \& Schaefer, D. R. (2007). Building solidarity through generalized exchange : A theory of reciprocity. American Journal of Sociology, 113(1), 205-242.

Moren-Cross, J. L., \& Lin, N. (2006). Social networks and health. In R. H. Binstock, L. K. George, S. Cutler, J. Hendricks, \& J. Schulz (Eds.), Handbook of aging and the social sciences (6th éd., pp. 111126). Cambridge, MA : Academic Press.

Moreno, J. L. (1934). Who shall survive? A new approach to the problem of human interrelations. Washington, D.C. : Nervous and mental disease publishing.

Morgan, D. H. J. (1996). Family connections : An introduction to family studies. Cambridge, UK : Polity Press.

Mueller, M. M., Wilhelm, B., \& Elder, G. H. (2002). Variations in grandparenting. Research on Aging, 24(3), 360-388.

Murdock, G. P. (1949). Social structure. New York, NY : Macmillan. 
Nicolet, M., \& Oris, M. (2016). Mesures et capture de la vulnérabilité dans une enquête sur les conditions de vie et de santé des personnes âgées. L'expérience de VLV (Vivre-Leben-Vivere) en Suisse. In M. Oris, P. Cordazzo, G. Bellis, E. Brown, \& A. Parant (Eds.), Les populations vulnérables. Actes du XVIe colloque national de démographie, Aix-en-Provence. Bordeaux : CUDEP (Conférence Universitaire de Démographie et d'Étude des Populations).

Offer, S. (2012). The burden of reciprocity : Processes of exclusion and withdrawal from personal networks among low-income families. Current Sociology, 60(6), 788-805.

Oris, M., Guichard, E., Nicolet, M., Gabriel, R., Tholomier, A., Monnot, C., ... Joye, D. (2016). Representation of vulnerability and the elderly. A total survey error perspective on the VLV survey. In M. Oris, C. Roberts, D. Joye, \& M. Ernst Stähli (Eds.), Surveying human vulnerabilities across the life course (pp. 27-64). New York, NY : Springer.

Pahl, R., \& Spencer, L. (2004). Personal communities : Not simply families of 'fate'or 'choice'. Current Sociology, 52(2), 199-221.

Parlebas, P. (1992). Sociométrie, réseaux et communication. Paris : Presses universitaires de France.

Parrott, T. M., \& Bengtson, V. L. (1999). The effects of earlier intergenerational affection, normative expectations, and family conflict on contemporary exchanges of help and support. Research on Aging, 21(1), 73-105.

Parsons, T., \& Bales, R. F. (1956). Family socialization and interaction process. London, UK : Routledge.

Paulle, B., Van Heerikhuizen, B., \& Emirbayer, M. (2012). Elias and Bourdieu. Journal of Classical Sociology, 12(1), 69-93.

Pezzin, L. E., Pollak, R. A., \& Schone, B. S. (2008). Parental marital disruption, family type, and transfers to disabled elderly parents. The Journals of Gerontology, Series B : Psychological Sciences and Social Sciences, 63(6), S349-S358.

Pinquart, M., \& Sörensen, S. (2000). Influences of socioeconomic status, social network, and competence on subjective well-being in later life : A meta-analysis. Psychology and Aging, 15(2), 187-224. 


\section{Références}

Pinquart, M., \& Sörensen, S. (2006). Gender differences in caregiver stressors, social resources, and health : An updated meta-analysis. The Journals of Gerontology, Series B : Psychological Sciences and Social Sciences, 61B(1), P33-P45.

Pollet, T. V., Kuppens, T., \& Dunbar, R. (2006). When nieces and nephews become important : Differences between childless women and mothers in relationships with nieces and nephews. Journal of Cultural and Evolutionary Psychology, 4(2), 83-94.

Popenoe, D. (1993). American family decline, 1960-1990 : A review and appraisal. Journal of Marriage and Family, 55(3), 527-542.

Portes, A. (1998). Social capital : Its origins and applications in modern sociology. Annual Review of Sociology, 24(1), 1-24.

Pudrovska, T., Schieman, S., \& Carr, D. (2006). Strains of singlehood in later life : Do race and gender matter? The Journals of Gerontology Series B : Psychological Sciences and Social Sciences, 61B(6), S315$\mathrm{S} 322$.

Putnam, R. D. (2000). Bowling alone: The collapse and revival of American community. New York, NY : Simon and Schuster.

Pyke, K. D., \& Bengtson, V. L. (1996). Caring more or less : Individualistic and collectivist systems of family eldercare. Journal of Marriage and Family, 58(2), 379-392.

Quintaneiro, T. (2005). The concept of figuration or configuration in Norbert Elias' sociological theory. Teoria \& Sociedade, 12(1), 54-69.

R Core Team. (2015). R : A language and environment for statistical computing [Manuel de logiciel]. Vienna, Austria. https ://www.Rproject.org/

Rao, A. R., \& Bandyopadhyay, S. (1987). Measures of reciprocity in a social network. Sankhyā : The Indian Journal of Statistics, Series A (1961-2002), 49(2), 141-188.

Rappoport, A., \& Lowenstein, A. (2007). A possible innovative association between the concept of inter-generational ambivalence and the emotions of guilt and shame in care-giving. European Journal of Ageing, 4(1), 13-21.

Riley, M. W. (1983). The family in an aging society : A matrix of latent relationships. Journal of Family Issues, 4(3), 439-454. 
Riley, M. W., \& Riley, J. W. (1996). Generational relations : A future perspective. In T. K. Hareven (Ed.), Aging and generational relations : Life-course and cross-cultural perspectives (pp. 283-291). New York, NY : Aldine de Gruyter.

Roberts, R. E., \& Bengtson, V. L. (1990). Is intergenerational solidarity a unidimensional construct? A second test of a formal model. Journal of Gerontology, 45(1), S12-S20.

Robles, T. F., \& Kiecolt-Glaser, J. K. (2003). The physiology of marriage : Pathways to health. Physiology \& Behavior, 79(3), 409-416.

Rosow, I., \& Breslau, N. (1966). A Guttman health scale for the aged. Journal of Gerontology, 21(4), 556-559.

Rossi, A. S., \& Rossi, P. H. (1990). Of human bonding : Parent-child relations across the life course. Piscataway, NJ : Aldine Transaction.

Rousseaux, E.,Deville, M., \& Ritschard, G. (2014). The SPNET package : Plotting networks on maps with R. Consulté sur http ://emmanuel. rousseaux.me/rousseaux2014spnet-rencontresr3.pdf.

Ryan, A. K., \& Willits, F. K. (2007). Family ties, physical health, and psychological well-being. Journal of Aging and Health, 19(6), 907920.

Sahlins, M. D. (1965). On the sociology of primitive exchange. In M. Banton (Ed.), The relevance of models for social anthropology (pp. 139-236). New York, NY : Frederick A. Praeger.

Sapin, M., Spini, D., \& Widmer, E. D. (2007). Les parcours de vie : De l'adolescence au grand âge. Lausanne : Presses polytechniques et universitaires romandes.

Sapin, M., Widmer, E. D., \& Radulescu, C. (2008). Social isolation or relational instability? Family configurations of women at risk of child abandonment. In E. D. Widmer \& R. Jallinoja (Eds.), Beyond the nuclear family: Families in a configurational perspective (pp. 303-328). Bern : Peter Lang.

Saracco, J., Chavent, M., \& Kuentz, V. (2010). Rotation in Multiple Correspondence Analysis : A planar rotation iterative procedure. Cahiers $d u$ GREThA (Groupe de Recherche en Economie Théorique et Appliquée de l'Université de Bordeaux), 2010(4), 1-19.

Schmeeckle, M., Giarrusso, R., Feng, D., \& Bengtson, V. L. (2006). What makes someone family? Adult children's perceptions of current and former stepparents. Journal of Marriage and Family, 68(3), 595-610. 


\section{Références}

Schnettler, S., \& Wöhler, T. (2014). On the supporting role of friendship for parents and non-parents in later life. A comparative analysis using data from three waves of the German Aging Survey. In M. Löw (Ed.), Vielfalt und zusammenhalt: Verhandlungen des 36. kongresses der Deutschen Gesellschaft für Soziologie in Bochum 2012 (pp. 1-26). Frankfurt am Main, Germany : Campus Verlag.

Scott, J. P. (2000). Social network analysis : A handbook (2nd éd.). London, UK : Sage.

Shalizi, C. (2009). Distances between clustering, hierarchical clustering (Lectures notes No 8). Pittsburgh, PA : Carnegie Mellon University.

Shapiro, A., \& Cooney, T. M. (2007). Divorce and intergenerational relations across the life course. In T. J. Owens \& J. J. Suitor (Eds.), Interpersonal relations across the life course (Vol. 12, pp. 191-219). Oxford, UK : Elsevier.

Shaw, B. A., \& Janevic, M. (2004). Associations between anticipated support, physical functioning, and education level among a nationally representative sample of older adults. Journal of Aging and Health, 16(4), 539-561.

Shaw, B. A., Krause, N., Liang, J., \& Bennett, J. (2007). Tracking changes in social relations throughout late life. The Journals of Gerontology, Series B : Psychological Sciences and Social Sciences, 62B(2), S90S99.

Shor, E., Roelfs, D. J., \& Yogev, T. (2013). The strength of family ties : A meta-analysis and meta-regression of self-reported social support and mortality. Social Networks, 35(4), 626-638.

Sillars, A., Canary, D. J., \& Tafoya, M. (2004). Communication, conflict, and the quality of family relationships. In A. L. Vangelisti (Ed.), Handbook offamily communication (pp. 413-446). Mahwah, NJ : Lawrence Erlbaum Associates.

Silverstein, M., \& Bengtson, V. L. (1994). Does intergenerational social support influence the psychological well-being of older parents? The contingencies of declining health and widowhood. Social science \& Medicine, 38(7), 943-957.

Silverstein, M., \& Bengtson, V. L. (1997). Intergenerational solidarity and the structure of adult child-parent relationships in American families. American Journal of Sociology, 103(2), 429-460. 
Silverstein, M., Chen, X., \& Heller, K. (1996). Too much of a good thing? Intergenerational social support and the psychological well-being of older parents. Journal of Marriage and Family, 58(4), 970-982.

Silverstein, M., Conroy, S. J., Wang, H., Giarrusso, R., \& Bengtson, V. L. (2002). Reciprocity in parent-child relations over the adult life course. The Journals of Gerontology, Series B : Psychological Sciences and Social Sciences, 57B(1), S3-S13.

Silverstein, M., Gans, D., \& Yang, F. M. (2006). Intergenerational support to aging parents : The role of norms and needs. Journal of Family Issues, 27(8), 1068-1084.

Silverstein, M., \& Giarrusso, R. (2010). Aging and family life : A decade review. Journal of Marriage and Family, 72(5), 1039-1058.

Silverstein, M., Lawton, L., \& Bengtson, V. L. (1994). Types of relations between parents and adult children. In V. L. Bengtson \& R. A. Harootyan (Eds.), Intergenerational linkages : Hidden connections in American society (1st éd., pp. 43-76). New York, NY : Springer.

Silverstein, M., \& Marenco, A. (2001). How Americans enact the grandparent role across the family life course. Journal of Family Issues, 22(4), 493-522.

Smart, C. (2007). Personal life : New directions in sociological thinking. Cambridge, UK : Polity Press.

Song, L., Son, J., \& Lin, N. (2011). Social support. In J. Scott \& P. J. Carrington (Eds.), The SAGE handbook of social network analysis (1st éd., pp. 116-128). London, UK : Sage.

Sprey, J. (1999). Family dynamics : An essay on conflict and power. In M. B. Sussman, S. K. Steinmetz, \& G. W. Peterson (Eds.), Handbook of marriage and the family (2nd éd., pp. 667-685). New York, NY : Plenum Press.

Stevens, N. L., \& Van Tilburg, T. G. (2011). Cohort differences in having and retaining friends in personal networks in later life. Journal of Social and Personal Relationships, 28(1), 24-43.

Street, D., Burge, S., Quadagno, J., \& Barrett, A. (2007). The salience of social relationships for resident well-being in assisted living. The Journals of Gerontology, Series B : Psychological Sciences and Social Sciences, 62B(2), S129-S134. 


\section{Références}

Studer, M. (2013). Weightedcluster library manual : A practical guide to creating typologies of trajectories in the social sciences with $R$ (LIVES Working Paper No 24). Lausanne : Swiss National Center of Competence in Research LIVES.

Suanet, B., Van Tilburg, T. G., \& Broese Van Groenou, M. I. (2013). Nonkin in older adults' personal networks : More important among later cohorts? The Journals of Gerontology, Series B : Psychological Sciences and Social Sciences, 68(4), 633-643.

Suitor, J. J., Sechrist, J., Gilligan, M., \& Pillemer, K. (2011). Intergenerational relations in later-life families. In R. A. Settersten \& J. L. Angel (Eds.), Handbook of sociology of aging (pp. 161-178). New York, NY : Springer.

Suzman, R., \& Riley, M. W. (1985). Introducing the "oldest old". The Milbank Memorial Fund Quarterly. Health and Society, 63(2), 175186.

Szydlik, M. (2008). Intergenerational solidarity and conflict. Journal of Comparative Family Studies, 39(1), 97-114.

Tabachnick, B. G., \& Fidell, L. S. (1996). Using multivariate statistics (4th éd.). New York, NY : HarperCollins.

Thoits, P. A. (2011). Mechanisms linking social ties and support to physical and mental health. Journal of Health and Social Behavior, 52(2), $145-161$.

Treas, J., \& Marcum, C. S. (2011). Diversity and family relations in an aging society. In R. A. Settersten \& J. L. Angel (Eds.), Handbook of sociology of aging (pp. 131-141). New York : Springer.

Tucker, J. S. (2002). Health-related social control within older adults' relationships. The Journals of Gerontology, Series B : Psychological Sciences and Social Sciences, 57B(5), P387-P395.

Vachon, M., Beaulieu-Prévost, D., Ouellette, A., \& Achille, M. (2005). Analyse de classification hiérarchique et qualité de vie. Tutorials in Quantitative Methods for Psychology, 1(1), 25-30.

Van der Pas, S., \& Van Tilburg, T. G. (2010). The influence of family structure on the contact between older parents and their adult biological children and stepchildren in the Netherlands. The Journals of Gerontology, Series B : Psychological Sciences and Social Sciences, 65B(2), 236-245. 
Van Tilburg, T. G. (1998). Losing and gaining in old age : Changes in personal network size and social support in a four-year longitudinal study. The Journals of Gerontology, Series B : Psychological Sciences and Social Sciences, 53B(6), S313-S323.

Van Tilburg, T. G., \& Thomése, F. (2010). Societal dynamics in personal networks. In D. Dannefer \& C. Phillipson (Eds.), The SAGE handbook of social gerontology (pp. 215-225). London, UK : Sage.

Voorpostel, M. (2013). Just like family : Fictive kin relationships in the Netherlands. The Journals of Gerontology, Series B : Psychological Sciences and Social Sciences, 68(5), 816-824.

Voorpostel, M., \& Van Der Lippe, T. (2007). Support between siblings and between friends : Two worlds apart? Journal of Marriage and Family, 69(5), 1271-1282.

Waite, L., \& Das, A. (2010). Families, social life, and well-being at older ages. Demography, 47(1), S87-S109.

Walker, A. J., Pratt, C. C., \& Eddy, L. (1995). Informal caregiving to aging family members : A critical review. Family Relations, 44(4), 402-411.

Wall, K., Aboim, S., Cunha, V., \& Vasconcelos, P. (2001). Families and informal support networks in Portugal : The reproduction of inequality. Journal of European Social Policy, 11(3), 213-233.

Wall, K., Aboim, S., \& Marinho, S. (2008). Family configurations from the male perspective : Exploring diversity over the life course. In E. D. Widmer \& R. Jallinoja (Eds.), Beyond the nuclear family : Families in a configurational perspective (pp. 207-229). Bern : Peter Lang.

Wanner, P., Sauvain-Dugerdil, C., Guilley, E., \& Hussy, C. (2005). Ages et générations: La vie après 50 ans en Suisse. Neuchâtel : OFS (Office fédéral de la statistique).

Weeks, J., Heaphy, B., \& Donovan, C. (2001). Same sex intimacies : Families of choice and other life experiments (1st éd.). London, UK : Routledge.

Wenger, G. C. (1997). Social networks and the prediction of elderly people at risk. Aging \& Mental Health, 1(4), 311-320.

Widmer, E. D. (1999a). Family contexts as cognitive networks : A structural approach of family relationships. Personal Relationships, 6(4), 487503.

Widmer, E. D. (1999b). Les relations fraternelles des adolescents. Paris : Presses Universitaires de France. 


\section{Références}

Widmer, E. D. (2006). Who are my family members? Bridging and binding social capital in family configurations. Journal of Social and Personal Relationships, 23(6), 979-998.

Widmer, E. D. (2016). Family configurations : A structural approach to family diversity (2nd éd.). London, UK : Routledge.

Widmer, E. D., Aeby, G., \& Sapin, M. (2013). Collecting family network data. International Review of Sociology / Revue Internationale de Sociologie, 23(1), 27-46.

Widmer, E. D., Castrén, A.-M., Jallinoja, R., \& Ketokivi, K. (2008). Introduction. In E. D. Widmer \& R. Jallinoja (Eds.), Beyond the nuclear family : Families in a configurational perspective (pp. 1-10). Bern : Peter Lang.

Widmer, E. D., Giudici, F., Le Goff, J.-M., \& Pollien, A. (2009). From support to control : A configurational perspective on conjugal quality. Journal of marriage and family, 71(3), 437-448.

Widmer, E. D., \& Jallinoja, R. (2008). Beyond the nuclear family : Families in a configurational perspective. Bern : Peter Lang.

Widmer, E. D., Kellerhals, J., \& Levy, R. (2004). Types of conjugal networks, conjugal conflict and conjugal quality. European Sociological Review, 20(1), 63-77.

Widmer, E. D., Kempf-Constantin, N., \& Carminati, G. G. (2010). Exploring perceptions of family relationships by individuals with intellectual disability and psychiatric disorders. Families in Society : The Journal of Contemporary Social Services, 91(4), 378-384.

Widmer, E. D., \& La Farga, L.-A. (2000). Family networks : A sociometric method to study relationships in families. Field Methods, 12(2), 108128.

Widmer, E. D., Le Goff, J.-M., Levy, R., Hammer, R., \& Kellerhals, J. (2006). Embedded parenting? The influence of conjugal networks on parent-child relationships. Journal of Social and Personal Relationships, 23(3), 387-406.

Widmer, E. D., \& Ritschard, G. (2009). The de-standardization of the life course : Are men and women equal? Advances in Life Course Research, 14(1), 28-39. 
Widmer, E. D., \& Sapin, M. (2008). Families on the move. Insights on family configurations of individuals undergoing psychotherapy. In E. D. Widmer \& R. Jallinoja (Eds.), Beyond the nuclear family : $\mathrm{Fa}$ milies in a configurational perspective (pp. 279-302). Bern : Peter Lang.

Willson, A. E., Shuey, K. M., Elder, G. H., \& Wickrama, K. A. S. (2006). Ambivalence in mother-adult child relations : A dyadic analysis. Social Psychology Quarterly, 69(3), 235-252. 
